\title{
Evaluation of the Initial Isothermal Physics Measurements at the Fast Flux Test Facility, a Prototypic Liquid Metal Fast Breeder Reactor
}

John D. Bess

Sam Bays

Richard M. Lell

Richard D. McKnight

James A. Morman

David W. Wootan

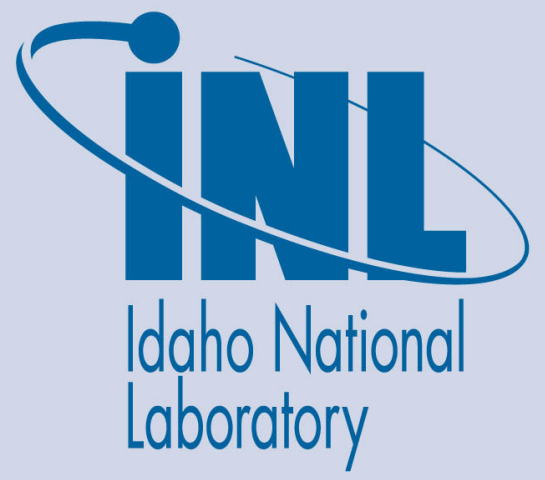

March 2010

The INL is a U.S. Department of Energy National Laboratory operated by Battelle Energy Alliance 
INL/EXT-09-16524

\section{Evaluation of the Initial Isothermal Physics Measurements at the Fast Flux Test Facility, a Prototypic Liquid Metal Fast Breeder Reactor}

${ }^{1}$ Argonne National Laboratory

${ }^{2}$ Pacific Northwest National Laboratory

John D. Bess

Sam Bays

Richard M. Lell ${ }^{1}$

Richard D. McKnight ${ }^{1}$

James A. Morman ${ }^{1}$

David W. Wootan ${ }^{2}$

March 2010

Idaho National Laboratory

Idaho Falls, Idaho 83415

http://www.inl.gov

Prepared for the

U.S. Department of Energy

Office of Nuclear Energy

Under DOE Idaho Operations Office

Contract DE-AC07-05ID14517 


\title{
EVALUATION OF THE INITIAL ISOTHERMAL PHYSICS MEASUREMENTS AT THE FAST FLUX TEST FACILITY, A PROTOTYPIC LIQUID METAL FAST BREEDER REACTOR
}

\author{
Evaluator \\ John D. Bess \\ Idaho National Laboratory \\ Internal Reviewer \\ Sam Bays
}

Independent Reviewers

Richard M. Lell

Richard D. McKnight

James A. Morman

Argonne National Laboratory

David W. Wootan

Pacific Northwest National Laboratory 
NEA/NSC/DOC(2006)1

Liquid Metal Fast Reactor - LMFR

FFTF-LMFR-RESR-001

CRIT-SPEC-REAC-COEF-MISC

Status of Compilation / Evaluation / Peer Review

\begin{tabular}{|c|c|c|c|c|c|}
\hline \multicolumn{2}{|r|}{ Section 1} & \multirow{2}{*}{$\begin{array}{c}\text { Compiled } \\
\text { YES } \\
\end{array}$} & \multirow{2}{*}{$\begin{array}{c}\begin{array}{c}\text { Independent } \\
\text { Review }\end{array} \\
\text { YES }\end{array}$} & \multirow{2}{*}{$\begin{array}{c}\begin{array}{c}\text { Working Group } \\
\text { Review }\end{array} \\
\text { YES }\end{array}$} & \multirow{2}{*}{$\begin{array}{c}\text { Approved } \\
\text { YES }\end{array}$} \\
\hline 1.0 & DETAILED DESCRIPTION & & & & \\
\hline 1.1 & $\begin{array}{l}\text { Description of the Critical and / or } \\
\text { Subcritical Configuration }\end{array}$ & YES & YES & YES & YES \\
\hline 1.2 & $\begin{array}{l}\text { Description of Buckling and } \\
\text { Extrapolation Length Measurements }\end{array}$ & $\mathrm{N} / \mathrm{A}$ & N/A & $\mathrm{N} / \mathrm{A}$ & N/A \\
\hline 1.3 & $\begin{array}{l}\text { Description of Spectral Characteristics } \\
\text { Measurements }\end{array}$ & YES & YES & YES & YES \\
\hline 1.4 & $\begin{array}{l}\text { Description of Reactivity Effects } \\
\text { Measurements }\end{array}$ & YES & YES & YES & YES \\
\hline 1.5 & $\begin{array}{l}\text { Description of Reactivity Coefficient } \\
\text { Measurements }\end{array}$ & YES & YES & YES & YES \\
\hline 1.6 & Description of Kinetics Measurements & N/A & N/A & N/A & N/A \\
\hline 1.7 & $\begin{array}{l}\text { Description of Reaction-Rate } \\
\text { Distribution Measurements }\end{array}$ & N/A & N/A & N/A & N/A \\
\hline 1.8 & $\begin{array}{l}\text { Description of Power Distribution } \\
\text { Measurements }\end{array}$ & N/A & N/A & N/A & N/A \\
\hline 1.9 & Description of Isotopic Measurements & $\mathrm{N} / \mathrm{A}$ & $\mathrm{N} / \mathrm{A}$ & N/A & N/A \\
\hline 1.10 & $\begin{array}{l}\text { Description of Other Miscellaneous } \\
\text { Types of Measurements }\end{array}$ & YES & YES & YES & YES \\
\hline & Section 2 & Evaluated & $\begin{array}{c}\text { Independent } \\
\text { Review }\end{array}$ & $\begin{array}{l}\text { Working Group } \\
\text { Review }\end{array}$ & Approved \\
\hline & $\begin{array}{l}\text { EVALUATION OF EXPERIMENTAL } \\
\text { DATA }\end{array}$ & YES & YES & YES & YES \\
\hline 2.1 & $\begin{array}{l}\text { Evaluation of Critical and / or Subcritical } \\
\text { Configuration Data }\end{array}$ & YES & YES & YES & YES \\
\hline 2.2 & $\begin{array}{l}\text { Evaluation of Buckling and Extrapolation } \\
\text { Length Data }\end{array}$ & N/A & N/A & N/A & N/A \\
\hline 2.3 & $\begin{array}{l}\text { Evaluation of Spectral Characteristics } \\
\text { Data }\end{array}$ & YES & YES & YES & YES \\
\hline 2.4 & Evaluation of Reactivity Effects Data & YES & YES & YES & YES \\
\hline 2.5 & Evaluation of Reactivity Coefficient Data & YES & YES & YES & YES \\
\hline & $\begin{array}{l}\text { Evaluation of Kinetics Measurements } \\
\text { Data }\end{array}$ & N/A & N/A & N/A & N/A \\
\hline & $\begin{array}{l}\text { Evaluation of Reaction Rate } \\
\text { Distributions }\end{array}$ & N/A & N/A & N/A & N/A \\
\hline 2.8 & Evaluation of Power Distribution Data & N/A & $\mathrm{N} / \mathrm{A}$ & N/A & N/A \\
\hline 2.9 & Evaluation of Isotopic Measurements & N/A & N/A & N/A & N/A \\
\hline 2.10 & $\begin{array}{l}\text { Evaluation of Other Miscellaneous Types } \\
\text { of Measurements }\end{array}$ & YES & YES & YES & YES \\
\hline
\end{tabular}


NEA/NSC/DOC(2006)1

Liquid Metal Fast Reactor - LMFR

FFTF-LMFR-RESR-001

CRIT-SPEC-REAC-COEF-MISC

\begin{tabular}{|c|c|c|c|c|c|}
\hline \multicolumn{2}{|r|}{ Section 3} & \multirow{2}{*}{$\begin{array}{c}\text { Compiled } \\
\text { YES }\end{array}$} & \multirow{2}{*}{$\begin{array}{c}\text { Independent Review } \\
\text { YES }\end{array}$} & \multirow{2}{*}{$\begin{array}{c}\begin{array}{c}\text { Working } \\
\text { Group Review }\end{array} \\
\text { YES }\end{array}$} & \multirow{2}{*}{$\begin{array}{c}\text { Approved } \\
\text { YES }\end{array}$} \\
\hline 3.0 & BENCHMARK SPECIFICATIONS & & & & \\
\hline 3.1 & $\begin{array}{l}\text { Benchmark-Model Specifications for } \\
\text { Critical and / or Subcritical } \\
\text { Measurements }\end{array}$ & YES & YES & YES & YES \\
\hline 3.2 & $\begin{array}{l}\text { Benchmark-Model Specifications for } \\
\text { Buckling and Extrapolation Length } \\
\text { Measurements }\end{array}$ & N/A & N/A & N/A & N/A \\
\hline 3.3 & $\begin{array}{l}\text { Benchmark-Model Specifications for } \\
\text { Spectral Characteristics Measurements }\end{array}$ & YES & YES & YES & YES \\
\hline 3.4 & $\begin{array}{l}\text { Benchmark-Model Specifications for } \\
\text { Reactivity Effects Measurements }\end{array}$ & YES & YES & YES & YES \\
\hline 3.5 & $\begin{array}{l}\text { Benchmark-Model Specifications for } \\
\text { Reactivity Coefficient Measurements }\end{array}$ & YES & YES & YES & YES \\
\hline 3.6 & $\begin{array}{l}\text { Benchmark-Model Specifications for } \\
\text { Kinetics Measurements }\end{array}$ & N/A & N/A & N/A & N/A \\
\hline 3.7 & $\begin{array}{l}\text { Benchmark-Model Specifications for } \\
\text { Reaction-Rate Distribution } \\
\text { Measurements }\end{array}$ & N/A & N/A & $\mathrm{N} / \mathrm{A}$ & N/A \\
\hline 3.8 & $\begin{array}{l}\text { Benchmark-Model Specifications for } \\
\text { Power Distribution Measurements }\end{array}$ & N/A & N/A & N/A & N/A \\
\hline 3.9 & $\begin{array}{l}\text { Benchmark-Model Specifications for } \\
\text { Isotopic Measurements }\end{array}$ & N/A & N/A & N/A & N/A \\
\hline 3.10 & $\begin{array}{l}\text { Benchmark-Model Specifications of } \\
\text { Other Miscellaneous Types of } \\
\text { Measurements }\end{array}$ & YES & YES & YES & YES \\
\hline & Section 4 & Compiled & Independent Review & $\begin{array}{c}\text { Working } \\
\text { Group Review }\end{array}$ & Approved \\
\hline 4.0 & $\begin{array}{l}\text { RESULTS OF SAMPLE } \\
\text { CALCULATIONS }\end{array}$ & YES & YES & YES & YES \\
\hline 4.1 & $\begin{array}{l}\text { Results of Calculations of the Critical or } \\
\text { Subcritical Configurations }\end{array}$ & YES & YES & YES & YES \\
\hline 4.2 & $\begin{array}{l}\text { Results of Buckling and Extrapolation } \\
\text { Length Calculations }\end{array}$ & N/A & N/A & N/A & N/A \\
\hline 4.3 & $\begin{array}{l}\text { Results of Spectral Characteristics } \\
\text { Calculations }\end{array}$ & YES & YES & YES & YES \\
\hline 4.4 & Results of Reactivity Effect Calculations & YES & YES & YES & YES \\
\hline 4.5 & $\begin{array}{l}\text { Results of Reactivity Coefficient } \\
\text { Calculations }\end{array}$ & YES & YES & YES & YES \\
\hline 4.6 & $\begin{array}{l}\text { Results of Kinetics Parameter } \\
\text { Calculations }\end{array}$ & N/A & N/A & N/A & N/A \\
\hline 4.7 & $\begin{array}{l}\text { Results of Reaction-Rate Distribution } \\
\text { Calculations }\end{array}$ & N/A & N/A & N/A & N/A \\
\hline 4.8 & $\begin{array}{l}\text { Results of Power Distribution } \\
\text { Calculations }\end{array}$ & $\mathrm{N} / \mathrm{A}$ & N/A & N/A & N/A \\
\hline 4.9 & Results of Isotopic Calculations & $\mathrm{N} / \mathrm{A}$ & $\mathrm{N} / \mathrm{A}$ & $\mathrm{N} / \mathrm{A}$ & $\mathrm{N} / \mathrm{A}$ \\
\hline 4.10 & $\begin{array}{l}\text { Results of Calculations of Other } \\
\text { Miscellaneous Types of Measurements }\end{array}$ & YES & YES & YES & YES \\
\hline & Section 5 & Compiled & Independent Review & Working Group Review & Approved \\
\hline 5.0 & REFERENCES & YES & YES & YES & YES \\
\hline $\begin{array}{l}\text { Apper } \\
\text { and T }\end{array}$ & $\begin{array}{l}\text { endix A: Computer Codes, Cross Sections, } \\
\text { Typical Input Listings }\end{array}$ & YES & YES & YES & YES \\
\hline
\end{tabular}


NEA/NSC/DOC(2006)1

Liquid Metal Fast Reactor - LMFR

FFTF-LMFR-RESR-001

CRIT-SPEC-REAC-COEF-MISC

\title{
EVALUATION OF THE INITIAL ISOTHERMAL PHYSICS MEASUREMENTS AT THE FAST FLUX TEST FACILITY, A PROTOTYPIC LIQUID METAL FAST BREEDER REACTOR
}

\author{
IDENTIFICATION NUMBER: FFTF-LMFR-RESR-001 \\ CRIT-SPEC-REAC-COEF-MISC
}

KEY WORDS: fast reactor, HEX-Z partially homogenized, Inconel 600 reflected, isothermal, sodium coolant, uranium-plutonium mixed oxide (MOX) fuel

\section{SUMMARY INFORMATION}

\subsection{DETAILED DESCRIPTION}

The Fast Flux Test Facility (FFTF) was a 400-MWt, sodium-cooled, low-pressure, high-temperature, fast-neutron flux, nuclear fission reactor plant designed for the irradiation testing of nuclear reactor fuels and materials for the development of liquid metal fast breeder reactors (LMFBRs). The Fast Test Reactor (FTR) was fueled with plutonium-uranium mixed oxide (MOX) and reflected by Inconel 600. Westinghouse Hanford Company operated the FFTF as part of the Hanford Engineering Development Laboratory (HEDL) for the U.S. Department of Energy on the Hanford Site near Richland, Washington. Although the FFTF was a testing facility not specifically designed to breed fuel or produce electricity, it did provide valuable information for LMFBR projects and base technology programs in the areas of plant system and component design, component fabrication, prototype testing, and site construction (Ref. 5, pp. 1-3 and 1-4). The major objectives of the FFTF were to provide a strong, disciplined engineering base for the LMFBR program, provide fast flux testing for other U.S. programs, and contribute to the development of a viable self-sustaining competitive U.S. LMFBR industry. ${ }^{\text {a }}$ During its ten years of operation, the FFTF acted as a national research facility to test advanced nuclear fuels, materials, components, systems, nuclear power plant operating and maintenance procedures, and active and passive reactor safety technologies; it also produced a large number of isotopes for medical and industrial users, tested materials for the U.S. fusion research program, and participated in cooperative, international research work. ${ }^{\mathrm{b}}$

Experimental and analytical methods for assessing the initial FFTF configuration were developed using the full-scale Engineering Mockup Critical experimental program in the Zero Power Reactor (ZPR-9) facility at Argonne National Laboratory. ${ }^{\text {cd }}$

Prior to the implementation of the reactor characterization program, a series of isothermal physics measurements were performed; this acceptance testing program consisted of a series of measurements of control rod worths, critical rod positions, subcriticality, maximum reactivity addition rates, shutdown margins, excess reactivity, and isothermal temperature coefficient (Ref. 2, p. 16). The results of these experiments were of particular importance because they provided extensive information which can be

\footnotetext{
a United States Atomic Energy Commission, "Environmental Statement: Fast Flux Test Facility, Richland, Washington," WASH-1510, (May 1972).

${ }^{\mathrm{b}}$ Department of Energy Richland Operations Office, Fast Flux Test Facility, http://www.hanford.gov/RL/?page=304\&parent=0, Last Updated 09/14/2005, Accessed 07/16/2009.
}

${ }^{\text {c }}$ P. A. Ombrellaro, R. A. Bennett, J. W. Daughtry, K. D. Dobbin, R. A. Harris, J. W. Nelson, R. E. Peterson, and R. B. Rothrock, "Biases for Current FFTF Calculational Methods," HEDL-SA-1393 (CONF-780401-9), Proc. Advances in Reactor Physics, Gatlinburg, TN, April 9 (January 1978).

${ }^{d}$ R. A. Bennett, J. W. Daughtry, R. A. Harris, D. H. Jones, J. W. Nelson, J. A. Rawlins, R. B. Rothrock, R. A. Sevenich, and B. D. Zimmerman, "Nuclear Startup, Testing and Core Management of the FTR," HEDL-SA-1729 (CONF-790933-3), Proc. IAEA International Symposium on Fast Reactor Physics, Aix-en-Provence, France, September 24-28 (1979).

Revision: 0

Page 1 of 304

Date: March 31, 2010 
NEA/NSC/DOC(2006)1

\section{Liquid Metal Fast Reactor - LMFR}

FFTF-LMFR-RESR-001

CRIT-SPEC-REAC-COEF-MISC

directly applied to the design of large LMFBRs (Ref. 4, p. 1). It should be recognized that the data presented in the initial report (Ref. 1) were evaluated only to the extent necessary to ensure that adequate data were obtained. Later reports provided further interpretation and detailed comparisons with prediction techniques. The conclusion of the isothermal physics measurements was that the FFTF nuclear characteristics were within design specifications and all safety requirements were satisfied. From a neutronic point of view, the FFTF was qualified to proceed into power operation mode (Ref. 1, p. 2). A listing of the activities performed for the initial isothermal physics tests is provided in Table 1.1.1.

A reactor characterization program was then implemented to provide accurate neutronic characterization in the FFTF and measure a variety of neutron-induced reaction rates that were important to fuels and materials testing, as well as reactor operation. This program provided high quality neutronic, gamma ray, and thermal hydraulic data that could be used as a basis for adjustment of calculational tools used for FFTF analysis. This program also provided benchmark data for the U.S. LMFBR Programs (Ref. 4, p. 1). The program consisted of three phases prior to the initiation of routine operation. The first phase consisted of low power measurements of fission rates and spectra by active and passive techniques in a controlled temperature environment. The second phase consisted of the irradiation of passive sensors in fuel-pin cladding contained within reactor characterization assemblies that were designed to resemble normal reactor components. The third phase entailed a full-power irradiation of passive sensors in characterizers for eight, full-power days. ${ }^{\text {a }}$

The FFTF was completed in 1978 and first achieved criticality on February 9, 1980. Upon completion of the isothermal physics and reactor characterization programs, the FFTF operated for ten years from April 1982 to April 1992. Reactor operations of the FFTF were terminated and the reactor facility was then defueled, deactivated, and placed into cold standby condition. Deactivation of the reactor was put on hold from 1996 to 2000 while the U.S. Department of Energy examined alternative uses for the FFTF but then announced the permanent deactivation of the FFTF in December 2001. Its core support basket was later drilled in May 2005, so as to remove all of the remaining sodium coolant. ${ }^{b}$ On April 17, 2006, the American Nuclear Society designated the FFTF as a "National Nuclear Historic Landmark".

A more detailed description of the operational highlights and merits of the FFTF may be read elsewhere. ${ }^{\mathrm{d}}$

Many reactor facilities implement the same name for both the reactor core and facility. The term FFTF originally referred to the entire reactor plant with the term FTR referring to just the reactor core and other components within the reactor vessel. By the time operations started, the term FFTF was almost universally applied to both the reactor and plant. ${ }^{\mathrm{e}}$ The distinction of one term over the other is not significant, therefore the term FFTF will be used throughout this report.

a J. A. Rawlins, "FFTF Reactor Characterization Program: Neutronic Experiments," TC-1904 Part I, (April 1981).

${ }^{\mathrm{b}}$ M. S. Gerber, On the Home Front: The Cold War Legacy of the Hanford Nuclear Site, University of Nebraska Press, (2007).

c American Nuclear Society News Release, "Nuclear Landmark Status,” http://www.ans.org/pi/media/releases/r-1145465480, (April 19, 2006).

d D. B. Klos, K. Greenwell, and D. L. Nielsen, "Fast Flux Test Facility - A History of Safety and Operational Excellence," FFTF-20083, Fluor Hanford (February 2004).

e Personal Communication with David W. Wootan at Pacific Northwest National Laboratory (December 16, 2009).

Revision: 0

Page 2 of 304

Date: March 31, 2010 
NEA/NSC/DOC(2006)1

Liquid Metal Fast Reactor - LMFR

FFTF-LMFR-RESR-001

CRIT-SPEC-REAC-COEF-MISC

Table 1.1.1. FFTF Testing Sequence (Ref. 7).

\begin{tabular}{|c|c|}
\hline Testing & Date \\
\hline First Fuel Assembly Loaded & November 27, 1979 \\
\hline Trisector \#3 Loading Complete & December 23, 1979 \\
\hline Trisector \#1 Loading Complete & January 26, 1980 \\
\hline Trisector \#2 Loaded for Criticality (59 Assemblies) & February 3, 1980 \\
\hline Initial Criticality & February 9, 1980 \\
\hline Full Core Loadout Complete & February 19,1980 \\
\hline $\begin{array}{l}\text { Core Characterization } \\
\text { - Gamma Energy Spectrum (100 keV }-5 \mathrm{MeV}) \\
\text { - Neutron Energy Spectrum (Proton Recoil Chambers) } \\
\text { - } \text { Neutron Energy Spectrum (Proton Recoil Emulsions) } \\
\text { Gamma Ray Energy Spectrum (100 keV to } 9 \mathrm{MeV})\end{array}$ & February 29,1980 \\
\hline $\begin{array}{l}\text { Shutdown Reactivity Monitoring } \\
\text { - Modified Source Multiplication (MSM) Technique } \\
\text { - Inverse Kinetic Rod Drops }\end{array}$ & March 2, 1980 \\
\hline Primary and Secondary Rod System Worths & March 3, 1980 \\
\hline Individual Rod Worths & March 5, 1980 \\
\hline Primary and Secondary System Shutdown Margins & March 6, 1980 \\
\hline Maximum Reactivity Addition Rate & March 6, 1980 \\
\hline Excess Reactivity & March 6, 1980 \\
\hline Temperature Coefficient of Reactivity & March 7, 1980 \\
\hline Flow Induced Reactivity Effect Measurement & March 14, 1980 \\
\hline
\end{tabular}

\subsection{Description of the Critical and / or Subcritical Configuration}

\subsubsection{Overview of Experiment}

The FFTF was operated by Westinghouse Hanford Company for the U.S. Department of Energy. During the period from November 27, 1979 to March 8, 1980, fuel was loaded into the FFTF core, initial criticality was achieved, and several subcritical physics measurements were performed (Ref. 1, p. 1).

At 3:45 pm on February 9, 1980, the first self-sustaining nuclear chain reaction occurred in the reactor core of the FFTF. Fifty-nine fuel assemblies were installed in the core with the three primary safety rods fully withdrawn to a height of 36.5 inches $(92.71 \mathrm{~cm})^{\text {a }}$ and the six secondary control rods banked at a nominal height of 31.3 inches $(79.502 \mathrm{~cm})$ (Ref. 1, p. 2).

On March 8, 1980, at 8:13 am, the reactor achieved criticality for the first time after initial full-core loading. The three primary rods had been fully withdrawn to a height of 36.5 inches $(92.71 \mathrm{~cm})$, and the secondary rods were banked at a height of 14 inches $(35.56 \mathrm{~cm})$. Rod 4 was then pulled to a height of

\footnotetext{
${ }^{a}$ Retention of additional digits in unit conversion does not imply additional precision. See first paragraph in Section 1.1.2.
} 
NEA/NSC/DOC(2006)1

Liquid Metal Fast Reactor - LMFR

FFTF-LMFR-RESR-001

CRIT-SPEC-REAC-COEF-MISC

15.4 inches $(39.116 \mathrm{~cm})$ to achieve criticality, which would have occurred for an extrapolated critical rod bank height of 14.1 inches $(35.814 \mathrm{~cm})$. Seventy-three fuel assemblies were in the core (Ref. 1, pp. 4446).

From March 1 to 7, 1980, several near-critical zero power core physics measurements were performed, including individual rod worths, total worths of the primary and secondary control rod systems, the maximum reactivity addition rate, primary and secondary systems shutdown margins, excess reactivity, and isothermal temperature coefficient of reactivity. To measure differential worths of each secondary control rod, the reactor was taken critical several times during the period of March 8 to 10. On March 11, the reactor was taken to a fission power level of approximately $10 \mathrm{~kW}$ to characterize ex-vessel flux monitor response with various neutron spectrum modifiers. The reactor was taken critical several times to powers less than $1 \mathrm{~kW}$ between March 11 to 15 to gain operator training and experience before terminating the initial isothermal testing (Ref. 2, p. 16).

The critical reactor physics benchmark evaluated in this section pertains to the data available for the initial isothermal fully-loaded core configuration on March 8, 1980 (Ref. 1 and 2). The total uncertainty in the benchmark eigenvalue of 0.9993 is \pm 0.0021 .

The initial isothermal partially-loaded core critical configuration on February 9, 1980 (Ref. 1 and 2) has not been evaluated. Seven subcritical configurations, created during core reactivity measurements, are reported in Reference 3 and 4, but they have not yet been evaluated.

\subsubsection{Geometry of the Experiment Configuration and Measurement Procedure}

The dimensions obtained from most of the references were reported in feet and inches. Where referenced in this report, the original dimensions are then followed by their converted values in units of meters or centimeters, in parentheses. The number of decimal places in the converted units does not imply additional precision, but preservation of the original measurement. Much of the information in Section 1 of this report was taken as directly from the references as possible, with some modification to either reduce the volume of information or provide further clarification of the data.

Information is preserved in Appendix E regarding the general FFTF facility and experimental measurements not assessed in this benchmark evaluation such as initial fuel loading, partially-loaded core critical, and subcritical configurations.

\section{Reactor Core}

The 400-MW reactor core was comprised of a vertical array of 199 replaceable hexagonal core assemblies. While the general design of the core assemblies was similar, the handling sockets and nozzles differed, so as to assure proper positioning of the various types of assemblies within the core. The fast neutron flux was provided by the $\mathrm{PuO}_{2}-\mathrm{UO}_{2}$ driver fuel assemblies and the reactor was controlled by nine $\mathrm{B}_{4} \mathrm{C}$ control rod absorber assemblies. A variable number of fixed shim absorber assemblies may also be used in the core during a particular fuel cycle. Inconel reflectors were utilized to reduce neutron leakage from the fueled portions of the core. The core accommodated up to eight independently instrumented test assemblies that were used for testing either fissioning materials such as advanced fuels or nonfissioning materials. The core assemblies were typically $12 \mathrm{ft}(3.6576 \mathrm{~m})$ long (Ref. 5, pp. 3-3 and 3-9).

The inlet receptacles in the core support plate and spacer pads at two elevations along the hexagonal ducts maintained proper spacing of the assemblies. The radial spacing of the core components was controlled at the elevation of the inlet nozzles by the nominal 4.730 -inch $(12.0142 \mathrm{~cm})$ room temperature hexagonal spacing of the inlet receptacles in the core support structure; the hexagonal pitch was 12.051 


\section{Liquid Metal Fast Reactor - LMFR \\ FFTF-LMFR-RESR-001 \\ CRIT-SPEC-REAC-COEF-MISC}

$\mathrm{cm}$ at $400^{\circ} \mathrm{F}\left(\sim 204^{\circ} \mathrm{C}\right)$, the temperature at which the isothermal physics measurements were performed (Ref. 3, pp. 1-2).

The FFTF was an irradiation test reactor; therefore the composition and arrangement of the core was changed subject to the requirements for varying testing procedures. The typical core arrangement is described in Table 1.1.2 and Figure 1.1.1. A more detailed map of the FFTF core design is shown in Figure 1.1.2. The core was arranged in the form of concentric hexagonal rings surrounding a central assembly position. The central assembly is known as "Row 1" with subsequent rows numbered from "Row 2" up to "Row 9." The first four rows were known as the "inner enrichment zone" and Rows 5 and 6 were known as the "outer enrichment zone." These first six rows constituted the active fueled region of the core, which was $3 \mathrm{ft}(91.44 \mathrm{~cm})$ in axial length and had an equivalent diameter of 47.2 in $(1.19888 \mathrm{~m})$. These first six rows also contained nine control rod assemblies and up to eight instrumented test assemblies. Three rows of assemblies surrounded the active core. Rows 7 through 9 contained the reflectors and fixed shim absorber assemblies. These rows were then surrounded by the segmented radial shielding, core restraints, and the core barrel (Ref. 5, pp. 3-10 through 3-12).

Table 1.1.2. Typical Core Arrangement (Ref. 5, p. 3-10).

\begin{tabular}{||c|cccccccccc||}
\hline Type of Assembly & \multicolumn{11}{|c||}{ Row } \\
\hline \hline & 1 & 2 & 3 & 4 & 5 & 6 & 7 & 8 & 9 & Total \\
\cline { 2 - 11 } & 1 & 4 & 9 & 15 & 18 & 27 & -- & -- & -- & 74 \\
Driver Fuel Positions & & & 3 & & & & & & & 3 \\
Primary Control Rods & & & & & & & & & & 6 \\
Secondary Control Rods & & & & & 6 & & & & & 9 \\
Potential Control Rod Positions & & & & -- & & & 9 & -- & -- & 9 \\
Fixed Shim Absorber Positions & & & & & & & 6 & -- & -- & 6 \\
Reflector Positions & & & & & & & & & \\
Open Test Positions & -- & 1 & -- & 1 & -- & 2 & -- & -- & -- & 4 \\
Closed Loop Positions & -- & 1 & -- & 2 & -- & 1 & -- & -- & -- & 4 \\
\hline Total & 1 & 6 & 12 & 18 & 24 & 30 & 36 & 42 & 30 & 199 \\
\hline \hline
\end{tabular}


NEA/NSC/DOC(2006)1

Liquid Metal Fast Reactor - LMFR

FFTF-LMFR-RESR-001

CRIT-SPEC-REAC-COEF-MISC

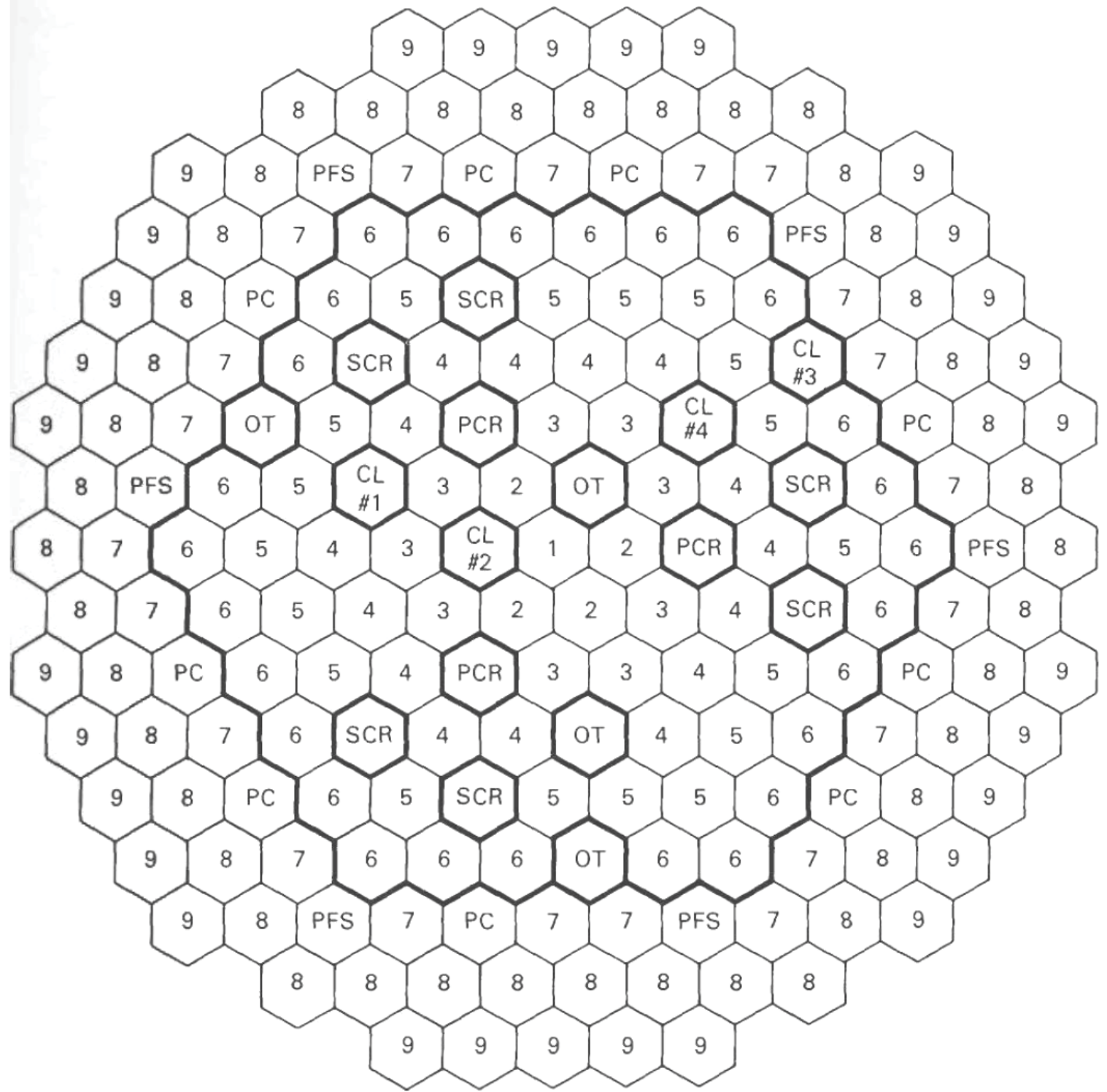

1-9: ROW NUMBER FOR CORE ASSEMBLY. ROWS 1-6 ARE THE FUELED ZONE; ROWS 7-9 ARE THE REFLECTOR ZONE.

CL - CLOSED LOOP POSITION.

SCR - SECONDARY CONTROL ROD ABSORBER POSITION. SIX ASSEMBLIES WERE INSTALLED INITIALLY.

OT - OPEN TEST ASSEMBLY POSITION.

PC - POSITIONS IN WHICH CONTROL RODS MAY BE INSTALLED.

PFS - PERIPHERAL FIXED SHIM ABSORBERS.

PCR - PRIMARY CONTROL RODS. THREE RODS WERE INSTALLED INITIALLY.

Figure 1.1.1. Core Map Showing Typical Arrangement (Ref. 5, Fig. 3-9). 


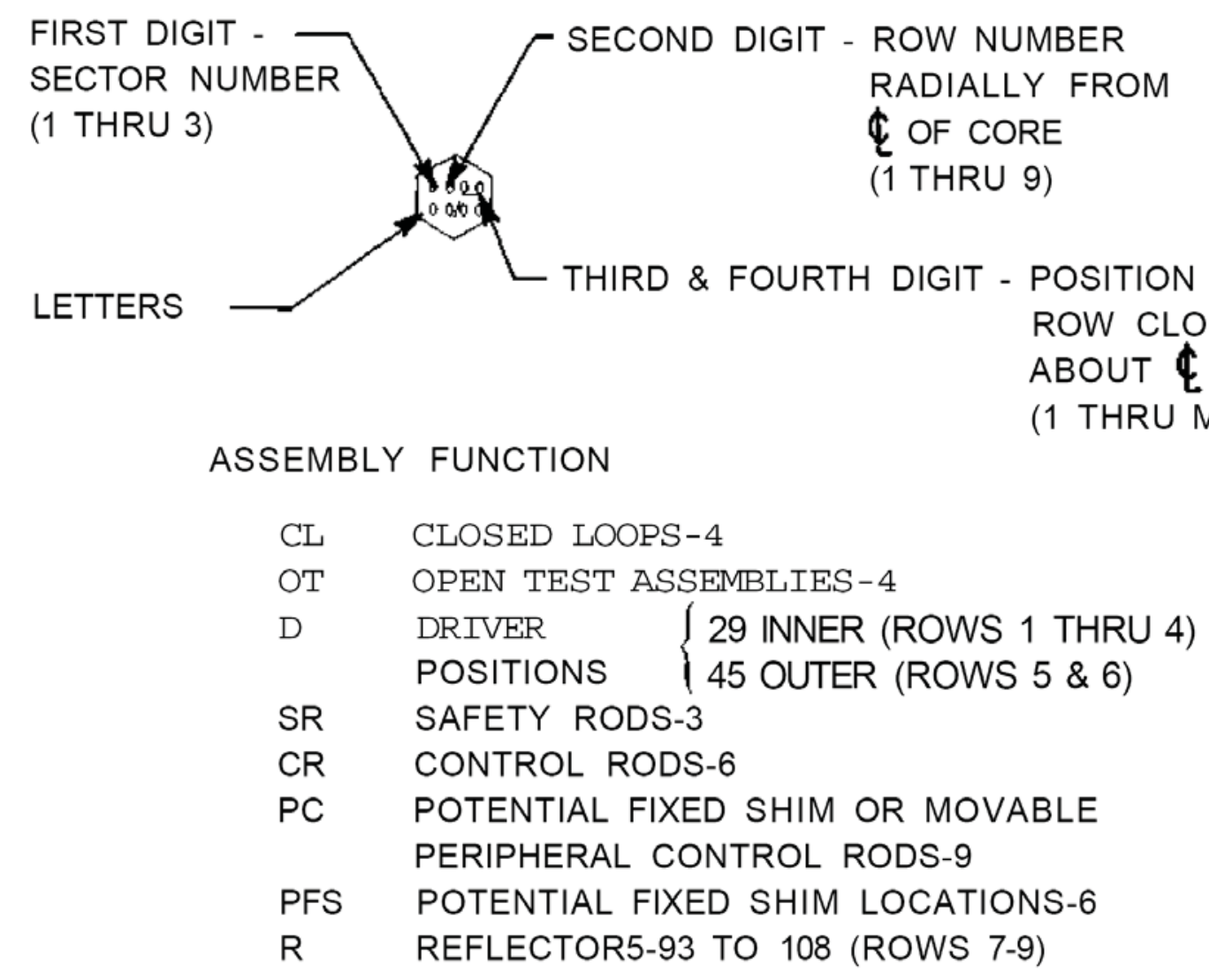

REACTOR VESSEL HEAD PENETRATIONS (REF)

A1-A3 SAFETY RODS-3

A4-A9 CONTROL RODS-6

A10-A18 POTENTIAL FIXED SHIM OR MOVABLE PERIPHERAL CONTROL RODS-9

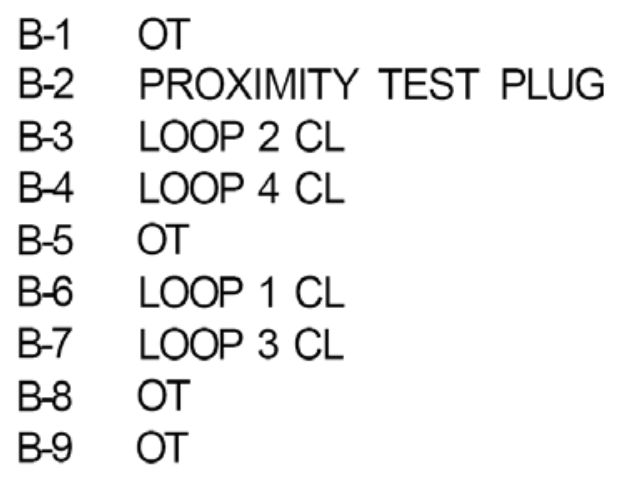

Figure 1.1.2. Detailed Map of FFTF Core (Ref. 3, Figs. A-1 and A-2). 
NEA/NSC/DOC(2006)1

Liquid Metal Fast Reactor - LMFR

FFTF-LMFR-RESR-001

CRIT-SPEC-REAC-COEF-MISC

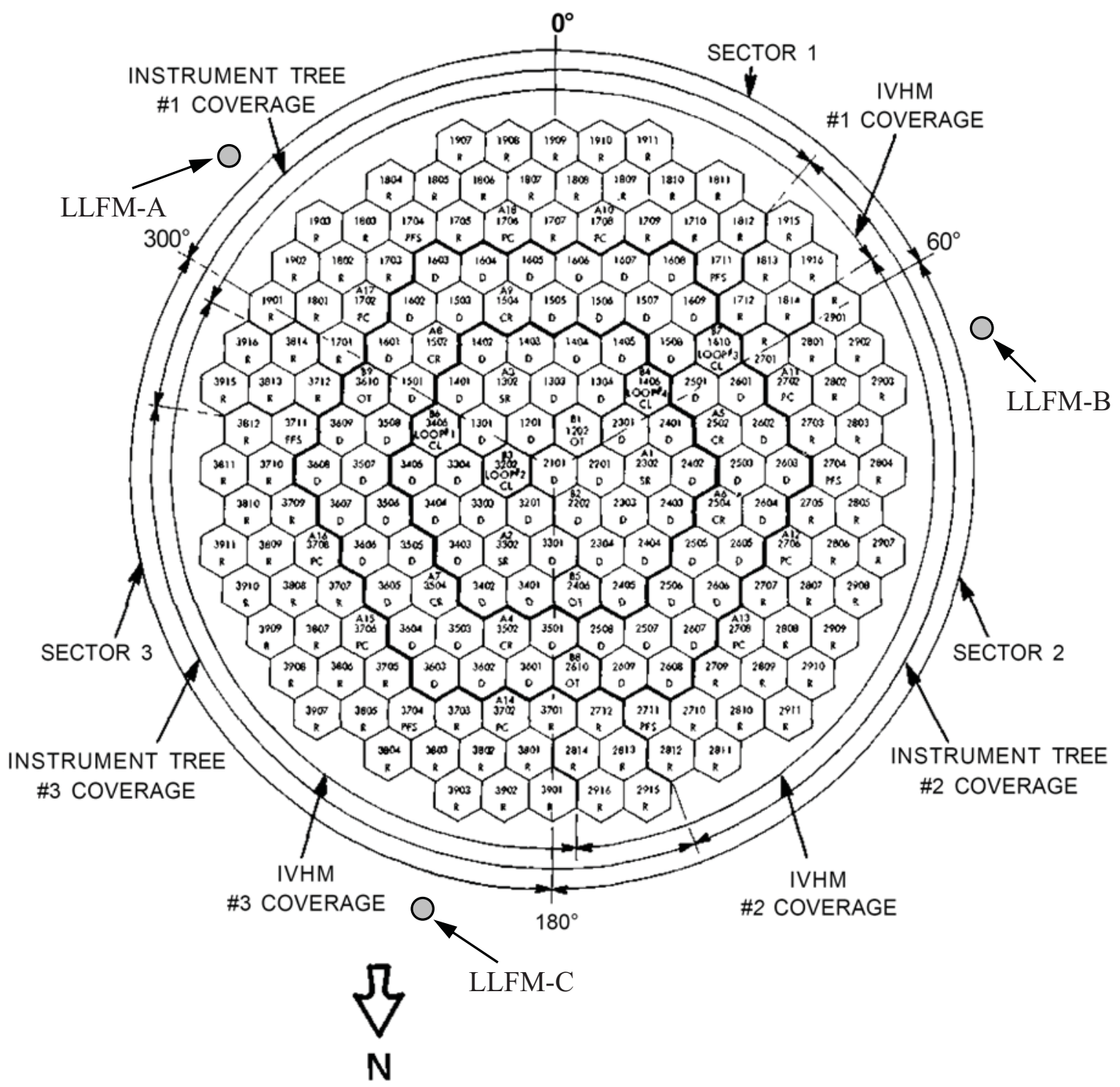

Figure 1.1.2 (cont'd.). Detailed Map of FFTF Core (Ref. 3, Figs. A-1 and A-2).

\section{Driver Fuel Assemblies (DFA)}

The FFTF driver fuel assemblies (Figures 1.1.3 and 1.1.4) were hexagonally-shaped components, 4.715 inches $(11.9761 \mathrm{~cm})$ across outside load pad flats, and $12 \mathrm{ft}(3.6576 \mathrm{~m})$ long. An assembly contained a bundle of 217 fuel pins, 0.230 -inch $(0.5842-\mathrm{cm}) \mathrm{OD}$, each containing mixed oxide $\left(\mathrm{PuO}_{2}-\mathrm{UO}_{2}\right)$ pellets; a surrounding duct; and lower and upper end hardware. The driver fuel pins (Figures 1.1.5 and 1.1.6) were approximately 94 inches $(2.3876 \mathrm{~m})$ long and positioned in a hexagonal array. They were spaced by 0.056-inch $(0.14224-\mathrm{cm})$ diameter helical-wrapped wires. The pin end caps were designed to assure that the pins of the proper enrichment were placed in each fuel assembly. Each pin contained a "tag gas" capsule. Inside the tag gas capsule was a penetrator, which was magnetically actuated to permit the gas to escape into the plenum after the clad tube has been sealed (Ref. 5, pp. 3-13 and 3-14). Monitoring of the reactor cover gas allowed for the detection and isolation of a driver fuel assembly in the event of gas release from a rupture. 
FFTF-LMFR-RESR-001

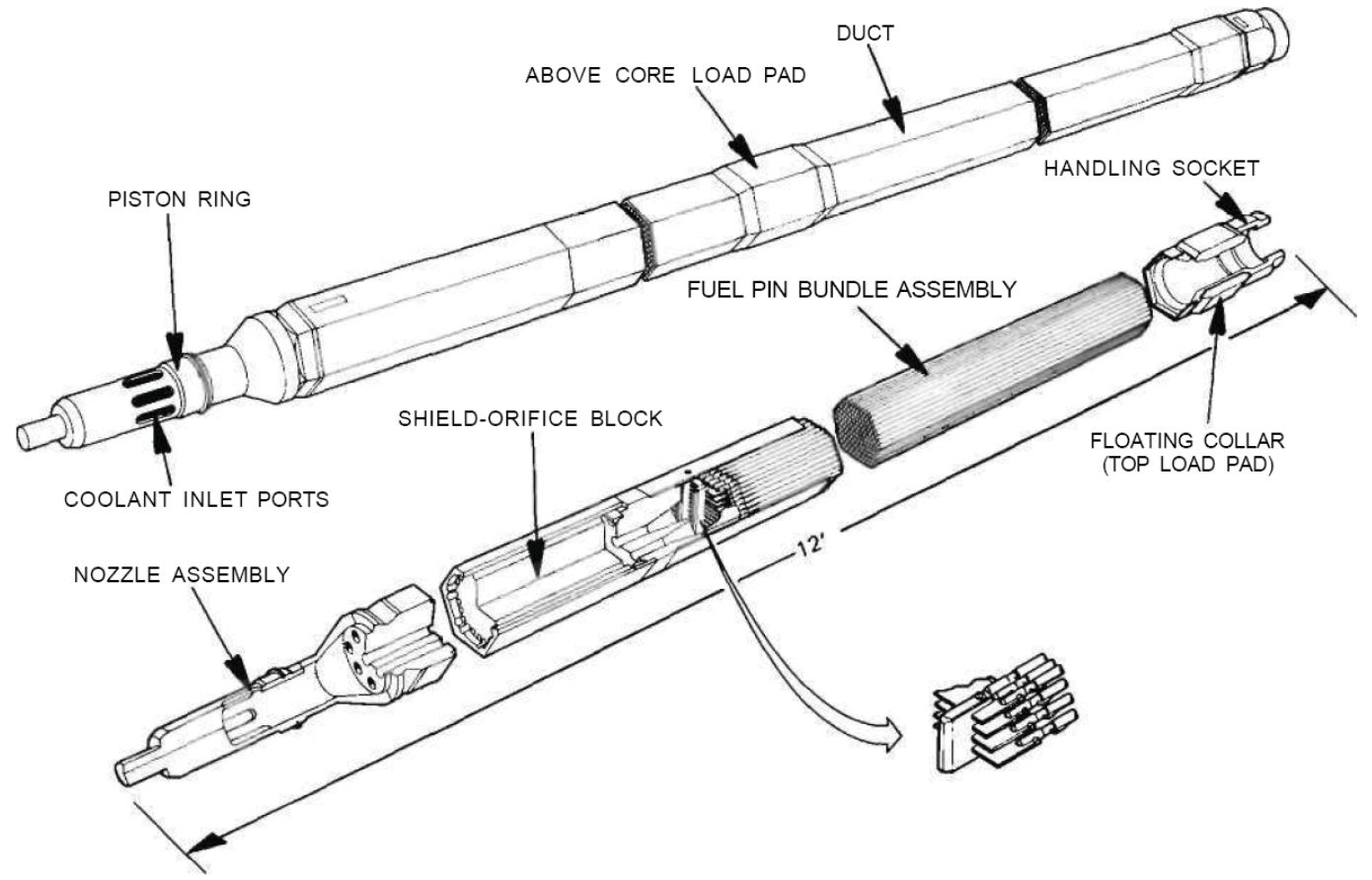

Figure 1.1.3. Driver Fuel Assembly (DFA) (Ref. 5, Fig. 3-11).

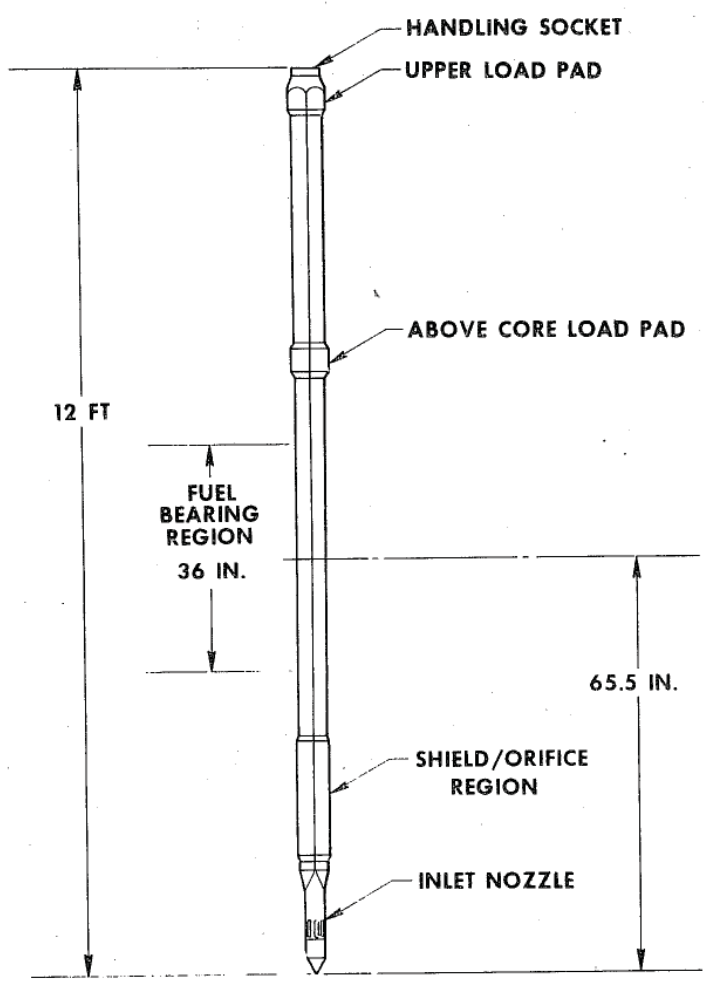

Figure 1.1.4. Driver Fuel Assembly. ${ }^{a}$

\footnotetext{
a Figure II.B.2.6 (p. II-32) from United States Atomic Energy Commission, "Environmental Statement: Fast Flux Test Facility, Richland, Washington,” WASH-1510, (May 1972).
} 
FFTF-LMFR-RESR-001

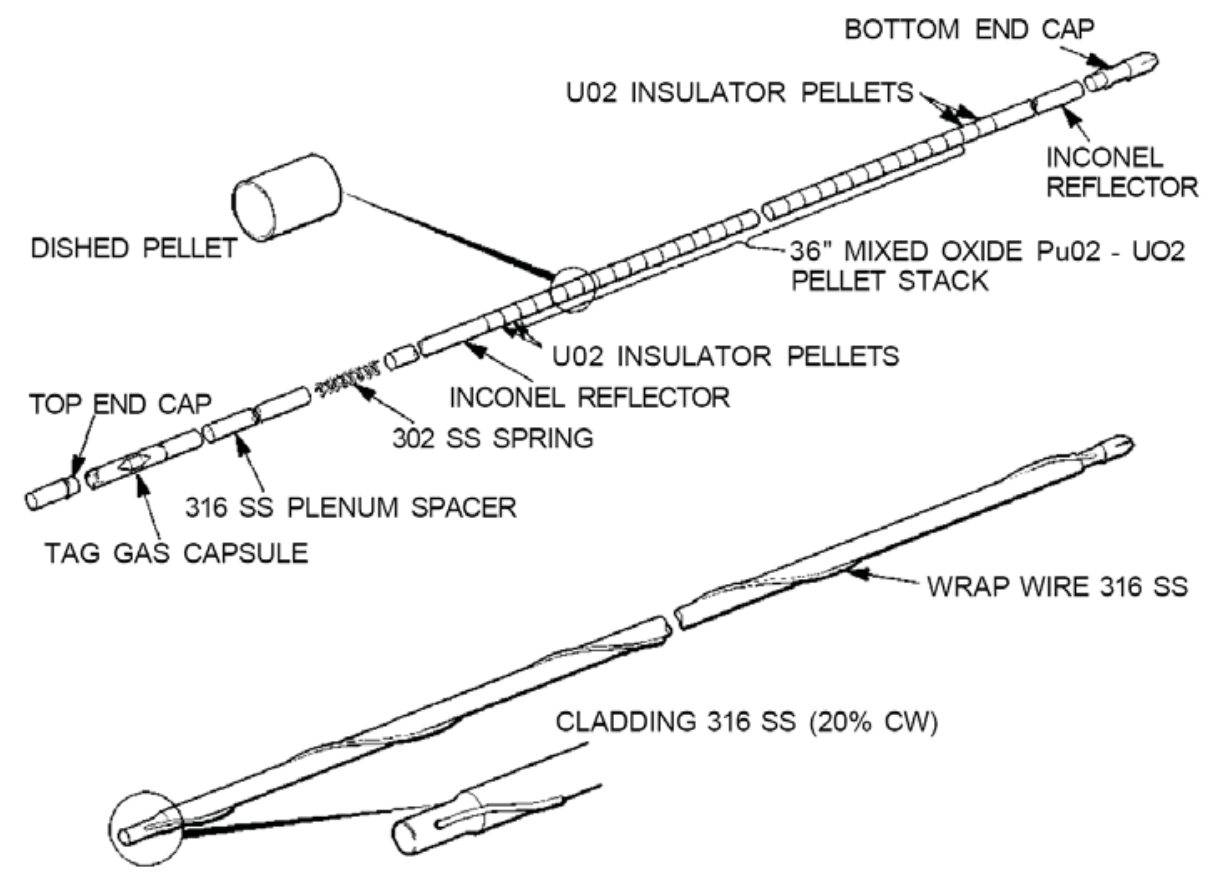

Figure 1.1.5. Driver Fuel Pin (Ref. 5 Fig. 3-12).
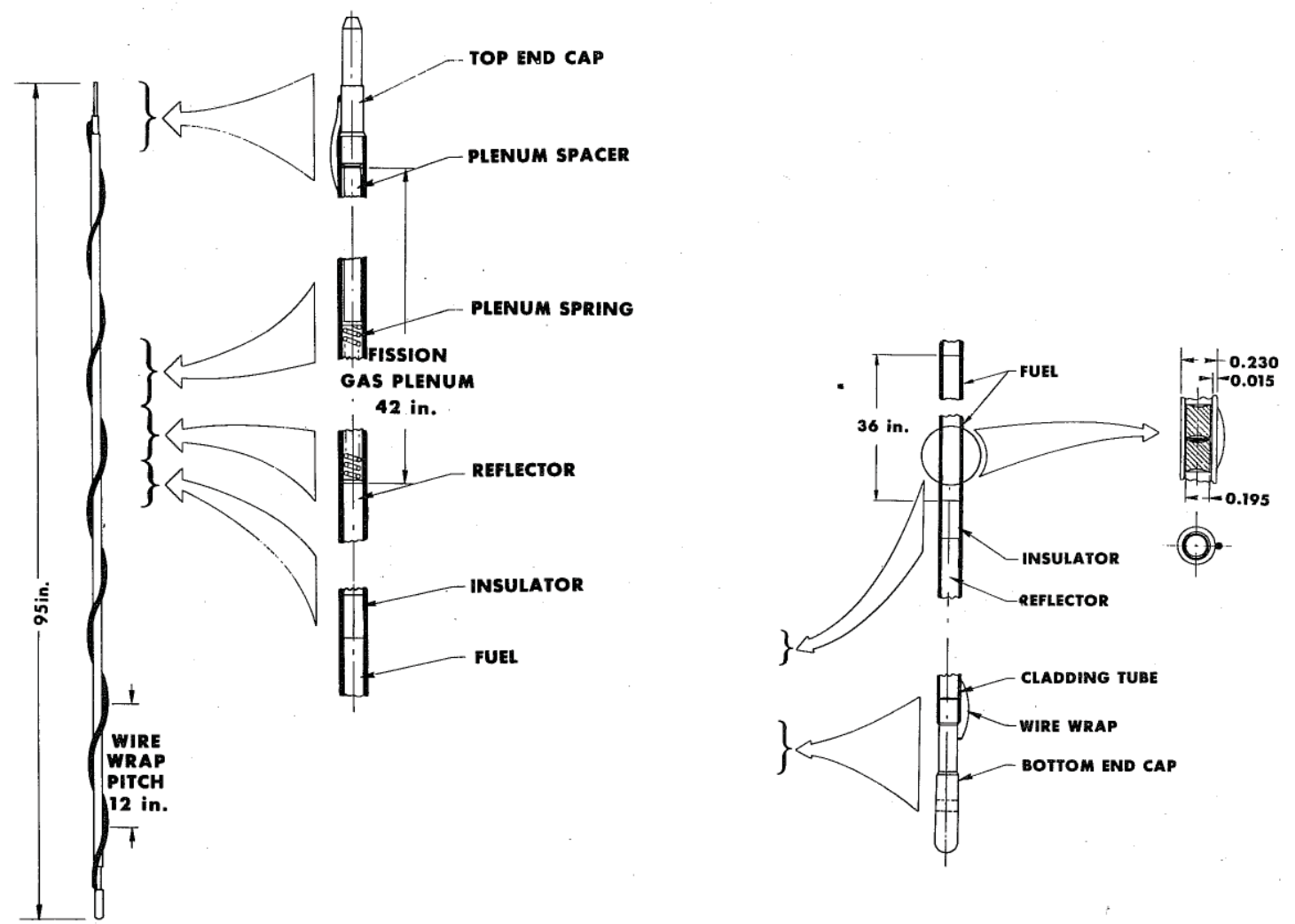

Figure 1.1.6. Driver Fuel Pin. ${ }^{\mathrm{a}}$

\footnotetext{
${ }^{a}$ Figure II.B.2.7 (p. II-34) from United States Atomic Energy Commission, "Environmental Statement: Fast Flux Test Facility, Richland, Washington," WASH-1510, (May 1972).
} 
NEA/NSC/DOC(2006)1

\section{Liquid Metal Fast Reactor - LMFR}

FFTF-LMFR-RESR-001

CRIT-SPEC-REAC-COEF-MISC

Each assembly was assigned a set of tag ratios of xenon and krypton isotopes, and all 217 pins within that assembly contained a small amount of tag gas mixture conforming to these specific isotopic ratios. In the event of a fission gas leak from a fuel pin, some of the tag gas mixture would also escape into the reactor cover gas, where it could be sampled and analyzed be mass spectrometry to determine the identity of the leaker. Eighty different sets of tag ratios were provided, compared with a nominal equilibrium core loading of 76 fuel assemblies, so that it was possible to load the reactor without duplication of gas tags. The total fuel inventory at startup, counting both the original and follow-on fuel purchases, consisted of four sets or 'cores' of from 77 to 80 fuel assemblies each, all with gas tags conforming to the same set of 80 different isotopic ratios. The fuel assemblies were also divided into three different combinations of enrichment/flow zones, each with an associated set of gas tag ratios. ${ }^{a}$

The fuel assembly cross section at midplane is shown in Figure 1.1.7, and key dimensions of the DFA are provided in Table 1.1.3. The pellets were nominally 0.25 -inch $(0.635-\mathrm{cm})$ high with dished ends and the fuel pellet stacks were assembled to give a fuel column length of $36 \pm 1 / 8$ inches $(91.44 \pm 0.3175 \mathrm{~cm})$. On either end of the fuel column were two, 0.4-inch $(1.016-\mathrm{cm}) \mathrm{UO}_{2}$ insulator pellets, and beyond the insulator pellets were axial reflectors. These were 0.1895 -inch $(0.48133-\mathrm{cm})$ diameter Inconel 600 rods, 5.7-inches $(14.478 \mathrm{~cm})$ long. Other fuel pin internal hardware included spacers, springs, and end fittings, as well as structural materials such as the duct, cladding, and spacer wires (Ref. 3, p. 4).

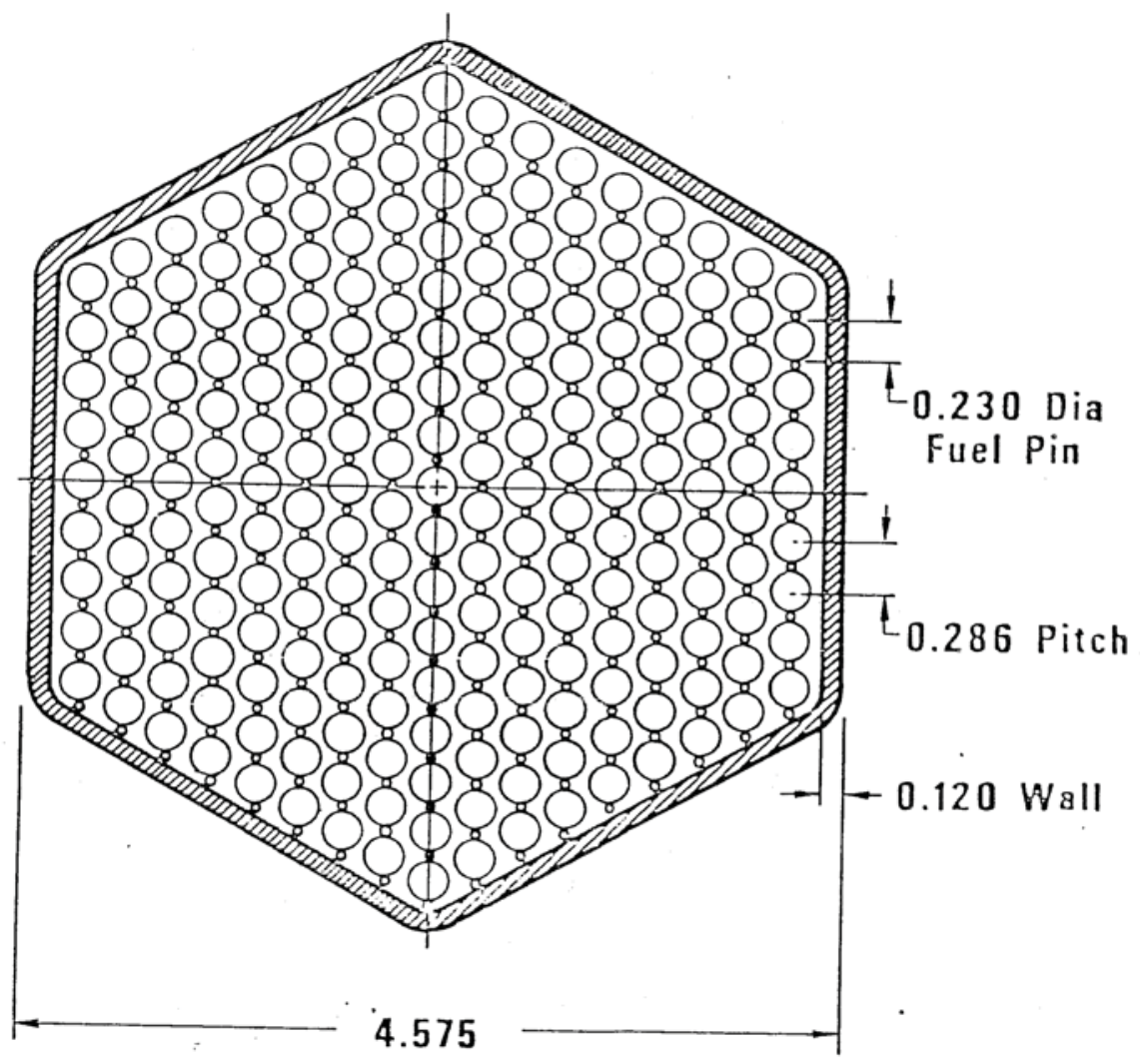

Figure 1.1.7. Driver Fuel Assembly Cross Section (Dimensions in Inches) (Ref. 3 and Ref. 4, Fig. 2).

\footnotetext{
${ }^{a}$ R. A. Bennett, J. W. Daughtry, R. A. Harris, D. H. Jones, J. W. Nelson, J. A. Rawlins, R. B. Rothrock, R. A. Sevenich, and B. D. Zimmerman, "Nuclear Startup, Testing and Core Management of the FTR," HEDL-SA-1729 (CONF-790933-3), Proc. IAEA International Symposium on Fast Reactor Physics, Aix-en-Provence, France, September 24-28 (1979).
}

Revision: 0

Page 11 of 304

Date: March 31, 2010 
NEA/NSC/DOC(2006)1

Liquid Metal Fast Reactor - LMFR

FFTF-LMFR-RESR-001

CRIT-SPEC-REAC-COEF-MISC

Table 1.1.3. DFA Nominal Dimensions at Room Temperature (Ref. 3, p. 5).

\begin{tabular}{||c|c||}
\hline Number of pins per assembly & 217 \\
Fuel pellet end geometry & Dished \\
Fuel pellet OD & 0.1945 in. $(0.49403 \mathrm{~cm})$ \\
Planar smeared density & 0.855 \\
Fuel composition & $(\mathrm{U}, \mathrm{Pu}) \mathrm{O}_{1.96}$ \\
Cladding and duct material & SS-316 \\
Cladding tube ID & 0.200 in. $(0.508 \mathrm{~cm})$ \\
Cladding tube OD & 0.230 in. $(0.5842 \mathrm{~cm})$ \\
Spacer system & Spiral wire wrap \\
Spacer wire diameter & 0.056 in. $(0.14224 \mathrm{~cm})$ \\
Spacer wire pitch & 12 in. $(30.48 \mathrm{~cm})$ \\
Duct tube inner dimension & 4.335 in. $(11.0109 \mathrm{~cm})$ \\
Duct wall thickness & 0.120 in. $(0.3048 \mathrm{~cm})$ \\
Fuel pellet stack length & $36 \pm 1 / 8$ in. $(91.44 \pm 0.3175 \mathrm{~cm})$ \\
Insulator pellet stack length & 0.8 in. $(2.032 \mathrm{~cm})$ each end \\
Axial reflector length & 5.7 in. $(14.478 \mathrm{~cm})$ each end \\
Axial reflector material & Inconel 600 \\
Axial reflector geometry & Cylindrical rod \\
Axial reflector OD & 0.1895 in. $(0.48133 \mathrm{~cm})$ \\
\hline \hline
\end{tabular}

The manufacturing tolerances for both the inner and outer diameters of the cladding were reported as two different values in the same source: \pm 0.001 inches $( \pm 0.00254 \mathrm{~cm})$ and $/$ or \pm 0.0005 inches $( \pm 0.00127$ $\mathrm{cm})^{\mathrm{a}}$

The manufacturing tolerances for the diameter and height of the Inconel reflectors were reported as \pm 0.005 inches $( \pm 0.00127 \mathrm{~cm})$ and \pm 0.0005 inches $( \pm 0.00127 \mathrm{~cm})$, respectively.

The manufacturing tolerance for the diameter of the spacer wire was reported as \pm 0.0005 inches $( \pm 0.00127 \mathrm{~cm}){ }^{\mathrm{c}}$

Lateral spacing between the FFTF fuel pins was maintained with a spiral wire wrap composed of $17 \pm$ $2 \%$ cold-work type 316 stainless steel with a diameter between 0.0565 inches $(0.14351 \mathrm{~mm})$ and 0.0570 in $(0.14478 \mathrm{~cm})$. About $500 \mathrm{~kg}$, or $39 \mathrm{~km}$, of fuel pin wrap wire was used in each core loading. The finished wire was subjected to multiple examinations prior to being shipped to HEDL: chemistry, mechanical, grain size, hardness, residual halides, corrosion resistance, intergranular attack, carbide precipitation, surface roughness, and dimensional. Many of these tests were then repeated at HEDL and

\footnotetext{
${ }^{a}$ NE Standard, Fast Flux Test Facility Driver Fuel Pin Seamless Cladding Tube, NE E 13-8T (June 1971).

${ }^{\mathrm{b}}$ NE Standard, Fast Flux Test Facility Driver Fuel Pin Reflectors, NE E 13-10T (June 1971).

${ }^{c}$ NE Standard, Fast Flux Test Facility Driver Fuel Pin Wrap Wire, NE E 13-13T (June 1971).

Revision: 0

Page 12 of 304

Date: March 31, 2010
} 
NEA/NSC/DOC(2006)1

Liquid Metal Fast Reactor - LMFR

FFTF-LMFR-RESR-001

CRIT-SPEC-REAC-COEF-MISC

the wire was also subjected to eddy current tests to check for surface defects such as inclusions, gall marks, and residual drawing lubricant. Defective portions of the wire were removed from use. ${ }^{\mathrm{a}}$

\section{Fuels Open Test Assemblies (FOTA)}

The fuels open test assemblies (Figure 1.1.8) allowed for the irradiation of highly-instrumented fuel assemblies in reactor coolant. Sodium exit temperatures could be approximately $120^{\circ} \mathrm{F}$ higher than the sodium exit temperature from adjacent fuel assemblies. Flow was controlled using an inlet orifice. The FOTA used a standard driver fuel duct and an attached stalk. The overall assembly length was $40 \mathrm{ft}$ $(12.192 \mathrm{~m})$. Temperatures of the sodium, clad, duct, and fuel centerline were monitored, as well as the fission gas pressure within the pin. Transducers were used to measure the differential pressure within the fuel bundle. Up to fifty-one leads for pressure, temperature, and electrical connections were used (Ref. 5, pp. 3-14 through 3-16).

Two FOTA were provided with special instrumentation for in-core temperature measurements in core positions 1202 and 3610 (see Figure 1.1.2). They were considered identical to standard (noninstrumented) DFA for nuclear analysis purposes (Ref. 3, p. 5).

Care was taken to minimize the impact of the additional instrumentation in the FOTA. All 90 thermocouples in the two FOTA could measure sodium temperature to within $\pm 2{ }^{\circ} \mathrm{F}\left( \pm 1{ }^{\circ} \mathrm{C}\right)$ during isothermal flow. The thermocouples were Type $\mathrm{K}$ ungrounded and centerless ground to provide two diameters: $1.42 \mathrm{~mm}$ to fit the fuel pin spacing geometry, and $2.36 \mathrm{~mm}$ to form the pressure boundary weld in the instrument stalk. Further details regarding the instrumentation and design of the FOTA are provided in the reference. ${ }^{\mathrm{b}}$

\footnotetext{
${ }^{a}$ E. M. Epperson, "Fabrication of FFTF Fuel Pin Wire Wrap,” HEDL-SA-1999 (CONF-800607-73), Proc. ANS Annual Meeting, Las Vegas, NV, June 8-13 (1980).

${ }^{\mathrm{b}}$ L. V. Feigenbutz and C. W. Hoth, "Instrumented Fuels Test for FFTF," HEDL-SA-2007 (CONF-800607-62), Proc. ANS Annual Meeting, Las Vegas, NV, June 8-13 (1980).
} 
FFTF-LMFR-RESR-001
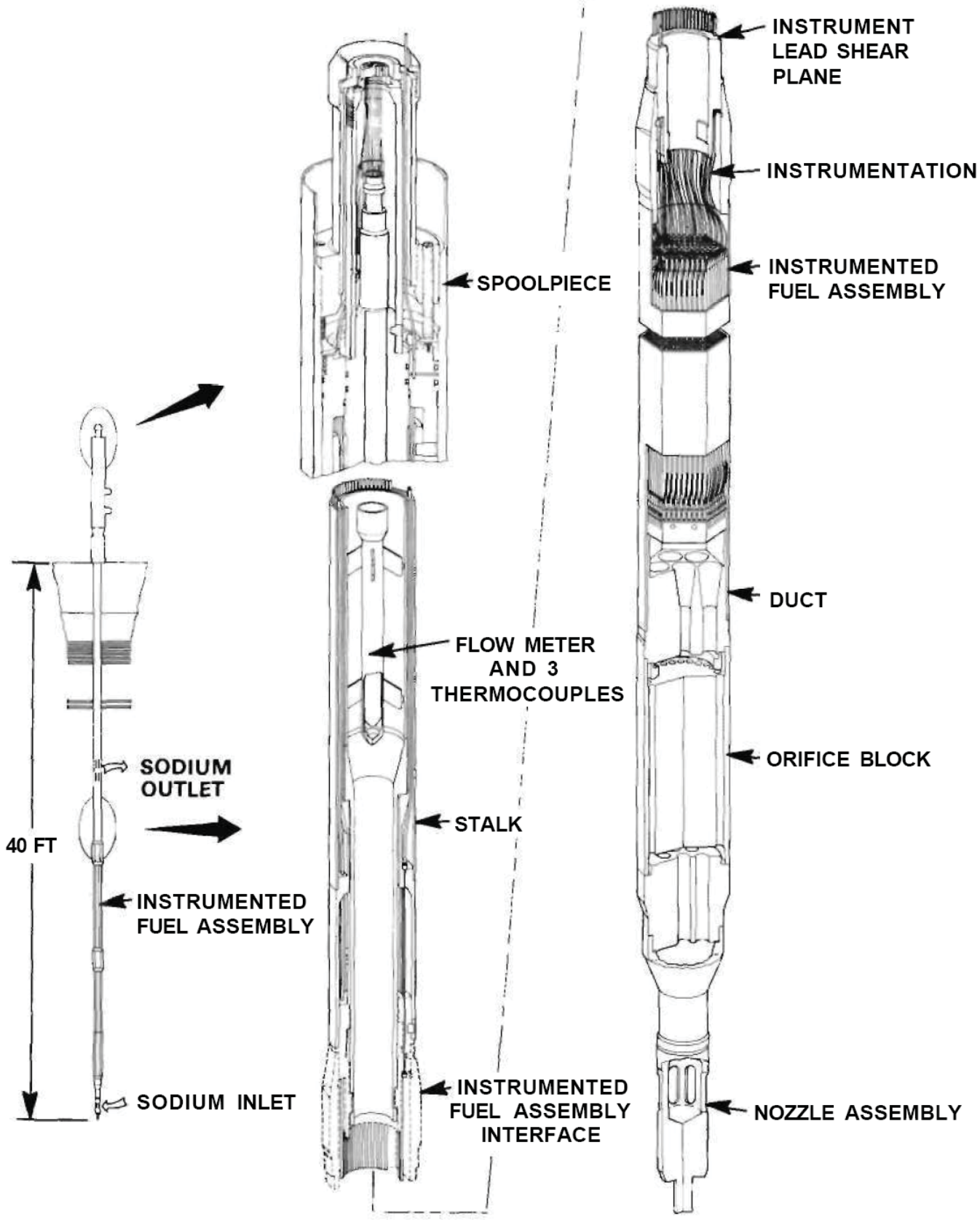

Figure 1.1.8. Fuels Open Test Assembly (FOTA) (Ref. 5, Fig. 3-13).

\section{Vibration Open Test Assembly (VOTA)}

The vibration open test assembly was an instrumented facility for monitoring core vibrations. It also contained thermocouples, calorimeters, gamma ion chambers, and self-powered neutron and gamma detectors. It had an outer duct similar to a DFA, containing a vertical pipe on which the instruments were mounted. The instrumentation in the VOTA had a complex geometry but rather small mass (Ref. 3, p. $10)$. 
NEA/NSC/DOC(2006)1

Liquid Metal Fast Reactor - LMFR

FFTF-LMFR-RESR-001

CRIT-SPEC-REAC-COEF-MISC

\section{In-Reactor Thimble (IRT)}

An in-reactor thimble was installed in core position 3202 to provide access near the core center for a variety of instruments used in core monitoring and characterization. This component was only used during very low power operation, and was subsequently removed from the reactor. In the vicinity of the core, the IRT had the configuration of a 4-inch $(10.16-\mathrm{cm})$ ID stainless steel pipe, with a 0.25 -inch $(0.635-\mathrm{cm})$ wall thickness. The interior of the pipe was essentially void, when no experimental equipment was installed. During measurements using the IRT, shield plugs were ordinarily installed above and below the fueled region to reduce streaming. The IRT was typically empty during most of the zero power physics measurements (Ref. 3, p. 11).

The interior of the IRT was insulated from the reactor sodium coolant by a 40-ft (12.192-m) long vacuum bottle that was part of the Instrument Cooling System (ICS). The interior space was cooled by continuous flushing with cooled gaseous nitrogen, providing an instrument operating temperature of about $40{ }^{\circ} \mathrm{F}\left(\sim 4{ }^{\circ} \mathrm{C}\right)$ at the core center with the reactor operating at a temperature of about $400{ }^{\circ} \mathrm{F}(\sim 204$ $\left.{ }^{\circ} \mathrm{C}\right),(\operatorname{Ref} 2, \mathrm{p} .65)$.

A Startup Chamber Holder ( $\mathrm{SCH})$ containing three fission chambers was installed in the IRT near the core centerline for the initial fuel loading process. It was removed upon completion of fuel loading (Ref. 2 , p. 5). The chambers IRT-1, -2 , and -3 , were placed 18 inches $(45.72 \mathrm{~cm})$ above core midplane, at the core midplane, and 18 inches $(45.72 \mathrm{~cm})$ below core midplane, respectively. The startup chambers in the IRT were removed sometime between February 9 and February 22, 1980, leaving the empty IRT in place during the complete core loading criticality (Ref. 10, pp. 17-18).

\section{Absorber Assemblies}

The reactivity of the FFTF reactor core was controlled using nine control rods, neutron absorber assemblies containing pins of boron carbide. These assemblies contained boron carbide in a movable pin bundle assembly, and provided control over the local neutron flux through adjustment of the axial elevation of the pin bundle. The control rod assembly was a hexagonally-shaped component (Figure 1.1.9) consisting of an orifice region, duct tube, load pads, handling socket, and pin bundle. The pin bundle was mounted in an inner duct that was attached to, or detached from, the drive mechanisms through an absorber coupling at the top of the absorber assembly. They were uncoupled prior to refueling operations and recoupled to resume reactor operations. The disconnected pin bundle remained in the core. Fixed shim absorber assemblies were similar to the control rod absorber assemblies. However, the pin bundle did not move, and there was no coupling section (Ref. 5, pp. 3-18 and 3-19).

Of the nine in-core control rods, the three control rod positions in Row 3 were designated as safety rods (SR), and were normally fully withdrawn during reactor operations. The six control rods (CR) in Row 5 were used to compensate for power defect and burnup reactivity loss. They were partially inserted during normal operations. The fixed shim control rods (CRFS) were used in Rows 6 and 7 for minor core reactivity adjustments. (Ref. 3, p. 7)

Each absorber assembly contained 61 sealed absorber pins containing natural $\mathrm{B}_{4} \mathrm{C}$ pellets, arranged in a hexagonal perforated steel inner duct. The inner duct and pin bundle were contained within an outer duct, of the same dimensions as the DFA flow duct. A cross section of the absorber assembly is shown in Figure 1.1.10 and key dimensions are provided in Table 1.1.4 (Ref. 3, p. 7). 
Liquid Metal Fast Reactor - LMFR

FFTF-LMFR-RESR-001

CRIT-SPEC-REAC-COEF-MISC
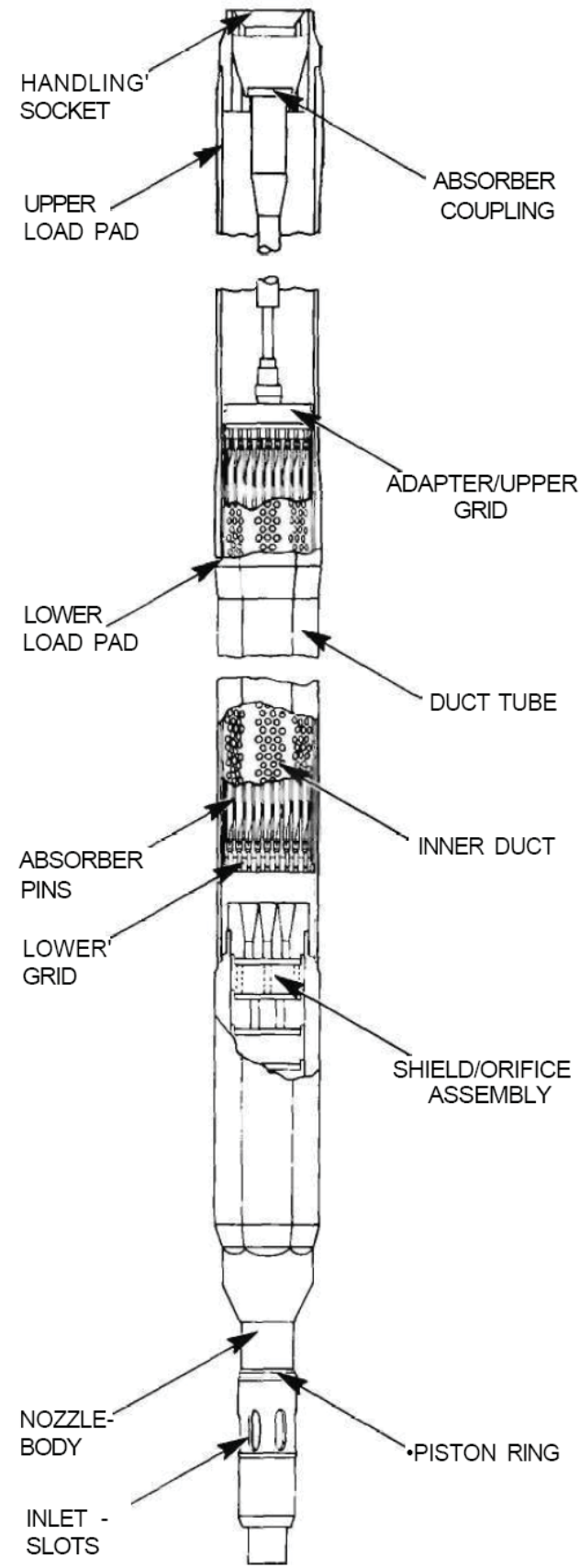

Figure 1.1.9. Control Rod Absorber Assembly (Ref. 5, Fig. 3-16). 
NEA/NSC/DOC(2006)1

Liquid Metal Fast Reactor - LMFR

FFTF-LMFR-RESR-001

CRIT-SPEC-REAC-COEF-MISC

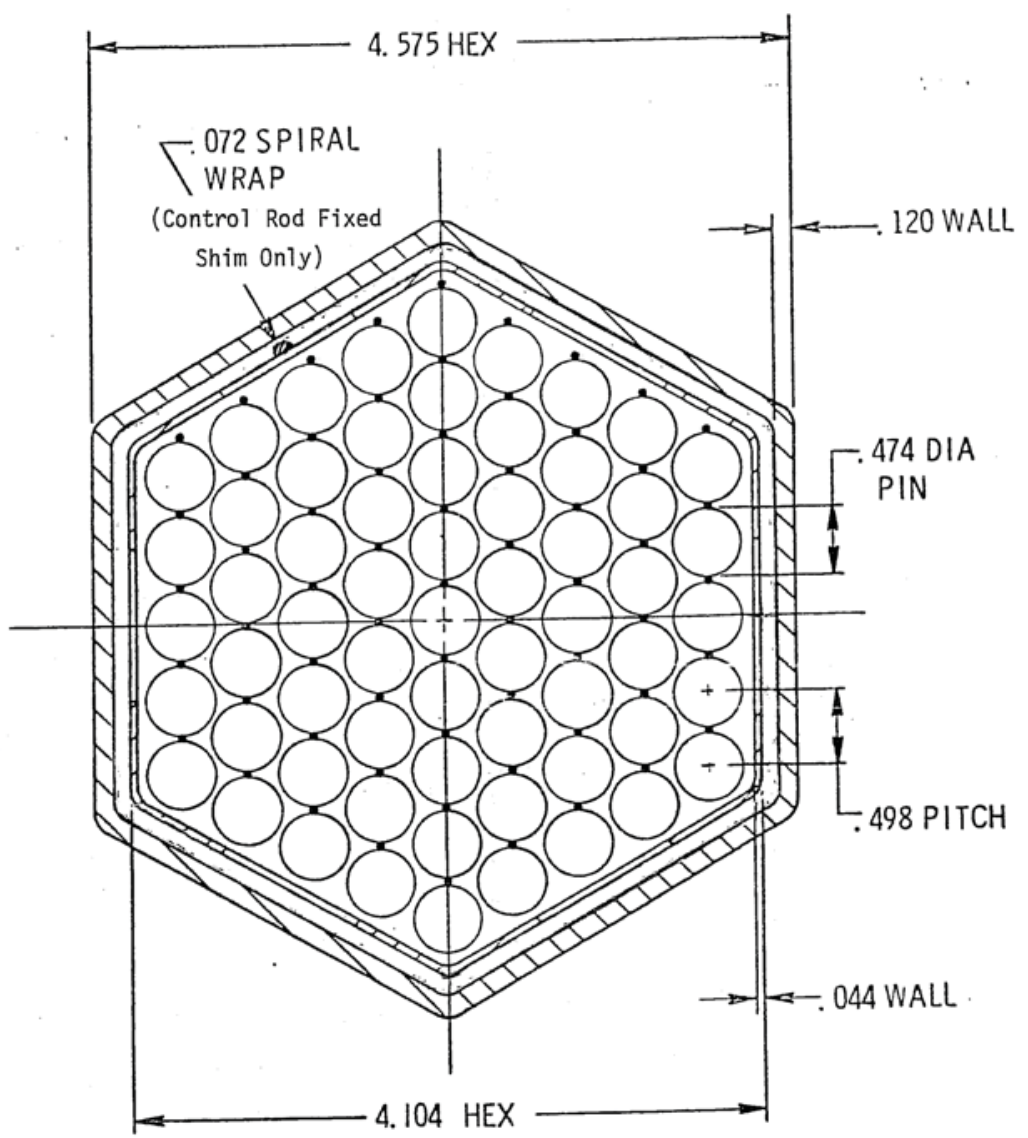

Figure 1.1.10. Control Rod Absorber and Control Rod Fixed Shim (Dimensions in Inches) (Ref. 3 and Ref. 4, Fig. 3).

Table 1.1.4. Absorber Assembly Parameters at Nominal Room Temperature (Ref. 3, p. 10).

\begin{tabular}{||c|c||}
\hline Number of pins per assembly & 61 \\
Absorber pellet composition & $\mathrm{B}_{4} \mathrm{C}$ \\
Boron isotopic composition & Natural \\
Absorber pellet diameter & 0.362 in. $(0.91948 \mathrm{~cm})$ \\
Absorber pellet column length & 36.0 in. $(91.44 \mathrm{~cm})$ \\
Absorber pellet density & $92 \pm 2 \% \mathrm{~T} . \mathrm{D}$. \\
Absorber pin cladding ID & 0.372 in. $(0.94488 \mathrm{~cm})$ \\
Absorber pin cladding OD & 0.474 in. $(1.20396 \mathrm{~cm})$ \\
Absorber pin pitch & 0.498 in. $(1.26492 \mathrm{~cm})$ \\
Outer duct inner dimension & 4.335 in. $(11.0109 \mathrm{~cm}) \mathrm{across}$ flats \\
Outer duct wall thickness & 0.120 in. $(0.3048 \mathrm{~cm})$ \\
Inner duct outer dimension & 4.104 in. $(10.42416 \mathrm{~cm}) \mathrm{across}$ flats \\
Inner duct wall thickness & 0.044 in. $(0.11176 \mathrm{~cm})$ \\
\hline
\end{tabular}


NEA/NSC/DOC(2006)1

Liquid Metal Fast Reactor - LMFR

FFTF-LMFR-RESR-001

CRIT-SPEC-REAC-COEF-MISC

The control rod absorber pins (Figure 1.1.11) were 63.63-inches (1.616202-m) long, including the end caps. The cladding was 0.474 -inches $(1.20396-\mathrm{cm})$ nominal OD by 0.051 -inches $(0.12954-\mathrm{cm})$ thick for the reference design. The portion of the pins containing absorber material consisted of a stack of boron carbide pellets 36-inches $(91.44-\mathrm{cm})$ long. The nominal absorber pellet stack consisted of 60 pellets, each 0.6-inches $(1.524-\mathrm{cm})$ long and 0.362-inches $(0.91948-\mathrm{cm})$ in diameter, of hot-pressed and sintered boron carbide produced by a solid-state process. A gas plenum with an effective length of 21.24 inches $(53.9496 \mathrm{~cm})$ was provided so as to limit the internal gas pressure buildup caused by helium generation. Within the upper plenum were a spacer, tag gas capsule, and a spring to keep the pellets and lower plenum spacer compressed during handing prior to installation in the reactor. The pins for the fixed shim absorbers were generally similar to the control rod absorber pins, but were 95.83 inches $(2.434082 \mathrm{~m})$ long (Ref. 5, p. 3-19).

The absorber stack length was measured to the nearest 0.020 inches $(0.0508 \mathrm{~cm}){ }^{\mathrm{a}}$

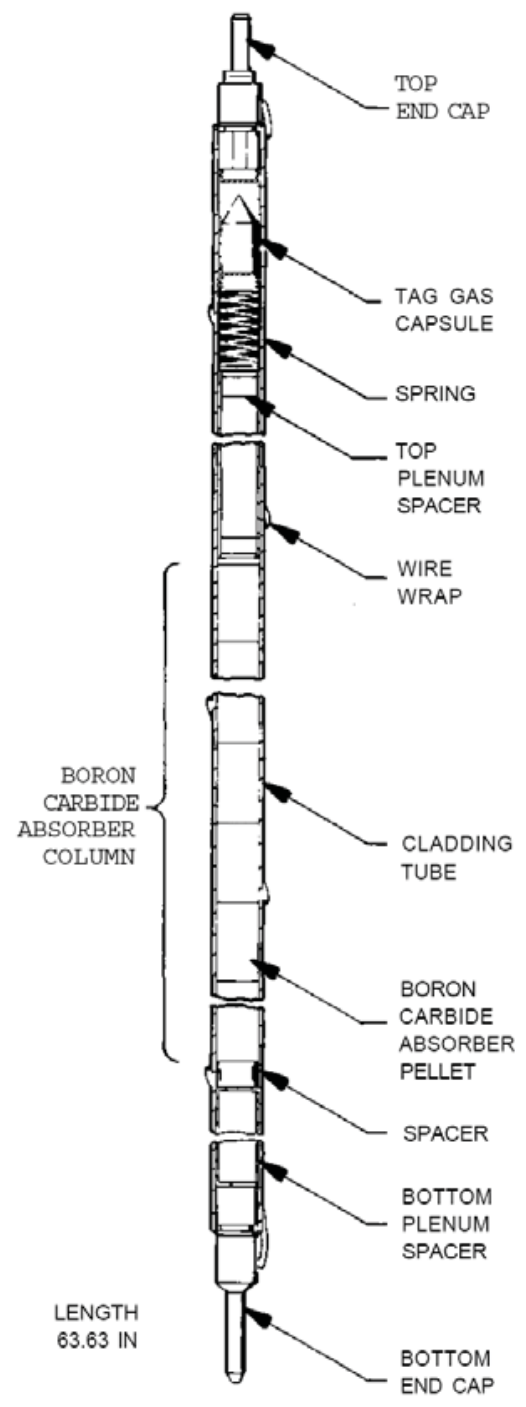

Figure 1.1.11. Control Rod Absorber Pin (Ref. 5, Fig. 3-17).

\footnotetext{
${ }^{a}$ NE Standard, Control Rod Absorber Pin for Liquid Metal Fast Breeder Reactors, NE E 6-25T (January 1977).
} 


\section{Liquid Metal Fast Reactor - LMFR \\ FFTF-LMFR-RESR-001 \\ CRIT-SPEC-REAC-COEF-MISC}

\section{Radial Reflectors (RR)}

The radial reflectors (Figure 1.1.12) consisted of hexagonal reflector assemblies in Rows 7, 8, and 9. The inner assemblies were supported by the core basket and the outer assemblies were supported by the inner radial shield assembly. Reflector assemblies were composed of a section of Inconel 600 and upper and lower shield sections of stainless steel 316. The Inconel section and the upper shield sections consisted of blocks stacked on a vertical array of parallel coolant and structural tubes (Ref. 5, p. 3-20).

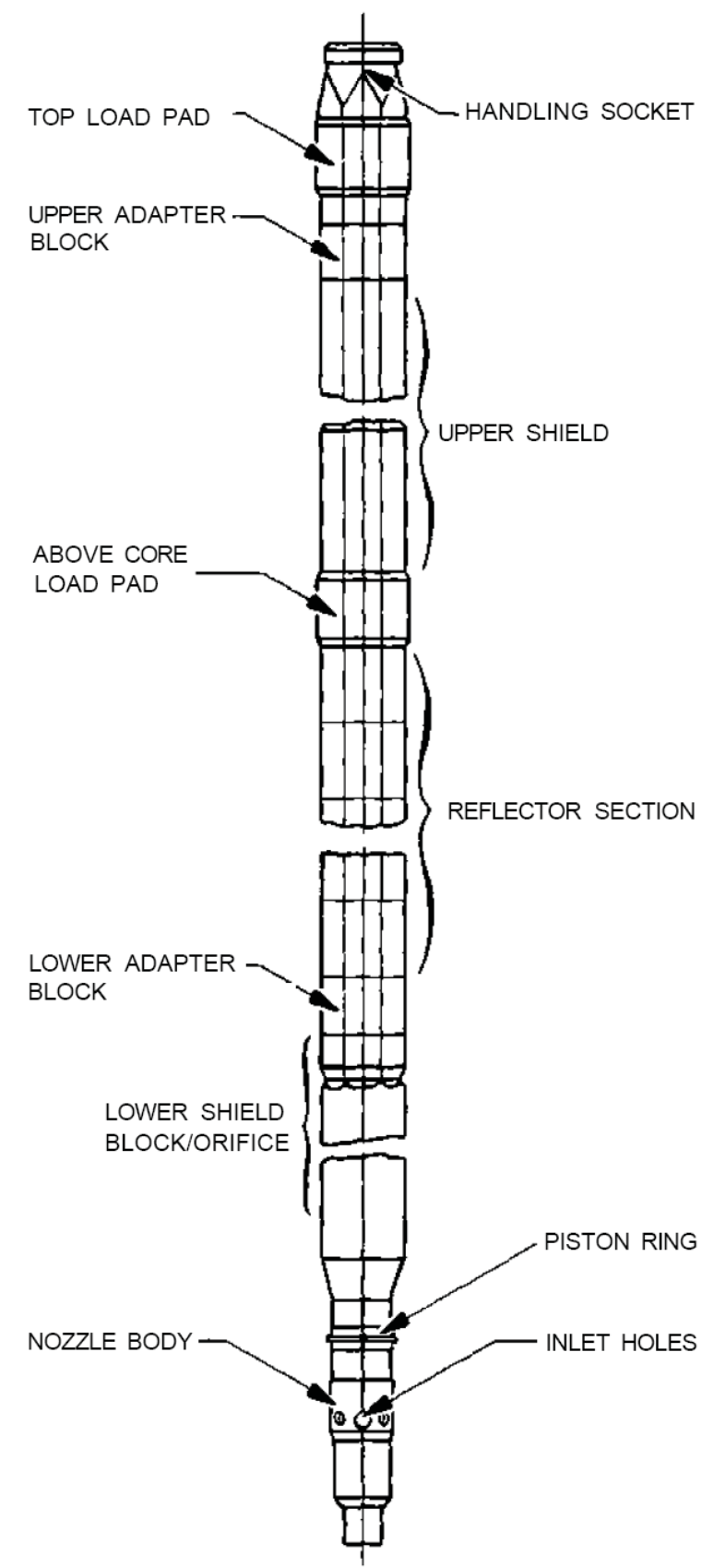

Figure 1.1.12. Radial Reflector Assembly (Ref. 5, Fig. 3-18). 
FFTF-LMFR-RESR-001

CRIT-SPEC-REAC-COEF-MISC

The radial reflector assemblies contained a 48-inch (1.2192-m) stack of hexagonal reflector blocks made of Inconel 600, approximately centered axially on the core midplane, with upper and lower end sections for shielding and mating with the core inlet region and the upper handling socket. The stack consisted of 12 blocks, with holes for interconnecting steel rods that provided structural strength and coolant flow passages. Due to the different heating rates, the reflectors designed for use in Row 7 (Figure 1.1.13) had a different number, size, and spacing of holes compared to those used in Rows 8 and 9 (Figure 1.1.14). A map showing the locations of each type of reflector in the core is shown in Figure 1.1.15 (Ref. 3, p. 11).

These assemblies served as a radial neutron reflector, shielding, and a straight, but flexible, core boundary. The stack of 4-inch $(10.16-\mathrm{cm})$ high blocks was centered along the core midplane. The central tie rod was designed to transmit all tensile loads and the other three tie rods were designed to provide backup structural support for the central member. The coolant flow tubes were not designed to support tensile loads. These tubes started in the plenum above the orifice assembly and terminated in the plenum within the above-core load pad block. Above this upper plenum, the coolant fed into three large coolant channels that were used in conjunction with the four structural members to cool the upper stainless steel shield blocks. ${ }^{a}$

\footnotetext{
${ }^{a}$ B. J. Makenas, R. G. Trenchard, S. L. Hecht, J. M. McCarthy, and F. A. Garner, "The Effect of Swelling in Inconel 600 on the Performance of FFTF Reflector Assemblies," HEDL-SA-3388-FP (CONF-860605-46), Proc. ASTM - Radiation Effects on Materials, Seattle, WA, June (February 1986).
} 
NEA/NSC/DOC(2006)1

Liquid Metal Fast Reactor - LMFR

FFTF-LMFR-RESR-001

CRIT-SPEC-REAC-COEF-MISC

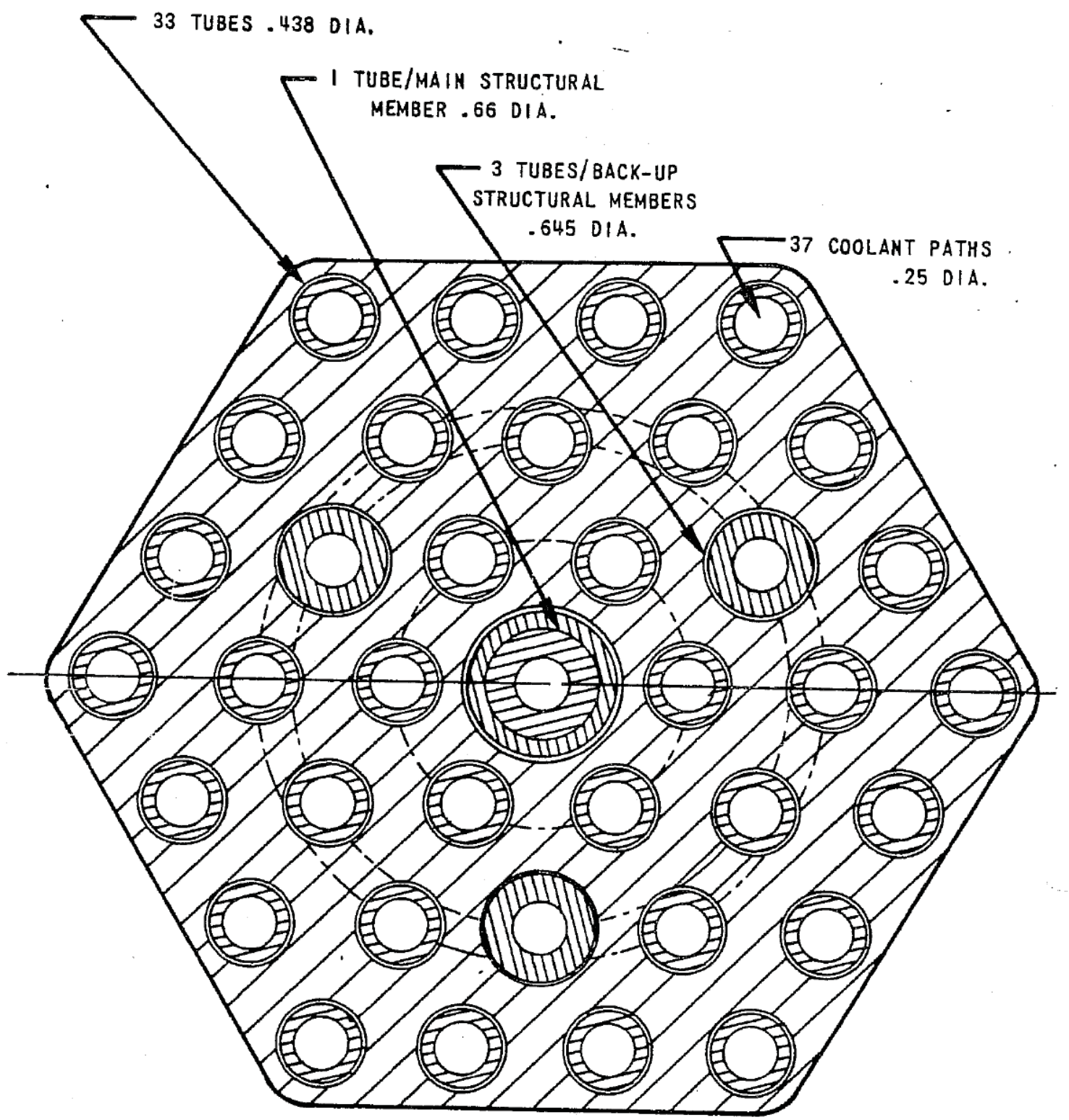

Figure 1.1.13. Row 7 Radial Reflector Assembly (Dimensions in Inches) (Ref. 3, Fig. 5A and Ref. 4, Fig. 5). 
NEA/NSC/DOC(2006)1

Liquid Metal Fast Reactor - LMFR

FFTF-LMFR-RESR-001

CRIT-SPEC-REAC-COEF-MISC

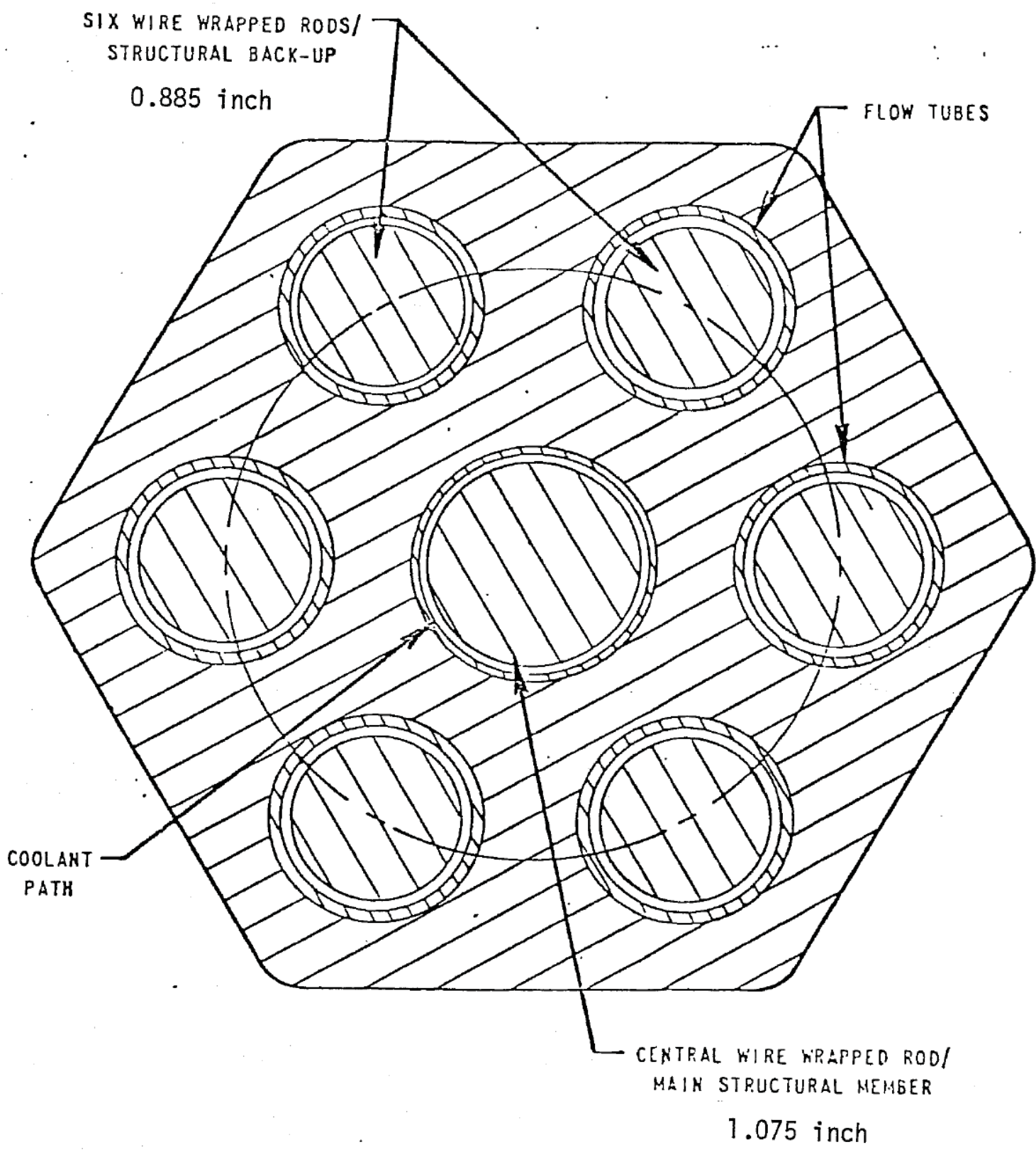

Figure 1.1.14. Rows 8 and 9 Radial Reflector Assembly (Dimensions in Inches)

(Ref. 3, Fig. 5B and Ref. 4, Fig. 6). 
Liquid Metal Fast Reactor - LMFR

FFTF-LMFR-RESR-001

CRIT-SPEC-REAC-COEF-MISC

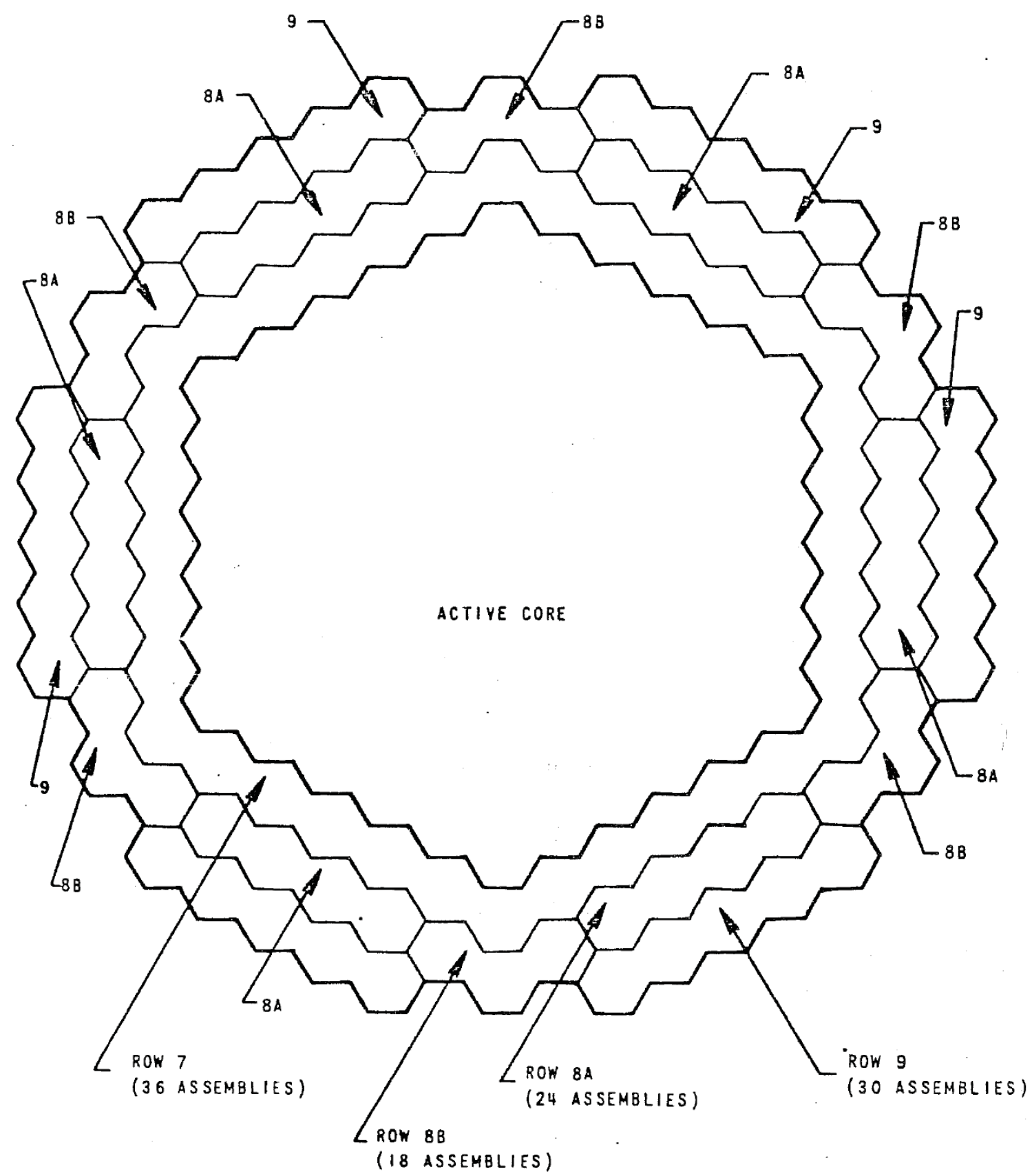

Figure 1.1.15. Radial Reflector Locations (Ref. 3, Fig. 5C). 
NEA/NSC/DOC(2006)1

Liquid Metal Fast Reactor - LMFR

FFTF-LMFR-RESR-001

CRIT-SPEC-REAC-COEF-MISC

\section{In-Core Shim Assemblies (ICSA)}

The in-core shim assemblies were nonfueled assemblies that were installed in the core in substitution for fuel or test assemblies during reactor operation. An ICSA was a stainless-steel, hexagonally-shaped component, 4.715 inches $(11.9761 \mathrm{~cm})$ across load pad flats, and 12-ft $(3.6576-\mathrm{m})$ long. These assemblies were used at initial startup to compensate for the excess reactivity that would otherwise have existed in the initial core, and then later used as necessary (Ref. 5, p. 3-20). The bundle of steel rods was contained in a standard driver fuel flow duct, as shown in Figure 1.1.16 (Ref. 3, p. 7).

An ICSA replaced the IRT after the initial physics experiments and before the reactor was taken to full power. This occurred in January 1981. ${ }^{\mathrm{a}}$

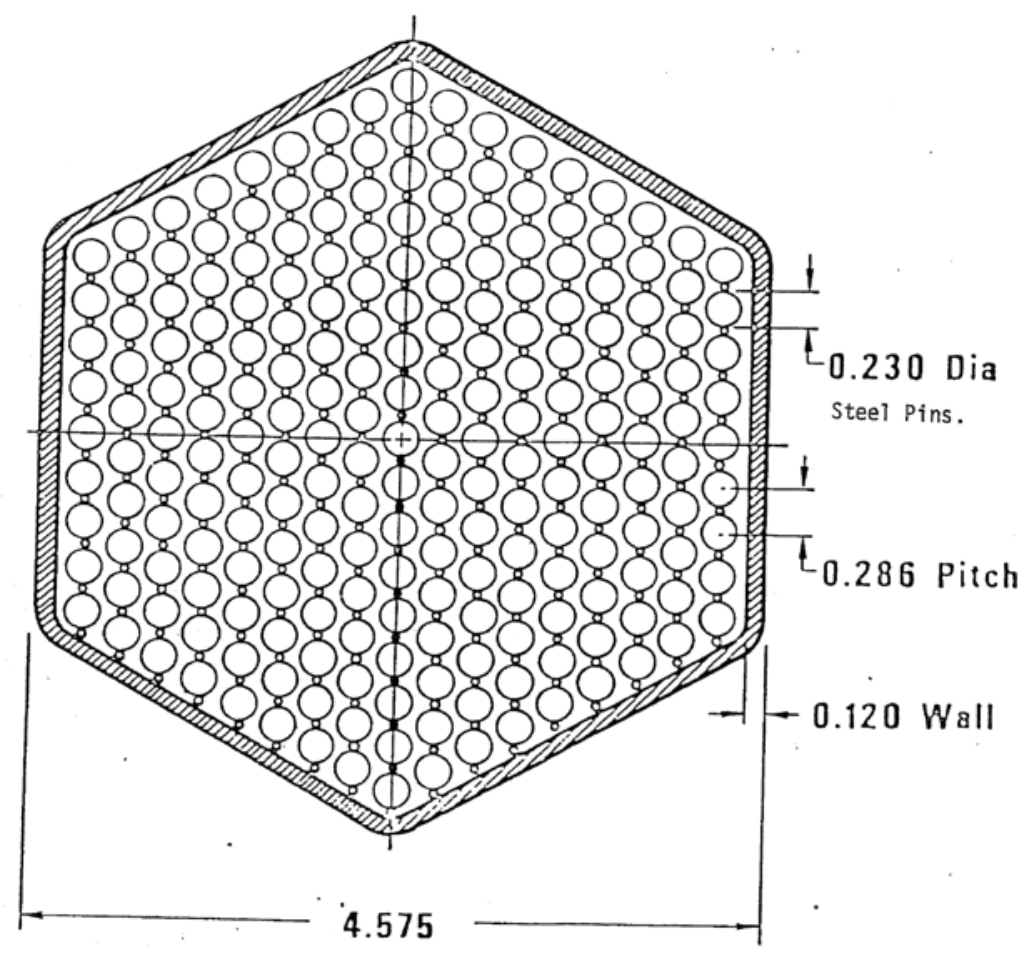

Figure 1.1.16. In-Core Shim Assembly Cross Section (Dimensions in Inches) (Ref. 3, Fig. 4).

\subsubsection{Reported Uncertainties}

Axial alignment of core components (e.g., fuel and absorber pellet stacks) was controlled by (1) dimensional tolerances in the components; (2) seating of the assemblies by the in-vessel handling machine (IVHM); and (3) relative thermal expansion. For the case of the initial isothermal physics tests on the unirradiated core, all assemblies were assumed to be properly seated, since all components were relatively straight and there was no interference from adjacent, distorted assemblies that might prevent proper seating. This assumption was supported by records of IVHM load-stroke characteristics which show little resistance during downward motion of each assembly into the core, until it is seated. Since the reactor was isothermal, no additional misalignment was introduced by differential thermal expansion, and dimensional variations in the components which locate the bottom of the fuel pellet stack were not expected to produce a significant misalignment in the unirradiated condition. The tolerance on the fuel

\footnotetext{
a Personal Communication with David W. Wootan at Pacific Northwest National Laboratory (September 30, 2009).
} 
NEA/NSC/DOC(2006)1

Liquid Metal Fast Reactor - LMFR

FFTF-LMFR-RESR-001

CRIT-SPEC-REAC-COEF-MISC

pellet stack length accounted for a $\pm 1 / 8$-inch $( \pm 0.3175$-cm) variation in the location of the top of the stack (Ref. 3, p.25).

Radial alignment of core assemblies was controlled by the 4.730-inch (12.0142-cm, at room temperature) hole spacing in the core support plate, the 4.715 -inch $(11.9761-\mathrm{cm}$, at room temperature) outer dimension of the spacer pads on the core components, and the core restraint yokes external to the reflector which had an average diametral spacing (across corners) of 71.17 inches $(1.807718 \mathrm{~m})$. These dimensions provided clearance under isothermal conditions for the withdrawal of bowed assemblies. Consequently, the exact radial geometry of the core during the isothermal physics tests was not precisely determined. Analysis of the as-built core dimension showed that the active core diameter at the midplane (dimension across the core diagonal, to the outer edge of Row 6 could increase by a maximum of 0.19 inches $(0.4826$ $\mathrm{cm}$ ) or decrease by a maximum of 0.10 inches $(0.254 \mathrm{~cm})$, from the nominal value inferred from the core support plate hole pitch (Ref. 3, pp. 25-26). It is unclear whether thermal expansion effects of the grid plate due to local or global temperature effects were included in the uncertainty of the core diagonal dimension.

Control rod positions were ordinarily recorded from the digital relative rod position indicator (RRPI) system. These indicators were reset to zero when each rod was latched (at fully inserted condition), and they indicated the rod position by counting pulses sent to the control rod drive motors. One increment, 0.100 inches $(0.254 \mathrm{~cm})$, of rod position was registered for every four rod drive motor pulses; ordinarily the exact position of the rod, between digitized increments, was not known. The estimated uncertainty in the inferred rod position was 0.04 inches $(0.1016 \mathrm{~cm})$, at the $1 \sigma$ level, under isothermal conditions (Ref. 3, p. 29).

Rod heights were maintained within 0.1 inches $(0.254 \mathrm{~cm})$ of the preplanned height (Ref. 2, p. 12).

'Critical' rod positions were inferred from a sequence of count rate measurements at increasing rod withdrawal, by plotting reciprocal count rate and extrapolating to the point where the reciprocal count rate vanishes. The net uncertainty in a critical rod position measurement, including the rod position measurement uncertainty, was estimated as 0.06 inches (0.1524) at $1 \sigma$ (Ref. 3, p.29). This larger uncertainty was only for analyses involving critical rod positions that were extrapolated instead of actually performed.

\subsubsection{Description of Criticality Measurements}

On March 8, 1980, at 8:13 am, following completion of various subcritical measurements, the reactor achieved criticality for the first time following the completion of core loading. Rod movements were performed in a manner similar to the criticality approach on February 22, until a secondary rod bank height from full insertion of 13 inches $(33.02 \mathrm{~cm})$ was achieved. Then each secondary rod was pulled one inch $(2.54 \mathrm{~cm})$ to a 14 -inches $(35.56-\mathrm{cm})$ height, with count data taken after each individual rod pull. With all secondary rods banked at 14 inches $(35.56 \mathrm{~cm})$, rod 4 was pulled to a 15.4 -inches $(36.116-\mathrm{cm})$ height, achieving criticality and a startup rate of 0.3 decade per minute. Startup was terminated prior to reaching $1 \mathrm{~kW}$ of fission power by reinsertion of rod 4 (Ref. 1, p.46). The purpose of this critical operation was to verify operator proficiency using a nuclear startup procedure to one kilowatt of fission power (Ref. 2, p. 16).

Safety rods were fully withdrawn and the reactor temperature was approximately $400{ }^{\circ} \mathrm{F}(\sim 478 \mathrm{~K})$ with a core layout as shown in Figure 1.1.17. 
NEA/NSC/DOC(2006)1

Liquid Metal Fast Reactor - LMFR

FFTF-LMFR-RESR-001

CRIT-SPEC-REAC-COEF-MISC

FULLY LOADED FFTF CORE

FEBRUARY 19, 1980
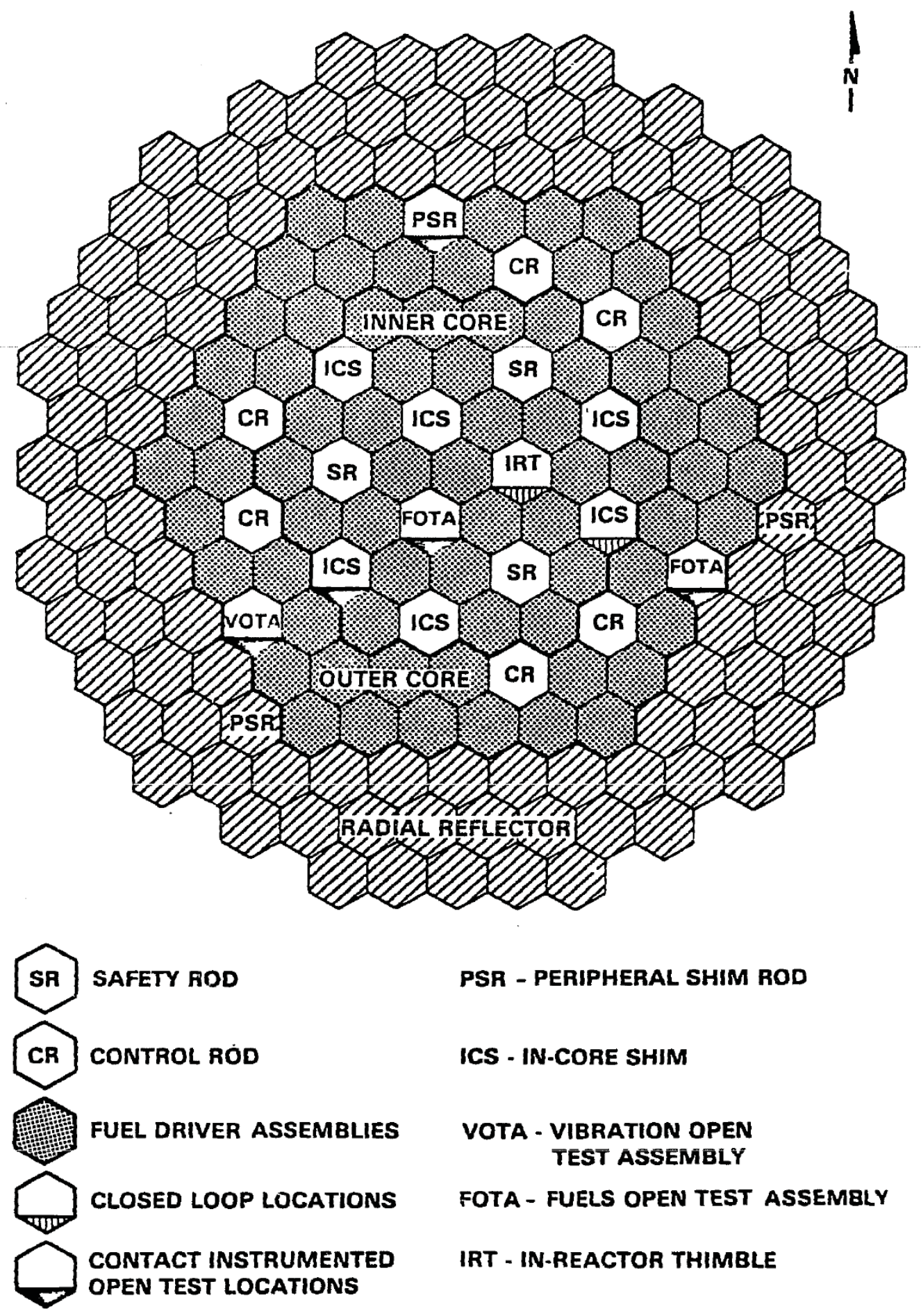

Figure 1.1.17. Fully-Loaded Core Layout (Ref. 1, Fig. 28). 
NEA/NSC/DOC(2006)1

Liquid Metal Fast Reactor - LMFR

FFTF-LMFR-RESR-001

CRIT-SPEC-REAC-COEF-MISC

\subsubsection{Material Data}

\section{Sodium Coolant}

The normal sodium purity was $<1$ ppm oxygen and $<0.04$ ppm hydrogen. ${ }^{a}$

During the first months of measurements, the oxygen and hydrogen content of the sodium remained at $<2$ and $0.1 \mathrm{ppm}$, respectively. Trace metals, boron, chlorine, fluorine, and sulfur were below the specified limit of $10 \mathrm{ppm}$ with only iron exceeding the limit. The hydrogen in the argon cover gas was measured as high as $120 \mathrm{vppm}$ (parts per million by volume) but typically between 5 and $64 \mathrm{vppm}$ during testing. Trace methane $<10 \mathrm{ppm}$ was detected in the cover gas, as well as $<50 \mathrm{ppm}$ nitrogen, indicating no air leakage. Bulk sodium samples measured trace elements $<0.5 \mathrm{ppm}$ except iron that was between 10 and 40 ppm. $^{\text {b }}$

Oxygen, hydrogen, and carbon in the primary sodium averaged $1.5 \mathrm{ppm} \mathrm{O}, 0.05 \mathrm{ppm} \mathrm{H}$, and $0.6 \mathrm{ppm} \mathrm{C}$. They averaged $0.97 \mathrm{ppm} \mathrm{O}, 0.04 \mathrm{ppm} \mathrm{H}$, and $0.033 \mathrm{ppm} \mathrm{C}$ in the secondary sodium. Trace metal impurities in the primary and secondary sodium were within acceptable limits and were $<5 \mathrm{ppm}$ for each element. Hydrogen in the primary cover gas increased from $\sim 3 \mathrm{ppm}$ at $204^{\circ} \mathrm{C}$ to a maximum of $54 \mathrm{ppm}$ and then decreased to $\sim 20 \mathrm{ppm}$ and then $\sim 4 \mathrm{ppm}$. Methane in the cover gas remained below the detectable limit of $\sim 2 \mathrm{ppm}$ by gas chromatography. Other impurities in the primary cover gas were $<20$ ppm. $^{\mathrm{c}}$

The sodium in the core was purified during the months of December 1978 to February 1979 with the core systems at $400^{\circ} \mathrm{F}\left(\sim 204^{\circ} \mathrm{C}\right)$. Oxygen, hydrogen, and carbon were measured in the sodium by metal equilibration methods and trace metals were analyzed on bulk sodium samples. The oxygen and hydrogen levels were constant with saturation solubilities indicated from plugging temperature measurements. Impurity quantities in sodium are shown in Table 1.1.5. ${ }^{\mathrm{d}}$

\footnotetext{
a C. L. Peckinpaugh, R. A. Bennett, and W. R. Wycoff, "FFTF Operational Results," HEDL-SA-2694 (CONF820914-4)/IAEA-CN-42/276, Proc. IAEA Int. Cong. Nuclear Power Experience, Vienna, Austria, September 1317 (July 1982).

b J. J. McCown, G. B. Barton, G. R. Bloom, G. E. Meadows, G. W. Mettler, "FFTF Sodium and Cover-Gas Chemistry," Trans. Am. Nuc. Soc., 34, 697-698 (1980).

c J. M. Atwood, "Sodium Technology Progress Report: July - September 1980," HEDL-TME-80-32, (December 1980).

d J. J. McCown, G. R. Bloom, G. E. Meadows, and G. W. Mettler, "FFTF Sodium and Cover Gas Characterization and Purification," HEDL-SA-1874-FP (CONF-800401-16), Proc. $2^{\text {nd }}$ Int. Conf. Liquid Metal Technology in Energy Production, Richland, WA, April 20-24 (February 1980).
}

Revision: 0

Page 27 of 304

Date: March 31, 2010 
NEA/NSC/DOC(2006)1

Liquid Metal Fast Reactor - LMFR

FFTF-LMFR-RESR-001

CRIT-SPEC-REAC-COEF-MISC

Table 1.1.5. Sodium Impurity Levels in Primary Loop (ppm by weight) - 1979.. ${ }^{\text {(a) }}$

\begin{tabular}{|c|ccc|c|}
\hline Element & Low & High & Ave. & $\begin{array}{c}\text { No. } \\
\text { Samples }\end{array}$ \\
\hline \hline $\mathrm{Al}$ & $<.01$ & 0.63 & 0.17 & 16 \\
$\mathrm{Au}$ & $<.01$ & 0.07 & $<.01$ & 14 \\
$\mathrm{Ag}$ & $<.01$ & 0.03 & $<.01$ & 16 \\
$\mathrm{~B}$ & $<.002$ & 0.01 & .006 & 2 \\
$\mathrm{Ba}$ & $<.01$ & 0.04 & $<.01$ & 5 \\
$\mathrm{Bi}$ & $<.01$ & 0.04 & .01 & 15 \\
$\mathrm{Ca}$ & $<.01$ & 0.19 & .02 & 15 \\
$\mathrm{Cd}$ & $<.01$ & $<.01$ & $<.01$ & 1 \\
$\mathrm{Cs}$ & $<.02$ & $<.02$ & $<.02$ & 1 \\
$\mathrm{Cl}$ & .01 & 3 & 1.5 & 2 \\
$\mathrm{Cr}$ & .02 & 1.8 & 0.37 & 15 \\
$\mathrm{Co}$ & $<.01$ & 0.06 & 0.02 & 15 \\
$\mathrm{Cu}$ & $<.01$ & 0.06 & 0.02 & 16 \\
$\mathrm{~F}$ & $<.01$ & 0.10 & 0.10 & 2 \\
$\mathrm{Fe}$ & 0.1 & 170.0 & 12.9 & 15 \\
$\mathrm{~Pb}$ & $<.01$ & 0.07 & 0.05 & 16 \\
$\mathrm{Li}$ & $<.01$ & $<.01$ & $<.01$ & 7 \\
$\mathrm{Mg}$ & $<.01$ & 0.18 & 0.04 & 16 \\
\hline \hline
\end{tabular}

\begin{tabular}{||c|ccc|c||}
\hline Element & Low & High & Ave. & $\begin{array}{c}\text { No. } \\
\text { Samples }\end{array}$ \\
\hline \hline $\mathrm{Mn}$ & $<.01$ & 0.4 & 0.09 & 16 \\
$\mathrm{Mo}$ & $<.01$ & 0.44 & 0.06 & 16 \\
$\mathrm{Ni}$ & .02 & 1.2 & 0.29 & 16 \\
$\mathrm{~K}$ & - & 310 & - & 1 \\
$\mathrm{Rb}$ & - & $<.02$ & - & 1 \\
$\mathrm{Si}$ & .02 & 0.1 & 0.06 & 2 \\
$\mathrm{~S}$ & - & 0.01 & - & 1 \\
$\mathrm{Sn}$ & $<.01$ & 0.2 & 0.04 & 14 \\
$\mathrm{Sm}$ & $<.01$ & $<.01$ & $<.01$ & 16 \\
$\mathrm{Ti}$ & $<.01$ & 0.04 & 0.02 & 16 \\
$\mathrm{U}$ & - & .002 & - & 1 \\
$\mathrm{~V}$ & $<.01$ & 0.04 & $0.02^{(b)}$ & 16 \\
$\mathrm{Zn}$ & - & $<.01$ & - & 1 \\
$\mathrm{P}$ & - & 0.2 & - & 1 \\
$\mathrm{O}$ & 0.41 & 2.8 & 1.48 & 11 \\
$\mathrm{H}$ & 0.27 & 0.08 & 0.14 & 10 \\
$\mathrm{C}$ & $<.05$ & 0.43 & 0.16 & 7 \\
\hline \hline
\end{tabular}

(a) J. J. McCown, G. R. Bloom, G. E. Meadows, and G. W. Mettler, "FFTF Sodium and Cover Gas Characterization and Purification," HEDL-SA-1874-FP (CONF-80040116), Proc. $2^{\text {nd }}$ Int. Conf. Liquid Metal Technology in Energy Production, Richland, WA, April 20-24 (February 1980).

(b) This value was omitted from the table for the primary coolant but included in the table for the secondary coolant measurements. All other values in this row were identical.

\section{Driver Fuel Assemblies (DFA)}

Two commercial vendors manufactured the driver fuel pins using the same specifications but different fabrication processes: Kerr-McGee (KM) Corporation and Babcock \& Wilcox/Nuclear Materials Division $(\mathrm{B} \& \mathrm{~W})$. The KM fuels were fabricated by a high-pressure preslugging method which resulted in a fuel structure with regions of high and low density separated by interconnected porosity. The B\&W fuels were produced by a low-pressure preslugging process with a pore former that resulted in fairly uniform fuel matrix with varying sizes of closed spherical pores. Although the microstructures were different, the fuel from both manufacturers was acceptable. ${ }^{a}$ Five hundred qualification fuel pins and

\footnotetext{
a J. W. Hales and R. B. Baker, "Evaluation of FFTF Reference Driver Fuel to $100 \mathrm{MWd} / \mathrm{kgM}$ Burnup," Proc. International Conference on Reliable Fuels for Liquid Metal Reactors, Tucson, Arizona, September 7-11 (1986).
} 
NEA/NSC/DOC(2006)1

\section{Liquid Metal Fast Reactor - LMFR \\ FFTF-LMFR-RESR-001 \\ CRIT-SPEC-REAC-COEF-MISC}

18,000 FFTF fuel pins were requested from the two vendors; they were approved to begin production in 1973 for these Core 1 and 2 fuel pins, and fabrication was completed in the fall of $1975 .^{\text {a }}$

The fuel was designed for a peak burnup of $80 \mathrm{MWd} / \mathrm{kg}$ and experienced no breaches below 100 $\mathrm{MWd} / \mathrm{kg}$. Some assemblies reached $120 \mathrm{MWd} / \mathrm{kg}$ before they had to be removed from the reactor due to duct distortion. Only a single rod breached at a burnup fluence of $103 \mathrm{MWd} / \mathrm{kg}$ and $16 \times 10^{22} \mathrm{n} / \mathrm{cm}^{2}$. Testing had shown that irradiation-induced swelling was the life-limiting phenomenon for the driver fuel assemblies, which induced elongation of the duct. Other distortions included bow and dilation, which affected rod withdrawal, depending on swelling, irradiation creep, and thermal creep effects. ${ }^{\text {b }}$ More than 48,000 pins had been irradiated of the series I and II driver fuel used in the first two core loadings of the FFTF. ${ }^{\mathrm{c}}$

The supplier contracts required that the fuel for the initial two core loadings were within $0.5 \%$ of the total requested fissile plutonium content. Fifty-eight fuel pin shipments were received, with an average of $\sim 695$ pins per shipment. The plutonium content was between 30 and 39 grams per pin with an approximate target of 37.14 and 30.46 grams of fissile plutonium per pin for the outer and inner core positions, respectively. Fifteen inner core pins were measured with chemical assay and determined to have a total $\mathrm{Pu}$ fissile mass of $447.68 \mathrm{~g}$, and each pin has a mean mass of $29.845 \pm 0.2261 \mathrm{~g}$. The outer core fuel pins (34 analyzed in total) had a total Pu fissile mass of $1240.83 \mathrm{~g}$ and a mean mass of 36.495 $\pm 0.2853 \mathrm{~g} .{ }^{\mathrm{d}}$ The actual date of this assay is unclear, but it occurred somewhere during the years of 1976 and 1977.

The FFTF driver fuel assemblies for the first two core designs contained 22.43 and 27.37 wt.\% $\mathrm{Pu} /(\mathrm{U}+\mathrm{Pu})$ for the inner and outer enrichment zones, respectively. The plutonium used had a fissile content of approximately $88 \mathrm{wt} . \%\left({ }^{239} \mathrm{Pu}+{ }^{241} \mathrm{Pu}\right)$. Natural uranium was used. Later reload driver fuel deviated from these compositions as operational requirements and feed material assays were changed (Ref. 5, pp. 3-13).

FFTF driver fuel pins identical to the type used in the isothermal physics experiments were used in various series of critical experiments, including water-flooded and water-reflected (Ref. A) ${ }^{\mathrm{e}}$ and also moderation/reflection with organic matter (Ref. B). ${ }^{f}$ Data provided from these experiments are useful in describing the geometry and physical properties of the driver fuel pins.

Two types of FFTF fuel pins were available for use in the critical experiments: Type 3.1 and Type 3.2. Composition of each type fuel pin is given in Figures 1.1.18 and 1.1.19. The data in Figure 1.1.19 are based on measured data from pellet lots used in fabricating the fuel pins and are more accurate than those reported in Figure 1.1.18. Physically, both types of fuel pins were essentially identical. A diagram giving both the common dimensions and those dimensions that differ between the two types of pins is also shown. Both types of fuel pins were $\sim 2.4 \mathrm{~m}$ in length. In either fuel pin, the actual fuel region was

a J. F. Williams and L. H. Rice, "Fuel Pin Fabrication for the FFTF," Trans. Am. Nucl. Soc., 34, 221-223 (1980).

${ }^{\mathrm{b}}$ R. D. Leggett and L. C. Walters, "Status of LMR Fuel Development in the United States of America," J. Nucl. Mater., 204, 23-32 (1993).

${ }^{\mathrm{c}}$ R. B. Baker, F. E. Bard, and J. L. Ethridge, "Performance of Fast Flux Test Facility Driver and Prototype Driver Fuels,” WHC-SA-0974, Proc. ANS Winter Meeting, Wasshington, D.C., November 11-15 (1990).

d P. Goris, “Rod Scanner Assay of FFTF Fuel,” HEDL-TME 77-29 (January 1978).

e B. M. Durst, S. R. Bierman, E. D. Clayton, J. F. Mincey, and R. T. Primm III, "Consolidated Fuel Reprocessing Program: Summary of Experimental Data for Critical Arrays of Water Moderated Fast Test Reactor Fuel," PNL3313, (May 1981).

${ }^{\mathrm{f}}$ S. R. Bierman, "Consolidated Fuel Reprocessing Program: Criticality Experiments with Fast Test Reactor Fuel Pins in an Organic Moderator,” PNL-5803, (December 1986). 


\section{Liquid Metal Fast Reactor - LMFR \\ FFTF-LMFR-RESR-001 \\ CRIT-SPEC-REAC-COEF-MISC}

restricted to a $\sim 91.4 \mathrm{~cm}$ length near the bottom of the pin. The remainder of the pin consisted of end caps and other types of hardware (Ref. A, p. 7 and Ref. B, p. 2.4).

The primary differences between the two types of fuel pins were in the composition of the $\mathrm{PuO}_{2}-\mathrm{UO}_{2}$ mixtures. The Type 3.1 fuel pins contained 24.39 wt.\% plutonium in the $\mathrm{PuO}_{2}-\mathrm{UO}_{2}$, while the Type 3.2 fuel pins contained $19.84 \mathrm{wt.} \%$ plutonium. The plutonium in either type of fuel pin contained $11.5 \mathrm{wt} . \%$ ${ }^{240} \mathrm{Pu}$ per total plutonium. The uranium in the $\mathrm{PuO}_{2}-\mathrm{UO}_{2}$ mixture was natural uranium for both the Type 3.1 and Type 3.2 fuel pins (Ref. A, p. 10 and Ref. B, pp. 2.4 and 2.8).

Material properties for various components of the DFA pins are shown in Figures 1.1.18 and 1.1.19. 
NEA/NSC/DOC(2006)1

Liquid Metal Fast Reactor - LMFR

FFTF-LMFR-RESR-001

CRIT-SPEC-REAC-COEF-MISC

\section{SIMPLIFIED DESCRIPTION OF FAST TEST REACTOR FUEL PIN}

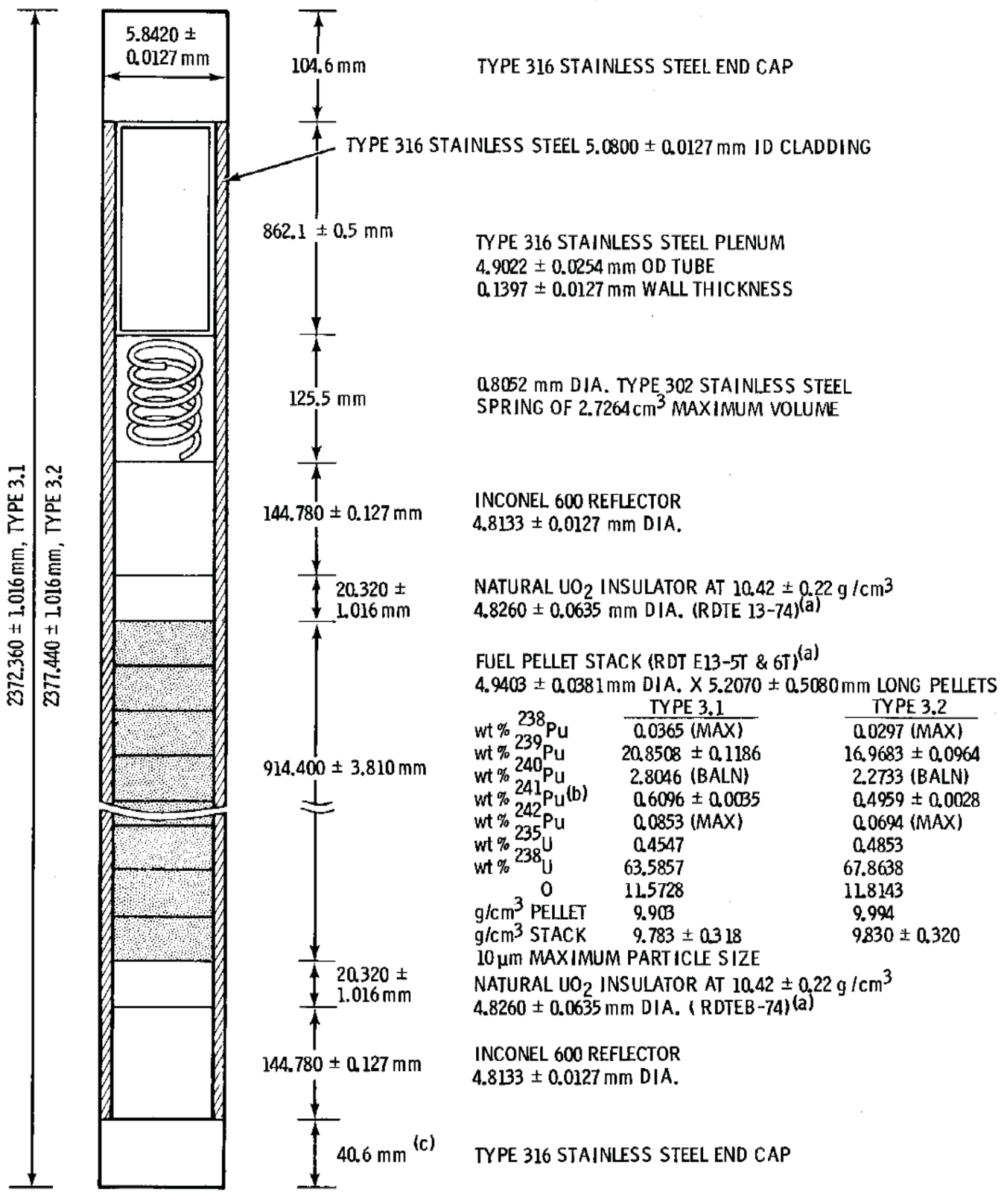

(a) Referenced Division of Reactor Development and Technology Standard

(b) 241 Am content of Total Plutonium was Measured to be $0.13 \mathrm{Wt} \%$ during April and May of 1972 .

(c) $35.6 \mathrm{~mm}$ for Type 3.1 Fuel Pins

Figure 1.1.18. Simplified Description of Fast Test Reactor Fuel Pin (Water-Reflected Experiments)

(Ref. A, Fig. 3). 
NEA/NSC/DOC(2006)1

Liquid Metal Fast Reactor - LMFR

FFTF-LMFR-RESR-001

CRIT-SPEC-REAC-COEF-MISC

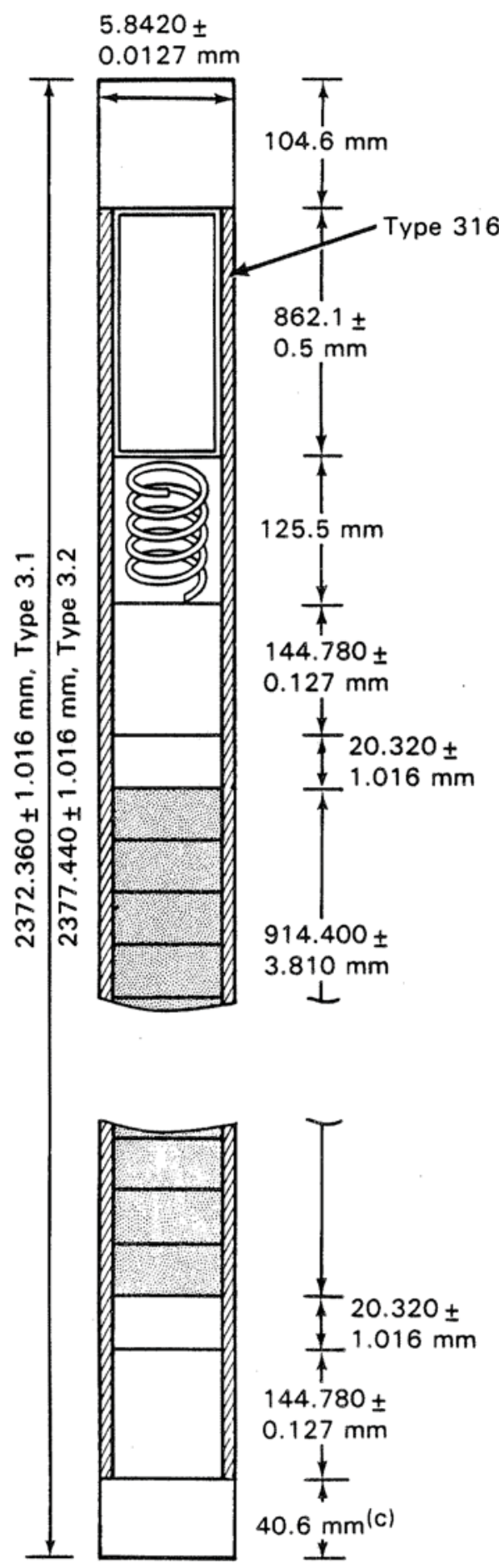

Type 316 Stainless Steel End Cap

$0.8052 \mathrm{~mm}$ Dia. Type 302 Stainless Steel Spring of $2.7264 \mathrm{~cm}^{3}$ Maximum Volume

Inconel 600 Reflector

$4.8133 \pm 0.0127 \mathrm{~mm}$ Dia.

Natural $\mathrm{UO}_{2}$ Insulator at $10.42 \pm 0.22 \mathrm{~g} / \mathrm{cm}^{3}$

$4.8260 \pm 0.0635 \mathrm{~mm}$ Dia. (RDT E13-7T)(a)

Fuel Pellet Stack (RDT E13-5T and 6T) (a)

$4.9403 \pm 0.0381 \mathrm{~mm}$ Dia. $\times 5.2070 \pm 0.5080 \mathrm{~mm}$ Long Pellets

\begin{tabular}{|c|c|c|}
\hline & Type $3.1^{\text {(b) }}$ & Type $3.2^{(b)}$ \\
\hline Wt\% & 0.0130 & 0.0111 \\
\hline Wt\% & 21.0641 & 17.1251 \\
\hline Wt $\%$ & 2.8606 & 2.3150 \\
\hline $\mathrm{Wt} \%{ }^{241} \mathrm{Pu}$ & 0.3461 & 0.2876 \\
\hline $\mathrm{Wt} \%{ }^{241} \mathrm{Am}$ & 0.0504 & 0.0597 \\
\hline Wt $\%{ }^{2}$ & 0.0526 & 0.0381 \\
\hline $\mathrm{Wt} \%{ }_{238}^{235} \mathrm{U}$ & 0.4547 & 0.4853 \\
\hline$W t \%{ }^{238} U$ & 63.5857 & 67.8638 \\
\hline 0 & 11.5728 & 11.8143 \\
\hline $\mathrm{g} / \mathrm{cm}_{3}^{3}$ Pellet & 9.903 & 9.994 \\
\hline $\mathrm{g} / \mathrm{cm}^{3}$ Stack & $9.783 \pm 0.318$ & $9.830 \pm 0.320$ \\
\hline
\end{tabular}

$10 \mu \mathrm{m}$ Maximum Particle Size

Natural $\mathrm{UO}_{2}$ Insulator at $10.42 \pm 0.22 \mathrm{~g} / \mathrm{cm}^{3}$

$4.8260 \pm 0.0635 \mathrm{~mm} \mathrm{Dia}$. (RDT E13-7T) ${ }^{(\mathrm{a})}$

Inconel 600 Reflector

$4.8133 \pm 0.0127 \mathrm{~mm}$ Dia.

Type 316 Stainless Steel End Cap

\footnotetext{
(a) Referenced Division of Reactor Development and Technology standard.

(b) Isotopics based on measured data from pellet lots. Americium-241 contents as of January 1, 1976 based on Plutonium-241 half life of 14.35 yrs.

(c) $35.6 \mathrm{~mm}$ for type 3.1 fuel pins.
}

Figure 1.1.19. Simplified Description of Fast Test Reactor Fuel Pin (Organic Moderated Experiments)

(Ref. B, Fig. 2.5). 
NEA/NSC/DOC(2006)1

\section{Liquid Metal Fast Reactor - LMFR}

FFTF-LMFR-RESR-001

CRIT-SPEC-REAC-COEF-MISC

For the initial two cores of the FFTF, 104 unique tags were prepared ( 80 for fuel assemblies and 24 for control assemblies). Five unique ratios of ${ }^{126} \mathrm{Xe} /{ }^{129} \mathrm{Xe}$ were used with nominal values of $0.0100,0.0122$, $0.0149,0.0182$, and 0.0222 . Corresponding to each xenon ratio was a set of ratios of ${ }^{82} \mathrm{Kr}{ }^{80} \mathrm{Kr}$ and ${ }^{78} \mathrm{Kr} /{ }^{80} \mathrm{Kr}$. The ratios are sufficiently separated that single assembly failures can be diagnosed without the aid of burnup analysis of the tag gas. After partial fuel reloading, two fuel assemblies with the same tag gas ratios can be distinguished via an "age tag", which is the ratio of ${ }^{129} \mathrm{Xe}^{124} \mathrm{Xe}$ that varies strongly with fuel burnup. ${ }^{a}$ Two milliliters of gas was needed for each tag gas canister, nominally consisting of $1 \mathrm{~mL}$ each of $\mathrm{Kr}$ and Xe isotopes. ${ }^{\mathrm{b}}$ An anticipated leak of $\sim 5 \%$ of the $2 \mathrm{~cm}^{3}$ (STP) initial loading would be sufficient to cause, in the $1.5 \times 10^{7} \mathrm{~cm}^{3}$ (STP) of argon cover gas, a concentration of at least $3 \mathrm{vppb}$ (volume parts per billion) of $\mathrm{Xe}$ or $\mathrm{Kr}$, considerably above the prescribed background levels of 0.25 and $0.24 \mathrm{vppb}$, respectively. Further discussion of the mixing ratios is presented elsewhere. ${ }^{\mathrm{c}}$

The bonding gas used during the fuel pin filling process is helium plus hydrogen with a concentration of $98 \%$, excluding the tag gas. It was required that at least $95 \%$ of the fuel pins had a helium plus hydrogen concentration no less that $95 \%$. The pressure of the bonding gas after fuel pin closure is $1.0 \pm 0.1 \mathrm{~atm}$ $(100 \pm 10 \mathrm{kPa})$ at room temperature. ${ }^{\mathrm{d}}$

The total impurities in the fuel pellets, excluding americium, were not to exceed $4000 \mu \mathrm{g} / \mathrm{g}$ by weight during manufacturing unless otherwise specified. Typical impurities are listed in Table 1.1.6. The oxygen-to-metal ratio was between 1.93:1 and 1.98:1 unless otherwise specified. Moisture content in the fuel pellets was to not exceed $20 \mu \mathrm{g} / \mathrm{g}$ of fuel. Fuel pellets were manufactured by cold pressing and sintering. No materials were added to the $(\mathrm{U}-\mathrm{Pu}) \mathrm{O}_{2}$ except for a small quantity of pore formers, organic binders, and organic lubricants needed for pelletization.

The total impurities in the uranium dioxide insulator pellets were not to exceed $3000 \mu \mathrm{g} / \mathrm{g}$ by weight during manufacturing unless otherwise specified. Typical impurities are listed in Table 1.1.7. The oxygen-to-metal ratio was between 1.995:1 and 2.010:1 unless otherwise specified. Moisture content in the fuel pellets was to not exceed $20 \mu \mathrm{g} / \mathrm{g}$ of fuel. Fuel pellets were manufactured by cold pressing and sintering. No materials were added to the $(\mathrm{U}-\mathrm{Pu}) \mathrm{O}_{2}$ except for a small quantity of pore formers, organic binders, and organic lubricants needed for pelletization. ${ }^{\mathrm{f}}$

The chemical composition requirements for the manufacture of the stainless steel 316 fuel cladding tubes are shown in Table 1.1.8. These requirements apply to all other fuel pin components comprised of stainless steel 316.

More than 80,000 cladding tubes were manufactured by Carpenter Technology Corporation, Special Products Division, for the first four cores of the FFTF. The tubes had the highest standards of technology, quality, and reliability for their manufacture. The tubes were formed from vacuum-melted type 316 stainless steel using a gun-drilled tube hollow manufacturing sequence through nine cold-drawn reduction stages. Bright annealing operations were incorporated after each drawing operation, except the

\footnotetext{
a N. J. McCormick, R. E.Schenter, and R. P. Omberg, "Gas Tag Design for Core I-IV of FFTF," Trans Am Nuc Soc, pp. 376-377, (1975).

${ }^{b}$ N. J. McCormick and R. E. Schenter, "Gas Tag Identification of Failed Fuel - I. Synergistic Use of Inert Gases," Nuc Tech, 24:149-155, (November 1974).

${ }^{c}$ N. J. McCormick, R. E. Schenter, and R. P. Omberg, "Gas Tag Identification of Failed Fuel - III. Tag Ratios for the Fast Flux Test Facility Cores I through IV,” Nuc Tech, 29:200-208 (May 1976).

${ }^{\mathrm{d}}$ NE Standard, Fast Breeder Reactor Fuel Pin, NE E 13-5T (January 1984).

${ }^{e}$ NE Standard, Fast Breeder Reactor Mixed Oxide Fuel Pellet, NE E 13-6T (January 1984).

${ }^{\mathrm{f}}$ NE Standard, Fast Breeder Reactor Uranium Dioxide Pellet, NE E 13-7T (January 1984).
} 


\section{Liquid Metal Fast Reactor - LMFR \\ FFTF-LMFR-RESR-001 \\ CRIT-SPEC-REAC-COEF-MISC}

final reduction, to incorporate $20 \%$ cold work into the finished tube. Ultrasonic testing and visual inspection were used to verify that the surfaces were free from defects. ${ }^{a}$

The driver fuel duct tubes were formed from seamless type 316 stainless steel hexagon sections fabricated to a $20 \pm 5 \%$ cold-work requirement, with further manufacturing overview provided elsewhere. ${ }^{\mathrm{b}}$

The chemical composition requirements for the manufacture of the Inconel 600 reflectors in the fuel pins are shown in Table 1.1.9.

a J. J. Inouye, "Manufacture of Driver Cladding Tubes for FFTF," Trans. Am. Nucl. Soc., 34, 217-218 (1980).

${ }^{b}$ D. R. Wozniak, “Fabrication of FFTF Driver Fuel Duct Tubes,” Trans. Am. Nucl. Soc., 34, 218-219 (1980). 
NEA/NSC/DOC(2006)1

Liquid Metal Fast Reactor - LMFR

FFTF-LMFR-RESR-001

CRIT-SPEC-REAC-COEF-MISC

Table 1.1.6. Fuel Pellet Impurity Limits. ${ }^{(a)}$

\begin{tabular}{|c|c|c|}
\hline Impurity Group & Element & $\begin{array}{c}\text { Maximum Impurity Limit } \\
(\mu \mathrm{g} / \mathrm{g})\end{array}$ \\
\hline 1 & $\begin{array}{c}\mathrm{Na} \\
\mathrm{K}\end{array}$ & $\begin{array}{c}\text { SUM }=700 \\
(\text { No one }>500)\end{array}$ \\
\hline 2 & $\begin{array}{l}\mathrm{Cl} \\
\mathrm{F}\end{array}$ & $\mathrm{SUM}=30$ \\
\hline 3 & $\begin{array}{l}\mathrm{C} \\
\mathrm{N}\end{array}$ & $\begin{array}{c}\text { SUM }=350 \\
(\text { No one }>200)\end{array}$ \\
\hline 4 & $\begin{array}{l}\mathrm{Sm} \\
\mathrm{Eu} \\
\mathrm{Gd} \\
\mathrm{Dy}\end{array}$ & SUM $=200$ \\
\hline 5 & $\begin{array}{l}\mathrm{Fe} \\
\mathrm{Cr} \\
\mathrm{Ni}\end{array}$ & $\begin{array}{c}\mathrm{SUM}=1500 \\
(\text { No one }>750)\end{array}$ \\
\hline 6 & $\begin{array}{l}\mathrm{P} \\
\mathrm{S}\end{array}$ & $\begin{array}{c}\mathrm{SUM}=400 \\
(\text { No one }>300)\end{array}$ \\
\hline 7 & $\begin{array}{l}\mathrm{Zn} \\
\mathrm{Pb} \\
\mathrm{Sn} \\
\mathrm{Cd}\end{array}$ & $\begin{array}{c}\text { SUM }=220 \\
(\text { No one }>100)\end{array}$ \\
\hline 8 & $\begin{array}{c}\mathrm{Be} \\
\mathrm{Ca} \\
\mathrm{Mg} \\
\mathrm{Al} \\
\mathrm{Si}\end{array}$ & $\begin{array}{c}\text { SUM }=1000 \\
(\text { No one }>500)\end{array}$ \\
\hline 9 & $\mathrm{~B}$ & 20 \\
\hline 10 & $\mathrm{Li}$ & 10 \\
\hline \multirow[t]{2}{*}{11} & $\begin{array}{c}\mathrm{Co} \\
\mathrm{Ti} \\
\mathrm{V} \\
\mathrm{Ta} \\
\mathrm{W} \\
\mathrm{Cu} \\
\mathrm{Ag} \\
\mathrm{Mo} \\
\mathrm{Mn}\end{array}$ & $\begin{array}{c}\mathrm{SUM}=1000 \\
(\text { No one }>500)\end{array}$ \\
\hline & $\mathrm{Zr}$ & 700 \\
\hline
\end{tabular}

(a) NE Standard, Fast Breeder Reactor Mixed Oxide Fuel Pellet, NE E 13-6T (January 1984). 
NEA/NSC/DOC(2006)1

Liquid Metal Fast Reactor - LMFR

FFTF-LMFR-RESR-001

CRIT-SPEC-REAC-COEF-MISC

Table 1.1.7. Insulator Pellet Impurity Limits. ${ }^{(a)}$

\begin{tabular}{|c|c|c|}
\hline Impurity Group & Element & $\begin{array}{c}\text { Maximum Impurity Limit } \\
(\mu \mathrm{g} / \mathrm{g})\end{array}$ \\
\hline 1 & $\begin{array}{l}\mathrm{Na} \\
\mathrm{K}\end{array}$ & $\begin{array}{c}\text { SUM }=700 \\
(\text { No one }>500)\end{array}$ \\
\hline 2 & $\begin{array}{l}\mathrm{Cl} \\
\mathrm{F}\end{array}$ & $\mathrm{SUM}=30$ \\
\hline 3 & $\begin{array}{l}\mathrm{C} \\
\mathrm{N} \\
\end{array}$ & $\begin{array}{c}\text { SUM }=350 \\
(\text { No one }>200)\end{array}$ \\
\hline 4 & $\begin{array}{l}\mathrm{Sm} \\
\mathrm{Eu} \\
\mathrm{Gd} \\
\mathrm{Dy}\end{array}$ & $\mathrm{SUM}=200$ \\
\hline 5 & $\begin{array}{l}\mathrm{Fe} \\
\mathrm{Cr} \\
\mathrm{Ni}\end{array}$ & $\begin{array}{c}\mathrm{SUM}=1500 \\
(\text { No one }>750)\end{array}$ \\
\hline 6 & $\begin{array}{l}\mathrm{P} \\
\mathrm{S}\end{array}$ & $\begin{array}{c}\text { SUM }=400 \\
(\text { No one }>300)\end{array}$ \\
\hline 7 & $\begin{array}{l}\mathrm{Zn} \\
\mathrm{Pb} \\
\mathrm{Sn} \\
\mathrm{Cd}\end{array}$ & $\begin{array}{c}\text { SUM }=220 \\
(\text { No one }>100)\end{array}$ \\
\hline 8 & $\begin{array}{c}\mathrm{Be} \\
\mathrm{Ca} \\
\mathrm{Mg} \\
\mathrm{Al} \\
\mathrm{Si} \\
\end{array}$ & $\begin{array}{c}\mathrm{SUM}=1000 \\
(\text { No one }>500)\end{array}$ \\
\hline 9 & $\mathrm{~B}$ & 20 \\
\hline 10 & $\mathrm{Li}$ & 10 \\
\hline 11 & $\begin{array}{c}\mathrm{Co} \\
\mathrm{Ti} \\
\mathrm{V} \\
\mathrm{Ta} \\
\mathrm{W} \\
\mathrm{Cu} \\
\mathrm{Ag} \\
\mathrm{Mo} \\
\mathrm{Mn} \\
\mathrm{Zr}\end{array}$ & $\begin{array}{c}\text { SUM }=1000 \\
(\text { No one }>500)\end{array}$ \\
\hline
\end{tabular}

(a) NE Standard, Fast Breeder Reactor Uranium Dioxide Pellet, NE E 13-7T (January 1984). 
Liquid Metal Fast Reactor - LMFR

FFTF-LMFR-RESR-001

CRIT-SPEC-REAC-COEF-MISC

Table 1.1.8. Cladding Alloy Composition (weight percent). ${ }^{(a)}$

\begin{tabular}{||c|c||}
\hline Chromium & $17.0-18.0$ \\
Nickel & $13.0-14.0$ \\
Carbon & $0.04-0.06$ \\
Molybdenum & $2.0-3.0$ \\
Manganese & $1.5-2.0$ \\
Nitrogen & 0.01 max. \\
Aluminum & 0.05 max. \\
Arsenic & 0.03 max. \\
Boron & 0.001 max. \\
Cobalt & 0.05 max. \\
Columbium & 0.05 max. \\
(Niobium) & 0.10 max. \\
Copper & 0.02 max. \\
Phosphorus & 0.75 max. \\
Silicon & 0.01 max. \\
Sulfur & 0.01 max. \\
Tantalum & 0.2 max. \\
Vanadium & Balance \\
Iron & Driv Fun Pan \\
\hline \hline
\end{tabular}

(a) NE Standard, Fast Flux Test Facility Driver Fuel Pin Seamless Cladding Tube, NE E 13-8T (June 1971). 
NEA/NSC/DOC(2006)1

Liquid Metal Fast Reactor - LMFR

FFTF-LMFR-RESR-001

CRIT-SPEC-REAC-COEF-MISC

Table 1.1.9. Inconel 600 Composition (weight percent). ${ }^{(a)}$

\begin{tabular}{|c|c|}
\hline Nickel & $72.0 \mathrm{~min}$. \\
(including Cobalt) & $14.0-17.0$ \\
Chromium & $6.0-10.0$ \\
Iron & $1.5-2.0$ \\
Manganese & 1.0 max. \\
Carbon & 0.08 max. \\
Copper & 0.50 max. \\
Silicon & 0.015 max. \\
Sulfur & 0.20 max. \\
Cobalt & \\
\hline
\end{tabular}

(a) NE Standard, Fast Flux Test Facility Driver Fuel Pin Reflectors, NE E 13-10T (June 1971).

\section{Fuels Open Test Assemblies (FOTA)}

These were considered identical to standard (non-instrumented) DFA for nuclear analysis (Ref. 3, p. 5).

\section{Vibration Open Test Assembly (VOTA)}

No additional information is currently available.

\section{$\underline{\text { In-Reactor Thimble (IRT) }}$}

The IRT was a stainless steel 304 pipe, typically empty except during zero power physics measurements (Ref. 3, p. 11). The fission chambers in the SCH were built by Reuter-Stokes, Inc. (Model RS-C3-2510114), each containing $1.3 \mathrm{~g}$ uranium-235 (Ref. 1, p. 3) with neutron sensitivity estimated at 0.7 cps/equivalent thermal neutron flux at a $1 \mathrm{cps}$ alpha-plus-noise cutoff discriminator setting (Ref. 10, p. 17).

\section{Absorber Assemblies}

The pellets in each absorber assembly were made of $\mathrm{B}_{4} \mathrm{C}$ (Ref. 3, p. 7). The boron enrichment was that of natural boron, $19.78 \%{ }^{10} \mathrm{~B}$. The pellet density was $92 \pm 2 \%$ of theoretical. The cladding on the control rod absorber pins was composed of $20 \%$ cold-drawn stainless steel 316 (Ref. 5, pp. 3-19).

The accuracy of the absorber stack weight was $\pm 0.1 \mathrm{~g}$ at the $95 \%$ confidence level. The pressure of the bonding gas during final closure was $1.0 \pm 0.1 \mathrm{~atm}$ absolute $(100 \pm 10 \mathrm{kPa}$ absolute $)$ at room temperature. ${ }^{\mathrm{a}}$

The impurity limits for the absorber pellets are listed in Table 1.1.10. Boron carbide powder was prepared by reacting boron and carbon, $\mathrm{B}_{2} \mathrm{O}_{3}$ and carbon, or boron $/ \mathrm{B}_{2} \mathrm{O}_{3}$ and carbon with a formed pellet boron concentration of $78.0 \pm 1.0$ weight percent, unless otherwise specified. Exclusive of soluble boron, the impurity sum in the pellets was not to exceed $5000 \mu \mathrm{g} / \mathrm{g}$ with $90 \%$ confidence. The boron to total

${ }^{a}$ NE Standard, Control Rod Absorber Pin for Liquid Metal Fast Breeder Reactors, NE E 6-25T (January 1977).

Revision: 0

Page 38 of 304

Date: March 31, 2010 
NEA/NSC/DOC(2006)1

Liquid Metal Fast Reactor - LMFR

FFTF-LMFR-RESR-001

CRIT-SPEC-REAC-COEF-MISC

carbon ratio in the finished pellets is $4.00 \pm 0.15$ with $90 \%$ confidence. The moisture content of a single pellet was to not exceed $50 \mu \mathrm{g} / \mathrm{g}^{\text {a }}$

Table 1.1.10. Absorber Pellet Impurity Limits. ${ }^{(a)}$

\begin{tabular}{|c|cc||}
\hline Impurity & $\begin{array}{c}\text { Maximum Lot } \\
\text { Average Concentration } \\
\text { in } \mu \mathrm{g} / \mathrm{g}(\mathrm{mg} / \mathrm{kg})\end{array}$ & $\begin{array}{c}\text { Maximum Individual } \\
\text { Pellet Concentration } \\
\text { in } \mu \mathrm{g} / \mathrm{g}(\mathrm{mg} / \mathrm{kg})\end{array}$ \\
\hline \hline Soluble carbon & 10000 & 15000 \\
$\mathrm{HNO}_{3}$ soluble boron & 3000 & 1500 \\
$\mathrm{HCl}$ soluble boron & 1500 & 2000 \\
$\mathrm{Al}$ & 1000 & 1500 \\
$\mathrm{Ca}$ & 1000 & 1500 \\
$\mathrm{Cr}$ & 300 & 500 \\
$\mathrm{Fe}$ & 3000 & 4500 \\
$\mathrm{Mg}$ & 500 & 750 \\
$\mathrm{Mn}$ & 200 & 300 \\
$\mathrm{~N}$ & 4000 & 5000 \\
$\mathrm{Ni}$ & 300 & 500 \\
$\mathrm{Si}$ & 2000 & 3000 \\
$\mathrm{Ti}$ & 300 & 500 \\
$\mathrm{Cl}$ & 25 & 40 \\
$\mathrm{~F}$ & 15 & 25 \\
\hline \hline
\end{tabular}

(a) NE Standard, Absorber Pin Boron Carbide Pellet, NE E 6-30T (September 1976).

\section{$\underline{\text { Radial Reflectors }}$}

Reflector assemblies were composed of a section of Inconel 600 and upper and lower shield sections of stainless steel 316 (Ref. 5, pp. 3-20).

\section{$\underline{\text { In-Core Shim Assemblies }}$}

An ICSA was a stainless-steel component with stainless steel simulated fuel pins (Ref. 5, pp. 3-20).

\subsubsection{Reported Uncertainties}

The isotopic fuel compositions, which were based on measured fuel pellet stack weights, pellet lot chemical analysis, and $\mathrm{Pu}$ lot mass spectrometric analyses, were estimated to be accurate to within $0.3 \%$ of the quoted values (Ref. 34, p. 26). A high resolution gamma spectrometer was used to measure the

${ }^{a}$ NE Standard, Absorber Pin Boron Carbide Pellet, NE E 6-30T (September 1976). 
NEA/NSC/DOC(2006)1

\section{Liquid Metal Fast Reactor - LMFR \\ FFTF-LMFR-RESR-001 \\ CRIT-SPEC-REAC-COEF-MISC}

plutonium isotopic ratios. Nondestructive assay with x-ray fluorescence was used to determine uranium and plutonium compositions with a precision of $0.3 \%$ or better. $^{a}$

The ${ }^{10} \mathrm{~B}$ control rod loadings, derived from similar manufacturing data, were estimated to be accurate to within $0.5 \%$ (Ref. 34, p. 26). Variation in the absorber content of control assemblies is illustrated by the following data (Table 1.1.11) for individual assemblies used in the isothermal physics tests (Ref. 3, p. 24).

Table 1.1.11. Absorber Assembly As-Built Loading (Ref. 3, p. 24).

\begin{tabular}{||cccc||}
\hline $\begin{array}{c}\text { Absorber Assembly } \\
\text { Serial Number }\end{array}$ & $\begin{array}{c}\text { Core } \\
\text { Position }\end{array}$ & $\begin{array}{c}{ }^{10} \mathrm{~B} \text { mass } \\
(\mathrm{g})\end{array}$ & $\begin{array}{c}\mathrm{B}_{4} \mathrm{C} \text { mass } \\
(\mathrm{g})\end{array}$ \\
\hline \hline 528 & 1302 & 1233 & 8452 \\
529 & 2302 & 1236 & 8439 \\
530 & 3302 & 1235 & 8432 \\
536 & 1504 & 1240 & 8465 \\
537 & 1502 & 1239 & 8459 \\
538 & 2502 & 1240 & 8463 \\
539 & 2504 & 1240 & 8454 \\
540 & 3502 & 1239 & 8448 \\
541 & 3504 & 1241 & 8456 \\
\hline
\end{tabular}

The dimensional measurements of the driver fuel assemblies were made along the assembly longitudinal axis, in the vertical plane, using laser interferometers. The limitations included up to a maximum bow in any $1-\mathrm{ft}(30.48-\mathrm{cm})$ section of 0.015 in $(0.0381 \mathrm{~cm})$, a maximum deviation from centerline at wear pad location of 0.050 inches $(0.127 \mathrm{~cm})$, a $0^{\circ} 50^{\prime}$ twist, and $142+0.040 /-0.080$ inches $(360.68+0.1016 /-$ $0.2032 \mathrm{~cm})$ overall length. The resolution of the interferometer was \pm 1 micro-inch $(0.0254 \mu \mathrm{m})$ and accuracy was well within \pm 0.0008 inches $( \pm 0.002032 \mathrm{~cm})$. The fuel pin OD gauge was a commercially available non-contacting laser device with an accuracy of \pm 0.0001 inches $( \pm 0.000254 \mathrm{~cm})$. A laser optical system with diode arrays could measure the length, diameter, and surface flaws of the pellets with a precision of \pm 0.0003 inches $( \pm 0.000762 \mathrm{~cm}), \pm 0.002$ inches $( \pm 0.00508 \mathrm{~cm})$, and $>0.006$ inches $(>0.01524 \mathrm{~cm})$. A commercial weigh scale with a precision of $\pm 5 \mathrm{mg}$ was used to measure the mass of the pellets. ${ }^{\mathrm{b}}$

\subsubsection{Temperature Data}

Experiments were performed at approximately $400{ }^{\circ} \mathrm{F}\left(\sim 204^{\circ} \mathrm{C}\right)$. Extrapolated critical rod positions in Table E.1.2 report the temperature of the fully loaded core during initial criticality as $404^{\circ} \mathrm{F}\left(\sim 207^{\circ} \mathrm{C}\right)$ and the temperature of the partially loaded core as $406^{\circ} \mathrm{F}\left(\sim 208^{\circ} \mathrm{C}\right)$.

\footnotetext{
${ }^{a}$ P. Goris, G. B. Frandsen, G. P. Gottschalk, M. C. Lambert, and J. A. Petty, "Nondestructive Assay of Plutonium Fuel for FFTF and Supporting Operations," HEDL-SA-2428-FP (CONF-811103-114), Proc. ANS Winter Meeting, San Francisco, CA, November 29 - December 4 (1981).

${ }^{\text {b }}$ H. G. Powars, D. R. McLemore, T. L. Kirchner, and G. P. Gottschalk, "Inspection Tehniques and Processes for Controlling FFTF Fuel Quality”, HEDL-SA-1593-FP (CONF-781105-82), Proc. ANS Winter Meeting, Washington, D.C., November (October 18, 1978).
} 
NEA/NSC/DOC(2006)1

Liquid Metal Fast Reactor - LMFR

FFTF-LMFR-RESR-001

CRIT-SPEC-REAC-COEF-MISC

During later full-power operations of the FFTF, a minimum temperature of $400{ }^{\circ} \mathrm{F}\left(\sim 204{ }^{\circ} \mathrm{C}\right)$ was required at the dump heat exchangers for reactor operation. The coolant passing through the core in the primary loop would have been at a much higher temperature. ${ }^{a}$ However, during the initial isothermal tests, the core was not in power operations and the entire primary system was held at a constant temperature of $400^{\circ} \mathrm{F}\left(\sim 204^{\circ} \mathrm{C}\right)$ using pump power. ${ }^{b}$

\subsubsection{Additional Information Relevant to Critical and Subcritical Measurements}

Additional information is not available.

\subsection{Description of Buckling and Extrapolation Length Measurements}

Buckling and extrapolation length measurements were not made.

\section{$1.3 \quad$ Description of Spectral Characteristics Measurements}

\subsubsection{Overview of Experiment}

The FFTF was operated by Westinghouse Hanford Company for the U.S. Department of Energy. During the period from November 27, 1979 to March 8, 1980, fuel was loaded into the FFTF core, initial criticality was achieved, and several subcritical physics measurements were performed (Ref. 1, p.1).

As part of the FFTF program, characterization measurements, including the measurement of neutron spectra by proton-recoil methods, were made during cold startup of the FFTF. The characterization testing was made possible by use of a special facility, the In-Reactor Thimble (IRT) inserted in one of the test locations near the center of the core. The IRT was fabricated and operated by HEDL, and a measurement program utilizing the IRT was initiated by HEDL. Results of one part of that program involving the measurement of neutron spectra by proportional counters was a continuation of ANL involvement with the physics of the FFTF initiated through the FFTF Engineering Mock-up criticalfacility program carried out at ANL in the late 1960's and early 1970's. ${ }^{\circ}$ The measurements were performed during the last week of February 1980, shortly after the FFTF had achieved criticality, but prior to any operation at a significant power level (Ref. 6. p. 1).

The spectra in the energy range of $5 \mathrm{keV}$ to $2 \mathrm{MeV}$ were measured with proportional counters at core midplane and in the lower axial shield region. Later nuclear emulsions were used to measure the spectra in the range of 0.8 to $10 \mathrm{MeV}$ at the same locations using the same experimental insert (Ref. 2, p. 35).

The reactor physics experiments evaluated from this section pertain to two neutron spectra measurements performed in the IRT near the radial center of the core at core midplane and $80 \mathrm{~cm}$ below core midplane using proportional counter detectors.

\footnotetext{
${ }^{a}$ Personal Communication with A. Nichole Ellis from Ellis Nuclear Engineering, LLC (October 20, 2009).

${ }^{b}$ Personal Communication with David W. Wootan at Pacific Northwest National Laboratory (November 10, 2009).

${ }^{\text {c }}$ P. A. Ombrellaro, R. A. Bennett, J. W. Daughtry, K. D. Dobbin, R. A. Harris, J. W. Nelson, R. E. Peterson, and R. B. Rothrock, "Biases for Current FFTF Calculational Methods," HEDL-SA-1393 (CONF-780401-9), Proc. Advances in Reactor Physics, Gatlinburg, TN, April 9 (January 1978).
} 
NEA/NSC/DOC(2006)1

Liquid Metal Fast Reactor - LMFR

FFTF-LMFR-RESR-001

CRIT-SPEC-REAC-COEF-MISC

\subsubsection{Geometry of the Experiment Configuration and Measurement Procedure}

The geometry of the core is that of the fully-loaded core configuration in Section 1.1.2 with modifications as stated below.

\section{In-Reactor Thimble (IRT)}

An in-reactor thimble was installed in core position 3202 to provide access near the core center for a variety of instruments used in core monitoring and characterization. This facility was only used during very low power operation, and was subsequently removed from the reactor. In the vicinity of the core, the IRT had the configuration of a 4 -inch $(10.16-\mathrm{cm})$ ID stainless steel pipe, with a 0.25 -inch $(0.635-\mathrm{cm})$ wall thickness. The interior of the pipe was essentially void when no experimental equipment was installed. During measurements using the IRT, shield plugs were ordinarily installed above and below the fueled region to reduce streaming. The IRT was typically empty during most of the zero power physics measurements (Ref. 3, p. 11).

The interior of the IRT was insulated from the reactor sodium coolant by a 40-ft (12.192-m) long vacuum bottle that is part of the Instrument Cooling System (ICS). The interior space was cooled by continuous flushing with cooled gaseous nitrogen, providing an instrument operating temperature of about $40{ }^{\circ} \mathrm{F}(\sim 4$ $\left.{ }^{\circ} \mathrm{C}\right)$ at the core center with the reactor operating at a temperature of about $400^{\circ} \mathrm{F}\left(\sim 204^{\circ} \mathrm{C}\right),(\operatorname{Ref} 2, \mathrm{p} .65)$.

Figure 1.3.1 shows the In Reactor Thimble (IRT) placed in the core for spectrum measurements. The IRT is shown in slightly more detail in Figure 1.3.2. Spectrum measurements were performed at the core midplane position and at a position $80 \mathrm{~cm}$ below the midplane in the lower axial reflector. A fission counter monitor was located in the upper axial reflector. The proton-recoil instrumentation rack and the IRT cooling system were located on the operating deck. Measurements were made shortly after the initial critical core loading and before any power runs to assure that the background from core activation was at a minimum (Ref. 6, p. 1). 
FFTF-LMFR-RESR-001

\section{IN-REACTOR THIMBLE (IRT)}

(IN POSITION)

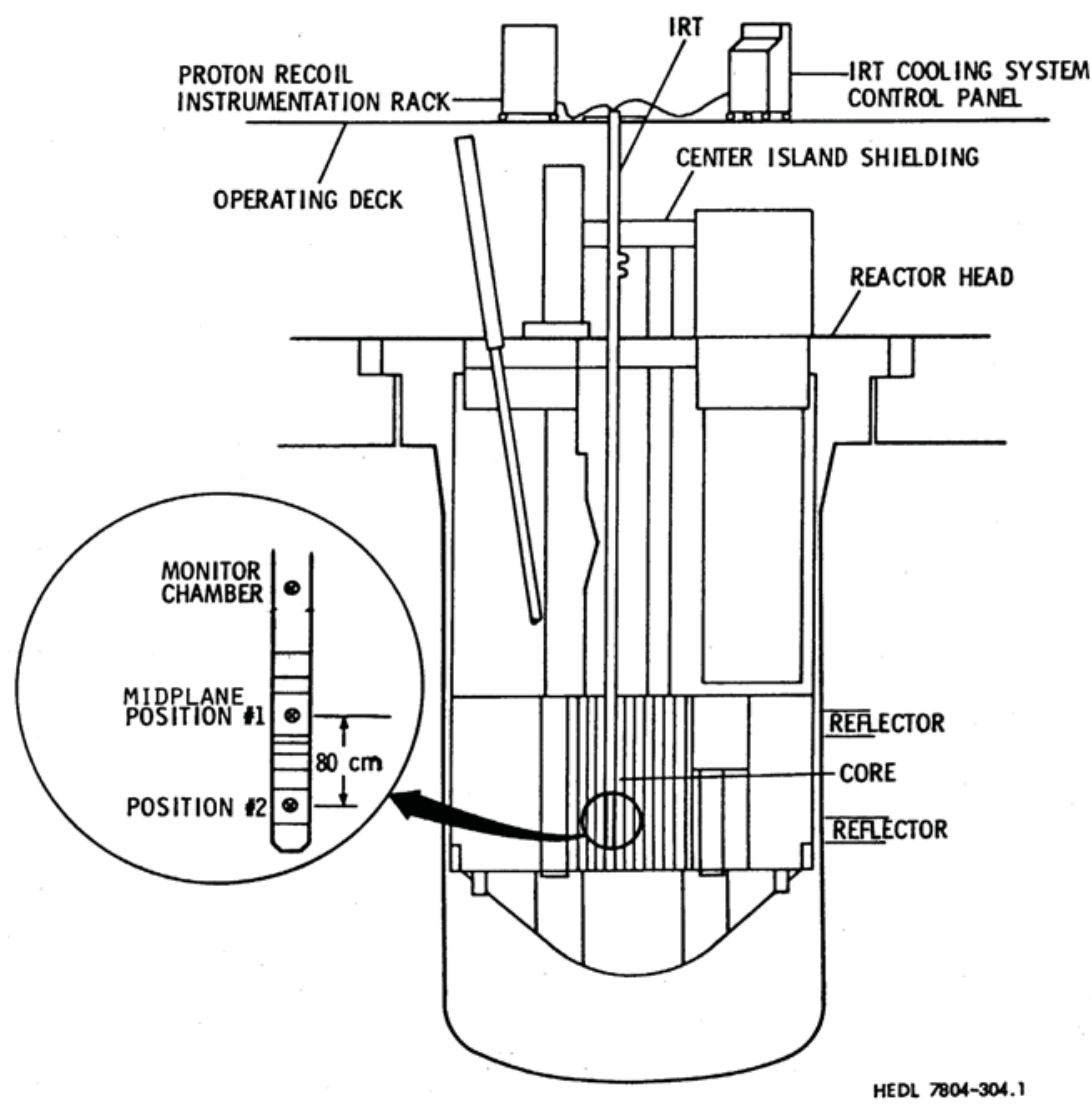

Figure 1.3.1. In-Reactor Thimble Installed in the FFTF (Ref. 6, Fig. 1). 


\section{IN - REACTOR THIMBLE AND INSTRUMENT COOLING SYSTEM SCHEMATIC}

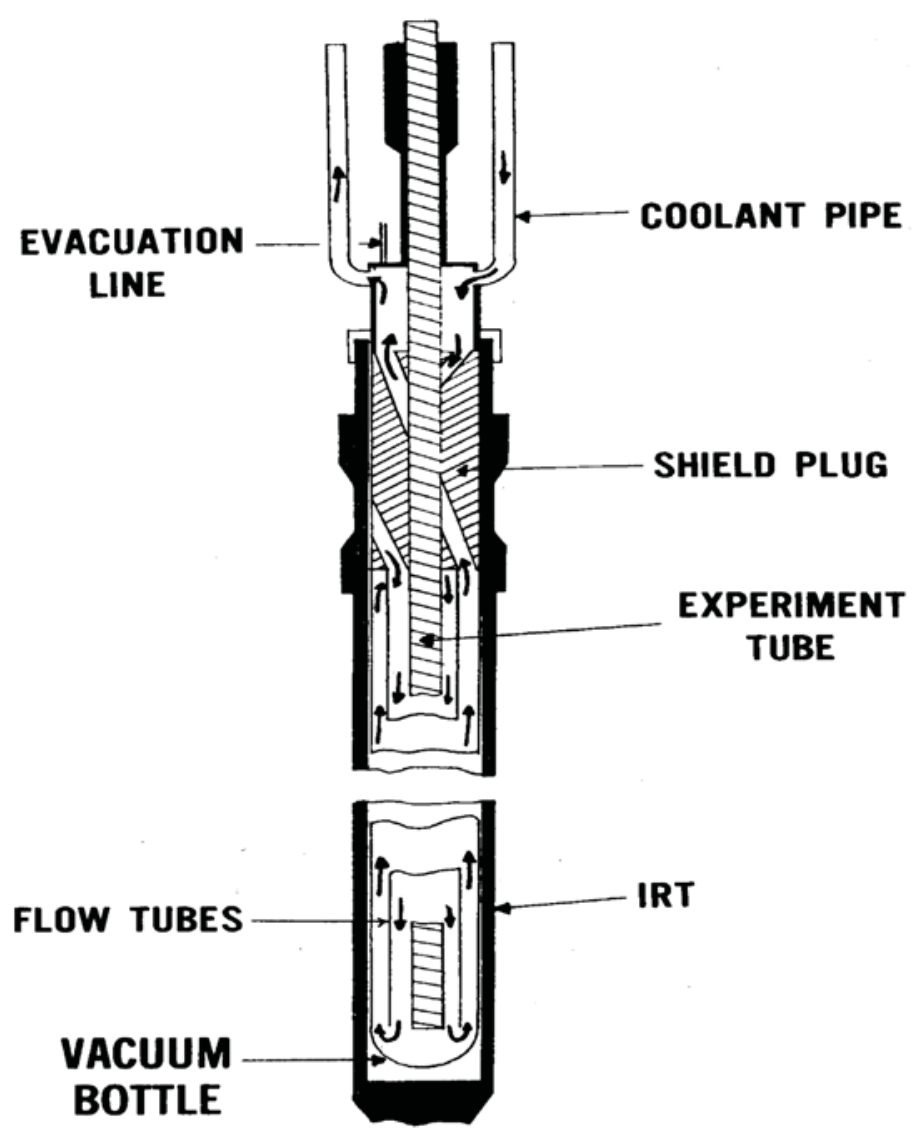

Figure 1.3.2. In-Reactor Thimble Schematic Diagram (Ref. 6, Fig. 2).

\section{Detectors}

A pair of proportional counters, mounted side-by-side, was used for the measurements at each of the two locations. One counter contained predominantly hydrogen gas for use below $\sim 100 \mathrm{keV}$; the other contained a mixture of methane and argon for neutron energies ranging from $100 \mathrm{keV}$ to several $\mathrm{MeV}$. A specially-designed preamplifier was used, which was suited to the limited space available in the IRT. The detector pair was input in parallel to the preamplifier and either could be enabled by attaching its bias-voltage cable to a voltage supply. The detectors and preamplifier were mounted in close proximity on a rigid frame to reduce microphonic sensitivity. The unit was electrically insulated from the experiment tube by the use of a small amount of soft rubber pad; this pad also provided acoustical isolation of the detector units from the experiment tube. A common ground for both detector units, together with signal and voltage leads, was run through the experiment tube to the data acquisition system (Ref. 6, p. 1). The methane/argon detectors were adequate for measurements extending to about 2 $\mathrm{MeV}$. The hydrogen gas detectors contained a small amount of methane for quenching (Ref. 6, p. 3).

The two types of counters had the same internal size with a $2.5-\mathrm{cm}$ effective length and $1.27-\mathrm{cm}$ diameter. Filling pressures were $\sim 7 \mathrm{~atm}$; operating bias voltages ranged from 3300 to 4600 volts. The relative proton content was selected to provide approximately equal maximum counting rates in the 
NEA/NSC/DOC(2006)1

Liquid Metal Fast Reactor - LMFR

FFTF-LMFR-RESR-001

CRIT-SPEC-REAC-COEF-MISC

central spectrum, allowing for a complete measurement without changing the reactor power. Pulseamplitude spectra were measured over a range of discrete bias-voltage settings; the composite protonrecoil spectrum was then used to derive the neutron spectrum. The measured spectra are absolute since the number of hydrogen atoms, the effective volume, and the $(n, p)$ elastic scattering cross section are all known (Ref. 6, p. 2).

The actual mechanical design of the counters is shown in Figure 1.3.3. The proportional counters were fabricated from a pair of ceramic electrical insulators attached to an adapter at each end. Stainless steel was used for the end adapters and for the thin-walled $(0.15-\mathrm{mm}$ thick) connecting tube which formed the cathode. The ceramic insulators are attached to the end adapters with high-melting soft solder. A 0.20$\mathrm{mm}$ O.D. brass field tube was inserted concentric with the adapter and soldered into place with one end flush with the adapter end. A length of stainless steel anode wire (0.0178 to $0.0254-\mathrm{mm}$ diameter) was threaded through the field tubes and the cathode tube before being soldered in place. The effective length of the counting region was $2.54 \mathrm{~cm}$, and the I.D. of the counter over the effective counting region was $1.27 \mathrm{~cm}$. The diameter of the end adapter over the field tube was reduced to $0.76 \mathrm{~cm}$ for improved electrical-field shaping. The counter was inserted into an outer cylindrical pressure containment tube with an O.D. of $1.44 \mathrm{~cm}$ and a wall thickness of $0.25 \mathrm{~mm}$. A wire was lead through a ceramic seal beam welded at one end of the pressure tube and soldered in place. At the other end of the outer pressure tube, a brass adapter was hard soldered to a cap, which was then beam welded to the outer pressure tube. A piece of $0.47-\mathrm{cm}$ O.D. soft copper pumpout tubing was soft soldered to the brass adapter and cleaning solvents were introduced into the counter to remove residual flux, etc. After filling to the desired gas pressure, the soft-copper pumpout tube was pinched off. Before using the counters in a reactor flux environment, lead foil about $0.5 \mathrm{~mm}$ thick was wrapped around the outer pressure tube over the sensitive region of the counter to provide adequate shielding again x-rays and soft photons to prevent increased gamma sensitivity of the detector. Table 1.3.1 contains a list of the composition and approximate masses of the complete counting tube (Ref. 6, p. 3).

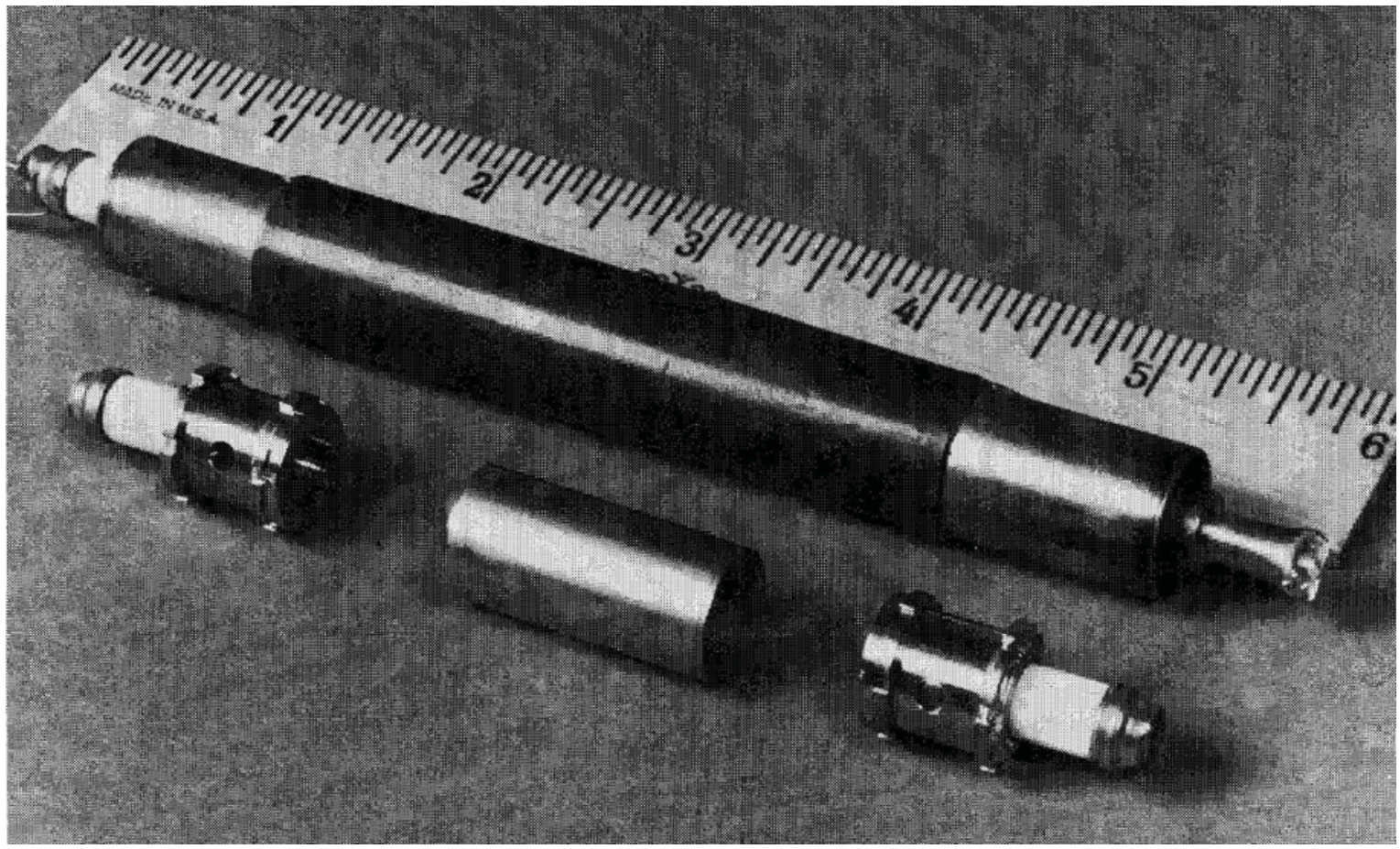

Figure 1.3.3. Component Layout of the Proton-Recoil Proportional Counter (Ref. 6, Fig. 3). 
NEA/NSC/DOC(2006)1

Liquid Metal Fast Reactor - LMFR

FFTF-LMFR-RESR-001

CRIT-SPEC-REAC-COEF-MISC

Table 1.3.1. Proton-Recoil Counter Component Mass Breakdown (Ref. 6, p. 27).

\begin{tabular}{|c|c|}
\hline Component & Mass (grams) \\
\hline \hline Interior Ceramic Seals & 2.4 \\
Interior End Adapters & 10.0 \\
Interior Cathode Tube & 1.2 \\
Pressure Tube & 10.7 \\
Pressure Tube End & 11.5 \\
Seals and Adapters & \\
Lead Foil Over & 20.6 \\
Pressure Tube & \\
\hline
\end{tabular}

Table 1.3.2 contains information regarding the proportional counters used in the IRT. The effective volume of all counters is approximately $3.01 \mathrm{~cm}^{3}( \pm 1 \%)$. Figures 1.3.4 and 1.3.5 show a detector package in place in the instrument canister; the steel tubing enclosing the package has been slipped back to reveal the detectors and electronics. Although the entire package was electrically insulated, some extraneous electrical noise was observed in the axial detector package and not the center detectors. No explanation could be determined; the disturbance magnitude did not prevent measurement in the axial location from being completed (Ref. 6, pp. 3-4).

Table 1.3.2. Counter Operating Specifications (Ref. 6, p. 28).

\begin{tabular}{|cccc||}
\hline Type & $\begin{array}{c}\text { Fill Gas in } \\
\text { Atm. at } 0^{\circ} \mathrm{C}\end{array}$ & $\begin{array}{c}\text { Anode } \\
\text { Diameter }\end{array}$ & $\begin{array}{c}\text { Operating } \\
\text { Voltages }\end{array}$ \\
\hline \hline \multirow{3}{*}{ Hydrogen } & $\mathrm{H}_{2}: 6.242$ & & $3350,3550,3750$, \\
& $\mathrm{CH}_{4}: 0.064$ & $0.0254 \mathrm{~mm}$ & $3950,4150,4300$, \\
& $\mathrm{N}_{2}: 0.064$ & & 4450 \\
\hline \multirow{3}{*}{ Methane } & $\mathrm{CH}_{4}: 6.137$ & & $3000,3200,3400$, \\
& $\begin{array}{c}\text { Ar: } 2.078 \\
\mathrm{~N}_{2}: 0.145\end{array}$ & $0.0178 \mathrm{~mm}$ & 3600 \\
\hline \hline
\end{tabular}


NEA/NSC/DOC(2006)1

Liquid Metal Fast Reactor - LMFR

FFTF-LMFR-RESR-001

CRIT-SPEC-REAC-COEF-MISC

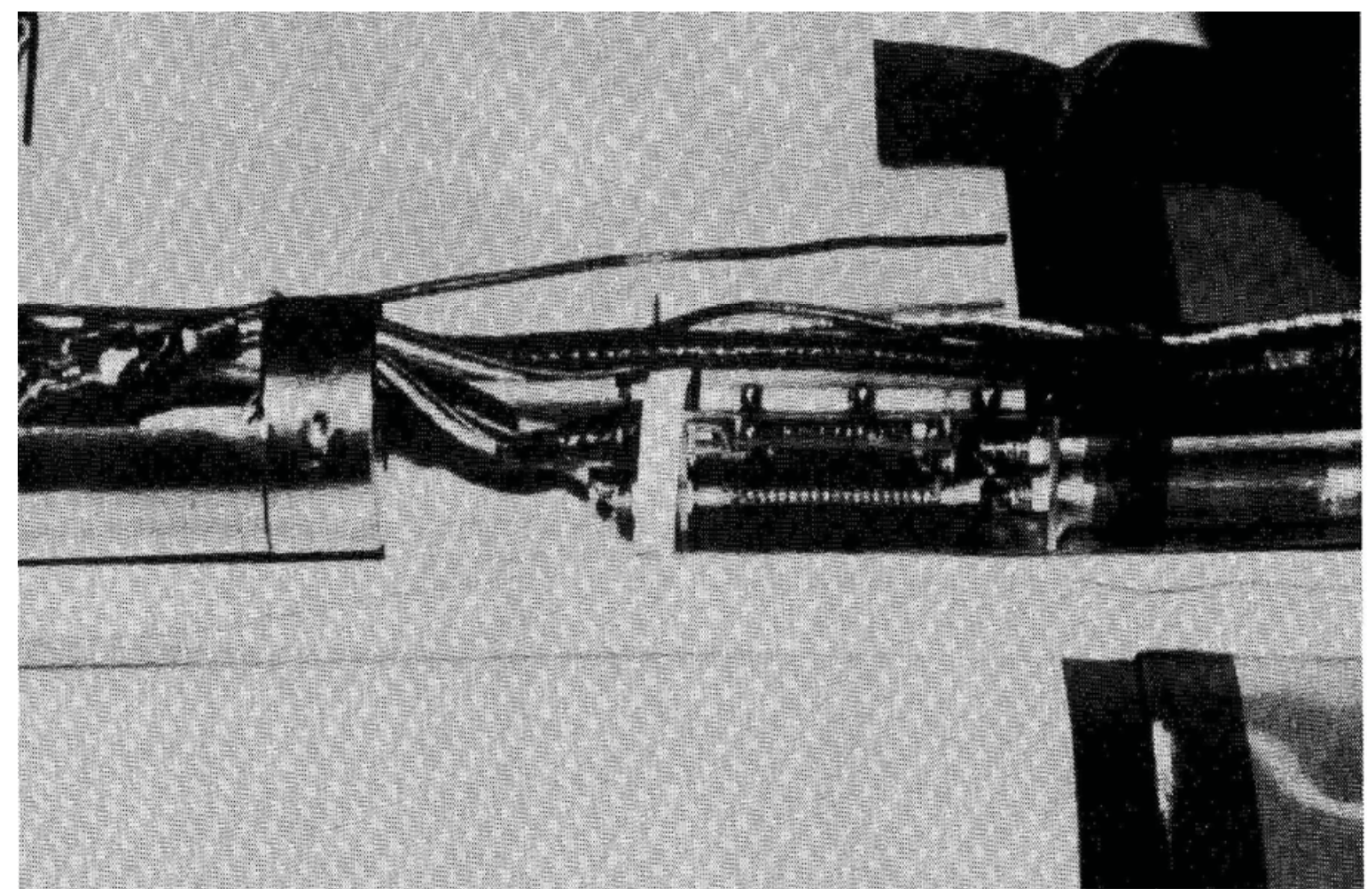

Figure 1.3.4. Photograph of the Detector-Preamplifier Package with Counters in Place (Ref. 6, Fig. 4).

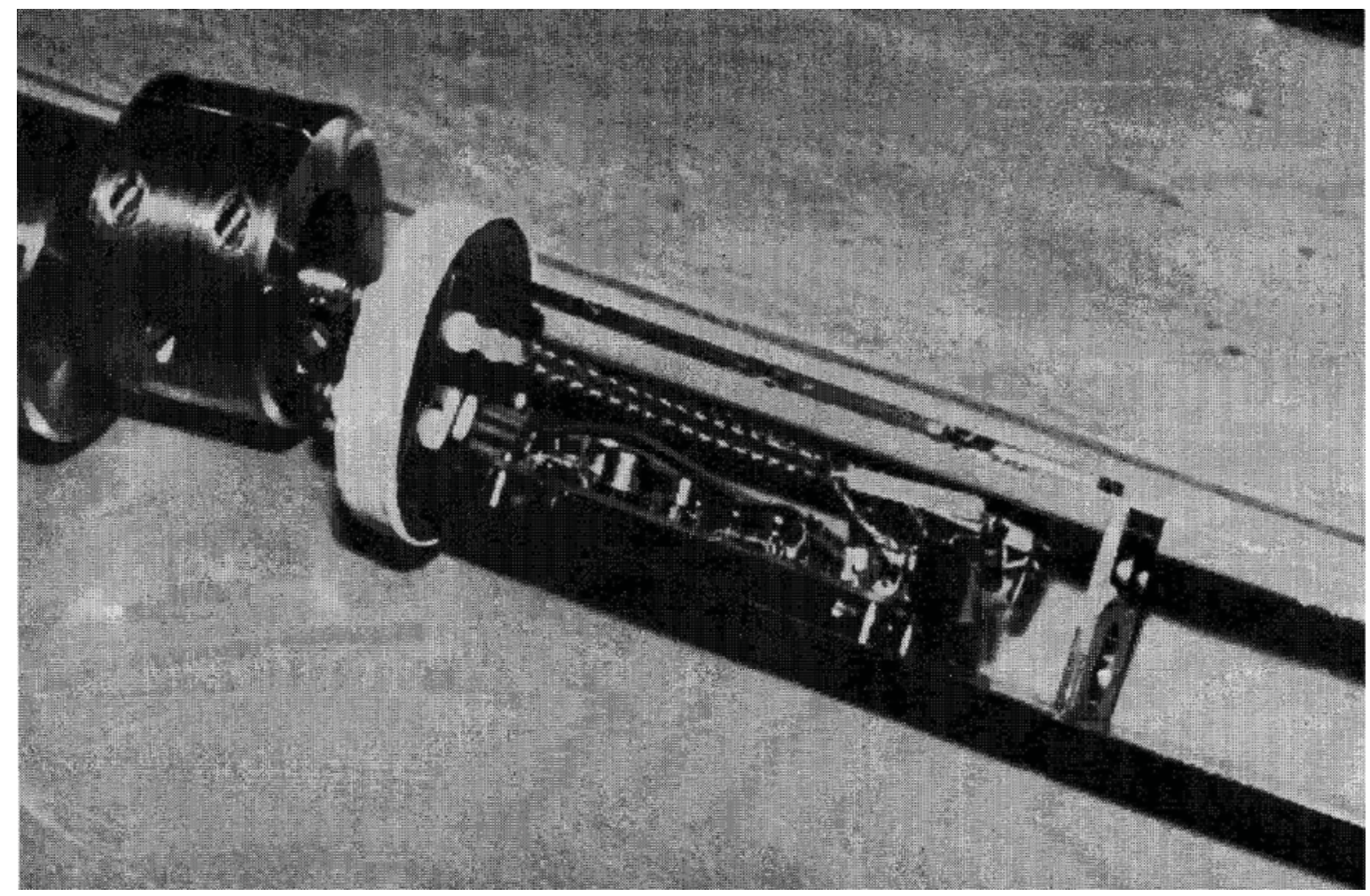

Figure 1.3.5. Photograph of the Preamplifier/Cable Layout with Counters Removed (Ref. 6, Fig. 5). 
NEA/NSC/DOC(2006)1

Liquid Metal Fast Reactor - LMFR

FFTF-LMFR-RESR-001

CRIT-SPEC-REAC-COEF-MISC

The proportional counters were calibrated prior to use. Approximately $1 \%$ nitrogen gas was added to each detector during the filling process. The detectors were placed in a well-thermalized neutron flux and mono-ionizing events of $\sim 615 \mathrm{keV}$ from the ${ }^{14} \mathrm{~N}(\mathrm{n}, \mathrm{p}){ }^{14} \mathrm{C}$ reaction were observed. These data indicated that the resolution (full width at half maximum) of the response was $\sim 6 \%$ and $\sim 10 \%$ for the hydrogen and methane/argon counters, respectively. The overall measurement resolution is affected by the intrinsic line width, which increases as energy decreases (due to the fewer number of ion pairs created in the gas) as well as by the slope-taking process used to infer the neutron energy spectrum from the measured pulse-amplitude spectrum of recoil ionization. Both detectors were also exposed to a $\mathrm{Co}^{60}$ source to assess the mean ionization from gamma-induced events and map out the relative variation in gas multiplication with bias voltage over the full range of multiplication required for the measurements (Ref. 6, pp. 4-5).

Additional calibration was carried out with mono-energetic neutrons produced from a ${ }^{7} \mathrm{Li}(\mathrm{p}, \mathrm{n})^{7} \mathrm{Be}$ reaction at a tandem accelerator. This confirmed the calibration for the methane/argon counter using the ${ }^{14} \mathrm{~N}(\mathrm{n}, \mathrm{p}){ }^{14} \mathrm{C}$ reaction to within a few percent from $150 \mathrm{keV}$ to $2 \mathrm{MeV}$. Additional calibration data for the hydrogen counter were taken with the mono-energetic neutron reaction down to $\sim 30 \mathrm{keV}$, but targetthickness-induced broadening of the neutron beam makes accurate calibration work difficult to perform in this manner below $\sim 50 \mathrm{keV}$. The calibration of the proportional counters became increasingly subject to error as the energy diminished below $10 \mathrm{keV}$ due to the uncertainty in which the proton energy loss per ion pair produced varied (Ref. 6, p. 5).

The fission product inventory was almost zero and gamma ray background was also acceptably low. Electron events due to gamma interactions were separated from proton recoil events by pulse shape discrimination. A modified National Bureau of Standards (NBS) absolute fission chamber containing a $471.6 \mu \mathrm{g}{ }^{239} \mathrm{Pu}$ deposit was used to normalize the proportional counter and emulsion irradiations. The monitor was located $54 \mathrm{~cm}$ above core midplane. The low-level flux monitors (LLFMs) were also used to obtain independent normalization information. The LLFMs were fully inserted during all proportional counter and emulsion irradiations. The core conditions for irradiation are shown in Table 1.3.3 (Ref. 2, pp. 35-36).

Table 1.3.3. Proton Recoil Measurement Conditions (Ref. 2, p. 37).

\begin{tabular}{|c|c|c|c|c|c|}
\hline Run & Detector & Location & $\begin{array}{c}\text { Control Rod } \\
\text { Configuration } \\
\end{array}$ & $\begin{array}{l}\text { Length } \\
\text { of Run }\end{array}$ & $\mathrm{k}_{\mathrm{eff}}^{(\mathrm{a})}$ \\
\hline $1-\mathrm{a}$ & $\mathrm{CH}_{4}, \mathrm{H}_{2}$ & Core midplane & $\begin{array}{l}3 \text { primary rods at } 23.5 \text { in } \\
6 \text { secondary rods at } 0 \text { in }\end{array}$ & $12 \mathrm{~h}$ & .958 \\
\hline $1-b$ & $\mathrm{CH}_{4}, \mathrm{H}_{2}$ & $80 \mathrm{~cm}$ below $\mathrm{n}$ & $\begin{array}{l}3 \text { primary rods at } 36 \text { in } \\
6 \text { secondary rods at } 12 \text { in }\end{array}$ & $12 \mathrm{~h}$ & .993 \\
\hline $2-a$ & Emulsions & Core 1 & All rods in & $30 \mathrm{~min}$ & .919 \\
\hline $2-b$ & Emulsions & $\begin{array}{l}\text { Core midplane, } \\
80 \mathrm{~cm} \text { below midplane }\end{array}$ & All rods in & $1.5 \mathrm{~h}$ & .919 \\
\hline $2-c$ & Emulsions & $80 \mathrm{~cm}$ below midplane & $\begin{array}{l}3 \text { primary rods at } 36 \text { in } \\
6 \text { secondary rods at } 0 \text { in }\end{array}$ & $4 \mathrm{~h}$ & .974 \\
\hline
\end{tabular}

(a) Calculated from LLFM count rates plus a near-critical rod drop calibration prior to the IRT experiment.

The experimental insert used for the proportional counter measurements was also used for the emulsions irradiations. Proton recoil emulsions, provided by HEDL Irradiation Environment, were loaded into dummy proportional counters and substituted for the active counters inside the insert. The configurations of the three experiments are provided in Table 1.3.3. ILFORD-type L4 emulsions, 200 and $400 \mu$ in thickness, were used in each irradiation. The distribution of the track lengths was measured with a Zeiss Universal Microscope by HEDL Irradiation Environment personnel. The track length distribution was 
NEA/NSC/DOC(2006)1

Liquid Metal Fast Reactor - LMFR

FFTF-LMFR-RESR-001

CRIT-SPEC-REAC-COEF-MISC

converted to a proton energy distribution, which was later unfolded to calculate the neutron energy spectrum. Preliminary scanning results indicated satisfactory track density and adequately low fogging due to gamma rays and low energy neutrons. Each irradiation yielded a neutron fluence in the range of 1 x $10^{9}$ to $3 \times 10^{9} \mathrm{n} / \mathrm{cm}^{2}$ (Ref. 2, p. 36).

\section{Measurement Procedure}

During the evening of Tuesday, February 26, 1980, the stalk of the IRT was withdrawn and the canister containing the proton-recoil experiment was attached. The canister was then re-introduced into the IRT and electrical checks made. FFTF control rod adjustments were then made to bring the reactor neutron flux level to a value acceptable for counting. Data collection for the center-position measurement began at 1:55 a.m. on Wednesday, February 27, 1980. Separate runs (of approximately one hour duration) were made at each of four bias voltages for the methane/argon counter, covering energies above $\sim 150 \mathrm{keV}$, at each of the seven bias voltages for the hydrogen counter, covering energies below $\sim 150 \mathrm{keV}$. No difficulties were experienced during the measurement sequence and the full set of data was recorded by 12:30 p.m. of that same day. No change in reactor power was required (Ref. 6, p. 6).

Following termination of the center run, the high-voltage bias and signal/test cables were reconnected to the detector pair in the axial reflector position $80 \mathrm{~cm}$ below the core midplane. Grounding changes were made to reduce spurious electrical noise pickup and perform data acquisition. The resultant data below $\sim 3 \mathrm{keV}$ were determined to be of poor quality and later discarded. The reactor was brought closer to critical (by a factor of 7.97 increase in neutron flux as determined by the center-position methane counter). Data acquisition at the axial position commenced February 27 at 6:50 p.m. The full set of four methane and seven hydrogen counter voltages (of which the two highest hydrogen counter voltages were later rejected) was completed at 6:40 a.m., February 28, 1980. Upon termination of the axial reflector measurements, the stalk was withdrawn and the proportional counters used for the measurements were removed from the canister. Identical "dummy" detectors were inserted for a sequence of foil-packet irradiations in the IRT at much higher flux levels (Ref. 6, p. 6).

Each irradiation lasted approximately twelve hours (Ref. 2, p. 35).

\section{Power Normalization}

The neutron flux levels in the FFTF were adjusted via control-rod movement to provide optimum counting rates at both the center and axial positions. The measured neutron spectrum is absolute at the power maintained during the neutron flux (emphasizing the high-energy neutrons from 0.65 to $2.9 \mathrm{MeV}$ ) at the two fixed levels used during the measurements. The normalization result was obtained by recording the spectrum of proton-recoil ionization from the methane/argon detector in the center position operated at the lowest voltage of the voltage sequence (which corresponds to the region of highest neutron energy). The count rate from this detector was quite low at the power selected for centerposition measurements, and did not increase unacceptably when power was adjusted to the higher level considered optimum for counting at the lower axial reflector position. The increase of the integrated spectrum of proton recoils (per unit live time) from the center counter was found to be 7.97 (with negligible statistical error) between the two levels. Also, the ratio of spectra recorded at the two levels did not change in excess of counting statistics indicating that the neutron spectrum up to about $2.9 \mathrm{MeV}$ was not dependent upon subcriticality over the range corresponding to the two levels (Ref. 6, p. 7).

Subcriticality of the reactor at either of the power levels used for counting could not be inferred from the proton-recoil results. Other information from operational measurements performed on the FFTF using data from the low-level startup neutron detectors and from the in-canister fission counter (both of which were monitored continuously during all measurements) is required to provide subcriticality. Any effects that occur are most pronounced at the higher energies, where the spectrum of spontaneous neutrons influences results, and at quite low energies (below $1 \mathrm{keV}$ ), where the effects of absorber control rods 
FFTF-LMFR-RESR-001

CRIT-SPEC-REAC-COEF-MISC

begin to appear. Based upon an analysis of the proton-recoil measurements in the interval from 0.65 to $2.9 \mathrm{MeV}$, it would appear that subcriticality effects must constitute a rather small perturbation to the spectrum shape, at least over the energy region amenable to the proton-recoil technique (Ref. 6, p. 7).

\subsubsection{Material Data}

The materials in the core were those described in the fully-loaded core configuration in Section 1.1.3 with modifications as stated below.

\section{In-Reactor Thimble (IRT)}

The IRT was a stainless steel 304 pipe, typically with an empty interior except during zero power physics measurements (Ref. 3, p. 11). The fission chambers in the SCH were built by Reuter-Stokes, Inc. (Model RS-C3-2510-114), each containing 1.3 g uranium-235 (Ref. 1, p. 3) with neutron sensitivity estimated at $0.7 \mathrm{cps} /$ equivalent thermal neutron flux at a $1 \mathrm{cps}$ alpha-plus-noise cutoff discriminator setting (Ref. 10, p. 17).

See Table 1.1.11 for homogenized atom densities of the IRT.

\section{$\underline{\text { Detectors }}$}

Detector materials are discussed in Section 1.3.2.

The modified National Bureau of Standards (NBS) absolute fission chamber had the reported composition shown in Table 1.3.4.

Table 1.3.4. Composition of Plutonium Foil (Ref. 2, p. 41).

\begin{tabular}{|c|c|}
\hline Isotope & $\begin{array}{c}\text { Foil Assay } \\
\text { (at.\%) }\end{array}$ \\
\hline${ }^{239} \mathrm{Pu}$ & 99.978 \\
${ }^{240} \mathrm{Pu}$ & .021 \\
${ }^{241} \mathrm{Pu}$ & .005 \\
${ }^{242} \mathrm{Pu}$ & .005 \\
${ }^{244} \mathrm{Pu}$ & $<.002$ \\
\hline
\end{tabular}

\subsubsection{Temperature Data}

The temperature of the core was approximately $400^{\circ} \mathrm{F}\left(\sim 204^{\circ} \mathrm{C}\right)$. 
NEA/NSC/DOC(2006)1

Liquid Metal Fast Reactor - LMFR

FFTF-LMFR-RESR-001

CRIT-SPEC-REAC-COEF-MISC

\subsubsection{Additional Information Relevant to Spectral Characteristics Measurements}

\section{Carbon Recoils}

The methane/argon counter used for measurement of the neutron spectrum above $150 \mathrm{keV}$ was sensitive to recoiling carbon nuclei from scattering of neutrons in the high-energy tail of the spectrum onto carbon atoms in the methane gas. The recoiling carbon ions did not ionize as efficiently as did the recoil protons;, estimates had been made that a recoil proton would produce about five times the number of ion pairs in stopping in methane gas as would a carbon recoil of the same energy. Nevertheless, any measurement of neutron spectra using methane as a source of protons may have to contend with a small amount of contamination of the recoil-proton spectra by scattering on carbon. The procedure used was to accept the spectrum, as measured, up to $2 \mathrm{MeV}$ and then extrapolate above $2 \mathrm{MeV}$ using a simple prescription for the dependence of spectrum upon energy, consistent with information obtained from calculations, regarding the shape of the high-energy extrapolation. This result was then used to generate a computed ionization distribution from recoil-carbon scattering which was then subtracted from the observed distribution. The difference was considered to be carbon-recoil corrected (Ref. 6, pp. 7-8).

Although the procedure for doing the correction was simple, it is subject to uncertainty relating not only to uncertainty in the extrapolated spectrum assumed, but to the effective ionization ratio energy dependence (largely unknown) and to any simplifying assumptions regarding the cross section variation with energy for neutron scattering on carbon. For FFTF spectra (at the center position) the carbon recoil correction was less than $5 \%$ above $150 \mathrm{keV}$, which was the low-energy limit for use of methane counter data. The correction was negligible above about $0.7 \mathrm{MeV}$ but rose to almost $5 \%$ at $100 \mathrm{keV}$. This carbon correction had been made to the measurement results reported here. The measurement of the neutron spectrum in the lower axial reflector indicated few neutrons at higher energies, and no correction for carbon recoil was required under these circumstances (Ref. 6, p. 8).

\section{Wall-And-End Effects}

The proton-recoil ionization spectrum was distorted due to proton track lengths which were too long to be fully contained within the counter gas. Substantial truncation of proton tracks occurred increasingly as energy arose above $1 \mathrm{MeV}$; its effect upon the derived neutron spectra was modest up to about $2 \mathrm{MeV}$ for the detectors used in the FFTF measurements. These effects were relevant in methane/argon detectors above $100 \mathrm{keV}$ but corrections were unnecessary for the hydrogen detectors, especially at the low-energy region of the spectrum. Part of this was a consequence of the method used for gammaneutron discrimination, which tended to reject wall-and-end truncated events which were of relatively long range in the hydrogen counter data (Ref. 6, pp. 8-9).

\section{Results from Measurements}

The measured neutron spectra at the core center and in the lower axial reflector are shown in Figures 1.3.6 and 1.3.7, respectively. The spectra in the tables were absolute (in units of neutrons $/ \mathrm{cm}^{2}$-minute) at the constant power level during the measurement. The errors in the figures were entirely statistical, and an estimate of the energy resolution of the measurement is included in the data listed in Table 1.3.5 and 1.3.6, respectively. 
Liquid Metal Fast Reactor - LMFR

FFTF-LMFR-RESR-001

CRIT-SPEC-REAC-COEF-MISC

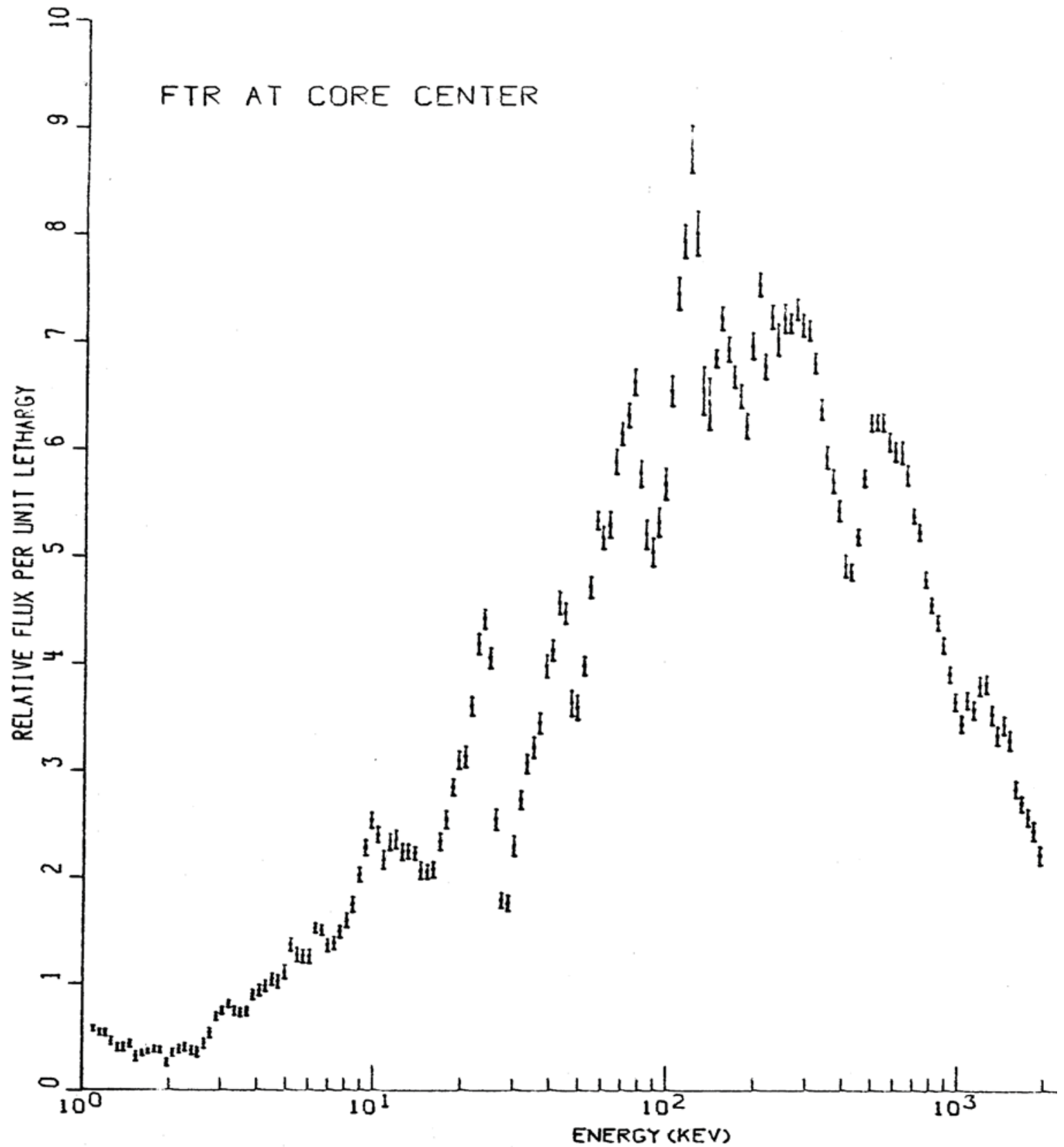

Figure 1.3.6. FFTF Core-Center Neutron Spectrum (Ref. 6, Fig. 11). 
Liquid Metal Fast Reactor - LMFR

FFTF-LMFR-RESR-001

CRIT-SPEC-REAC-COEF-MISC

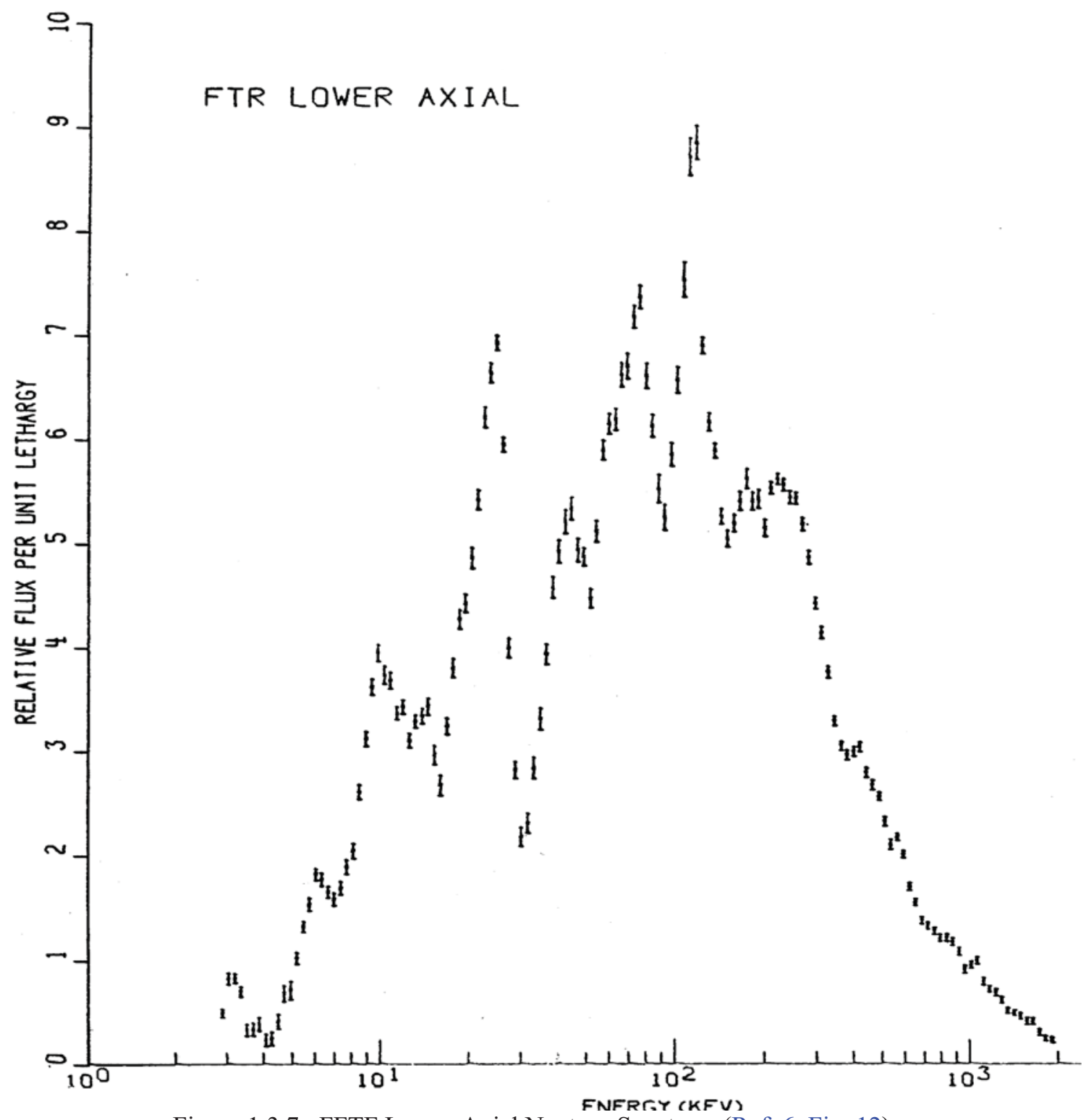

Figure 1.3.7. FFTF Lower-Axial Neutron Spectrum (Ref. 6, Fig. 12). 
NEA/NSC/DOC(2006)1

Liquid Metal Fast Reactor - LMFR

FFTF-LMFR-RESR-001

CRIT-SPEC-REAC-COEF-MISC

Table 1.3.5. Neutron Spectrum at the Core Center Location (Ref. 6, pp. 29-30).

\begin{tabular}{|c|c|c|c|c|c|c|c|}
\hline $\begin{array}{c}\text { Energy } \\
(\mathrm{keV})\end{array}$ & $\begin{array}{c}\text { Flux } \\
\left(\mathrm{n} / \mathrm{cm}^{2}-\mathrm{s}\right)\end{array}$ & Error & Resolution & $\begin{array}{c}\text { Energy } \\
(\mathrm{keV})\end{array}$ & $\begin{array}{c}\text { Flux } \\
\left(\mathrm{n} / \mathrm{cm}^{2}-\mathrm{s}\right)\end{array}$ & Error & Resolution \\
\hline 1903.548 & $2.2633 \mathrm{E} 07$ & .102E 06 & .100 & 1812.903 & ב.2906E 07 & $.102 \mathrm{E} 06$ & .100 \\
\hline 1725.574 & $.3057 \mathrm{E} 07$ & $.928 \mathrm{E} 05$ & .100 & 1644.356 & $.3210 \mathrm{E} 07$ & .873 E 05 & .101 \\
\hline 1566.054 & $.3368 \mathrm{E} 07$ & $.926 \mathrm{E} 05$ & .101 & 1491.480 & $.3967 \mathrm{E} 07$ & $.104 \mathrm{E} 06$ & .101 \\
\hline 1420.457 & $.4082 \mathrm{E} 07$ & $.967 \mathrm{E} 05$ & . 101 & 1352.816 & $.3967 \mathrm{E} 07$ & $.102 \mathrm{E} 06$ & . 101 \\
\hline 1288.396 & .4197E 07 & .103 E 06 & .101 & 1227.044 & $.4540 \mathrm{E} 07$ & $.993 \mathrm{E} 05$ & .101 \\
\hline 1168.613 & $.4317 \mathrm{E} 07$ & $.105 \mathrm{E} 06$ & . 101 & 1112.965 & $.4250 \mathrm{E} 07$ & $.996 \mathrm{E} 05$ & . 101 \\
\hline 1059.967 & $.4357 \mathrm{E} 07$ & .933 E 05 & . 101 & 1009.492 & $.4094 \mathrm{E} 07$ & $.936 \mathrm{E} 05$ & .101 \\
\hline 961.421 & .4339 E 07 & .932 E 05 & .101 & 915.639 & $.4648 \mathrm{E} 07$ & $.894 \mathrm{E} 05$ & .101 \\
\hline 872.037 & $.4974 \mathrm{E} 07$ & .840 E 05 & .101 & 830.512 & $.5229 \mathrm{E} 07$ & .832 E 05 & .101 \\
\hline 790.964 & $.5423 \mathrm{E} 07$ & $.804 \mathrm{E} 05$ & .101 & 753.299 & $.5703 \mathrm{E} 07$ & $.853 \mathrm{E} 05$ & .101 \\
\hline 717.427 & $.6253 \mathrm{E} 07$ & .890 E 05 & .101 & 683.264 & $.6415 \mathrm{E} 07$ & $.832 \mathrm{E} 05$ & .101 \\
\hline 650.728 & $.6870 \mathrm{E} 07$ & $.112 \mathrm{E} 06$ & .101 & 619.741 & $.7121 \mathrm{E} 07$ & $.121 \mathrm{E} 06$ & .101 \\
\hline 590.229 & $.7130 \mathrm{E} 07$ & .107 E 06 & 101 & 562.123 & $.7236 \mathrm{E} 07$ & $.102 \mathrm{E} 06$ & .102 \\
\hline 535.355 & $.7436 \mathrm{E} 07$ & $.961 \mathrm{E} 05$ & .102 & 509.862 & $.7458 \mathrm{E} 07$ & $.899 \mathrm{E} 05$ & .102 \\
\hline 485.583 & $.7450 \mathrm{E} 07$ & $.900 \mathrm{E} 05$ & .102 & 462.460 & $.6838 \mathrm{E} 07$ & .959 E 05 & .102 \\
\hline 440.438 & .6179 E 07 & $.916 \mathrm{E} 05$ & .102 & 419.465 & $.5786 \mathrm{E} 07$ & $.912 \mathrm{E} 05$ & .102 \\
\hline 399.490 & $.5858 \mathrm{E} 07$ & $.126 \mathrm{E} 06$ & .102 & 380.467 & $.6473 \mathrm{E} 07$ & $.116 \mathrm{E} 06$ & .102 \\
\hline 362.349 & $.6804 \mathrm{E} 07$ & $.131 \mathrm{E} 06$ & .102 & 345.095 & $.7068 \mathrm{E} 07$ & $.121 \mathrm{E} 06$ & .102 \\
\hline 328.662 & $.7602 \mathrm{E} 07$ & $.112 \mathrm{E} 06$ & .103 & 313.011 & $.8112 \mathrm{E} 07$ & $.111 \mathrm{E} 06$ & .103 \\
\hline 298.106 & $.8479 \mathrm{E} 07$ & $.109 \mathrm{E} 06$ & .103 & 283.910 & $.8535 \mathrm{E} 07$ & $.122 \mathrm{E} 06$ & .103 \\
\hline 270.391 & $.8710 \mathrm{E} 07$ & $.114 \mathrm{E} 06$ & .103 & 257.515 & $.8556 \mathrm{E} 07$ & $.105 \mathrm{E} 06$ & . 103 \\
\hline 245.252 & $.8610 \mathrm{E} 07$ & $.157 \mathrm{E} 06$ & .103 & 233.574 & $.8372 \mathrm{E} 07$ & $.177 \mathrm{E} 06$ & . 104 \\
\hline 222.451 & $.6826 \mathrm{E} 07$ & $.134 \mathrm{E} 06$ & .104 & 211.858 & $.8075 \mathrm{E} 07$ & $.137 \mathrm{E} 06$ & .104 \\
\hline 201.770 & $.8985 \mathrm{E} 07$ & $.129 \mathrm{E} 06$ & .104 & 192.162 & $.8305 \mathrm{E} 07$ & .145 E 06 & .104 \\
\hline 183.011 & .7418 E 07 & $.137 \mathrm{E} 06$ & .105 & 174.296 & $.7747 \mathrm{E} 07$ & $.129 \mathrm{E} 06$ & .105 \\
\hline 165.996 & $.7950 \mathrm{E} 07$ & $.119 \mathrm{E} 06$ & .105 & 158.092 & $.8267 \mathrm{E} 07$ & $.137 \mathrm{E} 06$ & .105 \\
\hline 150.564 & $.8611 \mathrm{E} 07$ & $.131 \mathrm{E} 06$ & .105 & 143.394 & $.8169 \mathrm{E} 07$ & .942 E 05 & .106 \\
\hline 136.566 & $.7664 \mathrm{E} 07$ & .287 E 06 & .106 & 130.063 & $.7810 \mathrm{E} 07$ & $.265 \mathrm{E} 06$ & .090 \\
\hline 123.869 & $.9539 \mathrm{E} 07$ & $.246 \mathrm{E} 06$ & .090 & 117.971 & $.1050 \mathrm{E} 08$ & $.267 \mathrm{E} 06$ & .091 \\
\hline 112.353 & $.9471 \mathrm{E} 07$ & $.186 \mathrm{E} 06$ & .091 & 107.003 & $.8888 \mathrm{E} 07$ & $.180 \mathrm{E} 06$ & .091 \\
\hline 101.907 & $.7606 \mathrm{E} 07$ & $.166 \mathrm{E} 06$ & .091 & 97.055 & $.6772 \mathrm{E} 07$ & .177 E 06 & .091 \\
\hline 92.433 & $.6349 \mathrm{E} 07$ & $.162 \mathrm{E} 06$ & .092 & 88.031 & $.6009 \mathrm{E} 07$ & $.156 \mathrm{E} 06$ & .092 \\
\hline 83.840 & $.6211 \mathrm{E} 07$ & $.161 \mathrm{E} 06$ & .092 & 79.847 & $.6888 \mathrm{E} 07$ & $.148 \mathrm{E} 06$ & .093 \\
\hline 76.045 & $.7908 \mathrm{E} 07$ & .145 E 06 & .093 & 72.424 & $.7539 \mathrm{E} 07$ & .133 E 06 & .093 \\
\hline 68.975 & $.7332 \mathrm{E} 07$ & $.123 \mathrm{E} 06$ & .093 & 65.690 & $.7021 \mathrm{E} 07$ & $.138 \mathrm{E} 06$ & .094 \\
\hline 62.562 & $.6319 \mathrm{E} 07$ & $.145 \mathrm{E} 06$ & .094 & 59.583 & $.6174 \mathrm{E} 07$ & $.124 \mathrm{E} 06$ & .094 \\
\hline 55.746 & $.6357 \mathrm{E} 07$ & $.104 \mathrm{E} 06$ & .095 & 54.044 & $.5622 \mathrm{E} 07$ & $.117 \mathrm{E} 06$ & .095 \\
\hline 51.470 & $.4742 \mathrm{E} 07$ & $.108 \mathrm{E} 06$ & .096 & 49.019 & $.4275 \mathrm{E} 07$ & $.138 \mathrm{E} 06$ & . 096 \\
\hline 46.685 & $.4326 \mathrm{E} 07$ & $.143 \mathrm{E} 06$ & .097 & 44.462 & $.5335 \mathrm{E} 07$ & $.116 \mathrm{E} 06$ & . 097 \\
\hline 42.345 & $.5449 \mathrm{E} 07$ & $.127 \mathrm{E} 06$ & .097 & 40.328 & $.4919 \mathrm{E} 07$ & $.116 \mathrm{E} 06$ & .098 \\
\hline 38.408 & $.4739 \mathrm{E} 07$ & $.130 \mathrm{E} 06$ & .096 & 36.579 & $.4101 \mathrm{E} 07$ & .119 E 06 & .099 \\
\hline 34.837 & $.3830 \mathrm{E} 07$ & $.116 \mathrm{E} 06$ & .100 & 33.178 & $.3652 \mathrm{E} 07$ & $.106 \mathrm{E} 06$ & .100 \\
\hline 31.598 & $.3248 \mathrm{E} 07$ & .103 E 06 & .101 & 30.094 & $.2733 \mathrm{E} 07$ & $.112 \mathrm{E} 06$ & 101 \\
\hline 28.661 & .2089 E 07 & $.858 \mathrm{E} 05$ & .102 & 27.296 & $.2123 \mathrm{E} 07$ & .841 E 05 & .103 \\
\hline 25.996 & $.3030 \mathrm{E} 07$ & $.117 \mathrm{E} 06$ & .103 & 24.758 & $.4834 \mathrm{E} 07$ & $.112 \mathrm{E} 06$ & .104 \\
\hline 23.579 & $.5271 \mathrm{E} 07$ & $.109 \mathrm{E} 06$ & .105 & 22.456 & $.4988 \mathrm{E} 07$ & $.115 \mathrm{E} 06$ & .105 \\
\hline 21.387 & $.4291 \mathrm{E} 07$ & $.106 \mathrm{E} 06$ & 107 & 20.368 & $.3730 \mathrm{E} 07$ & $.118 \mathrm{E} 06$ & .109 \\
\hline 19.399 & $.3693 \mathrm{E} 07$ & $.999 \mathrm{E} 05$ & .111 & 18.475 & $.3387 \mathrm{E} 07$ & $.922 \mathrm{E} 05$ & .113 \\
\hline 17.696 & $.3031 \mathrm{E} 07$ & $.986 \mathrm{E} 05$ & .115 & 16.757 & $.2781 \mathrm{E} 07$ & $.960 \mathrm{E} 05$ & .117 \\
\hline
\end{tabular}

Revision: 0

Page 54 of 304

Date: March 31, 2010 
NEA/NSC/DOC(2006)1

Liquid Metal Fast Reactor - LMFR

FFTF-LMFR-RESR-001

CRIT-SPEC-REAC-COEF-MISC

Table 1.3.5 (cont'd.). Neutron Spectrum at the Core Center Location (Ref. 6, pp. 29-30).

\begin{tabular}{|c|c|c|c|c|c|c|c|}
\hline $\begin{array}{c}\text { Energy } \\
(\mathrm{keV})\end{array}$ & $\begin{array}{c}\text { Flux } \\
\left(\mathrm{n} / \mathrm{cm}^{2}-\mathrm{s}\right)\end{array}$ & Error & Resolution & $\begin{array}{c}\text { Energy } \\
(\mathrm{keV})\end{array}$ & $\begin{array}{c}\text { Flux } \\
\left(\mathrm{n} / \mathrm{cm}^{2}-\mathrm{s}\right)\end{array}$ & Error & Resolution \\
\hline 15.959 & $.2468 \mathrm{E} 07$ & $.883 \mathrm{E} 05$ & .119 & 15.199 & (2445E 07 & $.814 \mathrm{E} 05$ & .122 \\
\hline 14.476 & $.2457 \mathrm{E} 07$ & .947 E 05 & .124 & 13.786 & $.2649 \mathrm{E} 07$ & $.724 \mathrm{E} 05$ & .126 \\
\hline 13.130 & $.2689 \mathrm{E} 07$ & .803 E 05 & .129 & 12.504 & $.2665 \mathrm{E} 07$ & $.943 \mathrm{E} 05$ & .131 \\
\hline 11.909 & $.2806 \mathrm{E} 07$ & $.101 \mathrm{E} 06$ & .134 & 11.342 & $.2780 \mathrm{E} 07$ & $.934 \mathrm{E} 05$ & .136 \\
\hline 10.802 & $.2576 \mathrm{E} 07$ & $.102 \mathrm{E} 06$ & .139 & 10.287 & $.2860 \mathrm{E} 07$ & .863 E 05 & .142 \\
\hline 9.793 & $.3021 \mathrm{E} 07$ & .913 E 05 & .145 & 9.331 & $.2713 \mathrm{E} 07$ & $.845 \mathrm{E} 05$ & .148 \\
\hline 8.887 & $.2415 \mathrm{E} 07$ & $.816 \mathrm{E} 05$ & .151 & 8.464 & $.2080 \mathrm{E} 07$ & $.870 \mathrm{E} 05$ & .154 \\
\hline 8.061 & $.1899 \mathrm{E} 07$ & $.803 \mathrm{E} 05$ & .157 & 7.677 & .1770 E 07 & $.665 \mathrm{E} 05$ & .160 \\
\hline 7.311 & $.1643 \mathrm{E} 07$ & $.715 \mathrm{E} 05$ & .164 & 6.963 & $.1620 \mathrm{E} 07$ & $.693 \mathrm{E} 05$ & .167 \\
\hline 6.631 & $.1701 \mathrm{E} 07$ & $.565 \mathrm{E} 05$ & .171 & 6.316 & $.1812 \mathrm{E} 07$ & .539 E 05 & .175 \\
\hline 6.015 & .1485 E 07 & .791 E 05 & .179 & 5.728 & $.1494 \mathrm{E} 07$ & $.728 \mathrm{E} 05$ & .182 \\
\hline 5.456 & .1513 E 07 & $.772 \mathrm{E} 05$ & .186 & 5.196 & $.1629 \mathrm{E} 07$ & $.712 \mathrm{E} 05$ & .191 \\
\hline 4.948 & $.1322 \mathrm{E} 07$ & .765 E 05 & .195 & 4.713 & $.1213 \mathrm{E} 07$ & .705 E 05 & 199 \\
\hline 4.488 & $.1238 \mathrm{E} 07$ & $.678 \mathrm{E} 05$ & .204 & 4.275 & $.1164 \mathrm{E} 07$ & $.622 \mathrm{E} 05$ & .208 \\
\hline 4.071 & $.1115 \mathrm{E} 07$ & $.651 \mathrm{E} 05$ & .213 & 3.877 & $.1065 \mathrm{E} 07$ & $.562 \mathrm{E} 05$ & .218 \\
\hline 3.693 & $.8730 \mathrm{E} 06$ & $.495 \mathrm{E} 05$ & .223 & 3.517 & $.8661 \mathrm{E} 06$ & $.474 \mathrm{E} 05$ & .228 \\
\hline 3.349 & $.8530 \mathrm{E} 06$ & $.484 \mathrm{E} 05$ & .233 & 3.190 & $.9634 \mathrm{E} 06$ & $.422 \mathrm{E} 05$ & .239 \\
\hline 3.038 & .8894 E 06 & .395 E 05 & .244 & 2.893 & .8223 E 06 & $.430 \mathrm{E} 05$ & .250 \\
\hline 2.755 & .6405 E 06 & .541 E 05 & .256 & 2.624 & $.5212 \mathrm{E} 06$ & $.546 \mathrm{E} 05$ & .261 \\
\hline 2.499 & $.4243 \mathrm{E} 06$ & $.524 \mathrm{E} 05$ & .268 & 2.380 & $.4455 \mathrm{E} 06$ & $.479 E 05$ & .274 \\
\hline 2.267 & $.4810 \mathrm{E} 06$ & $.438 \mathrm{E} 05$ & .280 & 2.159 & $.4550 \mathrm{E} 06$ & $.446 \mathrm{E} 05$ & 287 \\
\hline 2.056 & .4198E 06 & $.408 \mathrm{E} 05$ & .294 & 1.958 & $.3078 \mathrm{E} 06$ & .393 E 05 & .301 \\
\hline 1.865 & $.4463 \mathrm{E} 06$ & $.360 \mathrm{E} 05$ & .308 & 1.776 & $.4596 \mathrm{E} 06$ & $.369 \mathrm{E} 05$ & .315 \\
\hline 1.692 & $.4373 \mathrm{E} 06$ & $.316 \mathrm{E} 05$ & .323 & 1.611 & $.4142 \mathrm{E} 06$ & $.328 \mathrm{E} 05$ & .330 \\
\hline 1.534 & $.3752 \mathrm{E} 06$ & $.534 \mathrm{E} 05$ & .338 & 1.461 & $.5180 \mathrm{E} 06$ & $.424 \mathrm{E} 05$ & .346 \\
\hline 1.392 & $.4607 \mathrm{E} 06$ & $.447 \mathrm{E} 05$ & .355 & 1.325 & $.4776 \mathrm{E} 06$ & $.412 \mathrm{E} 05$ & .363 \\
\hline 1.262 & $.5468 \mathrm{E} 06$ & $.446 \mathrm{E} 05$ & .372 & 1.202 & $.6425 \mathrm{E} 06$ & $.409 \mathrm{E} 05$ & .381 \\
\hline 1.145 & $.6461 \mathrm{E} 06$ & $.377 \mathrm{E} 05$ & .390 & & & & \\
\hline
\end{tabular}


NEA/NSC/DOC(2006)1

Liquid Metal Fast Reactor - LMFR

FFTF-LMFR-RESR-001

CRIT-SPEC-REAC-COEF-MISC

Table 1.3.6. Neutron Spectrum at the Lower Axial Reflector Location (Ref. 6, pp. 31-32).

\begin{tabular}{|c|c|c|c|c|c|c|c|}
\hline $\begin{array}{c}\text { Energy } \\
(\mathrm{keV}) \\
\end{array}$ & $\begin{array}{c}\text { Flux } \\
\left(\mathrm{n} / \mathrm{cm}^{2}-\mathrm{s}\right) \\
\end{array}$ & Error & Resolution & $\begin{array}{c}\text { Energy } \\
(\mathrm{keV})\end{array}$ & $\begin{array}{c}\text { Flux } \\
\left(\mathrm{n} / \mathrm{cm}^{2}-\mathrm{s}\right) \\
\end{array}$ & Error & Resolution \\
\hline 1903.548 & $2.2817 \mathrm{E} 06$ & .308E 05 & .100 & 1812.903 & (3041E 06 & $.310 \mathrm{E} 05$ & .100 \\
\hline 1725.574 & $.3736 \mathrm{E} 06$ & $.365 \mathrm{E} 05$ & .100 & 1644.356 & $.5023 \mathrm{E} 06$ & $.366 \mathrm{E} 05$ & .101 \\
\hline 1566.054 & $.5060 \mathrm{E} 06$ & $.367 \mathrm{E} 05$ & .101 & 1491.480 & $.5681 \mathrm{E} 06$ & $.365 \mathrm{E} 05$ & .101 \\
\hline 1420.457 & $.6032 \mathrm{E} 06$ & $.326 \mathrm{E} 05$ & .101 & 1352.816 & $.6292 \mathrm{E} 06$ & $.321 \mathrm{E} 05$ & . 101 \\
\hline 1288.396 & $.7576 \mathrm{E} 06$ & $.632 \mathrm{E} 05$ & .101 & 1227.044 & $.8470 \mathrm{E} 06$ & $.412 \mathrm{E} 05$ & .101 \\
\hline 1168.613 & $.8849 \mathrm{E} 06$ & $.378 \mathrm{E} 05$ & . 101 & 1112.965 & $.9742 \mathrm{E} 06$ & $.409 \mathrm{E} 05$ & . 101 \\
\hline 1059.967 & $.1221 \mathrm{E} 07$ & $.400 \mathrm{E} 05$ & .101 & 1009.492 & $.1172 \mathrm{E} 07$ & $.389 \mathrm{E} 05$ & .101 \\
\hline 961.421 & $.1118 \mathrm{E} 07$ & .448 E 05 & .101 & 915.639 & $.1329 \mathrm{E} 07$ & $.431 \mathrm{E} 05$ & .101 \\
\hline 872.037 & .1442 E 07 & $.381 \mathrm{E} 05$ & .101 & 830.512 & $.1489 \mathrm{E} 07$ & .437 E 05 & .101 \\
\hline 790.964 & .1484 E 07 & $.396 \mathrm{E} 05$ & .101 & 753.299 & $.1568 \mathrm{E} 07$ & $.395 \mathrm{E} 05$ & .101 \\
\hline 717.427 & $.1625 \mathrm{E} 07$ & .403 E 05 & .101 & 683.264 & $.1689 \mathrm{E} 07$ & $.381 \mathrm{E} 05$ & .101 \\
\hline 650.728 & $.1898 \mathrm{E} 07$ & $.374 \mathrm{E} 05$ & .101 & 619.741 & .2085 E 07 & $.418 \mathrm{E} 05$ & .101 \\
\hline 590.229 & $.2464 \mathrm{E} 07$ & .402 E 05 & 101 & 562.123 & $.2665 \mathrm{E} 07$ & $.416 \mathrm{E} 05$ & .102 \\
\hline 535.355 & $.2530 \mathrm{E} 07$ & .579 E 05 & .102 & 509.862 & $.2851 \mathrm{E} 07$ & $.510 \mathrm{E} 05$ & .102 \\
\hline 485.583 & $.3146 \mathrm{E} 07$ & $.487 \mathrm{E} 05$ & .102 & 462.460 & $.3278 \mathrm{E} 07$ & $.557 \mathrm{E} 05$ & .102 \\
\hline 440.438 & $.3426 \mathrm{E} 07$ & $.540 \mathrm{E} 05$ & .102 & 419.465 & $.3728 \mathrm{E} 07$ & $.549 \mathrm{E} 05$ & .102 \\
\hline 399.490 & $.3672 \mathrm{E} 07$ & $.532 \mathrm{E} 05$ & .102 & 380.467 & $.3632 \mathrm{E} 07$ & $.564 \mathrm{E} 05$ & .102 \\
\hline 362.349 & $.3737 \mathrm{E} 07$ & $.498 \mathrm{E} 05$ & .102 & 345.095 & $.4035 \mathrm{E} 07$ & $.555 \mathrm{E} 05$ & .102 \\
\hline 328.662 & $.4616 \mathrm{E} 07$ & .708 E 05 & .103 & 313.011 & $.5081 \mathrm{E} 07$ & $.711 \mathrm{E} 05$ & .103 \\
\hline 296.106 & $.5432 \mathrm{E} 07$ & $.671 \mathrm{E} 05$ & .103 & 283.910 & $.5971 \mathrm{E} 07$ & $.790 \mathrm{E} 05$ & .103 \\
\hline 270.391 & $.6362 \mathrm{E} 07$ & $.747 \mathrm{E} 05$ & .103 & 257.515 & $.6671 \mathrm{E} 07$ & .712 E 05 & . 103 \\
\hline 245.252 & $.6662 \mathrm{E} 07$ & .793 E 05 & .103 & 233.574 & $.6826 \mathrm{E} 07$ & $.751 \mathrm{E} 05$ & . 104 \\
\hline 222.451 & $.6396 \mathrm{E} 07$ & $.655 \mathrm{E} 05$ & .104 & 211.858 & $.6791 \mathrm{E} 07$ & $.711 \mathrm{E} 05$ & .104 \\
\hline 201.770 & $.6319 \mathrm{E} 07$ & $.996 \mathrm{E} 05$ & .104 & 192.162 & $.6662 \mathrm{E} 07$ & .109 Е 06 & .104 \\
\hline 183.011 & $.6634 \mathrm{E} 07$ & $.104 \mathrm{E} 06$ & .105 & 174.296 & $.6902 \mathrm{E} 07$ & $.116 \mathrm{E} 06$ & .105 \\
\hline 165.996 & $.6539 \mathrm{E} 07$ & $.111 \mathrm{E} 06$ & .105 & 158.092 & $.6375 \mathrm{E} 07$ & .103 E 06 & .105 \\
\hline 150.564 & $.6195 \mathrm{E} 07$ & $.982 \mathrm{E} 05$ & .105 & 143.394 & $.6459 \mathrm{E} 07$ & .917 E 05 & .106 \\
\hline 136.566 & $.7233 \mathrm{E} 07$ & .879 E 05 & .106 & 130.063 & $.7569 \mathrm{E} 07$ & .105 E 06 & .106 \\
\hline 123.869 & $.8470 \mathrm{E} 07$ & $.994 \mathrm{E} 05$ & .107 & 117.971 & $.1087 \mathrm{E} 08$ & $.201 \mathrm{E} 06$ & 107 \\
\hline 112.353 & $.1070 \mathrm{E} 08$ & $.222 \mathrm{E} 06$ & .091 & 107.003 & .9248 E 07 & .209 E 06 & .091 \\
\hline 101.907 & $.8062 \mathrm{E} 07$ & $.153 \mathrm{E} 06$ & .091 & 97.055 & $.7187 \mathrm{E} 07$ & $.141 \mathrm{E} 06$ & .091 \\
\hline 92.433 & $.6441 \mathrm{E} 07$ & $.151 \mathrm{E} 06$ & .092 & 88.031 & $.6785 \mathrm{E} 07$ & $.164 \mathrm{E} 06$ & .092 \\
\hline 83.840 & $.7524 \mathrm{E} 07$ & $.134 \mathrm{E} 06$ & .092 & 79.847 & $.8112 \mathrm{E} 07$ & $.147 \mathrm{E} 06$ & .093 \\
\hline 76.045 & $.9046 \mathrm{E} 07$ & $.138 \mathrm{E} 06$ & .093 & 72.424 & $.8809 \mathrm{E} 07$ & $.134 \mathrm{E} 06$ & .093 \\
\hline 68.975 & $.8228 \mathrm{E} 07$ & $.152 \mathrm{E} 06$ & .093 & 65.690 & $.8127 \mathrm{E} 07$ & $.141 \mathrm{E} 06$ & .094 \\
\hline 62.562 & $.7602 \mathrm{E} 07$ & $.130 \mathrm{E} 06$ & .094 & 59.583 & $.7551 \mathrm{E} 07$ & $.121 \mathrm{E} 06$ & .094 \\
\hline 56.746 & $.7240 \mathrm{E} 07$ & $.118 \mathrm{E} 06$ & .095 & 54.044 & $.6278 \mathrm{E} 07$ & $.129 \mathrm{E} 06$ & .095 \\
\hline 51.470 & $.5489 \mathrm{E} 07$ & $.114 \mathrm{E} 06$ & .096 & 49.019 & $.5980 \mathrm{E} 07$ & $.106 \mathrm{E} 06$ & . 096 \\
\hline 46.685 & $.6060 \mathrm{E} 07$ & $.142 \mathrm{E} 06$ & .097 & 44.462 & $.6550 \mathrm{E} 07$ & $.136 \mathrm{E} 06$ & . 097 \\
\hline 42.345 & $.6397 \mathrm{E} 07$ & $.142 \mathrm{E} 06$ & .097 & 40.328 & $.6042 \mathrm{E} 07$ & $.138 \mathrm{E} 06$ & .098 \\
\hline 38.408 & $.5618 \mathrm{E} 07$ & $.127 \mathrm{E} 06$ & .098 & 36.579 & $.4827 \mathrm{E} 07$ & $.122 \mathrm{E} 06$ & .099 \\
\hline 34.837 & $.4058 \mathrm{E} 07$ & $.138 \mathrm{E} 06$ & .100 & 33.178 & .3477 E 07 & $.126 \mathrm{E} 06$ & .100 \\
\hline 31.598 & $.2830 \mathrm{E} 07$ & $.115 \mathrm{E} 06$ & .101 & 30.094 & $.2671 \mathrm{E} 07$ & $.110 \mathrm{E} 06$ & .101 \\
\hline 28.661 & $.3458 \mathrm{E} 07$ & $.969 \mathrm{E} 05$ & .102 & 27.296 & $.4903 \mathrm{E} 07$ & .113 E 06 & .103 \\
\hline 25.996 & $.7308 \mathrm{E} 07$ & $.875 \mathrm{E} 05$ & .103 & 24.758 & .8503 E 07 & $.887 \mathrm{E} 05$ & .104 \\
\hline 23.579 & $.8149 \mathrm{E} 07$ & $.117 \mathrm{E} 06$ & .105 & 22.456 & $.7628 \mathrm{E} 07$ & $.122 \mathrm{E} 06$ & .106 \\
\hline 21.387 & $.6637 \mathrm{E} 07$ & $.120 \mathrm{E} 06$ & 107 & 20.368 & $.5971 \mathrm{E} 07$ & $.126 \mathrm{E} 06$ & .109 \\
\hline 19.399 & $.5434 \mathrm{E} 07$ & $.105 \mathrm{E} 06$ & .111 & 18.475 & $.5244 \mathrm{E} 07$ & $.115 \mathrm{E} 06$ & .113 \\
\hline 17.595 & $.4664 \mathrm{E} 07$ & $.113 \mathrm{E} 06$ & .115 & 16.757 & $.3972 \mathrm{E} 07$ & .989 E 05 & .117 \\
\hline
\end{tabular}

Revision: 0

Page 56 of 304

Date: March 31, 2010 
NEA/NSC/DOC(2006)1

Liquid Metal Fast Reactor - LMFR

FFTF-LMFR-RESR-001

CRIT-SPEC-REAC-COEF-MISC

Table 1.3.6 (cont'd.). Neutron Spectrum at the Lower Axial Reflector Location (Ref. 6, pp. 31-32).

\begin{tabular}{|c|c|c|c|c|c|c|c|}
\hline $\begin{array}{c}\text { Energy } \\
(\mathrm{keV})\end{array}$ & $\begin{array}{c}\begin{array}{c}\text { Flux } \\
\left(\mathrm{n} / \mathrm{cm}^{2}-\mathrm{s}\right)\end{array} \\
\end{array}$ & Error & Resolution & $\begin{array}{c}\text { Energy } \\
(\mathrm{keV})\end{array}$ & $\begin{array}{c}\text { Flux } \\
\left(\mathrm{n} / \mathrm{cm}^{2}-\mathrm{s}\right)\end{array}$ & Error & Resolution \\
\hline 15.959 & .3276E 07 & .113E 06 & .119 & 15.199 & (.3636Е 07 & "109E 06 & .122 \\
\hline 14.476 & $.4211 \mathrm{E} 07$ & $.101 \mathrm{E} 06$ & .124 & 13.786 & $.4094 \mathrm{E} 07$ & $.928 \mathrm{E} 05$ & .126 \\
\hline 13.130 & $.4031 \mathrm{E} 07$ & $.785 \mathrm{E} 05$ & .129 & 12.504 & $.3802 \mathrm{E} 07$ & $.838 \mathrm{E} 05$ & .131 \\
\hline 11.909 & .4205 E 07 & $.832 \mathrm{E} 05$ & .134 & 11.342 & $.4129 \mathrm{E} 07$ & $.785 \mathrm{E} 05$ & .136 \\
\hline 10.802 & $.4521 \mathrm{E} 07$ & $.992 \mathrm{E} 05$ & .139 & 10.287 & $.4583 \mathrm{E} 07$ & .108 E 06 & .142 \\
\hline 9.798 & $.4844 \mathrm{E} 07$ & $.101 \mathrm{E} 06$ & .145 & 9.331 & .4443 E 07 & $.937 \mathrm{E} 05$ & .148 \\
\hline 8.887 & $.3326 \mathrm{E} 07$ & .875 E 05 & .151 & 8.464 & .3199 E 07 & .843 E 05 & .154 \\
\hline 8.061 & $.2509 \mathrm{E} 07$ & $.898 \mathrm{E} 05$ & 157 & 7.677 & $.2321 \mathrm{E} 07$ & $.866 \mathrm{E} 05$ & .160 \\
\hline 7.311 & .2069 E 07 & .760 E 05 & .164 & 6.963 & $.1936 \mathrm{E} 07$ & $.734 \mathrm{E} 05$ & .167 \\
\hline 6.631 & $.2021 \mathrm{E} 07$ & $.676 \mathrm{E} 05$ & .171 & 6.316 & $.2169 \mathrm{E} 07$ & $.768 \mathrm{E} 05$ & .175 \\
\hline 6.015 & $.2230 \mathrm{E} 07$ & .709 E 05 & .179 & 5.728 & $.1878 \mathrm{E} 07$ & .773 E 05 & .182 \\
\hline 5.456 & $.1618 \mathrm{E} 07$ & .631 E 05 & .180 & 5.196 & $.1249 \mathrm{E} 07$ & $.653 \mathrm{E} 05$ & .191 \\
\hline 4.948 & .8704 E 06 & $.105 \mathrm{E} 06$ & .195 & 4.713 & .8323 E 06 & .962 E 05 & .199 \\
\hline 4.488 & $.5033 \mathrm{E} 06$ & .879 E 05 & 204 & 4.275 & $.3011 \mathrm{E} 06$ & .803 E 05 & .208 \\
\hline 4.071 & $.2821 \mathrm{E} 06$ & .735 E 05 & .213 & 3.877 & $.4739 \mathrm{E} 06$ & $.821 \mathrm{E} 05$ & .218 \\
\hline 3.693 & $.4090 \mathrm{E} 06$ & .749 E 05 & .223 & 3.517 & .4035 E 06 & $.685 \mathrm{E} 05$ & .228 \\
\hline 3.349 & $.8565 \mathrm{E} 06$ & $.628 \mathrm{E} 05$ & .233 & 3.190 & .1015 E 07 & $.576 \mathrm{E} 05$ & .239 \\
\hline 3.038 & $.1010 \mathrm{E} 07$ & $.671 \mathrm{E} 05$ & .244 & & & & \\
\hline
\end{tabular}

Even within the modest $(\sim 10 \%)$ energy resolution of the measurement, a great deal of resonance scattering structure was apparent for both the center and axial result. There was an enhanced degree of resonance detail in the FFTF spectrum at the higher energies most likely due to the large amount of steel in the IRT enclosing the detectors. At lower energies, below $10 \mathrm{keV}$, the resonance depression due to the sodium appeared to have been largely washed out in the FFTF measurement. This was also presumably a consequence of the complex local environment of the FFTF detector. Unfortunately, the proton-recoil technique failed completely below $1 \mathrm{keV}$, and other methods were required to characterize the neutron spectrum at the low energies (Ref. 6, pp. 9-10).

\section{Measurement Uncertainties}

The neutron spectra contained estimates of statistical errors relating to the finite number of counts acquired during the measurements. Systematic errors arose from several effects, including:

1. Residual uncertainties in the energy-scale calibration.

2. Uncertainties in the wall-and-end effect distortion of the spectrum.

3. Uncertainty in the effect of carbon recoils when methane gas is used.

Systematic errors other than those listed above can also be estimated. The n-p scattering cross section was probably not known absolutely to better than 1 or 2 percent. The volume of the detectors, in consequence of small alignment variations during fabrication and the inherent uncertainty in definition of the effective counting volume of proportional counters of finite length (due to field fringing at ends) probably contributed another $2 \%$ uncertainty. These last-mentioned errors were primarily of concern for absolute spectra rather than as effects influencing the shape of spectra with energy (Ref. 6, p. 10).

A crude estimation of the likely systematic error in scale calibration as a function of energy is shown in Figure 1.3.8 (top graph). The uncertainty is small near the $28 \mathrm{keV}$ iron scattering resonance and at the $615 \mathrm{keV}{ }^{14} \mathrm{~N}(\mathrm{n}, \mathrm{p})^{14} \mathrm{C}$ reaction since these points were used to provide an absolute normalization for the other calibrations, which were primarily only of a relative nature. The quality of the calibration at other 
Liquid Metal Fast Reactor - LMFR

FFTF-LMFR-RESR-001

CRIT-SPEC-REAC-COEF-MISC

energies was tested by accelerator experiments and by the consistency of fine-structure detail. Below about $3 \mathrm{keV}$, the systematic uncertainty commenced to rise sharply in consequence of the largely speculative variation of energy loss per ion pair for protons in this low-energy region (Ref. 6, pp. 10-11).

Figure 1.3.8 (bottom graph) also shows the trend with energy of other systematic errors relating to uncertainties such as the wall-and-end effect, the effects of carbon recoil, and gamma-neutron discrimination. The peaking at $100 \mathrm{keV}$ was due to the uncertainty in the carbon recoil effect. The discontinuity at $100 \mathrm{keV}$ reflects the break between the use of data from hydrogen counters (below $\sim 100$ $\mathrm{keV}$ ) and use of the data from methane counters (above $\sim 100 \mathrm{keV}$ ). The monotonic increase at higher energies reflected the increasing uncertainty relating to wall-and-end effects. The uncertainty below 100 $\mathrm{keV}$ was mostly a reflection of gamma-neutron discrimination which may become increasingly difficult at the lowest energies depending upon the shape of the spectrum and the gamma background encountered, and uncertainty in the variation of energy loss per ion pair, which affected the amplitude as well as the energy-scale calibration of detectors (Ref. 6, p. 11). 
FFTF-LMFR-RESR-001

CRIT-SPEC-REAC-COEF-MISC

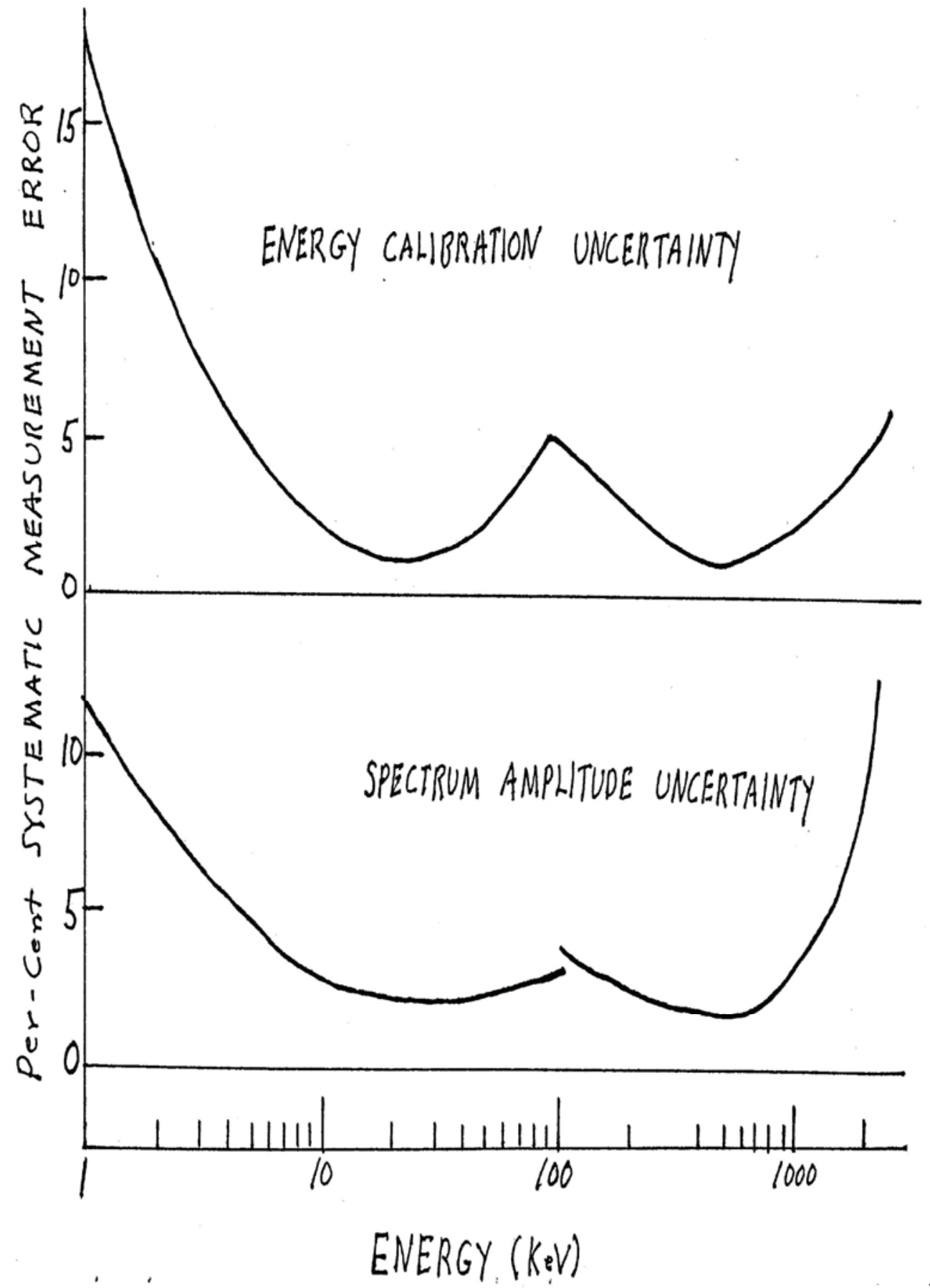

Figure 1.3.8. Approximate Systematic Errors in the Proton-Recoil Method (Ref. 6, Fig. 14). 
NEA/NSC/DOC(2006)1

Liquid Metal Fast Reactor - LMFR

FFTF-LMFR-RESR-001

CRIT-SPEC-REAC-COEF-MISC

\section{$1.4 \quad$ Description of Reactivity Effects Measurements}

\subsubsection{Overview of Experiment}

The FFTF was operated by Westinghouse Hanford Company for the U.S. Department of Energy. During the period from November 27, 1979 to March 8, 1980, fuel was loaded into the FFTF core, initial criticality was achieved, and several subcritical physics measurements were performed (Ref. 1, p.1).

On March 2, 1980, a near-critical, \$0.28 subcritical, core configuration was achieved with the three primary rods and control rod number 5 completely withdrawn and control rod number 7 withdrawn 28 inches $(71.12 \mathrm{~cm})$ above the base of the fuel. This configuration is the primary reference state from which individual and groups of rods were dropped (Ref. 1, p. 47, Ref. 3, p. 29, and Ref. 8, p 5).

Additional rod worth measurements were performed using other subcritical configurations of the core.

Reactivity effects measurements evaluated include a total of 21 control rod worth measurements (seven of which represent combinations of dropped rods), two control rod bank worth measurements, six differential control rod worth measurements, measured shutdown margin, and measured excess reactivity for the fully-loaded critical core configuration of the FFTF.

\subsubsection{Geometry of the Experiment Configuration and Measurement Procedure}

The geometry of the core was that of the fully-loaded core configuration in Section 1.1.2 with control rod movements as stated in the following subsections.

\subsubsection{Control Rod Worths}

The inverse kinetics rod drops (IKRD) method was used at the FFTF for precise measurements of subcriticality, for worth measurements of control rods, and to establish a calibration point for shutdown reactivity measurements using the modified source multiplication (MSM) technique. The rod drops were normally initiated from a reactivity state of about $10 \notin$ to $20 \notin$ subcritical, and were monitored with the three ex-core fission chambers (LLFM). This resulted in a predrop steady state count rate of about 10,000 to 20,000 counts/second on these detectors when inserted to the core midplane, with the intrinsic neutron source level of the fresh fuel, and initial detector electronics adjustments (Ref. 3, p. 26 and Ref. 9, p. 3).

Before any reactivity measurements could be made with MSM, it was necessary to calibrate the LLFMs by dropping control rod number 7 from the reference state of $\$ 0.28$ subcritical (Ref. 8, p. 5). Then the calibration constants, $\mathrm{Q}_{0}$, for the LLFMs could be obtained: 4769.23 $\pm 63.14,2843.99 \pm 51.03$, and 4755.06 \pm 61.50 \$-counts/sec for detector A, B, and C, respectively (Ref. 8, p. 6). Typical core configuration factors, $\mathrm{F}$, for various core states are shown in Table 1.4.1; generally, the $\mathrm{F}$ factors range from 0.87 to about 1.2 (Ref. 8, p. 8). Further information about the F factors can be found in the references. 
NEA/NSC/DOC(2006)1

Liquid Metal Fast Reactor - LMFR

FFTF-LMFR-RESR-001

CRIT-SPEC-REAC-COEF-MISC

Table 1.4.1. Typical Core Configuration Factors for Various Core States (Ref. 8, p. 9).

\begin{tabular}{|c|c|ccc||}
\hline $\begin{array}{c}\text { Rod(s) } \\
\text { Dropped }\end{array}$ & $\begin{array}{c}\text { Reactivity } \\
\text { Level (\$) }\end{array}$ & LLFM A & LLFM B & LLFM C \\
\hline \hline 0 & 0.28 & 1.0079 & 0.8858 & 1.0632 \\
3 & 4.65 & 1.0367 & 0.8765 & 1.1510 \\
1 & 6.04 & 1.1210 & 0.9183 & 1.040 \\
5,1 & 9.11 & 1.1898 & 0.9495 & 0.9456 \\
1,2 & 12.45 & 1.0893 & 1.0178 & 1.0723 \\
$5,1,2$ & 15.84 & 1.1603 & 1.0546 & 0.9745 \\
ARI $^{(\mathrm{a})}$ & 24.0 & 1.1036 & 1.1053 & 1.0929 \\
\hline \hline
\end{tabular}

(a) All Rods In.

The neutron level transient from each rod drop was analyzed via the IKRD method to obtain predrop and postdrop reactivities to determine the worth of the dropped $\operatorname{rod}(\mathrm{s})$. These analyses also yielded the effective neutron source strength, or calibration constants, required by the MSM technique. The constants obtained by dropping control rod 7 were used for all subsequent MSM assessments. These experiments and reactivity evaluations provided a direct comparison between the two methods for reactivities to about $\$ 16$ subcritical. The initial core configuration and combinations of rods to be dropped were selected such that significant (up to 19\%) detection efficiency changes would be introduced, to ensure that the MSM technique would be taxed to its anticipated limits (Ref. 1, p. 47).

Transient data collection from the three LLFM detectors was initiated by on-line computer shortly before the rod drop, and continued for about 60 seconds afterward. Data were collected from each LLFM at 0.01 -second intervals during the transient. The rod-drop neutron level transient data were processed at HEDL with the IKRD computer program on the FFTF Mod Comp-III computer, following the data collection phase. The recorded neutron level transient was normally sampled, for inverse kinetics calculations, every 0.3 seconds, and the delayed neutron parameters given in Table 1.4.2 were employed. This code employed the Yang-Albrecht algorithm ${ }^{\mathrm{a}}$ and processed the transient data from each detector separately. It required as input the change in detection efficiency for each detector due to the rod motion, and provided calculated values of pre- and post-drop reactivity, effective source strength (in units of \$counts/sec), and related uncertainties, for each of the three detectors (Ref. 3, p. 27 and Ref. 9, p. 3).

The reference state from which the effective source for each LLFM was obtained using the IKRD analysis is that with control rods 1,2,3, and 5 fully withdrawn; rods 4, 6, 8, and 9 fully inserted; and rod 7 withdrawn by 28 inches $(71.12 \mathrm{~cm})$ prior to being dropped. The coolant temperature was $404^{\circ} \mathrm{F}$ $\left(\sim 207^{\circ} \mathrm{C}\right)$. The results from the IKRD processing are shown in Table 1.4.3 (Ref. 3, p. 29).

\footnotetext{
${ }^{a}$ C. Y. Yangg and R. W. Albrecht, "Inverse Kinetics Determination of Sub-Criticality," Nucl. Tech., 22, 323 (1974).
}

Revision: 0

Page 61 of 304

Date: March 31, 2010 
NEA/NSC/DOC(2006)1

Liquid Metal Fast Reactor - LMFR

FFTF-LMFR-RESR-001

CRIT-SPEC-REAC-COEF-MISC

Table 1.4.2. Delayed Neutron Parameters used in IKRD Analysis (Ref. 3, p. 27).

\begin{tabular}{|c|cc||}
\hline $\begin{array}{c}\text { Neutron } \\
\text { Group }\end{array}$ & $\begin{array}{c}\text { Delayed } \\
\text { Neutron } \\
\text { Fraction }\end{array}$ & $\begin{array}{c}\text { Precursor } \\
\text { Decay } \\
\text { Constant }\end{array}$ \\
\hline \hline 1 & $0.8695 \times 10^{-4}$ & $0.01295 \mathrm{sec}^{-1}$ \\
2 & $0.71855 \times 10^{-3}$ & 0.03131 \\
3 & $0.61209 \times 10^{-3}$ & 0.13450 \\
4 & $0.11277 \times 10^{-2}$ & 0.3423 \\
5 & $0.48339 \times 10^{-3}$ & 1.3571 \\
6 & $0.15465 \times 10^{-3}$ & 3.6617 \\
\hline
\end{tabular}

Table 1.4.3. Results of IKRD Processing (Ref. 3, p. 30).

\begin{tabular}{|c|ccc||}
\hline & \multicolumn{3}{|c|}{ LLFM } \\
& A & B & C \\
\hline \hline Detector efficiency relative to reference state $(\mathrm{F})$ & 1.0079 & 0.8858 & 1.0632 \\
Change in detector efficiency due to rod drop & -0.096 & 0.0748 & 0.0379 \\
Pre-drop reactivity & $-\$ 0.276$ & $-\$ 0.273$ & $-\$ 0.268$ \\
Post-drop reactivity & $-\$ 3.189$ & $-\$ 3.200$ & $-\$ 3.120$ \\
Effective source (\$-counts/sec) & 4645.2 & 2774.9 & 4631.4 \\
Effective source, for reference state (\$-counts/sec) & 4608.8 & 3132.6 & 4356.1 \\
\hline
\end{tabular}

IKRD measurements were made of the worth of each control rod, and of the combined worth of the weakest two Row 3 rods. These measurements were all made from an initial state with the Row 3 rods fully withdrawn, and the Row 5 rods banked at a position to give a subcriticality of 10 to $20 \phi$. When the worth of a Row 5 rod was measured, it was fully withdrawn prior to dropping it and the remaining five Row 5 rods were inserted a sufficient amount to establish the desired pre-drop count rates. Results of these rod drop measurements are given in Table 1.4.4 (Ref. 3, p. 30 and Ref 9, p. 5).

The rod worths based on these measurements are shown in Table 1.4.5. 
FFTF-LMFR-RESR-001

CRIT-SPEC-REAC-COEF-MISC

Table 1.4.4. Results of IKRD Measurements (Ref. 3, p. 32 and Ref. 4, p. 39, and Ref. 9, pp. 6-7).

\begin{tabular}{|c|c|c|c|c|c|}
\hline \multirow{2}{*}{$\begin{array}{c}\text { Rod } \\
\text { Dropped }\end{array}$} & \multirow{2}{*}{$\begin{array}{c}\text { Row } 5 \\
\text { Bank Height }^{(\mathrm{a})} \\
\end{array}$} & & \multicolumn{3}{|c|}{ Detector } \\
\hline & & & A & $\mathrm{B}$ & $\mathrm{C}$ \\
\hline \multirow[t]{4}{*}{1} & \multirow{4}{*}{$\begin{array}{c}13.9 \text { in. } \\
(35.306 \mathrm{~cm})\end{array}$} & pre-drop count rate & 7163 & 4906 & 6959 \\
\hline & & detector efficiency change & 0.104 & 0.025 & -0.018 \\
\hline & & pre-drop reactivity & $-\$ 0.19$ & -0.19 & -0.19 \\
\hline & & post-drop reactivity & $-\$ 5.89$ & -6.18 & -5.94 \\
\hline \multirow[t]{4}{*}{2} & \multirow{4}{*}{$\begin{array}{c}13.9 \mathrm{in.} \\
(35.306 \mathrm{~cm})\end{array}$} & pre-drop count rate & 7243 & 4955 & 7035 \\
\hline & & detector efficiency change & -0.021 & 0.100 & 0.026 \\
\hline & & pre-drop reactivity & $-\$ 0.19$ & -0.19 & -0.19 \\
\hline & & post-drop reactivity & $-\$ 5.79$ & -5.62 & -5.70 \\
\hline \multirow[t]{4}{*}{3} & \multirow{4}{*}{$\begin{array}{c}13.9 \mathrm{in.} \\
(35.306 \mathrm{~cm})\end{array}$} & pre-drop count rate & 7123 & 4878 & 6931 \\
\hline & & detector efficiency change & 0.028 & -0.015 & 0.094 \\
\hline & & pre-drop reactivity & $-\$ 0.18$ & -0.19 & -0.19 \\
\hline & & post-drop reactivity & $-\$ 5.47$ & -5.69 & -5.59 \\
\hline \multirow[t]{4}{*}{4} & \multirow{4}{*}{$\begin{array}{c}9.7 \mathrm{in.} \\
(24.638 \mathrm{~cm})\end{array}$} & pre-drop count rate & 6936 & 5245 & 7324 \\
\hline & & detector efficiency change & 0.111 & -0.011 & -0.006 \\
\hline & & pre-drop reactivity & $-\$ 0.18$ & -0.18 & -0.18 \\
\hline & & post-drop reactivity & $-\$ 4.24$ & -4.28 & -4.21 \\
\hline \multirow[t]{4}{*}{5} & \multirow{4}{*}{$\begin{array}{c}9.6 \text { in. } \\
(24.384 \mathrm{~cm})\end{array}$} & pre-drop count rate & 6266 & 4477 & 7193 \\
\hline & & detector efficiency change & 0.106 & 0.048 & -0.102 \\
\hline & & pre-drop reactivity & $-\$ 0.20$ & -0.20 & -0.20 \\
\hline & & post-drop reactivity & $-\$ 4.26$ & -4.34 & -4.28 \\
\hline
\end{tabular}

(a) Except for rod being dropped. 
NEA/NSC/DOC(2006)1

\section{Liquid Metal Fast Reactor - LMFR \\ FFTF-LMFR-RESR-001 \\ CRIT-SPEC-REAC-COEF-MISC}

Table 1.4.4. (cont'd.). Results of IKRD Measurements

(Ref. 3, p. 32 and Ref. 4, p. 39, and Ref. 9, pp. 6-7).

\begin{tabular}{|c|c|c|c|c|c|}
\hline \multirow{2}{*}{$\begin{array}{c}\text { Rod } \\
\text { Dropped }\end{array}$} & \multirow{2}{*}{$\begin{array}{c}\text { Row } 5 \\
\text { Bank Height }^{(a)} \\
\end{array}$} & & \multicolumn{3}{|c|}{ Detector } \\
\hline & & & A & $\mathrm{B}$ & $\mathrm{C}$ \\
\hline \multirow[t]{4}{*}{6} & \multirow{4}{*}{$\begin{array}{c}10.4 \mathrm{in} . \\
(26.416 \mathrm{~cm})\end{array}$} & pre-drop count rate & 7149 & 4466 & 6928 \\
\hline & & detector efficiency change & -0.007 & 0.095 & -0.011 \\
\hline & & pre-drop reactivity & $-\$ 0.19$ & -0.19 & -0.20 \\
\hline & & post-drop reactivity & $-\$ 3.75$ & -3.73 & -3.81 \\
\hline \multirow[t]{4}{*}{7} & Rods $4,5,6,8-10.0$ in. & \multirow{4}{*}{$\begin{array}{l}\text { pre-drop count rate } \\
\text { detector efficiency change } \\
\text { pre-drop reactivity } \\
\text { post-drop reactivity }\end{array}$} & 7592 & 4368 & 6456 \\
\hline & $(25.4 \mathrm{~cm})$ & & -0.103 & 0.102 & 0.049 \\
\hline & $\operatorname{Rod} 9-9.9 \mathrm{in}$. & & $-\$ 0.19$ & -0.19 & -0.20 \\
\hline & $(25.146 \mathrm{~cm})$ & & $-\$ 4.02$ & -4.09 & -4.06 \\
\hline \multirow[t]{4}{*}{8} & \multirow{4}{*}{$\begin{array}{c}10.9 \mathrm{in} \\
(27.686 \mathrm{~cm})\end{array}$} & pre-drop count rate & 7242 & 4930 & 6512 \\
\hline & & detector efficiency change & -0.004 & -0.004 & 0.083 \\
\hline & & pre-drop reactivity & $-\$ 0.19$ & -0.19 & -0.19 \\
\hline & & post-drop reactivity & $-\$ 3.34$ & -3.43 & -3.31 \\
\hline \multirow[t]{4}{*}{9} & \multirow{4}{*}{$\begin{array}{c}10.1 \mathrm{in} . \\
(25.654 \mathrm{~cm})\end{array}$} & pre-drop count rate & 7692 & 5917 & 7190 \\
\hline & & detector efficiency change & 0.052 & -0.095 & 0.094 \\
\hline & & pre-drop reactivity & $-\$ 0.17$ & -0.17 & -0.17 \\
\hline & & post-drop reactivity & $-\$ 4.03$ & -3.98 & -3.98 \\
\hline \multirow[t]{4}{*}{$2 \& 3$} & \multirow{4}{*}{$\begin{array}{c}13.8 \mathrm{in} . \\
(35.052 \mathrm{~cm})\end{array}$} & pre-drop count rate & 6782 & 4607 & 6578 \\
\hline & & detector efficiency change & 0.0002 & 0.0872 & 0.122 \\
\hline & & pre-drop reactivity & $-\$ 0.20$ & -0.20 & -0.20 \\
\hline & & post-drop reactivity & $-\$ 11.53$ & -11.47 & -11.25 \\
\hline
\end{tabular}

(a) Except for rod being dropped. 
FFTF-LMFR-RESR-001

CRIT-SPEC-REAC-COEF-MISC

Table 1.4.5. Results of IKRD Measurements

(Ref. 2, p. 31, Ref. 3, p. 48 and Ref. 4, pp. 39-43, and Ref. 9, p. 13).

\begin{tabular}{||c|c|}
\hline $\begin{array}{c}\text { Rod Measured } \\
\text { (Position) }\end{array}$ & $\begin{array}{c}\text { Measured } \\
\text { Worth }(\$)^{(\mathrm{a})}\end{array}$ \\
\hline $1(1302)$ & 5.82 \\
$2(2302)$ & 5.52 \\
$3(3302)$ & 5.40 \\
$4(1502)$ & 4.07 \\
$5(1504)$ & 4.09 \\
$6(2502)$ & 3.57 \\
$7(2504)$ & 3.86 \\
$8(3502)$ & 3.17 \\
$9(3504)$ & 3.83 \\
$2 \& 3(2303 \& 3302)$ & 11.22 \\
\hline
\end{tabular}

(a) Relative uncertainty of approximately $1 \%$ in all measured values.

The reactivity worth of each of the three primary and six secondary control rods was measured using the IKRD rod drop technique (Table 1.4.6). Technical specifications on the FFTF required that no secondary control rod worth exceeded $\$ 5$ and no primary control rod worth exceeded $\$ 8$. The uncertainties quoted for the IKRD results include a contribution due to the detector efficiency changes, but are predominantly the result of the random noise of the neutron data. The estimated magnitude of this uncertainty was verified by repeating the measurement for control rod 5, five additional times. The variance in the data for the repeated measurements was consistent with the uncertainty shown in the table (Ref. 1, p. 51 and Ref. 2, pp. 30-31). 
NEA/NSC/DOC(2006)1

Liquid Metal Fast Reactor - LMFR

FFTF-LMFR-RESR-001

CRIT-SPEC-REAC-COEF-MISC

Table 1.4.6. Reactivity Worths of Individual Control Rods

(Ref. 1, p. 52, Ref. 2, p. 31, and Ref. 7).

\begin{tabular}{|c|ccc||}
\hline Rod & \multicolumn{3}{|c|}{ Rod Worth (\$) } \\
\hline \hline 1 & 5.82 & \pm & $0.08^{(\mathrm{b})}$ \\
2 & 5.52 & \pm & 0.07 \\
3 & 5.40 & \pm & 0.06 \\
4 & 4.07 & \pm & 0.04 \\
5 & $4.11^{(\mathrm{a})}$ & \pm & $0.02^{(\mathrm{b})}$ \\
6 & 3.57 & \pm & 0.04 \\
7 & 3.86 & \pm & $0.04^{(\mathrm{b})}$ \\
8 & 3.17 & \pm & $0.03^{(\mathrm{b})}$ \\
9 & 3.83 & \pm & 0.04 \\
\hline
\end{tabular}

(a) References 2 and 7 state that this value is $\$ 4.09$.

(b) Reference 7 states that the uncertainty is $0.04,0.05$, 0.06 , and 0.07 for Rods $1,5,7$, and 8 , respectively, but that they are based upon counting statistics only.

The results from some MSM and IKRD experiments are shown in Table 1.4.7. The MSM rod worths were accurate to within $5 \%$ even as far subcritical as $\sim \$ 16$ (Ref. 1, p. 47). Elsewhere, the primary worth values in this table were reported as reactivity changes averaged from the three LLFMs, and the worth for dropping Rod 7 from a height of 28 inches $(71.12 \mathrm{~cm})$ was also reported (Ref. 7).

The MSM method had requirements of obtaining results within $10 \%$ of the reactivity, $\rho$, for subcritical levels ranging from near critical to 5 dollars. For reactivities ranging from 5 to 15 dollars, the accuracy requirement was $20 \%(2 \sigma)$ in reactivity. For reactivities greater than 15 dollars, especially from 25 to 40 dollars, the accuracy was to be within $20 \%(2 \sigma)$ in reactivity and rod worths evaluated by MSM were to be accurate to within one dollar (Ref. 8, p. 5). The values in Table 1.4.7 demonstrate that the MSM technique agreed with the IKRD values to within 3\% for reactivities less than $\$ 6$ subcritical. They agreed within $5 \%$ for reactivities up to $\$ 15$ subcritical (Ref. 2, p. 28 and Ref. 8, p. 8). The accuracy of reactivites measured by MSM was generally well within the $20 \%$ limit prescribed for subcritical reactivities of about $\$ 23$ (Ref. 2, p. 28 and Ref. 8, p. 14). 
NEA/NSC/DOC(2006)1

Liquid Metal Fast Reactor - LMFR

FFTF-LMFR-RESR-001

CRIT-SPEC-REAC-COEF-MISC

Table 1.4.7. MSM Evaluation Experiments Near Critical

(Ref. 1, p. 48, Ref. 2, p. 26, Ref. 7, and Ref. 8, pp. 10-11).

\begin{tabular}{|c|c|c|c|ccc|ccc||}
\hline \multirow{2}{*}{ Experiment } & Rod(s) & Subcritical & IKRD/MSM & \multicolumn{5}{|c|}{ Rod Worths (\$) } \\
& Dropped & Reactivity Limit & Ratio & \multicolumn{3}{|c|}{ MSM } & \multicolumn{3}{|c|}{ IKRD } \\
\hline \hline 2 & 5 & & 1.024 & 3.75 & \pm & 0.07 & 3.84 & \pm & 0.05 \\
3 & 1 & \multirow{4}{*}{$\$ 6$} & 1.007 & 6.00 & \pm & 0.11 & 6.04 & \pm & 0.09 \\
4 & 2 & & $1.019^{(\mathrm{a})}$ & 5.78 & \pm & 0.09 & 5.89 & \pm & 0.09 \\
5 & 3 & & 1.013 & 4.59 & \pm & 0.07 & 4.65 & \pm & 0.07 \\
\hline 6 & 5,1 & & 1.012 & 8.89 & \pm & 0.19 & 9.00 & \pm & 0.16 \\
7 & 5,3 & & 1.007 & 8.74 & \pm & 0.16 & 8.80 & \pm & 0.14 \\
8 & 1,2 & & $1.038^{(\mathrm{a})}$ & 11.99 & \pm & 0.19 & 12.45 & \pm & 0.29 \\
9 & 1,3 & $-\$ 15$ & $1.024^{(\mathrm{a})}$ & 10.75 & \pm & 0.21 & 11.01 & \pm & 0.25 \\
10 & $5,1,2$ & & $1.047^{(\mathrm{a})(\mathrm{b})}$ & 15.13 & \pm & 0.29 & $15.84^{(\mathrm{b})}$ & \pm & 0.35 \\
11 & $5,2,3$ & & $1.028^{(\mathrm{a})}$ & 14.81 & \pm & 0.25 & 15.22 & \pm & 0.39 \\
\hline 1 & 7 (from 28 in) & -- & $0.993^{(\mathrm{c})}$ & 2.92 & -- & -- & 2.90 & -- & -- \\
\hline
\end{tabular}

(a) Reference 2 reports these values as $1.017,1.030,1.025,1.05$, and 1.017 , respectively.

(b) The IKRD value is reported as 15.85 in Reference 7, with an IKRD/MSM ratio of 1.048.

(c) The ratio is not 1.0 because the IKRD and MSM data were taken independently. The difference in these data $(0.7 \%)$ is the result of counting statistics.

\subsubsection{Control Rod Bank Worths}

The total reactivity worth of the three primary rods was determined by measuring the subcriticality of the reactor with the three primary rods fully withdrawn and then with the three primary rods fully inserted, taking the difference of the two measured subcriticality values. The six secondary control rods remained fully inserted. The worth was found to be $\$ 16.3$ at refueling temperature with an uncertainty of $\$ 0.1$ (Ref. 1, p. 50 and Ref. 2, p. 30). Elsewhere, the worth of the primary rod system has been reported as $\$ 16.34 \pm 0.12$ (Ref. 7).

The total reactivity worth of the six secondary control rods was determined with the primary control rods fully withdrawn and fully inserted. In the first configuration, the subcriticality of the reactor, with the secondary control rods fully inserted and the primary control rods fully withdrawn, was determined to be $\$ 7.32$ with an uncertainty of $\$ 0.06$ via the MSM technique. From the observed reactivity worth profile of the secondary control rods during the approach to critical with 59 fuel subassemblies loaded, it was estimated that $33.9 \pm 1.7 \%$ of the total worth would be realized by withdrawing the rods to the estimated critical position of $\sim 14.1$ inches $(35.814 \mathrm{~cm})$. This implied that the total secondary control rod worth is $\$ 22 \pm 1$ (Ref. 1, p. 50 and Ref. 2, p. 30). For the second configuration, the subcriticality of the reactor with all control rods fully inserted and only the six secondary control rods withdrawn was determined using the MSM technique. The difference between these reactivities and thus the worth of the secondary rods with the primary control rods fully inserted was $\$ 19.9 \pm 0.1$ (Ref. 1, p. 51 and Ref. 2, p. 30). Elsewhere, the worth of the secondary rod system has been reported as \$21.98 (Ref. 7).

\subsubsection{Differential Control Rod Worths}

The reactivity worth of moving individual secondary control rods a small increment from the mean secondary control rod bank position was assessed for a single bank position $\sim 13.7$ inches $(\sim 34.798 \mathrm{~cm})$ 
NEA/NSC/DOC(2006)1

Liquid Metal Fast Reactor - LMFR

FFTF-LMFR-RESR-001

CRIT-SPEC-REAC-COEF-MISC

withdrawn using the MSM technique. Each secondary control rod was first withdrawn one inch (2.54 $\mathrm{cm})$ and then inserted two inches $(5.08 \mathrm{~cm})$. The average differential reactivity worths are shown in Table 1.4.8. The uncertainties shown include a small contribution arising from the MSM measurements but are predominantly due to the uncertainty in positioning the control rods. The latter uncertainty is estimated to be \pm 0.025 inches $( \pm 0.0635 \mathrm{~cm})$ at the $1 \sigma$ level (Ref. 1, p. 55 and Ref. 2, pp. 33-34).

Table 1.4.8. Differential Control Rod Worths

(Ref. 1, p. 56 and Ref. 2, p. 34).

\begin{tabular}{|c|ccc||}
\hline \hline Rod & \multicolumn{3}{|c|}{ Diff. Worths $(\phi /$ in $)$} \\
\hline \hline 4 & 15.4 & \pm & 0.4 \\
5 & 15.7 & \pm & 0.4 \\
6 & 13.6 & \pm & 0.4 \\
7 & 14.5 & \pm & 0.4 \\
8 & 12.1 & \pm & 0.4 \\
9 & 14.3 & \pm & 0.4 \\
\hline \hline
\end{tabular}

\subsubsection{Shutdown Margin}

The all-control-rods-inserted reactivity assessment of the FFTF reactor was provided by the MSM method using calibration constants and equilibrium count rates measured for each of the three LLFMs located in the shield region of the core. The calibration constants were obtained from rod drop experiments with the core at $\$ 0.15$ subcritical: three primary rods fully withdrawn and all secondary rods banked at 13.9 inches $(35.306 \mathrm{~cm})$. The result of this MSM assessment demonstrated that the full shutdown reactivity of the core was $\$ 23.6$ with an uncertainty of $\$ 0.2$ (Ref. 1, p. 50 and Ref. 2, p. 29).

\subsubsection{Excess Reactivity}

The reactivity control remaining in the secondary control rod system after criticality at full power was reached determined how long the reactor could operate with the current fuel load. From previous estimates of the total reactivity worth of the secondary control rod system $(\$ 22 \pm 1)$ and the subcritical reactivity with the primary control rods fully withdrawn and the secondary control rods fully inserted $(\$ 7.32 \pm 0.06)$ it was estimated that the excess reactivity loss (power defect) of $\$ 3.2 \pm 0.6$ in attaining full power conditions yielded an estimated excess reactivity at full power of $\$ 11 \pm 1$ (Ref. 1, p. 54 and Ref. 2, p. 33). Elsewhere it was reported that the inferred excess reactivities at full power and $592^{\circ} \mathrm{F}\left(\sim 311^{\circ} \mathrm{C}\right)$ are $\$ 11.50$ and $\$ 13.96$, respectively. The measured excess reactivity at $400^{\circ} \mathrm{F}\left(\sim 204^{\circ} \mathrm{C}\right)$ was $\$ 14.66$ (Ref. 7).

\subsubsection{Material Data}

The materials in the core and control rods were those described in the fully-loaded core configuration in Section 1.1.3.

\subsubsection{Temperature Data}

The temperature of the core was approximately $400^{\circ} \mathrm{F}\left(\sim 204^{\circ} \mathrm{C}\right)$. 
NEA/NSC/DOC(2006)1

Liquid Metal Fast Reactor - LMFR

FFTF-LMFR-RESR-001

CRIT-SPEC-REAC-COEF-MISC

\subsubsection{Additional Information Relevant to Reactivity Effects Measurements}

Additional information is not available.

\subsection{Description of Reactivity Coefficient Measurements}

\subsubsection{Overview of Experiment}

The reactor physics experiments described in this section pertain to the data available for the isothermal temperature coefficient. The primary loop flow reactivity effect has not been evaluated.

\subsubsection{Isothermal Temperature Coefficient}

The isothermal temperature coefficient near the refueling temperature of $400^{\circ} \mathrm{F}\left(\sim 204^{\circ} \mathrm{C}\right)$ was determined to be negative and of the expected magnitude, $-0.7 \phi /{ }^{\circ} \mathrm{F}$ (Ref. 1, p. 1). It was measured over the temperature range of $383^{\circ} \mathrm{F}\left(\sim 195^{\circ} \mathrm{C}\right)$ to $417^{\circ} \mathrm{F}\left(\sim 214^{\circ} \mathrm{C}\right)$ using secondary pump work and Dump Heat Exchanger (DHX) adjustments to obtain the temperature increase. The change in reactivity of the core was measured by performing IKRD experiments before and after the temperature increase from reproducible initial control rod configurations. The difference between the pre-drop reactivities from the IKRD analyses yielded the desired reactivity changes (Ref. 1, p. 55 and Ref. 2, p. 33). The temperature coefficient is also reported as $-0.69 \phi /{ }^{\circ} \mathrm{F}$ (Ref. 2, p. 33) for this same experiment description. Elsewhere, the temperature coefficient of $-0.7 \phi /{ }^{\circ} \mathrm{F}$ is reported to have an uncertainty of $\pm 0.10 \phi /{ }^{\circ} \mathrm{F}$ (Ref. 7).

References 3 (p. 36) and 9 (p. 8) report that results from the subcriticality measurements could be adjusted with a measured isothermal temperature coefficient of $-0.6 \phi /{ }^{\circ} \mathrm{F}$.

Elsewhere the reactivity dependence upon isothermal temperature was reported to be linear with a slope of $-1.0 \pm 0.01$ cents $/{ }^{\circ} \mathrm{C}(1 \sigma)$ from the refueling temperature of $228^{\circ} \mathrm{C}$ to a hot standby temperature of $314^{\circ} \mathrm{C}$ using nuclear heat and reactivity measurements via IKRD and calibrated control rods. ${ }^{a}$

\subsubsection{Primary Loop Flow Reactivity Effect}

A measurement of reactivity change due to primary sodium coolant flow rate change was made by performing the IKRD assessments of the core reactivity with the primary coolant flow rate at $>90 \%$ and again at $8 \%$ of full flow. The difference in these reactivities was $4.00 \pm 0.64 \phi$. After correcting for a slight change in the coolant temperature at the core inlet, the total flow reactivity difference was found to be $5.1 \pm 0.7 \phi$ (Ref. 1, p. 56 and Ref. 2, p. 34). Elsewhere, this induced negative reactivity is said to range from "pony motor flow to 75 percent full flow" (Ref. 7).

\subsubsection{Geometry of the Experiment Configuration and Measurement Procedure}

The geometry of the core was that of the fully-loaded core configuration in Section 1.1.2.

\subsubsection{Material Data}

The materials in the core were those described in the fully-loaded core configuration in Section 1.1.3.

\footnotetext{
${ }^{a}$ C. L. Peckinpaugh, R. A. Bennett, and W. R. Wycoff, "FFTF Operational Results," HEDL-SA-2694 (CONF820914 4)/IAEA-CN-42/276, Proc. IAEA Int. Cong. Nuclear Power Experience, Vienna, Austria, September 1317 (July 1982).
} 
NEA/NSC/DOC(2006)1

Liquid Metal Fast Reactor - LMFR

FFTF-LMFR-RESR-001

CRIT-SPEC-REAC-COEF-MISC

\subsubsection{Temperature Data}

The refueling temperature coefficient of $-0.7 \phi /{ }^{\circ} \mathrm{F}$ at $400^{\circ} \mathrm{F}\left(\sim 204^{\circ} \mathrm{C}\right)$ was obtained using data measured over the temperature range of $383^{\circ} \mathrm{F}\left(\sim 195^{\circ} \mathrm{C}\right)$ to $417^{\circ} \mathrm{F}\left(\sim 214^{\circ} \mathrm{C}\right)$. The isothermal temperature coefficient of $-1.0+-0.01$ cents $/{ }^{\circ} \mathrm{C}$ was obtained from temperature measurements from $228^{\circ} \mathrm{C}$ to $314^{\circ} \mathrm{C}$.

\subsubsection{Additional Information Relevant to Reactivity Coefficient Measurements}

Additional information is not available.

\subsection{Description of Kinetics Measurements}

Kinetics measurements were not made.

\subsection{Description of Reaction-Rate Distribution Measurements}

Reaction-rate distribution measurements were not made.

\subsection{Description of Power Distribution Measurements}

Power distribution measurements were not made.

\subsection{Description of Isotopic Measurements}

Isotopic measurements were not made.

\subsection{Description of Other Miscellaneous Types of Measurements}

\subsubsection{Overview of Experiment}

Additional measurements in the FFTF include gamma spectra measurements in the In-Reactor Thimble (IRT) in preparation for establishing an accurate characterization of the gamma ray environment in the FFTF for irradiation experiments.

The reactor physics experiments evaluated in this section pertain to the low-energy electron and gammaray spectra measurements performed in the IRT near the axial and radial center of the core.

\subsubsection{Geometry of the Experiment Configuration and Measurement Procedure}

The geometry of the core was that of the fully-loaded core configuration described in Section 1.1.2 and 1.3.2 with modifications as stated below.

\section{Detectors}

The gamma ray spectrum measurements were made using a Compton recoil spectrometer comprised of a pair of solid-state, lithium drifted silicon ( $\mathrm{SiLi}$ ) detectors mounted face-to-face (Janus probe) and were cooled by a Peltier refrigerator to about $-30{ }^{\circ} \mathrm{F}\left(\sim-34{ }^{\circ} \mathrm{C}\right)$. The face-to-face arrangement provides a completely contained sensitive region permitting pulse-shape discrimination. Special electronics made 
NEA/NSC/DOC(2006)1

\section{Liquid Metal Fast Reactor - LMFR}

FFTF-LMFR-RESR-001

CRIT-SPEC-REAC-COEF-MISC

possible two complementary modes of operation: the non-coincidence mode for low energy $(<4 \mathrm{MeV})$ measurements, and the coincidence mode for higher energy measurements. The detector system allowed for measurements up to an energy of about $8 \mathrm{MeV}$ (Ref. 2, p. 66).

The gamma-ray spectrum measurements were the first experiments to be performed in the IRT following completion of fuel loading in the FFTF. They were made in three locations: $81 \mathrm{~cm}$ below core midplane in the lower axial reflector, at the core midplane elevation, and $64 \mathrm{~cm}$ above core midplane in the upper axial reflector. Background data were taken at each of these locations to minimize the effects of activation and fission products. The background count rate was over $10^{4}$ counts $/ \mathrm{sec}$. Data were obtained at two different subcritical power levels to cover the energy spectrum. Data were taken for at least four hours during each power stage to obtain valid statistical results and to permit pseudo-equilibrium buildup of fission products (Ref. 2, p. 66).

Figure 1.10.1 represents the lower energy electron spectrum measured at core midplane and Figure 1.10.2 displays the unfolded gamma-ray spectrum. These results are preliminary because analyses for finitesize effects, pair production, and experimental error have not been performed. Furthermore, the highenergy data has not been included.

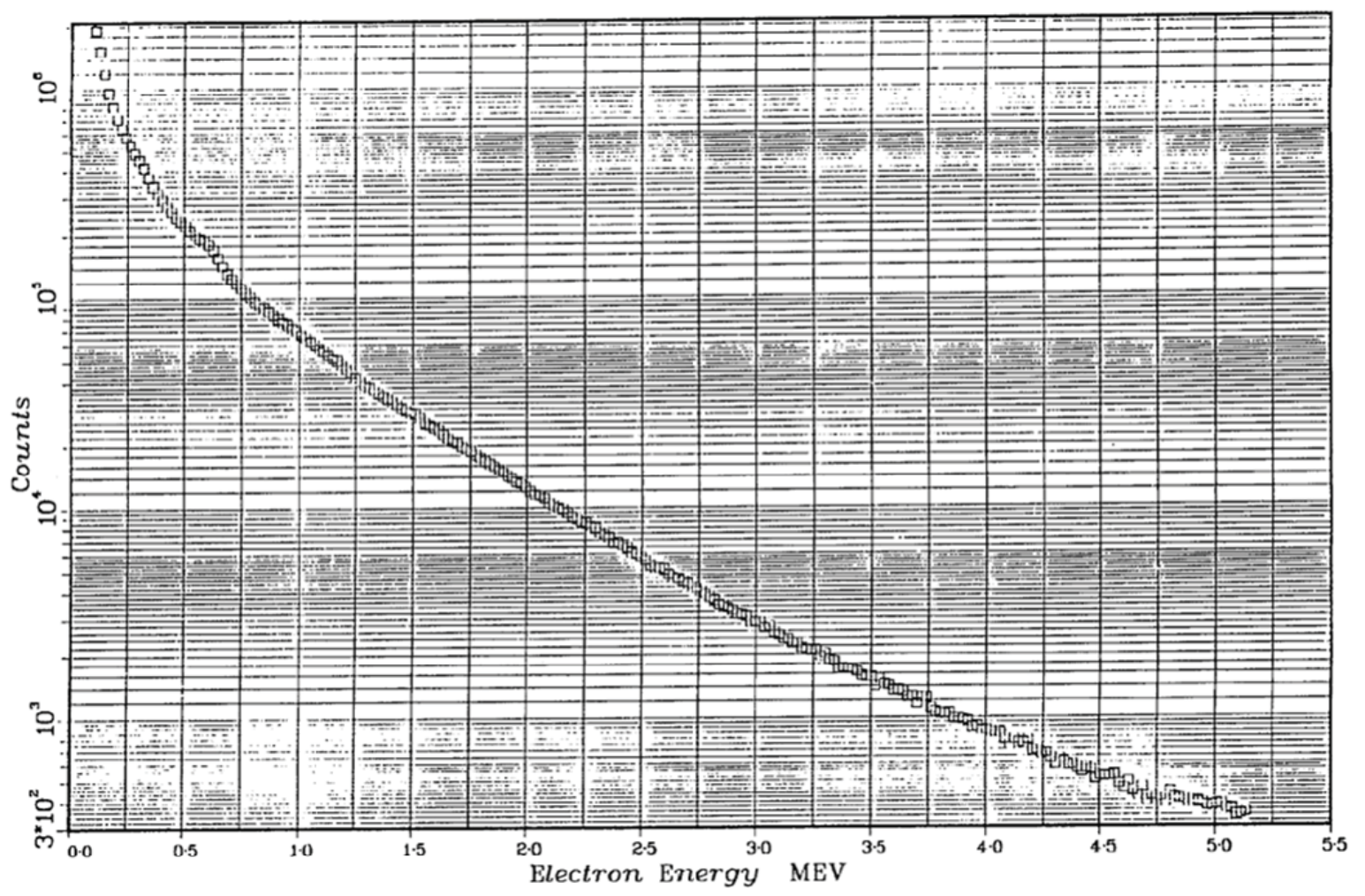

Figure 1.10.1. Low Energy Electron Spectrum at FFTF Core Midplane (Ref. 2, Fig. 20). 
NEA/NSC/DOC(2006)1

Liquid Metal Fast Reactor - LMFR

FFTF-LMFR-RESR-001

CRIT-SPEC-REAC-COEF-MISC

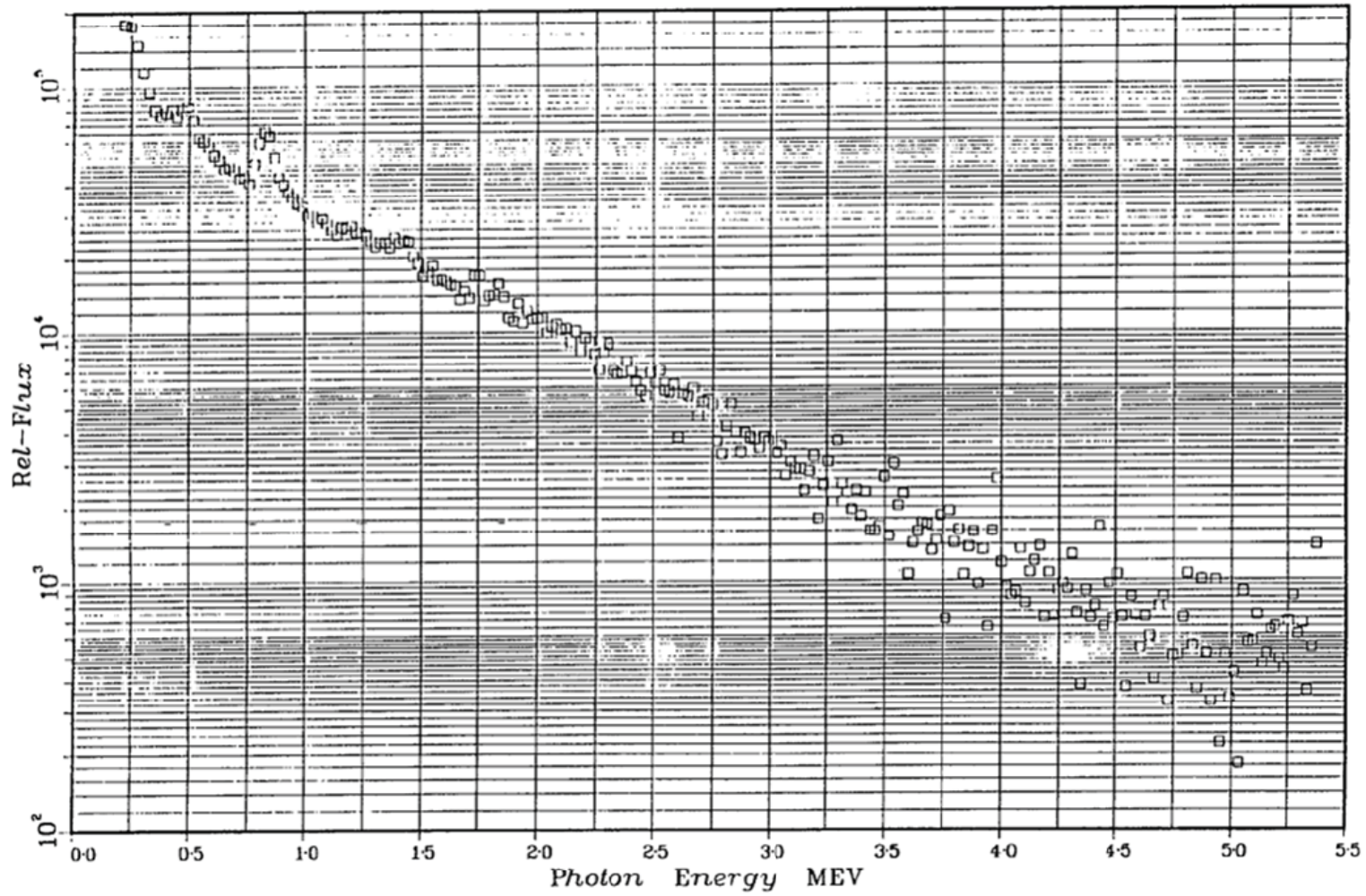

Figure 1.10.2. Unfolded Low-Energy Gamma-Ray Continuum at FFTF Core Midplane (Ref. 2, Fig. 21).

\subsubsection{Material Data}

The materials in the core were those described in the fully-loaded core configuration in Section 1.1.3 and 1.3.3.

\subsubsection{Temperature Data}

The temperature of the core was approximately $400^{\circ} \mathrm{F}\left(\sim 204^{\circ} \mathrm{C}\right)$.

\subsubsection{Additional Information Relevant to Reactivity Coefficient Measurements}

Additional information is not available. 
NEA/NSC/DOC(2006)1

Liquid Metal Fast Reactor - LMFR

FFTF-LMFR-RESR-001

CRIT-SPEC-REAC-COEF-MISC

\subsection{EVALUATION OF EXPERIMENTAL DATA}

\subsection{Evaluation of Critical and / or Subcritical Configuration Data}

Monte Carlo n-Particle (MCNP) version 5.1.51 calculations were utilized to estimate the biases and uncertainties associated with the experimental results for the FFTF fully-loaded critical configuration in this evaluation. MCNP is a general-purpose, continuous-energy, generalized-geometry, time-dependent, coupled n-particle Monte Carlo transport code. ${ }^{a}$ The Evaluated Neutron Data File library, ENDF/BVII.0, ${ }^{b}$ was utilized in analysis of the experiment and benchmark model biases and uncertainties. The nuclear data processing system, NJOY-99.296, ${ }^{\mathrm{c}}$ was used to process the cross section libraries to the desired analysis temperatures. Elemental data such as molecular weights and isotopic abundances were taken from the $16^{\text {th }}$ edition of the Chart of the Nuclides. ${ }^{\text {d }}$ These values are summarized in Appendix D.

The benchmark model provided in Section 3 was utilized with perturbations of the model parameters to estimate uncertainties in the benchmark configuration. All MCNP calculations have statistical uncertainties between 0.00005 and 0.00006 , resulting in $\Delta \mathrm{k}$ statistical uncertainties of approximately 0.00008 .

To test for correlation effects, several variations of the benchmark model were used in the uncertainty analysis as the final benchmark model was improved and refined. No noticeable variation in computed uncertainties was found. Furthermore, variation of the random number seed for the benchmark model presented in Section 3 was performed. The variation in the calculated $k_{\text {eff }}$ eigenvalues was not greater than the statistical standard deviation obtained in MCNP for the eigenvalues. Therefore it was judged that the correlation, if any, between the individual MCNP perturbation analyses was negligible compared to total uncertainty of the benchmark.

Typically both positive and negative perturbations were performed for each uncertainty, each resulting in a calculated $\Delta \mathrm{k}_{\text {eff }}$ uncertainty. The larger (in absolute magnitude) of the two values is selected to represent the uncertainty in perturbing a given parameter.

The term "Scaling Factor" denotes the necessary correction to adjust the calculated variation in $\mathrm{k}_{\text {eff }}$ to a $1 \sigma$ uncertainty value. Often a larger deviation is evaluated such that the calculated $\Delta \mathrm{k}$ value is greater than the statistical uncertainty in the analysis method. Then the calculated results are divided by the scaling factor to obtain the $1 \sigma$ uncertainty.

Some of the calculated uncertainties are poorly estimated because they are very small and on the order of the statistical uncertainty of the analysis method. However, these uncertainties are insignificant in magnitude compared to the total benchmark uncertainty. Reanalysis of most of these parameters with larger variations would not significantly reduce their uncertainties below the statistical uncertainty of the Monte Carlo calculations.

A stringent quality control assessment was maintained for components of the FFTF such that manufacturing tolerances were absolute limits on component requirements allowed in the initial core loading of the FFTF. "No non-conforming fuel ever made it into FFTF in the initial core loads." These

\footnotetext{
a X-5 Monte Carlo Team, "MCNP - a General Monte Carlo n-Particle Transport Code, version 5," LA-UR-031987, Los Alamos National Laboratory (2003).

${ }^{\text {b }}$ M. B. Chadwick, et al., "ENDF/B-VII.0: Next Generation Evaluated Nuclear Data Library for Nuclear Science and Technology," Nucl. Data Sheets, 107: 2931-3060 (2006).

c R. E. MacFarlane and D. W. Muir, “The NJOY Nuclear Data Processing System Version 91,” LA-12740-M (October 1994).

${ }^{\mathrm{d}}$ Nuclides and Isotopes: Chart of the Nuclides, $16^{\text {th }}$ edition, (2002).

Revision: 0

Page 73 of 304

Date: March 31, 2010
} 
NEA/NSC/DOC(2006)1

Liquid Metal Fast Reactor - LMFR

FFTF-LMFR-RESR-001

CRIT-SPEC-REAC-COEF-MISC

stringent requirements were later relaxed when it was experimentally shown that the results would have had negligible effects upon core operations. ${ }^{a}$

It is assumed that the manufacturing tolerances represent a $3 \sigma$ value, with normal distribution because those fabricating the FFTF components would desire to minimize rejected components. Therefore the measured average should fall approximately at the center of the tolerance bands. Most of the values reported for the FFTF are manufacturing tolerances. Unless otherwise specified, bounding limits are treated as aforementioned.

In many uncertainties, the variations apply to large numbers of objects; both systematic uncertainties (applying to all objects equally) and random uncertainties (different from one object to the next) will occur. Treating uncertainties as $100 \%$ random in nature (i.e., dividing by the square root of the number of objects) often statistically reduces the total uncertainty in a perturbed parameter to a negligible amount. It is assumed that the unknown systematic uncertainty is approximately $10 \%$ of the total assessed uncertainty to preserve some of the uncertainty. Evaluated uncertainties are listed as calculated, such that the readers may themselves adjust results according to some desired systematic-to-random uncertainty ratio. The summary of uncertainties tabulated in Section 2.1.5 is based upon a systematic uncertainty of $10 \%$ for all uncertainties with a random uncertainty component. The random uncertainty component is divided by the square-root of the number of objects varied during the perturbation analysis as discussed in a typical statistics text. ${ }^{\mathrm{b}}$

A systematic uncertainty of $10 \%$ is judged to appropriately depict actual measurement uncertainties of the components in the initial core loading of the FFTF. As discussed previously in this section, there were stringent requirements for the initial core load fueling. Similar stringent testing requirements would have been applied to all other components for the core. The user can adjust the total systematic contribution of uncertainties using the data summarized in Table 2.1.76.

A compilation of the total evaluated uncertainty in the FFTF fully-loaded critical configuration is shown in Table 2.1.76. The root-mean-square of all the uncertainties is used to obtain the total uncertainty in the benchmark eigenvalue. When there is a difference between the positive and negative perturbations of a parameter, the larger uncertainty value is included in the total uncertainty of the benchmark. Uncertainties less than or equal to 0.0001 are treated as negligible (neg). When calculated uncertainties in $\Delta \mathrm{k}_{\text {eff }}$ are less than their statistical uncertainties, the statistical uncertainties are used in the calculation of the total uncertainty, unless the absolute magnitude of the uncertainty is less than 0.0001 .

Most of the reported analysis uncertainties were believed to come from calculational approximations (Ref. 4, p. 10).

Many of the homogenized regions of the core described in Section 3.1 are based upon the \#-D Hex-Z model provided in Appendix F. There is insufficient publicly available data to analyze the heterogeneous effects in this regions, and many of them represent regions of low-worth, or neutronically unimportant, areas of the reactor.

\footnotetext{
${ }^{a}$ Personal communication with Robert D. Leggett, who served on the review board for the FFTF fuel fabrication (August 19, 2009).

${ }^{\mathrm{b}}$ G. F. Knoll, Radiation Detection and Measurement, $3^{\text {rd }}$ ed., "Chapter 3 - Counting Statistics and Error

Prediction," John Wiley \& Sons, New York, NY (2000).
}

Revision: 0

Page 74 of 304

Date: March 31, 2010 
NEA/NSC/DOC(2006)1

Liquid Metal Fast Reactor - LMFR

FFTF-LMFR-RESR-001

CRIT-SPEC-REAC-COEF-MISC

\subsubsection{Experimental Uncertainties}

\subsubsection{Temperature}

The reported temperature for the critical experiments is approximately $400^{\circ} \mathrm{F}\left(\sim 204^{\circ} \mathrm{C}\right.$ or $\left.\sim 478 \mathrm{~K}\right)$. Table E.1.2 reports the estimated critical rod bank temperature as $404{ }^{\circ} \mathrm{F}\left(\sim 207^{\circ} \mathrm{C}\right.$ or $\left.\sim 489 \mathrm{~K}\right)$. The MCNP input decks for the benchmark model were evaluated at a temperature of $477.59 \mathrm{~K}$ and implemented ENDF/B-VII.0 cross section data adjusted to $480 \mathrm{~K}$ using NJOY. Additional cross section data was processed for temperatures of 480 and $505 \mathrm{~K}$. An uncertainty in the temperature of $5 \mathrm{~K}$ was assumed. The uncertainty was evaluated with a perturbation of $25 \mathrm{~K}(5 \sigma)$ and then scaled to $1 \sigma$. The perturbation included adjustment of the temperature card in MCNP, analysis using the appropriate neutron cross section library at the evaluated temperature, and modification of the liquid sodium coolant density using the same data and equations from the report footnoted in Section 2.1.3.3. The core assembly pitch was maintained constant during this analysis; it is analyzed separately in Section 2.1.2.9.

As shown by the evaluation of the isothermal temperature coefficient (Section 2.5.1), the effects of the uncertainty in the temperature of the critical core configuration, as evaluated in this section, may only account for approximately half of the total uncertainty. This may be due to correlation effects with the uncertainty in the assembly pitch during core operations.

It was later determined that the effective measurement uncertainty in the isothermal temperature of the core by the 90 FOTA thermocouples was bound by $\pm 2{ }^{\circ} \mathrm{F}( \pm 1 \mathrm{~K})$. Therefore, the value assessed with the temperature adjustment of $5 \mathrm{~K}$ was divided by another factor of 5 and $\sqrt{3}$, because the uncertainty is bounding with uniform probability, to obtain the $1 \sigma$ value.

Results for the uncertainty in the temperature of the experiment are shown in Table 2.1.1.

The uncertainty in the temperature is negligible.

Table 2.1.1. Effect of Uncertainty in Temperature.

\begin{tabular}{||c|ccc|c|ccc||}
\hline \hline Deviation & $\Delta \mathrm{k}$ & \pm & $\sigma_{\Delta \mathrm{k}}$ & $\begin{array}{c}\text { Scaling } \\
\text { Factor }^{(\mathrm{a})}\end{array}$ & $\Delta \mathrm{k}_{\text {eff }}(1 \sigma)$ & \pm & $\sigma_{\Delta \mathrm{keff}}$ \\
\hline \hline$+25 \mathrm{~K}(25 \times$ limit $)$ & 0.00043 & \pm & 0.00008 & $25 \sqrt{ } 3$ & 0.00001 & \pm & 0.00000 \\
$-25 \mathrm{~K}(25 \times$ limit $)$ & -0.00055 & \pm & 0.00008 & $25 \sqrt{ } 3$ & 0.00001 & \pm & 0.00000 \\
\hline
\end{tabular}

(a) This uncertainty is bound with uniform distribution probability.

\subsubsection{Control Rod Positions}

\section{Primary Safety Control Rod Bank}

The uncertainty in the control rod positions $(91.44 \mathrm{~cm}$ withdrawn) is $\pm 0.1016 \mathrm{~cm}(1 \sigma)$. All three primary control rod positions were varied by $\pm 0.5080 \mathrm{~cm}(5 \sigma)$. The calculated results are shown in Table 2.1.2.

The total number of primary control rods used in the core is 3 . The uncertainty in the positions of the primary safety control rod bank is negligible. 
NEA/NSC/DOC(2006)1

Liquid Metal Fast Reactor - LMFR

FFTF-LMFR-RESR-001

CRIT-SPEC-REAC-COEF-MISC

Table 2.1.2. Uncertainty in Primary Control Rod Positions.

\begin{tabular}{||c|ccc|c|ccc||}
\hline \hline Deviation & $\Delta \mathrm{k}$ & \pm & $\sigma_{\Delta \mathrm{k}}$ & $\begin{array}{c}\text { Scaling } \\
\text { Factor }\end{array}$ & $\Delta \mathrm{k}_{\mathrm{eff}}(1 \sigma)$ & \pm & $\sigma_{\Delta \mathrm{keff}}$ \\
\hline \hline$+0.5080 \mathrm{~cm}(5 \sigma)$ & -0.00004 & \pm & 0.00008 & 5 & -0.00001 & \pm & 0.00002 \\
$-0.5080 \mathrm{~cm}(5 \sigma)$ & -0.00021 & \pm & 0.00008 & 5 & -0.00004 & \pm & 0.00002 \\
\hline
\end{tabular}

\section{Secondary Control Rod Bank}

The uncertainty in the secondary control rod positions (35.56 cm withdrawn) is $\pm 0.1016 \mathrm{~cm}(1 \sigma)$. All five secondary control rod positions (six minus the single rod used to obtain criticality) were varied by $\pm 0.5080 \mathrm{~cm}(5 \sigma)$. The calculated results are shown in Table 2.1.3.

The total number of secondary control rods used in the core is 5. For determining the random component of the uncertainty in Section 2.1.5, the results in Table 2.1.3 are divided by $\sqrt{5}$, as rods were individually positioned.

Table 2.1.3. Uncertainty in Secondary Control Rod Positions. ${ }^{(a)}$

\begin{tabular}{||c|ccc|c|ccc||}
\hline \hline Deviation & $\Delta \mathrm{k}$ & \pm & $\sigma_{\Delta \mathrm{k}}$ & $\begin{array}{c}\text { Scaling } \\
\text { Factor }\end{array}$ & $\Delta \mathrm{k}_{\mathrm{eff}}(1 \sigma)$ & \pm & $\sigma_{\Delta \mathrm{keff}}$ \\
\hline \hline$+0.5080 \mathrm{~cm}(5 \sigma)$ & 0.00034 & \pm & 0.00008 & 5 & 0.00007 & \pm & 0.00002 \\
$-0.5080 \mathrm{~cm}(5 \sigma)$ & -0.00056 & \pm & 0.00008 & 5 & -0.00011 & \pm & 0.00002 \\
\hline
\end{tabular}

(a) The random component of the uncertainty would be divided by $\sqrt{5}$.

\section{Critical Control Rod}

The uncertainty in the control rod position (36.116 cm withdrawn) of Rod 4, which was withdrawn to obtain criticality, is $\pm 0.1016 \mathrm{~cm}(1 \sigma)$. The control rod position was varied by $\pm 0.5080 \mathrm{~cm}(5 \sigma)$. The calculated results are shown in Table 2.1.4.

The uncertainty in the position of the critical control rod is negligible.

Table 2.1.4. Uncertainty in the Position of Control Rod 4.

\begin{tabular}{||c|ccc|c|ccc||}
\hline Deviation & $\Delta \mathrm{k}$ & \pm & $\sigma_{\Delta \mathrm{k}}$ & $\begin{array}{c}\text { Scaling } \\
\text { Factor }\end{array}$ & $\Delta \mathrm{k}_{\mathrm{eff}}(1 \sigma)$ & \pm & $\sigma_{\Delta \mathrm{keff}}$ \\
\hline \hline$+0.5080 \mathrm{~cm}(5 \sigma)$ & 0.00007 & \pm & 0.00008 & 5 & 0.00001 & \pm & 0.00002 \\
$-0.5080 \mathrm{~cm}(5 \sigma)$ & -0.00015 & \pm & 0.00008 & 5 & -0.00003 & \pm & 0.00002 \\
\hline
\end{tabular}

\section{Fixed Shim Control Rods}

The fixed shim control rods do not move; therefore an uncertainty in their movement was not performed. 
NEA/NSC/DOC(2006)1

Liquid Metal Fast Reactor - LMFR

FFTF-LMFR-RESR-001

CRIT-SPEC-REAC-COEF-MISC

\subsubsection{Measured Value of $k_{\text {eff }}$}

There is no additional information regarding the accuracy of $\mathrm{k}_{\mathrm{eff}}$ measurements. The reactor was held at critical and excess reactivity, if any, was not reported.

\subsubsection{Geometrical Properties}

\subsubsection{Driver Fuel Assemblies}

The uncertainties in the driver fuel assemblies were assessed by enrichment zone, although many of the components in both zones would be very similar, such as fuel cladding, insulator pellets, axial reflectors, wire wrap, ducts, etc. The purpose in separating analysis of the two regions was to investigate what effects might have more significant worth, if any, depending on its placement within a particular fueled region of the core. Furthermore, as fuel pins were fabricated in batches, there may have been some slight variations in the construction of the pins.

\section{Inner Enrichment Zone (Type 3.1 Fuel Pins)}

Inconel Axial Reflectors: Height

From Figure 1.1.19, the uncertainty in the height $(14.4780 \mathrm{~cm})$ of the axial reflectors is $\pm 0.0127 \mathrm{~cm}$. This value is representative of a manufacturing tolerance. Results are shown in Table 2.1.5.

The total number of axial reflectors in the inner enrichment zone is 11,718 . The uncertainty in the height of the Inconel axial reflectors is negligible.

Table 2.1.5. Uncertainty in the Height of the Inner-Fuel-Zone Axial Reflectors. ${ }^{(a)}$

\begin{tabular}{||c|ccc|c|ccc||}
\hline \hline Deviation & $\Delta \mathrm{k}$ & \pm & $\sigma_{\Delta \mathrm{k}}$ & $\begin{array}{c}\text { Scaling } \\
\text { Factor }\end{array}$ & $\Delta \mathrm{k}_{\mathrm{eff}}(1 \sigma)$ & \pm & $\sigma_{\Delta \mathrm{keff}}$ \\
\hline \hline$+0.0127 \mathrm{~cm}(3 \sigma)$ & -0.00020 & \pm & 0.00008 & 3 & -0.00007 & \pm & 0.00003 \\
$-0.0127 \mathrm{~cm}(3 \sigma)$ & -0.00015 & \pm & 0.00008 & 3 & -0.00005 & \pm & 0.00003 \\
\hline
\end{tabular}

(a) The random component of the uncertainty would be divided by $\sqrt{11,718}$.

\section{Inconel Axial Reflectors: Diameter}

From Figure 1.1.19, the uncertainty in the diameter $(0.48133 \mathrm{~cm})$ of the axial reflectors is $\pm 0.00127 \mathrm{~cm}$. This value is representative of a manufacturing tolerance. Results are shown in Table 2.1.6.

The total number of axial reflectors in the inner enrichment zone is 11,718. The uncertainty in the diameter of the Inconel axial reflectors is negligible. 
NEA/NSC/DOC(2006)1

Liquid Metal Fast Reactor - LMFR

FFTF-LMFR-RESR-001

CRIT-SPEC-REAC-COEF-MISC

Table 2.1.6. Uncertainty in the Diameter of the Inner-Fuel-Zone Axial Reflectors. ${ }^{(a)}$

\begin{tabular}{||c|ccc|c|ccc||}
\hline Deviation & $\Delta \mathrm{k}$ & \pm & $\sigma_{\Delta \mathrm{k}}$ & $\begin{array}{c}\text { Scaling } \\
\text { Factor }\end{array}$ & $\Delta \mathrm{k}_{\mathrm{eff}}(1 \sigma)$ & \pm & $\sigma_{\Delta \mathrm{keff}}$ \\
\hline \hline$+0.00127 \mathrm{~cm}(3 \sigma)$ & -0.00006 & \pm & 0.00008 & 3 & -0.00002 & \pm & 0.00003 \\
$-0.00127 \mathrm{~cm}(3 \sigma)$ & -0.00001 & \pm & 0.00008 & 3 & 0.00000 & \pm & 0.00003 \\
\hline
\end{tabular}

(a) The random component of the uncertainty would be divided by $\sqrt{11,718}$.

\section{$\mathrm{UO}_{2}$ Insulator Pellets: Height}

From Figure 1.1.19, the uncertainty in the height $(2.0320 \mathrm{~cm})$ of the insulator pellets is $\pm 0.1016 \mathrm{~cm}$. This value is representative of a manufacturing tolerance. Results are shown in Table 2.1.7.

The total number of insulator pellets in the inner enrichment zone is 23,436. The uncertainty in the height of the $\mathrm{UO}_{2}$ insulator pellets is negligible.

Table 2.1.7. Uncertainty in the Height of the Inner-Fuel-Zone Insulator Pellets. ${ }^{(a)}$

\begin{tabular}{||c|ccc|c|ccc||}
\hline Deviation & $\Delta \mathrm{k}$ & \pm & $\sigma_{\Delta \mathrm{k}}$ & $\begin{array}{c}\text { Scaling } \\
\text { Factor }\end{array}$ & $\Delta \mathrm{k}_{\mathrm{eff}}(1 \sigma)$ & \pm & $\sigma_{\Delta \mathrm{keff}}$ \\
\hline \hline$+0.1016 \mathrm{~cm}(3 \sigma)$ & -0.00027 & \pm & 0.00008 & 3 & -0.00009 & \pm & 0.00003 \\
$-0.1016 \mathrm{~cm}(3 \sigma)$ & -0.00005 & \pm & 0.00008 & 3 & -0.00002 & \pm & 0.00003 \\
\hline
\end{tabular}

(a) The random component of the uncertainty would be divided by $\sqrt{23,436}$.

\section{$\mathrm{UO}_{2}$ Insulator Pellets: Diameter}

From Figure 1.1.19, the uncertainty in the diameter $(0.48260 \mathrm{~cm})$ of the insulator pellets is $\pm 0.00635 \mathrm{~cm}$. This value is representative of a manufacturing tolerance. There are a total of 11,718 insulator pellets in the inner enrichment zone of the FFTF; therefore the effective uncertainty becomes negligible. Results are shown in Table 2.1.8.

The total number of insulator pellets in the inner enrichment zone is 11,718. The uncertainty in the diameter of the $\mathrm{UO}_{2}$ insulator pellets is negligible.

Table 2.1.8. Uncertainty in the Diameter of the Inner-Fuel-Zone Insulator Pellets. ${ }^{(a)}$

\begin{tabular}{|c|ccc|c|ccc||}
\hline \hline Deviation & $\Delta \mathrm{k}$ & \pm & $\sigma_{\Delta \mathrm{k}}$ & $\begin{array}{c}\text { Scaling } \\
\text { Factor }\end{array}$ & $\Delta \mathrm{k}_{\mathrm{eff}}(1 \sigma)$ & \pm & $\sigma_{\Delta \mathrm{keff}}$ \\
\hline \hline$+0.00635 \mathrm{~cm}(3 \sigma)$ & -0.00005 & \pm & 0.00008 & 3 & -0.00002 & \pm & 0.00003 \\
$-0.00635 \mathrm{~cm}(3 \sigma)$ & 0.00004 & \pm & 0.00008 & 3 & 0.00001 & \pm & 0.00003 \\
\hline
\end{tabular}

(a) The random component of the uncertainty would be divided by $\sqrt{11,718}$. 
NEA/NSC/DOC(2006)1

Liquid Metal Fast Reactor - LMFR

FFTF-LMFR-RESR-001

CRIT-SPEC-REAC-COEF-MISC

\section{Fuel Pellet Stack: Height}

From Figure 1.1.19, the uncertainty in the fuel pellet stack height $(91.4400 \mathrm{~cm})$ is $\pm 0.3810 \mathrm{~cm}$. This value is representative of a manufacturing tolerance. Elsewhere an uncertainty of $\pm 0.3175 \mathrm{~cm}$ is provided (Section 1.1.2 and Table 1.1.3); the larger value is used for the uncertainty analysis. Results are shown in Table 2.1.9.

The total number of fuel pellet stacks in the inner enrichment zone is 5,859. For determining the random component of the uncertainty in Section 2.1.5, the results in Table 2.1.9 are divided by $\sqrt{5,859}$.

Table 2.1.9. Uncertainty in the Inner-Fuel-Zone Fuel Pellet Stack Height. ${ }^{(a)}$

\begin{tabular}{||c|ccc|c|ccc||}
\hline \hline Deviation & $\Delta \mathrm{k}$ & \pm & $\sigma_{\Delta \mathrm{k}}$ & $\begin{array}{c}\text { Scaling } \\
\text { Factor }\end{array}$ & $\Delta \mathrm{k}_{\mathrm{eff}}(1 \sigma)$ & \pm & $\sigma_{\Delta \mathrm{keff}}$ \\
\hline \hline$+0.3810 \mathrm{~cm}(3 \sigma)$ & 0.00020 & \pm & 0.00008 & 3 & 0.00007 & \pm & 0.00003 \\
$-0.3810 \mathrm{~cm}(3 \sigma)$ & -0.00034 & \pm & 0.00008 & 3 & -0.00011 & \pm & 0.00003 \\
\hline
\end{tabular}

(a) The random component of the uncertainty would be divided by $\sqrt{5,589}$.

\section{Fuel Pellet Stack: Diameter}

From Figure 1.1.19, the uncertainty in the fuel pellet diameter $(0.49403 \mathrm{~cm})$ is $\pm 0.00381 \mathrm{~cm}$. This value is representative of a manufacturing tolerance. There are either 144 pellets in a stack (if each pellet is $0.635 \mathrm{~cm}$ high) or $\sim 176$ pellets in a stack (if each pellet is $0.52070 \mathrm{~cm}$ high). Conservatively, the lesser value is selected resulting in a total of 843,696 fuel pellets in the inner enrichment zone of the FFTF. Results are shown in Table 2.1.10.

The total number of fuel pellets in the inner enrichment zone is 843,696 . For determining the random component of the uncertainty in Section 2.1.5, the results in Table 2.1.10 are divided by $\sqrt{843,696}$.

Table 2.1.10. Uncertainty in the Inner-Fuel-Zone Fuel Pellet Diameter. ${ }^{(a)}$

\begin{tabular}{|c|ccc|c|ccc||}
\hline \hline Deviation & $\Delta \mathrm{k}$ & \pm & $\sigma_{\Delta \mathrm{k}}$ & $\begin{array}{c}\text { Scaling } \\
\text { Factor }\end{array}$ & $\Delta \mathrm{k}_{\mathrm{eff}}(1 \sigma)$ & \pm & $\sigma_{\Delta \mathrm{keff}}$ \\
\hline \hline$+0.00381 \mathrm{~cm}(3 \sigma)$ & 0.00318 & \pm & 0.00008 & 3 & 0.00106 & \pm & 0.00003 \\
$-0.00381 \mathrm{~cm}(3 \sigma)$ & -0.00345 & \pm & 0.00008 & 3 & -0.00115 & \pm & 0.00003 \\
\hline
\end{tabular}

(a) The random component of the uncertainty would be divided by $\sqrt{843,696}$.

\section{SS316 Cladding: Inner Diameter}

From Figure 1.1.19, the uncertainty in the fuel pin cladding inner diameter $(0.50800 \mathrm{~cm})$ is $\pm 0.00127 \mathrm{~cm}$. This value is representative of a manufacturing tolerance. Results are shown in Table 2.1.11. The mass and thickness of the cladding is not conserved, as variation in the manufacturing tolerance of both inner and outer clad diameters were reported, but not in the thickness or mass of the clad material. Therefore, the variation of the inner diameter of the cladding also changes the thickness and linear density of the cladding.

The total number of fuel pins in the inner enrichment zone is 5,859. The uncertainty in the inner diameter of the stainless steel cladding is negligible. 
NEA/NSC/DOC(2006)1

Liquid Metal Fast Reactor - LMFR

FFTF-LMFR-RESR-001

CRIT-SPEC-REAC-COEF-MISC

Table 2.1.11. Uncertainty in the Inner-Fuel-Zone Cladding Inner Diameter. ${ }^{(a)}$

\begin{tabular}{||c|ccc|c|ccc||}
\hline \hline Deviation & $\Delta \mathrm{k}$ & \pm & $\sigma_{\Delta \mathrm{k}}$ & $\begin{array}{c}\text { Scaling } \\
\text { Factor }\end{array}$ & $\Delta \mathrm{k}_{\mathrm{eff}}(1 \sigma)$ & \pm & $\sigma_{\Delta \mathrm{keff}}$ \\
\hline \hline$+0.00127 \mathrm{~cm}(3 \sigma)$ & -0.00007 & \pm & 0.00008 & 3 & -0.00002 & \pm & 0.00003 \\
$-0.00127 \mathrm{~cm}(3 \sigma)$ & -0.00022 & \pm & 0.00008 & 3 & -0.00007 & \pm & 0.00003 \\
\hline
\end{tabular}

(a) The random component of the uncertainty would be divided by $\sqrt{5,589}$.

\section{SS316 Cladding: Outer Diameter}

From Figure 1.1.19, the uncertainty in the fuel pin cladding outer diameter $(0.58420 \mathrm{~cm})$ is $\pm 0.00127 \mathrm{~cm}$. This value is representative of a manufacturing tolerance. Results are shown in Table 2.1.12. The mass and thickness of the cladding is not conserved, as variation in the manufacturing tolerance of both inner and outer clad diameters were reported, but not in the thickness or mass of the clad material. Therefore, the variation of the outer diameter of the cladding also changes the thickness and linear density of the cladding.

The total number of fuel pins in the inner enrichment zone is 5,859. The uncertainty in the outer diameter of the stainless steel cladding is negligible.

Table 2.1.12. Uncertainty in the Inner-Fuel-Zone Cladding Outer Diameter. ${ }^{(a)}$

\begin{tabular}{||c|ccc|c|ccc||}
\hline Deviation & $\Delta \mathrm{k}$ & \pm & $\sigma_{\Delta \mathrm{k}}$ & $\begin{array}{c}\text { Scaling } \\
\text { Factor }\end{array}$ & $\Delta \mathrm{k}_{\mathrm{eff}}(1 \sigma)$ & \pm & $\sigma_{\Delta \mathrm{keff}}$ \\
\hline \hline$+0.00127 \mathrm{~cm}(3 \sigma)$ & -0.00029 & \pm & 0.00008 & 3 & -0.00010 & \pm & 0.00003 \\
$-0.00127 \mathrm{~cm}(3 \sigma)$ & 0.00000 & \pm & 0.00008 & 3 & 0.00000 & \pm & 0.00003 \\
\hline
\end{tabular}

(a) The random component of the uncertainty would be divided by $\sqrt{5,589}$.

\section{SS316 Wire Wrap: Diameter}

The wire wrap woven between the fuel pins is not explicitly modeled in the benchmark. However, an uncertainty of $\pm 0.00127 \mathrm{~cm}$ applies to the wire diameter of $0.14224 \mathrm{~cm}$ (Table 1.1.3) and is analyzed by appropriately adjusting the volume fraction of the wire wrap steel in liquid sodium as discussed in Section 2.1.3.6. This value is representative of a manufacturing tolerance.

\section{Fuel Pin Assembly Lattice: Pitch}

The pitch of the fuel pin lattice is adjusted by the manufacturing tolerance of the wire wrap between the fuel pins; the fuel pitch of $0.72644 \mathrm{~cm}$ is adjusted by $\pm 0.00127 \mathrm{~cm}$. Results are shown in Table 2.1.13. An uncertainty in the fuel pin pitch due to variation in the grid-plate design, to which the fuel pins are attached, was not available. However, as seen in Figure 1.1.7, there is not sufficient room within the fuel assembly duct for larger pitch variations.

The total number of fuel pin wire wraps in the inner enrichment zone is 5,859 . The uncertainty in the fuel pin assembly lattice pitch is negligible. 
NEA/NSC/DOC(2006)1

Liquid Metal Fast Reactor - LMFR

FFTF-LMFR-RESR-001

CRIT-SPEC-REAC-COEF-MISC

Table 2.1.13. Uncertainty in the Inner-Fuel-Zone Fuel Pin Lattice Pitch. ${ }^{(a)}$

\begin{tabular}{|c|ccc|c|ccc||}
\hline Deviation & $\Delta \mathrm{k}$ & \pm & $\sigma_{\Delta \mathrm{k}}$ & $\begin{array}{c}\text { Scaling } \\
\text { Factor }\end{array}$ & $\Delta \mathrm{k}_{\text {eff }}(1 \sigma)$ & \pm & $\sigma_{\Delta \mathrm{keff}}$ \\
\hline \hline$+0.00127 \mathrm{~cm}(3 \sigma)$ & -0.00030 & \pm & 0.00008 & 3 & -0.00010 & \pm & 0.00003 \\
$-0.00127 \mathrm{~cm}(3 \sigma)$ & -0.00002 & \pm & 0.00008 & 3 & -0.00001 & \pm & 0.00003 \\
\hline
\end{tabular}

(a) The random component of the uncertainty would be divided by $\sqrt{5,589}$.

Fuel Assembly SS316 Duct: Flat-To-Flat Width

A manufacturing tolerance of \pm 0.015 in $(0.0381 \mathrm{~cm})$ across the flat-to-flat width $(11.0109 \mathrm{~cm})$ of the fuel assembly duct was selected. There are a total of 27 assembly ducts in the inner enrichment zone of the FFTF. The calculated results are shown in Table 2.1.14.

The total number of assembly ducts in the inner enrichment zone is 27 . For determining the random component of the uncertainty in Section 2.1.5, the results in Table 2.1.14 are divided by $\sqrt{ } 27$.

Table 2.1.14. Uncertainty in the Flat-To-Flat Width of the Inner-Fuel-Zone Ducts. ${ }^{(a)}$

\begin{tabular}{||c|ccc|c|ccc||}
\hline \hline Deviation & $\Delta \mathrm{k}$ & \pm & $\sigma_{\Delta \mathrm{k}}$ & $\begin{array}{c}\text { Scaling } \\
\text { Factor }\end{array}$ & $\Delta \mathrm{k}_{\mathrm{eff}}(1 \sigma)$ & \pm & $\sigma_{\Delta \mathrm{keff}}$ \\
\hline \hline$+0.0381 \mathrm{~cm}(3 \sigma)$ & -0.00049 & \pm & 0.00008 & 3 & -0.00016 & \pm & 0.00003 \\
$-0.0381 \mathrm{~cm}(3 \sigma)$ & 0.00059 & \pm & 0.00008 & 3 & 0.00020 & \pm & 0.00003 \\
\hline
\end{tabular}

(a) The random component of the uncertainty would be divided by $\sqrt{2} 2$.

\section{Axial Shield, Pin Attachment, and Gas Plenum Regions}

These regions have been homogenized as described in the Section 3.1, based upon the 3-D Hex-Z model provided in Appendix F. There is insufficient publicly available data to analyze the heterogeneous effects of these regions. Heterogeneous effects and the effect of homogenization are expected to represent an insignificant contribution to the total uncertainty of the benchmark model, as the uncertainties in other geometrical properties (Section 2.1.2) and density of the primary structural components (SS316, Inconel 600, and sodium in Sections 2.1.3.1 through 2.1.3.3) are relatively insignificant compared to other constituents of the total uncertainty. Therefore the uncertainty in the geometry of these regions is judged to be negligible.

\section{Outer Enrichment Zone (Type 3.2 Fuel Pins)}

Inconel Axial Reflectors: Height

From Figure 1.1.19, the uncertainty in the height $(14.4780 \mathrm{~cm})$ of the axial reflectors is $\pm 0.0127 \mathrm{~cm}$. This value is representative of a manufacturing tolerance. Results are shown in Table 2.1.15.

The total number of axial reflectors in the outer enrichment zone is 19,964 . The uncertainty in the height of the Inconel axial reflectors is negligible. 
NEA/NSC/DOC(2006)1

Liquid Metal Fast Reactor - LMFR

FFTF-LMFR-RESR-001

CRIT-SPEC-REAC-COEF-MISC

Table 2.1.15. Uncertainty in the Height of the Outer-Fuel-Zone Axial Reflectors. ${ }^{\text {(a) }}$

\begin{tabular}{||c|ccc|c|ccc||}
\hline Deviation & $\Delta \mathrm{k}$ & \pm & $\sigma_{\Delta \mathrm{k}}$ & $\begin{array}{c}\text { Scaling } \\
\text { Factor }\end{array}$ & $\Delta \mathrm{k}_{\mathrm{eff}}(1 \sigma)$ & \pm & $\sigma_{\Delta \mathrm{keff}}$ \\
\hline \hline$+0.0127 \mathrm{~cm}(3 \sigma)$ & -0.00012 & \pm & 0.00008 & 3 & -0.00004 & \pm & 0.00003 \\
$-0.0127 \mathrm{~cm}(3 \sigma)$ & 0.00002 & \pm & 0.00008 & 3 & 0.00001 & \pm & 0.00003 \\
\hline
\end{tabular}

(a) The random component of the uncertainty would be divided by $\sqrt{19,964}$.

\section{Inconel Axial Reflectors: Diameter}

From Figure 1.1.19, the uncertainty in the diameter $(0.48133 \mathrm{~cm})$ of the axial reflectors is $\pm 0.00127 \mathrm{~cm}$. This value is representative of a manufacturing tolerance. Results are shown in Table 2.1.16.

The total number of axial reflectors in the outer enrichment zone is 19,964. The uncertainty in the diameter of the Inconel axial reflectors is negligible.

Table 2.1.16. Uncertainty in the Diameter of the Outer-Fuel-Zone Axial Reflectors. ${ }^{(a)}$

\begin{tabular}{||c|ccc|c|ccc||}
\hline \hline Deviation & $\Delta \mathrm{k}$ & \pm & $\sigma_{\Delta \mathrm{k}}$ & $\begin{array}{c}\text { Scaling } \\
\text { Factor }\end{array}$ & $\Delta \mathrm{k}_{\text {eff }}(1 \sigma)$ & \pm & $\sigma_{\Delta \mathrm{keff}}$ \\
\hline \hline$+0.00127 \mathrm{~cm}(3 \sigma)$ & -0.00015 & \pm & 0.00008 & 3 & -0.00005 & \pm & 0.00003 \\
$-0.00127 \mathrm{~cm}(3 \sigma)$ & -0.00010 & \pm & 0.00008 & 3 & -0.00003 & \pm & 0.00003 \\
\hline
\end{tabular}

(a) The random component of the uncertainty would be divided by $\sqrt{19,964}$.

\section{$\mathrm{UO}_{2}$ Insulator Pellets: Height}

From Figure 1.1.19, the uncertainty in the height $(2.0320 \mathrm{~cm})$ of the insulator pellets is $\pm 0.1016 \mathrm{~cm}$. This value is representative of a manufacturing tolerance. Results are shown in Table 2.1.17.

The total number of insulator pellets in the outer enrichment zone is 39,928 . The uncertainty in the height of the $\mathrm{UO}_{2}$ insulator pellets is negligible.

Table 2.1.17. Uncertainty in the Height of the Outer-Fuel-Zone Insulator Pellets. ${ }^{\text {(a) }}$

\begin{tabular}{||c|ccc|c|ccc||}
\hline \hline Deviation & $\Delta \mathrm{k}$ & \pm & $\sigma_{\Delta \mathrm{k}}$ & $\begin{array}{c}\text { Scaling } \\
\text { Factor }\end{array}$ & $\Delta \mathrm{k}_{\mathrm{eff}}(1 \sigma)$ & \pm & $\sigma_{\Delta \mathrm{keff}}$ \\
\hline \hline$+0.1016 \mathrm{~cm}(3 \sigma)$ & -0.00011 & \pm & 0.00008 & 3 & -0.00004 & \pm & 0.00003 \\
$-0.1016 \mathrm{~cm}(3 \sigma)$ & 0.00006 & \pm & 0.00008 & 3 & 0.00002 & \pm & 0.00003 \\
\hline
\end{tabular}

(a) The random component of the uncertainty would be divided by $\sqrt{39,928}$. 
NEA/NSC/DOC(2006)1

Liquid Metal Fast Reactor - LMFR

FFTF-LMFR-RESR-001

CRIT-SPEC-REAC-COEF-MISC

\section{$\mathrm{UO}_{2}$ Insulator Pellets: Diameter}

From Figure 1.1.19, the uncertainty in the diameter $(0.48260 \mathrm{~cm})$ of the insulator pellets is $\pm 0.00635 \mathrm{~cm}$. This value is representative of a manufacturing tolerance. Results are shown in Table 2.1.18.

The total number of insulator pellets in the outer enrichment zone is 39,928 . The uncertainty in the diameter of the $\mathrm{UO}_{2}$ insulator pellets is negligible.

Table 2.1.18. Uncertainty in the Diameter of the Outer-Fuel-Zone Insulator Pellets. ${ }^{(a)}$

\begin{tabular}{||c|ccc|c|ccc||}
\hline \hline Deviation & $\Delta \mathrm{k}$ & \pm & $\sigma_{\Delta \mathrm{k}}$ & $\begin{array}{c}\text { Scaling } \\
\text { Factor }\end{array}$ & $\Delta \mathrm{k}_{\text {eff }}(1 \sigma)$ & \pm & $\sigma_{\Delta \mathrm{keff}}$ \\
\hline \hline$+0.00635 \mathrm{~cm}(3 \sigma)$ & -0.00016 & \pm & 0.00008 & 3 & -0.00005 & \pm & 0.00003 \\
$-0.00635 \mathrm{~cm}(3 \sigma)$ & -0.00013 & \pm & 0.00008 & 3 & -0.00004 & \pm & 0.00003 \\
\hline
\end{tabular}

(a) The random component of the uncertainty would be divided by $\sqrt{39}, 928$.

\section{Fuel Pellet Stack: Height}

From Figure 1.1.19, the uncertainty in the fuel pellet stack height $(91.4400 \mathrm{~cm})$ is $\pm 0.3810 \mathrm{~cm}$. This value is representative of a manufacturing tolerance. Elsewhere an uncertainty of $\pm 0.3175 \mathrm{~cm}$ is provided (Section 1.1.2 and Table 1.1.3); the larger value is used for the uncertainty analysis. Results are shown in Table 2.1.19.

The total number of fuel pellet stacks in the outer enrichment zone is 9,982. For determining the random component of the uncertainty in Section 2.1.5, the results in Table 2.1.19 are divided by $\sqrt{9,982}$.

Table 2.1.19. Uncertainty in the Outer-Fuel-Zone Fuel Pellet Stack Height. ${ }^{(a)}$

\begin{tabular}{||c|ccc|c|ccc||}
\hline Deviation & $\Delta \mathrm{k}$ & \pm & $\sigma_{\Delta \mathrm{k}}$ & $\begin{array}{c}\text { Scaling } \\
\text { Factor }\end{array}$ & $\Delta \mathrm{k}_{\mathrm{eff}}(1 \sigma)$ & \pm & $\sigma_{\Delta \mathrm{keff}}$ \\
\hline \hline$+0.3810 \mathrm{~cm}(3 \sigma)$ & 0.00013 & \pm & 0.00008 & 3 & 0.00004 & \pm & 0.00003 \\
$-0.3810 \mathrm{~cm}(3 \sigma)$ & -0.00043 & \pm & 0.00008 & 3 & -0.00014 & \pm & 0.00003 \\
\hline
\end{tabular}

(a) The random component of the uncertainty would be divided by $\sqrt{9,982}$.

\section{Fuel Pellet Stack: Diameter}

From Figure 1.1.19, the uncertainty in the fuel pellet diameter $(0.49403 \mathrm{~cm})$ is $\pm 0.00381 \mathrm{~cm}$. This value is representative of a manufacturing tolerance. There are either 144 pellets in a stack (if each pellet is $0.635 \mathrm{~cm}$ high) or $\sim 176$ pellets in a stack (if each pellet is $0.52070 \mathrm{~cm}$ high). Conservatively, the lesser value is selected, resulting in a total of 1,437,408 fuel pellets in the outer enrichment zone of the FFTF. Results are shown in Table 2.1.20.

The total number of fuel pellets in the outer enrichment zone is 1,437,408. For determining the random component of the uncertainty in Section 2.1.5, the results in Table 2.1.20 are divided by $\sqrt{1,437,408}$. 
NEA/NSC/DOC(2006)1

Liquid Metal Fast Reactor - LMFR

FFTF-LMFR-RESR-001

CRIT-SPEC-REAC-COEF-MISC

Table 2.1.20. Uncertainty in the Outer-Fuel-Zone Fuel Pellet Diameter. ${ }^{(a)}$

\begin{tabular}{|c|ccc|c|ccc||}
\hline Deviation & $\Delta \mathrm{k}$ & \pm & $\sigma_{\Delta \mathrm{k}}$ & $\begin{array}{c}\text { Scaling } \\
\text { Factor }\end{array}$ & $\Delta \mathrm{k}_{\text {eff }}(1 \sigma)$ & \pm & $\sigma_{\Delta \mathrm{keff}}$ \\
\hline \hline$+0.00381 \mathrm{~cm}(3 \sigma)$ & 0.00358 & \pm & 0.00008 & 3 & 0.00119 & \pm & 0.00003 \\
$-0.00381 \mathrm{~cm}(3 \sigma)$ & -0.00407 & \pm & 0.00008 & 3 & -0.00136 & \pm & 0.00003 \\
\hline
\end{tabular}

(a) The random component of the uncertainty would be divided by $\sqrt{1,437,408}$.

\section{SS316 Cladding: Inner Diameter}

From Figure 1.1.19, the uncertainty in the fuel pin cladding inner diameter $(0.50800 \mathrm{~cm})$ is $\pm 0.00127 \mathrm{~cm}$. This value is representative of a manufacturing tolerance. Results are shown in Table 2.1.21. The mass and thickness of the cladding is not conserved, as variation in the manufacturing tolerance of both inner and outer clad diameters were reported, but not in the thickness or mass of the clad material. Therefore, the variation of the inner diameter of the cladding also changes the thickness and linear density of the cladding.

The total number of fuel pins in the outer enrichment zone is 9,982. The uncertainty in the inner diameter of the stainless steel cladding is negligible.

Table 2.1.21. Uncertainty in the Outer-Fuel-Zone Cladding Inner Diameter. ${ }^{(a)}$

\begin{tabular}{||c|ccc|c|ccc||}
\hline Deviation & $\Delta \mathrm{k}$ & \pm & $\sigma_{\Delta \mathrm{k}}$ & $\begin{array}{c}\text { Scaling } \\
\text { Factor }\end{array}$ & $\Delta \mathrm{k}_{\text {eff }}(1 \sigma)$ & \pm & $\sigma_{\Delta \mathrm{keff}}$ \\
\hline \hline$+0.00127 \mathrm{~cm}(3 \sigma)$ & -0.00007 & \pm & 0.00008 & 3 & -0.00002 & \pm & 0.00003 \\
$-0.00127 \mathrm{~cm}(3 \sigma)$ & -0.00017 & \pm & 0.00008 & 3 & -0.00006 & \pm & 0.00003 \\
\hline
\end{tabular}

(a) The random component of the uncertainty would be divided by $\sqrt{9,982}$.

\section{SS316 Cladding: Outer Diameter}

From Figure 1.1.19, the uncertainty in the fuel pin cladding outer diameter $(0.58420 \mathrm{~cm})$ is $\pm 0.00127 \mathrm{~cm}$. This value is representative of a manufacturing tolerance. Results are shown in Table 2.1.22. The mass and thickness of the cladding is not conserved, as variation in the manufacturing tolerance of both inner and outer clad diameters were reported, but not in the thickness or mass of the clad material. Therefore, the variation of the outer diameter of the cladding also changes the thickness and linear density of the cladding.

The total number of fuel pins in the outer enrichment zone is 9,982. The uncertainty in the outer diameter of the stainless steel cladding is negligible.

Table 2.1.22. Uncertainty in the Outer-Fuel-Zone Cladding Outer Diameter. ${ }^{(a)}$

\begin{tabular}{||c|ccc|c|ccc||}
\hline Deviation & $\Delta \mathrm{k}$ & \pm & $\sigma_{\Delta \mathrm{k}}$ & $\begin{array}{c}\text { Scaling } \\
\text { Factor }\end{array}$ & $\Delta \mathrm{k}_{\mathrm{eff}}(1 \sigma)$ & \pm & $\sigma_{\Delta \mathrm{keff}}$ \\
\hline \hline$+0.00127 \mathrm{~cm}(3 \sigma)$ & -0.00017 & \pm & 0.00008 & 3 & -0.00006 & \pm & 0.00003 \\
$-0.00127 \mathrm{~cm}(3 \sigma)$ & -0.00006 & \pm & 0.00008 & 3 & -0.00002 & \pm & 0.00003 \\
\hline
\end{tabular}

(a) The random component of the uncertainty would be divided by $\sqrt{9,982}$. 
NEA/NSC/DOC(2006)1

Liquid Metal Fast Reactor - LMFR

FFTF-LMFR-RESR-001

CRIT-SPEC-REAC-COEF-MISC

\section{SS316 Wire Wrap: Diameter}

The wire wrap woven between the fuel pins is not explicitly modeled in the benchmark. However, an uncertainty of $\pm 0.00127 \mathrm{~cm}$ applies to the wire diameter of $0.14224 \mathrm{~cm}$ (Table 1.1.3) and is analyzed by appropriately adjusting the volume fraction of the wire wrap steel in liquid sodium as discussed in Section 2.1.3.6. This value is representative of a manufacturing tolerance.

\section{Fuel Pin Assembly Lattice: Pitch}

The pitch of the fuel pin lattice is adjusted by the manufacturing tolerance of the wire wrap between the fuel pins; the fuel pitch of $0.72644 \mathrm{~cm}$ is adjusted by $\pm 0.00127 \mathrm{~cm}$. Results are shown in Table 2.1.23. An uncertainty in the fuel pin pitch due to variation in the grid-plate design, to which the fuel pins are attached, was not available. However, as seen in Figure 1.1.7, there is not sufficient room within the fuel assembly duct for larger pitch variations.

The total number of fuel pins in the outer enrichment zone is 9,982. The uncertainty in the fuel pin assembly lattice pitch is negligible.

Table 2.1.23. Uncertainty in the Outer-Fuel-Zone Fuel Lattice Pitch. ${ }^{\text {(a) }}$

\begin{tabular}{|c|ccc|c|ccc||}
\hline Deviation & $\Delta \mathrm{k}$ & \pm & $\sigma_{\Delta \mathrm{k}}$ & $\begin{array}{c}\text { Scaling } \\
\text { Factor }\end{array}$ & $\Delta \mathrm{k}_{\mathrm{eff}}(1 \sigma)$ & \pm & $\sigma_{\Delta \mathrm{keff}}$ \\
\hline \hline$+0.00127 \mathrm{~cm}(3 \sigma)$ & -0.00019 & \pm & 0.00008 & 3 & -0.00006 & \pm & 0.00003 \\
$-0.00127 \mathrm{~cm}(3 \sigma)$ & 0.00009 & \pm & 0.00008 & 3 & 0.00003 & \pm & 0.00003 \\
\hline
\end{tabular}

(a) The random component of the uncertainty would be divided by $\sqrt{9}, 982$.

\section{Fuel Assembly SS316 Duct: Flat-To-Flat Width}

A manufacturing tolerance of \pm 0.015 in $(0.0381 \mathrm{~cm})$ across the flat-to-flat width $(11.0109 \mathrm{~cm})$ of the fuel assembly duct was selected. The calculated results are shown in Table 2.1.24.

The total number of assembly ducts in the outer enrichment zone is 46 . The uncertainty in the fuel assembly duct flat-to-flat width is negligible.

Table 2.1.24. Uncertainty in the Flat-To-Flat Width of the Outer-Fuel-Zone Ducts. ${ }^{(a)}$

\begin{tabular}{||c|ccc|c|ccc||}
\hline Deviation & $\Delta \mathrm{k}$ & \pm & $\sigma_{\Delta \mathrm{k}}$ & $\begin{array}{c}\text { Scaling } \\
\text { Factor }\end{array}$ & $\Delta \mathrm{k}_{\mathrm{eff}}(1 \sigma)$ & \pm & $\sigma_{\Delta \mathrm{keff}}$ \\
\hline \hline$+0.0381 \mathrm{~cm}(3 \sigma)$ & -0.00013 & \pm & 0.00008 & 3 & -0.00004 & \pm & 0.00003 \\
$-0.0381 \mathrm{~cm}(3 \sigma)$ & -0.00002 & \pm & 0.00008 & 3 & -0.00001 & \pm & 0.00003 \\
\hline
\end{tabular}

(a) The random component of the uncertainty would be divided by $\sqrt{ } 46$.

\section{Axial Shield, Pin Attachment, and Gas Plenum Regions}

These regions have been homogenized as described in the Section 3.1, based upon the 3-D Hex-Z model provided in Appendix F. There is insufficient publicly available data to analyze the heterogeneous effects of these regions. Heterogeneous effects and the effect of homogenization are expected to represent an insignificant contribution to the total uncertainty of the benchmark model, as the 
NEA/NSC/DOC(2006)1

Liquid Metal Fast Reactor - LMFR

FFTF-LMFR-RESR-001

CRIT-SPEC-REAC-COEF-MISC

uncertainties in other geometrical properties (Section 2.1.2) and density of the primary structural components (SS316, Inconel 600, and sodium in Sections 2.1.3.1 through 2.1.3.3) are relatively insignificant compared to other constituents of the total uncertainty. Therefore the uncertainty in the geometry of these regions is judged to be negligible.

\section{Fueled Open Test Assemblies}

Fueled open test assemblies are modeled identically as their respectively-zoned DFA assemblies; i.e., they do not include instrumentation. Insufficient information is available to assess a bias, if any, with its associated uncertainty. It is expected that neutronically the result would be negligible.

\subsubsection{Absorber Assemblies}

\section{All Control Rods}

When geometric and physical parameters were adjusted for the moveable control rods, they were also applied to the fixed shim control rods.

\section{Absorber Pellet Stack: Height}

The uncertainty in the absorber pellet stack height $(91.4400 \mathrm{~cm})$ is unknown. The manufacturing tolerance from the fuel pellet stack height $( \pm 0.3810 \mathrm{~cm})$ is used. Results are shown in Table 2.1.25.

The total number of absorber pellet stacks is 732 . The uncertainty in the height of the absorber pellet stack is negligible.

Table 2.1.25. Uncertainty in the Absorber Pellet Stack Height. ${ }^{(a)}$

\begin{tabular}{||c|ccc|c|ccc||}
\hline Deviation & $\Delta \mathrm{k}$ & \pm & $\sigma_{\Delta \mathrm{k}}$ & $\begin{array}{c}\text { Scaling } \\
\text { Factor }\end{array}$ & $\Delta \mathrm{k}_{\mathrm{eff}}(1 \sigma)$ & \pm & $\sigma_{\Delta \mathrm{keff}}$ \\
\hline \hline$+0.3810 \mathrm{~cm}(3 \sigma)$ & -0.00015 & \pm & 0.00008 & 3 & -0.00005 & \pm & 0.00003 \\
$-0.3810 \mathrm{~cm}(3 \sigma)$ & -0.00006 & \pm & 0.00008 & 3 & -0.00002 & \pm & 0.00003 \\
\hline
\end{tabular}

(a) The random component of the uncertainty would be divided by $\sqrt{732}$.

Absorber Pellet Stack: Diameter

The uncertainty in the absorber pellet diameter $(0.91948 \mathrm{~cm})$ is unknown. The manufacturing tolerance from the fuel pellet diameter $( \pm 0.00381 \mathrm{~cm})$ is used. Results are shown in Table 2.1.26.

The total number of absorber pellets is 43,920 . The uncertainty in the diameter of the absorber pellets is negligible. 
NEA/NSC/DOC(2006)1

Liquid Metal Fast Reactor - LMFR

FFTF-LMFR-RESR-001

CRIT-SPEC-REAC-COEF-MISC

Table 2.1.26. Uncertainty in the Absorber Pellet Diameter. ${ }^{(a)}$

\begin{tabular}{||c|ccc|c|ccc||}
\hline \hline Deviation & $\Delta \mathrm{k}$ & \pm & $\sigma_{\Delta \mathrm{k}}$ & $\begin{array}{c}\text { Scaling } \\
\text { Factor }\end{array}$ & $\Delta \mathrm{k}_{\mathrm{eff}}(1 \sigma)$ & \pm & $\sigma_{\Delta \mathrm{keff}}$ \\
\hline \hline$+0.00381 \mathrm{~cm}(3 \sigma)$ & -0.00027 & \pm & 0.00008 & 3 & -0.00009 & \pm & 0.00003 \\
$-0.00381 \mathrm{~cm}(3 \sigma)$ & 0.00021 & \pm & 0.00008 & 3 & 0.00007 & \pm & 0.00003 \\
\hline
\end{tabular}

(a) The random component of the uncertainty would be divided by $\sqrt{43,920}$.

\section{SS316 Cladding: Inner Diameter}

The uncertainty in the absorber pellet cladding inner diameter $(0.94488 \mathrm{~cm})$ is unknown. The manufacturing tolerance from the fuel pellet cladding inner diameter $\pm 0.00127 \mathrm{~cm}$ ) is used. Results are shown in Table 2.1.27. The mass and thickness of the cladding is not conserved, as variation in the manufacturing tolerance of both inner and outer clad diameters were reported, but not in the thickness or mass of the clad material.

The total number of absorber pins is 732 . The uncertainty in the inner diameter of the stainless steel cladding is negligible.

Table 2.1.27. Uncertainty in the Absorber Cladding Inner Diameter. ${ }^{(a)}$

\begin{tabular}{|c|ccc|c|ccc||}
\hline Deviation & $\Delta \mathrm{k}$ & \pm & $\sigma_{\Delta \mathrm{k}}$ & $\begin{array}{c}\text { Scaling } \\
\text { Factor }\end{array}$ & $\Delta \mathrm{k}_{\mathrm{eff}}(1 \sigma)$ & \pm & $\sigma_{\Delta \mathrm{keff}}$ \\
\hline \hline$+0.00127 \mathrm{~cm}(3 \sigma)$ & 0.00000 & \pm & 0.00008 & 3 & 0.00000 & \pm & 0.00003 \\
$-0.00127 \mathrm{~cm}(3 \sigma)$ & -0.00002 & \pm & 0.00008 & 3 & -0.00001 & \pm & 0.00003 \\
\hline
\end{tabular}

(a) The random component of the uncertainty would be divided by $\sqrt{732}$.

\section{SS316 Cladding: Outer Diameter}

The uncertainty in the absorber pellet cladding outer diameter $(1.20396 \mathrm{~cm})$ is unknown. The manufacturing tolerance from the fuel pellet cladding outer diameter $\pm 0.00127 \mathrm{~cm}$ ) is used. Results are shown in Table 2.1.28. The mass and thickness of the cladding is not conserved, as variation in the manufacturing tolerance of both inner and outer clad diameters were reported, but not in the thickness or mass of the clad material.

The total number of absorber pins is 732 . The uncertainty in the outer diameter of the stainless steel cladding is negligible.

Table 2.1.28. Uncertainty in the Absorber Cladding Outer Diameter. ${ }^{(a)}$

\begin{tabular}{||c|ccc|c|ccc||}
\hline \hline Deviation & $\Delta \mathrm{k}$ & \pm & $\sigma_{\Delta \mathrm{k}}$ & $\begin{array}{c}\text { Scaling } \\
\text { Factor }\end{array}$ & $\Delta \mathrm{k}_{\mathrm{eff}}(1 \sigma)$ & \pm & $\sigma_{\Delta \mathrm{keff}}$ \\
\hline \hline$+0.00127 \mathrm{~cm}(3 \sigma)$ & 0.00005 & \pm & 0.00008 & 3 & 0.00002 & \pm & 0.00003 \\
$-0.00127 \mathrm{~cm}(3 \sigma)$ & 0.00000 & \pm & 0.00008 & 3 & 0.00000 & \pm & 0.00003 \\
\hline
\end{tabular}

(a) The random component of the uncertainty would be divided by $\sqrt{732}$. 
NEA/NSC/DOC(2006)1

Liquid Metal Fast Reactor - LMFR

FFTF-LMFR-RESR-001

CRIT-SPEC-REAC-COEF-MISC

\section{SS316 Wire Wrap: Diameter}

The wire wrap woven between the absorber pins is not explicitly modeled in the benchmark. However, an uncertainty of $\pm 0.00127 \mathrm{~cm}$, taken from the uncertainty in the fuel assembly wire wraps, is applied to the wire diameter of $0.06096 \mathrm{~cm}$ and analyzed by appropriately adjusting the volume fraction of the wire wrap steel in liquid sodium as discussed in Section 2.1.3.7. This value is representative of a manufacturing tolerance.

\section{Absorber Pin Assembly Lattice: Pitch}

The pitch of the absorber pin lattice is adjusted by the manufacturing tolerance of the wire wrap between the absorber pins; the absorber pitch of $1.26492 \mathrm{~cm}$ is adjusted by $\pm 0.00127 \mathrm{~cm}$. Results are shown in Table 2.1.29. An uncertainty in the absorber pin pitch due to variation in the grid-plate design, to which the absorber pins are attached, was not available. However, as seen in Figure 1.1.10, there is not significant room within the absorber assembly duct for large pitch variations.

The total number of absorber pins is 732 . The uncertainty in the absorber pin lattice pitch is negligible.

Table 2.1.29. Uncertainty in the Absorber Pin Lattice Pitch. ${ }^{(a)}$

\begin{tabular}{|c|ccc|c|ccc||}
\hline \hline Deviation & $\Delta \mathrm{k}$ & \pm & $\sigma_{\Delta \mathrm{k}}$ & $\begin{array}{c}\text { Scaling } \\
\text { Factor }\end{array}$ & $\Delta \mathrm{k}_{\mathrm{eff}}(1 \sigma)$ & \pm & $\sigma_{\Delta \mathrm{keff}}$ \\
\hline \hline$+0.00127 \mathrm{~cm}(3 \sigma)$ & -0.00008 & \pm & 0.00008 & 3 & -0.00003 & \pm & 0.00003 \\
$-0.00127 \mathrm{~cm}(3 \sigma)$ & -0.00006 & \pm & 0.00008 & 3 & -0.00002 & \pm & 0.00002 \\
\hline
\end{tabular}

(a) The random component of the uncertainty would be divided by $\sqrt{732}$.

\section{Absorber Assembly SS316 Inner Duct: Flat-To-Flat Width}

A manufacturing tolerance of \pm 0.015 in $(0.0381 \mathrm{~cm})$ across the flat-to-flat width $(10.42416 \mathrm{~cm})$ of the absorber assembly inner duct was selected. The calculated results are shown in Table 2.1.30.

The total number of inner assembly ducts is 12 . The uncertainty in the absorber assembly inner duct flatto-flat width is negligible.

Table 2.1.30. Uncertainty in the Flat-To-Flat Width of the Inner Absorber Ducts. ${ }^{(a)}$

\begin{tabular}{||c|ccc|c|ccc||}
\hline Deviation & $\Delta \mathrm{k}$ & \pm & $\sigma_{\Delta \mathrm{k}}$ & $\begin{array}{c}\text { Scaling } \\
\text { Factor }\end{array}$ & $\Delta \mathrm{k}_{\mathrm{eff}}(1 \sigma)$ & \pm & $\sigma_{\Delta \mathrm{keff}}$ \\
\hline \hline$+0.0381 \mathrm{~cm}(3 \sigma)$ & 0.00007 & \pm & 0.00008 & 3 & 0.00002 & \pm & 0.00003 \\
$-0.0381 \mathrm{~cm}(3 \sigma)$ & -0.00031 & \pm & 0.00008 & 3 & -0.00010 & \pm & 0.00003 \\
\hline
\end{tabular}

(a) The random component of the uncertainty would be divided by $\sqrt{12}$.

Absorber Assembly SS316 Outer Duct: Flat-To-Flat Width

A manufacturing tolerance of \pm 0.015 in $(0.0381 \mathrm{~cm})$ across the flat-to-flat width $(10.42416 \mathrm{~cm})$ of the absorber assembly outer duct was selected. The calculated results are shown in Table 2.1.31. 
NEA/NSC/DOC(2006)1

Liquid Metal Fast Reactor - LMFR

FFTF-LMFR-RESR-001

CRIT-SPEC-REAC-COEF-MISC

The total number of outer assembly ducts is 12 . The uncertainty in the absorber assembly outer duct flatto-flat width is negligible.

Table 2.1.31. Uncertainty in the Flat-To-Flat Width of the Outer Absorber Ducts. ${ }^{(a)}$

\begin{tabular}{||c|ccc|c|ccc||}
\hline Deviation & $\Delta \mathrm{k}$ & \pm & $\sigma_{\Delta \mathrm{k}}$ & $\begin{array}{c}\text { Scaling } \\
\text { Factor }\end{array}$ & $\Delta \mathrm{k}_{\mathrm{eff}}(1 \sigma)$ & \pm & $\sigma_{\Delta \mathrm{keff}}$ \\
\hline \hline$+0.0381 \mathrm{~cm}(3 \sigma)$ & 0.00010 & \pm & 0.00008 & 3 & 0.00003 & \pm & 0.00003 \\
$-0.0381 \mathrm{~cm}(3 \sigma)$ & -0.00021 & \pm & 0.00008 & 3 & -0.00007 & \pm & 0.00003 \\
\hline
\end{tabular}

(a) The random component of the uncertainty would be divided by $\sqrt{12}$.

\section{Lower Shield, Below Poison, Above Poison, Driveline, and Withdrawn Absorber Regions}

These regions have been homogenized as described in the Section 3.1, based upon the 3-D Hex-Z model provided in Appendix F. There is insufficient publicly available data to analyze the heterogeneous effects of these regions. Heterogeneous effects and the effect of homogenization are expected to represent an insignificant contribution to the total uncertainty of the benchmark model, as the uncertainties in other geometrical properties (Section 2.1.2) and density of the primary structural components (SS316, Inconel 600, and sodium in Sections 2.1.3.1 through 2.1.3.3) are relatively insignificant compared to other constituents of the total uncertainty. Therefore the uncertainty in the geometry of these regions is judged to be negligible.

\section{Fixed Shim Control Rods Specifics}

Fixed shim control rods are modeled identical to fully inserted control rods. Insufficient information is available to assess a bias, if any, with its associated uncertainty. It is expected that neutronically the result would be negligible. The only significant difference between the fixed shim control rods and the primary and secondary control rods is that the fixed shim rod cannot be withdrawn, and a wire wrap is placed between the inner and outer ducts. When geometric and physical parameters were adjusted for the moveable control rods, they were also applied to the fixed shim control rods.

\section{Inter-Duct SS316 Wire Wrap: Diameter}

There is insufficient data to analyze the effective uncertainty in the diameter of the SS316 wire wrap placed between the inner and outer ducts of the fixed shim control rod assemblies. An approximation was performed to assess the uncertainty (see Section 2.1.3.7).

\subsubsection{Radial Reflectors}

\section{Row 7}

\section{Orifice, Lower Adapter, Reflector Blocks, Load Pad, and Upper Shield Regions}

These regions have been homogenized as described in the Section 3.1, based upon the 3-D Hex-Z model provided in Appendix F. There is insufficient publicly available data to analyze the heterogeneous effects of these regions. Heterogeneous effects and the effect of homogenization are expected to represent an insignificant contribution to the total uncertainty of the benchmark model, as the uncertainties in other geometrical properties (Section 2.1.2) and density of the primary structural components (SS316, Inconel 600, and sodium in Sections 2.1.3.1 through 2.1.3.3) are relatively insignificant compared to other constituents of the total uncertainty. Therefore the uncertainty in the geometry of these regions is judged to be negligible. 
NEA/NSC/DOC(2006)1

Liquid Metal Fast Reactor - LMFR

FFTF-LMFR-RESR-001

CRIT-SPEC-REAC-COEF-MISC

\section{Rows 8 and 9}

Orifice, Lower Adapter, Reflector Blocks, Load Pad, and Upper Shield Regions

These regions have been homogenized as described in the Section 3.1, based upon the 3-D Hex-Z model provided in Appendix F. There is insufficient publicly available data to analyze the heterogeneous effects of these regions. Heterogeneous effects and the effect of homogenization are expected to represent an insignificant contribution to the total uncertainty of the benchmark model, as the uncertainties in other geometrical properties (Section 2.1.2) and density of the primary structural components (SS316, Inconel 600, and sodium in Sections 2.1.3.1 through 2.1.3.3) are relatively insignificant compared to other constituents of the total uncertainty. Therefore the uncertainty in the geometry of these regions is judged to be negligible.

\subsubsection{Vibration Open Test Assembly}

\section{Orifice/Shield and Instruments/Housing Regions}

These regions have been homogenized as described in the Section 3.1, based upon the 3-D Hex-Z model provided in Appendix F. There is insufficient publicly available data to analyze the heterogeneous effects of these regions. Heterogeneous effects and the effect of homogenization are expected to represent an insignificant contribution to the total uncertainty of the benchmark model, as the uncertainties in other geometrical properties (Section 2.1.2) and density of the primary structural components (SS316, Inconel 600, and sodium in Sections 2.1.3.1 through 2.1.3.3) are relatively insignificant compared to other constituents of the total uncertainty. Therefore the uncertainty in the geometry of these regions is judged to be negligible.

\subsubsection{In-Core Shim Assemblies}

Orifice/Shield, Pin Attachment, and Simulated Fuel Bundle Regions

These regions have been homogenized as described in the Section 3.1, based upon the 3-D Hex-Z model provided in Appendix F. There is insufficient publicly available data to analyze the heterogeneous effects of these regions. Heterogeneous effects and the effect of homogenization are expected to represent an insignificant contribution to the total uncertainty of the benchmark model, as the uncertainties in other geometrical properties (Section 2.1.2) and density of the primary structural components (SS316, Inconel 600, and sodium in Sections 2.1.3.1 through 2.1.3.3) are relatively insignificant compared to other constituents of the total uncertainty. Therefore the uncertainty in the geometry of these regions is judged to be negligible.

\subsubsection{Simulated Core Assembly}

The fully-loaded core configuration of the FFTF does not contain simulated core assemblies.

\subsubsection{In-Reactor Thimble}

The fully-loaded core critical configuration contains the in-reactor thimble but without any inserts.

The in-reactor thimble has been homogenized as described in the Section 3.1, based upon the 3-D Hex-Z model provided in Appendix F. There is insufficient publicly available data to analyze the heterogeneous effects of the in-reactor thimble. Heterogeneous effects and the effect of homogenization are expected to represent an insignificant contribution to the total uncertainty of the benchmark model, as the uncertainties in other geometrical properties (Section 2.1.2) and density of the primary structural components (SS316, Inconel 600, and sodium in Sections 2.1.3.1 through 2.1.3.3) are relatively 
NEA/NSC/DOC(2006)1

Liquid Metal Fast Reactor - LMFR

FFTF-LMFR-RESR-001

CRIT-SPEC-REAC-COEF-MISC

insignificant compared to other constituents of the total uncertainty. Therefore the uncertainty in the geometry of the in-reactor thimble is judged to be negligible.

\subsubsection{Reactor Shielding}

Inner and Outer Radial Reactor Shielding Regions

These regions have been homogenized as described in the Section 3.1, based upon the 3-D Hex-Z model provided in Appendix F. There is insufficient publicly available data to analyze the heterogeneous effects of these regions. Heterogeneous effects and the effect of homogenization are expected to represent an insignificant contribution to the total uncertainty of the benchmark model, as the uncertainties in other geometrical properties (Section 2.1.2) and density of the primary structural components (SS316, Inconel 600, and sodium in Sections 2.1.3.1 through 2.1.3.3) are relatively insignificant compared to other constituents of the total uncertainty. Therefore the uncertainty in the geometry of these regions is judged to be negligible.

\section{Core Lattice Placement of Radial Reactor Shielding}

In the homogenization process of the radial reactor shielding, it is unclear exactly how many positions surrounding the reactor core should be modeled as shielding material. Furthermore, the actual coverage of shield plating adjacent to the core is unclear from currently available information. The benchmark configuration of Section 3 is shown in Figure 2.1.1 with a contingent of 54 inner shielding positions and 60 outer shielding positions. A second model was developed (Figure 2.1.2) which had a total of 48 shield positions (18 inner and 30 outer) replaced by liquid sodium. The result from this comparison is shown in Table 2.1.32 and is treated as a $1 \sigma$ uncertainty. Even with such an extreme analysis of this uncertainty, the effective uncertainty is relatively insignificant compared to other uncertainties in the benchmark model configuration. This analysis also accounts for any uncertainty due to streaming effects between the shielding sections that surround the core.

The uncertainty in the lattice placement of the radial reactor shielding is considered all systematic with no random component.

Table 2.1.32. Uncertainty in Location of Radial Reactor Shielding.

\begin{tabular}{||c|ccc|c|ccc||}
\hline Deviation & $\Delta \mathrm{k}$ & \pm & $\sigma_{\Delta \mathrm{k}}$ & $\begin{array}{c}\text { Scaling } \\
\text { Factor }\end{array}$ & $\Delta \mathrm{k}_{\mathrm{eff}}(1 \sigma)$ & \pm & $\sigma_{\Delta \mathrm{keff}}$ \\
\hline \hline Remove 48 Shield Positions & -0.00053 & \pm & 0.00008 & 1 & -0.00053 & \pm & 0.00008 \\
\hline
\end{tabular}




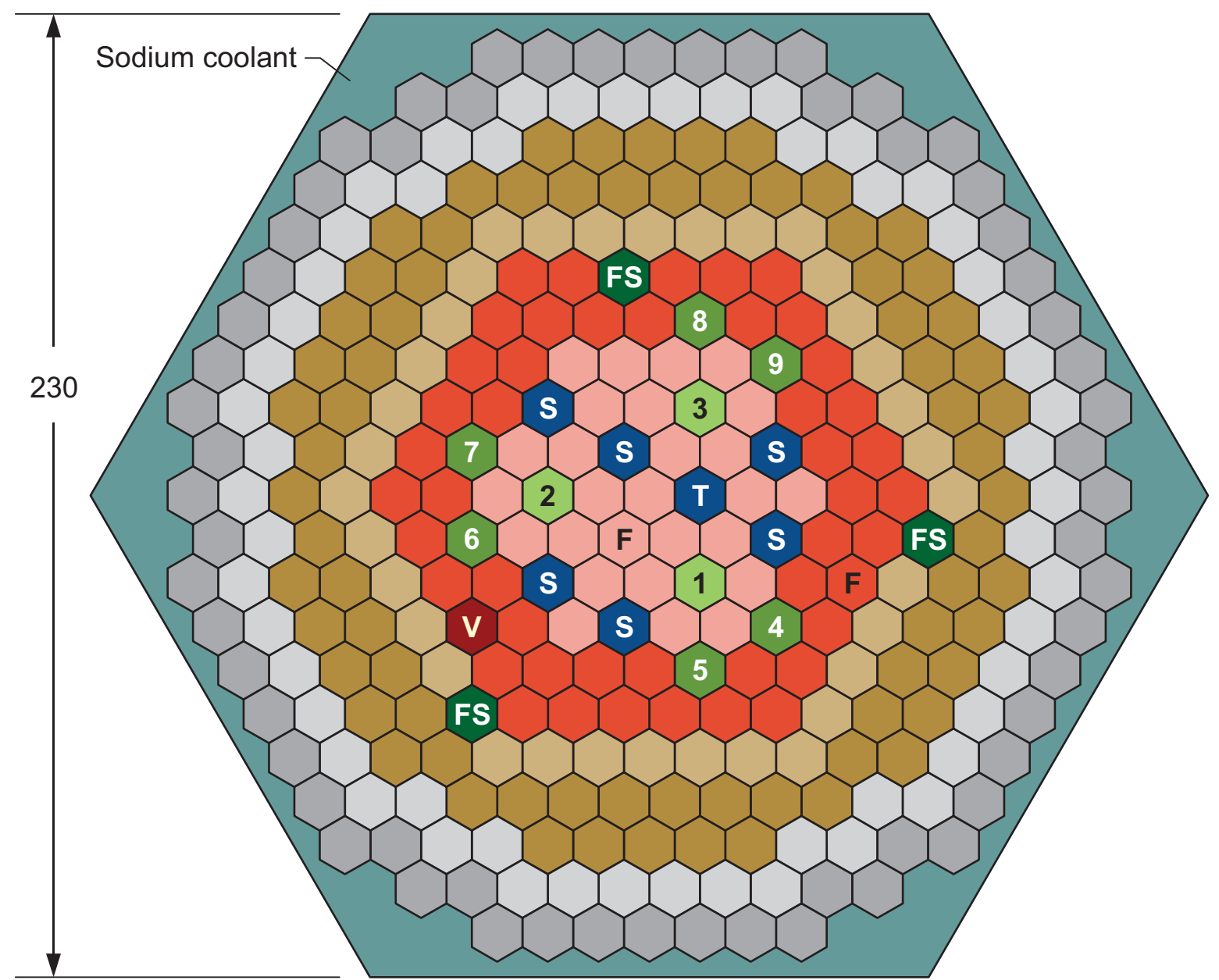

Outer radial shield

Inner radial shield

Radial reflectors in Row 8 and 9

Radial reflectors in Row 7

Driver fuel assembly in the Outer Enrichment Zone

Driver fuel assembly in the Inner Enrichment Zone

T Startup position of in reactor thimble (replaced by in-core shim assembly)
FS Fixed shim control rods

S In-core shim assemblies

\# Secondary control rods

\# Primary control rods

Fueled open test assembly

V Vibration open test assembly

Dimensions in $\mathrm{cm}$

09-GA50001-122

Figure 2.1.1. Diagram of FFTF Fully-Loaded Critical Core Configuration. 


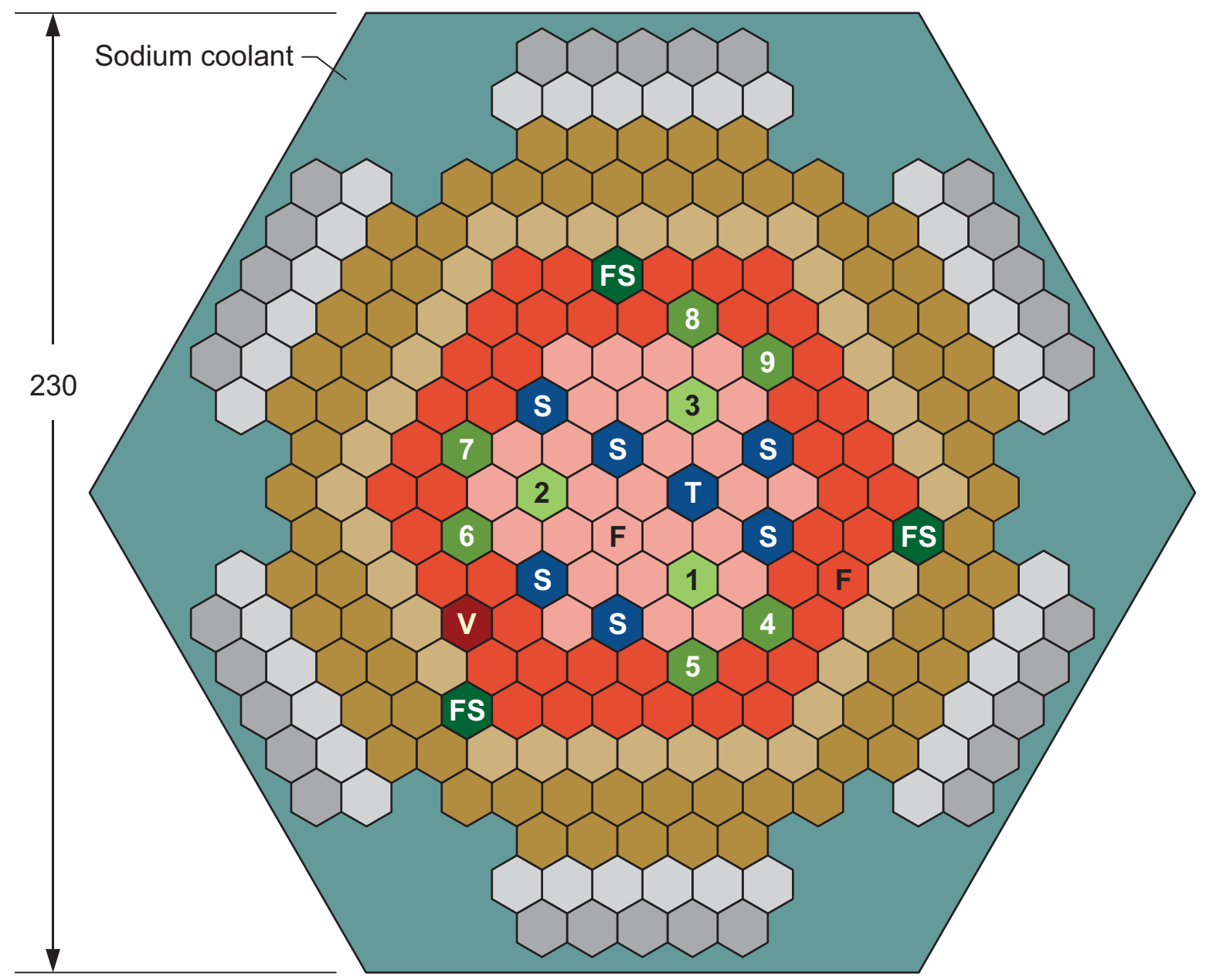

$\bigcirc$ Outer radial shield

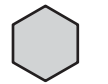

Inner radial shield

Radial reflectors in Row 8 and 9

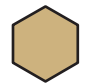

Radial reflectors in Row 7

Driver fuel assembly in the Outer Enrichment Zone

Driver fuel assembly in the Inner Enrichment Zone

Startup position of in reactor thimble (replaced by in-core shim assembly)
FS Fixed shim control rods

s In-core shim assemblies

\# Secondary control rods

\# Primary control rods

Fueled open test assembly

V Vibration open test assembly

Dimensions in cm 09-GA50001-122-2

Figure 2.1.2. Diagram of FFTF Fully-Loaded Critical Core Configuration with Less Shielding. 
NEA/NSC/DOC(2006)1

Liquid Metal Fast Reactor - LMFR

FFTF-LMFR-RESR-001

CRIT-SPEC-REAC-COEF-MISC

\subsubsection{Reactor Core Configuration}

\section{Core Lattice: Pitch}

The radial alignment of core assemblies is controlled by the $12.0142 \mathrm{~cm}$, at room temperature, hole spacing in the core support plate; the $11.9761 \mathrm{~cm}$, at room temperature, outer dimension of the spacer pads on the core components; and the core restraint yokes external to the reflector, which have an average diametral spacing (across corners) of $1.807718 \mathrm{~m}$.

The dimension across the core diagonal encompasses through Row 6 and can increase by a maximum of $0.4826 \mathrm{~cm}$ (causing the maximum core pitch) and decrease by a maximum of $0.254 \mathrm{~cm}$ (causing the minimum core pitch). The hexagonal pitch is $12.051 \mathrm{~cm}$ at the benchmark temperature. A core diagonal length of $1.807718 \mathrm{~m}$ is approximately equal to 15 positions across the core with a pitch of $12.051 \mathrm{~cm}$. The pitch can then vary by the bounding limits provided, divided by the number of pitch lengths, 11 . Therefore the uncertainty in the core lattice pitch is between $+0.044 \mathrm{~cm}$ (maximum) and $-0.023 \mathrm{~cm}$ (minimum). This limit is doubled for the analysis of the uncertainty and then scaled back to a $1 \sigma$ value. This bounding limit is treated with uniform probability distribution and divided by $\sqrt{ } 3$ instead of 3 . Results are shown in Table 2.1.33.

It is unclear whether thermal expansion effects of the grid plate due to either local or global temperature effects are included in the uncertainty of the core diagonal dimension. A core yoke system was used to try to minimize core expansion effects at elevated temperatures. It is assumed that temperature effects were included in the measurements.

It is assumed that any random component in grid plate spacing incurred during its manufacture is negligible.

The uncertainty in the core lattice pitch is considered all systematic with no random component.

Table 2.1.33. Uncertainty in the Core Lattice Pitch.

\begin{tabular}{||c|ccc|c|ccc||}
\hline \hline Deviation & $\Delta \mathrm{k}$ & \pm & $\sigma_{\Delta \mathrm{k}}$ & $\begin{array}{c}\text { Scaling } \\
\text { Factor }^{(\mathrm{a})}\end{array}$ & $\Delta \mathrm{k}_{\text {eff }}(1 \sigma)$ & \pm & $\sigma_{\Delta \mathrm{keff}}$ \\
\hline \hline $\begin{array}{c}+0.088 \mathrm{~cm} \\
(2 \times \text { maximum limit }) \\
\begin{array}{c}-0.046 \mathrm{~cm} \\
(2 \times \text { minimum limit })\end{array}\end{array}$ & -0.00393 & \pm & 0.00008 & $2 \sqrt{3}$ & -0.00113 & \pm & 0.00002 \\
\hline
\end{tabular}

(a) This uncertainty is treated as bounding with a uniform probability distribution.

\subsubsection{Compositional Variations}

\subsubsection{Stainless Steel 316}

\section{Composition}

The fabrication composition of stainless steel 316 is provided in Table 1.1.8. An average composition is used to represent the benchmark model (Table 2.1.34). To obtain the maximum uncertainty possible in the composition of the stainless steel, the iron content is maximized (by minimizing the content of all other constituents within the tolerance limits) and then minimized (by maximizing the content of all other constituents within the tolerance limits). This uncertainty is treated as a bounded limit with uniform probability distribution. The results are shown in Table 2.1.35. 
NEA/NSC/DOC(2006)1

Liquid Metal Fast Reactor - LMFR

FFTF-LMFR-RESR-001

CRIT-SPEC-REAC-COEF-MISC

The stainless steel composition of just the fuel pin cladding was assessed as well, and it was determined that approximately one-third of the uncertainty reported in Table 2.1 .35 , for maximizing/minimizing the total iron content, can be attributed to the cladding alone. The remaining uncertainty is due to the assembly ducts, core shielding, and the multitude of other components of the reactor. The application of this uncertainty analysis, where a single stainless steel component of the reactor does not dominate the total uncertainty of the stainless steel composition, overestimates the total uncertainty effect of compositional variation of stainless steel in the FFTF.

The most important secondary constituents (chromium, nickel, molybdenum, and manganese) of the stainless steel were then varied individually, while simultaneously adjusting the amount of iron to maintain the weight balance, to determine their respective worths. The results are added to Table 2.1.35. Molybdenum is the major contributor to the total uncertainty in the stainless steel composition. These uncertainties are similarly treated as bounding limits with uniform probability distribution. The squareroot of the sum of the squares of the largest uncertainty of each of these four individual contributors is used as the effective uncertainty in the stainless steel composition. While this method provides a smaller uncertainty than that obtained by adjusting all impurities to maximize/minimize the total iron content in the steel, it will still overestimate the contribution of the uncertainty in the composition of stainless steel to the overall uncertainty of the benchmark experiment.

The uncertainty in the stainless steel composition is considered all systematic with no random component.

Table 2.1.34. Stainless Steel 316 Composition in the Benchmark Model.

\begin{tabular}{||c|c|}
\hline Element & wt.\% \\
\hline \hline Chromium & 17.5 \\
Nickel & 13.5 \\
Carbon & 0.05 \\
Molybdenum & 2.5 \\
Manganese & 1.75 \\
Nitrogen & 0.005 \\
Aluminum & 0.025 \\
Arsenic & 0.015 \\
Boron & 0.0005 \\
Cobalt & 0.025 \\
Niobium & 0.025 \\
Copper & 0.05 \\
Phosphorus & 0.01 \\
Silicon & 0.375 \\
Sulfur & 0.005 \\
Tantalum & 0.005 \\
Vanadium & 0.1 \\
Iron & 64.0595 \\
\hline
\end{tabular}


NEA/NSC/DOC(2006)1

Liquid Metal Fast Reactor - LMFR

FFTF-LMFR-RESR-001

CRIT-SPEC-REAC-COEF-MISC

Table 2.1.35. Uncertainty in the Stainless Steel Composition.

\begin{tabular}{|c|c|c|c|c|c|c|c|}
\hline Deviation & $\Delta \mathrm{k}$ & \pm & $\sigma_{\Delta \mathrm{k}}$ & $\begin{array}{l}\text { Scaling } \\
\text { Factor }\end{array}$ & $\Delta \mathrm{k}_{\mathrm{eff}}(1 \sigma)$ & \pm & $\sigma_{\Delta \mathrm{keff}}$ \\
\hline Maxin & 00 & \pm & 0.00 & $\sqrt{3}$ & 0.00099 & \pm & 0.00005 \\
\hline Minimum Fe & -0.00169 & \pm & 0.00008 & $\sqrt{3}$ & -0.00098 & \pm & 0.00005 \\
\hline Max & 0.00019 & \pm & 0.00 & $\sqrt{3}$ & 0.00011 & \pm & 0.00005 \\
\hline Minimum $\mathrm{Cr}$ & -0.00006 & \pm & 0.00008 & $\sqrt{3}$ & -0.00003 & \pm & 0.00005 \\
\hline Maximu & -0.00012 & \pm & 0.00008 & $\sqrt{3}$ & -0.00007 & \pm & 0.00005 \\
\hline Minimum Mn & 0.00024 & \pm & 0.00007 & $\sqrt{3}$ & 0.00014 & \pm & 0.00004 \\
\hline Maxim & -0.00100 & \pm & 0.00007 & $\sqrt{3}$ & -0.00058 & \pm & 0.00004 \\
\hline Minimum Mo & 0.00129 & \pm & 0.00007 & $\sqrt{3}$ & 0.00074 & \pm & 0.00004 \\
\hline & 0.00002 & $I$ & 0.00008 & $\sqrt{3}$ & 0.00001 & \pm & 0.00005 \\
\hline Minimum Ni & 0.00026 & \pm & 0.00008 & $\sqrt{3}$ & 0.00015 & \pm & 0.00005 \\
\hline th & & & & & 0.00078 & \pm & .00009 \\
\hline
\end{tabular}

\section{Density}

The density of stainless steel 316 is reported in Table E. 1.2 to be $7.89 \mathrm{~g} / \mathrm{cm}^{3}$ at benchmark temperatures. Typical stainless steel 316 densities are between 7.9 and $8.0 \mathrm{~g} / \mathrm{cm}^{3}$ at room temperatures. The density was varied by $\pm 0.1 \mathrm{~g} / \mathrm{cm}^{3}$, which is twice the bounding limit, and treated with uniform distribution probability. The results are shown in Table 2.1.36.

The uncertainty in the stainless steel density is negligible.

Table 2.1.36. Uncertainty in the Stainless Steel Density.

\begin{tabular}{|c|ccc|c|ccc||}
\hline Deviation & $\Delta \mathrm{k}$ & \pm & $\sigma_{\Delta \mathrm{k}}$ & $\begin{array}{c}\text { Scaling } \\
\text { Factor }\end{array}$ & $\Delta \mathrm{k}_{\text {eff }}(1 \sigma)$ & \pm & $\sigma_{\Delta \mathrm{keff}}$ \\
\hline \hline$+0.1 \mathrm{~g} / \mathrm{cm}^{3}(2 \times$ limit $)$ & -0.00007 & \pm & 0.00008 & $2 \sqrt{3}$ & -0.00002 & \pm & 0.00002 \\
$-0.1 \mathrm{~g} / \mathrm{cm}^{3}(2 \times$ limit $)$ & 0.00006 & \pm & 0.00008 & $2 \sqrt{3}$ & 0.00002 & \pm & 0.00002 \\
\hline
\end{tabular}

\subsubsection{Inconel 600}

\section{Composition}

The fabrication composition of Inconel 600 is provided in Table 1.1.9. An average composition is used to represent the benchmark model (Table 2.1.37). To obtain the maximum uncertainty possible in the composition of the Inconel, the nickel content is maximized (by minimizing the content of all other constituents within the tolerance limits) and then minimizing the nickel content (by maximizing the content of all other constituents within the tolerance limits). This uncertainty is treated as a bounded limit with uniform probability distribution. The results are shown in Table 2.1.38. 
NEA/NSC/DOC(2006)1

\section{Liquid Metal Fast Reactor - LMFR \\ FFTF-LMFR-RESR-001 \\ CRIT-SPEC-REAC-COEF-MISC}

It was determined that the contribution from the axial reflectors in the fuel pins to the uncertainty reported in Table 2.1.38 is negligible. The effective uncertainty is due primarily to the compositional variation in the radial reflectors surrounding the core.

The most important secondary constituents (chromium, iron, and manganese) of the Inconel were then varied individually, while simultaneously adjusting the amount of nickel to maintain the weight balance, to determine their individual worths. The results are added to Table 2.1.38. These uncertainties are also similarly treated as bounding limits with uniform probability distribution. The square-root of the sum of the squares of the largest uncertainty of each of these three major contributors is used as the effective uncertainty in the Inconel 600 composition. While this method provides a smaller uncertainty than that obtained by adjusting all impurities to maximize/minimize the total nickel content in the Inconel, it will still overestimate the contribution of the uncertainty in the composition of Inconel 600 to the overall uncertainty of the benchmark experiment.

The uncertainty in the Inconel composition is considered all systematic with no random component.

Table 2.1.37. Inconel 600 Composition in the Benchmark Model.

\begin{tabular}{|c|c|}
\hline Element & wt.\% \\
\hline \hline Nickel & 73.6425 \\
Chromium & 15.5 \\
Iron & 8 \\
Manganese & 1.75 \\
Carbon & 0.5 \\
Copper & 0.04 \\
Silicon & 0.25 \\
Sulfur & 0.0075 \\
Cobalt & 0.1 \\
\hline
\end{tabular}

Table 2.1.38. Uncertainty in the Inconel Composition.

\begin{tabular}{||c|ccc|c|ccc||}
\hline \hline Deviation & $\Delta \mathrm{k}$ & \pm & $\sigma_{\Delta \mathrm{k}}$ & $\begin{array}{c}\text { Scaling } \\
\text { Factor }\end{array}$ & $\Delta \mathrm{k}_{\mathrm{eff}}(1 \sigma)$ & \pm & $\sigma_{\Delta \mathrm{keff}}$ \\
\hline \hline Maximum Ni & -0.00083 & \pm & 0.00008 & $\sqrt{ } 3$ & -0.00048 & \pm & 0.00005 \\
Minimum Ni & 0.00081 & \pm & 0.00008 & $\sqrt{ } 3$ & 0.00047 & \pm & 0.00005 \\
\hline \hline Maximum Cr & 0.00026 & \pm & 0.00007 & $\sqrt{3}$ & 0.00015 & \pm & 0.00004 \\
Minimum Cr & -0.00003 & \pm & 0.00008 & $\sqrt{3}$ & -0.00002 & \pm & 0.00005 \\
\hline Maximum Fe & 0.00007 & \pm & 0.00008 & $\sqrt{3}$ & 0.00004 & \pm & 0.00005 \\
Minimum Fe & 0.00008 & \pm & 0.00008 & $\sqrt{3}$ & 0.00005 & \pm & 0.00005 \\
\hline Maximum Mn & 0.00002 & \pm & 0.00007 & $\sqrt{ } 3$ & 0.00001 & \pm & 0.00004 \\
Minimum Mn & 0.00024 & \pm & 0.00007 & $\sqrt{3}$ & 0.00014 & \pm & 0.00004 \\
\hline \hline Total Uncertainty & & & & & 0.00021 & \pm & 0.00007 \\
\hline \hline
\end{tabular}


NEA/NSC/DOC(2006)1

Liquid Metal Fast Reactor - LMFR

FFTF-LMFR-RESR-001

CRIT-SPEC-REAC-COEF-MISC

\section{Density}

The density of Inconel 600 is reported in Table E.1.2 to be $8.34 \mathrm{~g} / \mathrm{cm}^{3}$ at benchmark temperatures. Typical Inconel 600 densities are between 8.43 and $8.47 \mathrm{~g} / \mathrm{cm}^{3}$ at room temperatures. The density was varied by $\pm 0.04 \mathrm{~g} / \mathrm{cm}^{3}$, which is twice the bounding limit, and treated with uniform distribution probability. The results are shown in Table 2.1.39.

The uncertainty in the Inconel density is negligible.

Table 2.1.39. Uncertainty in Inconel Density.

\begin{tabular}{||c|ccc|c|ccc||}
\hline Deviation & $\Delta \mathrm{k}$ & \pm & $\sigma_{\Delta \mathrm{k}}$ & $\begin{array}{c}\text { Scaling } \\
\text { Factor }\end{array}$ & $\Delta \mathrm{k}_{\text {eff }}(1 \sigma)$ & \pm & $\sigma_{\Delta \mathrm{keff}}$ \\
\hline \hline$+0.04 \mathrm{~g} / \mathrm{cm}^{3}(2 \times$ limit $)$ & 0.00002 & \pm & 0.00008 & $2 \sqrt{3}$ & 0.00001 & \pm & 0.00002 \\
$-0.04 \mathrm{~g} / \mathrm{cm}^{3}(2 \times$ limit $)$ & -0.00022 & \pm & 0.00008 & $2 \sqrt{3}$ & -0.00006 & \pm & 0.00002 \\
\hline
\end{tabular}

\subsubsection{Sodium Coolant}

Density

The density of the liquid sodium coolant at benchmark temperatures was determined using equations and data provided elsewhere. ${ }^{\mathrm{a}}$. The density is calculated to be $0.902 \mathrm{~g} / \mathrm{cm}^{3}$ at $478 \mathrm{~K}$. The uncertainty of the density was reported to be $\pm 0.3 \%$, which is equivalent to $\pm 0.0027 \mathrm{~g} / \mathrm{cm}^{3}(1 \sigma)$. The density of the sodium was varied and the results are shown in Table 2.1.40.

The uncertainty in the liquid sodium density is assumed to be \pm 0.00020 and considered all systematic with no random component.

Table 2.1.40. Uncertainty in Sodium Coolant Density.

\begin{tabular}{||c|ccc|c|ccc||}
\hline \hline Deviation & $\Delta \mathrm{k}$ & \pm & $\sigma_{\Delta \mathrm{k}}$ & $\begin{array}{c}\text { Scaling } \\
\text { Factor }\end{array}$ & $\Delta \mathrm{k}_{\text {eff }}(1 \sigma)$ & \pm & $\sigma_{\Delta \mathrm{keff}}$ \\
\hline \hline$+0.0027 \mathrm{~g} / \mathrm{cm}^{3}(1 \sigma)$ & -0.00020 & \pm & 0.00008 & 1 & -0.00020 & \pm & 0.00008 \\
$-0.0027 \mathrm{~g} / \mathrm{cm}^{3}(1 \sigma)$ & -0.00010 & \pm & 0.00008 & 1 & -0.00010 & \pm & 0.00008 \\
\hline
\end{tabular}

\section{Impurities}

The impurity composition in the sodium coolant is provided in Table 1.1.5. The maximum composition is used to represent the addition of impurities into the benchmark model (Table 2.1.41). This uncertainty is treated as a bounded limit with uniform probability distribution. The results are shown in Table 2.1.42.

The uncertainty in the liquid sodium impurities is negligible.

\footnotetext{
a J. K. Fink and L. Leibowitz, "Thermodyanmic and Transport Properties of Sodium Liquid and Vapor," ANL/RE95-2, Argonne National Laboratory (January 1995).
} 
NEA/NSC/DOC(2006)1

Liquid Metal Fast Reactor - LMFR

FFTF-LMFR-RESR-001

CRIT-SPEC-REAC-COEF-MISC

Table 2.1.41. Sodium Impurity Content (ppm by weight).

\begin{tabular}{||ccc|}
\hline \hline Element & Max & Ave. \\
\hline $\mathrm{Al}$ & 0.63 & 0.17 \\
$\mathrm{Au}$ & 0.07 & 0.005 \\
$\mathrm{Ag}$ & 0.03 & 0.005 \\
$\mathrm{~B}$ & 0.01 & 0.006 \\
$\mathrm{Ba}$ & 0.04 & 0.005 \\
$\mathrm{Bi}$ & 0.04 & 0.01 \\
$\mathrm{Ca}$ & 0.19 & 0.02 \\
$\mathrm{Cd}$ & 0.01 & 0.005 \\
$\mathrm{Cs}$ & 0.02 & 0.01 \\
$\mathrm{Cl}$ & 3 & 1.5 \\
$\mathrm{Cr}$ & 1.8 & 0.37 \\
$\mathrm{Co}$ & 0.06 & 0.02 \\
$\mathrm{Cu}$ & 0.06 & 0.02 \\
$\mathrm{~F}$ & 0.10 & 0.10 \\
$\mathrm{Fe}$ & 170.0 & 12.9 \\
$\mathrm{~Pb}$ & 0.07 & 0.05 \\
$\mathrm{Li}$ & 0.01 & 0.005 \\
$\mathrm{Mg}$ & 0.18 & 0.04 \\
\hline
\end{tabular}

\begin{tabular}{||ccc||}
\hline \hline Element & Max. & Ave. \\
\hline \hline $\mathrm{Mn}$ & 0.4 & 0.09 \\
$\mathrm{Mo}$ & 0.44 & 0.06 \\
$\mathrm{Ni}$ & 1.2 & 0.29 \\
$\mathrm{~K}$ & 310 & 310 \\
$\mathrm{Rb}$ & 0.02 & 0.01 \\
$\mathrm{Si}$ & 0.1 & 0.06 \\
$\mathrm{~S}$ & 0.01 & 0.01 \\
$\mathrm{Sn}$ & 0.2 & 0.04 \\
$\mathrm{Sm}$ & 0.01 & 0.005 \\
$\mathrm{Ti}$ & 0.04 & 0.02 \\
$\mathrm{U}$ & 0.002 & 0.002 \\
$\mathrm{~V}$ & 0.04 & 0.02 \\
$\mathrm{Zn}$ & 0.01 & 0.005 \\
$\mathrm{P}$ & 0.2 & 0.2 \\
$\mathrm{O}$ & 2.8 & 1.48 \\
$\mathrm{H}$ & 0.08 & 0.14 \\
$\mathrm{C}$ & 0.43 & 0.16 \\
\hline \hline
\end{tabular}

Table 2.1.42. Uncertainty in Sodium Impurity Content.

\begin{tabular}{||c|ccc|c|ccc||}
\hline Deviation & $\Delta \mathrm{k}$ & \pm & $\sigma_{\Delta \mathrm{k}}$ & $\begin{array}{c}\text { Scaling } \\
\text { Factor }\end{array}$ & $\Delta \mathrm{k}_{\text {eff }}(1 \sigma)$ & \pm & $\sigma_{\Delta \mathrm{keff}}$ \\
\hline \hline Maximum Impurities & -0.00016 & \pm & 0.00008 & $2 \sqrt{ } 3$ & -0.00005 & \pm & 0.00002 \\
\hline
\end{tabular}

\subsubsection{Helium-Filled Gaps}

The helium, and tag gas, that would be typically found between the pellets and cladding of the fuel and absorber pins was not included in the benchmark model. The effect of removing this material was approximated by including helium gas in all of the fuel and absorber pins. Assuming application of the ideal gas equation, the atomic density of helium gas inside the cladding is $2.0074 \mathrm{a} / \mathrm{b}-\mathrm{cm}$. The calculated results are shown in Table 2.1.43.

The uncertainty for the removal of helium from cladding gaps is negligible. 
NEA/NSC/DOC(2006)1

Liquid Metal Fast Reactor - LMFR

FFTF-LMFR-RESR-001

CRIT-SPEC-REAC-COEF-MISC

Table 2.1.43. Uncertainty in the Removal of Helium Gas from the Fuel and Absorber Pins.

\begin{tabular}{||c|ccc|c|ccc||}
\hline Deviation & $\Delta \mathrm{k}$ & \pm & $\sigma_{\Delta \mathrm{k}}$ & $\begin{array}{c}\text { Scaling } \\
\text { Factor }\end{array}$ & $\Delta \mathrm{k}_{\mathrm{eff}}(1 \sigma)$ & \pm & $\sigma_{\Delta \mathrm{keff}}$ \\
\hline \hline Add He Gas & 0.00002 & \pm & 0.00008 & 1 & 0.00002 & \pm & 0.00008 \\
\hline
\end{tabular}

\subsubsection{Moisture in Pellets}

The maximum moisture content in the fuel, insulator, and absorber pellets are 20,20 , and $50 \mu / \mathrm{g}$, respectively. This maximum content was added to all pellets in the core and treated as a bounding limit with uniform probability distribution. The results are shown in Table 2.1.44.

The uncertainty for the presence of moisture in the fuel and absorber pellets is negligible

Table 2.1.44. Uncertainty in Pellet Moisture Content.

\begin{tabular}{||c|ccc|c|ccc||}
\hline Deviation & $\Delta \mathrm{k}$ & \pm & $\sigma_{\Delta \mathrm{k}}$ & $\begin{array}{c}\text { Scaling } \\
\text { Factor }\end{array}$ & $\Delta \mathrm{k}_{\mathrm{eff}}(1 \sigma)$ & \pm & $\sigma_{\Delta \mathrm{keff}}$ \\
\hline \hline Maximum Moisture & 0.00015 & \pm & 0.00007 & $2 \sqrt{ } 3$ & 0.00004 & \pm & 0.00002 \\
\hline
\end{tabular}

\subsubsection{Driver Fuel Assemblies}

The uncertainties in the driver fuel assemblies were assessed by enrichment zone, although many of the components in both zones would be very similar, such as fuel cladding, insulator pellets, axial reflectors, wire wrap, ducts, etc. The purpose in separating analysis of the two regions was to investigate what effects might have more significant worth, if any, depending on its placement within a particular fueled region of the core. Furthermore, as fuel pins were fabricated in batches, there may have been some slight variations in the construction of the pins.

\section{Inner Enrichment Zone (Type 3.1 Fuel Pins)}

$\mathrm{UO}_{2}$ Insulator Pellets: Density

From Figure 1.1.19, the uncertainty in the density $\left(10.42 \mathrm{~g} / \mathrm{cm}^{3}\right)$ of the insulator pellets is $\pm 0.22 \mathrm{~g} / \mathrm{cm}^{3}$. This value is representative of a manufacturing tolerance. Results are shown in Table 2.1.45.

The total number of insulator pellets in the inner enrichment zone is 23,436 . The uncertainty in the $\mathrm{UO}_{2}$ insulator pellet density is negligible.

Table 2.1.45. Uncertainty in Inner-Fuel-Zone Insulator Pellet Density. ${ }^{(a)}$

\begin{tabular}{||c|ccc|c|ccc||}
\hline Deviation & $\Delta \mathrm{k}$ & \pm & $\sigma_{\Delta \mathrm{k}}$ & $\begin{array}{c}\text { Scaling } \\
\text { Factor }\end{array}$ & $\Delta \mathrm{k}_{\mathrm{eff}}(1 \sigma)$ & \pm & $\sigma_{\Delta \mathrm{keff}}$ \\
\hline \hline$+0.22 \mathrm{~g} / \mathrm{cm}^{3}(3 \sigma)$ & -0.00009 & \pm & 0.00008 & 3 & -0.00003 & \pm & 0.00003 \\
$-0.22 \mathrm{~g} / \mathrm{cm}^{3}(3 \sigma)$ & -0.00002 & \pm & 0.00008 & 3 & -0.00001 & \pm & 0.00003 \\
\hline
\end{tabular}

(a) The random component of the uncertainty would be divided by $\sqrt{23,436}$. 
NEA/NSC/DOC(2006)1

Liquid Metal Fast Reactor - LMFR

FFTF-LMFR-RESR-001

CRIT-SPEC-REAC-COEF-MISC

\section{$\mathrm{UO}_{2}$ Insulator Pellets: Isotopic Distribution}

It is unclear as to whether the insulator pellets are comprised of natural or depleted uranium material. The uranium isotopic distribution is adjusted such that the natural composition is reduced to contain 0.2 wt. $\%{ }^{235} \mathrm{U}$ and 0.0015 wt. $\%{ }^{234} \mathrm{U}$. Results are shown in Table 2.1.46.

The uncertainty in whether the insulator pellets are natural or depleted uranium is considered all systematic with no random component.

It was later determined that the initial fuel (Core 1 and 2) had natural uranium insulator pellets, and Core 3 and 4 had depleted uranium insulator pellets. ${ }^{\text {a }}$ Therefore, this uncertainty does not apply to the isothermal physics tests analyzed in this benchmark, and is only included here for future comparison.

Table 2.1.46. Uncertainty in the Isotopic Composition of the Inner-Fuel-Zone Insulator Pellets. ${ }^{(a)}$

\begin{tabular}{|c|ccc|c|ccc||}
\hline Deviation & $\Delta \mathrm{k}$ & \pm & $\sigma_{\Delta \mathrm{k}}$ & $\begin{array}{c}\text { Scaling } \\
\text { Factor }\end{array}$ & $\Delta \mathrm{k}_{\mathrm{eff}}(1 \sigma)$ & \pm & $\sigma_{\Delta \mathrm{keff}}$ \\
\hline \hline Depleted Uranium & -0.00019 & \pm & 0.00008 & 1 & -0.00019 & \pm & 0.00008 \\
\hline
\end{tabular}

(a) This uncertainty does not apply to the isothermal core physics tests of the FFTF..

\section{$\mathrm{UO}_{2}$ Insulator Pellets: Impurities}

The impurity composition in the insulator pellets is provided in Table 1.1.7. Half of the maximum composition is used to represent the addition of impurities into the benchmark model (Table 2.1.47). This uncertainty is treated as a bounded limit with uniform probability distribution. The uncertainty was also evaluated using the maximum limit, which was then reduced in half. The larger uncertainty is chosen to represent the uncertainty in the impurity content. The results are shown in Table 2.1.48.

The uncertainty in the insulator pellet impurity content is negligible.

\footnotetext{
${ }^{a}$ Personal Communication with David W. Wootan at Pacific Northwest National Laboratory (September 30, 2009).
} 
NEA/NSC/DOC(2006)1

Liquid Metal Fast Reactor - LMFR

FFTF-LMFR-RESR-001

CRIT-SPEC-REAC-COEF-MISC

Table 2.1.47. Insulator Pellet Impurity Content.

\begin{tabular}{|c|c|}
\hline Element & $\begin{array}{c}\text { Average Content } \\
(\mu \mathrm{g} / \mathrm{g})\end{array}$ \\
\hline $\mathrm{Na}$ & 350 \\
\hline $\mathrm{K}$ & 350 \\
\hline $\mathrm{Cl}$ & 7.5 \\
\hline F & 7.5 \\
\hline $\mathrm{C}$ & 175 \\
\hline $\mathrm{N}$ & 175 \\
\hline $\mathrm{Sm}$ & 25 \\
\hline $\mathrm{Eu}$ & 25 \\
\hline $\mathrm{Gd}$ & 25 \\
\hline Dy & 25 \\
\hline $\mathrm{Fe}$ & 250 \\
\hline $\mathrm{Cr}$ & 250 \\
\hline $\mathrm{Ni}$ & 250 \\
\hline $\mathrm{P}$ & 100 \\
\hline S & 100 \\
\hline $\mathrm{Zn}$ & 27.5 \\
\hline $\mathrm{Pb}$ & 27.5 \\
\hline $\mathrm{Sn}$ & 27.5 \\
\hline $\mathrm{Cd}$ & 27.5 \\
\hline $\mathrm{Be}$ & 100 \\
\hline $\mathrm{Ca}$ & 100 \\
\hline $\mathrm{Mg}$ & 100 \\
\hline $\mathrm{Al}$ & 100 \\
\hline $\mathrm{Si}$ & 100 \\
\hline B & 10 \\
\hline $\mathrm{Li}$ & 5 \\
\hline Co & 50 \\
\hline $\mathrm{Ti}$ & 50 \\
\hline V & 50 \\
\hline $\mathrm{Ta}$ & 50 \\
\hline W & 50 \\
\hline $\mathrm{Cu}$ & 50 \\
\hline $\mathrm{Ag}$ & 50 \\
\hline Мо & 50 \\
\hline $\mathrm{Mn}$ & 50 \\
\hline $\mathrm{Zr}$ & 50 \\
\hline
\end{tabular}

Table 2.1.48. Uncertainty in Inner-Fuel-Zone Insulator Pellet Impurity Content.

\begin{tabular}{||c|ccc|c|ccc||}
\hline Deviation & $\Delta \mathrm{k}$ & \pm & $\sigma_{\Delta \mathrm{k}}$ & $\begin{array}{c}\text { Scaling } \\
\text { Factor }\end{array}$ & $\Delta \mathrm{k}_{\mathrm{eff}}(1 \sigma)$ & \pm & $\sigma_{\Delta \mathrm{keff}}$ \\
\hline \hline Half Maximum Impurities & 0.00002 & \pm & 0.00008 & $\sqrt{ } 3$ & 0.00001 & \pm & 0.00005 \\
Maximum Impurities & -0.00017 & \pm & 0.00008 & $2 \sqrt{ } 3$ & -0.00005 & \pm & 0.00002 \\
\hline
\end{tabular}


NEA/NSC/DOC(2006)1

Liquid Metal Fast Reactor - LMFR

FFTF-LMFR-RESR-001

CRIT-SPEC-REAC-COEF-MISC

\section{$\mathrm{UO}_{2}$ Insulator Pellets: Oxygen-To-Uranium Ratio}

The nominal oxygen to uranium ratio in the insulator pellets is $2.000 \pm 0.005$. This ratio was varied within this tolerance limit and the results are shown in Table 2.1.49.

The uncertainty in the insulator pellet $\mathrm{O}: \mathrm{U}$ ratio is negligible.

Table 2.1.49. Uncertainty in O:U Ratio in the Inner-Fuel-Zone Insulator Pellets.

\begin{tabular}{||c|ccc|c|ccc||}
\hline \hline Deviation & $\Delta \mathrm{k}$ & \pm & $\sigma_{\Delta \mathrm{k}}$ & $\begin{array}{c}\text { Scaling } \\
\text { Factor }\end{array}$ & $\Delta \mathrm{k}_{\mathrm{eff}}(1 \sigma)$ & \pm & $\sigma_{\Delta \mathrm{keff}}$ \\
\hline \hline$+0.005 \mathrm{O}: \mathrm{U}(3 \sigma)$ & -0.00014 & \pm & 0.00008 & 3 & -0.00005 & \pm & 0.00003 \\
$-0.005 \mathrm{O}: \mathrm{U}(3 \sigma)$ & -0.00004 & \pm & 0.00008 & 3 & -0.00001 & \pm & 0.00003 \\
\hline
\end{tabular}

\section{Fuel Pellet Stack: Density}

From Figure 1.1.19, the uncertainty in the density $\left(9.783 \mathrm{~g} / \mathrm{cm}^{3}\right)$ of the fuel pellets is $\pm 0.318 \mathrm{~g} / \mathrm{cm}^{3}$. After performing the perturbation analysis, it was later determined that the measured variation in fuel mass for an individual pin is significantly smaller (about 25\%). ${ }^{a}$ Therefore, the computed results for the variation in fuel pellet stack density (mass) are scaled to correctly reflect the measured values. Results are shown in Table 2.1.50.

The total number of fuel pins that were chemical assayed was 15 . For determining the random component of the uncertainty in Section 2.1.5, the results in Table 2.1.50 are divided by $\sqrt{ } 15$.

Table 2.1.50. Uncertainty in the Inner-Fuel-Zone Fuel Pellet Stack Density. ${ }^{(a)}$

\begin{tabular}{||c|ccc|c|ccc||}
\hline Deviation & $\Delta \mathrm{k}$ & \pm & $\sigma_{\Delta \mathrm{k}}$ & $\begin{array}{c}\text { Scaling } \\
\text { Factor }\end{array}$ & $\Delta \mathrm{k}_{\text {eff }}(1 \sigma)$ & \pm & $\sigma_{\Delta \mathrm{keff}}$ \\
\hline \hline$+0.318 \mathrm{~g} / \mathrm{cm}^{3}(3 \sigma)$ & 0.00721 & \pm & 0.00008 & 4.266 & 0.00169 & \pm & 0.00002 \\
$-0.318 \mathrm{~g} / \mathrm{cm}^{3}(3 \sigma)$ & -0.00724 & \pm & 0.00008 & 4.266 & -0.00170 & \pm & 0.00002 \\
\hline
\end{tabular}

(a) The random component of the uncertainty would be divided by $\sqrt{ } 15$.

\section{Fuel Pellet Stack: Isotopic Distribution}

The uncertainty in the isotopic distribution of the fuel pellets (Figure 1.1.19) is reported to be within $0.3 \%$. This value is representative of a manufacturing tolerance. This uncertainty was evaluated by increasing or decreasing the ${ }^{239} \mathrm{Pu}$ content by $0.3 \%$. The content of the other plutonium isotopes were reduced or increased, respectively, such that the total content remained at $100 \%$; they were all adjusted equally. Results are shown in Table 2.1.51.

The uncertainty in the fuel pellet isotopic distribution is considered all systematic with no random component.

\footnotetext{
a P. Goris, “Rod Scanner Assay of FFTF Fuel,” HEDL-TME 77-29 (January 1978).
} 
NEA/NSC/DOC(2006)1

Liquid Metal Fast Reactor - LMFR

FFTF-LMFR-RESR-001

CRIT-SPEC-REAC-COEF-MISC

Table 2.1.51. Uncertainty in the Isotopic Distribution of the Inner-Fuel-Zone Fuel Pellets.

\begin{tabular}{||c|ccc|c|ccc||}
\hline Deviation & $\Delta \mathrm{k}$ & \pm & $\sigma_{\Delta \mathrm{k}}$ & $\begin{array}{c}\text { Scaling } \\
\text { Factor }\end{array}$ & $\Delta \mathrm{k}_{\text {eff }}(1 \sigma)$ & \pm & $\sigma_{\Delta \mathrm{keff}}$ \\
\hline \hline$+0.3 \%{ }^{239} \mathrm{Pu}(3 \sigma)$ & 0.00042 & \pm & 0.00007 & 3 & 0.00014 & \pm & 0.00002 \\
$-0.3 \%{ }^{239} \mathrm{Pu}(3 \sigma)$ & -0.00031 & \pm & 0.00007 & 3 & -0.00010 & \pm & 0.00002 \\
\hline
\end{tabular}

\section{Fuel Pellet Stack: Pu/(Pu+U) Ratio}

The plutonium content of the fuel was determined using the analysis of the isotopic distribution of the fuel pellets. The uncertainty is also within $0.3 \%$, and represents a manufacturing tolerance. This uncertainty was evaluated by increasing or decreasing the total plutonium content by $0.3 \%$ while reducing or increasing, respectively, the total uranium content. Results are shown in Table 2.1.52.

The uncertainty in the fuel pellet $\mathrm{Pu} /(\mathrm{Pu}+\mathrm{U})$ ratio is considered all systematic with no random component.

Table 2.1.52. Uncertainty in the $\mathrm{Pu} /(\mathrm{Pu}+\mathrm{U})$ Ratio of the Inner-Fuel-Zone Fuel Pellets.

\begin{tabular}{|c|ccc|c|ccc||}
\hline Deviation & $\Delta \mathrm{k}$ & \pm & $\sigma_{\Delta \mathrm{k}}$ & $\begin{array}{c}\text { Scaling } \\
\text { Factor }\end{array}$ & $\Delta \mathrm{k}_{\mathrm{eff}}(1 \sigma)$ & \pm & $\sigma_{\Delta \mathrm{keff}}$ \\
\hline \hline$+0.3 \% \mathrm{Pu}(3 \sigma)$ & 0.00255 & \pm & 0.00008 & 3 & 0.00085 & \pm & 0.00003 \\
$-0.3 \% \mathrm{Pu}(3 \sigma)$ & -0.00269 & \pm & 0.00007 & 3 & -0.00090 & \pm & 0.00003 \\
\hline
\end{tabular}

\section{Fuel Pellet Stack: Radioisotopic Decay}

The composition of the fuel reported in Figure 1.1.19 appears to represent measurements performed on January 1, 1976. The reported ${ }^{238} \mathrm{U}$ content was adjusted to account for ${ }^{234} \mathrm{U}$ that would be typically present in natural uranium. The actinides were then decayed from the apparent measurement date to the date of the critical experiment, March 8, 1980. The generation of ${ }^{237} \mathrm{~Np}$ was also included. The benchmark model includes the composition shown in Table 3.1.7.

The uncertainty in the actual composition of the material at the time of the experiment would depend upon the initial measured compositions and the uncertainty in the assay date. The material compositions of the fuel have been assessed via the other perturbation analyses in this section. Therefore the actual assay date will be investigated. It is unclear whether the assay was actually performed on 1/1/1976 or if only the year was recorded and the month and day were added by default by a computational database. Therefore, it is believed that the assay may have occurred somewhere in the year 1976 and the composition was varied by a decay time modified by \pm 1 year, which equates to an approximate change in the decay constant of approximately $\pm 24 \%$. The result is treated as a bounding uncertainty with uniform probability distribution. Results are shown in Table 2.1.53.

The uncertainty in the fuel pellet decay is considered all systematic with no random component. 
Liquid Metal Fast Reactor - LMFR

FFTF-LMFR-RESR-001

CRIT-SPEC-REAC-COEF-MISC

Table 2.1.53. Uncertainty in the Radioisotopic Decay of the Inner-Fuel-Zone Fuel Pellets.

\begin{tabular}{||c|ccc|c|ccc||}
\hline \hline Deviation & $\Delta \mathrm{k}$ & \pm & $\sigma_{\Delta \mathrm{k}}$ & $\begin{array}{c}\text { Scaling } \\
\text { Factor }\end{array}$ & $\Delta \mathrm{k}_{\mathrm{eff}}(1 \sigma)$ & \pm & $\sigma_{\Delta \mathrm{keff}}$ \\
\hline \hline+1 year & -0.00035 & \pm & 0.00007 & $\sqrt{ } 3$ & -0.00020 & \pm & 0.00004 \\
-1 year & 0.00018 & \pm & 0.00008 & $\sqrt{ } 3$ & 0.00010 & \pm & 0.00005 \\
\hline
\end{tabular}

Fuel Pellet Stack: Impurities

The impurity composition in the fuel pellets is provided in Table 1.1.6. Half of the maximum composition is used to represent the addition of impurities into the benchmark model (Table 2.1.54). This uncertainty is treated as a bounded limit with uniform probability distribution. The uncertainty was also evaluated using the maximum limit, which was then reduced in half. The larger uncertainty is chosen to represent the uncertainty in the impurity content. The results are shown in Table 2.1.55.

The uncertainty in the fuel pellet impurity content is considered all systematic with no random component. 
NEA/NSC/DOC(2006)1

Liquid Metal Fast Reactor - LMFR

FFTF-LMFR-RESR-001

CRIT-SPEC-REAC-COEF-MISC

Table 2.1.54. Fuel Pellet Impurity Content.

\begin{tabular}{|c|c|}
\hline Element & $\begin{array}{c}\text { Average Content } \\
(\mu \mathrm{g} / \mathrm{g})\end{array}$ \\
\hline $\mathrm{Na}$ & 350 \\
\hline $\mathrm{K}$ & 350 \\
\hline $\mathrm{Cl}$ & 7.5 \\
\hline $\mathrm{F}$ & 7.5 \\
\hline $\mathrm{C}$ & 175 \\
\hline $\mathrm{N}$ & 175 \\
\hline $\mathrm{Sm}$ & 25 \\
\hline $\mathrm{Eu}$ & 25 \\
\hline $\mathrm{Gd}$ & 25 \\
\hline Dy & 25 \\
\hline $\mathrm{Fe}$ & 250 \\
\hline $\mathrm{Cr}$ & 250 \\
\hline $\mathrm{Ni}$ & 250 \\
\hline P & 100 \\
\hline S & 100 \\
\hline $\mathrm{Zn}$ & 27.5 \\
\hline $\mathrm{Pb}$ & 27.5 \\
\hline $\mathrm{Sn}$ & 27.5 \\
\hline $\mathrm{Cd}$ & 27.5 \\
\hline $\mathrm{Be}$ & 100 \\
\hline $\mathrm{Ca}$ & 100 \\
\hline $\mathrm{Mg}$ & 100 \\
\hline $\mathrm{Al}$ & 100 \\
\hline $\mathrm{Si}$ & 100 \\
\hline B & 10 \\
\hline $\mathrm{Li}$ & 5 \\
\hline Co & 55.56 \\
\hline $\mathrm{Ti}$ & 55.56 \\
\hline V & 55.56 \\
\hline $\mathrm{Ta}$ & 55.56 \\
\hline W & 55.56 \\
\hline $\mathrm{Cu}$ & 55.56 \\
\hline $\mathrm{Ag}$ & 55.56 \\
\hline Mo & 55.56 \\
\hline $\mathrm{Mn}$ & 55.56 \\
\hline $\mathrm{Zr}$ & 350 \\
\hline
\end{tabular}

Table 2.1.55. Uncertainty in Inner-Fuel-Zone Fuel Pellet Impurity Content.

\begin{tabular}{|c|ccc|c|ccc||}
\hline Deviation & $\Delta \mathrm{k}$ & \pm & $\sigma_{\Delta \mathrm{k}}$ & $\begin{array}{c}\text { Scaling } \\
\text { Factor }\end{array}$ & $\Delta \mathrm{k}_{\mathrm{eff}}(1 \sigma)$ & \pm & $\sigma_{\Delta \mathrm{keff}}$ \\
\hline \hline Half Maximum Impurities & -0.00038 & \pm & 0.00008 & $\sqrt{ } 3$ & -0.00022 & \pm & 0.00005 \\
Maximum Impurities & -0.00079 & \pm & 0.00008 & $2 \sqrt{3}$ & -0.00023 & \pm & 0.00002 \\
\hline
\end{tabular}


NEA/NSC/DOC(2006)1

Liquid Metal Fast Reactor - LMFR

FFTF-LMFR-RESR-001

CRIT-SPEC-REAC-COEF-MISC

\section{Fuel Pellet Stack: Oxygen-To-Uranium/Plutonium Ratio}

The tolerance in the nominal oxygen to uranium/plutonium ratio in the fuel pellets is between $+0.02 /-$ 0.03. This ratio was varied within this tolerance limit and the results are shown in Table 2.1.56.

The uncertainty in the fuel pellet $\mathrm{O}:(\mathrm{U}, \mathrm{Pu})$ ratio is negligible.

Table 2.1.56. Uncertainty in O:(U,Pu) Ratio in the Inner-Fuel-Zone Fuel Pellets.

\begin{tabular}{||c|ccc|c|ccc||}
\hline Deviation & $\Delta \mathrm{k}$ & \pm & $\sigma_{\Delta \mathrm{k}}$ & $\begin{array}{c}\text { Scaling } \\
\text { Factor }\end{array}$ & $\Delta \mathrm{k}_{\text {eff }}(1 \sigma)$ & \pm & $\sigma_{\Delta \mathrm{keff}}$ \\
\hline \hline$+0.02 \mathrm{O}:(\mathrm{U}, \mathrm{Pu})(3 \sigma)$ & -0.00016 & \pm & 0.00007 & 3 & -0.00005 & \pm & 0.00002 \\
$-0.03 \mathrm{O}:(\mathrm{U}, \mathrm{Pu})(3 \sigma)$ & -0.00012 & \pm & 0.00008 & 3 & -0.00004 & \pm & 0.00003 \\
\hline
\end{tabular}

\section{SS316 Wire Wrap: Diameter (Homogenized)}

The wire wraps are homogenized with the sodium coolant surrounding the fuel pins. The effective volume fraction of the homogenized medium is varied according to the equivalent mass of material for variation in the wire wrap diameter of $\pm 0.00127 \mathrm{~cm}$ from the nominal value of $0.14224 \mathrm{~cm}$. This value is a manufacturing tolerance. Results are shown in Table 2.1.57.

Although most, if not all, of the $39 \mathrm{~km}$ of wire wrap was developed during a single fabrication process, there would be variability in the diameter of the wire during the drawing process. The total number of fuel pin wire wraps in the inner enrichment zone is 5,859. The uncertainty in the diameter of the stainless steel wire wrap is negligible.

Table 2.1.57. Uncertainty in the Inner-Fuel-Zone Wire Wrap Diameter. ${ }^{(a)}$

\begin{tabular}{||c|ccc|c|ccc||}
\hline Deviation & $\Delta \mathrm{k}$ & \pm & $\sigma_{\Delta \mathrm{k}}$ & $\begin{array}{c}\text { Scaling } \\
\text { Factor }\end{array}$ & $\Delta \mathrm{k}_{\mathrm{eff}}(1 \sigma)$ & \pm & $\sigma_{\Delta \mathrm{keff}}$ \\
\hline \hline$+0.00127 \mathrm{~cm}(3 \sigma)$ & 0.00002 & \pm & 0.00008 & 3 & 0.00001 & \pm & 0.00003 \\
$-0.00127 \mathrm{~cm}(3 \sigma)$ & -0.00016 & \pm & 0.00008 & 3 & -0.00005 & \pm & 0.00003 \\
\hline
\end{tabular}

(a) The random component of the uncertainty would be divided by $\sqrt{5,859}$.

\section{Axial Shield, Pin Attachment, and Gas Plenum Regions}

These regions have been homogenized as described in the Section 3.1, based upon the 3-D Hex-Z model provided in Appendix F. There is insufficient publicly available data to analyze the material properties of the heterogeneous components in these regions. Variation in the material properties in this region of the core are expected to represent an insignificant contribution to the total uncertainty of the benchmark model, as the uncertainties in other geometrical properties (Section 2.1.2) and density of the primary structural components (SS316, Inconel 600, and sodium in Sections 2.1.3.1 through 2.1.3.3) are relatively insignificant compared to other constituents of the total uncertainty. Therefore the uncertainty in the composition of these regions is judged to be negligible. 
NEA/NSC/DOC(2006)1

Liquid Metal Fast Reactor - LMFR

FFTF-LMFR-RESR-001

CRIT-SPEC-REAC-COEF-MISC

\section{Outer Enrichment Zone (Type 3.2 Fuel Pins)}

\section{$\mathrm{UO}_{2}$ Insulator Pellets: Density}

From Figure 1.1.19, the uncertainty in the density $\left(10.42 \mathrm{~g} / \mathrm{cm}^{3}\right)$ of the insulator pellets is $\pm 0.22 \mathrm{~g} / \mathrm{cm}^{3}$. This value is representative of a manufacturing tolerance. Results are shown in Table 2.1.58.

The total number of insulator pellets in the outer enrichment zone is 39,928 . The uncertainty in the $\mathrm{UO}_{2}$ insulator pellet density is negligible.

Table 2.1.58. Uncertainty in Outer-Fuel-Zone Insulator Pellet Density. ${ }^{(a)}$

\begin{tabular}{||c|rcc|c|ccc||}
\hline \hline Deviation & $\Delta \mathrm{k}$ & \pm & $\sigma_{\Delta \mathrm{k}}$ & $\begin{array}{c}\text { Scaling } \\
\text { Factor }\end{array}$ & $\Delta \mathrm{k}_{\mathrm{eff}}(1 \sigma)$ & \pm & $\sigma_{\Delta \mathrm{keff}}$ \\
\hline \hline$+0.22 \mathrm{~g} / \mathrm{cm}^{3}(3 \sigma)$ & -0.00001 & \pm & 0.00008 & 3 & 0.00000 & \pm & 0.00003 \\
$-0.22 \mathrm{~g} / \mathrm{cm}^{3}(3 \sigma)$ & -0.00007 & \pm & 0.00008 & 3 & -0.00002 & \pm & 0.00003 \\
\hline
\end{tabular}

(a) The random component of the uncertainty would be divided by $\sqrt{39,928}$.

\section{$\mathrm{UO}_{2}$ Insulator Pellets: Isotopic Distribution}

It is unclear as to whether the insulator pellets are comprised of natural or depleted uranium material. The uranium isotopic distribution is adjusted such that the natural composition is reduced to contain 0.2 wt. $\%{ }^{235} \mathrm{U}$ and 0.0015 wt. $\%{ }^{234} \mathrm{U}$. Results are shown in Table 2.1.59.

The uncertainty in whether the insulator pellets are natural or depleted uranium is considered all systematic with no random component.

It was later determined that the initial fuel (Core 1 and 2) had natural uranium insulator pellets, and Core 3 and 4 had depleted uranium insulator pellets. ${ }^{\text {a }}$ Therefore, this uncertainty does not apply to the isothermal physics tests analyzed in this benchmark, and is only included here for future comparison.

Table 2.1.59. Uncertainty in the Isotopic Composition of the Outer-Fuel-Zone Insulator Pellets. ${ }^{(a)}$

\begin{tabular}{||c|ccc|c|ccc||}
\hline Deviation & $\Delta \mathrm{k}$ & \pm & $\sigma_{\Delta \mathrm{k}}$ & $\begin{array}{c}\text { Scaling } \\
\text { Factor }\end{array}$ & $\Delta \mathrm{k}_{\mathrm{eff}}(1 \sigma)$ & \pm & $\sigma_{\Delta \mathrm{keff}}$ \\
\hline \hline Depleted Uranium & -0.00019 & \pm & 0.00008 & 1 & -0.00019 & \pm & 0.00008 \\
\hline
\end{tabular}

(a) This uncertainty does not apply to the isothermal core physics tests of the FFTF.

\section{$\mathrm{UO}_{2}$ Insulator Pellets: Impurities}

The impurity composition in the insulator pellets is provided in Table 1.1.7. Half of the maximum composition is used to represent the addition of impurities into the benchmark model (Table 2.1.47). This uncertainty is treated as a bounded limit with uniform probability distribution. The uncertainty was also evaluated using the maximum limit, which was then reduced in half. The larger uncertainty is chosen to represent the uncertainty in the impurity content. The results are shown in Table 2.1.60.

The uncertainty in the insulator pellet impurity content is negligible.

\footnotetext{
a Personal Communication with David W. Wootan at Pacific Northwest National Laboratory (September 30, 2009).
} 
NEA/NSC/DOC(2006)1

Liquid Metal Fast Reactor - LMFR

FFTF-LMFR-RESR-001

CRIT-SPEC-REAC-COEF-MISC

Table 2.1.60. Uncertainty in Outer-Fuel-Zone Insulator Pellet Impurity Content.

\begin{tabular}{||c|ccc|c|ccc||}
\hline Deviation & $\Delta \mathrm{k}$ & \pm & $\sigma_{\Delta \mathrm{k}}$ & $\begin{array}{c}\text { Scaling } \\
\text { Factor }\end{array}$ & $\Delta \mathrm{k}_{\text {eff }}(1 \sigma)$ & \pm & $\sigma_{\Delta \mathrm{keff}}$ \\
\hline \hline Half Maximum Impurities & 0.00002 & \pm & 0.00008 & $\sqrt{ } 3$ & 0.00001 & \pm & 0.00005 \\
Maximum Impurities & 0.00001 & \pm & 0.00008 & $\sqrt{ } 3$ & 0.00000 & \pm & 0.00002 \\
\hline
\end{tabular}

\section{$\mathrm{UO}_{2}$ Insulator Pellets: Oxygen-To-Uranium Ratio}

The nominal oxygen to uranium ratio in the insulator pellets is $2.000 \pm 0.005$. This ratio was varied within this tolerance limit and the results are shown in Table 2.1.61.

The uncertainty in the insulator pellet $\mathrm{O}: \mathrm{U}$ ratio is negligible.

Table 2.1.61. Uncertainty in O:U Ratio in the Outer-Fuel-Zone Insulator Pellets.

\begin{tabular}{||c|ccc|c|ccc||}
\hline Deviation & $\Delta \mathrm{k}$ & \pm & $\sigma_{\Delta \mathrm{k}}$ & $\begin{array}{c}\text { Scaling } \\
\text { Factor }\end{array}$ & $\Delta \mathrm{k}_{\text {eff }}(1 \sigma)$ & \pm & $\sigma_{\Delta \mathrm{keff}}$ \\
\hline \hline+0.005 O:U $(3 \sigma)$ & 0.00004 & \pm & 0.00008 & 3 & 0.00001 & \pm & 0.00003 \\
-0.005 O:U $(3 \sigma)$ & -0.00010 & \pm & 0.00008 & 3 & -0.00003 & \pm & 0.00003 \\
\hline
\end{tabular}

\section{Fuel Pellet Stack: Density}

From Figure 1.1.19, the uncertainty in the density $\left(9.830 \mathrm{~g} / \mathrm{cm}^{3}\right)$ of the fuel pellets is $\pm 0.320 \mathrm{~g} / \mathrm{cm}^{3}$. After performing the perturbation analysis, it was later determined that the measured variation in fuel mass for an individual pin is significantly smaller (about $25 \%$ ). ${ }^{\mathrm{a}}$ Therefore, the computed results for the variation in fuel pellet stack density (mass) are scaled to correctly reflect the measured values. Results are shown in Table 2.1.62.

The total number of fuel pins that were chemical assayed was 34 . For determining the random component of the uncertainty in Section 2.1.5, the results in Table 2.1.62 are divided by $\sqrt{34}$.

Table 2.1.62. Uncertainty in the Outer-Fuel-Zone Fuel Pellet Stack Density. ${ }^{(a)}$

\begin{tabular}{||c|ccc|c|ccc||}
\hline Deviation & $\Delta \mathrm{k}$ & \pm & $\sigma_{\Delta \mathrm{k}}$ & $\begin{array}{c}\text { Scaling } \\
\text { Factor }\end{array}$ & $\Delta \mathrm{k}_{\text {eff }}(1 \sigma)$ & \pm & $\sigma_{\Delta \mathrm{keff}}$ \\
\hline \hline$+0.320 \mathrm{~g} / \mathrm{cm}^{3}(3 \sigma)$ & 0.00825 & \pm & 0.00008 & 4.184 & 0.00197 & \pm & 0.00002 \\
$-0.320 \mathrm{~g} / \mathrm{cm}^{3}(3 \sigma)$ & -0.00845 & \pm & 0.00008 & 4.184 & -0.00202 & \pm & 0.00002 \\
\hline
\end{tabular}

(a) The random component of the uncertainty would be divided by $\sqrt{34}$.

${ }^{a}$ P. Goris, “Rod Scanner Assay of FFTF Fuel,” HEDL-TME 77-29 (January 1978).

Revision: 0 
NEA/NSC/DOC(2006)1

Liquid Metal Fast Reactor - LMFR

FFTF-LMFR-RESR-001

CRIT-SPEC-REAC-COEF-MISC

\section{Fuel Pellet Stack: Isotopic Distribution}

The uncertainty in the isotopic distribution of the fuel pellets (Figure 1.1.19) is reported to be within $0.3 \%$. This value is representative of a manufacturing tolerance. This uncertainty was evaluated by increasing or decreasing the ${ }^{239} \mathrm{Pu}$ content by $0.3 \%$. The content of the other plutonium isotopes were reduced or increased, respectively, such that the total content remained at $100 \%$; they were all adjusted equally. Results are shown in Table 2.1.63.

The uncertainty in the fuel pellet isotopic distribution is considered all systematic with no random component.

Table 2.1.63. Uncertainty in the Isotopic Distribution of the Outer-Fuel-Zone Fuel Pellets.

\begin{tabular}{||c|ccc|c|ccc||}
\hline \hline Deviation & $\Delta \mathrm{k}$ & \pm & $\sigma_{\Delta \mathrm{k}}$ & $\begin{array}{c}\text { Scaling } \\
\text { Factor }\end{array}$ & $\Delta \mathrm{k}_{\text {eff }}(1 \sigma)$ & \pm & $\sigma_{\Delta \mathrm{keff}}$ \\
\hline \hline$+0.3 \%{ }^{239} \mathrm{Pu}(3 \sigma)$ & 0.00042 & \pm & 0.00008 & 3 & 0.00014 & \pm & 0.00003 \\
$-0.3 \%{ }^{239} \mathrm{Pu}(3 \sigma)$ & -0.00040 & \pm & 0.00008 & 3 & -0.00013 & \pm & 0.00003 \\
\hline
\end{tabular}

Fuel Pellet Stack: Pu/(Pu+U) Ratio

The plutonium content of the fuel was determined using the analysis of the isotopic distribution of the fuel pellets. The uncertainty is also within $0.3 \%$, and represents a manufacturing tolerance. This uncertainty was evaluated by increasing or decreasing the total plutonium content by $0.3 \%$ while reducing or increasing, respectively, the total uranium content. Results are shown in Table 2.1.64.

The uncertainty in the fuel pellet $\mathrm{Pu} /(\mathrm{Pu}+\mathrm{U})$ ratio is considered all systematic with no random component.

Table 2.1.64. Uncertainty in the $\mathrm{Pu} /(\mathrm{Pu}+\mathrm{U})$ Ratio of the Outer-Fuel-Zone Fuel Pellets.

\begin{tabular}{||c|ccc|c|ccc||}
\hline \hline Deviation & $\Delta \mathrm{k}$ & \pm & $\sigma_{\Delta \mathrm{k}}$ & $\begin{array}{c}\text { Scaling } \\
\text { Factor }\end{array}$ & $\Delta \mathrm{k}_{\mathrm{eff}}(1 \sigma)$ & \pm & $\sigma_{\Delta \mathrm{keff}}$ \\
\hline \hline$+0.3 \% \mathrm{Pu}(3 \sigma)$ & 0.00202 & \pm & 0.00008 & 3 & 0.00067 & \pm & 0.00003 \\
$-0.3 \% \mathrm{Pu}(3 \sigma)$ & -0.00231 & \pm & 0.00007 & 3 & -0.00077 & \pm & 0.00002 \\
\hline
\end{tabular}

\section{Fuel Pellet Stack: Radioisotopic Decay}

The composition of the fuel reported in Figure 1.1.19 appears to represent measurements performed on January 1, 1976. The reported ${ }^{238} \mathrm{U}$ content was adjusted to account for ${ }^{234} \mathrm{U}$ that would be typically present in natural uranium. The actinides were then decayed from the apparent measurement date to the date of the critical experiment, March 8, 1980. The generation of ${ }^{237} \mathrm{~Np}$ was also included. The benchmark model includes the composition shown in Table 3.1.7.

The uncertainty in the actual composition of the material at the time of the experiment would depend upon the initial measured compositions and the uncertainty in the assay date. The material compositions of the fuel have been assessed via the other perturbation analyses in this section. Therefore the actual assay date will be investigated. It is unclear whether the assay was actually performed on 1/1/1976 or if only the year was recorded and the month and day were added by default by a computational database. 
NEA/NSC/DOC(2006)1

\section{Liquid Metal Fast Reactor - LMFR \\ FFTF-LMFR-RESR-001 \\ CRIT-SPEC-REAC-COEF-MISC}

Therefore, it is believed that the assay may have occurred somewhere in the year 1976 and the composition was varied by a decay time modified by \pm 1 year, which equates to an approximate change in the decay constant of approximately $\pm 24 \%$. The result is treated as a bounding uncertainty with uniform probability distribution. Results are shown in Table 2.1.65.

The uncertainty in the fuel pellet decay is considered all systematic with no random component.

Table 2.1.65. Uncertainty in the Radioisotopic Decay of the Outer-Fuel-Zone Fuel Pellets.

\begin{tabular}{||c|ccc|c|ccc||}
\hline \hline Deviation & $\Delta \mathrm{k}$ & \pm & $\sigma_{\Delta \mathrm{k}}$ & $\begin{array}{c}\text { Scaling } \\
\text { Factor }\end{array}$ & $\Delta \mathrm{k}_{\text {eff }}(1 \sigma)$ & \pm & $\sigma_{\Delta \mathrm{keff}}$ \\
\hline \hline+1 year & -0.00030 & \pm & 0.00008 & $\sqrt{ } 3$ & -0.00017 & \pm & 0.00005 \\
-1 year & 0.00012 & \pm & 0.00008 & $\sqrt{ } 3$ & 0.00007 & \pm & 0.00005 \\
\hline
\end{tabular}

\section{Fuel Pellet Stack: Impurities}

The impurity composition in the fuel pellets is provided in Table 1.1.6. Half of the maximum composition is used to represent the addition of impurities into the benchmark model (Table 2.1.54). This uncertainty is treated as a bounded limit with uniform probability distribution. The uncertainty was also evaluated using the maximum limit, which was then reduced in half. The larger uncertainty is chosen to represent the uncertainty in the impurity content. The results are shown in Table 2.1.66.

The uncertainty in the fuel pellet impurity content is considered all systematic with no random component.

Table 2.1.66. Uncertainty in Outer-Fuel-Zone Fuel Pellet Impurity Content.

\begin{tabular}{||c|ccc|c|ccc||}
\hline \hline Deviation & $\Delta \mathrm{k}$ & \pm & $\sigma_{\Delta \mathrm{k}}$ & $\begin{array}{c}\text { Scaling } \\
\text { Factor }\end{array}$ & $\Delta \mathrm{k}_{\mathrm{eff}}(1 \sigma)$ & \pm & $\sigma_{\Delta \mathrm{keff}}$ \\
\hline \hline Half Maximum Impurities & -0.00024 & \pm & 0.00008 & $\sqrt{ } 3$ & -0.00014 & \pm & 0.00005 \\
Maximum Impurities & -0.00066 & \pm & 0.00008 & $2 \sqrt{3}$ & -0.00019 & \pm & 0.00002 \\
\hline
\end{tabular}

\section{Fuel Pellet Stack: Oxygen-To-Uranium/Plutonium Ratio}

The tolerance in the nominal oxygen to uranium/plutonium ratio in the fuel pellets is between $+0.02 /-$ 0.03. This ratio was varied within this tolerance limit and the results are shown in Table 2.1.67.

The uncertainty in the fuel pellet O:(U,Pu)ratio is negligible.

Table 2.1.67. Uncertainty in O:(U,Pu) Ratio in the Outer-Fuel-Zone Fuel Pellets.

\begin{tabular}{||c|ccc|c|ccc||}
\hline Deviation & $\Delta \mathrm{k}$ & \pm & $\sigma_{\Delta \mathrm{k}}$ & $\begin{array}{c}\text { Scaling } \\
\text { Factor }\end{array}$ & $\Delta \mathrm{k}_{\text {eff }}(1 \sigma)$ & \pm & $\sigma_{\Delta \mathrm{keff}}$ \\
\hline \hline$+0.02 \mathrm{O}:(\mathrm{U}, \mathrm{Pu})(3 \sigma)$ & -0.00009 & \pm & 0.00007 & 3 & -0.00003 & \pm & 0.00002 \\
$-0.03 \mathrm{O}:(\mathrm{U}, \mathrm{Pu})(3 \sigma)$ & -0.00020 & \pm & 0.00007 & 3 & -0.00007 & \pm & 0.00002 \\
\hline
\end{tabular}


NEA/NSC/DOC(2006)1

Liquid Metal Fast Reactor - LMFR

FFTF-LMFR-RESR-001

CRIT-SPEC-REAC-COEF-MISC

\section{SS316 Wire Wrap: Diameter (Homogenized)}

The wire wraps are homogenized with the sodium coolant surrounding the fuel pins. The effective volume fraction of the homogenized medium is varied according to the equivalent mass of material for variation in the wire wrap diameter of $\pm 0.00127 \mathrm{~cm}$ from the nominal value of $0.14224 \mathrm{~cm}$. This value is a manufacturing tolerance. Results are shown in Table 2.1.68.

Although most, if not all, of the $39 \mathrm{~km}$ of wire wrap was developed during a single fabrication process, there would be variability in the diameter of the wire during the drawing process. The total number of fuel pin wire wraps in the outer enrichment zone is 9,982 . The uncertainty in the diameter of the stainless steel wire wrap is negligible.

Table 2.1.68. Uncertainty in the Outer-Fuel-Zone Wire Wrap Diameter. ${ }^{(a)}$

\begin{tabular}{||c|ccc|c|ccc||}
\hline Deviation & $\Delta \mathrm{k}$ & \pm & $\sigma_{\Delta \mathrm{k}}$ & $\begin{array}{c}\text { Scaling } \\
\text { Factor }\end{array}$ & $\Delta \mathrm{k}_{\text {eff }}(1 \sigma)$ & \pm & $\sigma_{\Delta \mathrm{keff}}$ \\
\hline \hline$+0.00127 \mathrm{~cm}(3 \sigma)$ & -0.00003 & \pm & 0.00008 & 3 & -0.00001 & \pm & 0.00003 \\
$-0.00127 \mathrm{~cm}(3 \sigma)$ & -0.00010 & \pm & 0.00008 & 3 & -0.00003 & \pm & 0.00003 \\
\hline
\end{tabular}

(a) The random component of the uncertainty would be divided by $\sqrt{9,982}$.

\section{Axial Shield, Pin Attachment, and Gas Plenum Regions}

These regions have been homogenized as described in the Section 3.1, based upon the 3-D Hex-Z model provided in Appendix F. There is insufficient publicly available data to analyze the material properties of the heterogeneous components in these regions. Variation in the material properties in this region of the core are expected to represent an insignificant contribution to the total uncertainty of the benchmark model, as the uncertainties in other geometrical properties (Section 2.1.2) and density of the primary structural components (SS316, Inconel 600, and sodium in Sections 2.1.3.1 through 2.1.3.3) are relatively insignificant compared to other constituents of the total uncertainty. Therefore the uncertainty in the composition of these regions is judged to be negligible.

\section{Fueled Open Test Assemblies}

Fueled open test assemblies are modeled identically as their respectively-zoned DFA assemblies; i.e., they do not include instrumentation. Insufficient information is available to assess a bias, if any, with its associated uncertainty. It is expected that neutronically the result would be negligible.

\subsubsection{Absorber Assemblies}

\section{All Control Rods}

When geometric and physical parameters were adjusted for the moveable control rods, they were also applied to the fixed shim control rods.

Absorber Pellet Stack: Density

From Table 1.1.4, the uncertainty in the density (92\%) of the absorber pellets is $\pm 2 \%$. This value is representative of a manufacturing tolerance. The theoretical density of $\mathrm{B}_{4} \mathrm{C}$ is $2.52 \mathrm{~g} / \mathrm{cm}^{3}$. 
NEA/NSC/DOC(2006)1

\section{Liquid Metal Fast Reactor - LMFR \\ FFTF-LMFR-RESR-001 \\ CRIT-SPEC-REAC-COEF-MISC}

An analysis of the as-built loading data for the nine control rods shows that the average density of the absorber pin stacks is $90.6 \pm 0.1 \%$ of the theoretical density for $\mathrm{B}_{4} \mathrm{C}$. Therefore, the assessed uncertainty of $\pm 2 \%$ was reduced by a factor of 20 . Results are shown in Table 2.1.69.

The total number of absorber assemblies utilized in the analysis of the average absorber density was 9 . The uncertainty in the density of the absorber pellets is negligible.

Table 2.1.69. Uncertainty in the Absorber Pellet Density.

\begin{tabular}{||c|ccc|c|ccc||}
\hline \hline Deviation & $\Delta \mathrm{k}$ & \pm & $\sigma_{\Delta \mathrm{k}}$ & $\begin{array}{c}\text { Scaling } \\
\text { Factor }\end{array}$ & $\Delta \mathrm{k}_{\text {eff }}(1 \sigma)$ & \pm & $\sigma_{\Delta \mathrm{keff}}$ \\
\hline \hline$+2 \% \mathrm{TD}(3 \sigma)$ & -0.00087 & \pm & 0.00008 & 20 & -0.00004 & \pm & 0.000000 \\
$-2 \% \mathrm{TD}(3 \sigma)$ & 0.00066 & \pm & 0.00008 & 20 & 0.00003 & \pm & 0.00000 \\
\hline
\end{tabular}

\section{Absorber Pellet Stack: ${ }^{10}$ B Mass Loading}

The absorber loading in the $\mathrm{B}_{4} \mathrm{C}$ pellets has a standard deviation of $\sim 0.173 \%$ (Table 1.1 .11 ) but is reported to be accurate within $0.5 \%$ (Section 1.1.3.1). The latter value is assumed to be bounding, and the $\mathrm{B}: \mathrm{C}$ ratio is varied while maintaining the isotopic abundance of ${ }^{10} \mathrm{~B}$ constant. Results are shown in Table 2.1.70.

The uncertainty in the absorber pellet ${ }^{10} \mathrm{~B}$ loading is considered all systematic with no random component.

Table 2.1.70. Uncertainty in the ${ }^{10} \mathrm{~B}$ Loading of the Absorber Pellets.

\begin{tabular}{||c|ccc|c|ccc||}
\hline \hline Deviation & $\Delta \mathrm{k}$ & \pm & $\sigma_{\Delta \mathrm{k}}$ & $\begin{array}{c}\text { Scaling } \\
\text { Factor }\end{array}$ & $\Delta \mathrm{k}_{\text {eff }}(1 \sigma)$ & \pm & $\sigma_{\Delta \mathrm{keff}}$ \\
\hline \hline$+0.5 \%(3 \sigma)$ & -0.00136 & \pm & 0.00008 & 3 & -0.00045 & \pm & 0.00003 \\
$-0.5 \%(3 \sigma)$ & 0.00141 & \pm & 0.00008 & 3 & 0.00047 & \pm & 0.00003 \\
\hline
\end{tabular}

\section{Absorber Pellet Stack: ${ }^{10}$ B Isotopic Abundance}

The ${ }^{10} \mathrm{~B}$ content in natural boron is nominally $19.9 \%$ but varies between 19.1 and $20.3 \%$. The isotopic abundance of ${ }^{10} \mathrm{~B}$ in the absorber pellets was varied while maintaining the total boron content constant. The results are shown in Table 2.1.71.

The uncertainty in the absorber pellet ${ }^{10} \mathrm{~B}$ isotopic abundance is negligible.

Table 2.1.71. Uncertainty in the Isotopic Abundance of Boron.

\begin{tabular}{||c|ccc|c|ccc||}
\hline \hline Deviation & $\Delta \mathrm{k}$ & \pm & $\sigma_{\Delta \mathrm{k}}$ & $\begin{array}{c}\text { Scaling } \\
\text { Factor }\end{array}$ & $\Delta \mathrm{k}_{\text {eff }}(1 \sigma)$ & \pm & $\sigma_{\Delta \mathrm{keff}}$ \\
\hline \hline $20.3 \%{ }^{10} \mathrm{~B}(3 \sigma)$ & -0.00019 & \pm & 0.00008 & 3 & -0.00006 & \pm & 0.00003 \\
$19.1 \%{ }^{10} \mathrm{~B}(3 \sigma)$ & -0.00010 & \pm & 0.00008 & 3 & -0.00003 & \pm & 0.00003 \\
\hline
\end{tabular}


NEA/NSC/DOC(2006)1

Liquid Metal Fast Reactor - LMFR

FFTF-LMFR-RESR-001

CRIT-SPEC-REAC-COEF-MISC

\section{Absorber Pellet Stack: Impurities}

The impurity composition in the absorber pellets is provided in Table 1.1.10. Half of the maximum lot average composition is used to represent the addition of impurities into the benchmark model (Table 2.1.72). This uncertainty is treated as a bounded limit with uniform probability distribution. The uncertainty was also evaluated using the maximum limit, which was then reduced in half. The larger uncertainty is chosen to represent the uncertainty in the impurity content. The results are shown in Table 2.1.73.

The uncertainty in the absorber pellet impurity content is negligible.

Table 2.1.72. Absorber Pellet Impurity Content.

\begin{tabular}{|cc|}
\hline Impurity & $\begin{array}{c}\text { Average Content } \\
\mu \mathrm{g} / \mathrm{g}\end{array}$ \\
\hline $\mathrm{Al}$ & 500 \\
$\mathrm{Ca}$ & 500 \\
$\mathrm{Cr}$ & 150 \\
$\mathrm{Fe}$ & 1500 \\
$\mathrm{Mg}$ & 250 \\
$\mathrm{Mn}$ & 100 \\
$\mathrm{~N}$ & 2000 \\
$\mathrm{Ni}$ & 150 \\
$\mathrm{Si}$ & 1000 \\
$\mathrm{Ti}$ & 150 \\
$\mathrm{Cl}$ & 12.5 \\
$\mathrm{~F}$ & 7.5 \\
\hline
\end{tabular}

Table 2.1.73. Uncertainty in the Absorber Pellet Impurity Content.

\begin{tabular}{||c|ccc|c|ccc||}
\hline Deviation & $\Delta \mathrm{k}$ & \pm & $\sigma_{\Delta \mathrm{k}}$ & $\begin{array}{c}\text { Scaling } \\
\text { Factor }\end{array}$ & $\Delta \mathrm{k}_{\text {eff }}(1 \sigma)$ & \pm & $\sigma_{\Delta \mathrm{keff}}$ \\
\hline \hline Half Maximum Impurities & 0.00008 & \pm & 0.00007 & $\sqrt{ } 3$ & 0.00005 & \pm & 0.00004 \\
Maximum Impurities & 0.00002 & \pm & 0.00008 & $2 \sqrt{ } 3$ & 0.00001 & \pm & 0.00002 \\
\hline
\end{tabular}

\section{SS316 Wire Wrap: Diameter (Homogenized)}

The wire wraps are homogenized with the sodium coolant surrounding the fuel pins. The effective volume fraction of the homogenized medium is varied according to the equivalent mass of material for variation in the wire wrap diameter of $\pm 0.00127 \mathrm{~cm}$ from the nominal value of $0.06096 \mathrm{~cm}$. This value is a manufacturing tolerance. Results are shown in Table 2.1.74.

Although most, if not all, of the wire wrap was developed during a single fabrication process, there would be variability in the diameter of the wire during the drawing process. The total number of 
NEA/NSC/DOC(2006)1

Liquid Metal Fast Reactor - LMFR

FFTF-LMFR-RESR-001

CRIT-SPEC-REAC-COEF-MISC

absorber pin wire wraps is 732 . The uncertainty in the diameter of the stainless steel wire wrap is negligible.

Table 2.1.74. Uncertainty in the Absorber Wire Wrap Diameter. ${ }^{(a)}$

\begin{tabular}{||c|ccc|c|ccc||}
\hline Deviation & $\Delta \mathrm{k}$ & \pm & $\sigma_{\Delta \mathrm{k}}$ & $\begin{array}{c}\text { Scaling } \\
\text { Factor }\end{array}$ & $\Delta \mathrm{k}_{\mathrm{eff}}(1 \sigma)$ & \pm & $\sigma_{\Delta \mathrm{keff}}$ \\
\hline \hline$+0.00127 \mathrm{~cm}(3 \sigma)$ & -0.00018 & \pm & 0.00008 & 3 & -0.00006 & \pm & 0.00003 \\
$-0.00127 \mathrm{~cm}(3 \sigma)$ & -0.00010 & \pm & 0.00008 & 3 & -0.00003 & \pm & 0.00003 \\
\hline \hline
\end{tabular}

(a) The random component of the uncertainty would be divided by $\sqrt{732}$.

\section{Lower Shield, Below Poison, Above Poison, Driveline, and Withdrawn Absorber Regions}

These regions have been homogenized as described in the Section 3.1, based upon the 3-D Hex-Z model provided in Appendix F. There is insufficient publicly available data to analyze the material properties of the heterogeneous components in these regions. Variation in the material properties in this region of the core are expected to represent an insignificant contribution to the total uncertainty of the benchmark model, as the uncertainties in other geometrical properties (Section 2.1.2) and density of the primary structural components (SS316, Inconel 600, and sodium in Sections 2.1.3.1 through 2.1.3.3) are relatively insignificant compared to other constituents of the total uncertainty. Therefore the uncertainty in the composition of these regions is judged to be negligible.

\section{Fixed Shim Control Rods}

Fixed shim control rods are modeled identical to fully inserted control rods. Insufficient information is available to assess a bias, if any, with its associated uncertainty. It is expected that neutronically the result would be negligible. The only significant difference between the fixed shim control rods and the primary and secondary control rods is that the fixed shim rod cannot be withdrawn, and a wire wrap is placed between the inner and outer ducts. When geometric and physical parameters were adjusted for the moveable control rods, they were also applied to the fixed shim control rods.

\section{Inter-Duct SS316 Wire Wrap}

There is insufficient data to analyze the effective uncertainty in the diameter of the SS316 wire wrap placed between the inner and outer ducts of the fixed shim control rod assemblies. An approximation is performed by replacing the material between the inner and outer assembly ducts with the homogenized wire wrap material around the absorber pins. This substitution was applied to all 12 control assemblies (although the safety and secondary control rods do not have an inter-duct wire wrap). This uncertainty is assumed to be bounding and uniformly distributed as the actual wire wrap content should be significantly less. The results are shown in Table 2.1.75.

The total number of absorber assemblies is 12 . The uncertainty in the stainless steel inter-duct wire wrap is negligible.

Table 2.1.75. Uncertainty in the Absorber Assembly Inter-Duct Wire Wrap.

\begin{tabular}{||c|ccc|c|ccc||}
\hline \hline Deviation & $\Delta \mathrm{k}$ & \pm & $\sigma_{\Delta \mathrm{k}}$ & $\begin{array}{c}\text { Scaling } \\
\text { Factor }\end{array}$ & $\Delta \mathrm{k}_{\text {eff }}(1 \sigma)$ & \pm & $\sigma_{\Delta \mathrm{keff}}$ \\
\hline \hline Add Wire Wrap Material & 0.00002 & \pm & 0.00007 & $\sqrt{ } 3$ & 0.00001 & \pm & 0.00004 \\
\hline
\end{tabular}

Revision: 0

Page 115 of 304

Date: March 31, 2010 
NEA/NSC/DOC(2006)1

Liquid Metal Fast Reactor - LMFR

FFTF-LMFR-RESR-001

CRIT-SPEC-REAC-COEF-MISC

\subsubsection{Radial Reflectors}

\section{Row 7}

Orifice, Lower Adapter, Reflector Blocks, Load Pad, and Upper Shielding Regions

These regions have been homogenized as described in the Section 3.1, based upon the 3-D Hex-Z model provided in Appendix F. There is insufficient publicly available data to analyze the material properties of the heterogeneous components in these regions. Variation in the material properties in this region of the core are expected to represent an insignificant contribution to the total uncertainty of the benchmark model, as the uncertainties in other geometrical properties (Section 2.1.2) and density of the primary structural components (SS316, Inconel 600, and sodium in Sections 2.1.3.1 through 2.1.3.3) are relatively insignificant compared to other constituents of the total uncertainty. Therefore the uncertainty in the composition of these regions is judged to be negligible.

\section{Rows 8 and 9}

Orifice, Lower Adapter, Reflector Blocks, Load Pad, and Upper Shielding Regions

These regions have been homogenized as described in the Section 3.1, based upon the 3-D Hex-Z model provided in Appendix F. There is insufficient publicly available data to analyze the material properties of the heterogeneous components in these regions. Variation in the material properties in this region of the core are expected to represent an insignificant contribution to the total uncertainty of the benchmark model, as the uncertainties in other geometrical properties (Section 2.1.2) and density of the primary structural components (SS316, Inconel 600, and sodium in Sections 2.1.3.1 through 2.1.3.3) are relatively insignificant compared to other constituents of the total uncertainty. Therefore the uncertainty in the composition of these regions is judged to be negligible.

\subsubsection{Vibration Open Test Assembly}

\section{Orifice/Shield and Instruments/Housing Regions}

These regions have been homogenized as described in the Section 3.1, based upon the 3-D Hex-Z model provided in Appendix F. There is insufficient publicly available data to analyze the material properties of the heterogeneous components in these regions. Variation in the material properties in this region of the core are expected to represent an insignificant contribution to the total uncertainty of the benchmark model, as the uncertainties in other geometrical properties (Section 2.1.2) and density of the primary structural components (SS316, Inconel 600, and sodium in Sections 2.1.3.1 through 2.1.3.3) are relatively insignificant compared to other constituents of the total uncertainty. Therefore the uncertainty in the composition of these regions is judged to be negligible.

\subsubsection{In-Core Shim Assemblies}

\section{Orifice/Shield, Pin Attachment, and Simulated Fuel Bundle Regions}

These regions have been homogenized as described in the Section 3.1, based upon the 3-D Hex-Z model provided in Appendix F. There is insufficient publicly available data to analyze the material properties of the heterogeneous components in these regions. Variation in the material properties in this region of the core are expected to represent an insignificant contribution to the total uncertainty of the benchmark model, as the uncertainties in other geometrical properties (Section 2.1.2) and density of the primary structural components (SS316, Inconel 600, and sodium in Sections 2.1.3.1 through 2.1.3.3) are relatively insignificant compared to other constituents of the total uncertainty. Therefore the uncertainty in the composition of these regions is judged to be negligible. 
NEA/NSC/DOC(2006)1

Liquid Metal Fast Reactor - LMFR

FFTF-LMFR-RESR-001

CRIT-SPEC-REAC-COEF-MISC

\subsubsection{Simulated Core Assembly}

The fully-loaded core configuration of the FFTF does not contain simulated core assemblies.

\subsubsection{In-Reactor Thimble}

The fully-loaded core critical configuration contains the in-reactor thimble but without any inserts.

The in-reactor thimble has been homogenized as described in the Section 3.1, based upon the 3-D Hex-Z model provided in Appendix F. There is insufficient publicly available data to analyze the material properties of the heterogeneous components in the in-reactor thimble region. Variation in the material properties in this region of the core are expected to represent an insignificant contribution to the total uncertainty of the benchmark model, as the uncertainties in other geometrical properties (Section 2.1.2) and density of the primary structural components (SS316, Inconel 600, and sodium in Sections 2.1.3.1 through 2.1.3.3) are relatively insignificant compared to other constituents of the total uncertainty. Therefore the uncertainty in the composition of the in-reactor thimble is judged to be negligible.

\subsubsection{Reactor Shielding}

Inner and Outer Radial Reactor Shielding Regions

These regions have been homogenized as described in the Section 3.1, based upon the 3-D Hex-Z model provided in Appendix F. There is insufficient publicly available data to analyze the material properties of the heterogeneous components in these regions. Variation in the material properties in this region of the core are expected to represent an insignificant contribution to the total uncertainty of the benchmark model, as the uncertainties in other geometrical properties (Section 2.1.2) and density of the primary structural components (SS316, Inconel 600, and sodium in Sections 2.1.3.1 through 2.1.3.3) are relatively insignificant compared to other constituents of the total uncertainty. Therefore the uncertainty in the composition of these regions is judged to be negligible.

\subsubsection{Systematic Biases and Uncertainties}

There were no systematic biases reported for these experiments. A systematic uncertainty of $10 \%$ is assumed for all uncertainties with a random uncertainty component due to the number of objects being perturbed. Some uncertainties were assumed to be $100 \%$ systematic because an appropriate means of accounting for the random component, if any, was not available.

\subsubsection{Total Experimental Uncertainty}

A compilation of the total evaluated uncertainty in the FFTF fully-loaded critical configuration is shown in Table 2.1.76. The root-mean-square of all the uncertainties is used to obtain the total uncertainty in the benchmark eigenvalue. When there is a difference between the positive and negative perturbations of a parameter, the larger uncertainty value is included in the total uncertainty of the benchmark. Uncertainties less than or equal to 0.0001 are treated as negligible (neg). When calculated uncertainties in $\Delta \mathrm{k}_{\mathrm{eff}}$ are less than their statistical uncertainties, the statistical uncertainties are used in the calculation of the total uncertainty, unless the absolute magnitude of the uncertainty is less than 0.0001. Table listings where calculations were not performed or otherwise not applicable are labeled with 'NA'. For uncertainties where a random component is not applicable, the uncertainty is denoted with '--'.

The uncertainty in possible room-return effects is analyzed in Section 3.1.1.1 but is considered negligible.

The experimental $\mathrm{k}_{\mathrm{eff}}$ is $1.0000 \pm 0.0021$. 


\section{Liquid Metal Fast Reactor - LMFR \\ FFTF-LMFR-RESR-001 \\ CRIT-SPEC-REAC-COEF-MISC}

The most significant contributions to the overall uncertainty from the systematic uncertainties include the fuel pellet $\mathrm{Pu} /(\mathrm{Pu}+\mathrm{U})$ ratios, the stainless steel composition, and the reactor core assembly pitch. All uncertainties providing at least $\sim 0.05 \% \Delta \mathrm{k}_{\text {eff }}$ or more are highlighted in gray in Table 2.1.76. All of the random uncertainties are $\sim 0.05 \% \Delta \mathrm{k}_{\text {eff. }}$. 
NEA/NSC/DOC(2006)1

Liquid Metal Fast Reactor - LMFR

FFTF-LMFR-RESR-001

CRIT-SPEC-REAC-COEF-MISC

Table 2.1.76. Total Experimental Uncertainty of the Fully-Loaded FFTF Core Critical.

\begin{tabular}{|c|c|c|c|c|}
\hline Varied Parameter & $\begin{array}{c}\text { Evaluated } \\
\text { Uncertainty } \\
\pm \Delta \mathrm{k}_{\text {eff }}(1 \sigma) \\
\end{array}$ & $\begin{array}{l}\text { Systematic } \\
\text { Uncertainty } \\
\pm \Delta \mathrm{k}_{\mathrm{eff}}(1 \sigma) \\
\end{array}$ & $\begin{array}{c}\text { Number of } \\
\text { Objects } \\
\mathrm{N} \\
\end{array}$ & $\begin{array}{c}\text { Random } \\
\text { Uncertainty } \\
\pm \Delta \mathrm{k}_{\text {eff }}(1 \sigma) \\
\end{array}$ \\
\hline Temperature & neg & neg & -- & -- \\
\hline Safety Rod Positions & neg & neg & 3 & neg \\
\hline Secondary Rod Positions & 0.00011 & neg & 5 & neg \\
\hline Critical (Rod 4) Position & neg & neg & -- & -- \\
\hline Fixed Shim Rod Positions & NA & NA & NA & NA \\
\hline Measured Value of $k_{\text {eff }}$ & NA & $\mathrm{NA}$ & NA & NA \\
\hline Inner Axial Reflector Height & neg & neg & 11718 & neg \\
\hline Inner Axial Reflector Diameter & neg & neg & 11718 & neg \\
\hline Inner Insulator Pellet Height & neg & neg & 23436 & neg \\
\hline Inner Insulator Pellet Diameter & neg & neg & 23436 & neg \\
\hline Inner Fuel Pellet Stack Height & 0.00011 & neg & 5859 & neg \\
\hline Inner Fuel Pellet Diameter & 0.00115 & 0.00011 & 843696 & neg \\
\hline Inner Cladding Inner Diameter & neg & neg & 5859 & neg \\
\hline Inner Cladding Outer Diameter & neg & neg & 5859 & neg \\
\hline Inner Wire Wrap Diameter & \multicolumn{4}{|c|}{ Discussed in Section 2.1.3.6 } \\
\hline Inner Fuel Pin Lattice Pitch & neg & neg & 5859 & neg \\
\hline Inner Assembly Duct Width & 0.00020 & neg & 27 & neg \\
\hline Inner Axial Shield Region & NA & NA & NA & NA \\
\hline Inner Pin Attachment Region & NA & NA & NA & NA \\
\hline Inner Gas Plenum Region & NA & NA & NA & NA \\
\hline Outer Axial Reflector Height & neg & neg & 19964 & neg \\
\hline Outer Axial Reflector Diameter & neg & neg & 19964 & neg \\
\hline Outer Insulator Pellet Height & neg & neg & 39928 & neg \\
\hline Outer Insulator Pellet Diameter & neg & neg & 39928 & neg \\
\hline Outer Fuel Pellet Stack Height & 0.00014 & neg & 9982 & neg \\
\hline Outer Fuel Pellet Diameter & 0.00136 & 0.00014 & 1437408 & neg \\
\hline Outer Cladding Inner Diameter & neg & neg & 9982 & neg \\
\hline Outer Cladding Outer Diameter & neg & neg & 9982 & neg \\
\hline Outer Wire Wrap Diameter & \multicolumn{4}{|c|}{ Discussed in Section 2.1.3.6 } \\
\hline Outer Fuel Pin Lattice Pitch & neg & neg & 9982 & neg \\
\hline Outer Assembly Duct Width & neg & neg & 46 & neg \\
\hline Outer Axial Shield Region & NA & NA & NA & NA \\
\hline Outer Pin Attachment Region & NA & NA & NA & NA \\
\hline Outer Gas Plenum Region & NA & $\mathrm{NA}$ & $\mathrm{NA}$ & $\mathrm{NA}$ \\
\hline
\end{tabular}


NEA/NSC/DOC(2006)1

Liquid Metal Fast Reactor - LMFR

FFTF-LMFR-RESR-001

CRIT-SPEC-REAC-COEF-MISC

Table 2.1.76 (cont'd). Total Experimental Uncertainty of the Fully-Loaded FFTF Core Critical.

\begin{tabular}{|c|c|c|c|c|}
\hline Varied Parameter & $\begin{array}{c}\text { Evaluated } \\
\text { Uncertainty } \\
\pm \Delta \mathrm{k}_{\text {eff }}(1 \sigma) \\
\end{array}$ & $\begin{array}{l}\text { Systematic } \\
\text { Uncertainty } \\
\pm \Delta \mathrm{k}_{\text {eff }}(1 \sigma) \\
\end{array}$ & $\begin{array}{c}\text { Number of } \\
\text { Objects } \\
\mathrm{N} \\
\end{array}$ & $\begin{array}{c}\text { Random } \\
\text { Uncertainty } \\
\pm \Delta \mathrm{k}_{\mathrm{eff}}(1 \sigma) \\
\end{array}$ \\
\hline Absorber Pellet Stack Height & neg & neg & 732 & neg \\
\hline Absorber Pellet Diameter & neg & neg & 43920 & neg \\
\hline Absorber Clad Inner Diameter & neg & neg & 732 & neg \\
\hline Absorber Clad Outer Diameter & neg & neg & 732 & neg \\
\hline Absorber Wire Wrap Diameter & \multicolumn{4}{|c|}{ Discussed in Section 2.1.3.7 } \\
\hline Absorber Pin Lattice Pitch & neg & neg & 732 & neg \\
\hline Absorber Inner Duct Width & neg & neg & 12 & neg \\
\hline Absorber Outer Duct Width & neg & neg & 12 & neg \\
\hline Absorber Lower Shield Region & NA & NA & NA & NA \\
\hline Absorber Below Poison Region & NA & NA & NA & NA \\
\hline Absorber Above Poison Region & NA & NA & NA & NA \\
\hline Absorber Driveline Region & NA & NA & NA & NA \\
\hline Withdrawn Absorber Region & NA & NA & NA & NA \\
\hline Fixed Shim Wire Wrap Diameter & \multicolumn{4}{|c|}{ Discussed in Section 2.1.3.7 } \\
\hline Row 7 Orifice Region & NA & NA & NA & NA \\
\hline Row 7 Lower Adapter Region & NA & NA & NA & NA \\
\hline Row 7 Reflector Blocks Region & NA & NA & NA & NA \\
\hline Row 7 Load Pad Region & NA & NA & NA & NA \\
\hline Row 7 Upper Shield Region & NA & NA & NA & NA \\
\hline Rows $8+9$ Orifice Region & NA & NA & NA & NA \\
\hline Rows 8+9 Lower Adapter Region & NA & NA & NA & NA \\
\hline Rows $8+9$ Reflector Blocks Region & NA & NA & NA & NA \\
\hline Rows 8+9 Load Pad Region & NA & NA & NA & NA \\
\hline Rows $8+9$ Upper Shield Region & NA & NA & NA & NA \\
\hline VOTA Orifice/Shield Region & NA & NA & NA & NA \\
\hline VOTA Instruments/Housing Region & NA & NA & NA & NA \\
\hline ICSA Orifice/Shield Region & NA & NA & NA & NA \\
\hline ICSA Pin Attachment Region & NA & NA & NA & NA \\
\hline ICSA Simulated Fuel Region & NA & NA & NA & NA \\
\hline In-Reactor Thimble & NA & NA & NA & NA \\
\hline Inner Radial Reactor Shielding & NA & NA & NA & NA \\
\hline Outer Radial Reactor Shielding & NA & NA & NA & NA \\
\hline Radial Reactor Shielding Placement & 0.00053 & 0.00053 & -- & -- \\
\hline Reactor Core Assembly Pitch & 0.00113 & 0.00113 & -- & -- \\
\hline
\end{tabular}


NEA/NSC/DOC(2006)1

Liquid Metal Fast Reactor - LMFR

FFTF-LMFR-RESR-001

CRIT-SPEC-REAC-COEF-MISC

Table 2.1.76 (cont'd). Total Experimental Uncertainty of the Fully-Loaded FFTF Core Critical.

\begin{tabular}{|c|c|c|c|c|}
\hline Varied Parameter & $\begin{array}{c}\text { Evaluated } \\
\text { Uncertainty } \\
\pm \Delta \mathrm{k}_{\mathrm{eff}}(1 \sigma) \\
\end{array}$ & $\begin{array}{l}\text { Systematic } \\
\text { Uncertainty } \\
\pm \Delta \mathrm{k}_{\text {eff }}(1 \sigma) \\
\end{array}$ & $\begin{array}{c}\text { Number of } \\
\text { Objects } \\
\mathrm{N} \\
\end{array}$ & $\begin{array}{c}\text { Random } \\
\text { Uncertainty } \\
\pm \Delta \mathrm{k}_{\text {eff }}(1 \sigma) \\
\end{array}$ \\
\hline SS316 Composition & 0.00078 & 0.00078 & -- & -- \\
\hline SS316 Density & neg & neg & -- & -- \\
\hline Inconel 600 Composition & 0.00021 & 0.00021 & -- & -- \\
\hline Inconel 600 Density & neg & neg & -- & -- \\
\hline Sodium Coolant Density & 0.00020 & 0.00020 & -- & -- \\
\hline Sodium Coolant Impurities & neg & neg & -- & -- \\
\hline Helium-Filled Gaps & neg & neg & -- & -- \\
\hline Moisture in Pellets & neg & neg & -- & -- \\
\hline Inner Insulator Pellet Density & neg & neg & 23436 & neg \\
\hline Inner Insulator Pellet Isotopics & \multicolumn{4}{|c|}{ Does Not Apply to Initial FFTF Core } \\
\hline Inner Insulator Pellet Impurities & neg & neg & -- & -- \\
\hline Inner Insulator Pellet O:U Ratio & neg & neg & -- & -- \\
\hline Inner Fuel Pellet Density & 0.00170 & 0.00017 & 15 & 0.00039 \\
\hline Inner Fuel Pellet Isotopics & 0.00014 & 0.00014 & -- & -- \\
\hline Inner Fuel Pellet $\mathrm{Pu} /(\mathrm{Pu}+\mathrm{U})$ Ratio & 0.00090 & 0.00090 & -- & -- \\
\hline Inner Fuel Pellet Decay & 0.00020 & 0.00020 & -- & -- \\
\hline Inner Fuel Pellet Impurities & 0.00023 & 0.00023 & -- & -- \\
\hline Inner Fuel Pellet O:(U,Pu) Ratio & neg & neg & -- & -- \\
\hline Inner Homogenized Wire Wrap & neg & neg & 5859 & neg \\
\hline Inner Axial Shield Region & NA & NA & NA & NA \\
\hline Inner Pin Attachment Region & NA & NA & NA & NA \\
\hline Inner Gas Plenum Region & NA & NA & NA & NA \\
\hline Outer Insulator Pellet Density & neg & neg & 39928 & neg \\
\hline Outer Insulator Pellet Isotopics & \multicolumn{4}{|c|}{ Does Not Apply to Initial FFTF Core } \\
\hline Outer Insulator Pellet Impurities & neg & neg & -- & -- \\
\hline Outer Insulator Pellet O:U Ratio & neg & neg & -- & -- \\
\hline Outer Fuel Pellet Density & 0.00202 & 0.00020 & 34 & 0.00031 \\
\hline Outer Fuel Pellet Isotopics & 0.00014 & 0.00014 & -- & -- \\
\hline Outer Fuel Pellet $\mathrm{Pu} /(\mathrm{Pu}+\mathrm{U})$ Ratio & 0.00077 & 0.00077 & -- & -- \\
\hline Outer Fuel Pellet Decay & 0.00017 & 0.00017 & -- & -- \\
\hline Outer Fuel Pellet Impurities & 0.00019 & 0.00019 & -- & -- \\
\hline Outer Fuel Pellet O:(U,Pu) Ratio & neg & neg & -- & -- \\
\hline Outer Homogenized Wire Wrap & neg & neg & 9982 & neg \\
\hline Outer Axial Shield Region & NA & NA & NA & NA \\
\hline
\end{tabular}


NEA/NSC/DOC(2006)1

Liquid Metal Fast Reactor - LMFR

FFTF-LMFR-RESR-001

CRIT-SPEC-REAC-COEF-MISC

Table 2.1.76 (cont'd). Total Experimental Uncertainty of the Fully-Loaded FFTF Core Critical.

\begin{tabular}{|c|c|c|c|c|}
\hline Varied Parameter & $\begin{array}{c}\text { Evaluated } \\
\text { Uncertainty } \\
\pm \Delta \mathrm{k}_{\text {eff }}(1 \sigma) \\
\end{array}$ & $\begin{array}{l}\text { Systematic } \\
\text { Uncertainty } \\
\pm \Delta \mathrm{k}_{\mathrm{eff}}(1 \sigma) \\
\end{array}$ & $\begin{array}{c}\text { Number of } \\
\text { Objects } \\
\mathrm{N}\end{array}$ & $\begin{array}{c}\text { Random } \\
\text { Uncertainty } \\
\pm \Delta \mathrm{k}_{\text {eff }}(1 \sigma) \\
\end{array}$ \\
\hline Outer Pin Attachment Region & NA & NA & NA & NA \\
\hline Outer Gas Plenum Region & NA & NA & NA & NA \\
\hline Absorber Pellet Density & neg & neg & 9 & neg \\
\hline Absorber Pellet ${ }^{10} \mathrm{~B}$ Mass Loading & 0.00047 & 0.00047 & -- & -- \\
\hline Absorber Pellet ${ }^{10} \mathrm{~B}$ Abundance & neg & neg & -- & -- \\
\hline Absorber Pellet Impurities & neg & neg & -- & -- \\
\hline Absorber Homogenized Wire Wrap & neg & neg & 732 & neg \\
\hline Absorber Lower Shield Region & NA & NA & NA & NA \\
\hline Absorber Below Poison Region & NA & NA & NA & NA \\
\hline Absorber Above Poison Region & NA & NA & NA & NA \\
\hline Absorber Driveline Region & NA & NA & NA & NA \\
\hline Withdrawn Absorber Region & NA & NA & NA & NA \\
\hline Fixed Shim Inter-Duct Wire Wrap & neg & neg & 12 & neg \\
\hline Row 7 Orifice Region & NA & NA & NA & NA \\
\hline Row 7 Lower Adapter Region & NA & NA & NA & NA \\
\hline Row 7 Reflector Blocks Region & NA & NA & NA & NA \\
\hline Row 7 Load Pad Region & NA & NA & NA & NA \\
\hline Row 7 Upper Shield Region & NA & NA & NA & NA \\
\hline Rows 8+9 Orifice Region & NA & NA & NA & NA \\
\hline Rows 8+9 Lower Adapter Region & NA & NA & NA & NA \\
\hline Rows 8+9 Reflector Blocks Region & NA & NA & NA & NA \\
\hline Rows 8+9 Load Pad Region & NA & NA & NA & NA \\
\hline Rows 8+9 Upper Shield Region & NA & NA & NA & NA \\
\hline VOTA Orifice/Shield Region & NA & NA & NA & NA \\
\hline VOTA Instruments/Housing Region & NA & NA & NA & NA \\
\hline ICSA Orifice/Shield Region & NA & NA & NA & NA \\
\hline ICSA Pin Attachment Region & NA & NA & NA & NA \\
\hline ICSA Simulated Fuel Region & NA & NA & NA & NA \\
\hline In-Reactor Thimble & NA & NA & NA & NA \\
\hline Inner Radial Reactor Shielding & NA & NA & NA & NA \\
\hline Outer Radial Reactor Shielding & NA & NA & NA & NA \\
\hline Room Return & neg & neg & -- & -- \\
\hline Uncertainty of Components & -- & 0.00205 & - & 0.00050 \\
\hline Evaluation Uncertainty & 0.00211 & & & \\
\hline
\end{tabular}


NEA/NSC/DOC(2006)1

Liquid Metal Fast Reactor - LMFR

FFTF-LMFR-RESR-001

CRIT-SPEC-REAC-COEF-MISC

\subsection{Evaluation of Buckling and Extrapolation Length Data}

Buckling and extrapolation length measurements were not made.

\subsection{Evaluation of Spectral Characteristics Data}

The reactor physics experiments evaluated in this section pertain to two neutron spectra measurements performed in the IRT near the axial center of the core at core midplane and $80 \mathrm{~cm}$ below core midplane using proportional counter detectors. There is insufficient data currently available to evaluate results from neutron spectra measurements performed with nuclear emulsions.

\subsubsection{Neutron Spectrum near Center of Fully-Loaded Core (Proportional Counters)}

The data portrayed in Figures 1.3.6 and 1.3.7 are derived from the data in Tables 1.3.5 and 1.3.6, respectively. The energy bins are spaced by a lethargy value of approximately 0.0488 . The relative flux values in the figures represent the point flux at a given lethargy energy divided by the maximum flux, which occurs at the energy of $117.971 \mathrm{keV}$ for both sets of data, and multiplied by the value 8.8. It is assumed that the scaling factor was used to spread the chart out such that it would fit onto a full sheet of computer printout paper; no further explanation is provided. The uncertainty in the neutron flux data reported in the tables can be generally approximated by Figure 1.3.8. However, it is assumed that the error values reported in the tables represent the true measurement uncertainty. The uncertainty in the neutron spectra ranges from 1.0 to $26.7 \%$ with an average of approximately $3.7 \%$. Additional uncertainty was not assessed beyond the measurement uncertainty of the neutron flux values.

A model of the FFTF with a homogenized IRT was developed using the data provided in Sections 1.1 and 1.3. Explicit modeling of the proportional counters was not performed as the exact placement within the IRT was originally unknown. The placement of the detectors in the IRT was found to be typically $\pm 1.5 \mathrm{~cm}$, from other experiments performed in the FFTF. ${ }^{a}$ Flux measurements were performed across a 2 inch $(5.08 \mathrm{~cm})$ high by 4 inch $(10.16 \mathrm{~cm})$ diameter space within the IRT, centered at the midplane position and also $80 \mathrm{~cm}$ below the core midplane. Control rod positions for the fully-loaded critical core configuration were maintained as information regarding rod movements was unavailable; as there were negligible burnup and temperature effects, this assumption is valid. It is assumed that the effect upon the neutron flux spectra would be insignificant.

The calculated neutron spectra are obtained by taking the variance-weighted average of results obtained using six variations of the input deck (Appendix A.1) with different random number seeds and tallies of the neutron flux (Appendix A.3). This approach was used to reduce the statistical uncertainty in the neutron flux tallies because the relative error values obtained can under-predict the true uncertainty in the calculated spectra. ${ }^{\mathrm{b}}$ The initial data point $(\mathrm{E}=1.145 \mathrm{keV}$ and $3.038 \mathrm{keV}$ for the midplane and belowmidplane measurements, respectively) were not included in the comparison as MCNP5 tracks all neutrons below that energy threshold while the proton-recoil detectors would have had an unknown minimum energy threshold for detection.

The neutron spectra calculations in MCNP are modified by a special tally feature, Gaussian Energy Broadening (GEB) that accounts for the resolution of the spectral measurement. This function uses the full width at half maximum (FWHM) data from the spectra to emulate energy broadening effects. A

\footnotetext{
${ }^{a}$ Personal Communication with David W. Wootan at Pacific Northwest National Laboratory (September 30, 2009).

${ }^{b}$ F. B. Brown, "A Review of Best Practices for Monte Carlo Criticality Calculations," Proc. NCSD 2009, Richland, WA, September 13-17 (2009).
}

Revision: 0

Page 123 of 304

Date: March 31, 2010 
NEA/NSC/DOC(2006)1

Liquid Metal Fast Reactor - LMFR

FFTF-LMFR-RESR-001

CRIT-SPEC-REAC-COEF-MISC

FWHM correlation is needed for this tally in the form of three constants, $a, b$, and c, that fit into Equation 2.3.1, where $\mathrm{E}$ is the energy, in $\mathrm{MeV}$, of the spectral measurement.

$$
F W H M=a+b \sqrt{E+c E^{2}} .
$$

The constants for Equation 2.3.1 are determined by first taking the resolution data, R, provided in Tables 1.3.5 and 1.3.6 and calculating the FWHM at each measurement energy, using Equation 2.3.2. ${ }^{\mathrm{a}}$

$$
R=\frac{F W H M}{E} .
$$

Next the FWHM values obtained from the measured data is fit with Equation 2.3.1 to obtain the three constants. A summary of these constants and the fit of the empirical formula to the actual measured data for the FWHM are provided in Table 2.3.1. The "best fit" parameter represents the square-root of the sum of the squares of the difference between the FWHM values obtained from the energy resolution data and the empirical formula; this value was minimized to obtain the empirical constants.

Table 2.3.1. Empirical Fitting Analysis for FWHM Values.

\begin{tabular}{|c|ccc|c||}
\hline \hline Position in Core & a & b & c & "best fit" \\
\hline Midplane & 0.0000 & 0.007035 & 204.42 & 0.0061 \\
$80 \mathrm{~cm}$ Below Midplane & 0.0000 & 0.007113 & 200.00 & 0.0055 \\
\hline \hline
\end{tabular}

The uncertainty in the energy of each data point is also obtained from the resolution data provided in Tables 1.3.5 and 1.3.6 using Equation 2.3.3. ${ }^{\mathrm{a}}$

$$
\sigma_{E}=\frac{E \cdot R}{2.35}
$$

The calculated and benchmark spectrum share the same general trend for measurements at the core midplane. The trending in the below midplane measurements appears to have slight agreement for energies less than about $150 \mathrm{keV}$. The calculated spectrum deviates significantly from the benchmark spectrum for larger energies. The results may be in part due to homogenization effects of core components below the fuel pin region. Homogenization of the IRT would have had a significant impact as the model doesn't fully account for scatter and streaming in the IRT.

\subsubsection{Neutron Spectrum near Center of Fully-Loaded Core (Nuclear Emulsions)}

Spectral characteristics measurements performed using the nuclear emulsions have not been evaluated. There is insufficient data currently available to evaluate results from neutron spectra measurements performed with nuclear emulsions.

\footnotetext{
${ }^{a}$ G. F. Knoll, Radiation Detection and Measurement, $3^{\text {rd }}$ ed., "Chapter 3 - Counting Statistics and Error Prediction," John Wiley \& Sons, New York, NY (2000).
} 
NEA/NSC/DOC(2006)1

Liquid Metal Fast Reactor - LMFR

FFTF-LMFR-RESR-001

CRIT-SPEC-REAC-COEF-MISC

\subsection{Evaluation of Reactivity Effects Data}

Reactivity effects measurements evaluated include a total of 21 control rod worth measurements (seven of which represent combinations of dropped rods), two control rod bank worth measurements, six differential control rod worth measurements, measured shutdown margin, and measured excess reactivity for the fully-loaded critical core configuration of the FFTF.

Reactivity worths were reported in $\$$ or $\phi$. The experimenters, engineers, and staff utilized a $\beta_{\text {eff }}$ value of 0.00318 (Table 1.1.8) to convert reactivity measurements to and from $\Delta \mathrm{k}$. This value was also used in this evaluation for the conversion of calculated reactivity worths to provide an equivalent comparison. Reference 4 reports $\beta_{\text {eff }}$ values of 0.003107 or 0.003097 in its calculations when calculating rod worths. However, rod worths reported in this reference do not differ from those reported in other references for Section 1.4.1. Use of the lowest $\beta_{\text {eff }}$ value, 0.003097 , results in a reactivity worth increase of $\sim 2.5 \%$ over that calculated using a $\beta_{\text {eff }}$ value of 0.00318 . A typical uncertainty of 3 to $5 \%$ is often attributed to the calculated value of $\beta_{\text {eff; }}$; the percent difference between the latter two values and that predominantly utilized in the references is on the order of 2 to $3 \%$.

It was believed at the time that an uncertainty of 3\% was appropriate for the $\beta_{\text {eff }}$ value used during the isothermal physics tests. This value was determined using ENDF/B-V. However, using later methods and neutron library data, the uncertainty might be reevaluated within $5 \%$ of the original values. It is also important to note that the value will change with different core loadings of the FFTF, and was recalculated for each cycle. ${ }^{\text {a }}$ Therefore, an additional 5\% uncertainty is added to all reactivity worth measurements in this section. This uncertainty is included by taking the square root of the sum of the squares of the experimental uncertainty and the uncertainty in $\beta_{\text {eff. }}$.

Where available, the IKRD measurements are preferred over the MSM measurements because of the higher confidence in the measurement. The IKRD method provided precise measurements for the calibration of the MSM method (Section 1.4.2.1). The uncertainties in the IKRD measurements include a contribution from detector efficiency changes, but are dominated by random noise in neutron data. An experiment was repeated to verify the random uncertainty component of the IKRD measurements (Section 1.4.2.1).

A systematic difference of $+2.67 \%$ was noted for use of MSM measurements instead of IKRD measurements (Section 1.1.2.5). MSM rod worths were shown to be accurate to within $3 \%$ for values as subcritical as $\$ 6$, and $5 \%$ for values as subcritical as $\$ 16$ (Section 1.4.2.1).

The uncertainties for many of the rod worth measurements using the IKRD method are typically between 1 to $3 \%$. It is assumed that the experimental measurement uncertainty is significantly larger than any uncertainties obtainable via computational analysis as comprehensive as that performed in Section 2.1 for the fully-loaded core critical. It is mentioned in the references (Section 1.4) that the IKRD measurement uncertainties included a contribution from the detector efficiency changes, but were dominated by the random noise of the neutron data (counting statistics); this was verified by performing repeatability measurements.

\subsubsection{Non-Critical Configurations}

There are a total of ten reference subcritical states from which reactor rod worth and differential rod worth measurements were performed (Table 2.4.1 and 2.4.2, respectively). Rod bank worths were determined by completely withdrawing either the primary or secondary control rod bank from the fully

\footnotetext{
a Personal Communication with David W. Wootan at Pacific Northwest National Laboratory (November 10, 2009).
} 
NEA/NSC/DOC(2006)1

\section{Liquid Metal Fast Reactor - LMFR \\ FFTF-LMFR-RESR-001 \\ CRIT-SPEC-REAC-COEF-MISC}

subcritical configuration. The excess reactivity of the reactor was estimated by fully withdrawing all control rods from the fully-loaded critical configuration (Section 3.1.2).

Table 2.4.1. Rod Positions for Control-Rod-Worth Reference Subcritical States.

\begin{tabular}{|c|ccccccccc||}
\hline $\begin{array}{c}\text { Control } \\
\text { Rod } \\
\text { Number }\end{array}$ & State 1 & State 2 & State 3 & State 4 & State 5 & State 6 & State 7 & State 8 & State 9 \\
\hline \hline 1 & 91.44 & 91.44 & 91.44 & 91.44 & 91.44 & 91.44 & 91.44 & 91.44 & 91.44 \\
2 & 91.44 & 91.44 & 91.44 & 91.44 & 91.44 & 91.44 & 91.44 & 91.44 & 91.44 \\
3 & 91.44 & 91.44 & 91.44 & 91.44 & 91.44 & 91.44 & 91.44 & 91.44 & 91.44 \\
4 & 0 & 35.306 & 91.44 & 24.384 & 26.416 & 25.4 & 27.686 & 25.654 & 35.052 \\
5 & 91.44 & 35.306 & 24.638 & 91.44 & 26.416 & 25.4 & 27.686 & 25.654 & 35.052 \\
6 & 0 & 35.306 & 24.638 & 24.384 & 91.44 & 25.4 & 27.686 & 25.654 & 35.052 \\
7 & 71.12 & 35.306 & 24.638 & 24.384 & 26.416 & 91.44 & 27.686 & 25.654 & 35.052 \\
8 & 0 & 35.306 & 24.638 & 24.384 & 26.416 & 25.4 & 91.44 & 25.654 & 35.052 \\
9 & 0 & 35.306 & 24.638 & 24.384 & 26.416 & 25.146 & 27.686 & 91.44 & 35.052 \\
\hline \hline
\end{tabular}

Table 2.4.2. Reference Subcritical State for Differential Rod Worth Measurements.

\begin{tabular}{||c|c||}
\hline $\begin{array}{c}\text { Control } \\
\text { Rod } \\
\text { Number }\end{array}$ & $\begin{array}{c}\text { Distance } \\
\text { Withdrawn }(\mathrm{cm})\end{array}$ \\
\cline { 2 - 3 } 1 & State 10 \\
\hline \hline 2 & 91.44 \\
3 & 91.44 \\
4 & 91.44 \\
5 & 34.798 \\
6 & 34.798 \\
7 & 34.798 \\
8 & 34.798 \\
9 & 34.798 \\
\hline
\end{tabular}

\subsubsection{Control Rod Worths}

A summary of the rod worth measurements performed per reference for each reference state in Table 2.4.1 is shown in Table 2.4.3. 
Liquid Metal Fast Reactor - LMFR

FFTF-LMFR-RESR-001

CRIT-SPEC-REAC-COEF-MISC

Table 2.4.3. Correlation of Rod Worth Tests with Subcritical Reference States.

\begin{tabular}{|c|cc|}
\hline Case & $\begin{array}{c}\text { Reference } \\
\text { State }\end{array}$ & $\begin{array}{c}\text { Rod(s) } \\
\text { Dropped }\end{array}$ \\
\hline 1 & 1 & 1 \\
2 & 1 & 2 \\
3 & 1 & 3 \\
4 & 1 & 5 \\
5 & 1 & 7 \\
6 & 1 & $1+2$ \\
7 & 1 & $1+3$ \\
8 & 1 & $1+5$ \\
9 & 1 & $3+5$ \\
10 & 1 & $1+2+5$ \\
11 & 1 & $2+3+5$ \\
12 & 2 & 1 \\
13 & 2 & 2 \\
14 & 2 & 3 \\
15 & 3 & 4 \\
16 & 4 & 5 \\
17 & 5 & 6 \\
18 & 6 & 7 \\
19 & 7 & 8 \\
20 & 8 & 9 \\
21 & 9 & \\
\hline & & 1 \\
\hline
\end{tabular}

From reference state one, a total of 11 rod worth measurements were performed (Tables 1.4.1, 1.4.3, and 1.4.7). The IKRD rod worths with their uncertainties were selected from Table 1.4 .7 for 10 cases. The rod worth of control rod 7 was reported in Table 1.4.3; its average value and uncertainty were computed from the three reported rod worth measurements. A comparison of the rod worth measurement data from Section 1.4 is provided in Table 2.4.4. Benchmark rod worth uncertainties have an additional $5 \%$ of the benchmark rod worth value included, to account for the uncertainty in $\beta_{\text {eff. }}$ 
FFTF-LMFR-RESR-001

CRIT-SPEC-REAC-COEF-MISC

Table 2.4.4. Comparison of IKRD Rod Worth Tests from the First Subcritical State.

\begin{tabular}{|c|c|ccc|ccc|ccc|ccc||}
\hline \multirow{2}{*}{ Case } & \multirow{2}{*}{ Rod(s) } & \multicolumn{2}{|c|}{ Table 1.4.1 } & \multicolumn{3}{|c|}{ Table 1.4.3 } & \multicolumn{3}{c|}{ Table 1.4.7 } & \multicolumn{3}{c|}{ Benchmark } \\
& Dropped & $\rho(\$)$ & \pm & $\sigma$ & $\rho$ & \pm & $\sigma$ & $\rho(\$)$ & \pm & $\sigma$ & $\rho(\$)$ & \pm & $\sigma$ \\
\hline \hline 1 & 1 & 6.04 & \pm & -- & -- & \pm & -- & 6.04 & \pm & 0.09 & 6.04 & \pm & 0.32 \\
2 & 2 & -- & \pm & -- & -- & \pm & -- & 5.89 & \pm & 0.09 & 5.89 & \pm & 0.31 \\
3 & 3 & 4.65 & \pm & -- & -- & \pm & -- & 4.65 & \pm & 0.07 & 4.65 & \pm & 0.25 \\
4 & 5 & -- & \pm & -- & -- & \pm & -- & 3.84 & \pm & 0.05 & 3.84 & \pm & 0.20 \\
5 & 7 & -- & \pm & -- & 2.90 & \pm & 0.04 & 2.90 & \pm & -- & 2.90 & \pm & 0.15 \\
6 & $1+2$ & 12.45 & \pm & -- & -- & \pm & -- & 12.45 & \pm & 0.29 & 12.45 & \pm & 0.69 \\
7 & $1+3$ & -- & \pm & -- & -- & \pm & -- & 11.01 & \pm & 0.25 & 11.01 & \pm & 0.60 \\
8 & $1+5$ & 9.11 & \pm & -- & -- & \pm & -- & 9.00 & \pm & 0.16 & 9.00 & \pm & 0.48 \\
9 & $3+5$ & -- & \pm & -- & -- & \pm & -- & 8.80 & \pm & 0.14 & 8.8 & \pm & 0.46 \\
10 & $1+2+5$ & 15.84 & \pm & -- & -- & \pm & -- & 15.85 & \pm & 0.35 & 15.84 & \pm & 0.87 \\
11 & $2+3+5$ & -- & \pm & -- & -- & \pm & -- & 15.22 & \pm & 0.39 & 15.22 & \pm & 0.86 \\
\hline
\end{tabular}

Three cases were performed from a second reference state, and then a single rod worth measurement performed for the remaining seven reference configurations. These data were obtained from Table 1.4.4, 1.4.5, and 1.4.6. Because the data in Table 1.4.4 were more comprehensive, it was used to determine the average rod worths and uncertainty for the latter ten cases. The uncertainty in the rod worth determined using the data in Table 1.4.4 was generally larger than the uncertainty reported in the other two tables. A comparison of the rod worth measurement data from Section 1.4 is provided in Table 2.4.5. Benchmark rod worth uncertainties have an additional $5 \%$ of the benchmark rod worth value included, to account for the uncertainty in $\beta_{\text {eff. }}$. 
NEA/NSC/DOC(2006)1

Liquid Metal Fast Reactor - LMFR

FFTF-LMFR-RESR-001

CRIT-SPEC-REAC-COEF-MISC

Table 2.4.5. Comparison of IKRD Rod Worth Tests from the Remaining Subcritical States.

\begin{tabular}{|c|c|c|c|c|c|c|c|c|c|c|c|c|c|}
\hline \multirow{2}{*}{ Case } & \multirow{2}{*}{$\begin{array}{l}\text { Rod(s) } \\
\text { Dropped }\end{array}$} & \multicolumn{3}{|c|}{ Table 1.4.4 } & \multicolumn{3}{|c|}{ Table 1.4.5 } & \multicolumn{3}{|c|}{ Table 1.4.6 } & \multicolumn{3}{|c|}{ Benchmark } \\
\hline & & $\rho(\$)$ & \pm & $\sigma$ & $\rho(\$)$ & \pm & $\sigma$ & $\rho(\$)$ & \pm & $\sigma$ & $\rho(\$)$ & \pm & $\sigma$ \\
\hline 12 & 1 & 5.81 & \pm & 0.16 & 5.82 & \pm & 0.06 & 5.82 & \pm & $\begin{array}{l}0.08 \\
0.04\end{array}$ & 5.81 & \pm & 0.33 \\
\hline 13 & 2 & 5.51 & \pm & 0.09 & 5.52 & \pm & 0.06 & 5.52 & \pm & 0.07 & 5.51 & \pm & 0.29 \\
\hline 14 & 3 & 5.40 & \pm & 0.11 & 5.40 & \pm & 0.05 & 5.40 & \pm & 0.06 & 5.40 & \pm & 0.29 \\
\hline 15 & 4 & 4.06 & \pm & 0.04 & 4.07 & \pm & 0.04 & 4.07 & \pm & 0.04 & 4.06 & \pm & 0.21 \\
\hline 16 & 5 & 4.09 & \pm & 0.04 & 4.09 & \pm & 0.04 & $\begin{array}{l}4.09 \\
4.11\end{array}$ & \pm & $\begin{array}{l}0.02 \\
0.05\end{array}$ & 4.09 & \pm & 0.21 \\
\hline 17 & 6 & 3.57 & \pm & 0.04 & 3.57 & \pm & 0.04 & 3.57 & \pm & 0.04 & 3.57 & \pm & 0.18 \\
\hline 18 & 7 & 3.86 & \pm & 0.04 & 3.86 & \pm & 0.04 & 3.86 & \pm & $\begin{array}{l}0.04 \\
0.06\end{array}$ & 3.86 & \pm & 0.20 \\
\hline 19 & 8 & 3.19 & \pm & 0.10 & 3.17 & \pm & 0.03 & 3.17 & \pm & $\begin{array}{l}0.03 \\
0.07\end{array}$ & 3.19 & \pm & 0.19 \\
\hline 20 & 9 & 3.83 & \pm & 0.03 & 3.83 & \pm & 0.04 & 3.83 & \pm & 0.04 & 3.83 & \pm & 0.19 \\
\hline 21 & $2+3$ & 11.22 & \pm & 0.15 & 11.22 & \pm & 0.11 & -- & \pm & -- & 11.22 & \pm & 0.58 \\
\hline
\end{tabular}

(a) The original references for this table provide slightly different information.

\subsubsection{Control Rod Bank Worths}

The control rod bank worth of the primary and secondary rods were determined by fully withdrawing the respective bank of rods from a fully subcritical core configuration of the fully-loaded core critical benchmark model.

In Section 1.4.2.2, two values are provided for the primary bank worth that are essentially the same within rounding error. The value of $\$ 16.34 \pm 0.12$ was selected because it provided more significant digits. With an increase in uncertainty to account for the uncertainty in $\beta_{\text {eff }}$, the total uncertainty in the primary bank worth is $\pm \$ 0.83$.

Of the various calculated and observed secondary bank worths reported in Section 1.4.2.2, the value $\$ 19.9 \pm 0.1$ obtained using the MSM technique appeared to be the most reliable. One of the other values $(\$ 22 \pm 1)$ was derived from a combination of measurements and calculations, while the pedigree of the third reported value (\$21.98) was unclear, and may be the same derived value. With an increase in uncertainty to account for the uncertainty in $\beta_{\text {eff }}$, the total uncertainty in the secondary bank worth is $\pm \$ 1.00$.

\subsubsection{Differential Control Rod Worths}

Differential control rod worths (Section 1.4.2.3) were obtained using the tenth reference subcritical configuration (Table 2.4.2). Only the rods in the secondary bank were assessed for a $\pm 2.54 \mathrm{~cm}$ perturbation from their withdrawn positions of $34.798 \mathrm{~cm}$. In order to reduce the uncertainty in the computational results, the rods were perturbed $\pm 5.08 \mathrm{~cm}$ to obtain differential rod worths, and then scaled back to the experimental value by dividing the worths in half. The differential rod worths reported in Table 2.4.6 were converted from inches into centimeters and then an additional uncertainty of $5 \%$ of the differential rod worth was added to the total uncertainty to account for uncertainty in $\beta_{\text {eff. }}$. 
NEA/NSC/DOC(2006)1

Liquid Metal Fast Reactor - LMFR

FFTF-LMFR-RESR-001

CRIT-SPEC-REAC-COEF-MISC

It is recognized that both the experimental measurements and initial analytical analyses for the differential control rod worths were not performed in a region of worth curve linearity. The analysis in this benchmark analysis was performed in conformance with the initial measurement method, and calculated results are in good agreement with the originally measured values and currently assessed uncertainties.

Table 2.4.6. Differential Control Rod Worths.

\begin{tabular}{|c|ccc|}
\hline Rod & \multicolumn{3}{|c|}{ Diff. Worths $(\phi / \mathrm{cm})$} \\
\hline \hline 4 & 6.1 & \pm & 0.3 \\
5 & 6.2 & \pm & 0.3 \\
6 & 5.4 & \pm & 0.3 \\
7 & 5.7 & \pm & 0.3 \\
8 & 4.8 & \pm & 0.3 \\
9 & 5.6 & \pm & 0.3 \\
\hline
\end{tabular}

\subsubsection{Shutdown Margin}

The shutdown margin was assessed from the first and second subcritical states. The first state (Table 1.4.4) had a reported reactivity worth of $\$ 24$ without any uncertainty. An uncertainty of 3\% was assumed for the measurement uncertainty. The shutdown margin from the second state was reported in Section 1.4.2.4 to be worth $\$ 23.6 \pm 0.2$. However, from the data in Table 1.1.7, the average worth can be calculated as $\$ 23.7 \pm 0.5$. This latter value was selected for the benchmark. An additional uncertainty was included for $\beta_{\text {eff, }}$ such that the respective uncertainties in the shutdown margins are increased to $\pm \$ 1.40$ and $\pm \$ 1.27$.

\subsubsection{Excess Reactivity}

The excess reactivity was estimated to be $\$ 11 \pm 1$ at full power, but was measured to be $\$ 14.66$ (Section 1.4.2.5). An uncertainty of $3 \%$ was assumed for the measurement uncertainty with an additional $5 \%$ for the $\beta_{\text {eff }}$ uncertainty. The total uncertainty is therefore $\pm \$ 0.85$ of the measured (benchmark) value of $\$ 14.66$.

\subsection{Evaluation of Reactivity Coefficient Data}

The reactor physics experiments evaluated in this section pertain to the data available for the isothermal temperature coefficient. The primary loop flow reactivity effect has not been evaluated.

\subsubsection{Isothermal Temperature Coefficient}

The isothermal temperature coefficient was evaluated using the results from the uncertainty analysis performed in Section 2.1.1.1 for the fully-loaded core critical. The calculated value is approximately half of the benchmark model. As discussed in Section 2.1.1.1, the core assembly pitch was not adjusted with the variation in core temperature. The model temperature, liquid sodium coolant density, and neutron cross section libraries were adjusted for the variation in temperature. The densities of major structural components were not adjusted. However, much of the core model is homogenized, and density effects would be negligible. There is not a correlation available between the core temperature and assembly pitch such that a more accurate calculation of the isothermal temperature coefficient could be evaluated. 
NEA/NSC/DOC(2006)1

Liquid Metal Fast Reactor - LMFR

FFTF-LMFR-RESR-001

CRIT-SPEC-REAC-COEF-MISC

The reported isothermal temperature coefficient value of $-0.7 \pm 0.10 \phi /{ }^{\circ} \mathrm{F}(1.26 \pm 0.18 \phi / \mathrm{K})$ was selected to represent the benchmark value.

If the average uncertainty in the core lattice pitch $\left( \pm 0.00086 \Delta \mathrm{k}_{\text {eff }}\right)$ from Section 2.1.2.9 is included with the calculation of the reactivity worth variation, then the calculated isothermal temperature coefficient is approximately $8.7 \%$ lower than the benchmark value. The uncertainty in the calculated value is increased due to the uncertainty in the core pitch worth. The uncertainty is determined by taking the square root of the sum of the squares of the calculated worth $\pm \$ 0.03$ from the initial isothermal temperature analysis and the uncertainty in the inclusion of the absolute core pitch worth $\pm \$ 0.085$ upon the calculations, which is determined by varying the calculated isothermal temperature coefficient using the $\Delta \mathrm{k}_{\text {eff }}(1 \sigma)$ values determined in Section 2.1.2.9.

As discussed in Section 2.4, an additional 5\% uncertainty is included to all reactivity worth measurements, such that the benchmark value is $-1.26 \pm 0.19 \phi / \mathrm{K}\left(-0.70 \pm 0.11 \phi /{ }^{\circ} \mathrm{F}\right)$.

The isothermal temperature coefficient for the refueling temperature was not evaluated.

\subsubsection{Primary Loop Flow Reactivity Effect}

The primary loop flow reactivity effect measurement has not been evaluated.

\subsection{Evaluation of Kinetics Measurements Data}

Kinetics measurements were not made.

\subsection{Evaluation of Reaction-Rate Distributions}

Reaction-rate distribution measurements were not made.

\subsection{Evaluation of Power Distribution Data}

Power distribution measurements were not made.

\subsection{Evaluation of Isotopic Measurements}

Isotopic measurements were not made.

\subsection{Evaluation of Other Miscellaneous Types of Measurements}

The reactor physics experiments evaluated in this section pertain to the low-energy electron and gammaray spectra measurements performed in the IRT near the axial and radial center of the core.

\subsubsection{Low-Energy Electron Spectrum}

As discussed in Section 2.7.1 for the neutron flux measurements performed for the neutron spectra measurements (Section 2.3), there is insufficient information to assess the absolute values reported for electron spectrum at the core center. Furthermore, a convenient table does not accompany the chart 
NEA/NSC/DOC(2006)1

Liquid Metal Fast Reactor - LMFR

FFTF-LMFR-RESR-001

CRIT-SPEC-REAC-COEF-MISC

(Figure 1.10.1). Therefore, the chart was digitized and the extracted data was then normalized to the maximum counts to produce normalized values to compare with computed results.

No uncertainty was reported for the experimental measurements; the detector system for counting electrons was also not discussed. An uncertainty of $10 \%$, as discussed for the gamma-ray measurements (Section 2.10.2), was selected to represent the uncertainty in the electron spectrum measurements. This uncertainty is assumed to include the additional uncertainty due to data digitization. It is recognized that the actual uncertainty in the measurements is energy dependent, but additional information is unavailable.

An evaluation similar to that for the neutron spectra (Section 2.3) was performed using MCNP5. The same model was utilized, with the IRT; however, the flux tally instead tracked the electron flux at the center of the core. The calculated electron spectra are obtained by taking the variance-weighted average of results obtained using six variations of the input deck (Appendix A.1) with different random number seeds and tallies of the electron flux (Appendix A.10). This approach was used to reduce the statistical uncertainty in the neutron flux tallies because the relative error values obtained can under-predict the true uncertainty in the calculated spectra. ${ }^{\mathrm{a}}$ The initial data point was not included in the comparison as MCNP5 tracks all electrons below that energy threshold while the detectors would have had an unknown minimum energy threshold for detection.

The digitized values for the low-energy electron spectrum at core center midplane and their approximate uncertainty are reported in Table 2.10.1 and shown in Figure 2.10.1.

The calculated values for the low-energy electron spectrum are shown in Table 2.10.2 and Figure 2.10.2. There is poor agreement between the calculated and benchmark low-energy electron spectra. Homogenization of the IRT would have had a significant impact as the model doesn't fully account for scatter and streaming in the IRT. Because insufficient data are available regarding the measurement detection method, and the results do not fit well, the electron spectrum data are not included as valid benchmark data.

${ }^{a}$ F. B. Brown, “A Review of Best Practices for Monte Carlo Criticality Calculations,” Proc. NCSD 2009, Richland, WA, September 13-17 (2009).

Revision: 0

Page 132 of 304

Date: March 31, 2010 
NEA/NSC/DOC(2006)1

Liquid Metal Fast Reactor - LMFR

FFTF-LMFR-RESR-001

CRIT-SPEC-REAC-COEF-MISC

Table 2.10.1. Experimental Data for FFTF Core Center Low-Energy Electron Spectrum.

\begin{tabular}{|c|c|c|c|c|c|c|c|}
\hline $\begin{array}{l}\text { Energy } \\
(\mathrm{MeV})\end{array}$ & $\begin{array}{c}\text { Normalized } \\
\text { Flux } \\
\varphi / \varphi_{\max }\end{array}$ & \pm & $\sigma$ & $\begin{array}{l}\text { Energy } \\
(\mathrm{MeV})\end{array}$ & $\begin{array}{c}\text { Normalized } \\
\text { Flux } \\
\varphi / \varphi_{\max }\end{array}$ & \pm & $\sigma$ \\
\hline 0.1077 & 1.00000 & \pm & 0.10000 & 0.9129 & 0.04245 & \pm & 0.00425 \\
\hline 0.1289 & 0.79873 & \pm & 0.07987 & 9307 & 04037 & \pm & .00404 \\
\hline 0.1461 & 0.62951 & \pm & 0.06295 & 0.9510 & 03983 & \pm & 0.00398 \\
\hline 0.1646 & 50455 & \pm & 0.05045 & 0.9741 & 03788 & \pm & 0.00379 \\
\hline 0.1842 & 2954 & \pm & 0.04295 & 0.9890 & & \pm & 0.00375 \\
\hline 0.2023 & 8 & \pm & 0.03769 & & & \pm & 00351 \\
\hline 0.2244 & & \pm & 0 . & & & \pm & 343 \\
\hline 0.2436 & & \pm & 0 & & & \pm & 329 \\
\hline 0.2656 & & \pm & 0 . & 1 & & I & 320 \\
\hline 0.2835 & & \pm & 0.02 & 77 & & $\perp$ & 00303 \\
\hline 0.3068 & & \pm & & & & \pm & \\
\hline 0.3260 & & \pm & 0 & & & \pm & \\
\hline 0.3467 & & \pm & 9 & & & $E$ & \\
\hline 0. & & \pm & & & & & \\
\hline 0 . & & \pm & 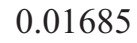 & & & & 56 \\
\hline 0.4071 & & \pm & 0 & & & & 45 \\
\hline 262 & & \pm & 4 & & & & 34 \\
\hline 0.4467 & & \pm & 8 & 1 & & & $0 .($ \\
\hline 0.4645 & 59 & \pm & 0.01 & 72 & & & 0.00213 \\
\hline 0.4863 & & \pm & 38 & 10 & & & 0.0 \\
\hline 0.5067 & 99 & \pm & 0.01 & 79 & 09 & \pm & 0.00201 \\
\hline 0.5285 & 8 & \pm & 0.01123 & 96 & 43 & \pm & 0.00194 \\
\hline 0.5516 & 59 & \pm & 0.01086 & 73 & 79 & \pm & 0.00188 \\
\hline 0.5707 & 57 & \pm & 0.01026 & 03 & 23 & \pm & 0.00182 \\
\hline 0.5896 & 20 & \pm & 0.01012 & 30 & .01763 & \pm & 0.00176 \\
\hline 0.6100 & 37 & \pm & 0.00 & 84 & .01699 & \pm & 0.00170 \\
\hline 0.6292 & 91 & \pm & 0.0 & 50 & 660 & \pm & 0.00166 \\
\hline 0.6511 & 31 & \pm & $0 .($ & 4 & 500 & \pm & 0.00160 \\
\hline 0.6704 & & \pm & & & 67 & \pm & 0.00157 \\
\hline 0.69 & 45 & \pm & 0.0 & 7 & 21 & \pm & 0.00152 \\
\hline 0.708 & 10 & \pm & 0.0 & 3 & 1 & \pm & 0.00150 \\
\hline 0.730 & 85 & \pm & 00 & & 32 & \pm & 0.00143 \\
\hline 0.7483 & 57 & \pm & 00 & 3 & 43 & \pm & 0.00134 \\
\hline 0.7729 & 8 & \pm & 000 & 99 & 30 & \pm & 0.00133 \\
\hline 0.7893 & 51 & \pm & 0.00 & 89 & 95 & \pm & 0.00129 \\
\hline 0.8097 & 05105 & \pm & 0.00511 & 79 & 56 & \pm & 0.00126 \\
\hline 0.8314 & 04953 & \pm & 0.00495 & 1.6423 & 01203 & \pm & 0.00120 \\
\hline 0.8504 & 04774 & \pm & 0.00477 & 1.6614 & .01140 & \pm & 0.00114 \\
\hline 0.8708 & 04586 & \pm & 0.00459 & 1679 & .01117 & \pm & 0.00112 \\
\hline & & \pm & & & & \pm & \\
\hline
\end{tabular}

Revision: 0 
NEA/NSC/DOC(2006)1

Liquid Metal Fast Reactor - LMFR

FFTF-LMFR-RESR-001

CRIT-SPEC-REAC-COEF-MISC

Table 2.10.1 (cont'd). Experimental Data for FFTF Core Center Low-Energy Electron Spectrum.

\begin{tabular}{|c|c|c|c|c|c|c|c|}
\hline $\begin{array}{l}\text { Energy } \\
(\mathrm{MeV})\end{array}$ & $\begin{array}{c}\text { Normalized } \\
\text { Flux } \\
\varphi / \varphi_{\max }\end{array}$ & \pm & $\sigma$ & $\begin{array}{l}\text { Energy } \\
(\mathrm{MeV})\end{array}$ & $\begin{array}{c}\text { Normalized } \\
\text { Flux } \\
\varphi / \varphi_{\max }\end{array}$ & \pm & $\sigma$ \\
\hline 1.7211 & 0.01045 & \pm & 0.00104 & 2.5397 & 0.00281 & \pm & 0.00028 \\
\hline 1.7374 & 01027 & \pm & 0.00103 & 2.5586 & 0.00275 & \pm & 0.00027 \\
\hline 1.7564 & 00997 & \pm & 0.00100 & 5816 & 0.00274 & \pm & 0.00027 \\
\hline 1.7781 & 00964 & \pm & 0.00096 & 2.5978 & 0.00271 & \pm & 0.00027 \\
\hline 1.7998 & 00932 & \pm & 0.00093 & & 0256 & \pm & .00026 \\
\hline 1.8188 & 08 & \pm & 0.00090 & & & \pm & 00025 \\
\hline 1.8365 & 0 & \pm & 0.00086 & & & \pm & 00025 \\
\hline 1.8582 & & \pm & 0.00 & & & \pm & 00024 \\
\hline 1.8799 & & \pm & 0.00 & & & \pm & 00023 \\
\hline 1.8976 & & \pm & 0.00 & & & \pm & 00022 \\
\hline 1.9 & & \pm & & & & \pm & \\
\hline 1.9 & & \pm & 3 & & & \pm & 21 \\
\hline 1. & & \pm & & & & \pm & 21 \\
\hline 1. & & \pm & & & & & 20 \\
\hline 1. & & \pm & 6 & & & & 19 \\
\hline 2.0158 & 36 & \pm & 4 & 8 & & & 18 \\
\hline 2.0389 & & \pm & 1 & 0 & & & 18 \\
\hline 2.0605 & & \pm & 60 & 3 & & & 0.00 \\
\hline 2.0808 & 1 & \pm & 0.00 & 2.9 & 73 & & 0.00017 \\
\hline 2.1000 & 4 & \pm & 00 & 2. & 67 & & 0.00017 \\
\hline 2.1176 & 7 & \pm & 0.00 & 2.9 & 65 & & 0.00017 \\
\hline 2.1379 & 9 & \pm & 0.00053 & 2.9 & 0.0 & & 0.00016 \\
\hline 2.1595 & 0523 & \pm & 0.00052 & 2. & 0.00152 & & 0.00015 \\
\hline 2.1799 & 0501 & \pm & 0.00 & 2.9 & 0.00152 & \pm & 0.00015 \\
\hline 2.2004 & 0478 & \pm & 0.0 & 3.0 & 0.00142 & \pm & 0.00014 \\
\hline 2.2424 & 53 & \pm & 5 & 3. & 0.00146 & \pm & 0.00015 \\
\hline 2.2572 & 3 & \pm & 0.00 & 4 & 0.00135 & \pm & 0.00013 \\
\hline 303 & 32 & \pm & 0.00 & 77 & 33 & \pm & 0.00013 \\
\hline 2. & 25 & \pm & & 41 & 27 & \pm & 0.00013 \\
\hline 2. & 06 & \pm & 0.00 & & 25 & \pm & 0.00013 \\
\hline 2. & 00394 & \pm & 0 & 7 & 21 & \pm & 0.00012 \\
\hline 2. & 00381 & \pm & 00 & 3 & 0.00121 & \pm & 0.00012 \\
\hline 2.3822 & 1 & \pm & 0.00 & 4 & 0 & \pm & 0.00011 \\
\hline 2.4011 & 2 & \pm & 0.00 & 3 & 13 & \pm & 0.00011 \\
\hline 2.4187 & 00354 & \pm & 0.00035 & 3.2433 & 0.00112 & \pm & 0.00011 \\
\hline 2.4404 & 00340 & \pm & 0.000 & 8 & 00111 & \pm & 0.00011 \\
\hline 2.4608 & 00326 & \pm & 0.00033 & 3.2814 & .00104 & \pm & 0.00010 \\
\hline 2.4798 & 00323 & \pm & 0.00032 & 3014 & .00107 & \pm & 0.00011 \\
\hline 2.5001 & 00313 & \pm & 0.0003 & & 0.00100 & \pm & 0.00010 \\
\hline & & \pm & & & & \pm & \\
\hline
\end{tabular}

Revision: 0 
NEA/NSC/DOC(2006)1

Liquid Metal Fast Reactor - LMFR

FFTF-LMFR-RESR-001

CRIT-SPEC-REAC-COEF-MISC

Table 2.10.1 (cont'd). Experimental Data for FFTF Core Center Low-Energy Electron Spectrum.

\begin{tabular}{|c|c|c|c|c|c|c|c|}
\hline $\begin{array}{l}\text { Energy } \\
(\mathrm{MeV})\end{array}$ & $\begin{array}{c}\text { Normalized } \\
\text { Flux } \\
\varphi / \varphi_{\max }\end{array}$ & \pm & $\sigma$ & $\begin{array}{l}\text { Energy } \\
(\mathrm{MeV})\end{array}$ & $\begin{array}{c}\text { Normalized } \\
\text { Flux } \\
\varphi / \varphi_{\max }\end{array}$ & \pm & $\sigma$ \\
\hline 3.3614 & 0.00091 & \pm & 0.00009 & .2241 & 0.00036 & \pm & 0.00004 \\
\hline 3.3803 & 00091 & \pm & 0.00009 & & 00036 & \pm & 00004 \\
\hline 3.4194 & 00090 & \pm & 0.00009 & & 00034 & \pm & 0.00003 \\
\hline 3.4398 & 06 & \pm & 0.00009 & & 00032 & \pm & 00003 \\
\hline 3.4588 & 0 & \pm & 0.00008 & & 33 & \pm & 00003 \\
\hline & 3 & \pm & 88 & & & \pm & 03 \\
\hline 3.4 & 3 & \pm & 8 & & 1 & \pm & 03 \\
\hline 3. & 5 & \pm & 77 & & 30 & \pm & 03 \\
\hline 3. & 0 & \pm & 8 & & 29 & \pm & 003 \\
\hline 3.5604 & & \pm & 08 & 2 & 30 & \pm & 03 \\
\hline 3.5833 & 75 & \pm & 08 & & 27 & \pm & 00003 \\
\hline 3.6038 & 71 & \pm & 0.00 & 9 & 28 & \pm & 0.00003 \\
\hline $3.61 \mathrm{~S}$ & 11 & \pm & 0. & & 28 & \pm & 0.00003 \\
\hline 3.6443 & 00070 & \pm & 0.00007 & 7 & 27 & \pm & 0.00003 \\
\hline 3.6634 & 0.00066 & \pm & 0.00007 & 4 & 0.00 & \pm & 0.00003 \\
\hline 3.6782 & 0.00066 & \pm & 0.00007 & 4.5 & 0.00025 & \pm & 0.00003 \\
\hline 3.7069 & 00061 & \pm & 0.00006 & 4.6086 & 0.00024 & \pm & 0.00002 \\
\hline 3.7416 & .00066 & \pm & 0.00007 & 4.6258 & 0.00026 & \pm & 0.00003 \\
\hline 3.7623 & 00059 & \pm & 0.00006 & 4.6478 & 0.00024 & \pm & 0.00002 \\
\hline 3.7855 & 00056 & \pm & 0.00006 & 4.6642 & 0.00022 & \pm & 0.00002 \\
\hline 3.8004 & 00055 & \pm & 0.00005 & 4.6 & 25 & \pm & 0.00002 \\
\hline 3.8179 & 00 & \pm & 0.00006 & 4.7 & 21 & \pm & 0.00002 \\
\hline 3.8394 & 00 & \pm & 0.00006 & 52 & 22 & \pm & 0.00002 \\
\hline 3.8639 & 3 & \pm & 0.00005 & 0 & 22 & \pm & 0.00002 \\
\hline 3.8814 & 3 & \pm & 0.00005 & 5 & 21 & \pm & 0.00002 \\
\hline 3.9044 & 52 & \pm & 0.00005 & $r$ & .00 & \pm & 0.00002 \\
\hline 3.9220 & 00051 & \pm & 0.00005 & 8 & .00022 & \pm & 0.00002 \\
\hline 3.9425 & 00048 & \pm & 0.00005 & 4.8 & .00021 & \pm & 0.00002 \\
\hline 3.9640 & 00048 & \pm & 0.00005 & 32 & 21 & \pm & 0.00002 \\
\hline 3.9805 & 00046 & \pm & 0.00005 & 58 & 21 & \pm & 0.00002 \\
\hline 4.0020 & 00046 & \pm & 0.00005 & 4 & 21 & \pm & 0.00002 \\
\hline 4.0411 & 15 & \pm & 0.00005 & 0 & 20 & \pm & 0.00002 \\
\hline 4.0641 & 5 & \pm & & & & \pm & 0.00002 \\
\hline 4.0833 & & \pm & 0.00004 & 97 & & \pm & 0.00002 \\
\hline 4.1051 & 39 & \pm & 0.00004 & 5.0476 & 00019 & \pm & 0.00002 \\
\hline 4.1237 & & \pm & & & & \pm & 0.00002 \\
\hline 4.1428 & & \pm & 0.00004 & & & \pm & 0.00002 \\
\hline 4.1576 & .00040 & \pm & 0.00004 & & 18 & \pm & 0.00002 \\
\hline 4.1844 & 0.00041 & \pm & 0.00004 & 5.1300 & 0.00019 & \pm & 0.00002 \\
\hline 4.2038 & 0.00036 & \pm & 0.00004 & & & & \\
\hline
\end{tabular}

Revision: 0 
NEA/NSC/DOC(2006)1

Liquid Metal Fast Reactor - LMFR

FFTF-LMFR-RESR-001

CRIT-SPEC-REAC-COEF-MISC

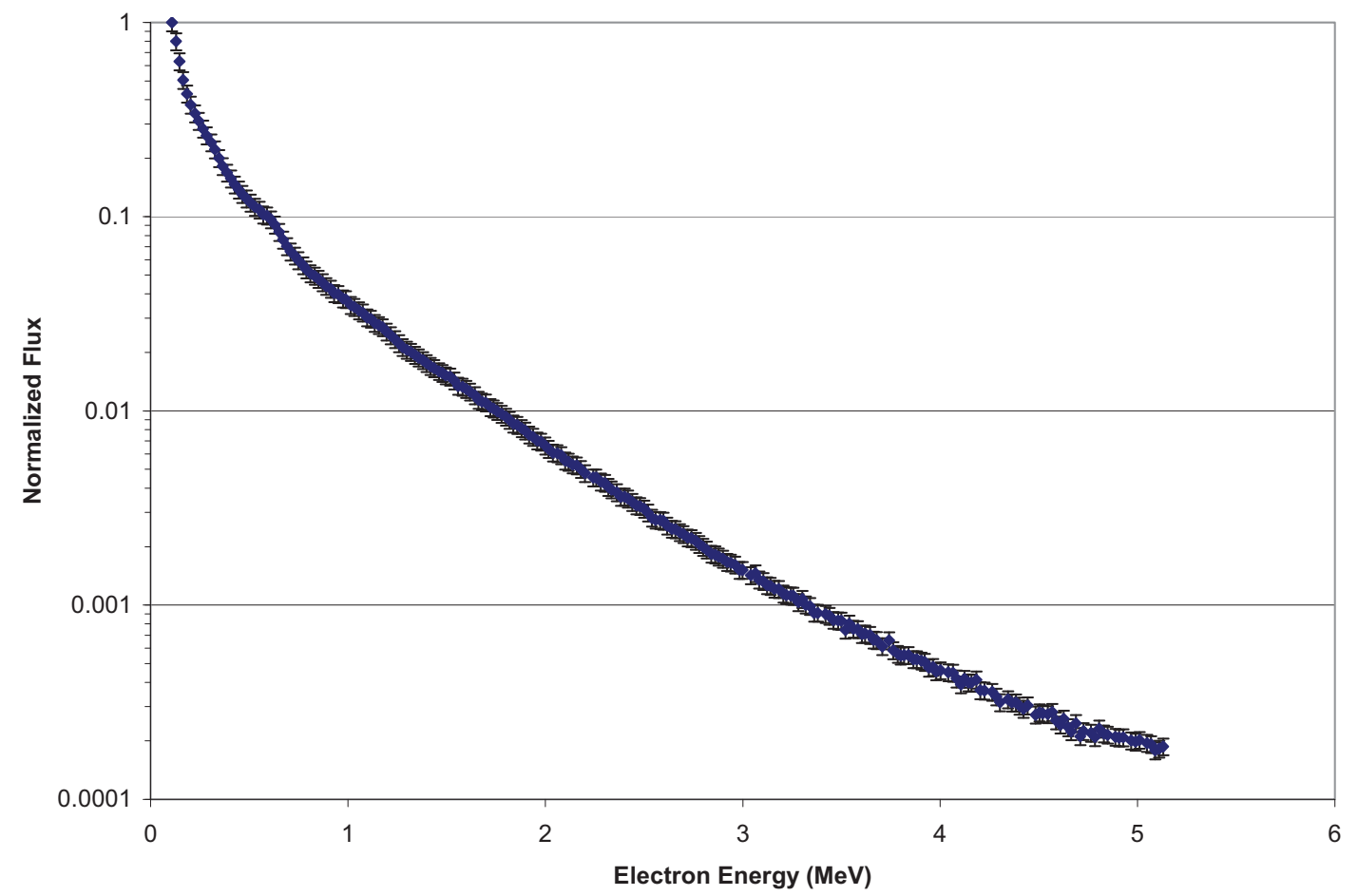

Figure 2.10.1. Low-Energy Electron Spectrum in FFTF Core Center. 
NEA/NSC/DOC(2006)1

Liquid Metal Fast Reactor - LMFR

FFTF-LMFR-RESR-001

CRIT-SPEC-REAC-COEF-MISC

Table 2.10.2. Calculated FFTF Core Center Low-Energy Electron Spectrum.

\begin{tabular}{|c|c|c|c|c|c|c|c|c|c|}
\hline $\begin{array}{l}\text { Energy } \\
(\mathrm{MeV})\end{array}$ & \multicolumn{2}{|c|}{$\begin{array}{l}\text { Calculated } \\
\text { Normalized }\end{array}$} & $\sigma$ & $\begin{array}{c}\text { Experi } \\
\text { Normalize } \\
\text { Flux } \\
\varphi / \varphi_{\max }\end{array}$ & enta & Data & & \multicolumn{2}{|l|}{$\mathrm{C} / \mathrm{E}$} \\
\hline 0.1077 & & -- & & 1.00000 & \pm & 0.10000 & & -- & \\
\hline 0.1289 & 1.0000 & \pm & 0.0018 & 0.79873 & \pm & 0.07987 & 1.25 & \pm & 0.13 \\
\hline 0.1461 & 0.7898 & \pm & 0.0015 & 0.62951 & \pm & 0.06295 & 1.25 & \pm & 0.13 \\
\hline 0.1646 & 0.8393 & \pm & 0.0016 & 0.50455 & \pm & 0.05045 & 1.66 & \pm & 0.17 \\
\hline 0.1842 & 0.8498 & \pm & 0.0017 & 0.42954 & \pm & 0.04295 & 1.98 & \pm & 0.20 \\
\hline 0.2023 & 0.7703 & \pm & 0.0016 & 0.37688 & \pm & 0.03769 & 2.04 & \pm & 0.20 \\
\hline 0.2244 & 0.9142 & \pm & 0.0019 & 0.33963 & \pm & 0.03396 & 2.69 & \pm & 0.27 \\
\hline 0.2436 & 0.7614 & \pm & 0.0016 & 0.31124 & \pm & 0.03112 & 2.45 & \pm & 0.24 \\
\hline 0.2656 & 0.8398 & \pm & 0.0018 & 0.28340 & \pm & 0.02834 & 2.96 & \pm & 0.30 \\
\hline 0.2835 & 0.6545 & \pm & 0.0014 & 0.26146 & \pm & 0.02615 & 2.50 & \pm & 0.25 \\
\hline 0.3068 & 0.8238 & \pm & 0.0018 & 0.24121 & \pm & 0.02412 & 3.42 & \pm & .34 \\
\hline 0.3260 & 0.6631 & \pm & 0.0015 & 22182 & \pm & 0.02218 & 2.99 & \pm & .30 \\
\hline 0.3467 & 83 & \pm & 0.0 & 4 & \pm & 0.0 & 3.29 & \pm & 33 \\
\hline 0.3646 & 71 & \pm & 0.0 & 1 & \pm & 20 & 3.06 & \pm & 31 \\
\hline 0.3865 & 6758 & \pm & 0.0 & 0 & \pm & 0.0 & 4.01 & \pm & 40 \\
\hline 0.4071 & 726 & \pm & 0.0015 & 0 & \pm & 0.01570 & 3.65 & \pm & 0.36 \\
\hline 0.4262 & 0.5359 & \pm & 0.0014 & 0.14 & \pm & 0.01464 & 3.66 & \pm & 0.37 \\
\hline 0.4467 & 0.5364 & \pm & 0.0015 & 0.13778 & \pm & 0.01378 & 3.89 & \pm & 0.39 \\
\hline 0.4645 & 0.4625 & \pm & 0.0012 & 0.13059 & \pm & 0.01306 & 3.54 & \pm & 0.35 \\
\hline 0.4863 & 0.5296 & \pm & 0.0015 & 0.12377 & \pm & 0.01238 & 4.28 & \pm & 0.43 \\
\hline 0.5067 & 0.4915 & \pm & 0.0014 & 0.11809 & \pm & 0.01181 & 4.16 & \pm & 0.42 \\
\hline 0.5285 & 0.4859 & \pm & 0.0014 & 0.11228 & \pm & 0.01123 & 4.33 & \pm & 0.43 \\
\hline 0.5516 & 0.5122 & \pm & 0.0015 & 0.10859 & \pm & 0.01086 & 4.72 & \pm & 0.47 \\
\hline 0.5707 & 0.3866 & \pm & 0.0012 & 0.10257 & \pm & 0.01026 & 3.77 & \pm & 0.38 \\
\hline 0.5896 & 0.3954 & \pm & 0.0012 & 0.10120 & \pm & 0.01012 & 3.91 & \pm & 0.39 \\
\hline 0.6100 & 0.3873 & \pm & 0.0012 & 0.09687 & \pm & 0.00969 & 4.00 & \pm & 0.40 \\
\hline 0.6292 & 0.3588 & \pm & 0.0011 & 0.09091 & \pm & 0.00909 & 3.95 & \pm & 0.39 \\
\hline 0.6511 & 0.4104 & \pm & 0.0013 & 0.08331 & \pm & 0.00833 & 4.93 & \pm & 0.49 \\
\hline 0.6704 & 0.3187 & \pm & 0.0011 & 0.07610 & \pm & 0.00761 & 4.19 & \pm & 0.42 \\
\hline 0.691 & 0.3522 & \pm & 0.0012 & 0.07045 & \pm & 0.00705 & 5.00 & \pm & 0.50 \\
\hline 0.7088 & 0.3033 & \pm & 0.0010 & 0.06610 & \pm & 0.00661 & 4.59 & \pm & 0.46 \\
\hline 0.7306 & 0.3284 & \pm & 0.0011 & 0.06285 & \pm & 0.00628 & 5.22 & \pm & 0.52 \\
\hline 0.7483 & 0.2733 & \pm & 0.0010 & 0.05957 & \pm & 0.00596 & 4.59 & \pm & 0.46 \\
\hline 0.7729 & 0.3775 & \pm & 0.0013 & 0.05608 & \pm & 0.00561 & 6.73 & \pm & 0.67 \\
\hline 0.7893 & 0.2227 & \pm & 0.0008 & 0.05351 & \pm & 0.00535 & 4.16 & \pm & 0.42 \\
\hline 0.8097 & 0.2822 & \pm & 0.0011 & 0.05105 & \pm & 0.00511 & 5.53 & \pm & 0.55 \\
\hline 0.8314 & 0.3045 & \pm & 0.0011 & 0.04953 & \pm & 0.00495 & 6.15 & \pm & 0.62 \\
\hline 0.8504 & 0.2480 & \pm & 0.0010 & 0.04774 & \pm & 0.00477 & 5.19 & \pm & 0.52 \\
\hline 0.8708 & 0.2478 & \pm & 0.0010 & 0.04586 & \pm & 0.00459 & 5.40 & \pm & 0.54 \\
\hline 0.8899 & 0.2376 & \pm & 0.0010 & 0.04390 & \pm & 0.00439 & 5.41 & \pm & 0.54 \\
\hline
\end{tabular}


NEA/NSC/DOC(2006)1

Liquid Metal Fast Reactor - LMFR

FFTF-LMFR-RESR-001

CRIT-SPEC-REAC-COEF-MISC

Table 2.10.2 (cont'd). Calculated FFTF Core Center Low-Energy Electron Spectrum.

\begin{tabular}{|c|c|c|c|c|c|c|c|c|c|}
\hline $\begin{array}{l}\text { Energy } \\
(\mathrm{MeV})\end{array}$ & \multicolumn{3}{|c|}{$\begin{array}{l}\text { Calculated } \\
\text { Normalized }\end{array}$} & $\begin{array}{c}\text { Experi } \\
\text { Normalize } \\
\text { Flux } \\
\varphi / \varphi_{\max }\end{array}$ & enta & Data & \multicolumn{3}{|c|}{$\mathrm{C} / \mathrm{E}$} \\
\hline 0.9129 & 0.2851 & 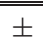 & 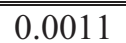 & 0.04245 & $\bar{~} \overline{ \pm}$ & 0.00425 & 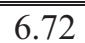 & 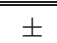 & 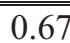 \\
\hline 0.9307 & 0.1996 & \pm & 0.0008 & 0.04037 & \pm & 0.00404 & 4.94 & \pm & 0.49 \\
\hline 0.9510 & 0.2218 & \pm & 0.0009 & 0.03983 & \pm & 0.00398 & 5.57 & \pm & 0.56 \\
\hline 0.9741 & 0.2571 & \pm & 0.0011 & 0.03788 & \pm & 0.00379 & 6.79 & \pm & 0.68 \\
\hline 0.9890 & 0.1645 & \pm & 0.0007 & 0.03750 & \pm & 0.00375 & 4.39 & \pm & 0.44 \\
\hline 1.0135 & 0.2487 & \pm & 0.0011 & 0.03507 & \pm & 0.00351 & 7.09 & \pm & 0.71 \\
\hline 1.0325 & 0.1832 & \pm & 0.0008 & 0.03425 & \pm & 0.00343 & 5.35 & \pm & 0.54 \\
\hline 1.0556 & 0.2249 & \pm & 0.0010 & 0.03290 & \pm & 0.00329 & 6.83 & \pm & 0.68 \\
\hline 1.0746 & 0.1855 & \pm & 0.0009 & 0.03203 & \pm & 0.00320 & 5.79 & \pm & 0.58 \\
\hline 1.0977 & 0.2137 & \pm & 0.0010 & 0.03026 & \pm & 0.00303 & 7.06 & \pm & 0.71 \\
\hline 1.1153 & 0.1465 & \pm & 0.0007 & 0.02975 & \pm & 0.00298 & 4.92 & \pm & 0.49 \\
\hline 1.1358 & 738 & \pm & 0.0009 & .02839 & \pm & 0.00284 & 6.12 & \pm & 0.61 \\
\hline 1.1561 & 0.1739 & \pm & 0.0009 & 0.02763 & \pm & 0.00276 & 6.29 & \pm & 0.63 \\
\hline 1.1764 & 0.1717 & \pm & 0.0008 & 0.02690 & \pm & 0.00269 & 6.38 & \pm & 0.64 \\
\hline 1.1955 & 0.1533 & \pm & 0.0008 & 0.02558 & \pm & 0.00256 & 5.99 & \pm & 0.60 \\
\hline 1.2159 & 0.1476 & \pm & 0.0008 & 0.02449 & \pm & 0.00245 & 6.03 & \pm & 0.60 \\
\hline 1.2391 & 0.1698 & \pm & 0.0009 & 0.02337 & \pm & 0.00234 & 7.27 & \pm & 0.73 \\
\hline 1.2581 & 0.1400 & \pm & 0.0007 & 0.02229 & \pm & 0.00223 & 6.28 & \pm & 0.63 \\
\hline 1.2772 & 0.1403 & \pm & 0.0008 & 0.02127 & \pm & 0.00213 & 6.59 & \pm & 0.66 \\
\hline 1.2976 & 0.1440 & \pm & 0.0008 & 0.02064 & \pm & 0.00206 & 6.98 & \pm & 0.70 \\
\hline 1.3179 & 0.1287 & \pm & 0.0007 & 0.02009 & \pm & 0.00201 & 6.41 & \pm & 0.64 \\
\hline 1.3396 & 0.1367 & \pm & 0.0008 & 0.01943 & \pm & 0.00194 & 7.03 & \pm & 0.70 \\
\hline 1.3573 & 0.1114 & \pm & 0.0006 & 0.01879 & \pm & 0.00188 & 5.93 & \pm & 0.59 \\
\hline 1.3803 & 0.1463 & \pm & 0.0008 & 0.01823 & \pm & 0.00182 & 8.02 & \pm & 0.80 \\
\hline 1.3980 & 0.1107 & \pm & 0.0007 & 0.01763 & \pm & 0.00176 & 6.28 & \pm & 0.63 \\
\hline 1.4184 & 0.1222 & \pm & 0.0007 & 0.01699 & \pm & 0.00170 & 7.19 & \pm & 0.72 \\
\hline 1.4360 & 0.0940 & \pm & 0.0006 & 0.01660 & \pm & 0.00166 & 5.66 & \pm & 0.57 \\
\hline 1.4604 & 0.1318 & \pm & 0.0008 & 0.01600 & \pm & 0.00160 & 8.24 & \pm & 0.83 \\
\hline 1.4781 & 0.0963 & \pm & 0.0006 & 0.01567 & \pm & 0.00157 & 6.14 & \pm & 0.62 \\
\hline 1.4957 & 0.0949 & \pm & 0.0006 & 0.01521 & \pm & 0.00152 & 6.24 & \pm & 0.63 \\
\hline 1.5173 & 0.1168 & \pm & 0.0007 & 0.01501 & \pm & 0.00150 & 7.78 & \pm & 0.78 \\
\hline 1.5378 & 0.1070 & \pm & 0.0007 & 0.01432 & \pm & 0.00143 & 7.47 & \pm & 0.75 \\
\hline 1.5583 & 0.0981 & \pm & 0.0006 & 0.01343 & \pm & 0.00134 & 7.30 & \pm & 0.73 \\
\hline 1.5799 & 0.0986 & \pm & 0.0006 & 0.01330 & \pm & 0.00133 & 7.41 & \pm & 0.74 \\
\hline 1.5989 & 0.0871 & \pm & 0.0006 & 0.01295 & \pm & 0.00129 & 6.73 & \pm & 0.67 \\
\hline 1.6179 & 0.0872 & \pm & 0.0006 & 0.01256 & \pm & 0.00126 & 6.94 & \pm & 0.70 \\
\hline 1.6423 & 0.1124 & \pm & 0.0008 & 0.01203 & \pm & 0.00120 & 9.35 & \pm & 0.94 \\
\hline 1.6614 & 0.0868 & \pm & 0.0006 & 0.01140 & \pm & 0.00114 & 7.61 & \pm & 0.76 \\
\hline 1.679 & 0.0764 & \pm & 0.0005 & 0.01117 & \pm & 0.00112 & 6.84 & \pm & 0.69 \\
\hline 1.698 & 0.0763 & \pm & 0.0005 & 0.01102 & \pm & 0.00110 & 6.92 & \pm & 0.69 \\
\hline
\end{tabular}

Revision: 0 
NEA/NSC/DOC(2006)1

Liquid Metal Fast Reactor - LMFR

FFTF-LMFR-RESR-001

CRIT-SPEC-REAC-COEF-MISC

Table 2.10.2 (cont'd). Calculated FFTF Core Center Low-Energy Electron Spectrum.

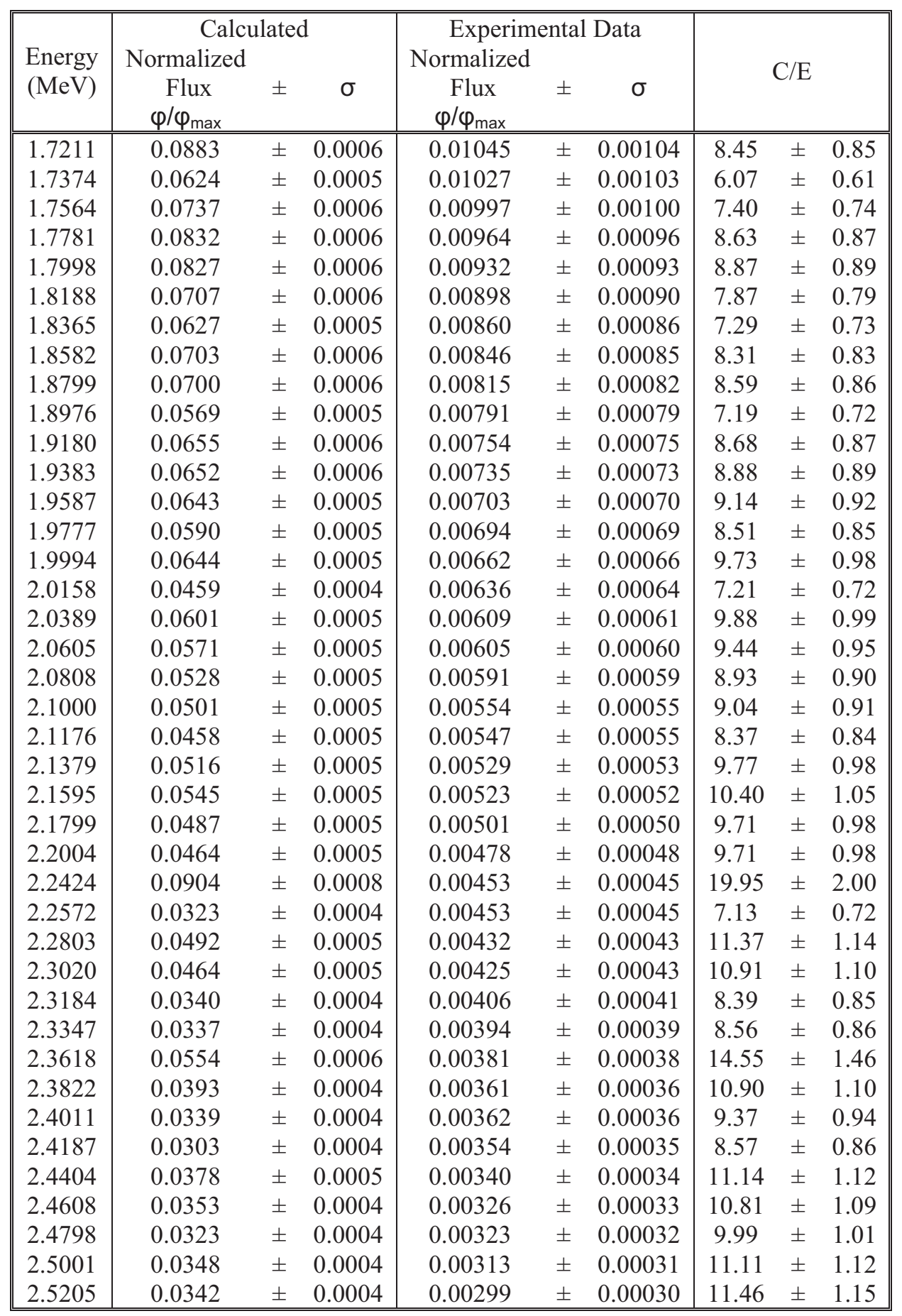


NEA/NSC/DOC(2006)1

Liquid Metal Fast Reactor - LMFR

FFTF-LMFR-RESR-001

CRIT-SPEC-REAC-COEF-MISC

Table 2.10.2 (cont'd). Calculated FFTF Core Center Low-Energy Electron Spectrum.

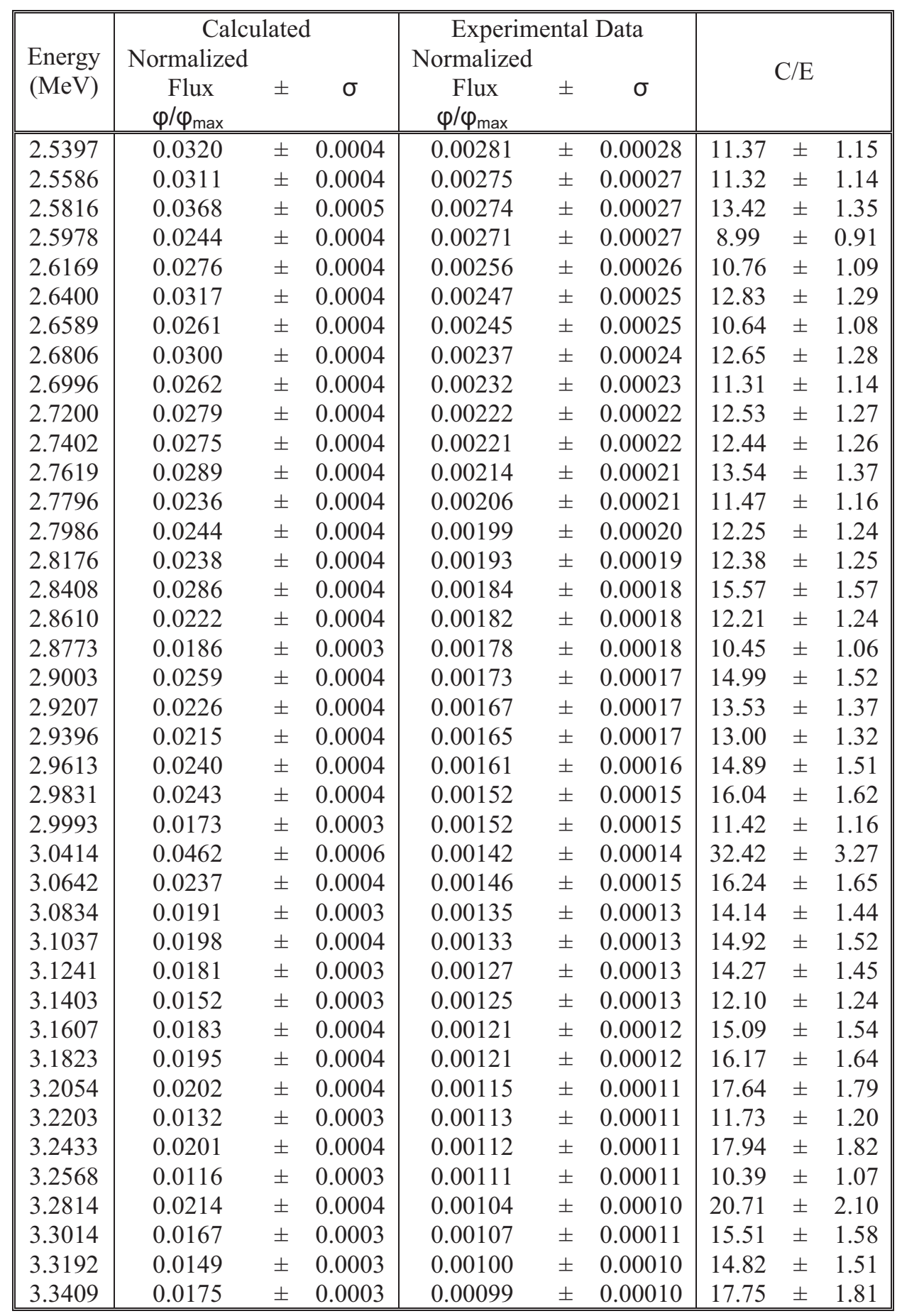


NEA/NSC/DOC(2006)1

Liquid Metal Fast Reactor - LMFR

FFTF-LMFR-RESR-001

CRIT-SPEC-REAC-COEF-MISC

Table 2.10.2 (cont'd). Calculated FFTF Core Center Low-Energy Electron Spectrum.

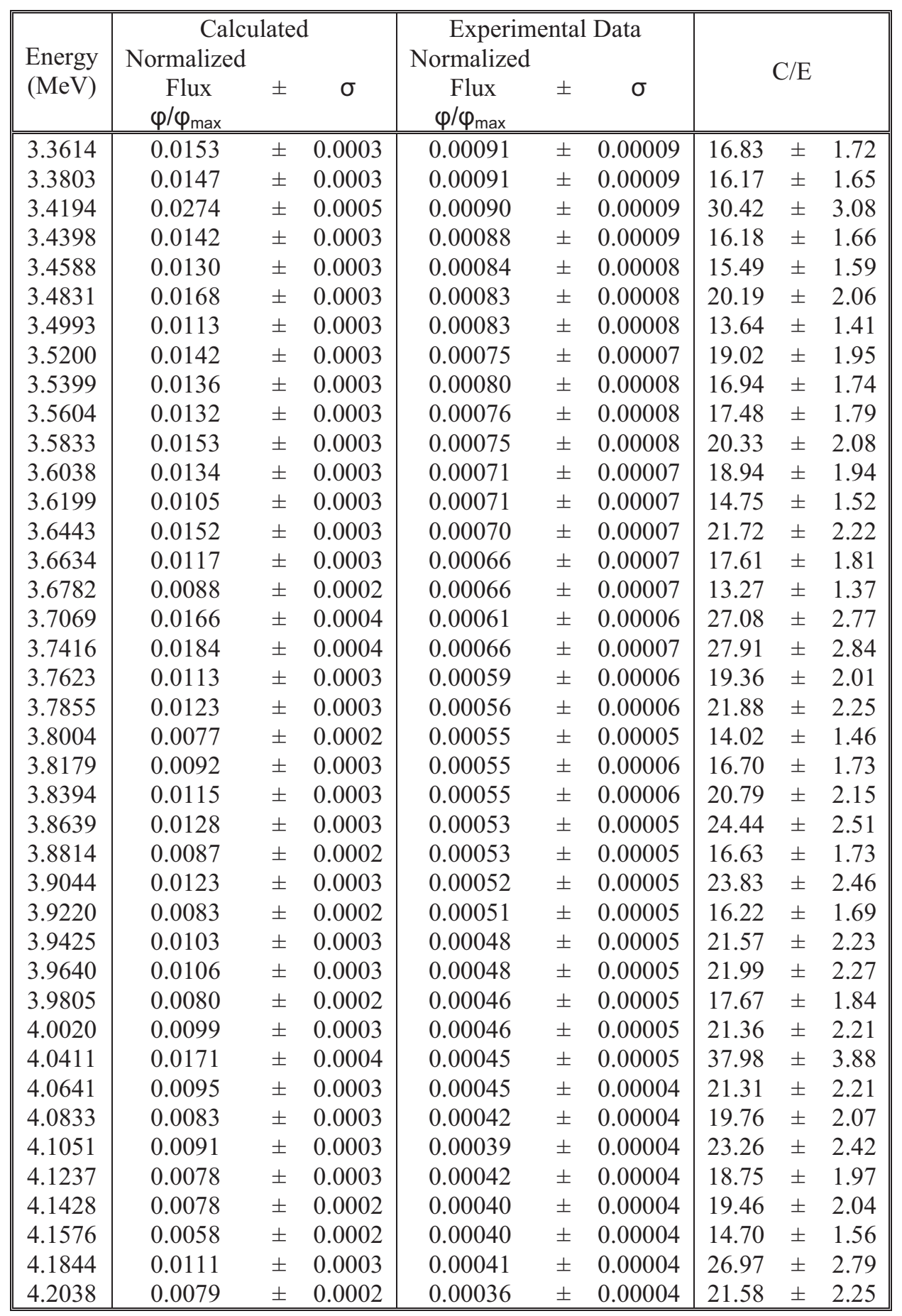

Revision: 0 
NEA/NSC/DOC(2006)1

Liquid Metal Fast Reactor - LMFR

FFTF-LMFR-RESR-001

CRIT-SPEC-REAC-COEF-MISC

Table 2.10.2 (cont'd). Calculated FFTF Core Center Low-Energy Electron Spectrum.

\begin{tabular}{|c|c|c|c|c|c|c|c|c|c|}
\hline $\begin{array}{l}\text { Energy } \\
(\mathrm{MeV})\end{array}$ & $\begin{array}{r}\text { Calc } \\
\text { Normalized } \\
\text { Flux } \\
\varphi / \varphi_{\max } \\
\end{array}$ & $\begin{array}{l}\text { Calculated } \\
\text { Normalized }\end{array}$ & $\sigma$ & $\begin{array}{c}\text { Experir } \\
\text { Normalizec } \\
\text { Flux } \\
\varphi / \varphi_{\max }\end{array}$ & nta & Data & \multicolumn{3}{|c|}{$\mathrm{C} / \mathrm{E}$} \\
\hline 2241 & 0.0083 & \pm & 0.0003 & 0.00036 & \pm & 0.00004 & 23.01 & \pm & 2.40 \\
\hline & 0.0154 & \pm & 0.0003 & 0.00036 & & 0.00004 & 43.16 & \pm & 4.43 \\
\hline 4.2850 & 0.0086 & \pm & 0.0003 & 0.00034 & \pm & 0.00003 & 25.49 & \pm & 2.65 \\
\hline 4.3029 & 0.0068 & \pm & 0.0002 & 0.00032 & \pm & 0.00003 & 21.51 & \pm & 2.26 \\
\hline 4.3431 & 0.0157 & \pm & 0.0004 & 0.00033 & \pm & 0.00003 & 48.12 & \pm & 4.93 \\
\hline 4.3635 & 0.0071 & \pm & 0.0002 & 0.00031 & \pm & 0.00003 & 22.71 & \pm & 2.39 \\
\hline 4.3837 & 0.0073 & \pm & 0.0002 & .00031 & \pm & 0.00003 & 23.34 & \pm & 2.45 \\
\hline 4.4014 & 0.0057 & \pm & 0.0002 & 0.00030 & \pm & 0.00003 & 18.65 & \pm & 1.99 \\
\hline 4.4218 & 0.0064 & \pm & 0.0002 & 0.00029 & \pm & 0.00003 & 21.88 & \pm & 2.32 \\
\hline 4.4432 & 0.0074 & \pm & 0.0003 & .00030 & \pm & 0.00003 & 24.22 & \pm & 2.56 \\
\hline 4.4841 & 0.0133 & \pm & 0.0003 & 0.00027 & \pm & 0.00003 & 48.79 & \pm & 5.03 \\
\hline 4.5029 & 0.0061 & \pm & 0.0002 & 000 & \pm & 0.00003 & 21.66 & \pm & 2.31 \\
\hline 4.5191 & 0.0056 & \pm & 0.0002 & 00 & \pm & 0.00003 & 20.11 & \pm & 2.16 \\
\hline 4.5447 & 0082 & \pm & 0.0003 & 00 & \pm & 0.00003 & 29.80 & \pm & 3.12 \\
\hline 4.5 & 0071 & \pm & 0.0002 & 00 & F & 0.00003 & 5.34 & \pm & 2.68 \\
\hline 4.5868 & 064 & \pm & 0.0002 & 0 . & \pm & 0.00003 & 5.02 & \pm & 2.66 \\
\hline 4.6086 & 0068 & \pm & 0.0002 & 4 & $=$ & 02 & 7.97 & \pm & 2.96 \\
\hline 4.6 & 3 & \pm & 0.0002 & 0 . & & 03 & 45 & \pm & 2.21 \\
\hline 4.6 & 9 & \pm & 0.0 & 0 . & & 22 & 20 & \pm & 3.09 \\
\hline 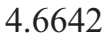 & 7 & \pm & 0.00 & 0 . & & & 5 & \pm & 2.26 \\
\hline & 3 & \pm & 0.0002 & 0.00 & & & 29.44 & \pm & 3 \\
\hline & 3 & \pm & 0.00 & 0 . & & & 30.07 & \pm & 3. \\
\hline 2 & 45 & + & 0.0002 & 00 & & 2 & 20.00 & \pm & 2. \\
\hline 540 & 0.0099 & \pm & 0.0003 & 00 & & 0.00002 & 45.13 & \pm & 4.69 \\
\hline 345 & 0.0055 & \pm & 0.0002 & 00 & & 0.00002 & 26.53 & \pm & 2. \\
\hline 3056 & 0.0051 & \pm & 0.0002 & 00 & & 0.00002 & 22.18 & \pm & 2. \\
\hline 288 & 0.0057 & \pm & 0.0002 & 0.000 & & 0.00002 & 25.95 & \pm & 2.77 \\
\hline 491 & 0.0051 & \pm & 0.0002 & 0.0002 & & 0.00002 & 23.68 & \pm & 2.58 \\
\hline 882 & 0.0096 & \pm & 0.0003 & 0.0002 & & 0.00002 & 45.62 & \pm & 4.76 \\
\hline & 0.0042 & \pm & 0.0002 & 0.00 & & 0.00002 & 20.03 & \pm & 2.21 \\
\hline 274 & 0.0051 & \pm & 0.0002 & 0.0002 & & 0.00002 & 24.43 & \pm & 2.64 \\
\hline & 0.0095 & \pm & 0.0003 & 00 & $I$ & 0.00002 & 47.12 & \pm & 4.9 \\
\hline & 0.0049 & \pm & 0.0 & 0.00 & 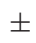 & 0.0 & 24.90 & \pm & 2.68 \\
\hline & 0 & \pm & 0.0 & 0.00020 & \lrcorner & 0.0 & 23.46 & \pm & 2.5 \\
\hline & 0 & \pm & 0.0 & 00019 & & 0.0 & 43.23 & \pm & 4.54 \\
\hline & (20 & \pm & $0.0 \mathrm{C}$ & 00 & & 000 & 25.62 & \pm & 2.7 \\
\hline & 0043 & \pm & 0.0002 & 00 & \pm & 0.00002 & 23.90 & \pm & 2.62 \\
\hline & 0044 & \pm & 0.0002 & 00018 & \pm & 0.00002 & 22.67 & \pm & 2.48 \\
\hline 5.1300 & 0.0046 & \pm & 0.0002 & 0.00019 & \pm & 0.00002 & 24.71 & \pm & 2.68 \\
\hline
\end{tabular}


NEA/NSC/DOC(2006)1

Liquid Metal Fast Reactor - LMFR

FFTF-LMFR-RESR-001

CRIT-SPEC-REAC-COEF-MISC

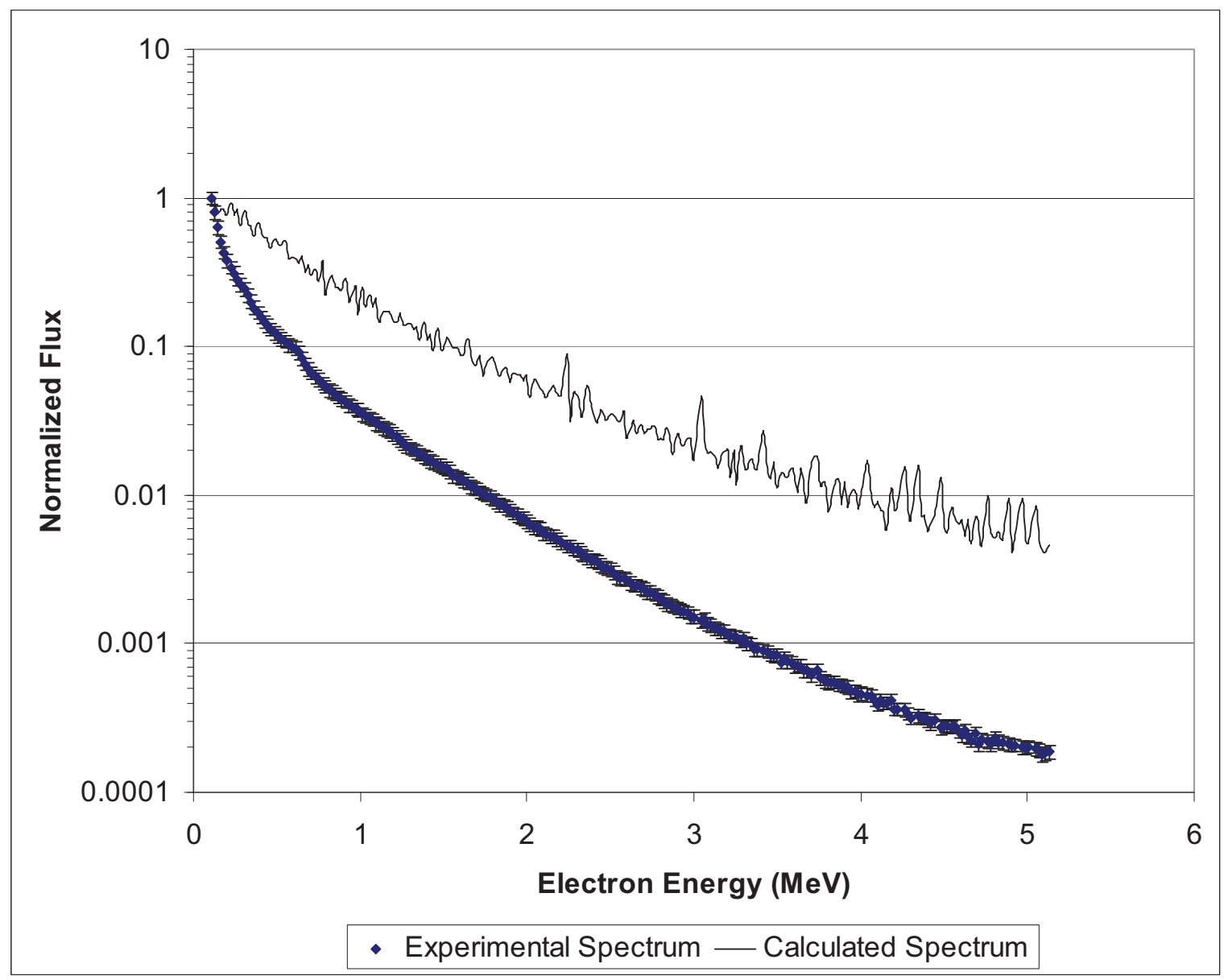

Figure 2.10.2. Low-Energy Electron Spectrum in FFTF Core Center.

\subsubsection{Low-Energy Gamma-Ray Spectrum}

As with the electron-spectrum, Figure 1.10.2 does not have an accompanying table with actual values. The means of normalization for the relative flux for the gamma-ray spectrum measurements is also unclear. Therefore, this chart was also digitized and the extracted data were renormalized to the second greatest value to produce a normalized set of data that can be compared against the calculated results. The data were not normalized to the maximum value because the minimum threshold energy for physical detection was unknown, resulting in a significant difference between the experimental and calculated values for the lowest energy data point.

No uncertainty was reported for the experimental measurements; the solid state SiLi detectors were used for counting gamma-rays. A brief literature search revealed measurement uncertainties of up to approximately $7-10 \%$ when using SiLi detectors. ${ }^{\mathrm{a}, \mathrm{b}}$ An additional uncertainty needs to be accounted for due to the effects of accurately digitizing the data from the figures. To assess the uncertainty in digitization, the gamma spectra charts were digitized and compared with their respective data. The

\footnotetext{
${ }^{a}$ N. J. Murray, A. D. Holland, D. R. Smith, J. P. Gow, P. J. Pool, and D. J. Burt, “The X-Ray Quantum Efficiency Measurement of High Resistivity CCDs," Nucl. Inst. Meth. A, 604, 180-182 (2009).

${ }^{\mathrm{b}}$ P. Yalcin, A. Sulun, A. Bastug, Y. Kurucu, and Y. Sahin, "The Efficieny Determination for Si(Li) Detector in the 3-723 keV Energy Range,” Can. J. Anal. Sci. Spect., 50, 108-115 (2005).
} 
NEA/NSC/DOC(2006)1

Liquid Metal Fast Reactor - LMFR

FFTF-LMFR-RESR-001

CRIT-SPEC-REAC-COEF-MISC

average uncertainty across all energy, flux, and uncertainty points was $\sim 1.8 \%$. An uncertainty of $10 \%$ was selected to represent the uncertainty in the gamma-ray spectrum measurements. This uncertainty is assumed to include the additional uncertainty due to data digitization. It is recognized that the actual uncertainty in the measurements is energy dependent, but additional information is unavailable.

An evaluation similar to that for the neutron spectra (Section 2.3) was performed using MCNP5. The same model was utilized, with the IRT; however, the flux tally instead tracked the photon flux at the center of the core. The calculated photon spectra are obtained by taking the variance-weighted average of results obtained using six variations of the input deck (Appendix A.1) with different random number seeds and tallies of the photon flux (Appendix A.10). This approach was used to reduce the statistical uncertainty in the neutron flux tallies because the relative error values obtained can under-predict the true uncertainty in the calculated spectra. ${ }^{a}$

The digitized values for the low-energy gamma-ray spectrum at core center midplane and their approximated uncertainty are reported in Table 3.10.2 and shown in Figure 3.10.2. The calculated results are shown in Table 4.10.1 and Figure 4.10.1. There is in general good correlation between the calculated and expected experimental benchmark values for the normalized photon energy spectrum. On average, the calculated normalized flux is $\sim 95 \%$ greater than the experimentally measured benchmark values. Homogenization of the IRT would have had a significant impact as the model doesn't fully account for scatter and streaming in the IRT.

Gamma production cross section data were not available for the isotopes ${ }^{238} \mathrm{Pu},{ }^{99} \mathrm{Mo}$, and ${ }^{100} \mathrm{Mo}$ in the ENDF/B-VII.0 library. These isotopes are minor constituents in the materials of the FFTF and their contribution to uncertainties in the gamma spectra analysis is considered negligible.

${ }^{a}$ F. B. Brown, “A Review of Best Practices for Monte Carlo Criticality Calculations,” Proc. NCSD 2009, Richland, WA, September 13-17 (2009).

Revision: 0

Page 144 of 304

Date: March 31, 2010 
NEA/NSC/DOC(2006)1

Liquid Metal Fast Reactor - LMFR

FFTF-LMFR-RESR-001

CRIT-SPEC-REAC-COEF-MISC

\subsection{BENCHMARK SPECIFICATIONS}

\subsection{Benchmark-Model Specifications for Critical and / or Subcritical Measurements}

Whereas insufficient information is publicly available, a finely-detailed benchmark model could not be established. A benchmark of the FFTF was prepared and analyzed with as much detail as feasible. A comprehensive simplification bias for this model could also not be appropriately determined. Once this information is made available, the FFTF benchmark could be adjusted as appropriate. However, provision of a more detailed FFTF benchmark model will probably not show a significant bias in eigenvalue calculations.

Sufficient details regarding the pin structure of the fuel and absorber assemblies are available. Typically fast neutron systems are insensitive to homogenization. However, the most significant bias would be that of homogenizing the pin lattice structures. Homogenization of the pin lattices in the absorber and fuel assemblies in the benchmark model incurred a calculational bias of approximately $-1.1 \% \Delta \mathrm{k}_{\text {eff }}$ compared to a heterogeneous model of the pin structures (see Appendix G); therefore, the fuel and absorber pinlattice structure was retained in the benchmark model. A HEX-Z partially-homogenized FFTF fullyloaded core critical configuration is provided. It is similar to the model developed by the engineers that worked with the initial isothermal physics testing (Appendix F). However, this model includes the fuel and absorber assemblies modeled with their respective heterogeneous pin-lattice structures.

\subsubsection{Description of the Benchmark Model Simplifications}

Significant simplifications were incurred to develop a general-use benchmark model of the FFTF because of a lack of information publicly available to determine dimensions and compositions. Fuel and absorber pin structures were modeled as heterogeneously as possible to retain their effects upon system criticality. The effect of homogenization upon other core components is much less significant.

Some simplifications include the substitution of the fueled open test assembly (FOTA) positions in the core with conventional fuel assemblies and the homogenization of the fuel and absorber pin wire wraps with the sodium coolant between the fuel pins. These effects are judged to be negligible. The inter-duct wire wrap in the fixed shim control rods is also not included in the benchmark model. Its removal was also determined to be negligible (Section 2.1.3.7).

Fixed shim control rods are modeled identical to fully inserted control rods. Insufficient information is available to assess a bias, if any, with its associated uncertainty. It is expected that neutronically the result would be negligible.

The exact placement of radial reactor shielding is unclear. An uncertainty was assessed for variation in reflector placement (Section 2.1.2.8), but no bias was applied. The magnitude of the uncertainty is believed to compensate for the uncertainty in whether application of a bias for this parameter is relevant.

Further simplifications include the absence of the rounding of edges, chamfers, welds, and dishing or various reactor components. This includes the stack of fuel pellets in each fuel pin that are modeled as a single pin with smeared density. It is assumed that the uncertainty and/or bias associated with this simplifications would be negligible compared to the total benchmark uncertainty.

The fuel and absorber pellets are modeled as stacks and not individual pellets. The bias is negligible.

The helium in the gaps between fuel or absorber pins and their respective cladding was not included in the benchmark model; they were instead modeled as void. The fuel gaps would also have contained a small amount of tag gas (not included in the model or analysis). The removal of the gas from the model was found to have negligible effects (Section 2.1.3.4). 
NEA/NSC/DOC(2006)1

Liquid Metal Fast Reactor - LMFR

FFTF-LMFR-RESR-001

CRIT-SPEC-REAC-COEF-MISC

The benchmark model includes only the core components and radial shielding; support structures, vessels, and the surrounding facility are not included. An approximation of room-return effects showed negligible results and not applied as a bias (Section 3.1.1.1).

The impurity content of the sodium coolant was evaluated in Section 2.1.3.3. The weight percent of the sodium was adjusted to contain the average composition of impurities. The maximum concentration of impurities was then evaluated to determine a $\Delta \mathrm{k}_{\text {eff }}$ of $-0.00016 \pm 0.00008$. Because the evaluated bias is not significantly greater than the uncertainty, it is neglected as a bias to the benchmark model and treated only as an uncertainty.

The impurities in the insulator and fuel pellets were also addressed in Sections 2.1.3.6 and 2.1.3.7 for the inner and outer fuel enrichment zones, respectively. The impurity content was addressed similar to the method described for the sodium coolant. The effective bias in the impurity content for the insulator pellets was found to be $-0.00017 \pm 0.00008$ and $0.00002 \pm 0.00008$ for the inner and outer fuel regions, respectively. As with the sodium coolant bias, these biases are considered negligible and included only as uncertainties.

The $\Delta \mathrm{k}_{\text {eff }}$ values determined for the inner and outer fuel pellets were $-0.00079 \pm 0.00008$ and $-0.00066 \pm$ 0.00008 , respectively. They are treated as half uncertainty and half bias.

The impurities in the absorber pellets were addressed in Section 2.1.3.8 and treated as described for the other pellet impurity contents. The effective bias in the impurity content of the absorber pellets was found to be $0.00008 \pm 0.00007$. Because the bias is the same as the statistical uncertainty, it is considered negligible and treated only as an uncertainty.

The fuel and absorber pins of the FFTF were not homogenized in the model. The effect of homogenization on these core components was performed (Section 2.1.4.2) but are not included in the benchmark model nor as a bias to the benchmark model.

There is insufficient data to analyze the heterogeneous effects or effects of homogenization for some of the core components. These components are typically above or below the fueled region of the core, or represent a small fraction of the in-core materials. Heterogeneous effects and the effect of homogenization are expected to represent an insignificant contribution to the total uncertainty of the benchmark model, as the uncertainties in other geometrical properties (Section 2.1.2) and density of the primary structural components (SS316, Inconel 600, and sodium in Sections 2.1.3.1 through 2.1.3.3) are relatively insignificant.

A summary of the bias assessment performed for the fully-loaded core critical benchmark model of the FFTF is provided in Table 3.1.1. 
NEA/NSC/DOC(2006)1

Liquid Metal Fast Reactor - LMFR

FFTF-LMFR-RESR-001

CRIT-SPEC-REAC-COEF-MISC

Table 3.1.1. Summary of Benchmark Model Biases for the Fully-Loaded FFTF.

\begin{tabular}{|c|c|}
\hline Simplification & $\Delta \mathrm{k}_{\mathrm{eff}}$ \\
\hline \hline Substitution of fueled assemblies for FOTA & neg \\
Homogenization of pin wire wraps & neg \\
Removal of fixed shim inter-duct wire wraps & neg \\
Placement of radial reactor shielding & $\mathrm{NA}$ \\
Geometric simplification & neg \\
Pellets modeled in stacks & neg \\
Void substituted for helium in clad gaps & neg \\
Room return effects & neg \\
Removal of Sodium Impurities & neg \\
Removal of Inner Insulator Pellet Impurities & neg \\
Removal of Outer Insulator Pellet Impurities & neg \\
Removal of Inner Fuel Pellet Impurities & -0.00040 \\
Removal of Outer Fuel Pellet Impurities & -0.00033 \\
Removal of Absorber Pellet Impurities & neg \\
Homogenization of non-fuel/absorber components & neg \\
Homogenization of fuel/absorber components & NA \\
\hline Total & $\mathbf{- 0 . 0 0 0 7 3}$ \\
\hline \hline
\end{tabular}

\subsubsection{Room Return}

Room return effects were approximated by modeling the reactor core within the reactor vessel shown in Figure E.1.4. Three feet of concrete was then added around all sides of the vessel to represent shielding and structural material. The vessel was modeled with stainless steel 316 and was filled with liquid sodium coolant. Type I Portland cement was used as the concrete material and its composition is shown in Table 3.1.2. Volatile material was supplemented to account for its loss ("ignition loss") and to bring the cement composition to $100 \mathrm{wt} . \%$ : $0.5 \mathrm{wt} . \%$ hydrogen and $1.8 \mathrm{wt} . \%$ sodium. The density of the cement was $1.5 \mathrm{~g} / \mathrm{cm}^{3}$. The room-return effects are shown in Table 3.1.3. The room configuration was not included in the benchmark models.

The uncertainty and bias in the room return are negligible. 
NEA/NSC/DOC(2006)1

Liquid Metal Fast Reactor - LMFR

FFTF-LMFR-RESR-001

CRIT-SPEC-REAC-COEF-MISC

Table 3.1.2. Type I Portland Cement Composition. ${ }^{(a)}$

\begin{tabular}{|c||c||}
\hline Compound & Weight Percent \\
\hline \hline$(\mathrm{CaO})_{3} \cdot \mathrm{SiO}_{2}$ & 55 \\
\hline$(\mathrm{CaO})_{2} \cdot \mathrm{SiO}_{2}$ & 19 \\
\hline$(\mathrm{CaO})_{3} \cdot \mathrm{Al}_{2} \mathrm{O}_{3}$ & 10 \\
\hline$(\mathrm{CaO})_{4} \cdot \mathrm{Al}_{2} \mathrm{O}_{3} \cdot \mathrm{Fe}_{2} \mathrm{O}_{3}$ & 7 \\
\hline $\mathrm{MgO}$ & 2.8 \\
\hline $\mathrm{SO}_{3}$ & 2.9 \\
\hline Free $\mathrm{CaO}$ & 1 \\
\hline Ignition Loss & 1 \\
\hline
\end{tabular}

(a) ASTM C150, ASTM International, West Conshohocken, Pennsylvania, (2007).

Table 3.1.3. Uncertainty in Room Return Effects.

\begin{tabular}{|c|ccc|c|ccc||}
\hline \hline Deviation & $\Delta \mathrm{k}$ & \pm & $\sigma_{\Delta \mathrm{k}}$ & $\begin{array}{c}\text { Scaling } \\
\text { Factor }\end{array}$ & $\Delta \mathrm{k}_{\mathrm{eff}}(1 \sigma)$ & \pm & $\sigma_{\Delta \mathrm{keff}}$ \\
\hline \hline Added Vessel, Sodium, and Concrete & 0.00006 & \pm & 0.00008 & 1 & 0.00006 & \pm & 0.00008 \\
\hline \hline
\end{tabular}

\subsubsection{Dimensions}

\subsubsection{Driver Fuel Assemblies}

\section{Driver Fuel Pin}

The driver fuel pins include a lower axial Inconel reflector, a lower $\mathrm{UO}_{2}$ insulator pellet, a stack of MOX fuel pellets, an upper $\mathrm{UO}_{2}$ insulator pellet, and an upper axial Inconel reflector. The pins are contained within stainless steel clad. The gap between the pellets and clad is filled with void space. Driver fuel pin dimensions and depiction are provided in Table 3.1.4 and Figure 3.1.1, respectively.

Table 3.1.4. Driver Fuel Pin Dimensions.

\begin{tabular}{|c|ccc||}
\hline Component & $\begin{array}{c}\text { Inner Diameter } \\
(\mathrm{cm})\end{array}$ & $\begin{array}{c}\text { Outer Diameter } \\
(\mathrm{cm})\end{array}$ & $\begin{array}{c}\text { Height } \\
(\mathrm{cm})\end{array}$ \\
\hline \hline Axial Inconel Reflector & -- & 0.48133 & 14.478 \\
$\mathrm{UO}_{2}$ Insulator Pellet & -- & 0.4826 & 2.032 \\
MOX Fuel Pellet Stack & -- & 0.49403 & 91.44 \\
Stainless Steel Cladding & 0.508 & 0.5842 & 124.46 \\
\hline
\end{tabular}


NEA/NSC/DOC(2006)1

Liquid Metal Fast Reactor - LMFR

FFTF-LMFR-RESR-001

CRIT-SPEC-REAC-COEF-MISC

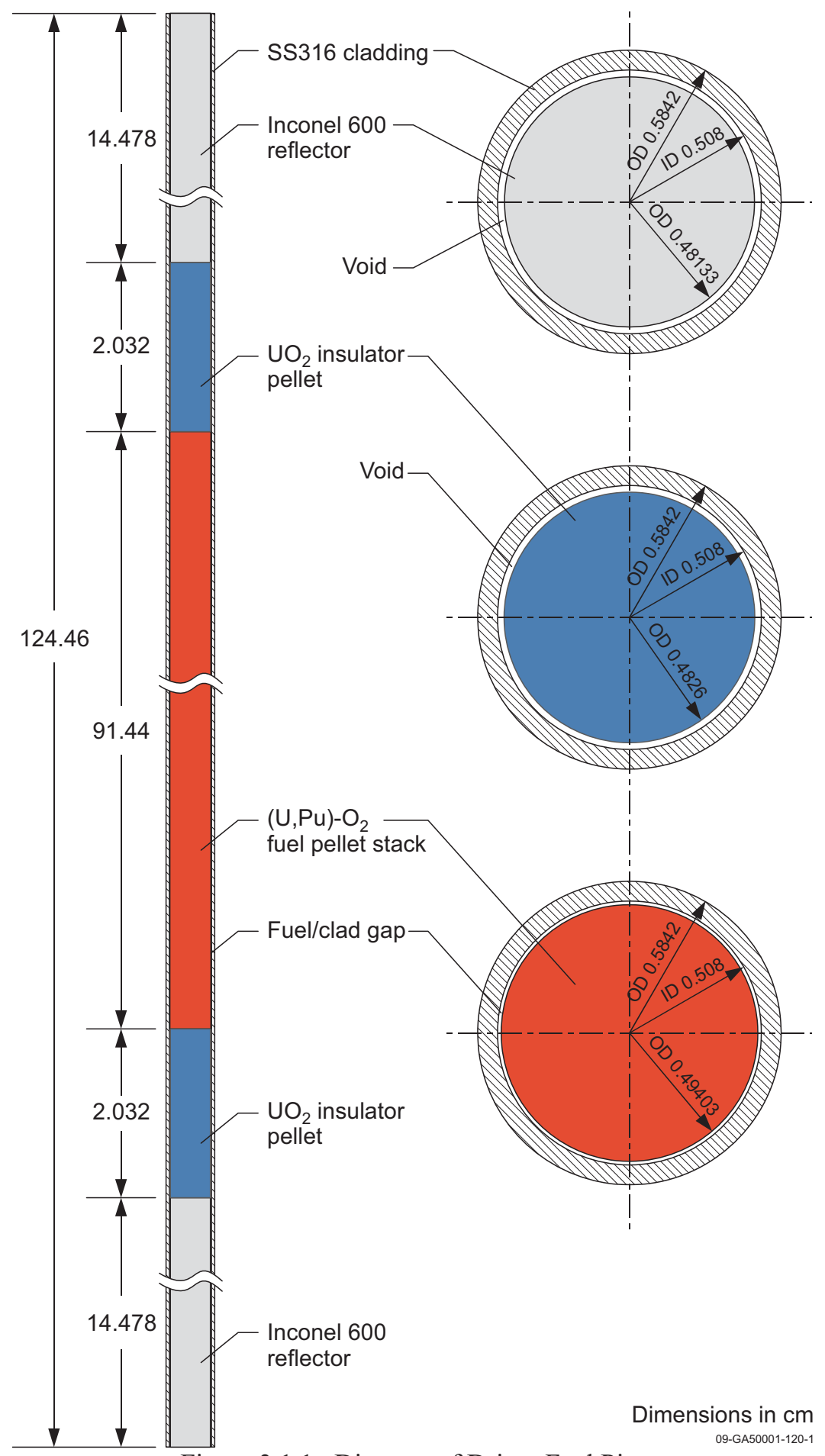

Figure 3.1.1. Diagram of Driver Fuel Pin. 
FFTF-LMFR-RESR-001

CRIT-SPEC-REAC-COEF-MISC

\section{Driver Fuel Assembly}

The driver fuel pins are placed in an array of 217 pins with a pitch of $0.72644 \mathrm{~cm}$ in a homogenized medium of sodium coolant and stainless steel wire wrap used for spacing the fuel pins. The fuel-pin lattice is contained within a hexagonal stainless steel duct with an inside flat-to-flat distance of 11.0109 $\mathrm{cm}$ and an outside flat-to-flat distance of $11.6205 \mathrm{~cm}$ (the duct thickness is $0.3048 \mathrm{~cm}$ ). The outside of the duct is surrounding by sodium coolant out to the core position hex flat-to-flat distance (i.e., pitch) of $12.051 \mathrm{~cm}$. A cross section of the driver fuel pin lattice is shown in Figure 3.1.2.

The fuel pin lattice is sandwiched between three homogenized layers in the driver fuel assembly. The bottom layers are the lower axial shield region and the fuel pin attachment region; the top layer is the gas plenum region, as shown in Figure 3.1.3. The lower axial shield region is $54.61 \mathrm{~cm}$ in length, the fuel pin attachment region is $10.16 \mathrm{~cm}$ in length, and the gas plenum region is $109.22 \mathrm{~cm}$ in length, with a total driver fuel assembly length of $298.45 \mathrm{~cm}$ in the model.

\section{Fueled Open Test Assemblies}

Fueled open test assemblies are modeled identically as their respectively-zoned driver fuel assemblies; i.e., they do not include instrumentation. 


\section{NEA/NSC/DOC(2006)1}

\section{Liquid Metal Fast Reactor - LMFR}

FFTF-LMFR-RESR-001

\section{CRIT-SPEC-REAC-COEF-MISC}

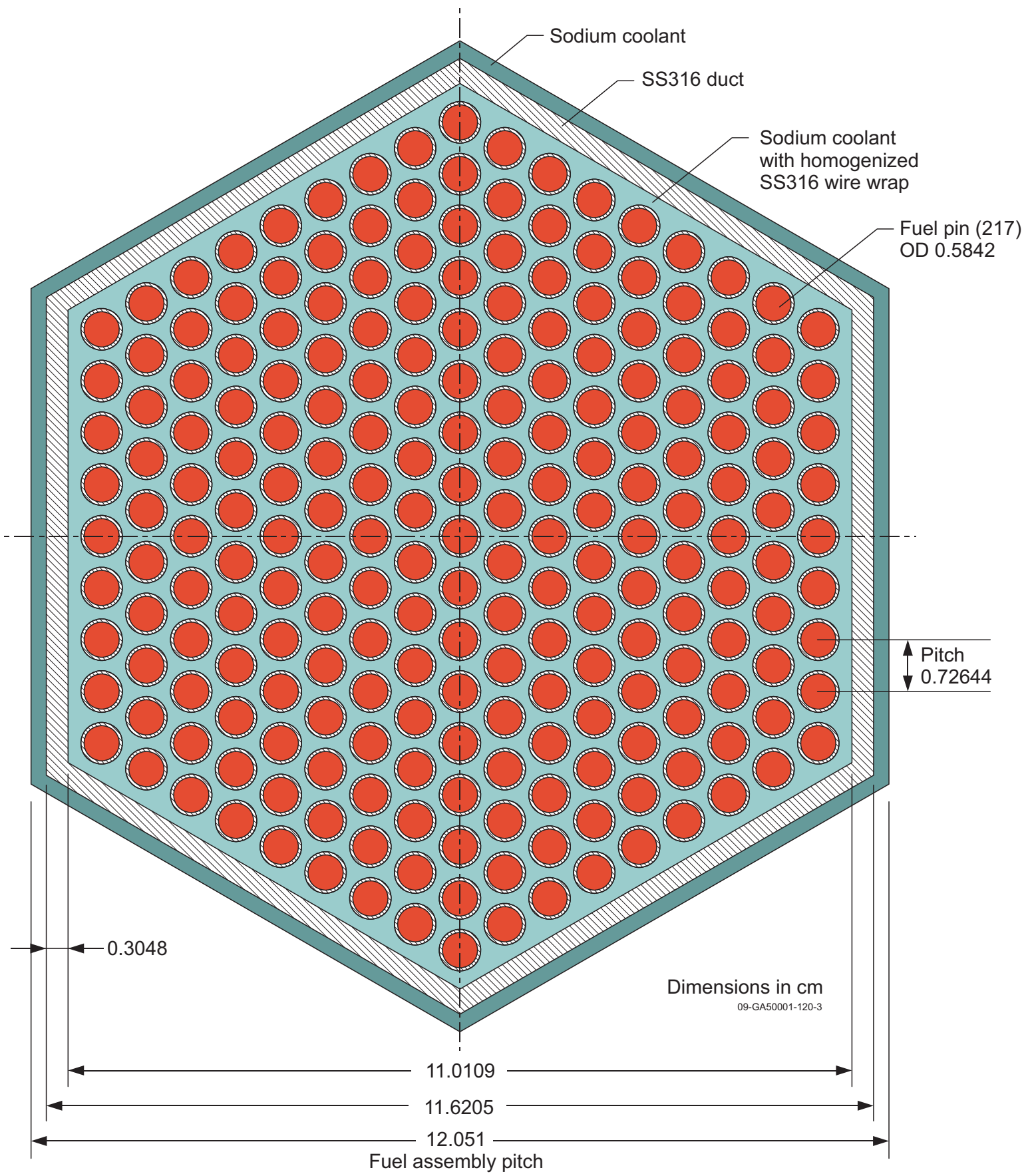

Figure 3.1.2. Diagram of Driver Fuel Pin Lattice. 
NEA/NSC/DOC(2006)1

Liquid Metal Fast Reactor - LMFR

FFTF-LMFR-RESR-001

CRIT-SPEC-REAC-COEF-MISC
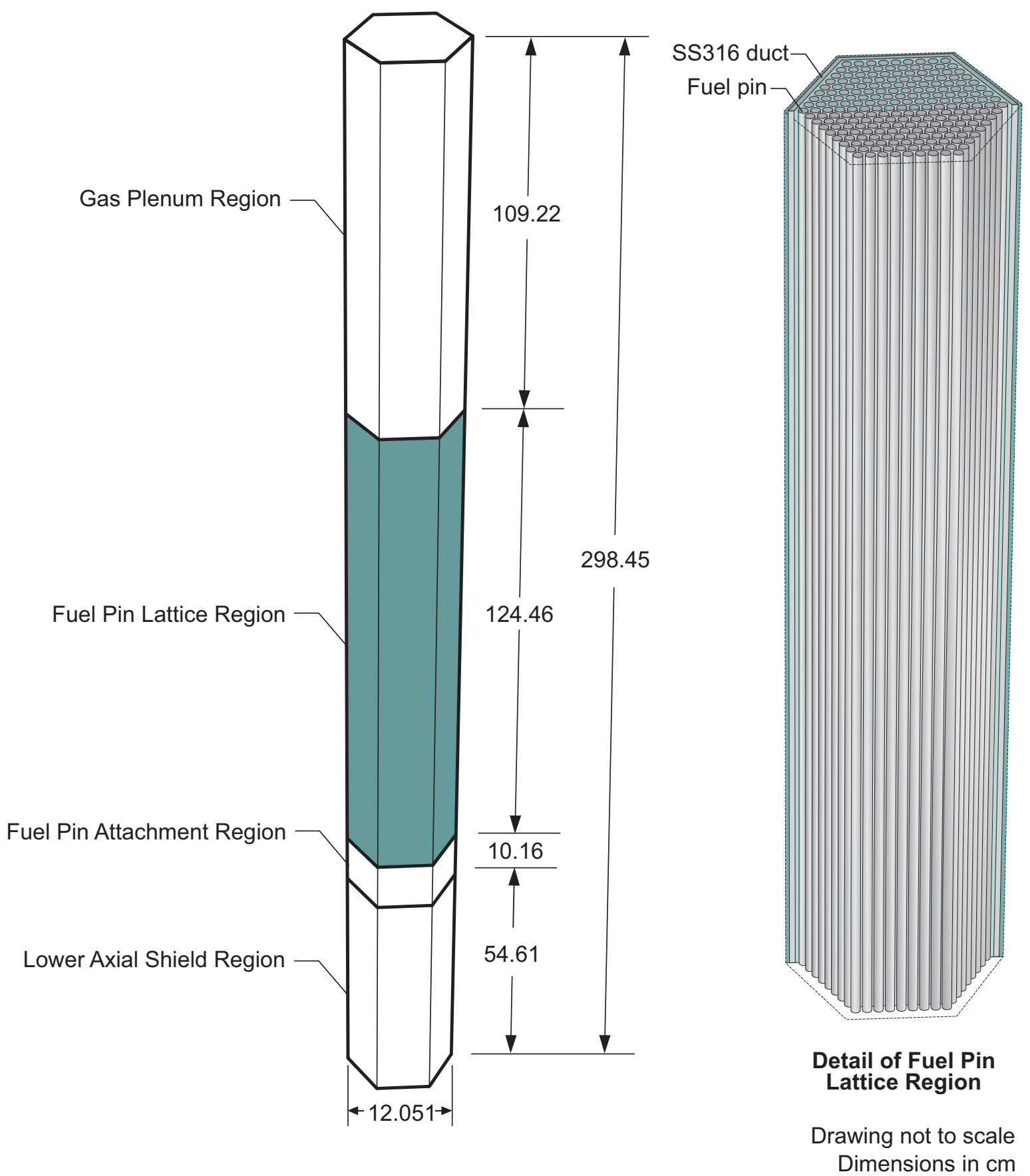

Figure 3.1.3. Diagram of Partially-Homogenized Driver Fuel Assembly. 
NEA/NSC/DOC(2006)1

Liquid Metal Fast Reactor - LMFR

FFTF-LMFR-RESR-001

CRIT-SPEC-REAC-COEF-MISC

\subsubsection{Absorber Assemblies}

\section{Absorber Pin}

The absorber fuel pins are comprised of a stack of absorber pellets with a diameter of $0.91948 \mathrm{~cm}$ and stack height of $91.44 \mathrm{~cm}$. The pins are contained within stainless steel clad. The gap between the pellets and clad is filled with void space. The clad is of the same height as the pellet stack with an inner and outer diameter of 0.94488 and $1.20396 \mathrm{~cm}$, respectively, as shown in Figure 3.1.4.

\section{Control Rod Assembly}

The absorber pins are placed in an array of 61 pins with a pitch of $1.26492 \mathrm{~cm}$ in a homogenized medium of sodium coolant and stainless steel wire wrap used for spacing the fuel pins. The fuel-pin lattice is contained within a hexagonal stainless steel duct with an inside flat-to-flat distance of $10.20064 \mathrm{~cm}$ and an outside flat-to-flat distance of $10.42416 \mathrm{~cm}$ (the duct thickness is 0.11176). Sodium coolant then separates this inner duct from the outer assembly duct with an inside flat-to-flat distance of $11.0109 \mathrm{~cm}$ and an outside flat-to-flat distance of $11.6205 \mathrm{~cm}$ (the duct thickness is $0.3048 \mathrm{~cm}$ ). The outside of the outer duct is surrounding by sodium coolant out to the core position hex flat-to-flat distance (i.e., pitch) of $12.051 \mathrm{~cm}$. A cross section of the absorber pin lattice is shown in Figure 3.1.5.

The absorber pin lattice is sandwiched between two homogenized layers in the absorber assembly. The bottom layer is the below poison region and the top layer is the above poison region. Below the absorber regions is the lower shield region, and above the absorber regions is the driveline region. A depiction of the absorber assembly is shown in Figure 3.1.6. The heights of each region are shown in Table 3.1.5, and the total absorber assembly length is $298.45 \mathrm{~cm}$ in the model. Figure 3.1 .6 represents a fully inserted control rod.

The control rods can be fully withdrawn $91.44 \mathrm{~cm}$. The lower shield region remains fixed and the volume between the lower shield and below poison regions is filled with the withdrawn absorber region, effectively shifting all vertical positions of the below poison, absorber pin lattice, above poison, and driveline regions. A depiction of a fully withdrawn control rod is shown in Figure 3.1.7. Because the overall height of the model is unchanged, the driveline region is not included in the model of a control rod that is fully withdrawn, and the above poison region length is reduced to $34.29 \mathrm{~cm}$.

\section{Fixed Shim Control Rods}

Fixed shim control rods are modeled identical to fully inserted control rods.

Table 3.1.5. Absorber Assembly Dimensions.

\begin{tabular}{||c|c|}
\hline Region & $\begin{array}{c}\text { Height } \\
(\mathrm{cm})\end{array}$ \\
\hline \hline Lower Shield & 61.92774 \\
Below Poison & 19.35226 \\
Absorber Pin Lattice & 91.44 \\
Above Poison & 46.1772 \\
Driveline & 79.5528 \\
\hline
\end{tabular}


Liquid Metal Fast Reactor - LMFR

FFTF-LMFR-RESR-001

CRIT-SPEC-REAC-COEF-MISC

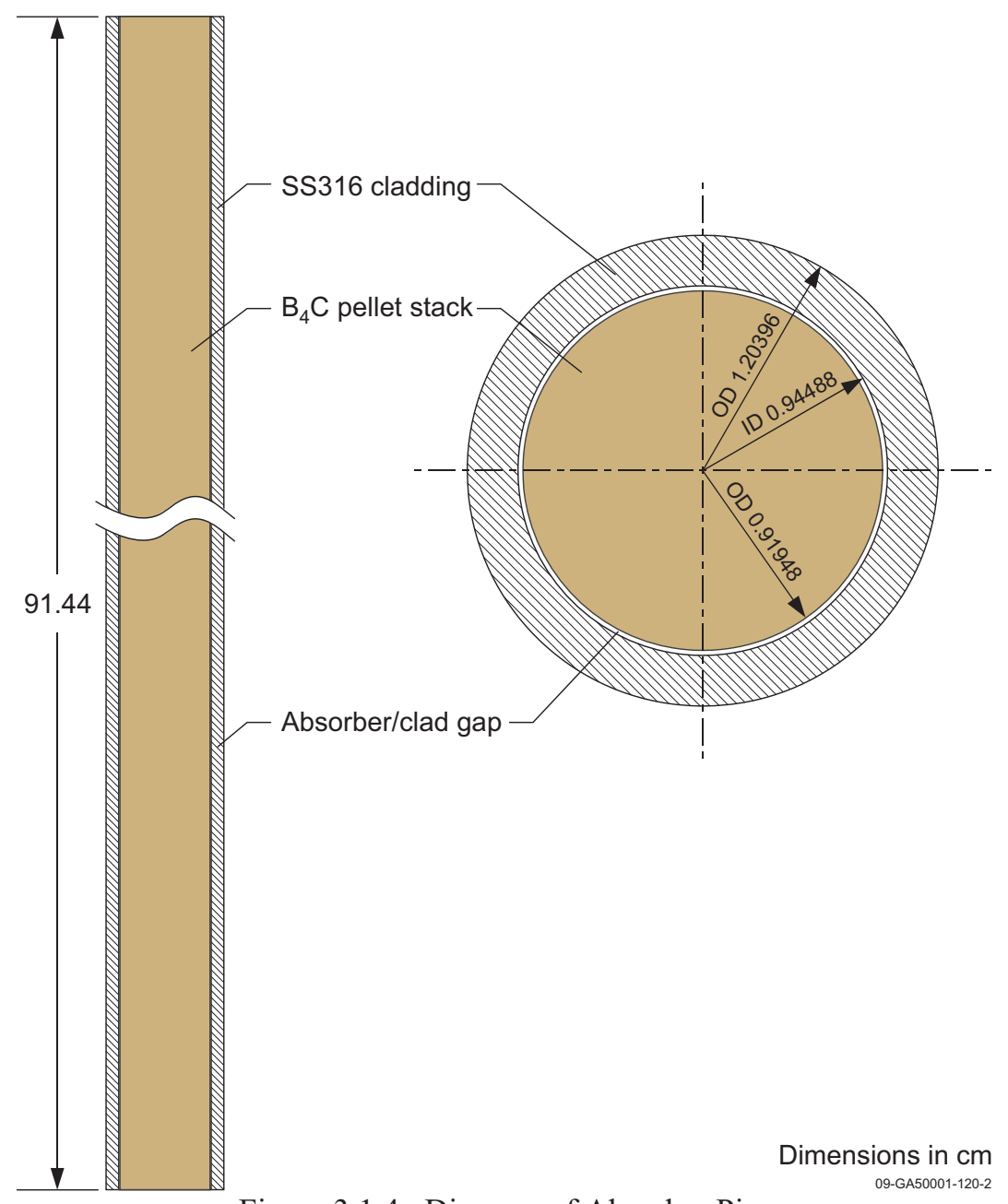

Figure 3.1.4. Diagram of Absorber Pin. 
NEA/NSC/DOC(2006)1

Liquid Metal Fast Reactor - LMFR

FFTF-LMFR-RESR-001

CRIT-SPEC-REAC-COEF-MISC

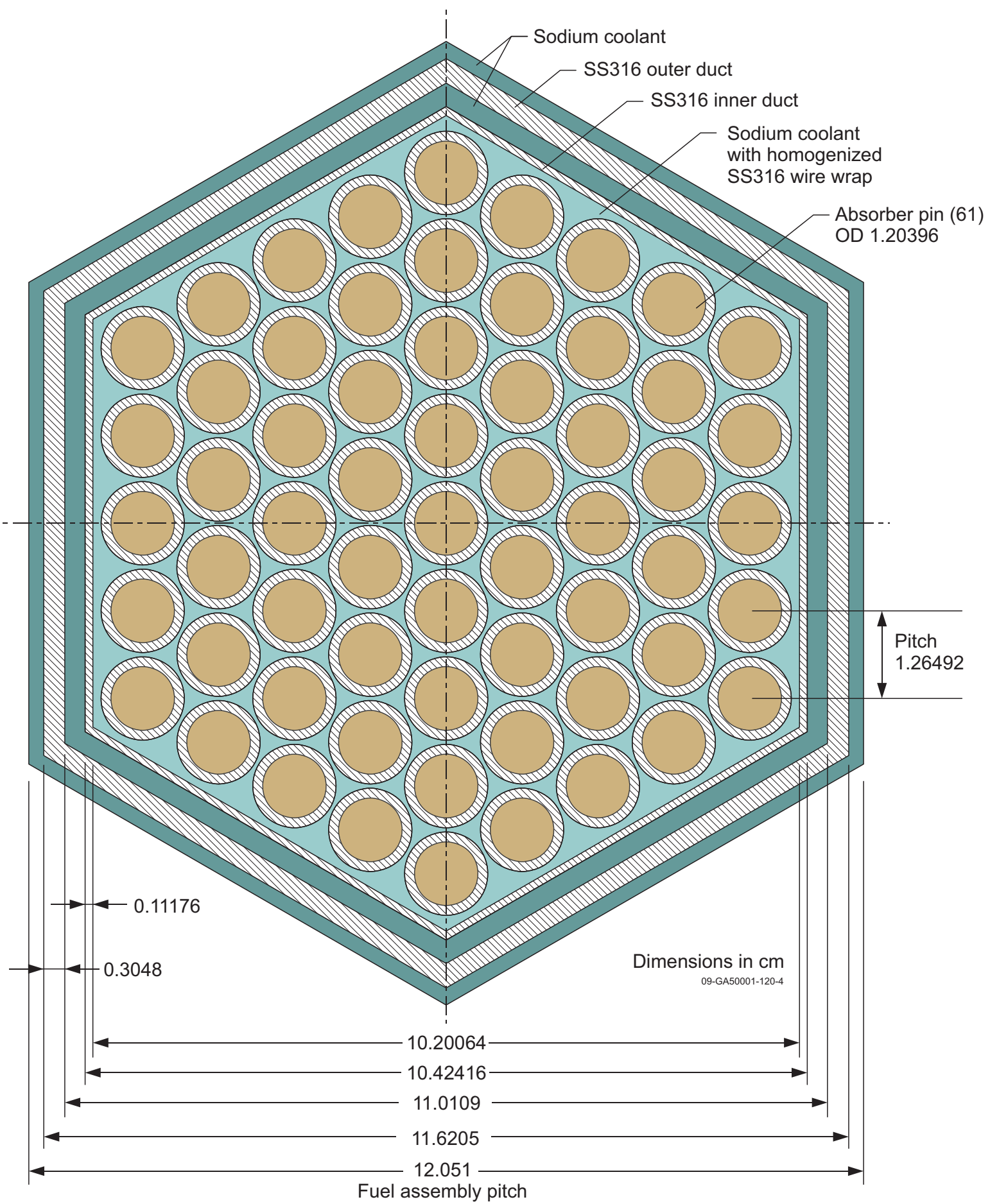

Figure 3.1.5. Diagram of Absorber Pin Lattice. 
NEA/NSC/DOC(2006)1

Liquid Metal Fast Reactor - LMFR

FFTF-LMFR-RESR-001

CRIT-SPEC-REAC-COEF-MISC

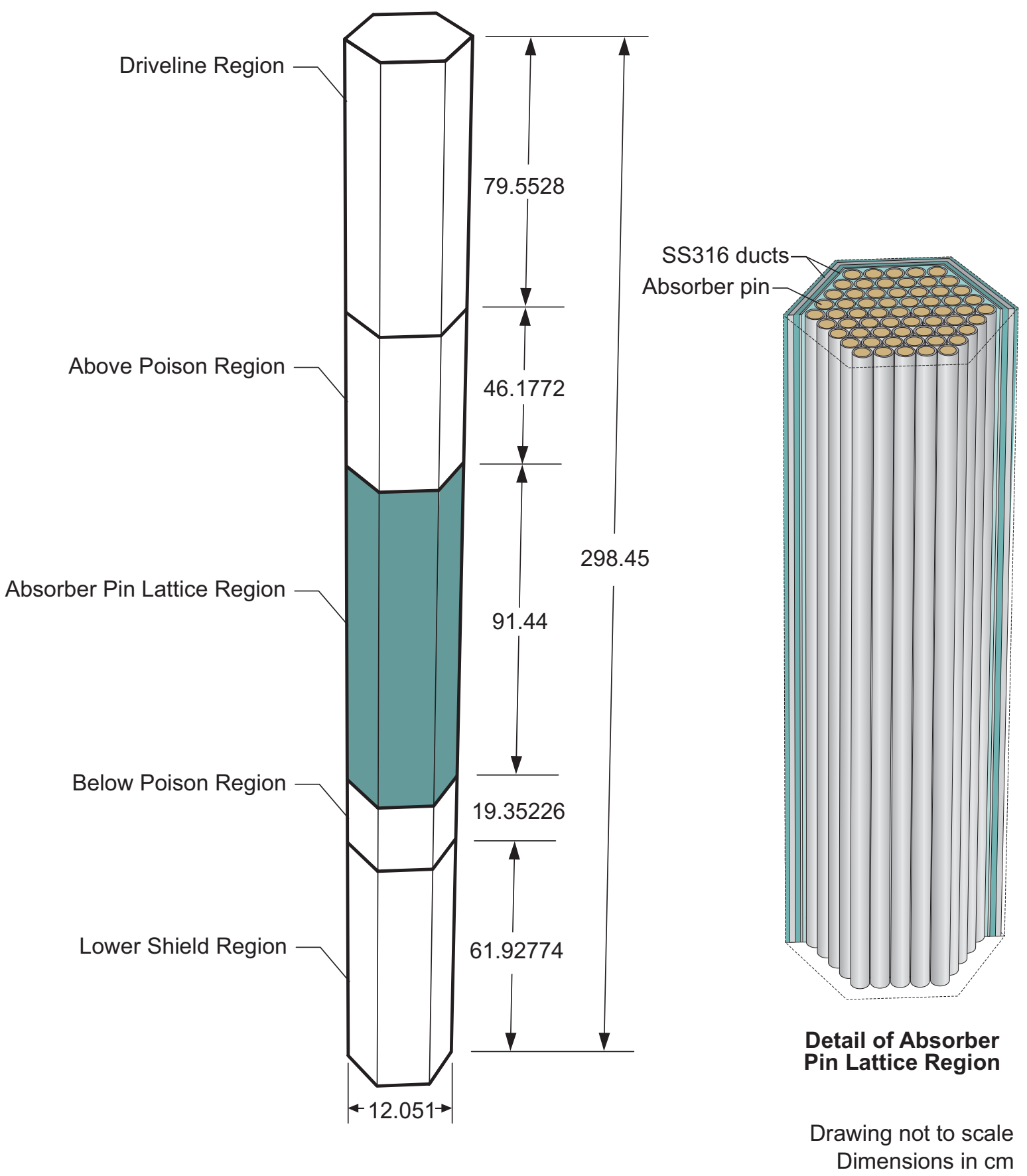

Figure 3.1.6. Diagram of Partially-Homogenized, Fully-Inserted Control Rod.

09-GA50001-121-2 
Liquid Metal Fast Reactor - LMFR

FFTF-LMFR-RESR-001

CRIT-SPEC-REAC-COEF-MISC

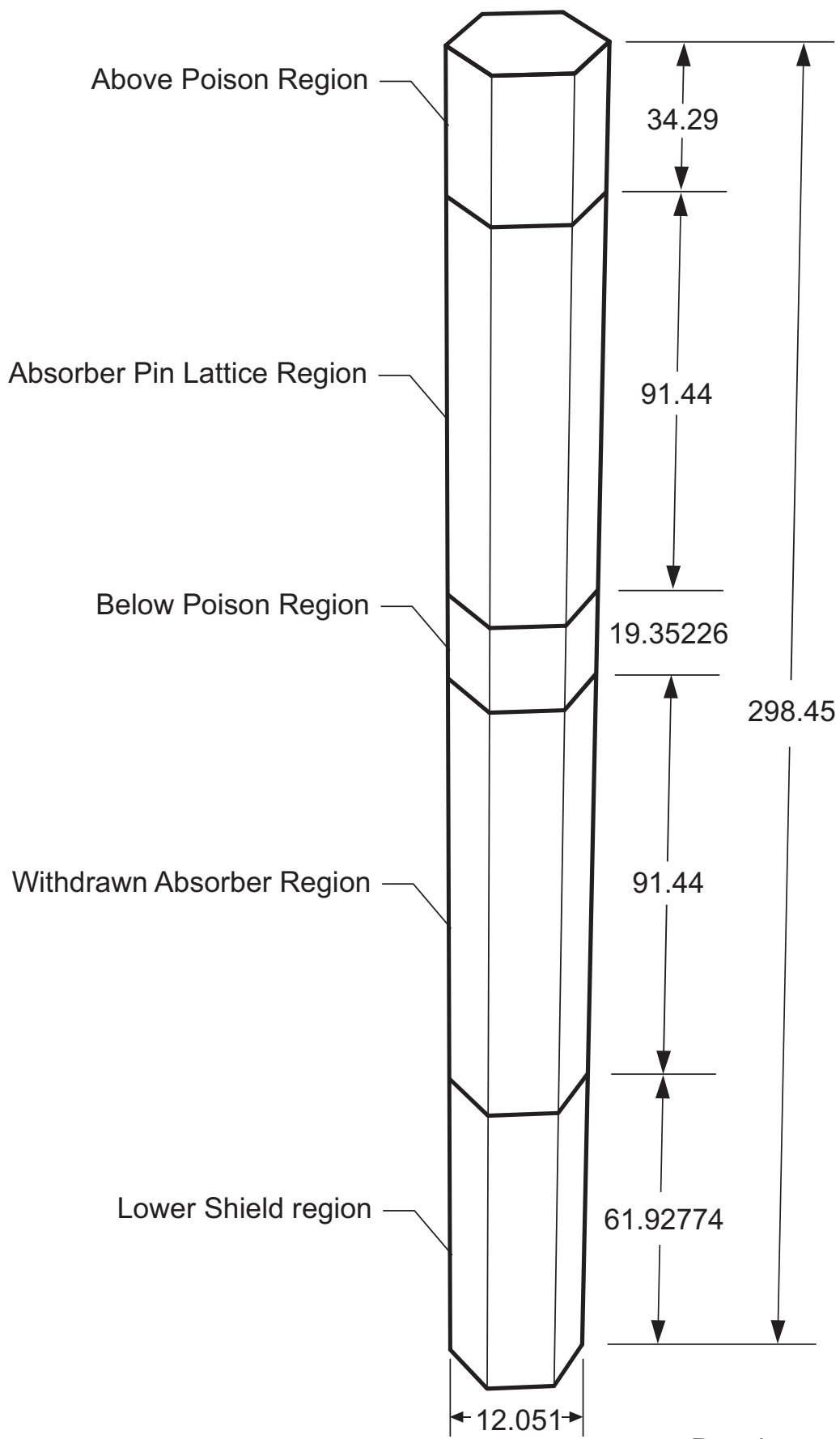

Drawing not to scale Dimensions in $\mathrm{cm}$ 09-GA50001-121-3

Figure 3.1.7. Diagram of Partially Homogenized, Fully-Withdrawn Control Rod. 


\section{Liquid Metal Fast Reactor - LMFR \\ FFTF-LMFR-RESR-001 \\ CRIT-SPEC-REAC-COEF-MISC}

\subsubsection{Radial Reflectors}

The radial reflectors are comprised of five homogenized layers: the orifice region $(58.1025 \mathrm{~cm})$, the lower adapter region $(7.62 \mathrm{~cm})$, the reflector blocks region $(121.92 \mathrm{~cm})$, the load pad region $(10.16 \mathrm{~cm})$, and the upper shield region $(100.6475 \mathrm{~cm})$. The total radial reflector length is $298.45 \mathrm{~cm}$ in the model. Figure 3.1.8 depicts the layers within the radial reflectors. The hexagonal flat-to-flat thickness of the homogenized regions is $12.051 \mathrm{~cm}$.

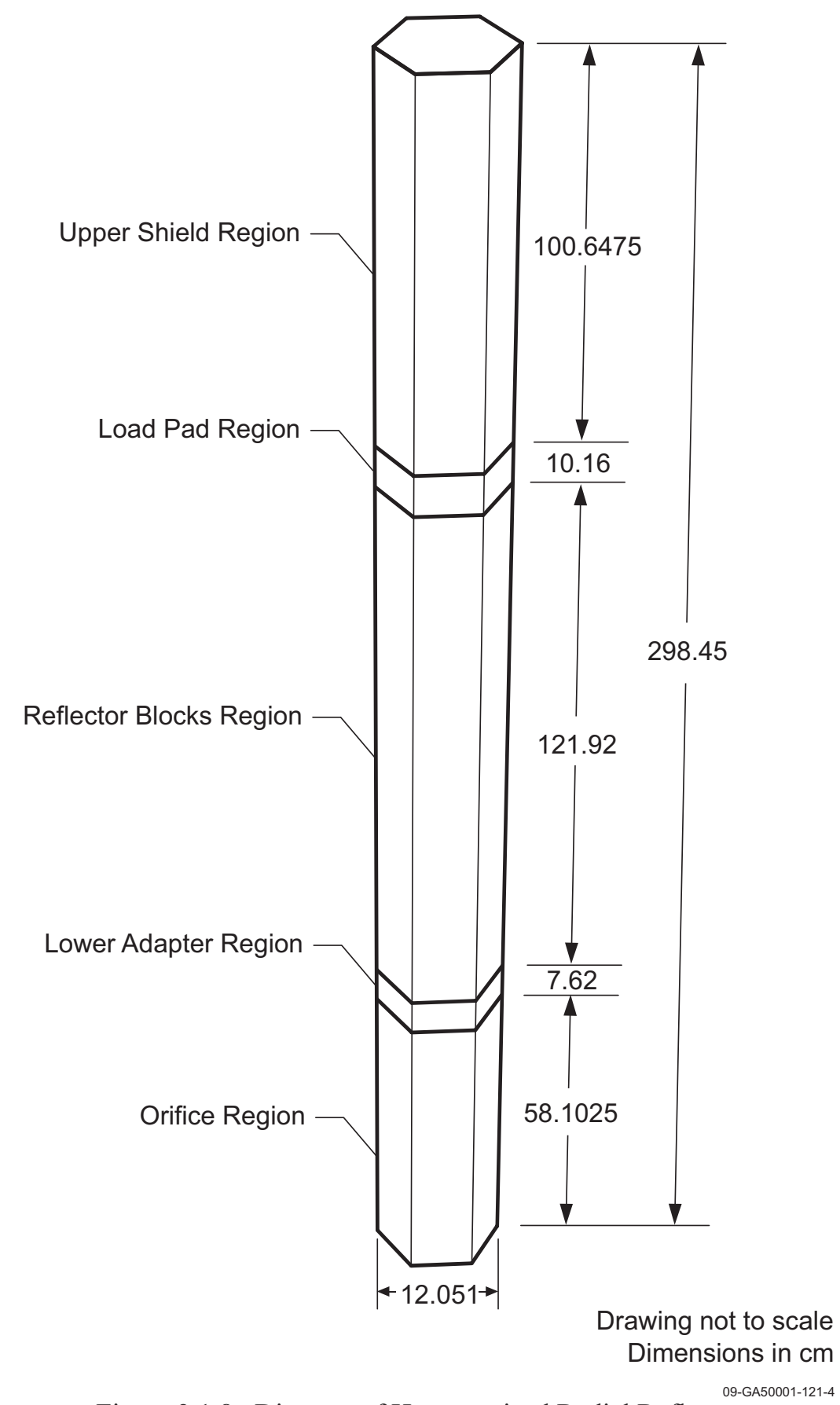

Figure 3.1.8. Diagram of Homogenized Radial Reflectors. 
FFTF-LMFR-RESR-001

CRIT-SPEC-REAC-COEF-MISC

\subsubsection{Vibration Open Test Assembly}

The vibration open test assembly consists of two homogenized layers: the orifice/shield region (57.15 $\mathrm{cm})$ and the instruments and housing region $(241.3 \mathrm{~cm})$. The total VOTA length is $298.45 \mathrm{~cm}$ in the model. Figure 3.1.9 depicts the layers within the VOTA. The hexagonal flat-to-flat thickness of the homogenized regions is $12.051 \mathrm{~cm}$.

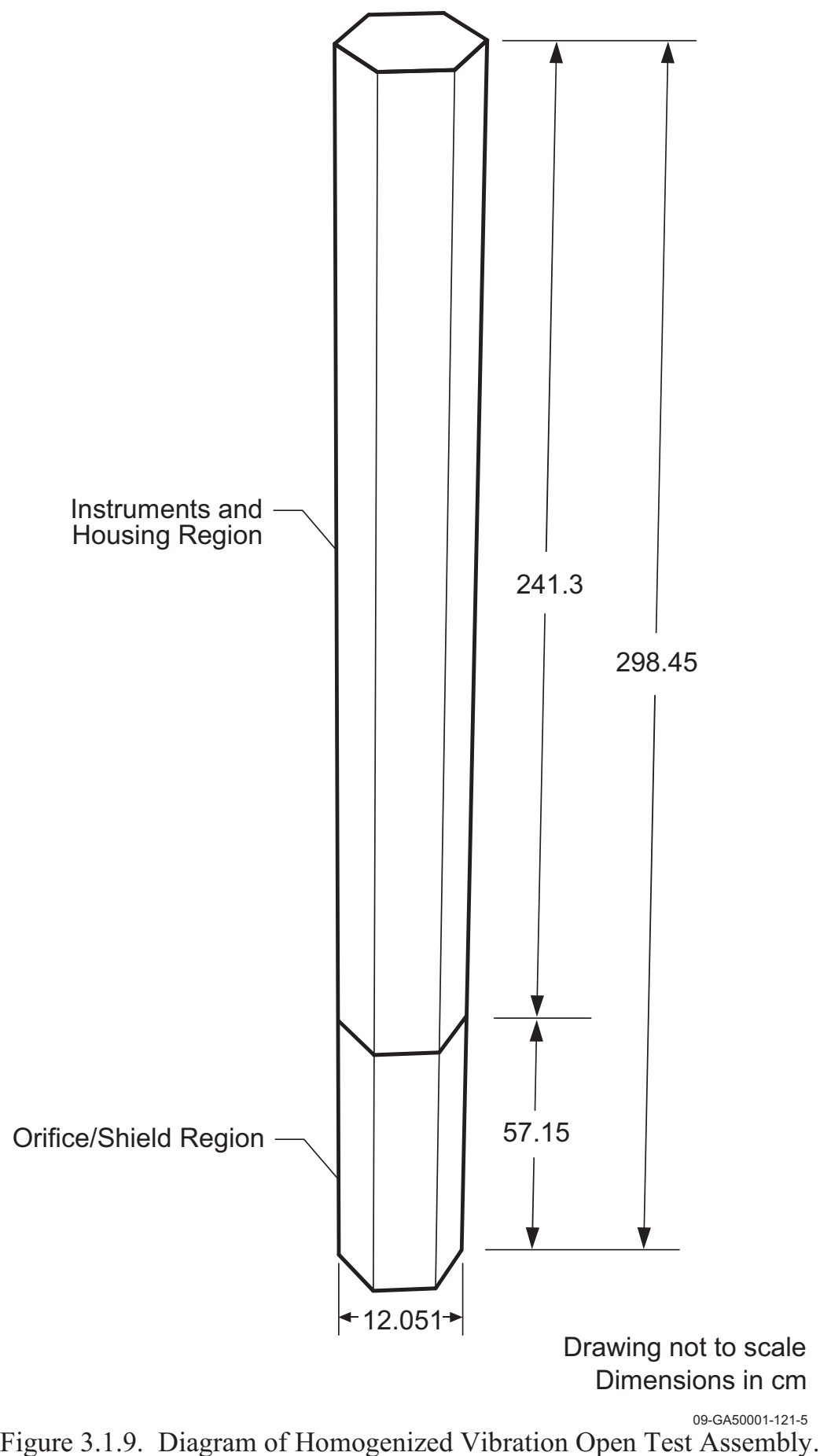


FFTF-LMFR-RESR-001

CRIT-SPEC-REAC-COEF-MISC

\subsubsection{In-Core Shim Assemblies}

The in-core shim assembly consists of three homogenized layers: the orifice/shield region $(54.61 \mathrm{~cm})$, the pin attachment region $(10.16 \mathrm{~cm})$ and the simulated fuel pin bundle region $(233.38 \mathrm{~cm})$. The total ICSA length is $298.45 \mathrm{~cm}$ in the model. Figure 3.1 .10 depicts the layers within the ICSA. The hexagonal flat-to-flat thickness of the homogenized regions is $12.051 \mathrm{~cm}$.

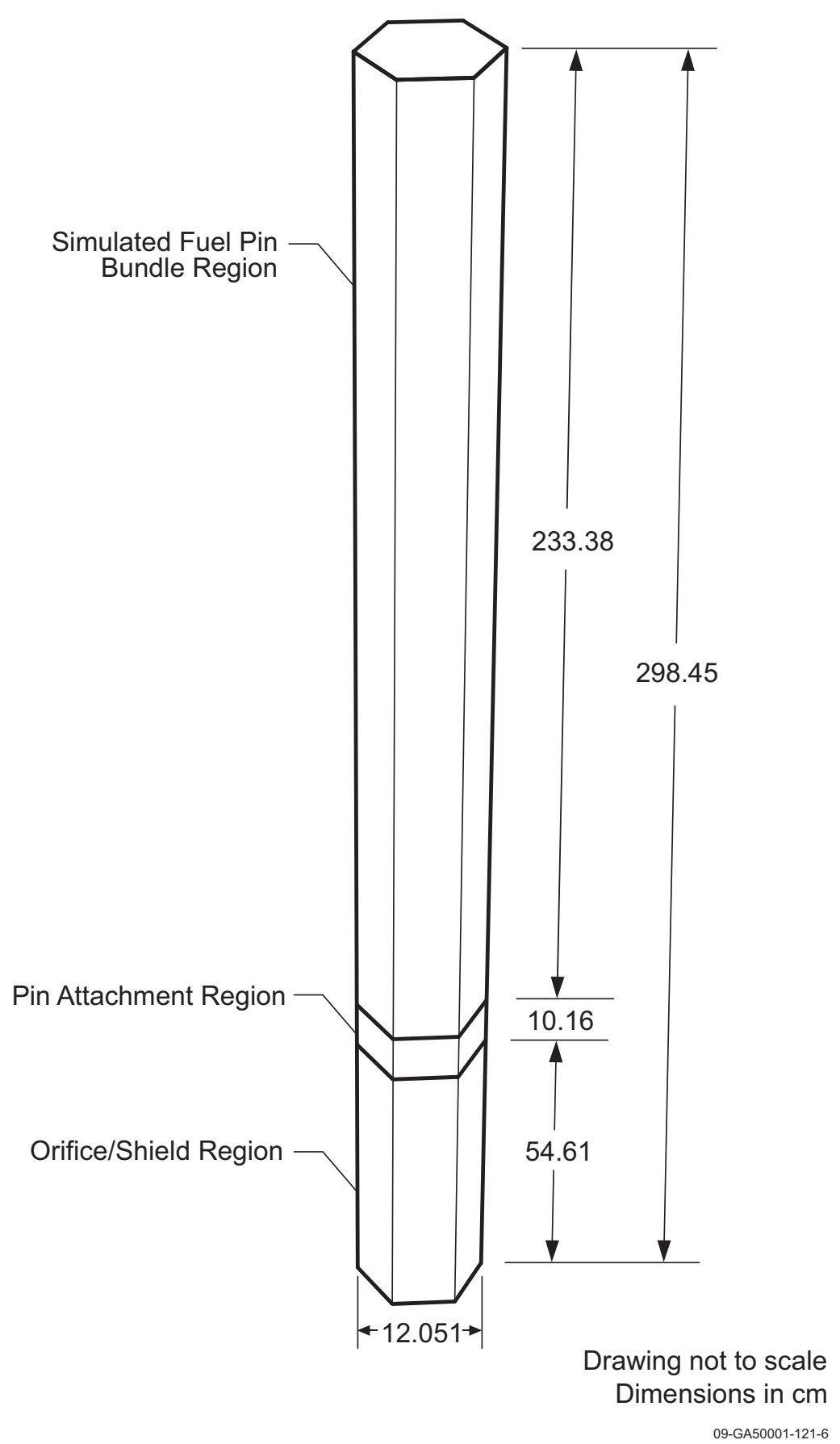

Figure 3.1.10. Diagram of Homogenized In-Core Shim Assemblies. 
FFTF-LMFR-RESR-001

CRIT-SPEC-REAC-COEF-MISC

\subsubsection{Simulated Core Assembly}

The fully-loaded core configuration of the FFTF does not contain simulated core assemblies.

\subsubsection{In-Reactor Thimble}

The in-reactor thimble consists of a single homogenized layer with a total length of $298.45 \mathrm{~cm}$ and a hexagonal flat-to-flat thickness of $12.051 \mathrm{~cm}$. Figure 3.1 .11 shows the homogenized in-reactor thimble region.

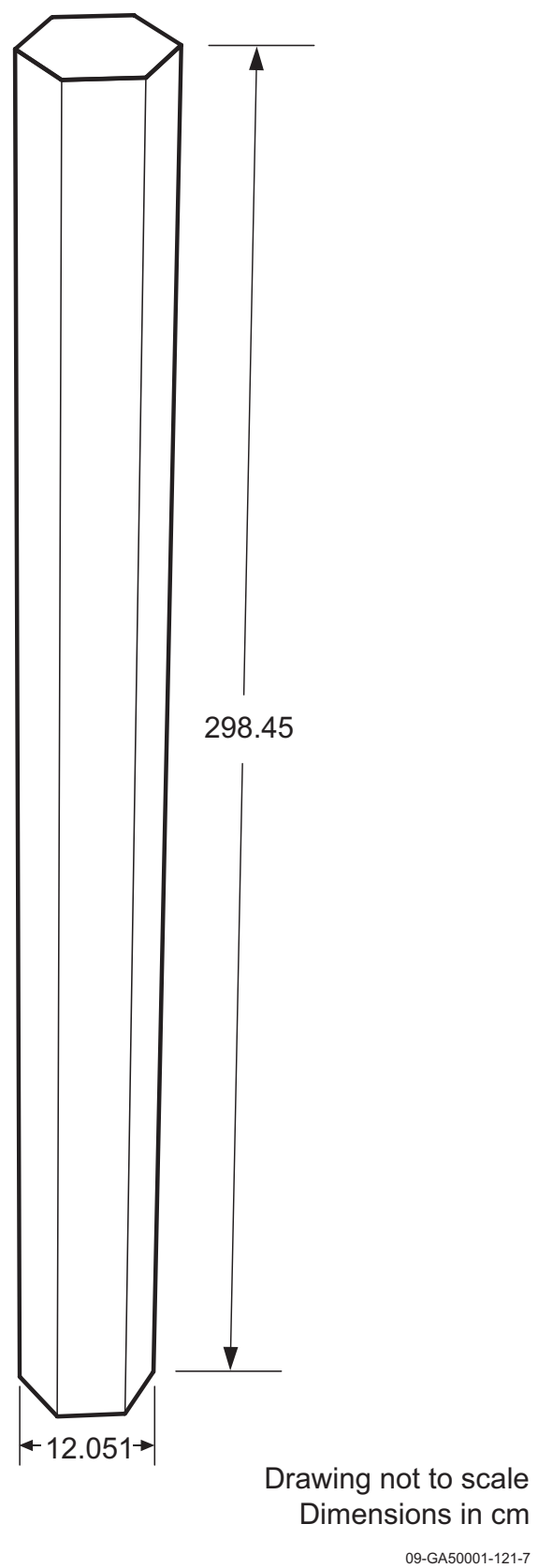

Figure 3.1.11. Diagram of Homogenized In-Reactor Thimble. 


\section{Liquid Metal Fast Reactor - LMFR \\ FFTF-LMFR-RESR-001 \\ CRIT-SPEC-REAC-COEF-MISC}

\subsubsection{Reactor Shielding}

The radial reactor shielding consists of a single homogenized layer with a total length of $298.45 \mathrm{~cm}$ and a hexagonal flat-to-flat thickness of $12.051 \mathrm{~cm}$. Figure 3.1 .12 shows the homogenized reactor shielding region.

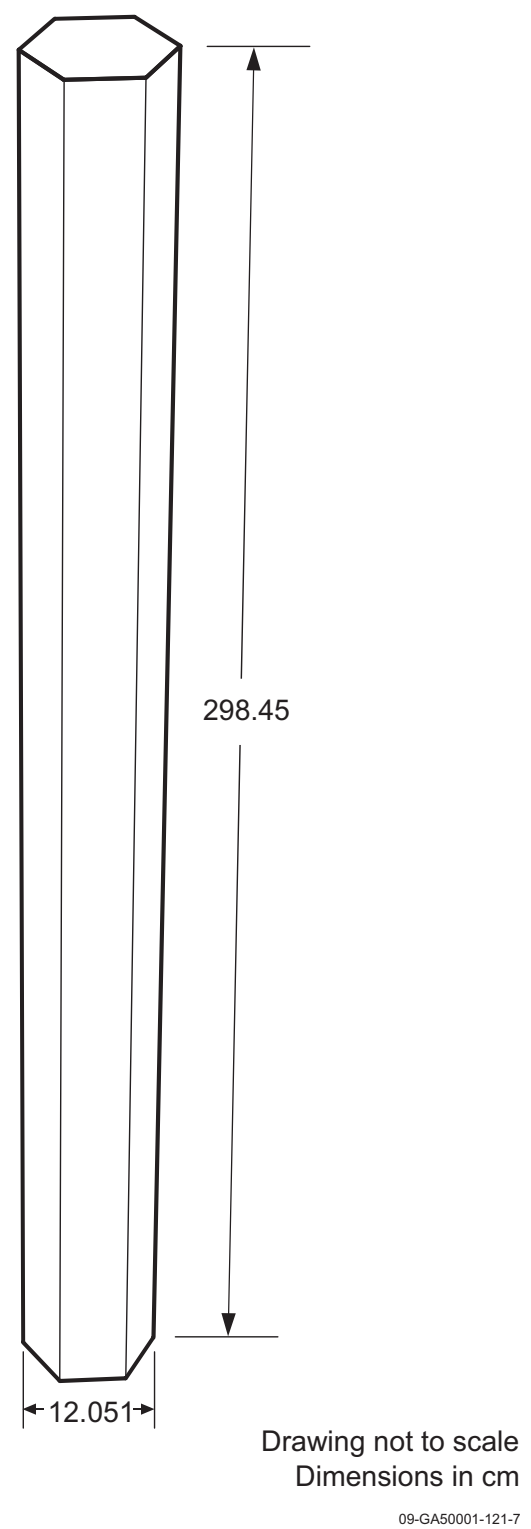

Figure 3.1.12. Diagram of Homogenized Reactor Shielding Region.

\subsubsection{Reactor Core Configuration}

The reactor core configuration is shown in Figure 3.1.13. The portion of the FFTF included in the benchmark model is the fueled portion of the core (including both inner and outer enrichment zones), the radial reflector region, and stainless steel shielding surrounded by sodium coolant. The boundaries of the model include the total height of $298.45 \mathrm{~cm}$ and a hexagonal footprint with flat-to-flat distance of 230 $\mathrm{cm}$. 


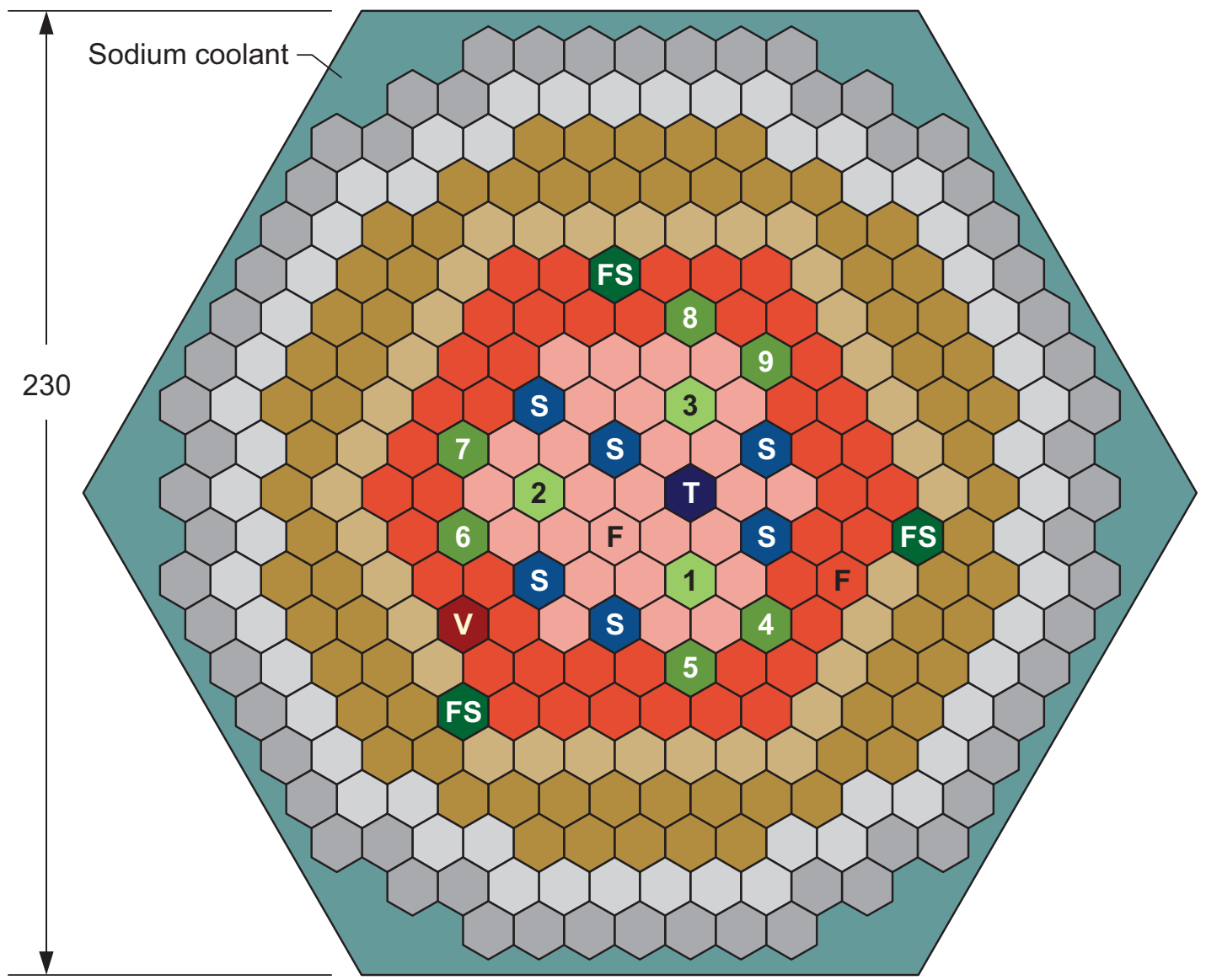

Outer radial shield

FS Fixed shim control rods

Inner radial shield

S In-core shim assemblies

Radial reflectors in Row 8 and 9

\# Secondary control rods

Radial reflectors in Row 7

\# Primary control rods

Driver fuel assembly in the Outer Enrichment Zone

F Fueled open test assembly

Driver fuel assembly in the Inner Enrichment Zone

Vibration open test assembly

T In reactor thimble

Dimensions in $\mathrm{cm}$

Figure 3.1.13. Diagram of FFTF Fully-Loaded Critical Core Configuration. 


\section{Liquid Metal Fast Reactor - LMFR \\ FFTF-LMFR-RESR-001 \\ CRIT-SPEC-REAC-COEF-MISC}

\subsubsection{Critical Rod Positions}

The critical rod positions in the fully-loaded critical core configuration of the FFTF benchmark model are listed in Table 3.1.6. Figure 3.1.14 shows a comparison of the control rod positions in the critical core configuration. Fixed shim control rods are fully inserted (and cannot be withdrawn).

Table 3.1.6. Control Rod Positions.

\begin{tabular}{|c|c|}
\hline $\begin{array}{c}\text { Control Rod } \\
\text { Number } \\
\text { (Type) }\end{array}$ & $\begin{array}{c}\text { Distance } \\
\text { Withdrawn } \\
\text { (cm) }\end{array}$ \\
\hline 1 (Safety) & 91.44 \\
2 (Safety) & 91.44 \\
3 (Safety) & 91.44 \\
4 (Control) & 36.116 \\
5 (Control) & 35.56 \\
6 (Control) & 35.56 \\
7 (Control) & 35.56 \\
8 (Control) & 35.56 \\
9 (Control) & 35.56 \\
Fixed Shims (3 Total) & 0 \\
\hline
\end{tabular}


NEA/NSC/DOC(2006)1

Liquid Metal Fast Reactor - LMFR

FFTF-LMFR-RESR-001

CRIT-SPEC-REAC-COEF-MISC

Safety Rods
1,2, and 3
(fully withdrawn)

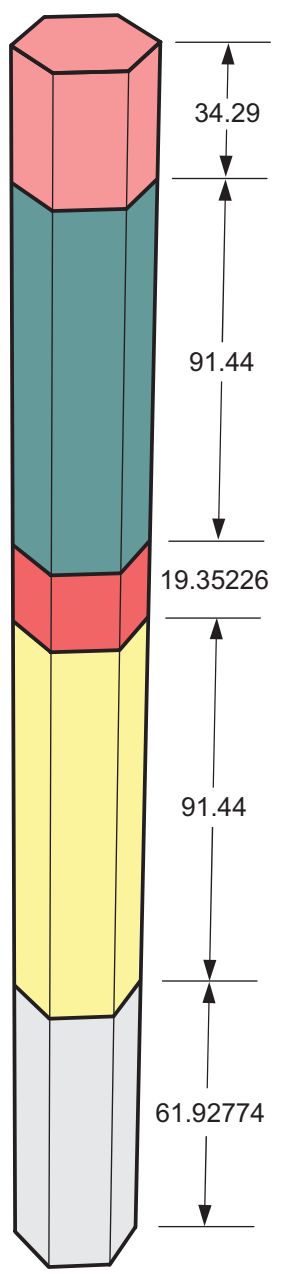

Lower Shield Region

Withdrawn Absorber Region $\underset{4}{\text { Control Rod }}$

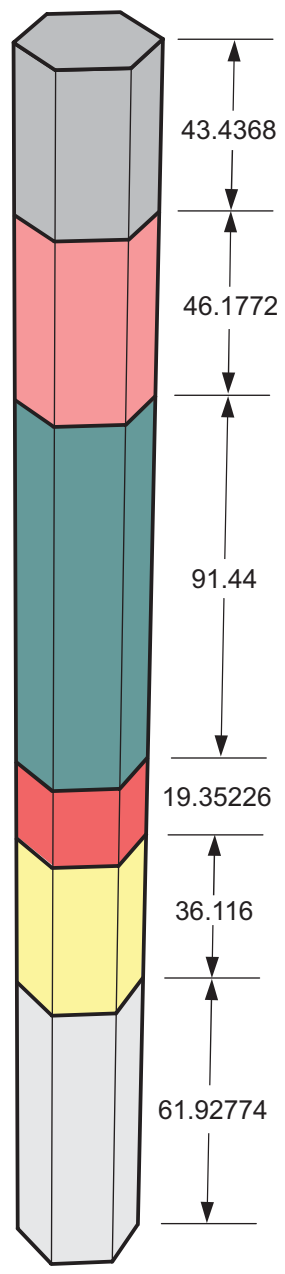

Below Poison Region

Absorber Pin Lattice Region
Control Rods
$5,6,7,8$ and 9

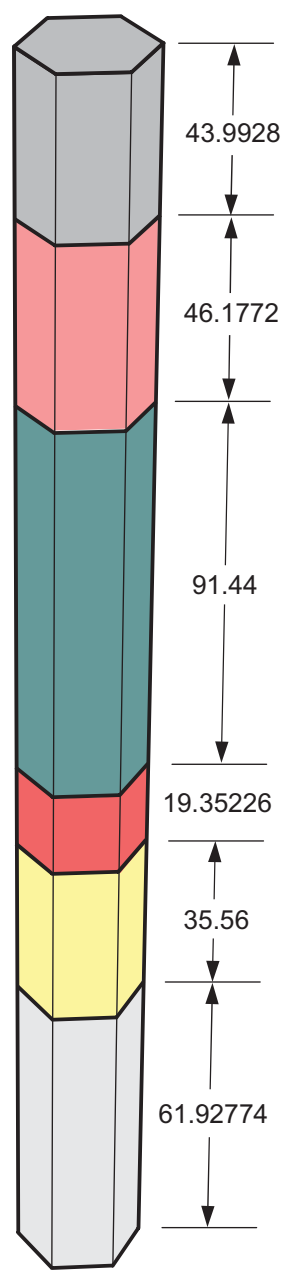

Above Poison Region

Driveline Region
Fixed Shim
Control Rods
3 Total
(fully inserted)

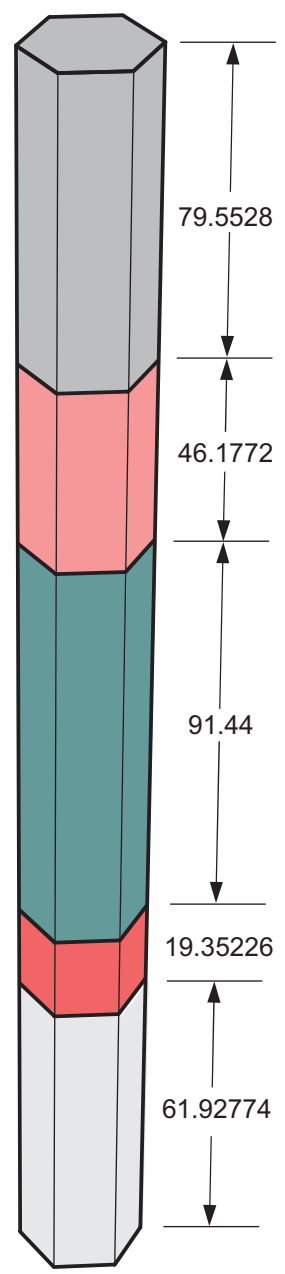

Drawing not to scale Dimensions in $\mathrm{cm}$ 09-CA50001-121-8

Figure 3.1.14. Diagram of Control Rod Positions.

\subsubsection{Material Data}

\subsubsection{Driver Fuel Assemblies}

\section{Driver Fuel Pin}

The driver fuel pins contain mixed oxide fuel pellets of two types. The pellets in the inner fuel region have lower plutonium content than those in the outer fuel region (see Figure 3.1.13 for location of fuel regions). The natural uranium insulator pellets have the same composition in both fuel regions of the core. The atomic composition of the oxide materials are provided in Table 3.1.7. 
NEA/NSC/DOC(2006)1

Liquid Metal Fast Reactor - LMFR

FFTF-LMFR-RESR-001

CRIT-SPEC-REAC-COEF-MISC

Table 3.1.7. Fuel and Insulator Pellet Compositions.

\begin{tabular}{|c|ccc||}
\hline \multirow{2}{*}{$\begin{array}{c}\text { Element } \\
\text { or Isotope }\end{array}$} & \multicolumn{3}{|c|}{ Atom Density (a/b-cm) } \\
& $\begin{array}{c}\text { Inner Fuel } \\
\text { (Type 3.1) }\end{array}$ & $\begin{array}{c}\text { Outer Fuel } \\
\text { (Type 3.2) }\end{array}$ & $\begin{array}{c}\text { Insulator } \\
\text { Pellets }\end{array}$ \\
\hline \hline $\mathrm{O}$ & $4.3372 \mathrm{E}-02$ & $4.2690 \mathrm{E}-02$ & $4.6351 \mathrm{E}-02$ \\
${ }^{234} \mathrm{U}$ & $1.0327 \mathrm{E}-06$ & $9.9319 \mathrm{E}-07$ & $1.2746 \mathrm{E}-06$ \\
${ }^{235} \mathrm{U}$ & $1.2127 \mathrm{E}-04$ & $1.1417 \mathrm{E}-04$ & $1.6686 \mathrm{E}-04$ \\
${ }^{238} \mathrm{U}$ & $1.6744 \mathrm{E}-02$ & $1.5764 \mathrm{E}-02$ & $2.3007 \mathrm{E}-02$ \\
${ }^{237} \mathrm{~Np}$ & $1.3313 \mathrm{E}-05$ & $1.6063 \mathrm{E}-05$ & -- \\
${ }^{238} \mathrm{Pu}$ & $2.6497 \mathrm{E}-06$ & $3.1181 \mathrm{E}-06$ & -- \\
${ }^{239} \mathrm{Pu}$ & $4.2072 \mathrm{E}-03$ & $5.1998 \mathrm{E}-03$ & -- \\
${ }^{240} \mathrm{Pu}$ & $5.6618 \mathrm{E}-04$ & $7.0298 \mathrm{E}-04$ & -- \\
${ }^{241} \mathrm{Pu}$ & $5.7297 \mathrm{E}-05$ & $6.9284 \mathrm{E}-05$ & -- \\
${ }^{242} \mathrm{Pu}$ & $9.2450 \mathrm{E}-06$ & $1.2825 \mathrm{E}-05$ & -- \\
${ }^{241} \mathrm{Am}$ & $1.4025 \mathrm{E}-05$ & $1.1744 \mathrm{E}-05$ & -- \\
\hline${ }_{\mathrm{T}}^{\mathrm{T}} \mathrm{Ptal}$ & $6.5108 \mathrm{E}-02$ & $6.4584 \mathrm{E}-02$ & $6.9526 \mathrm{E}-02$ \\
\hline \hline
\end{tabular}

The axial Inconel 600 reflectors have the composition shown in Table 3.1.8, and the stainless steel 316 cladding has the composition also provided in Table 3.1.8. 
NEA/NSC/DOC(2006)1

Liquid Metal Fast Reactor - LMFR

FFTF-LMFR-RESR-001

CRIT-SPEC-REAC-COEF-MISC

Table 3.1.8. Composition of Inconel 600 and Stainless Steel 316.

\begin{tabular}{|c|cc||}
\hline \multirow{2}{*}{$\begin{array}{c}\text { Element } \\
\text { or Isotope }\end{array}$} & \multicolumn{2}{|c|}{ Atom Density $(\mathrm{a} / \mathrm{b}-\mathrm{cm})$} \\
\hline \hline${ }^{10} \mathrm{~B}$ & -- & $4.3730 \mathrm{E}-07$ \\
${ }^{11} \mathrm{~B}$ & -- & $1.7602 \mathrm{E}-06$ \\
$\mathrm{C}$ & $2.0908 \mathrm{E}-03$ & $1.9780 \mathrm{E}-04$ \\
$\mathrm{~N}$ & -- & $1.6961 \mathrm{E}-05$ \\
$\mathrm{Al}$ & -- & $4.4025 \mathrm{E}-05$ \\
$\mathrm{Si}$ & $4.4707 \mathrm{E}-04$ & $6.3442 \mathrm{E}-04$ \\
$\mathrm{P}$ & -- & $1.5340 \mathrm{E}-05$ \\
$\mathrm{~S}$ & $1.1747 \mathrm{E}-05$ & $7.4091 \mathrm{E}-06$ \\
$\mathrm{~V}$ & -- & $9.3272 \mathrm{E}-05$ \\
$\mathrm{Cr}$ & $1.4972 \mathrm{E}-02$ & $1.5992 \mathrm{E}-02$ \\
$\mathrm{Mn}$ & $1.5998 \mathrm{E}-03$ & $1.5135 \mathrm{E}-03$ \\
$\mathrm{Fe}$ & $7.1948 \mathrm{E}-03$ & $5.4503 \mathrm{E}-02$ \\
$\mathrm{Co}$ & $8.5222 \mathrm{E}-05$ & $2.0156 \mathrm{E}-05$ \\
$\mathrm{Ni}$ & $6.3016 \mathrm{E}-02$ & $1.0929 \mathrm{E}-02$ \\
$\mathrm{Cu}$ & $1.9759 \mathrm{E}-04$ & $3.7386 \mathrm{E}-05$ \\
$\mathrm{As}$ & -- & $9.5128 \mathrm{E}-06$ \\
$\mathrm{Nb}$ & -- & $1.2786 \mathrm{E}-05$ \\
$\mathrm{Mo}$ & -- & $1.2381 \mathrm{E}-03$ \\
$\mathrm{Ta}$ & -- & $1.3129 \mathrm{E}-06$ \\
$\mathrm{Total}$ & $8.9615 \mathrm{E}-02$ & $8.5268 \mathrm{E}-02$ \\
\hline
\end{tabular}


FFTF-LMFR-RESR-001

CRIT-SPEC-REAC-COEF-MISC

\section{Driver Fuel Assembly}

The composition of the duct is that of stainless steel 316 in Table 3.1.8. The composition of the sodium coolant homogenized with the stainless steel 316 wire wrap that surrounds the driver fuel pins in the fuel pin lattice has the composition shown in Table 3.1.9. This composition is the same for fuel assemblies in both the inner and outer enrichment zones.

Table 3.1.9. Driver Fuel Assembly Homogenized Sodium with SS316 Wire Wrap.

\begin{tabular}{|c|c|}
\hline $\begin{array}{c}\text { Element } \\
\text { or Isotope }\end{array}$ & $\begin{array}{c}\text { Atom Density } \\
(\mathrm{a} / \mathrm{b}-\mathrm{cm})\end{array}$ \\
\hline \hline${ }^{10} \mathrm{~B}$ & $3.2283 \mathrm{E}-08$ \\
${ }^{11} \mathrm{~B}$ & $1.2994 \mathrm{E}-07$ \\
$\mathrm{C}$ & $1.4602 \mathrm{E}-05$ \\
$\mathrm{~N}$ & $1.2521 \mathrm{E}-06$ \\
$\mathrm{Na}$ & $2.1876 \mathrm{E}-02$ \\
$\mathrm{Al}$ & $3.2500 \mathrm{E}-06$ \\
$\mathrm{Si}$ & $4.6834 \mathrm{E}-05$ \\
$\mathrm{P}$ & $1.1325 \mathrm{E}-06$ \\
$\mathrm{~S}$ & $5.4696 \mathrm{E}-07$ \\
$\mathrm{~V}$ & $6.8856 \mathrm{E}-06$ \\
$\mathrm{Cr}$ & $1.1805 \mathrm{E}-03$ \\
$\mathrm{Mn}$ & $1.1173 \mathrm{E}-04$ \\
$\mathrm{Fe}$ & $4.0236 \mathrm{E}-03$ \\
$\mathrm{Co}$ & $1.4880 \mathrm{E}-06$ \\
$\mathrm{Ni}$ & $8.0679 \mathrm{E}-04$ \\
$\mathrm{Cu}$ & $2.7599 \mathrm{E}-06$ \\
$\mathrm{As}$ & $7.0226 \mathrm{E}-07$ \\
$\mathrm{Nb}$ & $9.4386 \mathrm{E}-07$ \\
$\mathrm{Mo}$ & $9.1402 \mathrm{E}-05$ \\
$\mathrm{Ta}$ & $9.6924 \mathrm{E}-08$ \\
\hline $\mathrm{Total}$ & $2.8171 \mathrm{E}-02$ \\
\hline
\end{tabular}

The homogenized layers of the driver fuel assembly have the compositions provided in Table 3.1.10. The composition of the lower axial shield region and the gas plenum region is identical for both inner and outer enrichment zones in the FFTF. The pin attachment region of the fuel assemblies is, however, different (see Figure 3.1.13 for location of fuel regions). 
NEA/NSC/DOC(2006)1

Liquid Metal Fast Reactor - LMFR

FFTF-LMFR-RESR-001

CRIT-SPEC-REAC-COEF-MISC

Table 3.1.10. Homogenized Compositions of the Driver Fuel Assemblies.

\begin{tabular}{|c|cccc||}
\hline \multirow{2}{*}{$\begin{array}{c}\text { Element } \\
\text { or Isotope }\end{array}$} & $\begin{array}{c}\text { Lower } \\
\text { Axial }\end{array}$ & $\begin{array}{c}\text { Atom Density }(\mathrm{a} / \mathrm{b}-\mathrm{cm}) \\
\text { Attachment }\end{array}$ & $\begin{array}{c}\text { Attach Pin } \\
\text { Attan }\end{array}$ & $\begin{array}{c}\text { Gas } \\
\text { Plenum }\end{array}$ \\
& Shield & Region & Region & Region \\
\hline \hline${ }^{10} \mathrm{~B}$ & $3.2491 \mathrm{E}-07$ & $1.8159 \mathrm{E}-07$ & $1.7764 \mathrm{E}-07$ & $1.2816 \mathrm{E}-07$ \\
${ }^{11} \mathrm{~B}$ & $1.3078 \mathrm{E}-06$ & $7.3091 \mathrm{E}-07$ & $7.1504 \mathrm{E}-07$ & $5.1585 \mathrm{E}-07$ \\
$\mathrm{C}$ & $1.4696 \mathrm{E}-04$ & $8.2135 \mathrm{E}-05$ & $8.0351 \mathrm{E}-05$ & $5.7968 \mathrm{E}-05$ \\
$\mathrm{~N}$ & $1.2602 \mathrm{E}-05$ & $7.0431 \mathrm{E}-06$ & $6.8901 \mathrm{E}-06$ & $4.9707 \mathrm{E}-06$ \\
$\mathrm{Na}$ & $6.1160 \mathrm{E}-03$ & $1.3847 \mathrm{E}-02$ & $1.4060 \mathrm{E}-02$ & $9.6390 \mathrm{E}-03$ \\
$\mathrm{Al}$ & $3.2710 \mathrm{E}-05$ & $1.8281 \mathrm{E}-05$ & $1.7884 \mathrm{E}-05$ & $1.2902 \mathrm{E}-05$ \\
$\mathrm{Si}$ & $4.7136 \mathrm{E}-04$ & $2.6344 \mathrm{E}-04$ & $2.5772 \mathrm{E}-04$ & $1.8592 \mathrm{E}-04$ \\
$\mathrm{P}$ & $1.1398 \mathrm{E}-05$ & $6.3699 \mathrm{E}-06$ & $6.2316 \mathrm{E}-06$ & $4.4957 \mathrm{E}-06$ \\
$\mathrm{~S}$ & $5.5048 \mathrm{E}-06$ & $3.0766 \mathrm{E}-06$ & $3.0097 \mathrm{E}-06$ & $2.1713 \mathrm{E}-06$ \\
$\mathrm{~V}$ & $6.9300 \mathrm{E}-05$ & $3.8731 \mathrm{E}-05$ & $3.7890 \mathrm{E}-05$ & $2.7335 \mathrm{E}-05$ \\
$\mathrm{Cr}$ & $1.1882 \mathrm{E}-02$ & $6.6404 \mathrm{E}-03$ & $6.4962 \mathrm{E}-03$ & $4.6866 \mathrm{E}-03$ \\
$\mathrm{Mn}$ & $1.1245 \mathrm{E}-03$ & $6.2848 \mathrm{E}-04$ & $6.1483 \mathrm{E}-04$ & $4.4356 \mathrm{E}-04$ \\
$\mathrm{Fe}$ & $4.0495 \mathrm{E}-02$ & $2.2632 \mathrm{E}-02$ & $2.2141 \mathrm{E}-02$ & $1.5973 \mathrm{E}-02$ \\
$\mathrm{Co}$ & $1.4976 \mathrm{E}-05$ & $8.3697 \mathrm{E}-06$ & $8.1879 \mathrm{E}-06$ & $5.9070 \mathrm{E}-06$ \\
$\mathrm{Ni}$ & $8.1199 \mathrm{E}-03$ & $4.5381 \mathrm{E}-03$ & $4.4395 \mathrm{E}-03$ & $3.2028 \mathrm{E}-03$ \\
$\mathrm{Cu}$ & $2.7777 \mathrm{E}-05$ & $1.5524 \mathrm{E}-05$ & $1.5187 \mathrm{E}-05$ & $1.0956 \mathrm{E}-05$ \\
$\mathrm{As}$ & $7.0679 \mathrm{E}-06$ & $3.9501 \mathrm{E}-06$ & $3.8643 \mathrm{E}-06$ & $2.7879 \mathrm{E}-06$ \\
$\mathrm{Nb}$ & $9.4995 \mathrm{E}-06$ & $5.3091 \mathrm{E}-06$ & $5.1938 \mathrm{E}-06$ & $3.7470 \mathrm{E}-06$ \\
$\mathrm{Mo}$ & $9.1991 \mathrm{E}-04$ & $5.1412 \mathrm{E}-04$ & $5.0296 \mathrm{E}-04$ & $3.6285 \mathrm{E}-04$ \\
$\mathrm{Ta}$ & $9.7549 \mathrm{E}-07$ & $5.4519 \mathrm{E}-07$ & $5.3334 \mathrm{E}-07$ & $3.8477 \mathrm{E}-07$ \\
\hline $\mathrm{Total}$ & $6.9469 \mathrm{E}-02$ & $4.9254 \mathrm{E}-02$ & $4.8698 \mathrm{E}-02$ & $3.4628 \mathrm{E}-02$ \\
\hline \hline
\end{tabular}


FFTF-LMFR-RESR-001

CRIT-SPEC-REAC-COEF-MISC

\subsubsection{Absorber Assemblies}

\section{Absorber Pin}

The composition of the absorber pins is provided in Table 3.1.11. The cladding of the absorber pins is stainless steel 316 (Table 3.1.8).

Table 3.1.11. Composition of Boron Carbide Absorber Pins.

\begin{tabular}{|c|c|}
\hline $\begin{array}{c}\text { Element } \\
\text { or Isotope }\end{array}$ & $\begin{array}{c}\text { Atom Density } \\
(\mathrm{a} / \mathrm{b}-\mathrm{cm})\end{array}$ \\
\hline \hline${ }^{10} \mathrm{~B}$ & $2.0297 \mathrm{E}-02$ \\
${ }^{11} \mathrm{~B}$ & $8.1700 \mathrm{E}-02$ \\
$\mathrm{C}$ & $2.3699 \mathrm{E}-02$ \\
\hline Total & $1.2570 \mathrm{E}-01$ \\
\hline
\end{tabular}

\section{Control Rod Assembly}

The composition of the ducts is that of stainless steel 316 in Table 3.1.8. The composition of the sodium coolant homogenized with the stainless steel 316 wire wrap that surrounds the absorber pins in the fuel pin lattice has the composition shown in Table 3.1.12. 
NEA/NSC/DOC(2006)1

Liquid Metal Fast Reactor - LMFR

FFTF-LMFR-RESR-001

CRIT-SPEC-REAC-COEF-MISC

Table 3.1.12. Absorber Assembly Homogenized Sodium with SS316 Wire Wrap.

\begin{tabular}{|c|c|}
\hline $\begin{array}{c}\text { Element } \\
\text { or Isotope }\end{array}$ & $\begin{array}{c}\text { Atom Density } \\
(\mathrm{a} / \mathrm{b}-\mathrm{cm})\end{array}$ \\
\hline \hline${ }^{10} \mathrm{~B}$ & $3.7987 \mathrm{E}-09$ \\
${ }^{11} \mathrm{~B}$ & $1.5290 \mathrm{E}-08$ \\
$\mathrm{C}$ & $1.7182 \mathrm{E}-06$ \\
$\mathrm{~N}$ & $1.4734 \mathrm{E}-07$ \\
$\mathrm{Na}$ & $2.3415 \mathrm{E}-02$ \\
$\mathrm{Al}$ & $3.8243 \mathrm{E}-07$ \\
$\mathrm{Si}$ & $5.5110 \mathrm{E}-06$ \\
$\mathrm{P}$ & $1.3326 \mathrm{E}-07$ \\
$\mathrm{~S}$ & $6.4361 \mathrm{E}-08$ \\
$\mathrm{~V}$ & $8.1023 \mathrm{E}-07$ \\
$\mathrm{Cr}$ & $1.3892 \mathrm{E}-04$ \\
$\mathrm{Mn}$ & $1.3148 \mathrm{E}-05$ \\
$\mathrm{Fe}$ & $4.7346 \mathrm{E}-04$ \\
$\mathrm{Co}$ & $1.7509 \mathrm{E}-07$ \\
$\mathrm{Ni}$ & $9.4935 \mathrm{E}-05$ \\
$\mathrm{Cu}$ & $3.2476 \mathrm{E}-07$ \\
$\mathrm{As}$ & $8.2636 \mathrm{E}-08$ \\
$\mathrm{Nb}$ & $1.1106 \mathrm{E}-07$ \\
$\mathrm{Mo}$ & $1.0755 \mathrm{E}-05$ \\
$\mathrm{Ta}$ & $1.1405 \mathrm{E}-08$ \\
\hline $\mathrm{Total}$ & $2.4155 \mathrm{E}-02$ \\
\hline & \\
\hline & \\
\hline
\end{tabular}

The homogenized layers of the absorber assembly have the compositions provided in Table 3.1.13. 
NEA/NSC/DOC(2006)1

Liquid Metal Fast Reactor - LMFR

FFTF-LMFR-RESR-001

CRIT-SPEC-REAC-COEF-MISC

Table 3.1.13. Homogenized Compositions of the Absorber Assemblies.

\begin{tabular}{|c|c|c|c|c|c|}
\hline \multirow[b]{2}{*}{$\begin{array}{l}\text { Element } \\
\text { or Isotope }\end{array}$} & \multicolumn{5}{|c|}{ Atom Density (a/b-cm) } \\
\hline & $\begin{array}{l}\text { Lower } \\
\text { Shield } \\
\text { Region }\end{array}$ & $\begin{array}{l}\text { Below } \\
\text { Poison } \\
\text { Region }\end{array}$ & $\begin{array}{l}\text { Above } \\
\text { Poison } \\
\text { Region }\end{array}$ & $\begin{array}{l}\text { Driveline } \\
\text { Region }\end{array}$ & $\begin{array}{c}\text { Withdrawn } \\
\text { Absorber } \\
\text { Region }\end{array}$ \\
\hline${ }^{10} \mathrm{~B}$ & 3.0703E-07 & $1.7405 \mathrm{E}-07$ & $1.6616 \mathrm{E}-07$ & $1.0681 \mathrm{E}-07$ & $4.1900 \mathrm{E}-08$ \\
\hline${ }^{11} \mathrm{~B}$ & $1.2358 \mathrm{E}-06$ & 7.0057E-07 & $6.6882 \mathrm{E}-07$ & 4.2993E-07 & $1.6865 \mathrm{E}-07$ \\
\hline $\mathrm{C}$ & $1.3888 \mathrm{E}-04$ & $7.8725 \mathrm{E}-05$ & 7.5157E-05 & 4.8313E-05 & $1.8952 \mathrm{E}-05$ \\
\hline $\mathrm{N}$ & 1.1909E-05 & $6.7507 \mathrm{E}-06$ & $6.4447 \mathrm{E}-06$ & 4.1429E-06 & $1.6252 \mathrm{E}-06$ \\
\hline $\mathrm{Na}$ & 7.0810E-03 & $1.4254 \mathrm{E}-02$ & $7.6440 \mathrm{E}-03$ & $1.7881 \mathrm{E}-02$ & $2.1370 \mathrm{E}-02$ \\
\hline $\mathrm{Al}$ & $3.0910 \mathrm{E}-05$ & $1.7522 \mathrm{E}-05$ & $1.6728 \mathrm{E}-05$ & $1.0753 \mathrm{E}-05$ & $4.2183 \mathrm{E}-06$ \\
\hline $\mathrm{Si}$ & 4.4543E-04 & $2.5250 \mathrm{E}-04$ & $2.4106 \mathrm{E}-04$ & $1.5496 \mathrm{E}-04$ & $6.0787 \mathrm{E}-05$ \\
\hline $\mathrm{P}$ & $1.0770 \mathrm{E}-05$ & $6.1055 \mathrm{E}-06$ & $5.8288 \mathrm{E}-06$ & 3.7469E-06 & $1.4698 \mathrm{E}-06$ \\
\hline S & 5.2019E-06 & $2.9488 \mathrm{E}-06$ & 2.8152E-06 & $1.8097 \mathrm{E}-06$ & 7.0990E-07 \\
\hline V & $6.5487 \mathrm{E}-05$ & $3.7123 \mathrm{E}-05$ & $3.5440 \mathrm{E}-05$ & $2.2782 \mathrm{E}-05$ & 8.9369E-06 \\
\hline $\mathrm{Cr}$ & $1.1228 \mathrm{E}-02$ & $6.3647 \mathrm{E}-03$ & $6.0763 \mathrm{E}-03$ & $3.9060 \mathrm{E}-03$ & $1.5322 \mathrm{E}-03$ \\
\hline $\mathrm{Mn}$ & $1.0627 \mathrm{E}-03$ & $6.0239 \mathrm{E}-04$ & $5.7509 \mathrm{E}-04$ & $3.6968 \mathrm{E}-04$ & $1.4502 \mathrm{E}-04$ \\
\hline $\mathrm{Fe}$ & $3.8267 \mathrm{E}-02$ & $2.1693 \mathrm{E}-02$ & 2.0709E-02 & $1.3313 \mathrm{E}-02$ & $5.2223 \mathrm{E}-03$ \\
\hline Co & $1.4152 \mathrm{E}-05$ & $8.0222 \mathrm{E}-06$ & $7.6586 \mathrm{E}-06$ & 4.9232E-06 & $1.9313 \mathrm{E}-06$ \\
\hline $\mathrm{Ni}$ & 7.6731E-03 & 4.3497E-03 & $4.1525 \mathrm{E}-03$ & 2.6694E-03 & $1.0471 \mathrm{E}-03$ \\
\hline $\mathrm{Cu}$ & $2.6249 \mathrm{E}-05$ & $1.4880 \mathrm{E}-05$ & $1.4205 \mathrm{E}-05$ & $9.1316 \mathrm{E}-06$ & $3.5821 \mathrm{E}-06$ \\
\hline As & $6.6790 \mathrm{E}-06$ & $3.7861 \mathrm{E}-06$ & $3.6146 \mathrm{E}-06$ & 2.3235E-06 & $9.1148 \mathrm{E}-07$ \\
\hline $\mathrm{Nb}$ & $8.9768 \mathrm{E}-06$ & $5.0887 \mathrm{E}-06$ & 4.8581E-06 & $3.1229 \mathrm{E}-06$ & $1.2251 \mathrm{E}-06$ \\
\hline Mo & 8.6929E-04 & 4.9278E-04 & 4.7045E-04 & $3.0242 \mathrm{E}-04$ & $1.1863 \mathrm{E}-04$ \\
\hline $\mathrm{Ta}$ & $9.2181 \mathrm{E}-07$ & $5.2255 \mathrm{E}-07$ & 4.9887E-07 & $3.2069 \mathrm{E}-07$ & $1.2580 \mathrm{E}-07$ \\
\hline Total & $6.6948 \mathrm{E}-02$ & $4.8191 \mathrm{E}-02$ & $4.0043 \mathrm{E}-02$ & $3.8708 \mathrm{E}-02$ & $2.9540 \mathrm{E}-02$ \\
\hline
\end{tabular}


NEA/NSC/DOC(2006)1

\section{Liquid Metal Fast Reactor - LMFR \\ FFTF-LMFR-RESR-001 \\ CRIT-SPEC-REAC-COEF-MISC}

\subsubsection{Radial Reflectors}

The homogenized layers of the radial reflectors in Row 7 have the compositions provided in Table 3.1.14. The compositions of the homogenized layers of the radial reflectors in Rows 8 and 9 are in Table 3.1.15. See Figure 3.1.13 for location of reflector regions.

Table 3.1.14. Homogenized Compositions of the Radial Reflectors in Row 7.

\begin{tabular}{|c|ccccc||}
\hline \multirow{2}{*}{$\begin{array}{c}\text { Element } \\
\text { or Isotope }\end{array}$} & \multicolumn{5}{|c}{ Atom Density (a/b-cm) } \\
& $\begin{array}{c}\text { Orifice } \\
\text { Region }\end{array}$ & $\begin{array}{c}\text { Lower } \\
\text { Adapter }\end{array}$ & $\begin{array}{c}\text { Reflector } \\
\text { Rlocks } \\
\text { Region }\end{array}$ & $\begin{array}{c}\text { Load } \\
\text { Pad }\end{array}$ & $\begin{array}{c}\text { Upper } \\
\text { Region }\end{array}$ \\
Region \\
\hline \hline${ }^{10} \mathrm{~B}$ & $3.4337 \mathrm{E}-07$ & $3.1698 \mathrm{E}-07$ & $9.6514 \mathrm{E}-08$ & $3.8369 \mathrm{E}-07$ & $3.7506 \mathrm{E}-07$ \\
${ }^{11} \mathrm{~B}$ & $1.3821 \mathrm{E}-06$ & $1.2759 \mathrm{E}-06$ & $3.8848 \mathrm{E}-07$ & $1.5444 \mathrm{E}-06$ & $1.5097 \mathrm{E}-06$ \\
$\mathrm{C}$ & $1.5531 \mathrm{E}-04$ & $1.4338 \mathrm{E}-04$ & $1.3432 \mathrm{E}-03$ & $1.7355 \mathrm{E}-04$ & $1.6965 \mathrm{E}-04$ \\
$\mathrm{~N}$ & $1.3318 \mathrm{E}-05$ & $1.2294 \mathrm{E}-05$ & $3.7434 \mathrm{E}-06$ & $1.4882 \mathrm{E}-05$ & $1.4547 \mathrm{E}-05$ \\
$\mathrm{Na}$ & $5.1210 \mathrm{E}-03$ & $6.5440 \mathrm{E}-03$ & $3.4400 \mathrm{E}-03$ & $2.9460 \mathrm{E}-03$ & $3.4120 \mathrm{E}-03$ \\
$\mathrm{Al}$ & $3.4568 \mathrm{E}-05$ & $3.1912 \mathrm{E}-05$ & $9.7165 \mathrm{E}-06$ & $3.8627 \mathrm{E}-05$ & $3.7758 \mathrm{E}-05$ \\
$\mathrm{Si}$ & $4.9814 \mathrm{E}-04$ & $4.5986 \mathrm{E}-04$ & $4.1789 \mathrm{E}-04$ & $5.5664 \mathrm{E}-04$ & $5.4411 \mathrm{E}-04$ \\
$\mathrm{P}$ & $1.2045 \mathrm{E}-05$ & $1.1119 \mathrm{E}-05$ & $3.3856 \mathrm{E}-06$ & $1.3459 \mathrm{E}-05$ & $1.3157 \mathrm{E}-05$ \\
$\mathrm{~S}$ & $5.8176 \mathrm{E}-06$ & $5.3705 \mathrm{E}-06$ & $8.9367 \mathrm{E}-06$ & $6.5007 \mathrm{E}-06$ & $6.3545 \mathrm{E}-06$ \\
$\mathrm{~V}$ & $7.3237 \mathrm{E}-05$ & $6.7609 \mathrm{E}-05$ & $2.0586 \mathrm{E}-05$ & $8.1837 \mathrm{E}-05$ & $7.9996 \mathrm{E}-05$ \\
$\mathrm{Cr}$ & $1.2557 \mathrm{E}-02$ & $1.1592 \mathrm{E}-02$ & $1.2835 \mathrm{E}-02$ & $1.4031 \mathrm{E}-02$ & $1.3715 \mathrm{E}-02$ \\
$\mathrm{Mn}$ & $1.1884 \mathrm{E}-03$ & $1.0971 \mathrm{E}-03$ & $1.3284 \mathrm{E}-03$ & $1.3280 \mathrm{E}-03$ & $1.2981 \mathrm{E}-03$ \\
$\mathrm{Fe}$ & $4.2796 \mathrm{E}-02$ & $3.9507 \mathrm{E}-02$ & $1.6501 \mathrm{E}-02$ & $4.7821 \mathrm{E}-02$ & $4.6745 \mathrm{E}-02$ \\
$\mathrm{Co}$ & $1.5826 \mathrm{E}-05$ & $1.4610 \mathrm{E}-05$ & $5.7417 \mathrm{E}-05$ & $1.7685 \mathrm{E}-05$ & $1.7287 \mathrm{E}-05$ \\
$\mathrm{Ni}$ & $8.5812 \mathrm{E}-03$ & $7.9217 \mathrm{E}-03$ & $4.1579 \mathrm{E}-02$ & $9.5888 \mathrm{E}-03$ & $9.3731 \mathrm{E}-03$ \\
$\mathrm{Cu}$ & $2.9355 \mathrm{E}-05$ & $2.7099 \mathrm{E}-05$ & $1.3106 \mathrm{E}-04$ & $3.2802 \mathrm{E}-05$ & $3.2064 \mathrm{E}-05$ \\
$\mathrm{As}$ & $7.4694 \mathrm{E}-06$ & $6.8954 \mathrm{E}-06$ & $2.0995 \mathrm{E}-06$ & $8.3465 \mathrm{E}-06$ & $8.1588 \mathrm{E}-06$ \\
$\mathrm{Nb}$ & $1.0039 \mathrm{E}-05$ & $9.2677 \mathrm{E}-06$ & $2.8218 \mathrm{E}-06$ & $1.1218 \mathrm{E}-05$ & $1.0966 \mathrm{E}-05$ \\
$\mathrm{Mo}$ & $9.7217 \mathrm{E}-04$ & $8.9746 \mathrm{E}-04$ & $2.7326 \mathrm{E}-04$ & $1.0863 \mathrm{E}-03$ & $1.0619 \mathrm{E}-03$ \\
$\mathrm{Ta}$ & $1.0309 \mathrm{E}-06$ & $9.5168 \mathrm{E}-07$ & $2.8977 \mathrm{E}-07$ & $1.1520 \mathrm{E}-06$ & $1.1260 \mathrm{E}-06$ \\
\hline $\mathrm{Total}$ & $7.2073 \mathrm{E}-02$ & $6.8351 \mathrm{E}-02$ & $7.7958 \mathrm{E}-02$ & $7.7760 \mathrm{E}-02$ & $7.6543 \mathrm{E}-02$ \\
\hline
\end{tabular}


NEA/NSC/DOC(2006)1

Liquid Metal Fast Reactor - LMFR

FFTF-LMFR-RESR-001

CRIT-SPEC-REAC-COEF-MISC

Table 3.1.15. Homogenized Compositions of the Radial Reflectors in Rows 8 and 9.

\begin{tabular}{|c|c|c|c|c|c|}
\hline \multirow[b]{2}{*}{$\begin{array}{l}\text { Element } \\
\text { or Isotope }\end{array}$} & \multicolumn{5}{|c|}{ Atom Density (a/b-cm) } \\
\hline & $\begin{array}{l}\text { Orifice } \\
\text { Region }\end{array}$ & $\begin{array}{l}\text { Lower } \\
\text { Adapter } \\
\text { Region } \\
\end{array}$ & $\begin{array}{c}\text { Reflector } \\
\text { Blocks } \\
\text { Region } \\
\end{array}$ & $\begin{array}{c}\text { Load } \\
\text { Pad } \\
\text { Region } \\
\end{array}$ & $\begin{array}{l}\text { Upper } \\
\text { Shield } \\
\text { Region } \\
\end{array}$ \\
\hline${ }^{10} \mathrm{~B}$ & $2.6281 \mathrm{E}-07$ & $3.3079 \mathrm{E}-07$ & $1.4214 \mathrm{E}-07$ & 4.0109E-07 & $3.9583 \mathrm{E}-07$ \\
\hline${ }^{11} \mathrm{~B}$ & $1.0578 \mathrm{E}-06$ & $1.3315 \mathrm{E}-06$ & $5.7214 \mathrm{E}-07$ & $1.6144 \mathrm{E}-06$ & $1.5933 \mathrm{E}-06$ \\
\hline $\mathrm{C}$ & $1.1887 \mathrm{E}-04$ & $1.4962 \mathrm{E}-04$ & $1.2472 \mathrm{E}-03$ & $1.8142 \mathrm{E}-04$ & $1.7904 \mathrm{E}-04$ \\
\hline $\mathrm{N}$ & $1.0193 \mathrm{E}-05$ & $1.2830 \mathrm{E}-05$ & 5.5132E-06 & $1.5557 \mathrm{E}-05$ & $1.5353 \mathrm{E}-05$ \\
\hline $\mathrm{Na}$ & $9.4660 \mathrm{E}-03$ & $5.7990 \mathrm{E}-03$ & $2.3240 \mathrm{E}-03$ & $2.0070 \mathrm{E}-03$ & $2.2910 \mathrm{E}-03$ \\
\hline $\mathrm{Al}$ & $2.6458 \mathrm{E}-05$ & $3.3302 \mathrm{E}-05$ & $1.4310 \mathrm{E}-05$ & 4.0379E-05 & $3.9850 \mathrm{E}-05$ \\
\hline $\mathrm{Si}$ & $3.8127 \mathrm{E}-04$ & $4.7989 \mathrm{E}-04$ & $4.5915 \mathrm{E}-04$ & $5.8188 \mathrm{E}-04$ & $5.7425 \mathrm{E}-04$ \\
\hline $\mathrm{P}$ & $9.2191 \mathrm{E}-06$ & $1.1604 \mathrm{E}-05$ & 4.9863E-06 & $1.4070 \mathrm{E}-05$ & $1.3885 \mathrm{E}-05$ \\
\hline $\mathrm{S}$ & 4.4527E-06 & $5.6044 \mathrm{E}-06$ & $9.0547 \mathrm{E}-06$ & $6.7955 \mathrm{E}-06$ & $6.7065 \mathrm{E}-06$ \\
\hline V & $5.6054 \mathrm{E}-05$ & $7.0554 \mathrm{E}-05$ & $3.0318 \mathrm{E}-05$ & $8.5549 \mathrm{E}-05$ & $8.4427 \mathrm{E}-05$ \\
\hline $\mathrm{Cr}$ & $9.6106 \mathrm{E}-03$ & $1.2096 \mathrm{E}-02$ & $1.3669 \mathrm{E}-02$ & $1.4667 \mathrm{E}-02$ & $1.4475 \mathrm{E}-02$ \\
\hline $\mathrm{Mn}$ & $9.0959 \mathrm{E}-04$ & $1.1449 \mathrm{E}-03$ & $1.3971 \mathrm{E}-03$ & $1.3882 \mathrm{E}-03$ & $1.3700 \mathrm{E}-03$ \\
\hline $\mathrm{Fe}$ & $3.2755 \mathrm{E}-02$ & $4.1228 \mathrm{E}-02$ & $2.1787 \mathrm{E}-02$ & 4.9990E-02 & $4.9335 \mathrm{E}-02$ \\
\hline Co & $1.2113 \mathrm{E}-05$ & $1.5247 \mathrm{E}-05$ & $5.4768 \mathrm{E}-05$ & $1.8487 \mathrm{E}-05$ & $1.8245 \mathrm{E}-05$ \\
\hline $\mathrm{Ni}$ & $6.5679 \mathrm{E}-03$ & $8.2668 \mathrm{E}-03$ & $3.9205 \mathrm{E}-02$ & $1.0024 \mathrm{E}-02$ & $9.8923 \mathrm{E}-03$ \\
\hline $\mathrm{Cu}$ & $2.2468 \mathrm{E}-05$ & $2.8280 \mathrm{E}-05$ & $1.2394 \mathrm{E}-04$ & $3.4290 \mathrm{E}-05$ & $3.3840 \mathrm{E}-05$ \\
\hline As & $5.7170 \mathrm{E}-06$ & $7.1957 \mathrm{E}-06$ & $3.0921 \mathrm{E}-06$ & $8.7251 \mathrm{E}-06$ & 8.6107E-06 \\
\hline $\mathrm{Nb}$ & 7.6838E-06 & $9.6713 \mathrm{E}-06$ & 4.1559E-06 & $1.1727 \mathrm{E}-05$ & $1.1573 \mathrm{E}-05$ \\
\hline Mo & 7.4408E-04 & $9.3655 \mathrm{E}-04$ & $4.0245 \mathrm{E}-04$ & $1.1356 \mathrm{E}-03$ & $1.1207 \mathrm{E}-03$ \\
\hline $\mathrm{Ta}$ & $7.8904 \mathrm{E}-07$ & $9.9313 \mathrm{E}-07$ & $4.2676 \mathrm{E}-07$ & $1.2042 \mathrm{E}-06$ & $1.1884 \mathrm{E}-06$ \\
\hline Total & $6.0710 \mathrm{E}-02$ & $7.0298 \mathrm{E}-02$ & $8.0742 \mathrm{E}-02$ & $8.0214 \mathrm{E}-02$ & $7.9473 \mathrm{E}-02$ \\
\hline
\end{tabular}


NEA/NSC/DOC(2006)1

Liquid Metal Fast Reactor - LMFR

FFTF-LMFR-RESR-001

CRIT-SPEC-REAC-COEF-MISC

\subsubsection{Vibration Open Test Assembly}

The homogenized layers of the vibration open test assembly have the compositions provided in Table 3.1.16.

Table 3.1.16. Homogenized Compositions of the Vibration Open Test Assembly.

\begin{tabular}{|c|cc||}
\hline \multirow{2}{*}{$\begin{array}{c}\text { Element } \\
\text { or Isotope }\end{array}$} & $\begin{array}{c}\text { Atom Density (a/b-cm) } \\
\text { Orifice/ } \\
\text { Shield } \\
\text { Region }\end{array}$ & $\begin{array}{c}\text { Instruments/ } \\
\text { Housing } \\
\text { Region }\end{array}$ \\
\hline \hline${ }^{10} \mathrm{~B}$ & $4.2124 \mathrm{E}-07$ & $7.6923 \mathrm{E}-08$ \\
${ }^{11} \mathrm{~B}$ & $1.6956 \mathrm{E}-06$ & $3.0963 \mathrm{E}-07$ \\
$\mathrm{C}$ & $1.9054 \mathrm{E}-04$ & $3.4794 \mathrm{E}-05$ \\
$\mathrm{~N}$ & $1.6338 \mathrm{E}-05$ & $2.9836 \mathrm{E}-06$ \\
$\mathrm{Na}$ & $1.4160 \mathrm{E}-03$ & $1.9249 \mathrm{E}-02$ \\
$\mathrm{Al}$ & $4.2408 \mathrm{E}-05$ & $7.7442 \mathrm{E}-06$ \\
$\mathrm{Si}$ & $6.1112 \mathrm{E}-04$ & $1.1160 \mathrm{E}-04$ \\
$\mathrm{P}$ & $1.4777 \mathrm{E}-05$ & $2.6984 \mathrm{E}-06$ \\
$\mathrm{~S}$ & $7.1370 \mathrm{E}-06$ & $1.3033 \mathrm{E}-06$ \\
$\mathrm{~V}$ & $8.9847 \mathrm{E}-05$ & $1.6407 \mathrm{E}-05$ \\
$\mathrm{Cr}$ & $1.5404 \mathrm{E}-02$ & $2.8130 \mathrm{E}-03$ \\
$\mathrm{Mn}$ & $1.4579 \mathrm{E}-03$ & $2.6624 \mathrm{E}-04$ \\
$\mathrm{Fe}$ & $5.2502 \mathrm{E}-02$ & $9.5874 \mathrm{E}-03$ \\
$\mathrm{Co}$ & $1.9416 \mathrm{E}-05$ & $3.5455 \mathrm{E}-06$ \\
$\mathrm{Ni}$ & $1.0527 \mathrm{E}-02$ & $1.9224 \mathrm{E}-03$ \\
$\mathrm{Cu}$ & $3.6013 \mathrm{E}-05$ & $6.5763 \mathrm{E}-06$ \\
$\mathrm{As}$ & $9.1635 \mathrm{E}-06$ & $1.6733 \mathrm{E}-06$ \\
$\mathrm{Nb}$ & $1.2316 \mathrm{E}-05$ & $2.2490 \mathrm{E}-06$ \\
$\mathrm{Mo}$ & $1.1927 \mathrm{E}-03$ & $2.1779 \mathrm{E}-04$ \\
$\mathrm{Ta}$ & $1.2647 \mathrm{E}-06$ & $2.3095 \mathrm{E}-07$ \\
\hline $\mathrm{Total}$ & $8.3553 \mathrm{E}-02$ & $3.4248 \mathrm{E}-02$ \\
\hline
\end{tabular}


NEA/NSC/DOC(2006)1

Liquid Metal Fast Reactor - LMFR

FFTF-LMFR-RESR-001

CRIT-SPEC-REAC-COEF-MISC

\subsubsection{In-Core Shim Assemblies}

The homogenized layers of the in-core shim assembly have the compositions provided in Table 3.1.17.

Table 3.1.17. Homogenized Compositions of the In-Core Shim Assemblies.

\begin{tabular}{|c|c|c|c|}
\hline $\begin{array}{l}\text { Element } \\
\text { or Isotope }\end{array}$ & $\begin{array}{c}\text { Orifice/ } \\
\text { Shield } \\
\text { Region } \\
\end{array}$ & $\begin{array}{l}\text { Density }(\mathrm{a} / \mathrm{b} \\
\text { Pin } \\
\text { Attachment } \\
\text { Region } \\
\end{array}$ & $\begin{array}{c}\text { Simulated } \\
\text { Fuel Pin } \\
\text { Region } \\
\end{array}$ \\
\hline${ }^{10} \mathrm{~B}$ & $4.2124 \mathrm{E}-07$ & $1.8159 \mathrm{E}-07$ & $2.5805 \mathrm{E}-07$ \\
\hline${ }^{11} \mathrm{~B}$ & $1.6956 \mathrm{E}-06$ & $7.3091 \mathrm{E}-07$ & $1.0387 \mathrm{E}-06$ \\
\hline $\mathrm{C}$ & $1.9054 \mathrm{E}-04$ & $8.2135 \mathrm{E}-05$ & $1.1672 \mathrm{E}-04$ \\
\hline $\mathrm{N}$ & $1.6338 \mathrm{E}-05$ & $7.0431 \mathrm{E}-06$ & $1.0009 \mathrm{E}-05$ \\
\hline $\mathrm{Na}$ & $1.4160 \mathrm{E}-03$ & $1.3847 \mathrm{E}-02$ & $9.7230 \mathrm{E}-03$ \\
\hline $\mathrm{Al}$ & $4.2408 \mathrm{E}-05$ & $1.8281 \mathrm{E}-05$ & $2.5979 \mathrm{E}-05$ \\
\hline $\mathrm{Si}$ & $6.1112 \mathrm{E}-04$ & $2.6344 \mathrm{E}-04$ & $3.7436 \mathrm{E}-04$ \\
\hline $\mathrm{P}$ & $1.4777 \mathrm{E}-05$ & $6.3699 \mathrm{E}-06$ & $9.0521 \mathrm{E}-06$ \\
\hline S & $7.1370 \mathrm{E}-06$ & $3.0766 \mathrm{E}-06$ & $4.3720 \mathrm{E}-06$ \\
\hline V & 8.9847E-05 & $3.8731 \mathrm{E}-05$ & $5.5039 \mathrm{E}-05$ \\
\hline $\mathrm{Cr}$ & $1.5404 \mathrm{E}-02$ & $6.6404 \mathrm{E}-03$ & $9.4365 \mathrm{E}-03$ \\
\hline $\mathrm{Mn}$ & $1.4579 \mathrm{E}-03$ & $6.2848 \mathrm{E}-04$ & $8.9312 \mathrm{E}-04$ \\
\hline $\mathrm{Fe}$ & $5.2502 \mathrm{E}-02$ & $2.2632 \mathrm{E}-02$ & $3.2162 \mathrm{E}-02$ \\
\hline Co & $1.9416 \mathrm{E}-05$ & $8.3697 \mathrm{E}-06$ & $1.1894 \mathrm{E}-05$ \\
\hline $\mathrm{Ni}$ & $1.0527 \mathrm{E}-02$ & $4.5381 \mathrm{E}-03$ & $6.4490 \mathrm{E}-03$ \\
\hline $\mathrm{Cu}$ & $3.6013 \mathrm{E}-05$ & $1.5524 \mathrm{E}-05$ & $2.2061 \mathrm{E}-05$ \\
\hline As & $9.1635 \mathrm{E}-06$ & $3.9501 \mathrm{E}-06$ & $5.6134 \mathrm{E}-06$ \\
\hline $\mathrm{Nb}$ & $1.2316 \mathrm{E}-05$ & $5.3091 \mathrm{E}-06$ & $7.5446 \mathrm{E}-06$ \\
\hline Mo & $1.1927 \mathrm{E}-03$ & $5.1412 \mathrm{E}-04$ & $7.3061 \mathrm{E}-04$ \\
\hline $\mathrm{Ta}$ & $1.2647 \mathrm{E}-06$ & $5.4519 \mathrm{E}-07$ & $7.7475 \mathrm{E}-07$ \\
\hline Total & $8.3553 \mathrm{E}-02$ & $4.9254 \mathrm{E}-02$ & $6.0039 \mathrm{E}-02$ \\
\hline
\end{tabular}


FFTF-LMFR-RESR-001

CRIT-SPEC-REAC-COEF-MISC

\subsubsection{Simulated Core Assembly}

The fully-loaded core configuration of the FFTF does not contain simulated core assemblies.

\subsubsection{In-Reactor Thimble}

The homogenized composition of the in-reactor thimble is shown in Table 3.1.18.

Table 3.1.18. Homogenized Composition of the In-Reactor Thimble.

\begin{tabular}{|c|c|}
\hline $\begin{array}{c}\text { Element } \\
\text { or Isotope }\end{array}$ & $\begin{array}{c}\text { Atom Density } \\
(\mathrm{a} / \mathrm{b}-\mathrm{cm})\end{array}$ \\
\hline \hline${ }^{10} \mathrm{~B}$ & $7.7205 \mathrm{E}-08$ \\
${ }^{11} \mathrm{~B}$ & $3.1076 \mathrm{E}-07$ \\
$\mathrm{C}$ & $3.4921 \mathrm{E}-05$ \\
$\mathrm{~N}$ & $2.9945 \mathrm{E}-06$ \\
$\mathrm{Na}$ & $1.9568 \mathrm{E}-02$ \\
$\mathrm{Al}$ & $7.7726 \mathrm{E}-06$ \\
$\mathrm{Si}$ & $1.1201 \mathrm{E}-04$ \\
$\mathrm{P}$ & $2.7083 \mathrm{E}-06$ \\
$\mathrm{~S}$ & $1.3081 \mathrm{E}-06$ \\
$\mathrm{~V}$ & $1.6467 \mathrm{E}-05$ \\
$\mathrm{Cr}$ & $2.8233 \mathrm{E}-03$ \\
$\mathrm{Mn}$ & $2.6721 \mathrm{E}-04$ \\
$\mathrm{Fe}$ & $9.6225 \mathrm{E}-03$ \\
$\mathrm{Co}$ & $3.5585 \mathrm{E}-06$ \\
$\mathrm{Ni}$ & $1.9295 \mathrm{E}-03$ \\
$\mathrm{Cu}$ & $6.6004 \mathrm{E}-06$ \\
$\mathrm{As}$ & $1.6795 \mathrm{E}-06$ \\
$\mathrm{Nb}$ & $2.2573 \mathrm{E}-06$ \\
$\mathrm{Mo}$ & $2.1859 \mathrm{E}-04$ \\
$\mathrm{Ta}$ & $2.3180 \mathrm{E}-07$ \\
\hline $\mathrm{Total}$ & $3.4622 \mathrm{E}-02$ \\
\hline
\end{tabular}


NEA/NSC/DOC(2006)1

Liquid Metal Fast Reactor - LMFR

FFTF-LMFR-RESR-001

CRIT-SPEC-REAC-COEF-MISC

\subsubsection{Reactor Shielding}

There are two types of radial reactor shielding regions in the core: inner and outer. The homogenized compositions of the reactor shielding are provided in Table 3.1.19 (see Figure 3.1.13 for location of shielding regions).

Table 3.1.19. Homogenized Compositions of the Radial Reactor Shielding.

\begin{tabular}{|c|cc||}
\hline \multirow{2}{*}{$\begin{array}{c}\text { Element } \\
\text { or Isotope }\end{array}$} & $\begin{array}{c}\text { Atom Density }(\mathrm{a} / \mathrm{b}-\mathrm{cm}) \\
\text { Inner } \\
\text { Radial } \\
\text { Shield }\end{array}$ & $\begin{array}{c}\text { Outer } \\
\text { Radial } \\
\text { Shield }\end{array}$ \\
\hline \hline${ }^{10} \mathrm{~B}$ & $3.5148 \mathrm{E}-07$ & $3.7558 \mathrm{E}-07$ \\
${ }^{11} \mathrm{~B}$ & $1.4147 \mathrm{E}-06$ & $1.5118 \mathrm{E}-06$ \\
$\mathrm{C}$ & $1.5898 \mathrm{E}-04$ & $1.6988 \mathrm{E}-04$ \\
$\mathrm{~N}$ & $1.3632 \mathrm{E}-05$ & $1.4567 \mathrm{E}-05$ \\
$\mathrm{Na}$ & $4.6830 \mathrm{E}-03$ & $3.3830 \mathrm{E}-03$ \\
$\mathrm{Al}$ & $3.5384 \mathrm{E}-05$ & $3.7811 \mathrm{E}-05$ \\
$\mathrm{Si}$ & $5.0990 \mathrm{E}-04$ & $5.4487 \mathrm{E}-04$ \\
$\mathrm{P}$ & $1.2329 \mathrm{E}-05$ & $1.3175 \mathrm{E}-05$ \\
$\mathrm{~S}$ & $5.9549 \mathrm{E}-06$ & $6.3633 \mathrm{E}-06$ \\
$\mathrm{~V}$ & $7.4966 \mathrm{E}-05$ & $8.0108 \mathrm{E}-05$ \\
$\mathrm{Cr}$ & $1.2853 \mathrm{E}-02$ & $1.3735 \mathrm{E}-02$ \\
$\mathrm{Mn}$ & $1.2165 \mathrm{E}-03$ & $1.2999 \mathrm{E}-03$ \\
$\mathrm{Fe}$ & $4.3806 \mathrm{E}-02$ & $4.6811 \mathrm{E}-02$ \\
$\mathrm{Co}$ & $1.6200 \mathrm{E}-05$ & $1.7311 \mathrm{E}-05$ \\
$\mathrm{Ni}$ & $8.7838 \mathrm{E}-03$ & $9.3862 \mathrm{E}-03$ \\
$\mathrm{Cu}$ & $3.0048 \mathrm{E}-05$ & $3.2109 \mathrm{E}-05$ \\
$\mathrm{As}$ & $7.6458 \mathrm{E}-06$ & $8.1701 \mathrm{E}-06$ \\
$\mathrm{Nb}$ & $1.0276 \mathrm{E}-05$ & $1.0981 \mathrm{E}-05$ \\
$\mathrm{Mo}$ & $9.9513 \mathrm{E}-04$ & $1.0634 \mathrm{E}-03$ \\
$\mathrm{Ta}$ & $1.0552 \mathrm{E}-06$ & $1.1276 \mathrm{E}-06$ \\
\hline $\mathrm{Total}$ & $7.3216 \mathrm{E}-02$ & $7.6616 \mathrm{E}-02$ \\
\hline & & \\
\hline
\end{tabular}


NEA/NSC/DOC(2006)1

Liquid Metal Fast Reactor - LMFR

FFTF-LMFR-RESR-001

CRIT-SPEC-REAC-COEF-MISC

\subsubsection{Sodium Coolant}

The composition of the sodium coolant is in Table 3.1.20.

Table 3.1.20. Sodium Coolant Composition.

\begin{tabular}{|c|c|}
\hline $\begin{array}{c}\text { Element } \\
\text { or Isotope }\end{array}$ & $\begin{array}{c}\text { Atom Density } \\
(\mathrm{a} / \mathrm{b}-\mathrm{cm})\end{array}$ \\
\hline \hline $\mathrm{Na}$ & $2.3620 \mathrm{E}-02$ \\
\hline Total & $2.3620 \mathrm{E}-02$ \\
\hline
\end{tabular}

\subsubsection{Temperature Data}

The benchmark model temperature is $478 \mathrm{~K}$.

\subsubsection{Experimental and Benchmark-Model $\mathbf{k}_{\text {eff }}$ and / or Subcritical Parameters}

The experimental $\mathrm{k}_{\mathrm{eff}}$ for the fully-loaded core critical was approximately unity, corresponding to delayed critical. A comprehensive bias assessment could not be performed for all homogenization effects but many biases are discussed in Section 3.1.1 and determined to be negligible and/or attributed to uncertainty in the benchmark model. The experimental $\mathrm{k}_{\text {eff }}$ value is adjusted with the total bias listed in Table 3.1.1 and includes the total uncertainty reported in Table 2.1.76. The benchmark model $\mathrm{k}_{\text {eff }}$ value is $0.9993 \pm 0.0021$.

\subsection{Benchmark-Model Specifications for Buckling and Extrapolation-Length Measurements}

Buckling and extrapolation length measurements were not made.

\subsection{Benchmark-Model Specifications for Spectral Characteristics Measurements}

The reactor physics experiments evaluated in this section pertain to two neutron spectra measurements performed in the IRT near the radial center of the core at core midplane and $80 \mathrm{~cm}$ below core midplane, in the lower axial shield region, using proportional counter detectors.

\subsubsection{Description of the Benchmark Model Simplifications}

Simplifications of the benchmark model for determination of the reactivity effects measurements in the FFTF are identical to those of the critical fully-loaded core configuration described in Section 3.1.1.

\subsubsection{Dimensions}

The dimensions of the benchmark model for determination of the reactivity effects measurements in the FFTF are identical to those of the critical fully-loaded core configuration described in Section 3.1.2. 
FFTF-LMFR-RESR-001

CRIT-SPEC-REAC-COEF-MISC

\subsubsection{Material Data}

The materials of the benchmark model for determination of the reactivity effects measurements in the FFTF are identical to those of the critical fully-loaded core configuration described in Section 3.1.3.

\subsubsection{Temperature Data}

The benchmark model temperature is $478 \mathrm{~K}$.

\subsubsection{Benchmark-Model Specification for Spectral Characteristics Parameters}

The benchmark values for the neutron spectra measurements near the radial center of the FFTF in the inreactor thimble at core midplane and at $80 \mathrm{~cm}$ below core midplane are provided in Tables 3.3 .2 and 3.3.3 and Figures 3.3.2 and 3.3.3, respectively. The benchmark values represent the experimental values with no bias correction.

The relative flux values represent the point flux at a given lethargy energy divided by the maximum flux, which occurs at the energy of $117.971 \mathrm{keV}$ for both sets of data, and multiplied by the value 8.8. It is assumed that the scaling factor was used to spread the chart out such that it would fit onto a full sheet of computer printout paper; no further explanation is provided.

The uncertainty in the energy is obtained from the resolution data, as explained in Section 2.3.1. 
NEA/NSC/DOC(2006)1

Liquid Metal Fast Reactor - LMFR

FFTF-LMFR-RESR-001

CRIT-SPEC-REAC-COEF-MISC

Table 3.3.2. Benchmark FFTF Core-Center Neutron Spectrum.

\begin{tabular}{|c|c|c|c|c|c|c|c|c|c|}
\hline $\begin{array}{l}\text { Energy } \\
(\mathrm{keV})\end{array}$ & $\sigma$ & $\begin{array}{c}\text { Relative Flux } \\
\varphi / \varphi_{\max } \times 8.8\end{array}$ & $\sigma$ & $\sigma(\%)$ & $\begin{array}{c}\text { Energy } \\
(\mathrm{keV})\end{array}$ & $\sigma$ & $\begin{array}{c}\text { Relative Flux } \\
\varphi / \varphi_{\max } \times 8.8\end{array}$ & $\sigma$ & $\sigma(\%)$ \\
\hline 11.145 & 0.190 & 0.54 & $\begin{array}{c}0.03 \\
\end{array}$ & 5.8 & 7.677 & 0.523 & 1.48 & 20.06 & 3.8 \\
\hline 1.202 & 0.195 & 0.54 & 0.03 & 6.4 & 8.061 & 0.539 & 1.59 & 0.07 & 4.2 \\
\hline 1.262 & 0.200 & 0.46 & 0.04 & 8.2 & 8.464 & 0.555 & 1.74 & 0.07 & 4.2 \\
\hline 1.325 & 0.205 & 0.40 & 0.03 & 8.6 & 8.887 & 0.571 & 2.02 & 0.07 & 3.4 \\
\hline 1.392 & 0.210 & 0.39 & 0.04 & 9.7 & 9.331 & 0.588 & 2.27 & 0.07 & 3.1 \\
\hline 1.461 & 0.215 & 0.43 & 0.04 & 8.2 & 9.798 & 0.605 & 2.53 & 0.08 & 3.0 \\
\hline 1.534 & 0.221 & 0.31 & 0.04 & 14.2 & 10.287 & 0.622 & 2.40 & 0.07 & 3.0 \\
\hline 1.611 & 0.226 & 0.35 & 0.03 & 7.9 & 10.802 & 0.639 & 2.16 & 0.09 & 4.0 \\
\hline 1.692 & 0.233 & 0.37 & 0.03 & 7.2 & 11.342 & 0.656 & 2.33 & 0.08 & 3.4 \\
\hline 1.776 & 0.238 & 0.39 & 0.03 & 8.0 & 11.909 & 0.679 & 2.35 & 0.08 & 3.6 \\
\hline 1.865 & 0.244 & 0.37 & 0.03 & 8.1 & 12.504 & 0.697 & 2.23 & 0.08 & 3.5 \\
\hline 1.958 & 0.251 & 0.26 & 0.03 & 12.8 & 13.13 & 0.721 & 2.25 & 0.07 & 3.0 \\
\hline 2.056 & 0.257 & 0.35 & 0.03 & 9.7 & 13.786 & 0.739 & 2.22 & 0.06 & 2.7 \\
\hline 2.159 & 0.264 & 0.38 & 0.04 & 9.8 & 14.476 & 0.764 & 2.06 & 0.08 & 3.9 \\
\hline 2.267 & 0.270 & 0.40 & 0.04 & 9.1 & 15.199 & 0.789 & 2.05 & 0.07 & 3.3 \\
\hline 2.380 & 0.277 & 0.37 & 0.04 & 10.8 & 15.959 & 0.808 & 2.07 & 0.07 & 3.6 \\
\hline 2.499 & 0.285 & 0.36 & 0.04 & 12.3 & 16.757 & 0.834 & 2.33 & 0.08 & 3.5 \\
\hline 2.624 & 0.291 & 0.44 & 0.05 & 10.5 & 17.595 & 0.861 & 2.54 & 0.08 & 3.3 \\
\hline 2.755 & 0.300 & 0.54 & 0.05 & 8.4 & 18.475 & 0.888 & 2.84 & 0.08 & 2.7 \\
\hline 2.893 & 0.308 & 0.69 & 0.04 & 5.2 & 19.399 & 0.916 & 3.10 & 0.08 & 2.7 \\
\hline 3.038 & 0.315 & 0.75 & 0.03 & 4.4 & 20.368 & 0.945 & 3.13 & 0.10 & 3.2 \\
\hline 3.190 & 0.324 & 0.81 & 0.04 & 4.4 & 21.387 & 0.974 & 3.60 & 0.09 & 2.5 \\
\hline 3.349 & 0.332 & 0.71 & 0.04 & 5.7 & 22.456 & 1.003 & 4.18 & 0.10 & 2.3 \\
\hline 3.517 & 0.341 & 0.73 & 0.04 & 5.5 & 23.579 & 1.054 & 4.42 & 0.09 & 2.1 \\
\hline 3.693 & 0.350 & 0.73 & 0.04 & 5.7 & 24.758 & 1.096 & 4.05 & 0.09 & 2.3 \\
\hline 3.877 & 0.360 & 0.89 & 0.05 & 5.3 & 25.996 & 1.139 & 2.54 & 0.10 & 3.9 \\
\hline 4.071 & 0.369 & 0.93 & 0.05 & 5.8 & 27.296 & 1.196 & 1.78 & 0.07 & 4.0 \\
\hline 4.275 & 0.378 & 0.98 & 0.05 & 5.3 & 28.661 & 1.244 & 1.75 & 0.07 & 4.1 \\
\hline 4.488 & 0.390 & 1.04 & 0.06 & 5.5 & 30.094 & 1.293 & 2.29 & 0.09 & 4.1 \\
\hline 4.713 & 0.399 & 1.02 & 0.06 & 5.8 & 31.598 & 1.358 & 2.72 & 0.09 & 3.2 \\
\hline 4.948 & 0.411 & 1.11 & 0.06 & 5.8 & 33.178 & 1.412 & 3.06 & 0.09 & 2.9 \\
\hline 5.196 & 0.422 & 1.37 & 0.06 & 4.4 & 34.837 & 1.482 & 3.21 & 0.10 & 3.0 \\
\hline 5.456 & 0.432 & 1.27 & 0.06 & 5.1 & 36.579 & 1.541 & 3.44 & 0.10 & 2.9 \\
\hline 5.728 & 0.444 & 1.25 & 0.06 & 4.9 & 38.408 & 1.569 & 3.97 & 0.11 & 2.7 \\
\hline 6.015 & 0.458 & 1.24 & 0.07 & 5.3 & 40.328 & 1.682 & 4.12 & 0.10 & 2.4 \\
\hline 6.316 & 0.470 & 1.52 & 0.05 & 3.0 & 42.345 & 1.748 & 4.57 & 0.11 & 2.3 \\
\hline 6.631 & 0.483 & 1.43 & 0.05 & 3.3 & 44.462 & 1.835 & 4.47 & 0.10 & 2.2 \\
\hline 6.963 & 0.495 & 1.36 & 0.06 & 4.3 & 46.685 & 1.927 & 3.63 & 0.12 & 3.3 \\
\hline 7.311 & 0.510 & 1.38 & 0.06 & 4.4 & 49.019 & 2.002 & 3.58 & 0.12 & 3.2 \\
\hline
\end{tabular}


NEA/NSC/DOC(2006)1

\section{Liquid Metal Fast Reactor - LMFR \\ FFTF-LMFR-RESR-001 \\ CRIT-SPEC-REAC-COEF-MISC}

Table 3.3.2 (cont'd). Benchmark FFTF Core-Center Neutron Spectrum.

\begin{tabular}{|c|c|c|c|c|c|c|c|c|c|}
\hline $\begin{array}{c}\text { Energy } \\
(\mathrm{keV})\end{array}$ & $\sigma$ & $\begin{array}{c}\text { Relative Flux } \\
\varphi / \varphi_{\max } \times 8.8 \\
\end{array}$ & $\sigma$ & $\sigma(\%)$ & $\begin{array}{c}\text { Energy } \\
(\mathrm{keV})\end{array}$ & $\sigma$ & $\begin{array}{c}\text { Relative Flux } \\
\varphi / \varphi_{\max } \times 8.8 \\
\end{array}$ & $\sigma$ & $\sigma(\%)$ \\
\hline 51.470 & 2.103 & 3.97 & 0.09 & 2.3 & 328.662 & 14.405 & 6.37 & 0.09 & 1.5 \\
\hline 54.044 & 2.185 & 4.71 & 0.10 & 2.1 & 345.095 & 14.979 & 5.92 & 0.10 & 1.7 \\
\hline 56.746 & 2.294 & 5.33 & 0.09 & 1.6 & 362.349 & 15.727 & 5.70 & 0.11 & 1.9 \\
\hline 59.583 & 2.383 & 5.17 & 0.10 & 2.0 & 380.467 & 16.514 & 5.42 & 0.10 & 1.8 \\
\hline 62.562 & 2.502 & 5.30 & 0.12 & 2.3 & 399.490 & 17.340 & 4.91 & 0.11 & 2.2 \\
\hline 65.690 & 2.628 & 5.88 & 0.12 & 2.0 & 419.465 & 18.207 & 4.85 & 0.08 & 1.6 \\
\hline 68.975 & 2.730 & 6.14 & 0.10 & 1.7 & 440.438 & 19.117 & 5.18 & 0.08 & 1.5 \\
\hline 72.424 & 2.866 & 6.32 & 0.11 & 1.8 & 462.460 & 20.073 & 5.73 & 0.08 & 1.4 \\
\hline 76.045 & 3.009 & 6.63 & 0.12 & 1.8 & 485.583 & 21.076 & 6.24 & 0.08 & 1.2 \\
\hline 79.847 & 3.160 & 5.77 & 0.12 & 2.1 & 509.862 & 22.130 & 6.25 & 0.08 & 1.2 \\
\hline 83.840 & 3.282 & 5.21 & 0.13 & 2.6 & 535.355 & 23.237 & 6.23 & 0.08 & 1.3 \\
\hline 88.031 & 3.446 & 5.04 & 0.13 & 2.6 & 562.123 & 24.399 & 6.06 & 0.09 & 1.4 \\
\hline 92.433 & 3.619 & 5.32 & 0.14 & 2.6 & 590.229 & 25.367 & 5.98 & 0.09 & 1.5 \\
\hline 97.055 & 3.758 & 5.68 & 0.15 & 2.6 & 619.741 & 26.636 & 5.97 & 0.10 & 1.7 \\
\hline 101.907 & 3.946 & 6.37 & 0.14 & 2.2 & 650.728 & 27.967 & 5.76 & 0.09 & 1.6 \\
\hline 107.003 & 4.144 & 7.45 & 0.15 & 2.0 & 683.264 & 29.366 & 5.38 & 0.07 & 1.3 \\
\hline 112.353 & 4.351 & 7.94 & 0.16 & 2.0 & 717.427 & 30.834 & 5.24 & 0.07 & 1.4 \\
\hline 117.971 & 4.568 & 8.80 & 0.22 & 2.5 & 753.299 & 32.376 & 4.78 & 0.07 & 1.5 \\
\hline 123.869 & 4.744 & 7.99 & 0.21 & 2.6 & 790.964 & 33.995 & 4.54 & 0.07 & 1.5 \\
\hline 130.063 & 4.981 & 6.55 & 0.22 & 3.4 & 830.512 & 35.694 & 4.38 & 0.07 & 1.6 \\
\hline 136.566 & 6.160 & 6.42 & 0.24 & 3.7 & 872.037 & 37.479 & 4.17 & 0.07 & 1.7 \\
\hline 143.394 & 6.468 & 6.85 & 0.08 & 1.2 & 915.639 & 39.353 & 3.90 & 0.07 & 1.9 \\
\hline 150.564 & 6.727 & 7.22 & 0.11 & 1.5 & 961.421 & 41.321 & 3.64 & 0.08 & 2.1 \\
\hline 158.092 & 7.064 & 6.93 & 0.11 & 1.7 & 1009.492 & 43.387 & 3.43 & 0.08 & 2.3 \\
\hline 165.996 & 7.417 & 6.66 & 0.10 & 1.5 & 1059.967 & 45.556 & 3.65 & 0.08 & 2.1 \\
\hline 174.296 & 7.788 & 6.49 & 0.11 & 1.7 & 1112.965 & 47.834 & 3.56 & 0.08 & 2.3 \\
\hline 183.011 & 8.177 & 6.22 & 0.11 & 1.8 & 1168.613 & 50.225 & 3.62 & 0.09 & 2.4 \\
\hline 192.162 & 8.504 & 6.96 & 0.12 & 1.7 & 1227.044 & 52.737 & 3.80 & 0.08 & 2.2 \\
\hline 201.770 & 8.929 & 7.53 & 0.11 & 1.4 & 1288.396 & 55.374 & 3.52 & 0.09 & 2.5 \\
\hline 211.858 & 9.376 & 6.77 & 0.11 & 1.7 & 1352.816 & 58.142 & 3.32 & 0.09 & 2.6 \\
\hline 222.451 & 9.845 & 5.72 & 0.11 & 2.0 & 1420.457 & 61.049 & 3.42 & 0.08 & 2.4 \\
\hline 233.574 & 10.337 & 7.02 & 0.15 & 2.1 & 1491.480 & 64.102 & 3.32 & 0.09 & 2.6 \\
\hline 245.252 & 10.749 & 7.22 & 0.13 & 1.8 & 1566.054 & 67.307 & 2.82 & 0.08 & 2.7 \\
\hline 257.515 & 11.287 & 7.17 & 0.09 & 1.2 & 1644.356 & 70.672 & 2.69 & 0.07 & 2.7 \\
\hline 270.391 & 11.851 & 7.30 & 0.10 & 1.3 & 1726.574 & 73.471 & 2.56 & 0.08 & 3.0 \\
\hline 283.910 & 12.444 & 7.15 & 0.10 & 1.4 & 1812.903 & 77.145 & 2.44 & 0.09 & 3.5 \\
\hline 298.106 & 13.066 & 7.11 & 0.09 & 1.3 & 1903.548 & 81.002 & 2.21 & 0.09 & 3.9 \\
\hline 313.011 & 13.719 & 6.80 & 0.09 & 1.4 & & & & & \\
\hline
\end{tabular}


NEA/NSC/DOC(2006)1

Liquid Metal Fast Reactor - LMFR

FFTF-LMFR-RESR-001

CRIT-SPEC-REAC-COEF-MISC

Table 3.3.3. Benchmark FFTF Lower-Axial Neutron Spectrum.

\begin{tabular}{|c|c|c|c|c|c|c|c|c|c|}
\hline $\begin{array}{c}\text { Energy } \\
(\mathrm{keV})\end{array}$ & $\sigma$ & $\begin{array}{c}\text { Relative Flux } \\
\varphi / \varphi_{\max } \times 8.8 \\
\end{array}$ & $\sigma$ & $\sigma(\%)$ & $\begin{array}{c}\text { Energy } \\
(\mathrm{keV}) \\
\end{array}$ & $\sigma$ & $\begin{array}{c}\text { Relative Flux } \\
\varphi / \varphi_{\max } \times 8.8\end{array}$ & $\sigma$ & $\sigma(\%)$ \\
\hline 3.038 & 0.315 & 0.82 & 0.05 & 6.6 & 20.368 & 0.945 & 4.83 & 0.10 & 2.1 \\
\hline 3.190 & 0.324 & 0.82 & 0.05 & 5.7 & 21.387 & 0.974 & 5.37 & 0.10 & 1.8 \\
\hline 3.349 & 0.332 & 0.69 & 0.05 & 7.3 & 22.456 & 1.013 & 6.18 & 0.10 & 1.6 \\
\hline 3.517 & 0.341 & 0.33 & 0.06 & 17.0 & 23.579 & 1.054 & 6.60 & 0.09 & 1.4 \\
\hline 3.693 & 0.350 & 0.33 & 0.06 & 18.3 & 24.758 & 1.096 & 6.88 & 0.07 & 1.0 \\
\hline 3.877 & 0.360 & 0.38 & 0.07 & 17.3 & 25.996 & 1.139 & 5.92 & 0.07 & 1.2 \\
\hline 4.071 & 0.369 & 0.23 & 0.06 & 26.1 & 27.296 & 1.196 & 3.97 & 0.09 & 2.3 \\
\hline 4.275 & 0.378 & 0.24 & 0.07 & 26.7 & 28.661 & 1.244 & 2.80 & 0.08 & 2.8 \\
\hline 4.488 & 0.390 & 0.41 & 0.07 & 17.5 & 30.094 & 1.293 & 2.16 & 0.09 & 4.1 \\
\hline 4.713 & 0.399 & 0.67 & 0.08 & 11.6 & 31.598 & 1.358 & 2.29 & 0.09 & 4.1 \\
\hline 4.948 & 0.411 & 0.70 & 0.09 & 12.1 & 33.178 & 1.412 & 2.81 & 0.10 & 3.6 \\
\hline 5.196 & 0.422 & 1.01 & 0.05 & 5.2 & 34.837 & 1.482 & 3.29 & 0.11 & 3.4 \\
\hline 5.456 & 0.418 & 1.31 & 0.05 & 3.9 & 36.579 & 1.541 & 3.91 & 0.10 & 2.5 \\
\hline 5.728 & 0.444 & 1.52 & 0.06 & 4.1 & 38.408 & 1.602 & 4.55 & 0.10 & 2.3 \\
\hline 6.015 & 0.458 & 1.81 & 0.06 & 3.2 & 40.328 & 1.682 & 4.89 & 0.11 & 2.3 \\
\hline 6.316 & 0.470 & 1.76 & 0.06 & 3.5 & 42.345 & 1.748 & 5.18 & 0.11 & 2.2 \\
\hline 6.631 & 0.483 & 1.64 & 0.05 & 3.3 & 44.462 & 1.835 & 5.30 & 0.11 & 2.1 \\
\hline 6.963 & 0.495 & 1.57 & 0.06 & 3.8 & 46.685 & 1.927 & 4.91 & 0.11 & 2.3 \\
\hline 7.311 & 0.510 & 1.67 & 0.06 & 3.7 & 49.019 & 2.002 & 4.84 & 0.09 & 1.8 \\
\hline 7.677 & 0.523 & 1.88 & 0.07 & 3.7 & 51.470 & 2.103 & 4.44 & 0.09 & 2.1 \\
\hline 8.061 & 0.539 & 2.03 & 0.07 & 3.6 & 54.044 & 2.185 & 5.08 & 0.10 & 2.1 \\
\hline 8.464 & 0.555 & 2.59 & 0.07 & 2.6 & 56.746 & 2.294 & 5.86 & 0.10 & 1.6 \\
\hline 8.887 & 0.571 & 2.69 & 0.07 & 2.6 & 59.583 & 2.383 & 6.11 & 0.10 & 1.6 \\
\hline 9.331 & 0.588 & 3.60 & 0.08 & 2.1 & 62.562 & 2.502 & 6.15 & 0.11 & 1.7 \\
\hline 9.798 & 0.605 & 3.92 & 0.08 & 2.1 & 65.690 & 2.628 & 6.58 & 0.11 & 1.7 \\
\hline 10.287 & 0.622 & 3.71 & 0.09 & 2.4 & 68.975 & 2.730 & 6.66 & 0.12 & 1.8 \\
\hline 10.802 & 0.639 & 3.66 & 0.08 & 2.2 & 72.424 & 2.866 & 7.13 & 0.11 & 1.5 \\
\hline 11.342 & 0.656 & 3.34 & 0.06 & 1.9 & 76.045 & 3.009 & 7.32 & 0.11 & 1.5 \\
\hline 11.909 & 0.679 & 3.40 & 0.07 & 2.0 & 79.847 & 3.160 & 6.57 & 0.12 & 1.8 \\
\hline 12.504 & 0.697 & 3.08 & 0.07 & 2.2 & 83.840 & 3.282 & 6.09 & 0.11 & 1.8 \\
\hline 13.130 & 0.721 & 3.26 & 0.06 & 1.9 & 88.031 & 3.446 & 5.49 & 0.13 & 2.4 \\
\hline 13.786 & 0.739 & 3.31 & 0.08 & 2.3 & 92.433 & 3.619 & 5.21 & 0.12 & 2.3 \\
\hline 14.476 & 0.764 & 3.41 & 0.08 & 2.4 & 97.055 & 3.758 & 5.82 & 0.11 & 2.0 \\
\hline 15.199 & 0.789 & 2.94 & 0.09 & 3.0 & 101.907 & 3.946 & 6.53 & 0.12 & 1.9 \\
\hline 15.959 & 0.808 & 2.65 & 0.09 & 3.4 & 107.003 & 4.144 & 7.49 & 0.17 & 2.3 \\
\hline 16.757 & 0.834 & 3.22 & 0.08 & 2.5 & 112.353 & 4.351 & 8.66 & 0.18 & 2.1 \\
\hline 17.595 & 0.861 & 3.78 & 0.09 & 2.4 & 117.971 & 5.371 & 8.80 & 0.16 & 1.8 \\
\hline 18.475 & 0.888 & 4.25 & 0.09 & 2.2 & 123.869 & 5.640 & 6.86 & 0.08 & 1.2 \\
\hline 19.399 & 0.916 & 4.40 & 0.09 & 1.9 & 130.063 & 5.867 & 6.13 & 0.09 & 1.4 \\
\hline
\end{tabular}


NEA/NSC/DOC(2006)1

Liquid Metal Fast Reactor - LMFR

FFTF-LMFR-RESR-001

CRIT-SPEC-REAC-COEF-MISC

Table 3.3.3 (cont'd). Benchmark FFTF Lower-Axial Neutron Spectrum.

\begin{tabular}{|cc|ccc||cc|ccc||}
\hline $\begin{array}{c}\text { Energy } \\
(\mathrm{keV})\end{array}$ & $\sigma$ & $\begin{array}{c}\text { Relative Flux } \\
\varphi / \varphi_{\max } \times 8.8\end{array}$ & $\sigma$ & $\sigma(\%)$ & $\begin{array}{c}\text { Energy } \\
(\mathrm{keV})\end{array}$ & $\sigma$ & $\begin{array}{c}\text { Relative Flux } \\
\varphi / \varphi_{\max } \times 8.8\end{array}$ & $\sigma$ & $\sigma(\%)$ \\
\hline \hline 136.566 & 6.160 & 5.86 & 0.07 & 1.2 & 535.355 & 23.237 & 2.05 & 0.05 & 2.3 \\
143.394 & 6.468 & 5.23 & 0.07 & 1.4 & 562.123 & 24.399 & 2.16 & 0.03 & 1.6 \\
150.564 & 6.727 & 5.02 & 0.08 & 1.6 & 590.229 & 25.367 & 1.99 & 0.03 & 1.6 \\
158.092 & 7.064 & 5.16 & 0.08 & 1.6 & 619.741 & 26.636 & 1.69 & 0.03 & 2.0 \\
165.996 & 7.417 & 5.29 & 0.09 & 1.7 & 650.728 & 27.967 & 1.54 & 0.03 & 2.0 \\
174.296 & 7.788 & 5.59 & 0.09 & 1.7 & 683.264 & 29.366 & 1.37 & 0.03 & 2.3 \\
183.011 & 8.177 & 5.37 & 0.08 & 1.6 & 717.427 & 30.834 & 1.32 & 0.03 & 2.5 \\
192.162 & 8.504 & 5.39 & 0.09 & 1.6 & 753.299 & 32.376 & 1.27 & 0.03 & 2.5 \\
201.770 & 8.929 & 5.12 & 0.08 & 1.6 & 790.964 & 33.995 & 1.20 & 0.03 & 2.7 \\
211.858 & 9.376 & 5.50 & 0.06 & 1.0 & 830.512 & 35.694 & 1.21 & 0.04 & 2.9 \\
222.451 & 9.845 & 5.18 & 0.05 & 1.0 & 872.037 & 37.479 & 1.17 & 0.03 & 2.6 \\
233.574 & 10.337 & 5.53 & 0.06 & 1.1 & 915.639 & 39.353 & 1.08 & 0.03 & 3.2 \\
245.252 & 10.749 & 5.39 & 0.06 & 1.2 & 961.421 & 41.321 & 0.91 & 0.04 & 4.0 \\
257.515 & 11.287 & 5.40 & 0.06 & 1.1 & 1009.492 & 43.387 & 0.95 & 0.03 & 3.3 \\
270.391 & 11.851 & 5.15 & 0.06 & 1.2 & 1059.967 & 45.556 & 0.99 & 0.03 & 3.3 \\
283.910 & 12.444 & 4.83 & 0.06 & 1.3 & 1112.965 & 47.834 & 0.79 & 0.03 & 4.2 \\
298.106 & 13.066 & 4.40 & 0.05 & 1.2 & 1168.613 & 50.225 & 0.72 & 0.03 & 4.3 \\
313.011 & 13.719 & 4.11 & 0.06 & 1.4 & 1227.044 & 52.737 & 0.69 & 0.03 & 4.9 \\
328.662 & 14.405 & 3.74 & 0.06 & 1.5 & 1288.396 & 55.374 & 0.61 & 0.05 & 8.3 \\
345.095 & 14.979 & 3.27 & 0.04 & 1.4 & 1352.816 & 58.142 & 0.51 & 0.03 & 5.1 \\
362.349 & 15.727 & 3.03 & 0.04 & 1.3 & 1420.457 & 61.049 & 0.49 & 0.03 & 5.4 \\
380.467 & 16.514 & 2.94 & 0.05 & 1.6 & 1491.480 & 64.102 & 0.46 & 0.03 & 6.4 \\
399.490 & 17.340 & 2.97 & 0.04 & 1.4 & 1566.054 & 67.307 & 0.41 & 0.03 & 7.3 \\
419.465 & 18.207 & 3.02 & 0.04 & 1.5 & 1644.356 & 70.672 & 0.41 & 0.03 & 7.3 \\
440.438 & 19.117 & 2.77 & 0.04 & 1.6 & 1726.574 & 73.471 & 0.30 & 0.03 & 9.8 \\
462.460 & 20.073 & 2.65 & 0.05 & 1.7 & 1812.903 & 77.145 & 0.25 & 0.03 & 10.2 \\
485.583 & 21.076 & 2.55 & 0.04 & 1.5 & 1903.548 & 81.002 & 0.23 & 0.02 & 10.9 \\
509.862 & 22.130 & 2.31 & 0.04 & 1.8 & & & & & \\
\hline
\end{tabular}


FFTF-LMFR-RESR-001

CRIT-SPEC-REAC-COEF-MISC

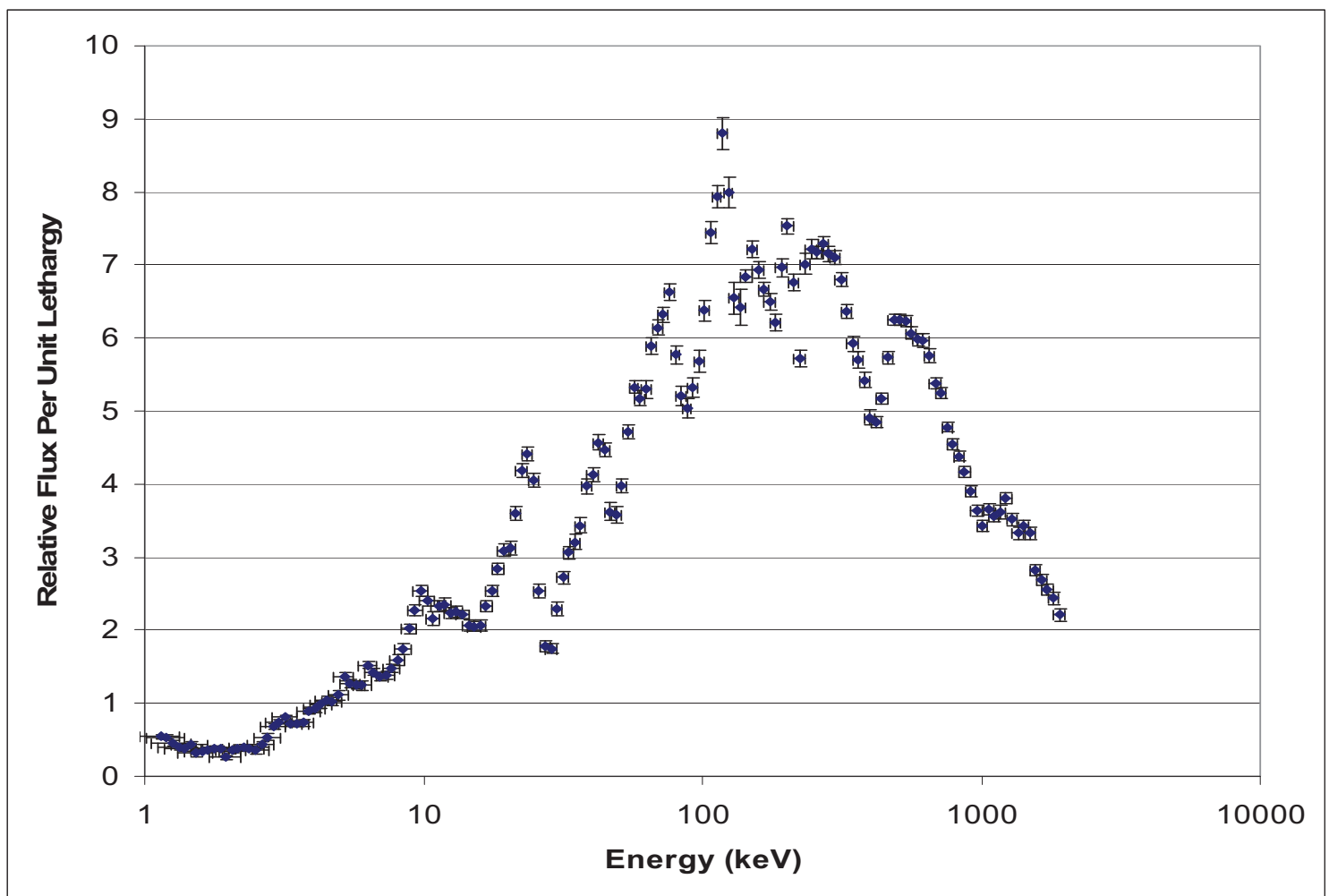

Figure 3.3.2. Neutron Spectrum in FFTF Core Center (at Midplane).

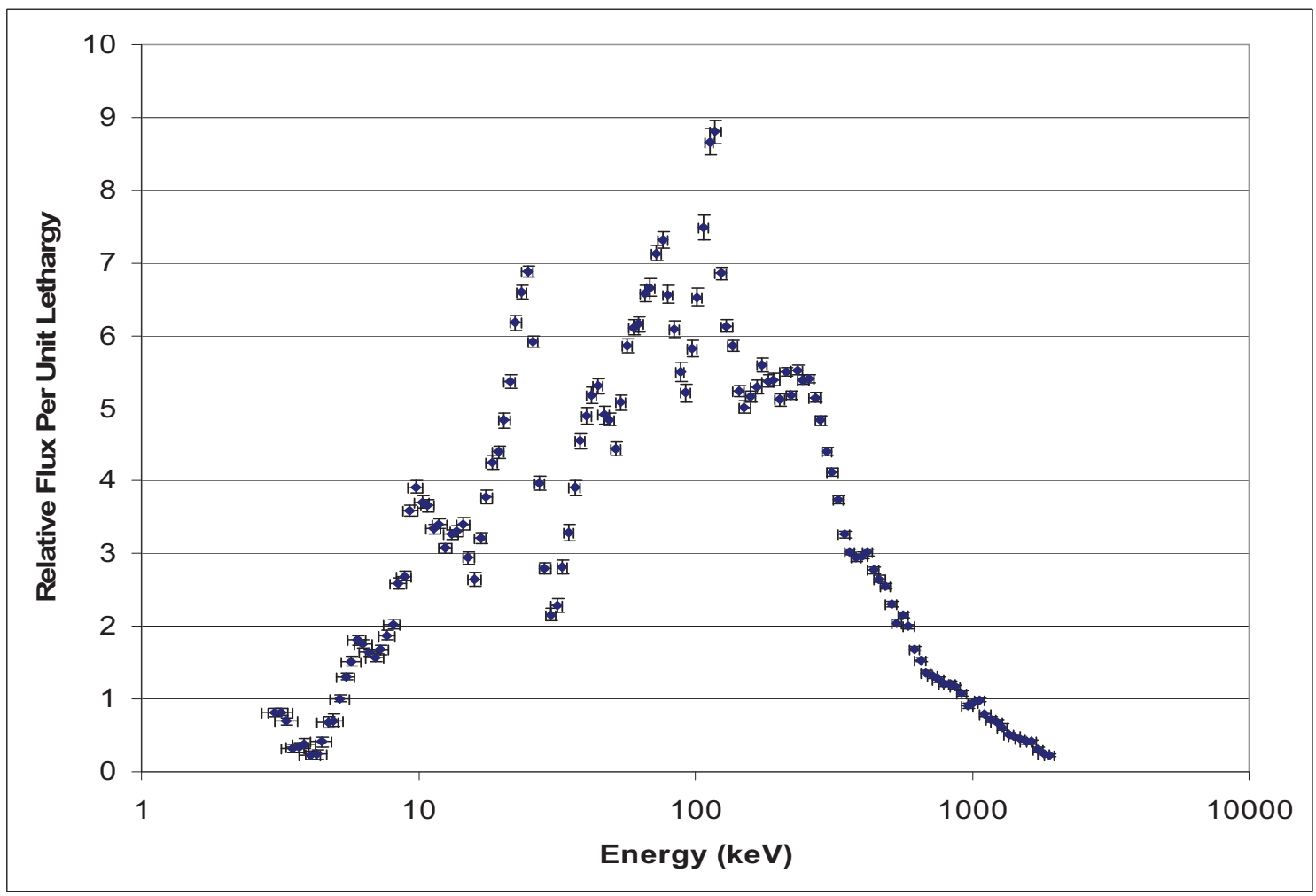

Figure 3.3.3. Neutron Spectrum in FFTF Core Center ( $80 \mathrm{~cm}$ Below Midplane). 
NEA/NSC/DOC(2006)1

Liquid Metal Fast Reactor - LMFR

FFTF-LMFR-RESR-001

CRIT-SPEC-REAC-COEF-MISC

\subsection{Benchmark-Model Specifications for Reactivity Effects Parameters}

Reactivity effects measurements evaluated include a total of 21 control rod worth measurements (seven of which represent combinations of dropped rods), two control rod bank worth measurements, six differential control rod worth measurements, measured shutdown margin, and measured excess reactivity for the fully-loaded critical core configuration of the FFTF.

\subsubsection{Description of the Benchmark Model Simplifications}

Simplifications of the benchmark model for determination of the reactivity effects measurements in the FFTF are identical to those of the critical fully-loaded core configuration described in Section 3.1.1.

\subsubsection{Dimensions}

The dimensions of the benchmark model for determination of the reactivity effects measurements in the FFTF are identical to those of the critical fully-loaded core configuration described in Section 3.1.2 with exception of the control rod positions.

\subsubsection{Control Rod Worths}

There are nine reference subcritical states from which control rod worths are evaluated. A summary of the rod positions for these states is provided in Table 3.4.1. One to three rods are then dropped from these reference states to ascertain their worth. A correlation of rod worth tests to their given reference state is provided in Table 3.4.2.

Table 3.4.1. Rod Positions for Control-Rod-Worth Reference Subcritical States.

\begin{tabular}{|c|ccccccccc||}
\hline \multirow{2}{*}{$\begin{array}{c}\text { Control } \\
\text { Rod } \\
\text { Number }\end{array}$} & State 1 & State 2 & State 3 & State 4 & State 5 & State 6 & State 7 & State 8 & State 9 \\
\cline { 2 - 10 } & 91.44 & 91.44 & 91.44 & 91.44 & 91.44 & 91.44 & 91.44 & 91.44 & 91.44 \\
2 & 91.44 & 91.44 & 91.44 & 91.44 & 91.44 & 91.44 & 91.44 & 91.44 & 91.44 \\
3 & 91.44 & 91.44 & 91.44 & 91.44 & 91.44 & 91.44 & 91.44 & 91.44 & 91.44 \\
4 & 0 & 35.306 & 91.44 & 24.384 & 26.416 & 25.4 & 27.686 & 25.654 & 35.052 \\
5 & 91.44 & 35.306 & 24.638 & 91.44 & 26.416 & 25.4 & 27.686 & 25.654 & 35.052 \\
6 & 0 & 35.306 & 24.638 & 24.384 & 91.44 & 25.4 & 27.686 & 25.654 & 35.052 \\
7 & 71.12 & 35.306 & 24.638 & 24.384 & 26.416 & 91.44 & 27.686 & 25.654 & 35.052 \\
8 & 0 & 35.306 & 24.638 & 24.384 & 26.416 & 25.4 & 91.44 & 25.654 & 35.052 \\
9 & 0 & 35.306 & 24.638 & 24.384 & 26.416 & 25.146 & 27.686 & 91.44 & 35.052 \\
\hline \hline
\end{tabular}


Liquid Metal Fast Reactor - LMFR

FFTF-LMFR-RESR-001

CRIT-SPEC-REAC-COEF-MISC

Table 3.4.2. Correlation of Rod Worth Tests with Subcritical Reference States.

\begin{tabular}{|c|cc||}
\hline Case & $\begin{array}{c}\text { Reference } \\
\text { State }\end{array}$ & $\begin{array}{c}\text { Rod(s) } \\
\text { Dropped }\end{array}$ \\
\hline 1 & 1 & 1 \\
2 & 1 & 2 \\
3 & 1 & 3 \\
4 & 1 & 5 \\
5 & 1 & 7 \\
6 & 1 & $1+2$ \\
7 & 1 & $1+3$ \\
8 & 1 & $1+5$ \\
9 & 1 & $3+5$ \\
10 & 1 & $1+2+5$ \\
11 & 1 & $2+3+5$ \\
12 & 2 & 1 \\
13 & 2 & 2 \\
14 & 2 & 3 \\
15 & 3 & 4 \\
16 & 4 & 5 \\
17 & 5 & 6 \\
18 & 6 & 7 \\
19 & 7 & 8 \\
20 & 8 & 9 \\
21 & 9 & \\
\hline
\end{tabular}

\subsubsection{Control Rod Bank Worths}

The rod bank worths of the three primary control rods and six secondary control rods are obtained from a comparison of adjusted subcritical core configurations with a fully subcritical core configuration. For the primary rod bank worth, a subcritical core with all rods fully inserted has the three primary safety rods fully withdrawn to provide a comparison. For the secondary rod bank worth, a subcritical core with all rods fully inserted has the six secondary rods fully withdrawn to provide a comparison. A summary of rod movements for the determination of the rod bank worths is provided in Table 3.4.3. 
Liquid Metal Fast Reactor - LMFR

FFTF-LMFR-RESR-001

CRIT-SPEC-REAC-COEF-MISC

Table 3.4.3. Rod Movements for the Determination of Rod Bank Worths.

\begin{tabular}{|c|c|c|c|}
\hline \multirow{2}{*}{$\begin{array}{c}\text { Control } \\
\text { Rod } \\
\text { Number }\end{array}$} & \multicolumn{3}{|c|}{ Distance Withdrawn (cm) } \\
\hline & $\begin{array}{l}\text { Fully Subcritical } \\
\text { (All Rods In) }\end{array}$ & $\begin{array}{c}\text { Primary Bank } \\
\text { Withdrawn (Case 22) }\end{array}$ & $\begin{array}{c}\text { Secondary Bank } \\
\text { Withdrawn (Case 23) }\end{array}$ \\
\hline 1 & 0 & 91.44 & 0 \\
\hline 2 & 0 & 91.44 & 0 \\
\hline 3 & 0 & 91.44 & 0 \\
\hline 4 & 0 & 0 & 91.44 \\
\hline 5 & 0 & 0 & 91.44 \\
\hline 6 & 0 & 0 & 91.44 \\
\hline 7 & 0 & 0 & 91.44 \\
\hline 8 & 0 & 0 & 91.44 \\
\hline 9 & 0 & 0 & 91.44 \\
\hline
\end{tabular}

\subsubsection{Differential Control Rod Worths}

A tenth reference subcritical state is utilized for the measurement of differential control rod worths (Table 3.4.4). Control rods $4,5,6,7,8$, and 9 are individually moved $\pm 2.54 \mathrm{~cm}$ from this configuration to obtain the differential worth measurements. These measurements represent Cases 24 through 29, respectively.

Table 3.4.4. Reference Subcritical State of

Differential Rod Worth Measurements.

\begin{tabular}{|c|c|}
\hline $\begin{array}{c}\text { Control } \\
\text { Rod } \\
\text { Number }\end{array}$ & $\begin{array}{c}\text { Distance } \\
\text { Withdrawn }(\mathrm{cm})\end{array}$ \\
\cline { 2 - 2 } & State 10 \\
\hline \hline 1 & 91.44 \\
2 & 91.44 \\
3 & 91.44 \\
4 & 34.798 \\
5 & 34.798 \\
6 & 34.798 \\
7 & 34.798 \\
8 & 34.798 \\
9 & 34.798 \\
\hline
\end{tabular}


NEA/NSC/DOC(2006)1

Liquid Metal Fast Reactor - LMFR

FFTF-LMFR-RESR-001

CRIT-SPEC-REAC-COEF-MISC

\subsubsection{Shutdown Margin}

The shutdown margin is evaluated using both the first and second reference subcritical states. The configurations of these two states in Table 3.4.1 is compared with the fully subcritical core configuration (Table 3.4.3) that is developed when all control rods are fully inserted. The evaluated shutdown margin values are Cases 30 and 31, respectively.

\subsubsection{Excess Reactivity}

The excess reactivity of the control rods remaining in the core is evaluated by using the fully-loaded critical core configuration of the FFTF and fully withdrawing all control rods (Table 3.4.5).

Table 3.4.5. Rod Positions for Evaluation of Core Excess Reactivity.

\begin{tabular}{||c|cc||}
\hline \multirow{2}{*}{$\begin{array}{c}\text { Control } \\
\text { Rod } \\
\text { Number }\end{array}$} & \multicolumn{2}{|c|}{ Distance Withdrawn (cm) } \\
\cline { 2 - 3 } & $\begin{array}{c}\text { Critical } \\
\text { Configuration }\end{array}$ & $\begin{array}{c}\text { All Rods } \\
\text { Withdrawn (Case 32) }\end{array}$ \\
\hline \hline 1 & 91.44 & 91.44 \\
2 & 91.44 & 91.44 \\
3 & 91.44 & 91.44 \\
4 & 36.116 & 91.44 \\
5 & 35.56 & 91.44 \\
6 & 35.56 & 91.44 \\
7 & 35.56 & 91.44 \\
8 & 35.56 & 91.44 \\
9 & 35.56 & 91.44 \\
\hline
\end{tabular}

\subsubsection{Material Data}

The materials of the benchmark model for determination of the reactivity effects measurements in the FFTF are identical to those of the critical fully-loaded core configuration described in Section 3.1.3.

\subsubsection{Temperature Data}

The benchmark model temperature is $478 \mathrm{~K}$.

\subsubsection{Benchmark-Model Specification for Reactivity Effects Parameters}

The benchmark values represent the experimental values without any bias correction. 
FFTF-LMFR-RESR-001

CRIT-SPEC-REAC-COEF-MISC

\subsubsection{Control Rod Worths}

The benchmark values for the control rod worths are provided in Table 3.4.6.

Table 3.4.6. Benchmark Control Rod Worths.

\begin{tabular}{|c|ccccc||}
\hline Case & $\begin{array}{c}\text { Reference } \\
\text { State }\end{array}$ & $\begin{array}{c}\text { Rod(s) } \\
\text { Dropped }\end{array}$ & $\rho(\$)$ & \pm & $\sigma$ \\
\hline \hline 1 & 1 & 1 & 6.04 & \pm & 0.32 \\
2 & 1 & 2 & 5.89 & \pm & 0.31 \\
3 & 1 & 3 & 4.65 & \pm & 0.25 \\
4 & 1 & 5 & 3.84 & \pm & 0.20 \\
5 & 1 & 7 & 2.90 & \pm & 0.15 \\
6 & 1 & $1+2$ & 12.45 & \pm & 0.69 \\
7 & 1 & $1+3$ & 11.01 & \pm & 0.60 \\
8 & 1 & $1+5$ & 9.00 & \pm & 0.48 \\
9 & 1 & $3+5$ & 8.8 & \pm & 0.46 \\
10 & 1 & $1+2+5$ & 15.84 & \pm & 0.87 \\
11 & 1 & $2+3+5$ & 15.22 & \pm & 0.86 \\
12 & 2 & 1 & 5.81 & \pm & 0.33 \\
13 & 2 & 2 & 5.51 & \pm & 0.29 \\
14 & 2 & 3 & 5.40 & \pm & 0.29 \\
15 & 3 & 4 & 4.06 & \pm & 0.21 \\
16 & 4 & 5 & 4.09 & \pm & 0.21 \\
17 & 5 & 6 & 3.57 & \pm & 0.18 \\
18 & 6 & 7 & 3.86 & \pm & 0.20 \\
19 & 7 & 8 & 3.19 & \pm & 0.19 \\
20 & 8 & 9 & 3.83 & \pm & 0.19 \\
21 & 9 & $2+3$ & 11.22 & \pm & 0.58 \\
\hline
\end{tabular}

\subsubsection{Control Rod Bank Worths}

The benchmark values for the control rod bank worths are provided in Table 3.4.7.

Table 3.4.7. Benchmark Control Rod Bank Worths.

\begin{tabular}{|c|cccccc||}
\hline Case & $\begin{array}{c}\text { Reference } \\
\text { State }\end{array}$ & $\begin{array}{c}\text { Rod(s) } \\
\text { Dropped }\end{array}$ & $\rho(\$)$ & \pm & $\sigma$ \\
\hline \hline 22 & Fully Subcritical & $1+2+3$ & 16.34 & \pm & 0.83 \\
23 & Fully Subcritical & $4+5+6+7+8+9$ & 19.9 & \pm & 1.00 \\
\hline
\end{tabular}


NEA/NSC/DOC(2006)1

Liquid Metal Fast Reactor - LMFR

FFTF-LMFR-RESR-001

CRIT-SPEC-REAC-COEF-MISC

\subsubsection{Differential Control Rod Worths}

The benchmark values for the differential control rod worths are provided in Table 3.4.8.

Table 3.4.8. Benchmark Differential Control Rod Worths.

\begin{tabular}{|c|cccccc||}
\hline Case & $\begin{array}{c}\text { Reference } \\
\text { State }\end{array}$ & $\begin{array}{c}\text { Rod } \\
\text { Movement }\end{array}$ & $\rho \phi / \mathrm{cm}$ & \pm & $\sigma$ \\
\hline \hline 24 & 10 & 4 & 6.1 & \pm & 0.3 \\
25 & 10 & 5 & 6.2 & \pm & 0.3 \\
26 & 10 & 6 & 5.4 & \pm & 0.3 \\
27 & 10 & 7 & 5.7 & \pm & 0.3 \\
28 & 10 & 8 & 4.8 & \pm & 0.3 \\
29 & 10 & 9 & 5.6 & \pm & 0.3 \\
\hline
\end{tabular}

\subsubsection{Shutdown Margin}

The benchmark values for the shutdown margin are provided in Table 3.4.9.

Table 3.4.9. Benchmark Shutdown Margins.

\begin{tabular}{|c|ccccc|}
\hline Case & $\begin{array}{c}\text { Reference } \\
\text { State }\end{array}$ & $\begin{array}{c}\text { Rods } \\
\text { Dropped }\end{array}$ & $\rho(\$)$ & \pm & $\sigma$ \\
\hline \hline 30 & 1 & All & 24.0 & \pm & 1.40 \\
31 & 2 & All & 23.7 & \pm & 1.27 \\
\hline
\end{tabular}

\subsubsection{Excess Reactivity}

The benchmark value for the excess reactivity (Case 32) is $\$ 14.66 \pm 0.85$, determined from the critical core configuration.

\subsection{Benchmark-Model Specifications for Reactivity Coefficient Measurements}

The isothermal temperature coefficient has been evaluated for the fully-loaded critical core configuration of the FFTF.

\subsubsection{Description of the Benchmark Model Simplifications}

Simplifications of the benchmark model for determination of the reactivity effects measurements in the FFTF are identical to those of the critical fully-loaded core configuration described in Section 3.1.1.

\subsubsection{Dimensions}

The dimensions of the benchmark model for determination of the reactivity effects measurements in the FFTF are identical to those of the critical fully-loaded core configuration described in Section 3.1.2. 
NEA/NSC/DOC(2006)1

Liquid Metal Fast Reactor - LMFR

FFTF-LMFR-RESR-001

CRIT-SPEC-REAC-COEF-MISC

\subsubsection{Material Data}

The materials of the benchmark model for determination of the reactivity effects measurements in the FFTF are identical to those of the critical fully-loaded core configuration described in Section 3.1.3.

The density of the liquid sodium coolant has been adjusted as shown in Table 3.5.1 for the temperature analysis.

Table 3.5.1. Sodium Coolant Composition.

\begin{tabular}{|c|c|}
\hline $\begin{array}{c}\text { Temperature } \\
(\mathrm{K})\end{array}$ & $\begin{array}{c}\text { Sodium Atom Density } \\
(\mathrm{a} / \mathrm{b}-\mathrm{cm})\end{array}$ \\
\hline \hline 453 & $2.3767 \mathrm{E}-02$ \\
478 & $2.3620 \mathrm{E}-02$ \\
503 & $2.3473 \mathrm{E}-02$ \\
\hline
\end{tabular}

\subsubsection{Temperature Data}

The fully-loaded core critical benchmark model temperature is $478 \mathrm{~K}$. The model temperature is varied $\pm 25 \mathrm{~K}$.

\subsubsection{Benchmark-Model Specification for Reactivity Coefficient Parameters}

The benchmark value for the isothermal temperature coefficient is $-1.26 \pm 0.19 \phi / \mathrm{K}\left(-0.7 \pm 0.11 \phi /{ }^{\circ} \mathrm{F}\right)$. The benchmark value represents the experimental value without any bias correction.

\subsection{Benchmark-Model Specifications for Kinetics Measurements}

Kinetics measurements were not made.

\subsection{Benchmark-Model Specifications for Reaction-Rate Distribution Measurements}

Reaction-rate distribution measurements were not made.

\subsection{Benchmark-Model Specifications for Power Distribution Measurements}

Power distribution measurements were not made.

\subsection{Benchmark-Model Specifications for Isotopic Measurements}

Isotopic measurements were not made. 
NEA/NSC/DOC(2006)1

Liquid Metal Fast Reactor - LMFR

FFTF-LMFR-RESR-001

CRIT-SPEC-REAC-COEF-MISC

\subsection{Benchmark-Model Specifications for Other Miscellaneous Types of Measurements}

The low-energy electron and gamma-ray spectra measurements performed in the IRT near the axial and radial center of the core have been evaluated for the fully-loaded critical core configuration of the FFTF.

\subsubsection{Description of the Benchmark Model Simplifications}

Simplifications of the benchmark model for determination of the reactivity effects measurements in the FFTF are identical to those of the critical fully-loaded core configuration described in Section 3.1.1.

\subsubsection{Dimensions}

The dimensions of the benchmark model for determination of the reactivity effects measurements in the FFTF are identical to those of the critical fully-loaded core configuration described in Section 3.1.2.

\subsubsection{Material Data}

The materials of the benchmark model for determination of the reactivity effects measurements in the FFTF are identical to those of the critical fully-loaded core configuration described in Section 3.1.3.

\subsubsection{Temperature Data}

The benchmark model temperature is $478 \mathrm{~K}$.

\subsubsection{Benchmark-Model Specification for Other Miscellaneous Types of Measurements}

The benchmark values for the low-energy gamma-ray spectra measurements are provided in Table 3.10.1 and Figure 3.10.1. The low-energy electron spectra measurements were evaluated, but judged to be not of benchmark quality (Section 2.10.1). The benchmark values represent the experimental values without any bias correction 
NEA/NSC/DOC(2006)1

Liquid Metal Fast Reactor - LMFR

FFTF-LMFR-RESR-001

CRIT-SPEC-REAC-COEF-MISC

Table 3.10.1. Benchmark FFTF Core Center Low-Energy Gamma-Ray Spectrum.

\begin{tabular}{|c|c|c|c|c|c|c|c|}
\hline $\begin{array}{l}\text { Energy } \\
(\mathrm{MeV})\end{array}$ & $\begin{array}{c}\text { Normalized } \\
\text { Flux } \\
\varphi / \varphi_{\max }\end{array}$ & \pm & $\sigma$ & $\begin{array}{l}\text { Energy } \\
(\mathrm{MeV})\end{array}$ & $\begin{array}{c}\text { Normalized } \\
\text { Flux } \\
\varphi / \varphi_{\max }\end{array}$ & \pm & $\sigma$ \\
\hline 0.2203 & 1.0148 & \pm & 0.1015 & 1.2364 & 0.1448 & \pm & 0.0145 \\
\hline 0.2485 & 1.0000 & \pm & 0.1000 & 1.2593 & 0.1419 & \pm & 0.0142 \\
\hline 0.2745 & 0.8341 & \pm & 0.0834 & 1.2809 & 0.1343 & \pm & 0.0134 \\
\hline 0.3006 & 0.6492 & \pm & 0.0649 & 1.3012 & 0.1275 & \pm & 0.0128 \\
\hline 0.3252 & 0.5383 & \pm & 0.0538 & 1.3186 & 0.1327 & \pm & 0.0133 \\
\hline 0.3498 & 0.4594 & \pm & 0.0459 & 1.3428 & 0.1330 & \pm & 0.0133 \\
\hline 0.3741 & 0.4335 & \pm & 0.0434 & 1.3617 & 0.1270 & \pm & 0.0127 \\
\hline 0.3955 & 0.4474 & \pm & 0.0447 & 1.3803 & 0.1396 & \pm & 0.0140 \\
\hline 0.4223 & 0.4615 & \pm & 0.0462 & 1.4005 & 0.1322 & \pm & 0.0132 \\
\hline 0.4426 & 0.4282 & \pm & 0.0428 & 1.4220 & 0.1348 & \pm & 0.0135 \\
\hline 0.4881 & 0.4746 & \pm & 0.0475 & 1.4435 & 0.1333 & \pm & 0.0133 \\
\hline 0.5152 & 0.4192 & \pm & 0.0419 & 1.4653 & 0.1170 & \pm & 0.0117 \\
\hline 0.5357 & 0.3568 & \pm & 0.0357 & 1.4843 & 0.1098 & \pm & 0.0110 \\
\hline 0.5546 & 0.3455 & \pm & 0.0346 & 1.5060 & 0.0982 & \pm & 0.0098 \\
\hline 0.5776 & 0.3261 & \pm & 0.0326 & 1.5274 & 0.1016 & \pm & 0.0102 \\
\hline 0.6033 & 0.3043 & \pm & 0.0304 & 1.5475 & 0.1073 & \pm & 0.0107 \\
\hline 0.6236 & 0.2897 & \pm & 0.0290 & 1.5666 & 0.0945 & \pm & 0.0094 \\
\hline 0.6452 & 0.2680 & \pm & 0.0268 & 1.5867 & 0.0944 & \pm & 0.0094 \\
\hline 0.6667 & 0.2718 & \pm & 0.0272 & 1.6056 & 0.0925 & \pm & 0.0093 \\
\hline 0.7140 & 0.2434 & \pm & 0.0243 & 1.6339 & 0.0901 & \pm & 0.0090 \\
\hline 0.7288 & 0.2427 & \pm & 0.0243 & 1.6500 & 0.0888 & \pm & 0.0089 \\
\hline 0.7299 & 0.2645 & \pm & 0.0265 & 1.6731 & 0.0782 & \pm & 0.0078 \\
\hline 0.7544 & 0.2304 & \pm & 0.0230 & 1.6904 & 0.0848 & \pm & 0.0085 \\
\hline 0.7768 & 0.2784 & \pm & 0.0278 & 1.7121 & 0.0796 & \pm & 0.0080 \\
\hline 0.7992 & 0.3365 & \pm & 0.0337 & 1.7358 & 0.0984 & \pm & 0.0098 \\
\hline 0.8205 & 0.3710 & \pm & 0.0371 & 1.7519 & 0.0984 & \pm & 0.0098 \\
\hline 0.8407 & 0.3604 & \pm & 0.0360 & 1.7726 & 0.0775 & \pm & 0.0077 \\
\hline 0.8626 & 0.2954 & \pm & 0.0295 & 1.7953 & 0.0809 & \pm & 0.0081 \\
\hline 0.8859 & 0.2457 & \pm & 0.0246 & 1.8128 & 0.0815 & \pm & 0.0082 \\
\hline 0.9035 & 0.2292 & \pm & 0.0229 & 1.8340 & 0.0909 & \pm & 0.0091 \\
\hline 0.9239 & 0.2127 & \pm & 0.0213 & 1.8558 & 0.0801 & \pm & 0.0080 \\
\hline 0.9442 & 0.2007 & \pm & 0.0201 & 1.8777 & 0.0662 & \pm & 0.0066 \\
\hline 0.9658 & 0.1911 & \pm & 0.0191 & 1.8966 & 0.0643 & \pm & 0.0064 \\
\hline 0.9873 & 0.1856 & \pm & 0.0186 & 1.9177 & 0.0758 & \pm & 0.0076 \\
\hline 1.0104 & 0.1707 & \pm & 0.0171 & 1.9370 & 0.0630 & \pm & 0.0063 \\
\hline 1.0722 & 0.1657 & \pm & 0.0166 & 1.9595 & 0.0705 & \pm & 0.0070 \\
\hline 1.0925 & 0.1591 & \pm & 0.0159 & 1.9758 & 0.0654 & \pm & 0.0065 \\
\hline 1.1127 & 0.1506 & \pm & 0.0151 & 2.0000 & 0.0659 & \pm & 0.0066 \\
\hline 1.1357 & 0.1430 & \pm & 0.0143 & 2.0188 & 0.0663 & \pm & 0.0066 \\
\hline 1.1544 & 0.1523 & \pm & 0.0152 & 2.0406 & 0.0574 & \pm & 0.0057 \\
\hline 1.1732 & 0.1518 & \pm & 0.0152 & 2.0647 & 0.0608 & \pm & 0.0061 \\
\hline 1.1946 & 0.1553 & \pm & 0.0155 & 2.0807 & 0.0625 & \pm & 0.0063 \\
\hline 1.2190 & 0.1445 & \pm & 0.0144 & 2.1010 & 0.0590 & \pm & 0.0059 \\
\hline
\end{tabular}

Revision: 0 
NEA/NSC/DOC(2006)1

Liquid Metal Fast Reactor - LMFR

FFTF-LMFR-RESR-001

CRIT-SPEC-REAC-COEF-MISC

Table 3.10.1 (cont'd). Benchmark FFTF Core Center Low-Energy Gamma-Ray Spectrum.

\begin{tabular}{|c|c|c|c|c|c|c|c|}
\hline $\begin{array}{l}\text { Energy } \\
(\mathrm{MeV})\end{array}$ & $\begin{array}{c}\text { Normalized } \\
\text { Flux } \\
\varphi / \varphi_{\max }\end{array}$ & \pm & $\sigma$ & $\begin{array}{l}\text { Energy } \\
(\mathrm{MeV})\end{array}$ & $\begin{array}{c}\text { Normalized } \\
\text { Flux } \\
\varphi / \varphi_{\max }\end{array}$ & \pm & $\sigma$ \\
\hline 2.1211 & 0.0600 & \pm & 0.0060 & 3.0307 & 0.0188 & \pm & 0.0019 \\
\hline 2.1443 & 0.0527 & \pm & 0.0053 & 3.0533 & 0.0200 & \pm & 0.0020 \\
\hline 2.1628 & 0.0583 & \pm & 0.0058 & 3.0728 & 0.0154 & \pm & 0.0015 \\
\hline 2.1821 & 0.0481 & \pm & 0.0048 & 3.0953 & 0.0172 & \pm & 0.0017 \\
\hline 2.2006 & 0.0550 & \pm & 0.0055 & 3.1116 & 0.0162 & \pm & 0.0016 \\
\hline 2.2249 & 0.0530 & \pm & 0.0053 & 3.1331 & 0.0162 & \pm & 0.0016 \\
\hline 2.2426 & 0.0473 & \pm & 0.0047 & 3.1524 & 0.0132 & \pm & 0.0013 \\
\hline 2.2631 & 0.0405 & \pm & 0.0041 & 3.1748 & 0.0157 & \pm & 0.0016 \\
\hline 2.2869 & 0.0483 & \pm & 0.0048 & 3.1906 & 0.0183 & \pm & 0.0018 \\
\hline 2.3068 & 0.0521 & \pm & 0.0052 & 3.2148 & 0.0102 & \pm & 0.0010 \\
\hline 2.3263 & 0.0404 & \pm & 0.0040 & 3.2315 & 0.0139 & \pm & 0.0014 \\
\hline 2.3438 & 0.0388 & \pm & 0.0039 & 3.2552 & 0.0172 & \pm & 0.0017 \\
\hline 2.3838 & 0.0442 & \pm & 0.0044 & 3.2749 & 0.0118 & \pm & 0.0012 \\
\hline 2.4069 & 0.0400 & \pm & 0.0040 & 3.2964 & 0.0209 & \pm & 0.0021 \\
\hline 2.4259 & 0.0361 & \pm & 0.0036 & 3.3148 & 0.0141 & \pm & 0.0014 \\
\hline 2.4463 & 0.0335 & \pm & 0.0034 & 3.3378 & 0.0130 & \pm & 0.0013 \\
\hline 2.4652 & 0.0319 & \pm & 0.0032 & 3.3570 & 0.0110 & \pm & 0.0011 \\
\hline 2.4889 & 0.0393 & \pm & 0.0039 & 3.3767 & 0.0133 & \pm & 0.0013 \\
\hline 2.5092 & 0.0361 & \pm & 0.0036 & 3.3988 & 0.0105 & \pm & 0.0010 \\
\hline 2.5264 & 0.0404 & \pm & 0.0040 & 3.4171 & 0.0130 & \pm & 0.0013 \\
\hline 2.5470 & 0.0333 & \pm & 0.0033 & 3.4381 & 0.0090 & \pm & 0.0009 \\
\hline 2.5659 & 0.0326 & \pm & 0.0033 & 3.4569 & 0.0091 & \pm & 0.0009 \\
\hline 2.5899 & 0.0355 & \pm & 0.0036 & 3.4783 & 0.0095 & \pm & 0.0009 \\
\hline 2.6072 & 0.0215 & \pm & 0.0022 & 3.4947 & 0.0150 & \pm & 0.0015 \\
\hline 2.6304 & 0.0322 & \pm & 0.0032 & 3.5161 & 0.0086 & \pm & 0.0009 \\
\hline 2.6479 & 0.0317 & \pm & 0.0032 & 3.5360 & 0.0169 & \pm & 0.0017 \\
\hline 2.6719 & 0.0341 & \pm & 0.0034 & 3.5571 & 0.0114 & \pm & 0.0011 \\
\hline 2.6913 & 0.0262 & \pm & 0.0026 & 3.5770 & 0.0128 & \pm & 0.0013 \\
\hline 2.7125 & 0.0300 & \pm & 0.0030 & 3.5989 & 0.0060 & \pm & 0.0006 \\
\hline 2.7326 & 0.0305 & \pm & 0.0031 & 3.6157 & 0.0081 & \pm & 0.0008 \\
\hline 2.7515 & 0.0292 & \pm & 0.0029 & 3.6369 & 0.0090 & \pm & 0.0009 \\
\hline 2.7698 & 0.0209 & \pm & 0.0021 & 3.6582 & 0.0098 & \pm & 0.0010 \\
\hline 2.7875 & 0.0185 & \pm & 0.0018 & 3.6744 & 0.0096 & \pm & 0.0010 \\
\hline 2.8124 & 0.0240 & \pm & 0.0024 & 3.6978 & 0.0075 & \pm & 0.0008 \\
\hline 2.8281 & 0.0295 & \pm & 0.0029 & 3.7190 & 0.0083 & \pm & 0.0008 \\
\hline 2.8488 & 0.0227 & \pm & 0.0023 & 3.7387 & 0.0105 & \pm & 0.0010 \\
\hline 2.8735 & 0.0187 & \pm & 0.0019 & 3.7611 & 0.0040 & \pm & 0.0004 \\
\hline 2.8918 & 0.0227 & \pm & 0.0023 & 3.7775 & 0.0110 & \pm & 0.0011 \\
\hline 2.9121 & 0.0216 & \pm & 0.0022 & 3.7984 & 0.0081 & \pm & 0.0008 \\
\hline 2.9336 & 0.0212 & \pm & 0.0021 & 3.8169 & 0.0091 & \pm & 0.0009 \\
\hline 2.9540 & 0.0195 & \pm & 0.0020 & 3.8380 & 0.0060 & \pm & 0.0006 \\
\hline 2.9699 & 0.0217 & \pm & 0.0022 & 3.8576 & 0.0078 & \pm & 0.0008 \\
\hline 2.9928 & 0.0207 & \pm & 0.0021 & 3.8801 & 0.0090 & \pm & 0.0009 \\
\hline
\end{tabular}

Revision: 0 
NEA/NSC/DOC(2006)1

Liquid Metal Fast Reactor - LMFR

FFTF-LMFR-RESR-001

CRIT-SPEC-REAC-COEF-MISC

Table 3.10.1 (cont'd). Benchmark FFTF Core Center Low-Energy Gamma-Ray Spectrum.

\begin{tabular}{|c|c|c|c|c|c|c|c|}
\hline $\begin{array}{l}\text { Energy } \\
(\mathrm{MeV})\end{array}$ & $\begin{array}{c}\text { Normalized } \\
\text { Flux } \\
\varphi / \varphi_{\max }\end{array}$ & \pm & $\sigma$ & $\begin{array}{l}\text { Energy } \\
(\mathrm{MeV})\end{array}$ & $\begin{array}{c}\text { Normalized } \\
\text { Flux } \\
\varphi / \varphi_{\max }\end{array}$ & \pm & $\sigma$ \\
\hline 3.9027 & 0.0055 & \pm & 0.0006 & 4.6468 & 0.0034 & \pm & 0.0003 \\
\hline 3.9181 & 0.0076 & \pm & 0.0008 & 4.6692 & 0.0023 & \pm & 0.0002 \\
\hline 3.9426 & 0.0037 & \pm & 0.0004 & 4.6878 & 0.0045 & \pm & 0.0004 \\
\hline 3.9607 & 0.0090 & \pm & 0.0009 & 4.7064 & 0.0049 & \pm & 0.0005 \\
\hline 3.9810 & 0.0147 & \pm & 0.0015 & 4.7247 & 0.0019 & \pm & 0.0002 \\
\hline 4.0003 & 0.0067 & \pm & 0.0007 & 4.7493 & 0.0028 & \pm & 0.0003 \\
\hline 4.0183 & 0.0054 & \pm & 0.0005 & 4.7654 & 0.0029 & \pm & 0.0003 \\
\hline 4.0400 & 0.0049 & \pm & 0.0005 & 4.7888 & 0.0040 & \pm & 0.0004 \\
\hline 4.0614 & 0.0051 & \pm & 0.0005 & 4.8080 & 0.0060 & \pm & 0.0006 \\
\hline 4.0793 & 0.0076 & \pm & 0.0008 & 4.8324 & 0.0031 & \pm & 0.0003 \\
\hline 4.1033 & 0.0046 & \pm & 0.0005 & 4.8508 & 0.0021 & \pm & 0.0002 \\
\hline 4.1214 & 0.0061 & \pm & 0.0006 & 4.8672 & 0.0057 & \pm & 0.0006 \\
\hline 4.1373 & 0.0068 & \pm & 0.0007 & 4.8890 & 0.0029 & \pm & 0.0003 \\
\hline 4.1639 & 0.0078 & \pm & 0.0008 & 4.9115 & 0.0019 & \pm & 0.0002 \\
\hline 4.1855 & 0.0041 & \pm & 0.0004 & 4.9290 & 0.0057 & \pm & 0.0006 \\
\hline 4.2021 & 0.0061 & \pm & 0.0006 & 4.9514 & 0.0013 & \pm & 0.0001 \\
\hline 4.2271 & 0.0041 & \pm & 0.0004 & 4.9682 & 0.0029 & \pm & 0.0003 \\
\hline 4.2427 & 0.0052 & \pm & 0.0005 & 4.9907 & 0.0019 & \pm & 0.0002 \\
\hline 4.2641 & 0.0056 & \pm & 0.0006 & 5.0089 & 0.0024 & \pm & 0.0002 \\
\hline 4.2844 & 0.0052 & \pm & 0.0005 & 5.0284 & 0.0010 & \pm & 0.0001 \\
\hline 4.3024 & 0.0072 & \pm & 0.0007 & 5.0475 & 0.0051 & \pm & 0.0005 \\
\hline 4.3225 & 0.0042 & \pm & 0.0004 & 5.0674 & 0.0032 & \pm & 0.0003 \\
\hline 4.3402 & 0.0022 & \pm & 0.0002 & 5.0888 & 0.0033 & \pm & 0.0003 \\
\hline 4.3650 & 0.0052 & \pm & 0.0005 & 5.1084 & 0.0042 & \pm & 0.0004 \\
\hline 4.3817 & 0.0041 & \pm & 0.0004 & 5.1323 & 0.0026 & \pm & 0.0003 \\
\hline 4.4029 & 0.0045 & \pm & 0.0005 & 5.1536 & 0.0029 & \pm & 0.0003 \\
\hline 4.4227 & 0.0095 & \pm & 0.0009 & 5.1706 & 0.0036 & \pm & 0.0004 \\
\hline 4.4464 & 0.0037 & \pm & 0.0004 & 5.1893 & 0.0037 & \pm & 0.0004 \\
\hline 4.4669 & 0.0055 & \pm & 0.0006 & 5.2115 & 0.0027 & \pm & 0.0003 \\
\hline 4.4852 & 0.0040 & \pm & 0.0004 & 5.2332 & 0.0025 & \pm & 0.0002 \\
\hline 4.5057 & 0.0060 & \pm & 0.0006 & 5.2510 & 0.0039 & \pm & 0.0004 \\
\hline 4.5254 & 0.0041 & \pm & 0.0004 & 5.2733 & 0.0049 & \pm & 0.0005 \\
\hline 4.5457 & 0.0021 & \pm & 0.0002 & 5.2943 & 0.0035 & \pm & 0.0003 \\
\hline 4.5653 & 0.0049 & \pm & 0.0005 & 5.3115 & 0.0038 & \pm & 0.0004 \\
\hline 4.5859 & 0.0041 & \pm & 0.0004 & 5.3304 & 0.0021 & \pm & 0.0002 \\
\hline 4.6081 & 0.0030 & \pm & 0.0003 & 5.3523 & 0.0030 & \pm & 0.0003 \\
\hline 4.6262 & 0.0041 & \pm & 0.0004 & 5.3702 & 0.0080 & \pm & 0.0008 \\
\hline
\end{tabular}

Revision: 0 
NEA/NSC/DOC(2006)1

Liquid Metal Fast Reactor - LMFR

FFTF-LMFR-RESR-001

CRIT-SPEC-REAC-COEF-MISC

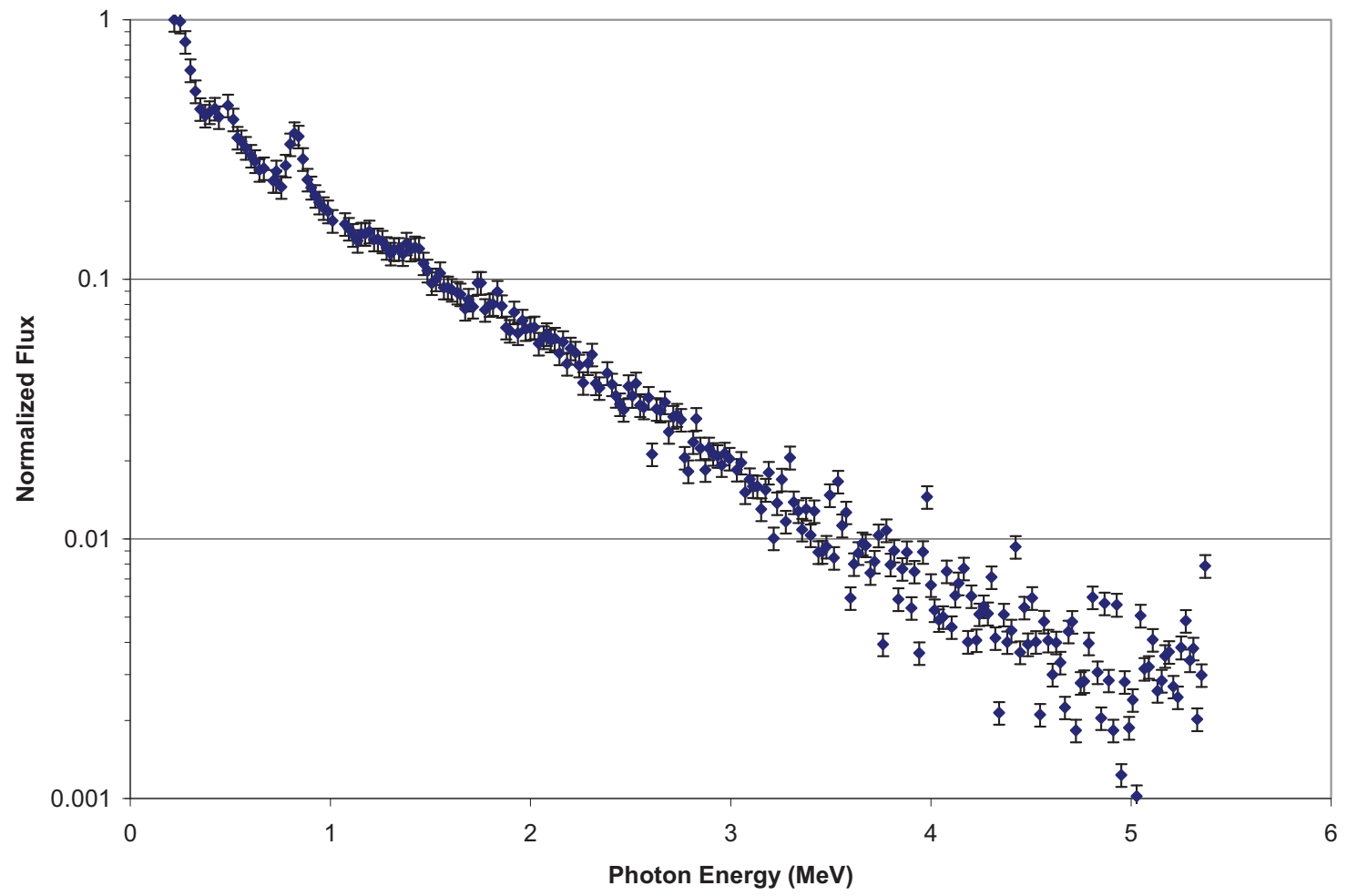

Figure 3.10.2. Low-energy Gamma-Ray Spectrum in FFTF Core Center. 
NEA/NSC/DOC(2006)1

Liquid Metal Fast Reactor - LMFR

FFTF-LMFR-RESR-001

CRIT-SPEC-REAC-COEF-MISC

\subsection{RESULTS OF SAMPLE CALCULATIONS}

For all analyses of the benchmark model, the MCNP5 input temperature is $478 \mathrm{~K}$. However, the neutron cross section library data for ENDF/B-VII.0 was processed at $480 \mathrm{~K}$ for most analyses. The ENDF/BVII.0 cross sections were processed at 455 and $505 \mathrm{~K}$ for analysis of the isothermal temperature coefficient.

\subsection{Results of Calculations of the Critical or Subcritical Configurations}

The calculated effective multiplication factor, $\mathrm{k}_{\mathrm{eff}}$, determined for the fully-loaded core critical configuration of the FFTF is shown in Table 4.1.1. Table C.1.1 contains spectral data calculated for this configuration.

Table 4.1.1. Eigenvalue for the FFTF Fully-Loaded Core Critical.

\begin{tabular}{|c|c|c|c|c|c|c|}
\hline \multicolumn{3}{|c|}{ Calculated } & \multicolumn{3}{|c|}{ Benchmark } & \multirow{2}{*}{$\begin{array}{c}(\mathrm{C}-\mathrm{E}) / \mathrm{E} \\
(\%)\end{array}$} \\
\hline $\mathrm{k}_{\mathrm{eff}}$ & \pm & $\sigma$ & $\mathrm{k}_{\mathrm{eff}}$ & \pm & $\sigma$ & \\
\hline 1.0031 & \pm & 0.0001 & 0.9993 & \pm & 0.0021 & $0.38 \pm 0.21$ \\
\hline
\end{tabular}

\subsection{Results of Buckling and Extrapolation Length Calculations}

Buckling and extrapolation length measurements were not made.

\subsection{Results of Spectral-Characteristics Calculations}

The benchmark model for the critical configuration described in Section 3.1 was modified as described in Section 3.3 and utilized in the analysis of the reactor physics experiments in Section 1.3.

The reactor physics experiments evaluated from this section pertain to two neutron spectra measurements performed in the IRT near the radial center of the core at core midplane and $80 \mathrm{~cm}$ below core midplane, in the lower axial shield region, using proportional counter detectors.

The corresponding calculated values for the neutron spectra measurements are provided in Tables 4.3.1 and 4.3.2 and Figures 4.3.1 and 4.3.2, respectively.

The relative flux values represent the point flux at a given lethargy energy divided by the maximum flux, which occurs at the energy of $117.971 \mathrm{keV}$ for both sets of data, and multiplied by the value 8.8 . It is assumed that the scaling factor was used to spread the chart out such that it would fit onto a full sheet of computer printout paper; no further explanation is provided.

The calculated neutron spectra are obtained by taking the variance-weighted average of results obtained using six variations of the input deck (Appendix A.1) with different random number seeds and tallies of the neutron flux (Appendix A.3). The initial data point ( $\mathrm{E}=1.145 \mathrm{keV}$ and $3.038 \mathrm{keV}$ for the midplane and below-midplane measurements, respectively) were not included in the comparison as MCNP5 tracks all neutrons below that energy threshold while the proton-recoil detectors would have had an unknown minimum energy threshold for detection. The neutron spectra calculations in MCNP are modified by a special tally feature, Gaussian Energy Broadening (GEB) that accounts for the resolution of the spectral measurement. This function is further discussed in Section 2.3.1. 


\section{Liquid Metal Fast Reactor - LMFR \\ FFTF-LMFR-RESR-001 \\ CRIT-SPEC-REAC-COEF-MISC}

The calculated and benchmark spectra share the same general trend for measurements at the core midplane. The trending in the below midplane measurements appears to have slight agreement for energies less than the about $150 \mathrm{keV}$. The calculated spectrum deviates significantly from the benchmark spectrum for higher energies. These results are possibly due to homogenization effects of core components below the fuel pin region. 
NEA/NSC/DOC(2006)1

Liquid Metal Fast Reactor - LMFR

FFTF-LMFR-RESR-001

CRIT-SPEC-REAC-COEF-MISC

Table 4.3.1. Calculated FFTF Core-Center Neutron Spectrum.

\begin{tabular}{|c|c|c|c|c|c|c|c|c|c|c|c|}
\hline $\begin{array}{c}\text { Energy } \\
(\mathrm{keV})\end{array}$ & \pm & $\sigma$ & $\begin{array}{l}\text { Calcul } \\
\text { Relative Flux } \\
\varphi / \varphi_{\max } \times 8.8\end{array}$ & ted & $\sigma$ & $\begin{array}{l}\text { Benchm } \\
\text { Relative Flux } \\
\varphi / \varphi_{\max } \times 8.8\end{array}$ & ark & $\sigma$ & $\mathrm{C} / \mathrm{E}$ & \pm & $\sigma$ \\
\hline 1.145 & \pm & 0.190 & & -- & & 0.54 & \pm & 0.03 & & -- & \\
\hline 1.202 & \pm & 0.195 & 1.656 & \pm & 0.013 & 0.54 & \pm & 0.03 & 3.08 & \pm & 0.20 \\
\hline 1.262 & \pm & 0.200 & 1.737 & \pm & 0.013 & 0.46 & \pm & 0.04 & 3.79 & \pm & 0.31 \\
\hline 1.325 & \pm & 0.205 & 1.869 & \pm & 0.014 & 0.40 & \pm & 0.03 & 4.67 & \pm & 0.40 \\
\hline 1.392 & \pm & 0.210 & 1.947 & \pm & 0.014 & 0.39 & \pm & 0.04 & 5.04 & \pm & 0.49 \\
\hline 1.461 & \pm & 0.215 & 1.964 & \pm & 0.014 & 0.43 & \pm & 0.04 & 4.52 & \pm & 0.37 \\
\hline 1.534 & \pm & 0.221 & 1.951 & \pm & 0.014 & 0.31 & \pm & 0.04 & 6.20 & \pm & 0.88 \\
\hline 1.611 & \pm & 0.226 & 1.945 & \pm & 0.014 & 0.35 & \pm & 0.03 & 5.60 & \pm & 0.45 \\
\hline 1.692 & \pm & 0.233 & 1.907 & \pm & 0.014 & 0.37 & \pm & 0.03 & 5.20 & \pm & 0.38 \\
\hline 1.776 & \pm & 0.238 & 1.766 & \pm & 0.013 & 0.39 & \pm & 0.03 & 4.59 & \pm & 0.37 \\
\hline 1.865 & \pm & 0.244 & 1.653 & \pm & 0.012 & 0.37 & \pm & 0.03 & 4.42 & \pm & 0.36 \\
\hline 1.958 & \pm & 0.251 & 1.453 & \pm & 0.011 & 0.26 & \pm & 0.03 & 5.63 & \pm & 0.72 \\
\hline 2.056 & \pm & 0.257 & 1.241 & \pm & 0.010 & 0.35 & \pm & 0.03 & 3.53 & \pm & 0.34 \\
\hline 2.159 & \pm & 0.264 & 1.003 & \pm & 0.009 & 0.38 & \pm & 0.04 & 2.63 & \pm & 0.26 \\
\hline 2.267 & \pm & 0.270 & 0.778 & \pm & 0.007 & 0.40 & \pm & 0.04 & 1.93 & \pm & 0.18 \\
\hline 2.380 & \pm & 0.277 & 0.554 & \pm & 0.006 & 0.37 & \pm & 0.04 & 1.48 & \pm & 0.16 \\
\hline 2.499 & \pm & 0.285 & 0.382 & \pm & 0.004 & 0.36 & \pm & 0.04 & 1.07 & \pm & 0.13 \\
\hline 2.624 & \pm & 0.291 & 0.240 & \pm & 0.003 & 0.44 & \pm & 0.05 & 0.55 & \pm & 0.06 \\
\hline 2.755 & \pm & 0.300 & 0.156 & \pm & 0.002 & 0.54 & \pm & 0.05 & 0.29 & \pm & 0.02 \\
\hline 2.893 & \pm & 0.308 & 0.126 & \pm & 0.002 & 0.69 & \pm & 0.04 & 0.18 & \pm & 0.01 \\
\hline 3.038 & \pm & 0.315 & 0.153 & \pm & 0.002 & 0.75 & \pm & 0.03 & 0.20 & \pm & 0.01 \\
\hline 3.190 & \pm & 0.324 & 0.227 & \pm & 0.003 & 0.81 & \pm & 0.04 & 0.28 & \pm & 0.01 \\
\hline 3.349 & \pm & 0.332 & 0.344 & \pm & 0.004 & 0.71 & \pm & 0.04 & 0.48 & \pm & 0.03 \\
\hline 3.517 & \pm & 0.341 & 0.487 & \pm & 0.005 & 0.73 & \pm & 0.04 & 0.67 & \pm & 0.04 \\
\hline 3.693 & \pm & 0.350 & 0.636 & \pm & 0.006 & 0.73 & \pm & 0.04 & 0.87 & \pm & 0.05 \\
\hline 3.877 & \pm & 0.360 & 0.805 & \pm & 0.007 & 0.89 & \pm & 0.05 & 0.90 & \pm & 0.05 \\
\hline 4.071 & \pm & 0.369 & 0.943 & \pm & 0.008 & 0.93 & \pm & 0.05 & 1.01 & \pm & 0.06 \\
\hline 4.275 & \pm & 0.378 & 1.070 & \pm & 0.009 & 0.98 & \pm & 0.05 & 1.10 & \pm & 0.06 \\
\hline 4.488 & \pm & 0.390 & 1.210 & \pm & 0.010 & 1.04 & \pm & 0.06 & 1.17 & \pm & 0.06 \\
\hline 4.713 & \pm & 0.399 & 1.336 & \pm & 0.011 & 1.02 & \pm & 0.06 & 1.31 & \pm & 0.08 \\
\hline 4.948 & \pm & 0.411 & 1.440 & \pm & 0.011 & 1.11 & \pm & 0.06 & 1.30 & \pm & 0.08 \\
\hline 5.196 & \pm & 0.422 & 1.569 & \pm & 0.012 & 1.37 & \pm & 0.06 & 1.15 & \pm & 0.05 \\
\hline 5.456 & \pm & 0.432 & 1.666 & \pm & 0.013 & 1.27 & \pm & 0.06 & 1.31 & \pm & 0.07 \\
\hline 5.728 & \pm & 0.444 & 1.722 & \pm & 0.013 & 1.25 & \pm & 0.06 & 1.37 & \pm & 0.07 \\
\hline 6.015 & \pm & 0.458 & 1.818 & \pm & 0.013 & 1.24 & \pm & 0.07 & 1.46 & \pm & 0.08 \\
\hline 6.316 & \pm & 0.470 & 1.887 & \pm & 0.014 & 1.52 & \pm & 0.05 & 1.24 & \pm & 0.04 \\
\hline 6.631 & \pm & 0.483 & 1.943 & \pm & 0.014 & 1.43 & \pm & 0.05 & 1.36 & \pm & 0.05 \\
\hline 6.963 & \pm & 0.495 & 1.974 & \pm & 0.014 & 1.36 & \pm & 0.06 & 1.45 & \pm & 0.06 \\
\hline 7.311 & \pm & 0.510 & 1.899 & \pm & 0.014 & 1.38 & \pm & 0.06 & 1.38 & \pm & 0.06 \\
\hline
\end{tabular}


NEA/NSC/DOC(2006)1

Liquid Metal Fast Reactor - LMFR

FFTF-LMFR-RESR-001

CRIT-SPEC-REAC-COEF-MISC

Table 4.3.1 (cont'd). Calculated FFTF Core-Center Neutron Spectrum.

\begin{tabular}{|c|c|c|c|c|c|c|c|c|c|c|c|}
\hline \multirow{2}{*}{$\begin{array}{l}\text { Energy } \\
(\mathrm{keV})\end{array}$} & \multirow[b]{2}{*}{ \pm} & \multirow[b]{2}{*}{$\sigma$} & \multicolumn{3}{|c|}{ Calculated } & \multicolumn{3}{|c|}{ "Benchmark } & \multirow[b]{2}{*}{$\mathrm{C} / \mathrm{E}$} & \multirow[b]{2}{*}{ \pm} & \multirow[b]{2}{*}{$\sigma$} \\
\hline & & & $\begin{array}{c}\text { Relative Flux } \\
\varphi / \varphi_{\max } \times 8.8\end{array}$ & \pm & $\sigma$ & $\begin{array}{c}\text { Relative Flux } \\
\varphi / \varphi_{\max } \times 8.8\end{array}$ & \pm & $\sigma$ & & & \\
\hline 7.677 & \pm & 0.523 & 1.817 & \pm & 0.014 & 1.48 & \pm & 0.06 & 1.22 & \pm & 0.05 \\
\hline 8.061 & \pm & 0.539 & 1.737 & \pm & 0.013 & 1.59 & \pm & 0.07 & 1.09 & \pm & 0.05 \\
\hline 8.464 & \pm & 0.555 & 1.794 & \pm & 0.013 & 1.74 & \pm & 0.07 & 1.03 & \pm & 0.04 \\
\hline 8.887 & \pm & 0.571 & 2.001 & \pm & 0.015 & 2.02 & \pm & 0.07 & 0.99 & \pm & 0.03 \\
\hline 9.331 & \pm & 0.588 & 2.283 & \pm & 0.016 & 2.27 & \pm & 0.07 & 1.00 & \pm & 0.03 \\
\hline 9.798 & \pm & 0.605 & 2.587 & \pm & 0.017 & 2.53 & \pm & 0.08 & 1.02 & \pm & 0.03 \\
\hline 10.287 & \pm & 0.622 & 2.803 & \pm & 0.018 & 2.40 & \pm & 0.07 & 1.17 & \pm & 0.04 \\
\hline 10.802 & \pm & 0.639 & 2.957 & \pm & 0.019 & 2.16 & \pm & 0.09 & 1.37 & \pm & 0.05 \\
\hline 11.342 & \pm & 0.656 & 3.040 & \pm & 0.019 & 2.33 & \pm & 0.08 & 1.30 & \pm & 0.04 \\
\hline 11.909 & \pm & 0.679 & 3.030 & \pm & 0.019 & 2.35 & \pm & 0.08 & 1.29 & \pm & 0.05 \\
\hline 12.504 & \pm & 0.697 & 2.882 & \pm & 0.019 & 2.23 & \pm & 0.08 & 1.29 & \pm & 0.05 \\
\hline 13.130 & \pm & 0.721 & 2.874 & \pm & 0.019 & 2.25 & \pm & 0.07 & 1.28 & \pm & 0.04 \\
\hline 13.786 & \pm & 0.739 & 3.019 & \pm & 0.019 & 2.22 & \pm & 0.06 & 1.36 & \pm & 0.04 \\
\hline 14.476 & \pm & 0.764 & 3.126 & \pm & 0.020 & 2.06 & \pm & 0.08 & 1.52 & \pm & 0.06 \\
\hline 15.199 & \pm & 0.789 & 2.894 & \pm & 0.019 & 2.05 & \pm & 0.07 & 1.41 & \pm & 0.05 \\
\hline 15.959 & \pm & 0.808 & 2.633 & \pm & 0.018 & 2.07 & \pm & 0.07 & 1.27 & \pm & 0.05 \\
\hline 16.757 & \pm & 0.834 & 2.811 & \pm & 0.019 & 2.33 & \pm & 0.08 & 1.21 & \pm & 0.04 \\
\hline 17.595 & \pm & 0.861 & 3.240 & \pm & 0.021 & 2.54 & \pm & 0.08 & 1.28 & \pm & 0.04 \\
\hline 18.475 & \pm & 0.888 & 3.649 & \pm & 0.022 & 2.84 & \pm & 0.08 & 1.29 & \pm & 0.04 \\
\hline 19.399 & \pm & 0.916 & 4.025 & \pm & 0.024 & 3.10 & \pm & 0.08 & 1.30 & \pm & 0.04 \\
\hline 20.368 & \pm & 0.945 & 4.239 & \pm & 0.025 & 3.13 & \pm & 0.10 & 1.36 & \pm & 0.04 \\
\hline 21.387 & \pm & 0.974 & 4.382 & \pm & 0.025 & 3.60 & \pm & 0.09 & 1.22 & \pm & 0.03 \\
\hline 22.456 & \pm & 1.003 & 4.717 & \pm & 0.026 & 4.18 & \pm & 0.10 & 1.13 & \pm & 0.03 \\
\hline 23.579 & \pm & 1.054 & 5.034 & \pm & 0.027 & 4.42 & \pm & 0.09 & 1.14 & \pm & 0.02 \\
\hline 24.758 & \pm & 1.096 & 5.556 & \pm & 0.029 & 4.05 & \pm & 0.09 & 1.37 & \pm & 0.03 \\
\hline 25.996 & \pm & 1.139 & 5.715 & \pm & 0.030 & 2.54 & \pm & 0.10 & 2.25 & \pm & 0.09 \\
\hline 27.296 & \pm & 1.196 & 4.480 & \pm & 0.026 & 1.78 & \pm & 0.07 & 2.52 & \pm & 0.10 \\
\hline 28.661 & \pm & 1.244 & 2.768 & \pm & 0.019 & 1.75 & \pm & 0.07 & 1.58 & \pm & 0.07 \\
\hline 30.094 & \pm & 1.293 & 2.516 & \pm & 0.018 & 2.29 & \pm & 0.09 & 1.10 & \pm & 0.05 \\
\hline 31.598 & \pm & 1.358 & 3.186 & \pm & 0.021 & 2.72 & \pm & 0.09 & 1.17 & \pm & 0.04 \\
\hline 33.178 & \pm & 1.412 & 3.969 & \pm & 0.024 & 3.06 & \pm & 0.09 & 1.30 & \pm & 0.04 \\
\hline 34.837 & \pm & 1.482 & 4.414 & \pm & 0.026 & 3.21 & \pm & 0.10 & 1.38 & \pm & 0.04 \\
\hline 36.579 & \pm & 1.541 & 4.666 & \pm & 0.027 & 3.44 & \pm & 0.10 & 1.36 & \pm & 0.04 \\
\hline 38.408 & \pm & 1.569 & 4.967 & \pm & 0.028 & 3.97 & \pm & 0.11 & 1.25 & \pm & 0.04 \\
\hline 40.328 & \pm & 1.682 & 5.218 & \pm & 0.029 & 4.12 & \pm & 0.10 & 1.27 & \pm & 0.03 \\
\hline 42.345 & \pm & 1.748 & 5.496 & \pm & 0.030 & 4.57 & \pm & 0.11 & 1.20 & \pm & 0.03 \\
\hline 44.462 & \pm & 1.835 & 5.798 & \pm & 0.031 & 4.47 & \pm & 0.10 & 1.30 & \pm & 0.03 \\
\hline 46.685 & \pm & 1.927 & 6.171 & \pm & 0.032 & 3.63 & \pm & 0.12 & 1.70 & \pm & 0.06 \\
\hline 49.019 & \pm & 2.002 & 5.687 & \pm & 0.031 & 3.58 & \pm & 0.12 & 1.59 & \pm & 0.05 \\
\hline
\end{tabular}


NEA/NSC/DOC(2006)1

Liquid Metal Fast Reactor - LMFR

FFTF-LMFR-RESR-001

CRIT-SPEC-REAC-COEF-MISC

Table 4.3.1 (cont'd). Calculated FFTF Core-Center Neutron Spectrum.

\begin{tabular}{|c|c|c|c|c|c|c|c|c|c|c|c|}
\hline $\begin{array}{c}\text { Energy } \\
(\mathrm{keV})\end{array}$ & \pm & $\sigma$ & $\begin{array}{r}\text { Calcul } \\
\text { Relative Flux } \\
\varphi / \varphi_{\max } \times 8.8\end{array}$ & ted & $\sigma$ & $\begin{array}{r}\text { Benchn } \\
\text { Relative Flux } \\
\varphi / \varphi_{\max } \times 8.8\end{array}$ & \pm & $\sigma$ & $\mathrm{C} / \mathrm{E}$ & \pm & $\sigma$ \\
\hline 51.470 & \pm & 2.103 & 4.396 & \pm & 0.026 & 3.97 & \pm & 0.09 & 1.11 & \pm & 0.03 \\
\hline 54.044 & \pm & 2.185 & 3.591 & \pm & 0.023 & 4.71 & \pm & 0.10 & 0.76 & \pm & 0.02 \\
\hline 56.746 & \pm & 2.294 & 4.476 & \pm & 0.027 & 5.33 & \pm & 0.09 & 0.84 & \pm & 0.01 \\
\hline 59.583 & \pm & 2.383 & 5.801 & \pm & 0.032 & 5.17 & \pm & 0.10 & 1.12 & \pm & 0.02 \\
\hline 62.562 & \pm & 2.502 & 6.172 & \pm & 0.033 & 5.30 & \pm & 0.12 & 1.17 & \pm & 0.03 \\
\hline 65.690 & \pm & 2.628 & 6.033 & \pm & 0.033 & 5.88 & \pm & 0.12 & 1.03 & \pm & 0.02 \\
\hline 68.975 & \pm & 2.730 & 6.118 & \pm & 0.033 & 6.14 & \pm & 0.10 & 1.00 & \pm & 0.02 \\
\hline 72.424 & \pm & 2.866 & 6.476 & \pm & 0.034 & 6.32 & \pm & 0.11 & 1.02 & \pm & 0.02 \\
\hline 76.045 & \pm & 3.009 & 6.393 & \pm & 0.034 & 6.63 & \pm & 0.12 & 0.96 & \pm & 0.02 \\
\hline 79.847 & \pm & 3.160 & 6.862 & \pm & 0.036 & 5.77 & \pm & 0.12 & 1.19 & \pm & 0.03 \\
\hline 83.840 & \pm & 3.282 & 7.003 & \pm & 0.036 & 5.21 & \pm & 0.13 & 1.35 & \pm & 0.04 \\
\hline 88.031 & \pm & 3.446 & 6.452 & \pm & 0.035 & 5.04 & \pm & 0.13 & 1.28 & \pm & 0.03 \\
\hline 92.433 & \pm & 3.619 & 6.598 & \pm & 0.035 & 5.32 & \pm & 0.14 & 1.24 & \pm & 0.03 \\
\hline 97.055 & \pm & 3.758 & 6.425 & \pm & 0.034 & 5.68 & \pm & 0.15 & 1.13 & \pm & 0.03 \\
\hline 101.907 & \pm & 3.946 & 6.271 & \pm & 0.034 & 6.37 & \pm & 0.14 & 0.98 & \pm & 0.02 \\
\hline 107.003 & \pm & 4.144 & 6.665 & \pm & 0.035 & 7.45 & \pm & 0.15 & 0.89 & \pm & 0.02 \\
\hline 112.353 & \pm & 4.351 & 7.207 & \pm & 0.037 & 7.94 & \pm & 0.16 & 0.91 & \pm & 0.02 \\
\hline 117.971 & \pm & 4.568 & 7.738 & \pm & 0.039 & 8.80 & \pm & 0.22 & 0.88 & \pm & 0.02 \\
\hline 123.869 & \pm & 4.744 & 8.172 & \pm & 0.040 & 7.99 & \pm & 0.21 & 1.02 & \pm & 0.03 \\
\hline 130.063 & \pm & 4.981 & 8.632 & \pm & 0.042 & 6.55 & \pm & 0.22 & 1.32 & \pm & 0.05 \\
\hline 136.566 & \pm & 6.160 & 8.800 & \pm & 0.042 & 6.42 & \pm & 0.24 & 1.37 & \pm & 0.05 \\
\hline 143.394 & \pm & 6.468 & 7.771 & \pm & 0.040 & 6.85 & \pm & 0.08 & 1.14 & \pm & 0.01 \\
\hline 150.564 & \pm & 6.727 & 829 & \pm & 0.037 & 7.22 & \pm & 0.11 & 0.95 & \pm & 0.02 \\
\hline 158.092 & \pm & 7.064 & 7.436 & \pm & 0.039 & 6.93 & \pm & 0.11 & 1.07 & \pm & 0.02 \\
\hline 165.996 & \pm & 7.417 & 7.958 & \pm & 0.040 & 6.66 & \pm & 0.10 & 1.19 & \pm & 0.02 \\
\hline 174.296 & \pm & 7.788 & 8.355 & \pm & 0.042 & 6.49 & \pm & 0.11 & 1.29 & \pm & 0.02 \\
\hline 183.011 & \pm & 8.177 & 8.661 & \pm & 0.042 & 6.22 & E & 0.11 & 1.39 & \pm & 0.03 \\
\hline 192.162 & \pm & 8.504 & 7.651 & \pm & 0.040 & 6.96 & t & 0.12 & 1.10 & \pm & 0.02 \\
\hline 201.770 & \pm & 8.929 & 6.411 & \pm & 0.036 & 7.53 & \pm & 0.11 & 0.85 & \pm & 0.01 \\
\hline 211.858 & \pm & 9.376 & 6.402 & \pm & 0.036 & 6.77 & \pm & 0.11 & 0.95 & \pm & 0.02 \\
\hline 222.451 & \pm & 9.845 & 6.753 & \pm & 0.037 & 5.72 & \pm & 0.11 & 1.18 & \pm & 0.02 \\
\hline 233.574 & \pm & 10.337 & 6.753 & \pm & 0.038 & 7.02 & \pm & 0.15 & 0.96 & \pm & 0.02 \\
\hline 245.252 & \pm & 10.749 & 6.625 & \pm & 0.037 & 7.22 & \pm & 0.13 & 0.92 & \pm & 0.02 \\
\hline 257.515 & \pm & 11.287 & 7.389 & \pm & 0.040 & 7.17 & \pm & 0.09 & 1.03 & \pm & 0.01 \\
\hline 270.391 & \pm & 11.851 & 8.570 & \pm & 0.043 & 7.30 & \pm & 0.10 & 1.17 & \pm & 0.02 \\
\hline 283.910 & \pm & 12.444 & 8.569 & \pm & 0.044 & 7.15 & \pm & 0.10 & 1.20 & \pm & 0.02 \\
\hline 298.106 & \pm & 13.066 & 8.360 & \pm & 0.043 & 7.11 & \pm & 0.09 & 1.18 & \pm & 0.02 \\
\hline 313.011 & \pm & 13.719 & 8.170 & \pm & 0.043 & 6.80 & \pm & 0.09 & 1.20 & \pm & 0.02 \\
\hline
\end{tabular}


NEA/NSC/DOC(2006)1

Liquid Metal Fast Reactor - LMFR

FFTF-LMFR-RESR-001

CRIT-SPEC-REAC-COEF-MISC

Table 4.3.1 (cont'd). Calculated FFTF Core-Center Neutron Spectrum.

\begin{tabular}{|c|c|c|c|c|c|c|c|c|c|c|c|}
\hline $\begin{array}{c}\text { Energy } \\
(\mathrm{keV})\end{array}$ & \pm & $\sigma$ & $\begin{array}{r}\text { Calcul } \\
\text { Relative Flux } \\
\varphi / \varphi_{\max } \times 8.8 \\
\end{array}$ & & $\sigma$ & $\begin{array}{c}\text { Benchm } \\
\text { Relative Flux } \\
\varphi / \varphi_{\max } \times 8.8 \\
\end{array}$ & \pm & $\sigma$ & $\mathrm{C} / \mathrm{E}$ & \pm & $\sigma$ \\
\hline 328.662 & \pm & 14.405 & 7.430 & \pm & 0.041 & 6.37 & \pm & 0.09 & 1.17 & \pm & 0.02 \\
\hline 345.095 & \pm & 14.979 & 7.064 & \pm & 0.040 & 5.92 & \pm & 0.10 & 1.19 & \pm & 0.02 \\
\hline 362.349 & \pm & 15.727 & 6.933 & \pm & 0.039 & 5.70 & \pm & 0.11 & 1.22 & \pm & 0.02 \\
\hline 380.467 & \pm & 16.514 & 6.170 & \pm & 0.037 & 5.42 & \pm & 0.10 & 1.14 & \pm & 0.02 \\
\hline 399.490 & \pm & 17.340 & 5.403 & \pm & 0.034 & 4.91 & \pm & 0.11 & 1.10 & \pm & 0.02 \\
\hline 419.465 & \pm & 18.207 & 5.103 & \pm & 0.033 & 4.85 & \pm & 0.08 & 1.05 & \pm & 0.02 \\
\hline 440.438 & \pm & 19.117 & 5.356 & \pm & 0.034 & 5.18 & \pm & 0.08 & 1.03 & \pm & 0.02 \\
\hline 462.460 & \pm & 20.073 & 6.271 & \pm & 0.038 & 5.73 & \pm & 0.08 & 1.09 & \pm & 0.02 \\
\hline 485.583 & \pm & 21.076 & 7.436 & \pm & 0.041 & 6.24 & \pm & 0.08 & 1.19 & \pm & 0.02 \\
\hline 509.862 & \pm & 22.130 & 8.227 & \pm & 0.044 & 6.25 & \pm & 0.08 & 1.32 & \pm & 0.02 \\
\hline 535.355 & \pm & 23.237 & 8.249 & \pm & 0.044 & 6.23 & \pm & 0.08 & 1.32 & \pm & 0.02 \\
\hline 562.123 & \pm & 24.399 & 7.658 & \pm & 0.042 & 6.06 & \pm & 0.09 & 1.26 & \pm & 0.02 \\
\hline 590.229 & \pm & 25.367 & 7.178 & \pm & 0.041 & 5.98 & \pm & 0.09 & 1.20 & \pm & 0.02 \\
\hline 619.741 & \pm & 26.636 & 6.708 & \pm & 0.039 & 5.97 & \pm & 0.10 & 1.12 & \pm & 0.02 \\
\hline 650.728 & \pm & 27.967 & 87 & \pm & 0.037 & 5.76 & \pm & 0.09 & 1.06 & \pm & 0.02 \\
\hline 683.264 & \pm & 29.366 & 02 & \pm & 0.035 & 5.38 & \pm & 0.07 & 1.02 & \pm & 0.01 \\
\hline 717.427 & \pm & 30.834 & 65 & \pm & 0.033 & 5.24 & \pm & 0.07 & 0.95 & \pm & 0.01 \\
\hline 753.299 & \pm & 32.376 & 21 & \pm & 0.032 & 4.78 & \pm & 0.07 & 0.97 & \pm & 0.02 \\
\hline 790.964 & \pm & 33.995 & 99 & \pm & 0.032 & 4.54 & \pm & 0.07 & 0.99 & \pm & 0.02 \\
\hline 830.512 & \pm & 35.694 & 24 & \pm & 0.032 & 4.38 & \pm & 0.07 & 0.99 & \pm & 0.02 \\
\hline 87 & \pm & 3 & 9 & & 0 & 4.17 & \pm & 0.07 & .00 & \pm & 0.02 \\
\hline 915. & \pm & 3 & 1 & & $0 .($ & 3.90 & \pm & 0.07 & 0.98 & \pm & 0.02 \\
\hline 961.421 & \pm & 41.3 & 1 & & 0.0 & 3.64 & \pm & 0.08 & 0.97 & \pm & 0.02 \\
\hline 1009.492 & \pm & 4 & 1 & & 0.0 & 3.43 & \pm & 0.08 & 0.98 & \pm & 0.02 \\
\hline 1059.967 & \pm & 45.5 & 1 & + & 0.029 & 3.65 & \pm & 0.08 & 0.91 & \pm & 0.02 \\
\hline 1112.965 & \pm & 47.8 & 7 & & 0.029 & 3.56 & \pm & 0.08 & 0.98 & \pm & 0.02 \\
\hline 1168.613 & \pm & 50.225 & 3.714 & $I$ & 0.030 & 3.62 & \pm & 0.09 & 1.03 & \pm & 0.03 \\
\hline 1227.044 & \pm & 52.737 & 3.691 & $\perp$ & 0.030 & 3.80 & \pm & 0.08 & 0.97 & \pm & 0.02 \\
\hline 1288.396 & \pm & 55.374 & 3.434 & $\perp$ & 0.029 & 3.52 & \pm & 0.09 & 0.98 & \pm & 0.03 \\
\hline 1352.816 & \pm & 58.142 & 3.318 & \pm & 0.029 & 3.32 & \pm & 0.09 & 1.00 & \pm & 0.03 \\
\hline 1420.457 & \pm & 61.049 & 3.254 & \pm & 0.028 & 3.42 & \pm & 0.08 & 0.95 & \pm & 0.02 \\
\hline 1491.480 & \pm & 64.102 & 3.192 & \pm & 0.028 & 3.32 & \pm & 0.09 & 0.96 & \pm & 0.03 \\
\hline 1566.054 & \pm & 67.307 & 3.031 & \pm & 0.027 & 2.82 & \pm & 0.08 & 1.07 & \pm & 0.03 \\
\hline 1644.356 & \pm & 70.672 & 2.906 & \pm & 0.027 & 2.69 & \pm & 0.07 & 1.08 & \pm & 0.03 \\
\hline 1726.574 & \pm & 73.471 & 2.792 & \pm & 0.026 & 2.56 & \pm & 0.08 & 1.09 & \pm & 0.03 \\
\hline 1812.903 & \pm & 77.145 & 2.644 & \pm & 0.026 & 2.44 & \pm & 0.09 & 1.09 & \pm & 0.04 \\
\hline 1903.548 & \pm & 81.002 & 2.572 & \pm & 0.026 & 2.21 & \pm & 0.09 & 1.17 & \pm & 0.05 \\
\hline
\end{tabular}


NEA/NSC/DOC(2006)1

Liquid Metal Fast Reactor - LMFR

FFTF-LMFR-RESR-001

CRIT-SPEC-REAC-COEF-MISC

Table 4.3.2. Calculated FFTF Lower-Axial Neutron Spectrum.

\begin{tabular}{|c|c|c|c|c|c|c|c|c|c|c|c|}
\hline \multirow[b]{2}{*}{$\begin{array}{l}\text { Energy } \\
(\mathrm{keV})\end{array}$} & \multirow[b]{2}{*}{ \pm} & \multirow[b]{2}{*}{$\sigma$} & \multicolumn{3}{|c|}{ Calculated } & \multicolumn{3}{|c|}{ Benchmark } & \multirow[b]{2}{*}{$\mathrm{C} / \mathrm{E}$} & \multirow[b]{2}{*}{ \pm} & \multirow[b]{2}{*}{$\sigma$} \\
\hline & & & $\begin{array}{c}\text { Relative Flux } \\
\varphi / \varphi_{\max } \times 8.8\end{array}$ & \pm & $\sigma$ & $\begin{array}{c}\text { Relative Flux } \\
\varphi / \varphi_{\max } \times 8.8\end{array}$ & \pm & $\sigma$ & & & \\
\hline 3.038 & \pm & 0.315 & & -- & & 0.82 & \pm & 0.05 & & -- & \\
\hline 3.190 & \pm & 0.324 & 0.224 & \pm & 0.002 & 0.82 & \pm & 0.05 & 0.27 & \pm & 0.02 \\
\hline 3.349 & \pm & 0.332 & 0.338 & \pm & 0.003 & 0.69 & \pm & 0.05 & 0.49 & \pm & 0.04 \\
\hline 3.517 & \pm & 0.341 & 0.483 & \pm & 0.004 & 0.33 & \pm & 0.06 & 1.48 & \pm & 0.25 \\
\hline 3.693 & \pm & 0.350 & 0.632 & \pm & 0.005 & 0.33 & \pm & 0.06 & 1.91 & \pm & 0.35 \\
\hline 3.877 & \pm & 0.360 & 0.776 & \pm & 0.006 & 0.38 & \pm & 0.07 & 2.02 & \pm & 0.35 \\
\hline 4.071 & \pm & 0.369 & 0.915 & \pm & 0.007 & 0.23 & \pm & 0.06 & 4.01 & \pm & 1.04 \\
\hline 4.275 & \pm & 0.378 & 1.046 & \pm & 0.008 & 0.24 & \pm & 0.07 & 4.29 & \pm & 1.15 \\
\hline 4.488 & \pm & 0.390 & 1.178 & \pm & 0.009 & 0.41 & \pm & 0.07 & 2.89 & \pm & 0.51 \\
\hline 4.713 & \pm & 0.399 & 1.310 & \pm & 0.009 & 0.67 & \pm & 0.08 & 1.94 & \pm & 0.23 \\
\hline 4.948 & \pm & 0.411 & 1.411 & \pm & 0.010 & 0.70 & \pm & 0.09 & 2.00 & \pm & 0.24 \\
\hline 5.196 & \pm & 0.422 & 1.535 & \pm & 0.011 & 1.01 & \pm & 0.05 & 1.52 & \pm & 0.08 \\
\hline 5.456 & \pm & 0.418 & 1.610 & \pm & 0.011 & 1.31 & \pm & 0.05 & 1.23 & \pm & 0.05 \\
\hline 5.728 & \pm & 0.444 & 1.658 & \pm & 0.011 & 1.52 & \pm & 0.06 & 1.09 & \pm & 0.05 \\
\hline 6.015 & \pm & 0.458 & 1.746 & \pm & 0.012 & 1.81 & \pm & 0.06 & 0.97 & \pm & 0.03 \\
\hline 6.316 & \pm & 0.470 & 1.843 & \pm & 0.012 & 1.76 & \pm & 0.06 & 1.05 & \pm & 0.04 \\
\hline 6.631 & \pm & 0.483 & 1.892 & \pm & 0.012 & 1.64 & \pm & 0.05 & 1.16 & \pm & 0.04 \\
\hline 6.963 & \pm & 0.495 & 1.936 & \pm & 0.012 & 1.57 & \pm & 0.06 & 1.24 & \pm & 0.05 \\
\hline 7.311 & \pm & 0.510 & 1.879 & \pm & 0.012 & 1.67 & \pm & 0.06 & 1.12 & \pm & 0.04 \\
\hline 7.677 & \pm & 0.523 & 1.743 & \pm & 0.012 & 1.88 & \pm & 0.07 & 0.93 & \pm & 0.04 \\
\hline 8.061 & \pm & 0.539 & 1.709 & \pm & 0.011 & 2.03 & \pm & 0.07 & 0.84 & \pm & 0.03 \\
\hline 8.464 & \pm & 0.555 & 1.792 & \pm & 0.012 & 2.59 & \pm & 0.07 & 0.69 & \pm & 0.02 \\
\hline 8.887 & \pm & 0.571 & 1.966 & \pm & 0.013 & 2.69 & \pm & 0.07 & 0.73 & \pm & 0.02 \\
\hline 9.331 & \pm & 0.588 & 2.245 & \pm & 0.014 & 3.60 & \pm & 0.08 & 0.62 & \pm & 0.01 \\
\hline 9.798 & \pm & 0.605 & 2.565 & \pm & 0.015 & 3.92 & \pm & 0.08 & 0.65 & \pm & 0.01 \\
\hline 10.287 & \pm & 0.622 & 2.797 & \pm & 0.016 & 3.71 & \pm & 0.09 & 0.75 & \pm & 0.02 \\
\hline 10.802 & \pm & 0.639 & 2.949 & \pm & 0.017 & 3.66 & \pm & 0.08 & 0.81 & \pm & 0.02 \\
\hline 11.342 & \pm & 0.656 & 2.986 & \pm & 0.017 & 3.34 & \pm & 0.06 & 0.89 & \pm & 0.02 \\
\hline 11.909 & \pm & 0.679 & 2.931 & \pm & 0.017 & 3.40 & \pm & 0.07 & 0.86 & \pm & 0.02 \\
\hline 12.504 & \pm & 0.697 & 2.840 & \pm & 0.016 & 3.08 & \pm & 0.07 & 0.92 & \pm & 0.02 \\
\hline 13.130 & \pm & 0.721 & 2.847 & \pm & 0.016 & 3.26 & \pm & 0.06 & 0.87 & \pm & 0.02 \\
\hline 13.786 & \pm & 0.739 & 3.007 & \pm & 0.017 & 3.31 & \pm & 0.08 & 0.91 & \pm & 0.02 \\
\hline 14.476 & \pm & 0.764 & 3.065 & \pm & 0.017 & 3.41 & \pm & 0.08 & 0.90 & \pm & 0.02 \\
\hline 15.199 & \pm & 0.789 & 2.815 & \pm & 0.016 & 2.94 & \pm & 0.09 & 0.96 & \pm & 0.03 \\
\hline 15.959 & \pm & 0.808 & 2.591 & \pm & 0.016 & 2.65 & \pm & 0.09 & 0.98 & \pm & 0.03 \\
\hline 16.757 & \pm & 0.834 & 2.740 & \pm & 0.016 & 3.22 & \pm & 0.08 & 0.85 & \pm & 0.02 \\
\hline 17.595 & \pm & 0.861 & 3.185 & \pm & 0.018 & 3.78 & \pm & 0.09 & 0.84 & \pm & 0.02 \\
\hline 18.475 & \pm & 0.888 & 3.599 & \pm & 0.019 & 4.25 & \pm & 0.09 & 0.85 & \pm & 0.02 \\
\hline 19.399 & \pm & 0.916 & 3.939 & \pm & 0.020 & 4.40 & \pm & 0.09 & 0.90 & \pm & 0.02 \\
\hline
\end{tabular}


NEA/NSC/DOC(2006)1

Liquid Metal Fast Reactor - LMFR

FFTF-LMFR-RESR-001

CRIT-SPEC-REAC-COEF-MISC

Table 4.3.2 (cont'd). Calculated FFTF Lower-Axial Neutron Spectrum.

\begin{tabular}{|c|c|c|c|c|c|c|c|c|c|c|c|}
\hline \multirow{2}{*}{$\begin{array}{l}\text { Energy } \\
(\mathrm{keV})\end{array}$} & \multirow[b]{2}{*}{ \pm} & \multirow[b]{2}{*}{$\sigma$} & \multicolumn{3}{|c|}{ Calculated } & \multicolumn{3}{|c|}{ Benchmark } & \multirow[b]{2}{*}{$\mathrm{C} / \mathrm{E}$} & \multirow[b]{2}{*}{ \pm} & \multirow[b]{2}{*}{$\sigma$} \\
\hline & & & $\begin{array}{c}\text { Relative Flux } \\
\varphi / \varphi_{\max } \times 8.8\end{array}$ & \pm & $\sigma$ & $\begin{array}{c}\text { Relative Flux } \\
\varphi / \varphi_{\max } \times 8.8\end{array}$ & \pm & $\sigma$ & & & \\
\hline 20.368 & \pm & 0.945 & 4.172 & \pm & 0.021 & 4.83 & \pm & 0.10 & 0.86 & \pm & 0.02 \\
\hline 21.387 & \pm & 0.974 & 4.374 & \pm & 0.022 & 5.37 & \pm & 0.10 & 0.81 & \pm & 0.02 \\
\hline 22.456 & \pm & 1.013 & 4.622 & \pm & 0.023 & 6.18 & \pm & 0.10 & 0.75 & \pm & 0.01 \\
\hline 23.579 & \pm & 1.054 & 4.944 & \pm & 0.024 & 6.60 & \pm & 0.09 & 0.75 & \pm & 0.01 \\
\hline 24.758 & \pm & 1.096 & 5.500 & \pm & 0.025 & 6.88 & \pm & 0.07 & 0.80 & \pm & 0.01 \\
\hline 25.996 & \pm & 1.139 & 5.658 & \pm & 0.026 & 5.92 & \pm & 0.07 & 0.96 & \pm & 0.01 \\
\hline 27.296 & \pm & 1.196 & 4.430 & \pm & 0.022 & 3.97 & \pm & 0.09 & 1.12 & \pm & 0.03 \\
\hline 28.661 & \pm & 1.244 & 2.767 & \pm & 0.017 & 2.80 & \pm & 0.08 & 0.99 & \pm & 0.03 \\
\hline 30.094 & \pm & 1.293 & 2.492 & \pm & 0.016 & 2.16 & \pm & 0.09 & 1.15 & \pm & 0.05 \\
\hline 31.598 & \pm & 1.358 & 3.182 & \pm & 0.018 & 2.29 & \pm & 0.09 & 1.39 & \pm & 0.06 \\
\hline 33.178 & \pm & 1.412 & 3.890 & \pm & 0.021 & 2.81 & \pm & 0.10 & 1.38 & \pm & 0.05 \\
\hline 34.837 & \pm & 1.482 & 4.333 & \pm & 0.022 & 3.29 & \pm & 0.11 & 1.32 & \pm & 0.05 \\
\hline 36.579 & \pm & 1.541 & 4.592 & \pm & 0.023 & 3.91 & \pm & 0.10 & 1.18 & \pm & 0.03 \\
\hline 38.408 & \pm & 1.602 & 4.915 & \pm & 0.024 & 4.55 & \pm & 0.10 & 1.08 & \pm & 0.03 \\
\hline 40.328 & \pm & 1.682 & 5.194 & \pm & 0.025 & 4.89 & \pm & 0.11 & 1.06 & \pm & 0.02 \\
\hline 42.345 & \pm & 1.748 & 5.407 & \pm & 0.026 & 5.18 & \pm & 0.11 & 1.04 & \pm & 0.02 \\
\hline 44.462 & \pm & 1.835 & 5.762 & \pm & 0.027 & 5.30 & \pm & 0.11 & 1.09 & \pm & 0.02 \\
\hline 46.685 & \pm & 1.927 & 6.082 & \pm & 0.028 & 4.91 & \pm & 0.11 & 1.24 & \pm & 0.03 \\
\hline 49.019 & \pm & 2.002 & 5.664 & \pm & 0.027 & 4.84 & \pm & 0.09 & 1.17 & \pm & 0.02 \\
\hline 51.470 & \pm & 2.103 & 4.343 & \pm & 0.023 & 4.44 & \pm & 0.09 & 0.98 & \pm & 0.02 \\
\hline 54.044 & \pm & 2.185 & 3.546 & \pm & 0.020 & 5.08 & \pm & 0.10 & 0.70 & \pm & 0.01 \\
\hline 56.746 & \pm & 2.294 & 4.411 & \pm & 0.024 & 5.86 & \pm & 0.10 & 0.75 & \pm & 0.01 \\
\hline 59.583 & \pm & 2.383 & 5.672 & \pm & 0.027 & 6.11 & \pm & 0.10 & 0.93 & \pm & 0.02 \\
\hline 62.562 & \pm & 2.502 & 6.127 & \pm & 0.029 & 6.15 & \pm & 0.11 & 1.00 & \pm & 0.02 \\
\hline 65.690 & \pm & 2.628 & 5.928 & \pm & 0.028 & 6.58 & \pm & 0.11 & 0.90 & \pm & 0.02 \\
\hline 68.975 & \pm & 2.730 & 6.112 & \pm & 0.029 & 6.66 & \pm & 0.12 & 0.92 & \pm & 0.02 \\
\hline 72.424 & \pm & 2.866 & 6.457 & \pm & 0.030 & 7.13 & \pm & 0.11 & 0.91 & \pm & 0.01 \\
\hline 76.045 & \pm & 3.009 & 6.344 & \pm & 0.030 & 7.32 & \pm & 0.11 & 0.87 & \pm & 0.01 \\
\hline 79.847 & \pm & 3.160 & 6.809 & \pm & 0.031 & 6.57 & \pm & 0.12 & 1.04 & \pm & 0.02 \\
\hline 83.840 & \pm & 3.282 & 6.996 & \pm & 0.032 & 6.09 & \pm & 0.11 & 1.15 & \pm & 0.02 \\
\hline 88.031 & \pm & 3.446 & 6.382 & \pm & 0.030 & 5.49 & \pm & 0.13 & 1.16 & \pm & 0.03 \\
\hline 92.433 & \pm & 3.619 & 6.475 & \pm & 0.030 & 5.21 & \pm & 0.12 & 1.24 & \pm & 0.03 \\
\hline 97.055 & \pm & 3.758 & 6.393 & \pm & 0.030 & 5.82 & \pm & 0.11 & 1.10 & \pm & 0.02 \\
\hline 101.907 & \pm & 3.946 & 6.243 & \pm & 0.030 & 6.53 & \pm & 0.12 & 0.96 & \pm & 0.02 \\
\hline 107.003 & \pm & 4.144 & 6.636 & \pm & 0.031 & 7.49 & \pm & 0.17 & 0.89 & \pm & 0.02 \\
\hline 112.353 & \pm & 4.351 & 7.108 & \pm & 0.032 & 8.66 & \pm & 0.18 & 0.82 & \pm & 0.02 \\
\hline 117.971 & \pm & 5.371 & 7.682 & \pm & 0.034 & 8.80 & \pm & 0.16 & 0.87 & \pm & 0.02 \\
\hline 123.869 & \pm & 5.640 & 8.083 & \pm & 0.035 & 6.86 & \pm & 0.08 & 1.18 & \pm & 0.01 \\
\hline 130.063 & \pm & 5.867 & 8.512 & \pm & 0.036 & 6.13 & \pm & 0.09 & 1.39 & \pm & 0.02 \\
\hline
\end{tabular}


NEA/NSC/DOC(2006)1

Liquid Metal Fast Reactor - LMFR

FFTF-LMFR-RESR-001

CRIT-SPEC-REAC-COEF-MISC

Table 4.3.2 (cont'd). Calculated FFTF Lower-Axial Neutron Spectrum.

\begin{tabular}{|c|c|c|c|c|c|c|c|c|c|c|c|}
\hline $\begin{array}{c}\text { Energy } \\
(\mathrm{keV})\end{array}$ & \pm & $\sigma$ & $\begin{array}{r}\text { Calcul } \\
\text { Relative Flux } \\
\varphi / \varphi_{\max } \times 8.8 \\
\end{array}$ & ted & $\sigma$ & $\begin{array}{r}\text { Benchn } \\
\text { Relative Flux } \\
\varphi / \varphi_{\max } \times 8.8 \\
\end{array}$ & ark & $\sigma$ & $\mathrm{C} / \mathrm{E}$ & \pm & $\sigma$ \\
\hline 136.566 & \pm & 6.160 & 8.800 & \pm & 0.037 & 5.86 & \pm & 0.07 & 1.50 & \pm & 0.02 \\
\hline 143.394 & \pm & 6.468 & 7.660 & \pm & 0.034 & 5.23 & \pm & 0.07 & 1.46 & \pm & 0.02 \\
\hline 150.564 & \pm & 6.727 & 6.785 & \pm & 0.032 & 5.02 & \pm & 0.08 & 1.35 & \pm & 0.02 \\
\hline 158.092 & \pm & 7.064 & 7.287 & \pm & 0.033 & 5.16 & \pm & 0.08 & 1.41 & \pm & 0.02 \\
\hline 165.996 & \pm & 7.417 & 7.907 & \pm & 0.035 & 5.29 & \pm & 0.09 & 1.49 & \pm & 0.03 \\
\hline 174.296 & \pm & 7.788 & 8.259 & \pm & 0.036 & 5.59 & \pm & 0.09 & 1.48 & \pm & 0.03 \\
\hline 183.011 & \pm & 8.177 & 8.507 & \pm & 0.037 & 5.37 & \pm & 0.08 & 1.58 & \pm & 0.03 \\
\hline 192.162 & \pm & 8.504 & 7.619 & \pm & 0.035 & 5.39 & \pm & 0.09 & 1.41 & \pm & 0.02 \\
\hline 201.770 & \pm & 8.929 & 6.353 & \pm & 0.031 & 5.12 & \pm & 0.08 & 1.24 & \pm & 0.02 \\
\hline 211.858 & \pm & 9.376 & 6.365 & \pm & 0.031 & 5.50 & \pm & 0.06 & 1.16 & \pm & 0.01 \\
\hline 222.451 & \pm & 9.845 & 6.685 & \pm & 0.032 & 5.18 & \pm & 0.05 & 1.29 & \pm & 0.01 \\
\hline 233.574 & \pm & 10.337 & 6.567 & \pm & 0.032 & 5.53 & \pm & 0.06 & 1.19 & \pm & 0.01 \\
\hline 245.252 & \pm & 10.749 & 6.488 & \pm & 0.0 & 5.35 & \pm & 0.06 & 1.20 & \pm & 0.02 \\
\hline 257.515 & \pm & 11.287 & 55 & \pm & 0.0 & 5.4 & \pm & 0.06 & 1.36 & \pm & 0.02 \\
\hline 270.391 & \pm & 11.851 & 84 & \pm & 0.0 & 5.1 & \pm & 0.06 & 1.65 & \pm & 0.02 \\
\hline 283.910 & \pm & 12.444 & 8.392 & \pm & 0.0 & 4.83 & \pm & 0.06 & 1.74 & \pm & 0.02 \\
\hline 298.106 & \pm & 13.066 & 8.152 & \pm & 0.037 & 4.4( & \pm & 0.05 & 1.85 & \pm & 0.02 \\
\hline 313.011 & \pm & 13.719 & 29 & \pm & 0.037 & 4.1 & \pm & 0.06 & 1.98 & \pm & 0.03 \\
\hline 328.662 & \pm & 14.405 & 90 & \pm & 0.036 & 3.7 & \pm & 0.06 & 1.98 & \pm & 0.03 \\
\hline 345.095 & \pm & 14.979 & 7.045 & \pm & 0.035 & 3.27 & \pm & 0.04 & 2.16 & \pm & 0.03 \\
\hline 362.349 & \pm & 15.727 & 54 & \pm & 0.034 & 3.03 & \pm & 0.04 & 2.27 & \pm & 0.03 \\
\hline 380.467 & \pm & 16.514 & 6.195 & \pm & 0.033 & 2.94 & \pm & 0.05 & 2.11 & \pm & 0.03 \\
\hline 399.490 & \pm & 17.340 & 5.346 & \pm & 0.030 & 2.97 & \pm & 0.04 & 1.80 & \pm & 0.03 \\
\hline 419.465 & \pm & 18.207 & 5.055 & \pm & 0.029 & 3.02 & \pm & 0.04 & 1.67 & \pm & 0.03 \\
\hline 440.438 & \pm & 19.117 & 5.256 & \pm & 0.030 & 2.77 & \pm & 0.04 & 1.89 & \pm & 0.03 \\
\hline 462.460 & \pm & 20.073 & 6.220 & \pm & 0.033 & 2.65 & \pm & 0.05 & 2.34 & \pm & 0.04 \\
\hline 485.583 & \pm & 21.076 & 7.406 & \pm & 0.036 & 2.55 & t & 0.04 & 2.91 & \pm & 0.05 \\
\hline 509.862 & \pm & 22.130 & 8.170 & \pm & 0.038 & 2.31 & \pm & 0.04 & 3.54 & \pm & 0.07 \\
\hline
\end{tabular}


NEA/NSC/DOC(2006)1

Liquid Metal Fast Reactor - LMFR

FFTF-LMFR-RESR-001

CRIT-SPEC-REAC-COEF-MISC

Table 4.3.2 (cont'd). Calculated FFTF Lower-Axial Neutron Spectrum.

\begin{tabular}{|c|c|c|c|c|c|c|c|c|c|c|c|}
\hline \multirow{2}{*}{$\begin{array}{l}\text { Energy } \\
(\mathrm{keV})\end{array}$} & \multirow[b]{2}{*}{ \pm} & \multirow[b]{2}{*}{$\sigma$} & \multicolumn{3}{|c|}{ Calculated } & \multicolumn{3}{|c|}{ "Benchmark } & \multirow[b]{2}{*}{$\mathrm{C} / \mathrm{E}$} & \multirow[b]{2}{*}{ \pm} & \multirow[b]{2}{*}{$\sigma$} \\
\hline & & & $\begin{array}{c}\text { Relative Flux } \\
\varphi / \varphi_{\max } \times 8.8\end{array}$ & \pm & $\sigma$ & $\begin{array}{c}\text { Relative Flux } \\
\varphi / \varphi_{\max } \times 8.8\end{array}$ & \pm & $\sigma$ & & & \\
\hline 535.355 & \pm & 23.237 & 8.201 & \pm & 0.038 & 2.05 & \pm & 0.05 & 4.00 & \pm & 0.09 \\
\hline 562.123 & \pm & 24.399 & 7.597 & \pm & 0.037 & 2.16 & \pm & 0.03 & 3.52 & \pm & 0.06 \\
\hline 590.229 & \pm & 25.367 & 7.083 & \pm & 0.035 & 1.99 & \pm & 0.03 & 3.55 & \pm & 0.06 \\
\hline 619.741 & \pm & 26.636 & 6.580 & \pm & 0.034 & 1.69 & \pm & 0.03 & 3.90 & \pm & 0.08 \\
\hline 650.728 & \pm & 27.967 & 6.083 & \pm & 0.033 & 1.54 & \pm & 0.03 & 3.96 & \pm & 0.08 \\
\hline 683.264 & \pm & 29.366 & 5.525 & \pm & 0.031 & 1.37 & \pm & 0.03 & 4.04 & \pm & 0.09 \\
\hline 717.427 & \pm & 30.834 & 4.939 & \pm & 0.029 & 1.32 & \pm & 0.03 & 3.75 & \pm & 0.10 \\
\hline 753.299 & \pm & 32.376 & 4.582 & \pm & 0.028 & 1.27 & \pm & 0.03 & 3.61 & \pm & 0.09 \\
\hline 790.964 & \pm & 33.995 & 4.535 & \pm & 0.028 & 1.20 & \pm & 0.03 & 3.78 & \pm & 0.10 \\
\hline 830.512 & \pm & 35.694 & 4.376 & \pm & 0.028 & 1.21 & \pm & 0.04 & 3.63 & \pm & 0.11 \\
\hline 872.037 & \pm & 37.479 & 4.213 & \pm & 0.028 & 1.17 & \pm & 0.03 & 3.61 & \pm & 0.10 \\
\hline 915.639 & \pm & 39.353 & 3.866 & \pm & 0.027 & 1.08 & \pm & 0.03 & 3.59 & \pm & 0.12 \\
\hline 961.421 & \pm & 41.321 & 3.579 & \pm & 0.026 & 0.91 & \pm & 0.04 & 3.95 & \pm & 0.16 \\
\hline 1009.492 & \pm & 43.387 & 3.337 & \pm & 0.025 & 0.95 & \pm & 0.03 & 3.52 & \pm & 0.12 \\
\hline 1059.967 & \pm & 45.556 & 3.362 & \pm & 0.025 & 0.99 & \pm & 0.03 & 3.40 & \pm & 0.11 \\
\hline 1112.965 & \pm & 47.834 & 3.546 & \pm & 0.026 & 0.79 & \pm & 0.03 & 4.50 & \pm & 0.19 \\
\hline 1168.613 & \pm & 50.225 & 3.765 & \pm & 0.027 & 0.72 & \pm & 0.03 & 5.26 & \pm & 0.23 \\
\hline 1227.044 & \pm & 52.737 & 3.779 & \pm & 0.027 & 0.69 & \pm & 0.03 & 5.51 & \pm & 0.27 \\
\hline 1288.396 & \pm & 55.374 & 3.506 & \pm & 0.026 & 0.61 & \pm & 0.05 & 5.72 & \pm & 0.48 \\
\hline 1352.816 & \pm & 58.142 & 3.321 & \pm & 0.025 & 0.51 & \pm & 0.03 & 6.52 & \pm & 0.34 \\
\hline 1420.457 & \pm & 61.049 & 3.319 & \pm & 0.025 & 0.49 & \pm & 0.03 & 6.80 & \pm & 0.37 \\
\hline 1491.480 & \pm & 64.102 & 3.191 & \pm & 0.025 & 0.46 & \pm & 0.03 & 6.94 & \pm & 0.45 \\
\hline 1566.054 & \pm & 67.307 & 3.061 & \pm & 0.024 & 0.41 & \pm & 0.03 & 7.47 & \pm & 0.55 \\
\hline 1644.356 & \pm & 70.672 & 2.932 & \pm & 0.024 & 0.41 & \pm & 0.03 & 7.21 & \pm & 0.53 \\
\hline 1726.574 & \pm & 73.471 & 2.794 & \pm & 0.023 & 0.30 & \pm & 0.03 & 9.24 & \pm & 0.91 \\
\hline 1812.903 & \pm & 77.145 & 2.704 & \pm & 0.023 & 0.25 & \pm & 0.03 & 10.98 & \pm & 1.12 \\
\hline 1903.548 & \pm & 81.002 & 2.578 & \pm & 0.022 & 0.23 & \pm & 0.02 & 11.31 & \pm & 1.24 \\
\hline
\end{tabular}


NEA/NSC/DOC(2006)1

Liquid Metal Fast Reactor - LMFR

FFTF-LMFR-RESR-001

CRIT-SPEC-REAC-COEF-MISC

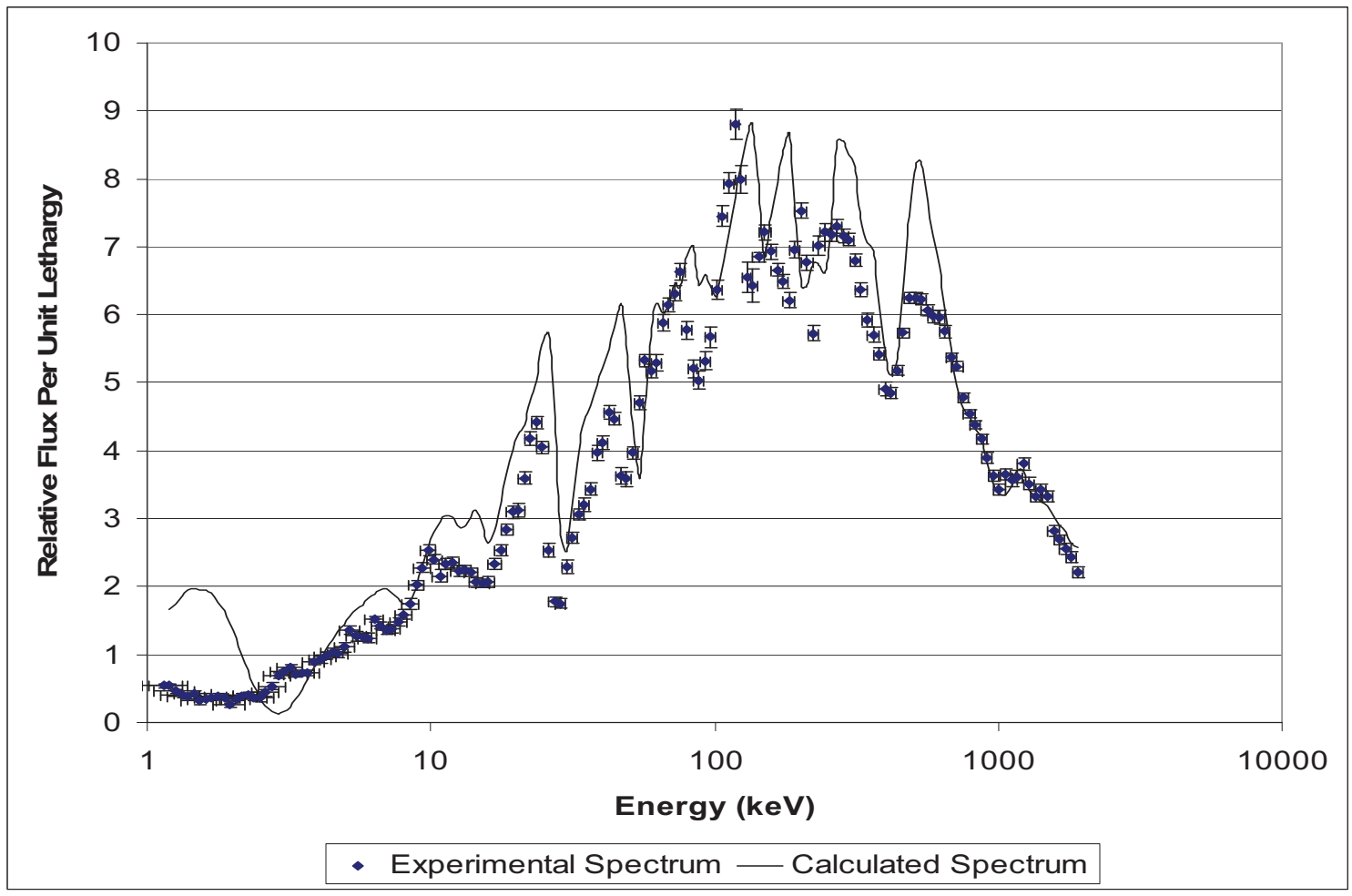

Figure 4.3.1. Neutron Spectrum in FFTF Core Center (at Midplane).

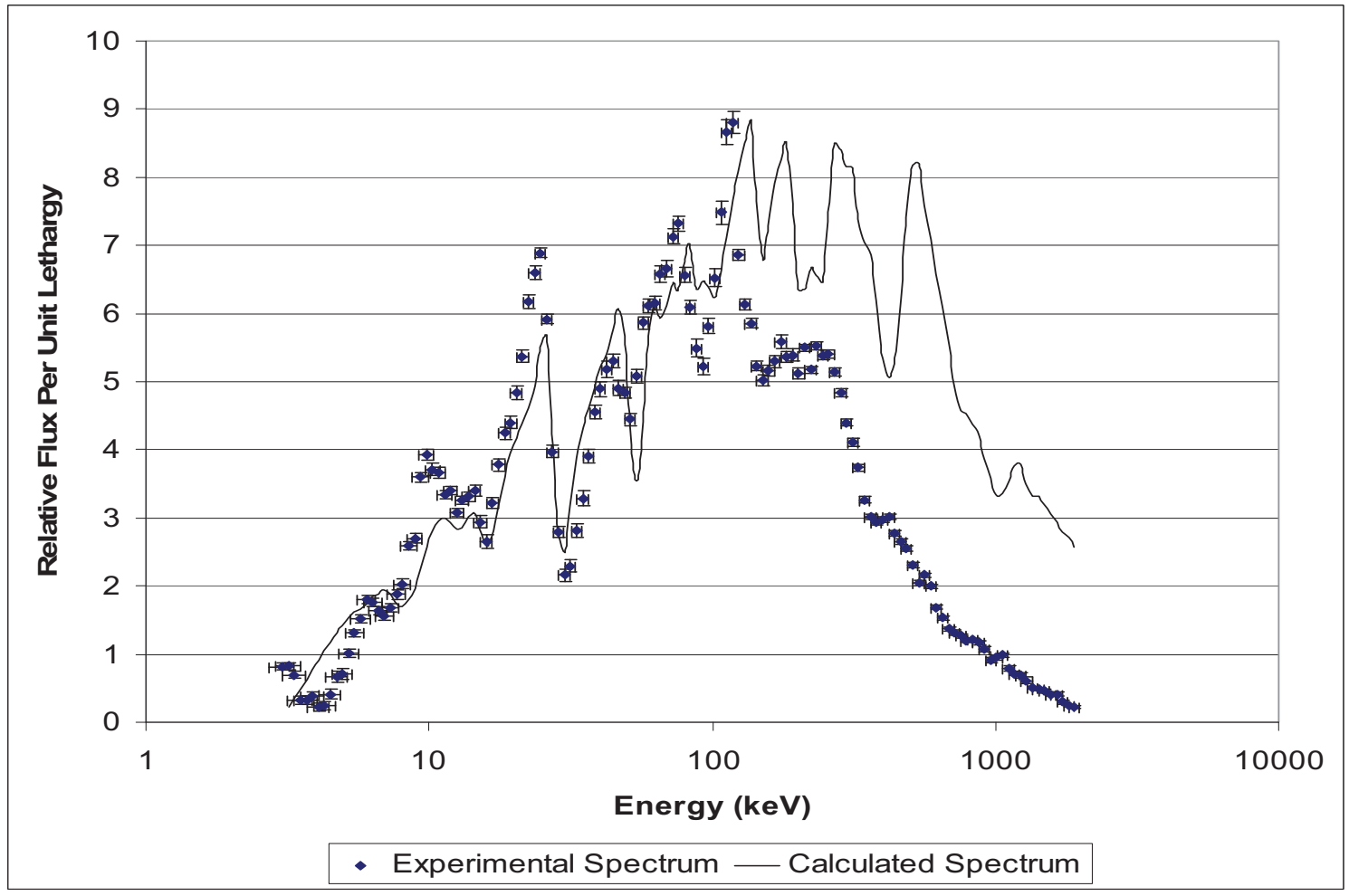

Figure 4.3.2. Neutron Spectrum in FFTF Core Center ( $80 \mathrm{~cm}$ Below Midplane). 
FFTF-LMFR-RESR-001

CRIT-SPEC-REAC-COEF-MISC

\subsection{Results of Reactivity-Effects Calculations}

The benchmark model for the critical configuration described in Section 3.1 was utilized in the analysis of the reactor physics experiments in Section 1.4.

Reactivity effects measurements evaluated include a total of 21 control rod worth measurements (seven of which represent combinations of dropped rods), two control rod bank worth measurements, six differential control rod worth measurements, measured shutdown margin, and measured excess reactivity for the fully-loaded critical core configuration of the FFTF.

Reactivity worths were determined using a typical $\beta_{\text {eff }}$ value of 0.00318 ; this value corresponds to the delayed neutron precursor data used in the IKRD rod worth measurements. Use of another reported value, 0.003097 , results in an increase in the calculated rod worths by approximately $2.5 \%$, bringing them closer into agreement with the benchmark rod worths. Because of the uncertainty in the calculated $\beta_{\text {eff }}$ value, an additional $5 \%$ uncertainty was included in the reported benchmark worths. 


\subsubsection{Control Rod Worths}

The calculated values for the control rod worths are provided in Table 4.4.1. Calculated control rod worths are consistently $2-6 \%$ lower than the benchmark control rod worths, but within $1 \sigma$ of the benchmark value.

Table 4.4.1. Control Rod Worths for the FFTF Fully-Loaded Core Critical.

\begin{tabular}{|c|c|c|c|c|c|c|c|c|c|c|c|}
\hline \multirow{2}{*}{ Case } & \multirow{2}{*}{$\begin{array}{c}\text { Reference } \\
\text { State }\end{array}$} & \multirow{2}{*}{$\begin{array}{c}\text { Rod(s) } \\
\text { Dropped }\end{array}$} & \multicolumn{3}{|c|}{ Calculated } & \multicolumn{3}{|c|}{ Benchmark } & \multirow{2}{*}{$\mathrm{C} / \mathrm{E}$} & \multirow{2}{*}{ \pm} & \multirow{2}{*}{$\sigma$} \\
\hline & & & $\rho(\$)$ & \pm & $\sigma$ & $\rho(\$)$ & \pm & $\sigma$ & & & \\
\hline 1 & 1 & 1 & 5.92 & \pm & 0.02 & 6.04 & \pm & 0.32 & 0.98 & \pm & 0.05 \\
\hline 2 & 1 & 2 & 5.65 & \pm & 0.02 & 5.89 & \pm & 0.31 & 0.96 & \pm & 0.05 \\
\hline 3 & 1 & 3 & 4.36 & \pm & 0.02 & 4.65 & \pm & 0.25 & 0.94 & \pm & 0.05 \\
\hline 4 & 1 & 5 & 3.63 & \pm & 0.02 & 3.84 & \pm & 0.20 & 0.95 & \pm & 0.05 \\
\hline 5 & 1 & 7 & 2.85 & \pm & 0.02 & 2.90 & \pm & 0.15 & 0.98 & \pm & 0.05 \\
\hline 6 & 1 & $1+2$ & 12.08 & \pm & 0.02 & 12.45 & \pm & 0.69 & 0.97 & \pm & 0.05 \\
\hline 7 & 1 & $1+3$ & 10.57 & \pm & 0.03 & 11.01 & \pm & 0.60 & 0.96 & \pm & 0.05 \\
\hline 8 & 1 & $1+5$ & 8.61 & \pm & 0.02 & 9.00 & \pm & 0.48 & 0.96 & \pm & 0.05 \\
\hline 9 & 1 & $3+5$ & 8.47 & \pm & 0.02 & 8.8 & \pm & 0.46 & 0.96 & \pm & 0.05 \\
\hline 10 & 1 & $1+2+5$ & 15.07 & \pm & 0.03 & 15.84 & \pm & 0.87 & 0.95 & \pm & 0.05 \\
\hline 11 & 1 & $2+3+5$ & 14.94 & \pm & 0.02 & 15.22 & \pm & 0.86 & 0.98 & \pm & 0.06 \\
\hline 12 & 2 & 1 & 5.64 & \pm & 0.02 & 5.81 & \pm & 0.33 & 0.97 & \pm & 0.06 \\
\hline 13 & 2 & 2 & 5.36 & \pm & 0.02 & 5.51 & \pm & 0.29 & 0.97 & \pm & 0.06 \\
\hline 14 & 2 & 3 & 5.18 & \pm & 0.02 & 5.40 & \pm & 0.29 & 0.96 & \pm & 0.05 \\
\hline 15 & 3 & 4 & 3.97 & \pm & 0.02 & 4.06 & \pm & 0.21 & 0.98 & \pm & 0.05 \\
\hline 16 & 4 & 5 & 3.97 & \pm & 0.03 & 4.09 & \pm & 0.21 & 0.97 & \pm & 0.05 \\
\hline 17 & 5 & 6 & 3.48 & \pm & 0.02 & 3.57 & \pm & 0.18 & 0.97 & \pm & 0.05 \\
\hline 18 & 6 & 7 & 3.76 & \pm & 0.02 & 3.86 & \pm & 0.20 & 0.97 & \pm & 0.05 \\
\hline 19 & 7 & 8 & 3.01 & \pm & 0.02 & 3.19 & \pm & 0.19 & 0.94 & \pm & 0.06 \\
\hline 20 & 8 & 9 & 3.62 & \pm & 0.02 & 3.83 & \pm & 0.19 & 0.95 & \pm & 0.05 \\
\hline 21 & 9 & $2+3$ & 10.89 & \pm & 0.03 & 11.22 & \pm & 0.58 & 0.97 & \pm & 0.05 \\
\hline
\end{tabular}


NEA/NSC/DOC(2006)1

Liquid Metal Fast Reactor - LMFR

FFTF-LMFR-RESR-001

CRIT-SPEC-REAC-COEF-MISC

\subsubsection{Control Rod Bank Worths}

The calculated values for the control rod bank worths are provided in Table 4.4.2. Calculated control rod bank worths are slightly greater than the benchmark control rod bank worths and well within $1 \sigma$.

Table 4.4.2. Control Rod Bank Worths for the FFTF Fully-Loaded Core Critical.

\begin{tabular}{|c|cc|ccc|ccc|ccc|}
\hline \multirow{2}{*}{ Case } & \multirow{2}{*}{$\begin{array}{c}\text { Reference } \\
\text { State }\end{array}$} & Rods & \multicolumn{2}{|c|}{ Calculated } & \multicolumn{2}{|c|}{ Benchmark } & \multirow{2}{*}{ C/E } & \pm & $\sigma$ \\
& & $\rho(\$)$ & \pm & $\sigma$ & $\rho(\$)$ & \pm & $\sigma$ & & & \\
\hline \hline 22 & Fully Subcritical & $1+2+3$ & 16.76 & \pm & 0.03 & 16.34 & \pm & 0.83 & 1.03 & \pm & 0.05 \\
23 & Fully Subcritical & $4+5+6+7+8+9$ & 20.04 & \pm & 0.03 & 19.9 & \pm & 1.00 & 1.01 & \pm & 0.05 \\
\hline
\end{tabular}

\subsubsection{Differential Control Rod Worths}

The calculated values for the differential control rod worths are provided in Table 4.4.3. Calculated differential control rod bank worths are approximately $4-8 \%$ lower than the benchmark differential control rod worths and generally within $1 \sigma$.

Table 4.4.3. Differential Control Rod Worths for the FFTF Fully-Loaded Core Critical.

\begin{tabular}{|c|ccc|ccc|ccc|ccc||}
\hline \multirow{2}{*}{ Case } & \multirow{2}{*}{$\begin{array}{c}\text { Reference } \\
\text { State }\end{array}$} & Rod & \multicolumn{2}{|c|}{ Calculated } & \multicolumn{2}{|c|}{ Benchmark } & \multicolumn{2}{|c||}{ C/E } & \pm & $\sigma$ \\
& & $\rho \phi / \mathrm{cm}$ & \pm & $\sigma$ & $\rho \notin / \mathrm{cm}$ & \pm & $\sigma$ & & & \\
\hline \hline 24 & 10 & 4 & 5.9 & \pm & 0.2 & 6.1 & \pm & 0.3 & 0.97 & \pm & 0.05 \\
25 & 10 & 5 & 5.7 & \pm & 0.2 & 6.2 & \pm & 0.3 & 0.92 & \pm & 0.05 \\
26 & 10 & 6 & 5.0 & \pm & 0.2 & 5.4 & \pm & 0.3 & 0.93 & \pm & 0.05 \\
27 & 10 & 7 & 5.3 & \pm & 0.2 & 5.7 & \pm & 0.3 & 0.94 & \pm & 0.05 \\
28 & 10 & 8 & 4.5 & \pm & 0.3 & 4.8 & \pm & 0.3 & 0.95 & \pm & 0.06 \\
29 & 10 & 9 & 5.5 & \pm & 0.2 & 5.6 & \pm & 0.3 & 0.97 & \pm & 0.05 \\
\hline
\end{tabular}

\subsubsection{Shutdown Margin}

The calculated value for the shutdown margin is provided in Table 4.4.4. The calculated and benchmark

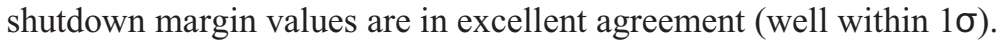

Table 4.4.4. Shutdown Margin Values for the FFTF Fully-Loaded Core Critical.

\begin{tabular}{|c|c|c|c|c|c|c|c|c|c|c|c|}
\hline \multirow{2}{*}{ Case } & \multirow{2}{*}{$\begin{array}{l}\text { Reference } \\
\text { State }\end{array}$} & \multirow{2}{*}{$\begin{array}{c}\text { Rods } \\
\text { Dropped }\end{array}$} & \multicolumn{3}{|c|}{ Calculated } & \multicolumn{3}{|c|}{ Benchmark } & \multirow{2}{*}{$\mathrm{C} / \mathrm{E}$} & \multirow{2}{*}{ \pm} & \multirow{2}{*}{$\sigma$} \\
\hline & & & $\rho(\$)$ & \pm & $\sigma$ & $\rho(\$)$ & \pm & $\sigma$ & & & \\
\hline 30 & 1 & & 23.67 & $\perp$ & 0.02 & 24.0 & \pm & 1.40 & 0.99 & \pm & 0.06 \\
\hline 31 & 2 & All & 23.76 & \pm & 0.03 & 23.7 & \pm & 1.27 & 1.00 & \pm & 0.05 \\
\hline
\end{tabular}


NEA/NSC/DOC(2006)1

Liquid Metal Fast Reactor - LMFR

FFTF-LMFR-RESR-001

CRIT-SPEC-REAC-COEF-MISC

\subsubsection{Excess Reactivity}

The calculated value for the excess reactivity is provided in Table 4.4.5. There is a 7\% difference between the calculated and benchmark values for the excess reactivity, which is within $2 \sigma$.

Table 4.4.5. Excess Reactivity for the FFTF Fully-Loaded Core Critical.

\begin{tabular}{|c|c|c|c|c|c|c|c|c|c|c|}
\hline \multirow{2}{*}{ Case } & \multirow{2}{*}{$\begin{array}{l}\text { Reference } \\
\text { State }\end{array}$} & \multirow{2}{*}{$\begin{array}{c}\text { Rods } \\
\text { Withdrawn }\end{array}$} & \multicolumn{3}{|c|}{ Calculated } & \multicolumn{3}{|c|}{ Benchmark } & \multirow{2}{*}{\multicolumn{2}{|c|}{$\mathrm{C} / \mathrm{E}$}} \\
\hline & & & $\rho(\$)$ & \pm & $\sigma$ & $\rho(\$)$ & 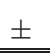 & $\sigma$ & & \\
\hline 32 & Critical & All & 13.66 & \pm & 0.03 & 14.66 & \pm & 0.85 & 0.93 & \pm 0.05 \\
\hline
\end{tabular}

\subsection{Results of Reactivity Coefficient Calculations}

The benchmark model for the critical configuration described in Section 3.1 was utilized in the analysis of the reactor physics experiments in Section 1.5.

The calculated value for the isothermal temperature coefficient is provided in Table 4.5.1. The calculated value is approximately half of the benchmark model. As discussed in Section 2.1.1.1, the core assembly pitch was not adjusted with the variation in core temperature. The model temperature, liquid sodium coolant density, and neutron cross section libraries were adjusted for the variation in temperature. The densities of major structural components were not adjusted. However, much of the core model is homogenized, and density effects would be negligible. There is no correlation available between the core temperature and assembly pitch such that a more accurate calculation of the isothermal temperature coefficient could be evaluated.

If the average uncertainty in the core lattice pitch $\left( \pm 0.00086 \Delta \mathrm{k}_{\text {eff }}\right)$ determined in Section 2.1.2.9 is included with the calculation of the reactivity worth variation, then the calculated isothermal temperature coefficient is approximately $8.7 \%$ lower than the benchmark value. However, this is an estimated value with a larger uncertainty, and an exact correlation between the core temperature effects and core lattice pitch has not been established.

Table 4.5.1. Isothermal Temperature Coefficient for the FFTF Fully-Loaded Core Critical.

\begin{tabular}{|c|c|c|c|c|c|c|c|c|c|}
\hline $\begin{array}{l}\text { Core Pitch } \\
\text { Effects }\end{array}$ & $\begin{array}{r}\text { Calcu } \\
\rho_{\mathrm{T}}(\rho \notin / K)\end{array}$ & latec & $\sigma$ & $\begin{array}{r}\text { Bench } \\
\rho_{\mathrm{T}}(\rho \notin / K)\end{array}$ & marl & $\sigma$ & $\mathrm{C} / \mathrm{E}$ & \pm & $\sigma$ \\
\hline None & -0.61 & \pm & 0.05 & -1.26 & \pm & 0.19 & 0.48 & \pm & 0.09 \\
\hline Approximated & -1.15 & \pm & 0.18 & -1.26 & \pm & 0.19 & 0.91 & \pm & 0.20 \\
\hline
\end{tabular}

\subsection{Results of Kinetics Parameter Calculations}

Kinetics measurements were not made.

\subsection{Results of Reaction-Rate Distribution Calculations}

Reaction-rate distribution measurements were not made. 
NEA/NSC/DOC(2006)1

Liquid Metal Fast Reactor - LMFR

FFTF-LMFR-RESR-001

CRIT-SPEC-REAC-COEF-MISC

\subsection{Results of Power Distribution Calculations}

Power distribution measurements were not made.

\subsection{Results of Isotopic Calculations}

Isotopic measurements were not made.

\subsection{Results of Calculations for Other Miscellaneous Types of Measurements}

The benchmark model for the critical configuration described in Section 3.1 was modified as described in Section 3.10 and utilized in the analysis of the reactor physics experiments in Section 1.10.

The reactor physics experiments evaluated from this section pertain to low-energy gamma-ray spectra measurements performed in the IRT near the radial center of the core at core midplane. The low-energy electron spectra measurements were evaluated but judged to not be of benchmark quality (Section 2.10.1).

The calculated photon spectra are obtained by taking the variance-weighted average of results obtained using six variations of the input deck (Appendix A.1) with different random number seeds and tallies of the photon flux (Appendix A.10). This approach was used to reduce the statistical uncertainty in the neutron flux tallies because the relative error values obtained can underpredict the true uncertainty in the calculated spectra. ${ }^{a}$ The initial data point was not included in the comparison as MCNP5 tracks all photons below that energy threshold while the detectors would have had an unknown minimum energy threshold for detection.

The calculated values for the gamma-ray spectra measurements near the radial center of the FFTF in the in-reactor thimble at core midplane are provided in Table 4.10.1 and Figure 4.10.1. There is in general good correlation between the calculated and benchmark values for the normalized photon energy spectrum. On average, the calculated normalized flux is $\sim 37 \%$ greater than the experimentally measured benchmark values.

Gamma production cross section data were not available for the isotopes ${ }^{238} \mathrm{Pu},{ }^{99} \mathrm{Mo}$, and ${ }^{100} \mathrm{Mo}$ is the ENDF/B-VII.0 library. These isotopes are minor constituents in the materials of the FFTF and their contribution to uncertainties in the gamma spectra analysis is considered negligible.

${ }^{a}$ F. B. Brown, “A Review of Best Practices for Monte Carlo Criticality Calculations,” Proc. NCSD 2009, Richland, WA, September 13-17 (2009). 
NEA/NSC/DOC(2006)1

Liquid Metal Fast Reactor - LMFR

FFTF-LMFR-RESR-001

CRIT-SPEC-REAC-COEF-MISC

Table 4.10.1. Calculated FFTF Core Center Low-Energy Gamma-Ray Spectrum.

\begin{tabular}{|c|c|c|c|c|c|c|c|c|c|}
\hline $\begin{array}{l}\text { Energy } \\
(\mathrm{MeV})\end{array}$ & $\begin{array}{c}\text { Calc } \\
\text { Normalized } \\
\text { Flux } \\
\varphi / \varphi_{\max }\end{array}$ & \pm & $\sigma$ & $\begin{array}{c}\text { Benc } \\
\text { Normalized } \\
\text { Flux } \\
\varphi / \varphi_{\max }\end{array}$ & \pm & $\sigma$ & $\mathrm{C} / \mathrm{E}$ & \pm & $\sigma$ \\
\hline 0.2203 & & -- & & 1.0148 & $\bar{~} \overline{ \pm}$ & 0.1015 & & $\bar{~}$ & \\
\hline 0.2485 & 0.7289 & \pm & 0.0042 & 1.0000 & \pm & 0.1000 & 0.73 & \pm & 0.07 \\
\hline 0.2745 & 0.6069 & \pm & 0.0038 & 0.8341 & \pm & 0.0834 & 0.73 & \pm & 0.07 \\
\hline 0.3006 & 0.5586 & \pm & 0.0037 & 0.6492 & \pm & 0.0649 & 0.86 & \pm & 0.09 \\
\hline 0.3252 & 0.4940 & \pm & 0.0034 & 0.5383 & \pm & 0.0538 & 0.92 & \pm & 0.09 \\
\hline 0.3498 & 0.4866 & \pm & 0.0035 & 0.4594 & \pm & 0.0459 & 1.06 & \pm & 0.11 \\
\hline 0.3741 & 0.4800 & \pm & 0.0035 & 0.4335 & \pm & 0.0434 & 1.11 & \pm & 0.11 \\
\hline 0.3955 & 0.4076 & \pm & 0.0032 & 0.4474 & \pm & 0.0447 & 0.91 & \pm & 0.09 \\
\hline 0.4223 & 0.5295 & \pm & 0.0036 & 0.4615 & \pm & 0.0462 & 1.15 & \pm & 0.11 \\
\hline 0.4426 & 0.8352 & \pm & 0.0045 & 0.4282 & \pm & 0.0428 & 1.95 & \pm & 0.20 \\
\hline 0.4881 & 0.8402 & \pm & 0.0047 & 0.4746 & \pm & 0.0475 & 1.77 & \pm & 0.18 \\
\hline 0.5152 & 1.0000 & \pm & 0.0054 & 0.4192 & \pm & 0.0419 & 2.39 & \pm & 0.24 \\
\hline 0.5357 & 0.2844 & \pm & 0.0027 & 0.3568 & \pm & 0.0357 & 0.80 & \pm & 0.08 \\
\hline 0.5546 & 0.2625 & \pm & 0.0027 & 0.3455 & \pm & 0.0346 & 0.76 & \pm & 0.08 \\
\hline 0.5776 & 0.3319 & \pm & 0.0031 & 0.3261 & \pm & 0.0326 & 1.02 & \pm & 0.10 \\
\hline 0.6033 & 0.3715 & \pm & 0.0033 & 0.3043 & \pm & 0.0304 & 1.22 & \pm & 0.12 \\
\hline 0.6236 & 0.2752 & \pm & 0.0028 & 2897 & \pm & 0.0290 & 0.95 & \pm & 0.10 \\
\hline 0.6452 & 0.2840 & \pm & 0.0029 & 0.2680 & \pm & 0.0268 & 1.06 & \pm & 0.11 \\
\hline 0.6667 & 0.2771 & \pm & 0.0029 & 0.2718 & \pm & 0.0272 & 1.02 & \pm & 0.10 \\
\hline 0.7140 & 0.5717 & \pm & 0.0042 & 0.2434 & \pm & 0.0243 & 2.35 & \pm & 0.24 \\
\hline 0.7288 & 0.1705 & \pm & 0.0023 & 0.2427 & \pm & 0.0243 & 0.70 & \pm & 0.07 \\
\hline 0.7299 & 0.0115 & \pm & 0.0006 & 0.2645 & \pm & 0.0265 & 0.04 & \pm & 0.00 \\
\hline 0.7544 & 0.2773 & \pm & 0.0029 & 0.2304 & \pm & 0.0230 & 1.20 & \pm & 0.12 \\
\hline 0.7768 & 0.2481 & \pm & 0.0027 & 0.2784 & \pm & 0.0278 & 0.89 & \pm & 0.09 \\
\hline 0.7992 & 0.2517 & \pm & 0.0028 & 0.3365 & \pm & 0.0337 & 0.75 & \pm & 0.08 \\
\hline 0.8205 & 0.2373 & \pm & 0.0028 & 0.3710 & \pm & 0.0371 & 0.64 & \pm & 0.06 \\
\hline 0.8407 & 0.2188 & \pm & 0.0026 & 0.3604 & \pm & 0.0360 & 0.61 & \pm & 0.06 \\
\hline 0.8626 & 0.8506 & \pm & 0.0048 & 0.2954 & \pm & 0.0295 & 2.88 & \pm & 0.29 \\
\hline 0.8859 & 0.2221 & \pm & 0.0027 & 0.2457 & \pm & 0.0246 & 0.90 & \pm & 0.09 \\
\hline 0.9035 & 0.1570 & \pm & 0.0023 & 0.2292 & \pm & 0.0229 & 0.68 & \pm & 0.07 \\
\hline 0.9239 & 0.1737 & \pm & 0.0024 & 0.2127 & \pm & 0.0213 & 0.82 & \pm & 0.08 \\
\hline 0.9442 & 0.1755 & \pm & 0.0024 & 0.2007 & \pm & 0.0201 & 0.87 & \pm & 0.09 \\
\hline 0.9658 & 0.1774 & \pm & 0.0024 & 0.1911 & \pm & 0.0191 & 0.93 & \pm & 0.09 \\
\hline 0.9873 & 0.1725 & \pm & 0.0024 & 0.1856 & \pm & 0.0186 & 0.93 & \pm & 0.09 \\
\hline 1.0104 & 0.1891 & \pm & 0.0025 & 0.1707 & \pm & 0.0171 & 1.11 & \pm & 0.11 \\
\hline 1.0722 & 0.4680 & \pm & 0.0040 & 0.1657 & \pm & 0.0166 & 2.82 & \pm & 0.28 \\
\hline 1.0925 & 0.1478 & \pm & 0.0022 & 0.1591 & \pm & 0.0159 & 0.93 & \pm & 0.09 \\
\hline 1.1127 & 0.1463 & \pm & 0.0022 & 0.1506 & \pm & 0.0151 & 0.97 & \pm & 0.10 \\
\hline 1.1357 & 0.1615 & \pm & 0.0023 & 0.1430 & \pm & 0.0143 & 1.13 & \pm & 0.11 \\
\hline 1.1544 & 0.1312 & \pm & 0.0021 & 0.1523 & \pm & 0.0152 & 0.86 & \pm & 0.09 \\
\hline 1.1732 & 0.1351 & \pm & 0.0021 & 0.1518 & \pm & 0.0152 & 0.89 & \pm & 0.09 \\
\hline 1.1946 & 0.1495 & \pm & 0.0023 & 0.1553 & \pm & 0.0155 & 0.96 & \pm & 0.10 \\
\hline 1.2190 & 0.1675 & \pm & 0.0024 & 0.1445 & \pm & 0.0144 & 1.16 & \pm & 0.12 \\
\hline
\end{tabular}

Revision: 0 
NEA/NSC/DOC(2006)1

Liquid Metal Fast Reactor - LMFR

FFTF-LMFR-RESR-001

CRIT-SPEC-REAC-COEF-MISC

Table 4.10.1 (cont'd). Calculated FFTF Core Center Low-Energy Gamma-Ray Spectrum.

\begin{tabular}{|c|c|c|c|c|c|c|c|c|c|}
\hline $\begin{array}{l}\text { Energy } \\
(\mathrm{MeV})\end{array}$ & $\begin{array}{c}\text { Calc } \\
\text { Normalized } \\
\text { Flux } \\
\varphi / \varphi_{\max }\end{array}$ & ate & $\sigma$ & $\begin{array}{c}\text { Benc } \\
\text { Normalized } \\
\text { Flux } \\
\varphi / \varphi_{\max }\end{array}$ & $\mathrm{ma}$ & $\sigma$ & $\mathrm{C} / \mathrm{E}$ & \pm & $\sigma$ \\
\hline 1.2364 & 0.1158 & \pm & 0.0020 & 0.1448 & \pm & 0.0145 & 0.80 & \pm & 0.08 \\
\hline 1.2593 & 0.1919 & \pm & 0.0025 & 0.1419 & \pm & 0.0142 & 1.35 & \pm & 0.14 \\
\hline 1.2809 & 0.1375 & \pm & 0.0022 & 0.1343 & \pm & 0.0134 & 1.02 & \pm & 0.10 \\
\hline 1.3012 & 0.1336 & \pm & 0.0021 & 0.1275 & \pm & 0.0128 & 1.05 & \pm & 0.11 \\
\hline 1.3186 & 0.1047 & \pm & 0.0019 & 0.1327 & \pm & 0.0133 & 0.79 & \pm & 0.08 \\
\hline 1.3428 & 0.1823 & \pm & 0.0024 & 0.1330 & \pm & 0.0133 & 1.37 & \pm & 0.14 \\
\hline 1.3617 & 0.1111 & \pm & 0.0020 & 0.1270 & \pm & 0.0127 & 0.87 & \pm & 0.09 \\
\hline 1.3803 & 0.1053 & \pm & 0.0019 & 0.1396 & \pm & 0.0140 & 0.75 & \pm & 0.08 \\
\hline 1.4005 & 0.1093 & \pm & 0.0020 & 0.1322 & \pm & 0.0132 & 0.83 & \pm & 0.08 \\
\hline 1.4220 & 0.1428 & \pm & 0.0022 & 0.1348 & \pm & 0.0135 & 1.06 & \pm & 0.11 \\
\hline 1.4435 & 0.2458 & \pm & 0.0028 & 0.1333 & \pm & 0.0133 & 1.84 & \pm & 0.19 \\
\hline 1.4653 & 0.1736 & \pm & 0.0023 & 0.1170 & \pm & 0.0117 & 1.48 & \pm & 0.15 \\
\hline 1.4843 & 0.0940 & \pm & 0.0019 & 0.1098 & \pm & 0.0110 & 0.86 & \pm & 0.09 \\
\hline 1.5060 & 0.1036 & \pm & 0.0019 & 0.0982 & \pm & 0.0098 & 1.06 & \pm & 0.11 \\
\hline 1.5274 & 0.1012 & \pm & 0.0019 & 0.1016 & \pm & 0.0102 & 1.00 & \pm & 0.10 \\
\hline 1.5475 & 0.1019 & \pm & 0.0019 & 0.1073 & \pm & 0.0107 & 0.95 & \pm & 0.10 \\
\hline 1.5666 & 0.0886 & \pm & 0.0018 & 0.0945 & \pm & 0.0094 & 0.94 & \pm & 0.10 \\
\hline 1.5867 & 0.0983 & \pm & 0.0019 & 0.0944 & \pm & 0.0094 & 1.04 & \pm & 0.11 \\
\hline 1.6056 & 0.0871 & \pm & 0.0018 & 0.0925 & \pm & 0.0093 & 0.94 & \pm & 0.10 \\
\hline 1.6339 & 0.1314 & \pm & 0.0022 & 0.0901 & \pm & 0.0090 & 1.46 & \pm & 0.15 \\
\hline 1.6500 & 0.0736 & \pm & 0.0016 & 0.0888 & \pm & 0.0089 & 0.83 & \pm & 0.08 \\
\hline 1.6731 & 0.1028 & \pm & 0.0019 & 0.0782 & \pm & 0.0078 & 1.31 & \pm & 0.13 \\
\hline 1.6904 & 0.0757 & \pm & 0.0016 & 0.0848 & \pm & 0.0085 & 0.89 & \pm & 0.09 \\
\hline 1.7121 & 0.0903 & \pm & 0.0018 & 0.0796 & \pm & 0.0080 & 1.14 & \pm & 0.12 \\
\hline 1.7358 & 0.1021 & \pm & 0.0019 & 0.0984 & \pm & 0.0098 & 1.04 & \pm & 0.11 \\
\hline 1.7519 & 0.0666 & \pm & 0.0015 & 0.0984 & \pm & 0.0098 & 0.68 & \pm & 0.07 \\
\hline 1.7726 & 0.0809 & \pm & 0.0017 & 0.0775 & \pm & 0.0077 & 1.04 & \pm & 0.11 \\
\hline 1.7953 & 0.0845 & \pm & 0.0017 & 0.0809 & \pm & 0.0081 & 1.05 & \pm & 0.11 \\
\hline 1.8128 & 0.0962 & \pm & 0.0018 & 0.0815 & \pm & 0.0082 & 1.18 & \pm & 0.12 \\
\hline 1.8340 & 0.0766 & \pm & 0.0017 & 0.0909 & \pm & 0.0091 & 0.84 & \pm & 0.09 \\
\hline 1.8558 & 0.0766 & \pm & 0.0017 & 0.0801 & \pm & 0.0080 & 0.96 & \pm & 0.10 \\
\hline 1.8777 & 0.0788 & \pm & 0.0017 & 0.0662 & \pm & 0.0066 & 1.19 & \pm & 0.12 \\
\hline 1.8966 & 0.0669 & \pm & 0.0015 & 0.0643 & \pm & 0.0064 & 1.04 & \pm & 0.11 \\
\hline 1.9177 & 0.0703 & \pm & 0.0016 & 0.0758 & \pm & 0.0076 & 0.93 & \pm & 0.10 \\
\hline 1.9370 & 0.0627 & \pm & 0.0015 & 0.0630 & \pm & 0.0063 & 1.00 & \pm & 0.10 \\
\hline 1.9595 & 0.0724 & \pm & 0.0016 & 0.0705 & \pm & 0.0070 & 1.03 & \pm & 0.11 \\
\hline 1.9758 & 0.0563 & \pm & 0.0014 & 0.0654 & \pm & 0.0065 & 0.86 & \pm & 0.09 \\
\hline 2.0000 & 0.0828 & \pm & 0.0017 & 0.0659 & \pm & 0.0066 & 1.26 & \pm & 0.13 \\
\hline 2.0188 & 0.0598 & \pm & 0.0015 & 0.0663 & \pm & 0.0066 & 0.90 & \pm & 0.09 \\
\hline 2.0406 & 0.0688 & \pm & 0.0016 & 0.0574 & \pm & 0.0057 & 1.20 & \pm & 0.12 \\
\hline 2.0647 & 0.0721 & \pm & 0.0016 & 0.0608 & \pm & 0.0061 & 1.19 & \pm & 0.12 \\
\hline 2.0807 & 0.0487 & \pm & 0.0013 & 0.0625 & \pm & 0.0063 & 0.78 & \pm & 0.08 \\
\hline 2.1010 & 0.0691 & \pm & 0.0016 & 0.0590 & \pm & 0.0059 & 1.17 & \pm & 0.12 \\
\hline
\end{tabular}

Revision: 0 
NEA/NSC/DOC(2006)1

Liquid Metal Fast Reactor - LMFR

FFTF-LMFR-RESR-001

CRIT-SPEC-REAC-COEF-MISC

Table 4.10.1 (cont'd). Calculated FFTF Core Center Low-Energy Gamma-Ray Spectrum.

\begin{tabular}{|c|c|c|c|c|c|c|c|c|c|}
\hline $\begin{array}{l}\text { Energy } \\
(\mathrm{MeV})\end{array}$ & $\begin{array}{c}\text { Calc } \\
\text { Normalized } \\
\text { Flux } \\
\varphi / \varphi_{\max }\end{array}$ & ate & $\sigma$ & $\begin{array}{c}\text { Benc } \\
\text { Normalized } \\
\text { Flux } \\
\varphi / \varphi_{\max }\end{array}$ & $\mathrm{ma}$ & $\sigma$ & $\mathrm{C} / \mathrm{E}$ & \pm & $\sigma$ \\
\hline 2.1211 & 0.0782 & \pm & 0.0016 & 0.0600 & \pm & 0.0060 & 1.30 & \pm & 0.13 \\
\hline 2.1443 & 0.0659 & \pm & 0.0015 & 0.0527 & \pm & 0.0053 & 1.25 & \pm & 0.13 \\
\hline 2.1628 & 0.0538 & \pm & 0.0014 & 0.0583 & \pm & 0.0058 & 0.92 & \pm & 0.10 \\
\hline 2.1821 & 0.0537 & \pm & 0.0014 & 0.0481 & \pm & 0.0048 & 1.12 & \pm & 0.12 \\
\hline 2.2006 & 0.0512 & \pm & 0.0014 & 0.0550 & \pm & 0.0055 & 0.93 & \pm & 0.10 \\
\hline 2.2249 & 0.0661 & \pm & 0.0015 & 0.0530 & \pm & 0.0053 & 1.25 & \pm & 0.13 \\
\hline 2.2426 & 0.0441 & \pm & 0.0013 & 0.0473 & \pm & 0.0047 & 0.93 & \pm & 0.10 \\
\hline 2.2631 & 0.0557 & \pm & 0.0014 & 0.0405 & \pm & 0.0041 & 1.38 & \pm & 0.14 \\
\hline 2.2869 & 0.0716 & \pm & 0.0016 & 0.0483 & \pm & 0.0048 & 1.48 & \pm & 0.15 \\
\hline 2.3068 & 0.0504 & \pm & 0.0014 & 0.0521 & \pm & 0.0052 & 0.97 & \pm & 0.10 \\
\hline 2.3263 & 0.0484 & \pm & 0.0013 & 0.0404 & \pm & 0.0040 & 1.20 & \pm & 0.12 \\
\hline 2.3438 & 0.0423 & \pm & 0.0012 & 0.0388 & \pm & 0.0039 & 1.09 & \pm & 0.11 \\
\hline 2.3838 & 0.0979 & \pm & 0.0019 & 0.0442 & \pm & 0.0044 & 2.22 & \pm & 0.23 \\
\hline 2.4069 & 0.0539 & \pm & 0.0014 & 0.0400 & \pm & 0.0040 & 1.35 & \pm & 0.14 \\
\hline 2.4259 & 0.0436 & \pm & 0.0013 & 0.0361 & \pm & 0.0036 & 1.21 & \pm & 0.13 \\
\hline 2.4463 & 0.0439 & \pm & 0.0013 & 0.0335 & \pm & 0.0034 & 1.31 & \pm & 0.14 \\
\hline 2.4652 & 0.0408 & \pm & 0.0012 & 0.0319 & \pm & 0.0032 & 1.28 & \pm & 0.13 \\
\hline 2.4889 & 0.0484 & \pm & 0.0013 & 0.0393 & \pm & 0.0039 & 1.23 & \pm & 0.13 \\
\hline 2.5092 & 0.0415 & \pm & 0.0012 & 0.0361 & \pm & 0.0036 & 1.15 & \pm & 0.12 \\
\hline 2.5264 & 0.0471 & \pm & 0.0013 & 0.0404 & \pm & 0.0040 & 1.17 & \pm & 0.12 \\
\hline 2.5470 & 0.0418 & \pm & 0.0012 & 0.0333 & \pm & 0.0033 & 1.26 & \pm & 0.13 \\
\hline 2.5659 & 0.0380 & \pm & 0.0012 & 0.0326 & \pm & 0.0033 & 1.17 & \pm & 0.12 \\
\hline 2.5899 & 0.0472 & \pm & 0.0013 & 0.0355 & \pm & 0.0036 & 1.33 & \pm & 0.14 \\
\hline 2.6072 & 0.0432 & \pm & 0.0012 & 0.0215 & \pm & 0.0022 & 2.01 & \pm & 0.21 \\
\hline 2.6304 & 0.0437 & \pm & 0.0013 & 0.0322 & \pm & 0.0032 & 1.36 & \pm & 0.14 \\
\hline 2.6479 & 0.0326 & \pm & 0.0011 & 0.0317 & \pm & 0.0032 & 1.03 & \pm & 0.11 \\
\hline 2.6719 & 0.0446 & \pm & 0.0013 & 0.0341 & \pm & 0.0034 & 1.31 & \pm & 0.14 \\
\hline 2.6913 & 0.0341 & \pm & 0.0011 & 0.0262 & \pm & 0.0026 & 1.30 & \pm & 0.14 \\
\hline 2.7125 & 0.0377 & \pm & 0.0012 & 0.0300 & \pm & 0.0030 & 1.25 & \pm & 0.13 \\
\hline 2.7326 & 0.0312 & \pm & 0.0011 & 0.0305 & \pm & 0.0031 & 1.02 & \pm & 0.11 \\
\hline 2.7515 & 0.0303 & \pm & 0.0011 & 0.0292 & \pm & 0.0029 & 1.04 & \pm & 0.11 \\
\hline 2.7698 & 0.0362 & \pm & 0.0011 & 0.0209 & \pm & 0.0021 & 1.73 & \pm & 0.18 \\
\hline 2.7875 & 0.0248 & \pm & 0.0010 & 0.0185 & \pm & 0.0018 & 1.34 & \pm & 0.14 \\
\hline 2.8124 & 0.0385 & \pm & 0.0012 & 0.0240 & \pm & 0.0024 & 1.61 & \pm & 0.17 \\
\hline 2.8281 & 0.0233 & \pm & 0.0009 & 0.0295 & \pm & 0.0029 & 0.79 & \pm & 0.08 \\
\hline 2.8488 & 0.0309 & \pm & 0.0011 & 0.0227 & \pm & 0.0023 & 1.36 & \pm & 0.14 \\
\hline 2.8735 & 0.0368 & \pm & 0.0012 & 0.0187 & \pm & 0.0019 & 1.97 & \pm & 0.21 \\
\hline 2.8918 & 0.0271 & \pm & 0.0010 & 0.0227 & \pm & 0.0023 & 1.19 & \pm & 0.13 \\
\hline 2.9121 & 0.0284 & \pm & 0.0010 & 0.0216 & \pm & 0.0022 & 1.32 & \pm & 0.14 \\
\hline 2.9336 & 0.0319 & \pm & 0.0011 & 0.0212 & \pm & 0.0021 & 1.51 & \pm & 0.16 \\
\hline 2.9540 & 0.0266 & \pm & 0.0010 & 0.0195 & \pm & 0.0020 & 1.36 & \pm & 0.14 \\
\hline 2.9699 & 0.0199 & \pm & 0.0008 & 0.0217 & \pm & 0.0022 & 0.92 & \pm & 0.10 \\
\hline 2.9928 & 0.0328 & \pm & 0.0011 & 0.0207 & \pm & 0.0021 & 1.58 & \pm & 0.17 \\
\hline
\end{tabular}


NEA/NSC/DOC(2006)1

Liquid Metal Fast Reactor - LMFR

FFTF-LMFR-RESR-001

CRIT-SPEC-REAC-COEF-MISC

Table 4.10.1 (cont'd). Calculated FFTF Core Center Low-Energy Gamma-Ray Spectrum.

\begin{tabular}{|c|c|c|c|c|c|c|c|c|c|}
\hline $\begin{array}{l}\text { Energy } \\
(\mathrm{MeV})\end{array}$ & $\begin{array}{c}\text { Calc } \\
\text { Normalized } \\
\text { Flux } \\
\varphi / \varphi_{\max }\end{array}$ & late & $\sigma$ & $\begin{array}{c}\text { Benc } \\
\text { Normalized } \\
\text { Flux } \\
\varphi / \varphi_{\max }\end{array}$ & $\mathrm{ma}$ & $\sigma$ & $\mathrm{C} / \mathrm{E}$ & \pm & $\sigma$ \\
\hline 3.0307 & 0.0478 & \pm & 0.0013 & 0.0188 & \pm & 0.0019 & 2.55 & \pm & 0.26 \\
\hline 3.0533 & 0.0275 & \pm & 0.0010 & 0.0200 & \pm & 0.0020 & 1.38 & \pm & 0.15 \\
\hline 3.0728 & 0.0195 & \pm & 0.0008 & 0.0154 & \pm & 0.0015 & 1.27 & \pm & 0.14 \\
\hline 3.0953 & 0.0249 & \pm & 0.0010 & 0.0172 & \pm & 0.0017 & 1.44 & \pm & 0.15 \\
\hline 3.1116 & 0.0189 & \pm & 0.0008 & 0.0162 & \pm & 0.0016 & 1.17 & \pm & 0.13 \\
\hline 3.1331 & 0.0223 & \pm & 0.0009 & 0.0162 & \pm & 0.0016 & 1.38 & \pm & 0.15 \\
\hline 3.1524 & 0.0211 & \pm & 0.0009 & 0.0132 & \pm & 0.0013 & 1.60 & \pm & 0.17 \\
\hline 3.1748 & 0.0244 & \pm & 0.0009 & 0.0157 & \pm & 0.0016 & 1.55 & \pm & 0.17 \\
\hline 3.1906 & 0.0149 & \pm & 0.0007 & 0.0183 & \pm & 0.0018 & 0.82 & \pm & 0.09 \\
\hline 3.2148 & 0.0262 & \pm & 0.0010 & 0.0102 & \pm & 0.0010 & 2.57 & \pm & 0.27 \\
\hline 3.2315 & 0.0157 & \pm & 0.0007 & 0.0139 & \pm & 0.0014 & 1.13 & \pm & 0.12 \\
\hline 3.2552 & 0.0253 & \pm & 0.0010 & 0.0172 & \pm & 0.0017 & 1.47 & \pm & 0.16 \\
\hline 3.2749 & 0.0199 & \pm & 0.0008 & 0.0118 & \pm & 0.0012 & 1.68 & \pm & 0.18 \\
\hline 3.2964 & 0.0189 & \pm & 0.0008 & 0.0209 & \pm & 0.0021 & 0.90 & \pm & 0.10 \\
\hline 3.3148 & 0.0158 & \pm & 0.0008 & 0.0141 & \pm & 0.0014 & 1.12 & \pm & 0.13 \\
\hline 3.3378 & 0.0206 & \pm & 0.0009 & 0.0130 & \pm & 0.0013 & 1.58 & \pm & 0.17 \\
\hline 3.3570 & 0.0171 & \pm & 0.0008 & 0.0110 & \pm & 0.0011 & 1.55 & \pm & 0.17 \\
\hline 3.3767 & 0.0179 & \pm & 0.0008 & 0.0133 & \pm & 0.0013 & 1.35 & \pm & 0.15 \\
\hline 3.3988 & 0.0177 & \pm & 0.0008 & 0.0105 & \pm & 0.0010 & 1.68 & \pm & 0.19 \\
\hline 3.4171 & 0.0151 & \pm & 0.0007 & 0.0130 & \pm & 0.0013 & 1.16 & \pm & 0.13 \\
\hline 3.4381 & 0.0162 & \pm & 0.0008 & 0.0090 & \pm & 0.0009 & 1.80 & \pm & 0.20 \\
\hline 3.4569 & 0.0188 & \pm & 0.0008 & 0.0091 & \pm & 0.0009 & 2.08 & \pm & 0.23 \\
\hline 3.4783 & 0.0156 & \pm & 0.0007 & 0.0095 & \pm & 0.0009 & 1.64 & \pm & 0.18 \\
\hline 3.4947 & 0.0108 & \pm & 0.0006 & 0.0150 & \pm & 0.0015 & 0.72 & \pm & 0.08 \\
\hline 3.5161 & 0.0142 & \pm & 0.0007 & 0.0086 & \pm & 0.0009 & 1.66 & \pm & 0.19 \\
\hline 3.5360 & 0.0148 & \pm & 0.0008 & 0.0169 & \pm & 0.0017 & 0.88 & \pm & 0.10 \\
\hline 3.5571 & 0.0197 & \pm & 0.0008 & 0.0114 & \pm & 0.0011 & 1.72 & \pm & 0.19 \\
\hline 3.5770 & 0.0144 & \pm & 0.0007 & 0.0128 & \pm & 0.0013 & 1.12 & \pm & 0.13 \\
\hline 3.5989 & 0.0188 & \pm & 0.0008 & 0.0060 & \pm & 0.0006 & 3.14 & \pm & 0.34 \\
\hline 3.6157 & 0.0175 & \pm & 0.0008 & 0.0081 & \pm & 0.0008 & 2.15 & \pm & 0.23 \\
\hline 3.6369 & 0.0155 & \pm & 0.0008 & 0.0090 & \pm & 0.0009 & 1.73 & \pm & 0.19 \\
\hline 3.6582 & 0.0154 & \pm & 0.0007 & 0.0098 & \pm & 0.0010 & 1.58 & \pm & 0.18 \\
\hline 3.6744 & 0.0112 & \pm & 0.0006 & 0.0096 & \pm & 0.0010 & 1.17 & \pm & 0.13 \\
\hline 3.6978 & 0.0165 & \pm & 0.0008 & 0.0075 & \pm & 0.0008 & 2.20 & \pm & 0.24 \\
\hline 3.7190 & 0.0143 & \pm & 0.0007 & 0.0083 & \pm & 0.0008 & 1.73 & \pm & 0.19 \\
\hline 3.7387 & 0.0109 & \pm & 0.0006 & 0.0105 & \pm & 0.0010 & 1.04 & \pm & 0.12 \\
\hline 3.7611 & 0.0121 & \pm & 0.0007 & 0.0040 & \pm & 0.0004 & 3.03 & \pm & 0.35 \\
\hline 3.7775 & 0.0087 & \pm & 0.0006 & 0.0110 & \pm & 0.0011 & 0.80 & \pm & 0.09 \\
\hline 3.7984 & 0.0128 & \pm & 0.0007 & 0.0081 & \pm & 0.0008 & 1.58 & \pm & 0.18 \\
\hline 3.8169 & 0.0101 & \pm & 0.0006 & 0.0091 & \pm & 0.0009 & 1.11 & \pm & 0.13 \\
\hline 3.8380 & 0.0143 & \pm & 0.0007 & 0.0060 & \pm & 0.0006 & 2.40 & \pm & 0.27 \\
\hline 3.8576 & 0.0102 & \pm & 0.0006 & 0.0078 & \pm & 0.0008 & 1.31 & \pm & 0.15 \\
\hline 3.8801 & 0.0133 & \pm & 0.0007 & 0.0090 & \pm & 0.0009 & 1.47 & \pm & 0.17 \\
\hline
\end{tabular}


NEA/NSC/DOC(2006)1

Liquid Metal Fast Reactor - LMFR

FFTF-LMFR-RESR-001

CRIT-SPEC-REAC-COEF-MISC

Table 4.10.1 (cont'd). Calculated FFTF Core Center Low-Energy Gamma-Ray Spectrum.

\begin{tabular}{|c|c|c|c|c|c|c|c|c|c|}
\hline $\begin{array}{l}\text { Energy } \\
(\mathrm{MeV})\end{array}$ & $\begin{array}{c}\text { Calc } \\
\text { Normalized } \\
\text { Flux } \\
\varphi / \varphi_{\max }\end{array}$ & Iate & $\sigma$ & $\begin{array}{c}\text { Benc } \\
\text { Normalized } \\
\text { Flux } \\
\varphi / \varphi_{\max } \\
\end{array}$ & \pm & $\sigma$ & $\mathrm{C} / \mathrm{E}$ & \pm & $\sigma$ \\
\hline 3.9027 & 0.0127 & 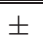 & 0.0007 & 0.0055 & 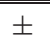 & 0.0006 & 2.30 & \pm & 0.26 \\
\hline 3.9181 & 0.0077 & \pm & 0.0005 & 0.0076 & \pm & 0.0008 & 1.02 & \pm & 0.12 \\
\hline 3.9426 & 0.0131 & \pm & 0.0007 & 0.0037 & \pm & 0.0004 & 3.55 & \pm & 0.40 \\
\hline 3.9607 & 0.0103 & \pm & 0.0006 & 0.0090 & \pm & 0.0009 & 1.14 & \pm & 0.13 \\
\hline 3.9810 & 0.0122 & \pm & 0.0007 & 0.0147 & \pm & 0.0015 & 0.83 & \pm & 0.10 \\
\hline 4.0003 & 0.0161 & \pm & 0.0008 & 0.0067 & \pm & 0.0007 & 2.39 & \pm & 0.26 \\
\hline 4.0183 & 0.0131 & \pm & 0.0007 & 0.0054 & \pm & 0.0005 & 2.43 & \pm & 0.27 \\
\hline 4.0400 & 0.0145 & \pm & 0.0007 & 0.0049 & \pm & 0.0005 & 2.94 & \pm & 0.33 \\
\hline 4.0614 & 0.0156 & \pm & 0.0007 & 0.0051 & \pm & 0.0005 & 3.07 & \pm & 0.34 \\
\hline 4.0793 & 0.0148 & \pm & 0.0007 & 0.0076 & \pm & 0.0008 & 1.94 & \pm & 0.22 \\
\hline 4.1033 & 0.0174 & \pm & 0.0008 & 0.0046 & \pm & 0.0005 & 3.74 & \pm & 0.41 \\
\hline 4.1214 & 0.0121 & \pm & 0.0006 & 0.0061 & \pm & 0.0006 & 1.97 & \pm & 0.22 \\
\hline 4.1373 & 0.0108 & \pm & 0.0006 & 0.0068 & \pm & 0.0007 & 1.58 & \pm & 0.18 \\
\hline 4.1639 & 0.0146 & \pm & 0.0007 & 0.0078 & \pm & 0.0008 & 1.86 & \pm & 0.21 \\
\hline 4.1855 & 0.0090 & \pm & 0.0006 & 0.0041 & \pm & 0.0004 & 2.21 & \pm & 0.26 \\
\hline 4.2021 & 0.0081 & \pm & 0.0006 & 0.0061 & \pm & 0.0006 & 1.33 & \pm & 0.16 \\
\hline 4.2271 & 0.0110 & \pm & 0.0006 & 0.0041 & \pm & 0.0004 & 2.67 & \pm & 0.31 \\
\hline 4.2427 & 0.0061 & \pm & 0.0005 & 0.0052 & \pm & 0.0005 & 1.16 & \pm & 0.15 \\
\hline 4.2641 & 0.0080 & \pm & 0.0005 & 0.0056 & \pm & 0.0006 & 1.43 & \pm & 0.17 \\
\hline 4.2844 & 0.0069 & \pm & 0.0005 & 0.0052 & \pm & 0.0005 & 1.31 & \pm & 0.16 \\
\hline 4.3024 & 0.0046 & \pm & 0.0004 & 0.0072 & \pm & 0.0007 & 0.64 & \pm & 0.08 \\
\hline 4.3225 & 0.0066 & \pm & 0.0005 & 0.0042 & \pm & 0.0004 & 1.57 & \pm & 0.20 \\
\hline 4.3402 & 0.0053 & \pm & 0.0004 & 0.0022 & \pm & 0.0002 & 2.45 & \pm & 0.32 \\
\hline 4.3650 & 0.0094 & \pm & 0.0006 & 0.0052 & \pm & 0.0005 & 1.81 & \pm & 0.21 \\
\hline 4.3817 & 0.0050 & \pm & 0.0004 & 0.0041 & \pm & 0.0004 & 1.23 & \pm & 0.16 \\
\hline 4.4029 & 0.0066 & \pm & 0.0005 & 0.0045 & \pm & 0.0005 & 1.46 & \pm & 0.18 \\
\hline 4.4227 & 0.0065 & \pm & 0.0005 & 0.0095 & \pm & 0.0009 & 0.69 & \pm & 0.09 \\
\hline 4.4464 & 0.0071 & \pm & 0.0005 & 0.0037 & \pm & 0.0004 & 1.91 & \pm & 0.24 \\
\hline 4.4669 & 0.0063 & \pm & 0.0005 & 0.0055 & \pm & 0.0006 & 1.14 & \pm & 0.14 \\
\hline 4.4852 & 0.0057 & \pm & 0.0004 & 0.0040 & \pm & 0.0004 & 1.42 & \pm & 0.18 \\
\hline 4.5057 & 0.0065 & \pm & 0.0005 & 0.0060 & \pm & 0.0006 & 1.08 & \pm & 0.13 \\
\hline 4.5254 & 0.0059 & \pm & 0.0005 & 0.0041 & \pm & 0.0004 & 1.44 & \pm & 0.19 \\
\hline 4.5457 & 0.0052 & \pm & 0.0004 & 0.0021 & \pm & 0.0002 & 2.45 & \pm & 0.32 \\
\hline 4.5653 & 0.0050 & \pm & 0.0004 & 0.0049 & \pm & 0.0005 & 1.03 & \pm & 0.13 \\
\hline 4.5859 & 0.0056 & \pm & 0.0004 & 0.0041 & \pm & 0.0004 & 1.36 & \pm & 0.17 \\
\hline 4.6081 & 0.0064 & \pm & 0.0005 & 0.0030 & \pm & 0.0003 & 2.10 & \pm & 0.27 \\
\hline 4.6262 & 0.0042 & \pm & 0.0004 & 0.0041 & \pm & 0.0004 & 1.05 & \pm & 0.15 \\
\hline
\end{tabular}


NEA/NSC/DOC(2006)1

Liquid Metal Fast Reactor - LMFR

FFTF-LMFR-RESR-001

CRIT-SPEC-REAC-COEF-MISC

Table 4.10.1 (cont'd). Calculated FFTF Core Center Low-Energy Gamma-Ray Spectrum.

\begin{tabular}{|c|c|c|c|c|c|c|c|c|c|}
\hline $\begin{array}{l}\text { Energy } \\
(\mathrm{MeV})\end{array}$ & $\begin{array}{c}\text { Calc } \\
\text { Normalized } \\
\text { Flux } \\
\varphi / \varphi_{\max }\end{array}$ & late & $\sigma$ & $\begin{array}{c}\text { Benc } \\
\text { Normalized } \\
\text { Flux } \\
\varphi / \varphi_{\max }\end{array}$ & \pm & $\sigma$ & $\mathrm{C} / \mathrm{E}$ & \pm & $\sigma$ \\
\hline 4.6468 & 0.0049 & 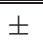 & 0.0004 & 0.0034 & 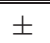 & 0.0003 & 1.46 & \pm & 0.19 \\
\hline 4.6692 & 0.0054 & \pm & 0.0004 & 0.0023 & \pm & 0.0002 & 2.37 & \pm & 0.31 \\
\hline 4.6878 & 0.0050 & \pm & 0.0004 & 0.0045 & \pm & 0.0004 & 1.12 & \pm & 0.15 \\
\hline 4.7064 & 0.0042 & \pm & 0.0004 & 0.0049 & \pm & 0.0005 & 0.86 & \pm & 0.12 \\
\hline 4.7247 & 0.0052 & \pm & 0.0004 & 0.0019 & \pm & 0.0002 & 2.81 & \pm & 0.36 \\
\hline 4.7493 & 0.0051 & \pm & 0.0004 & 0.0028 & \pm & 0.0003 & 1.81 & \pm & 0.23 \\
\hline 4.7654 & 0.0037 & \pm & 0.0004 & 0.0029 & \pm & 0.0003 & 1.28 & \pm & 0.18 \\
\hline 4.7888 & 0.0039 & \pm & 0.0004 & 0.0040 & \pm & 0.0004 & 0.98 & \pm & 0.14 \\
\hline 4.8080 & 0.0034 & \pm & 0.0003 & 0.0060 & \pm & 0.0006 & 0.56 & \pm & 0.08 \\
\hline 4.8324 & 0.0066 & \pm & 0.0005 & 0.0031 & \pm & 0.0003 & 2.12 & \pm & 0.27 \\
\hline 4.8508 & 0.0041 & \pm & 0.0004 & 0.0021 & \pm & 0.0002 & 1.96 & \pm & 0.26 \\
\hline 4.8672 & 0.0028 & \pm & 0.0003 & 0.0057 & \pm & 0.0006 & 0.49 & \pm & 0.07 \\
\hline 4.8890 & 0.0053 & \pm & 0.0004 & 0.0029 & \pm & 0.0003 & 1.85 & \pm & 0.24 \\
\hline 4.9115 & 0.0047 & \pm & 0.0004 & 0.0019 & \pm & 0.0002 & 2.55 & \pm & 0.35 \\
\hline 4.9290 & 0.0027 & \pm & 0.0003 & 0.0057 & \pm & 0.0006 & 0.48 & \pm & 0.07 \\
\hline 4.9514 & 0.0051 & \pm & 0.0004 & 0.0013 & \pm & 0.0001 & 4.07 & \pm & 0.53 \\
\hline 4.9682 & 0.0025 & \pm & 0.0003 & 0.0029 & \pm & 0.0003 & 0.89 & \pm & 0.14 \\
\hline 4.9907 & 0.0042 & \pm & 0.0004 & 0.0019 & \pm & 0.0002 & 2.19 & \pm & 0.30 \\
\hline 5.0089 & 0.0023 & \pm & 0.0003 & 0.0024 & \pm & 0.0002 & 0.93 & \pm & 0.14 \\
\hline 5.0284 & 0.0057 & \pm & 0.0005 & 0.0010 & \pm & 0.0001 & 5.54 & \pm & 0.71 \\
\hline 5.0475 & 0.0034 & \pm & 0.0004 & 0.0051 & \pm & 0.0005 & 0.66 & \pm & 0.10 \\
\hline 5.0674 & 0.0041 & \pm & 0.0004 & 0.0032 & \pm & 0.0003 & 1.28 & \pm & 0.17 \\
\hline 5.0888 & 0.0038 & \pm & 0.0004 & 0.0033 & \pm & 0.0003 & 1.18 & \pm & 0.16 \\
\hline 5.1084 & 0.0036 & \pm & 0.0004 & 0.0042 & \pm & 0.0004 & 0.87 & \pm & 0.13 \\
\hline 5.1323 & 0.0040 & \pm & 0.0004 & 0.0026 & \pm & 0.0003 & 1.53 & \pm & 0.21 \\
\hline 5.1536 & 0.0038 & \pm & 0.0004 & 0.0029 & \pm & 0.0003 & 1.32 & \pm & 0.19 \\
\hline 5.1706 & 0.0025 & \pm & 0.0003 & 0.0036 & \pm & 0.0004 & 0.70 & \pm & 0.11 \\
\hline 5.1893 & 0.0042 & \pm & 0.0004 & 0.0037 & \pm & 0.0004 & 1.14 & \pm & 0.15 \\
\hline 5.2115 & 0.0043 & \pm & 0.0004 & 0.0027 & \pm & 0.0003 & 1.55 & \pm & 0.20 \\
\hline 5.2332 & 0.0049 & \pm & 0.0004 & 0.0025 & \pm & 0.0002 & 1.96 & \pm & 0.26 \\
\hline 5.2510 & 0.0029 & \pm & 0.0003 & 0.0039 & \pm & 0.0004 & 0.76 & \pm & 0.11 \\
\hline 5.2733 & 0.0049 & \pm & 0.0004 & 0.0049 & \pm & 0.0005 & 1.01 & \pm & 0.13 \\
\hline 5.2943 & 0.0038 & \pm & 0.0004 & 0.0035 & \pm & 0.0003 & 1.11 & \pm & 0.15 \\
\hline 5.3115 & 0.0031 & \pm & 0.0003 & 0.0038 & \pm & 0.0004 & 0.79 & \pm & 0.11 \\
\hline 5.3304 & 0.0033 & \pm & 0.0003 & 0.0021 & \pm & 0.0002 & 1.61 & \pm & 0.23 \\
\hline 5.3523 & 0.0032 & \pm & 0.0003 & 0.0030 & \pm & 0.0003 & 1.07 & \pm & 0.15 \\
\hline 5.3702 & 0.0035 & \pm & 0.0004 & 0.0080 & \pm & 0.0008 & 0.44 & \pm & 0.06 \\
\hline
\end{tabular}


NEA/NSC/DOC(2006)1

Liquid Metal Fast Reactor - LMFR

FFTF-LMFR-RESR-001

CRIT-SPEC-REAC-COEF-MISC

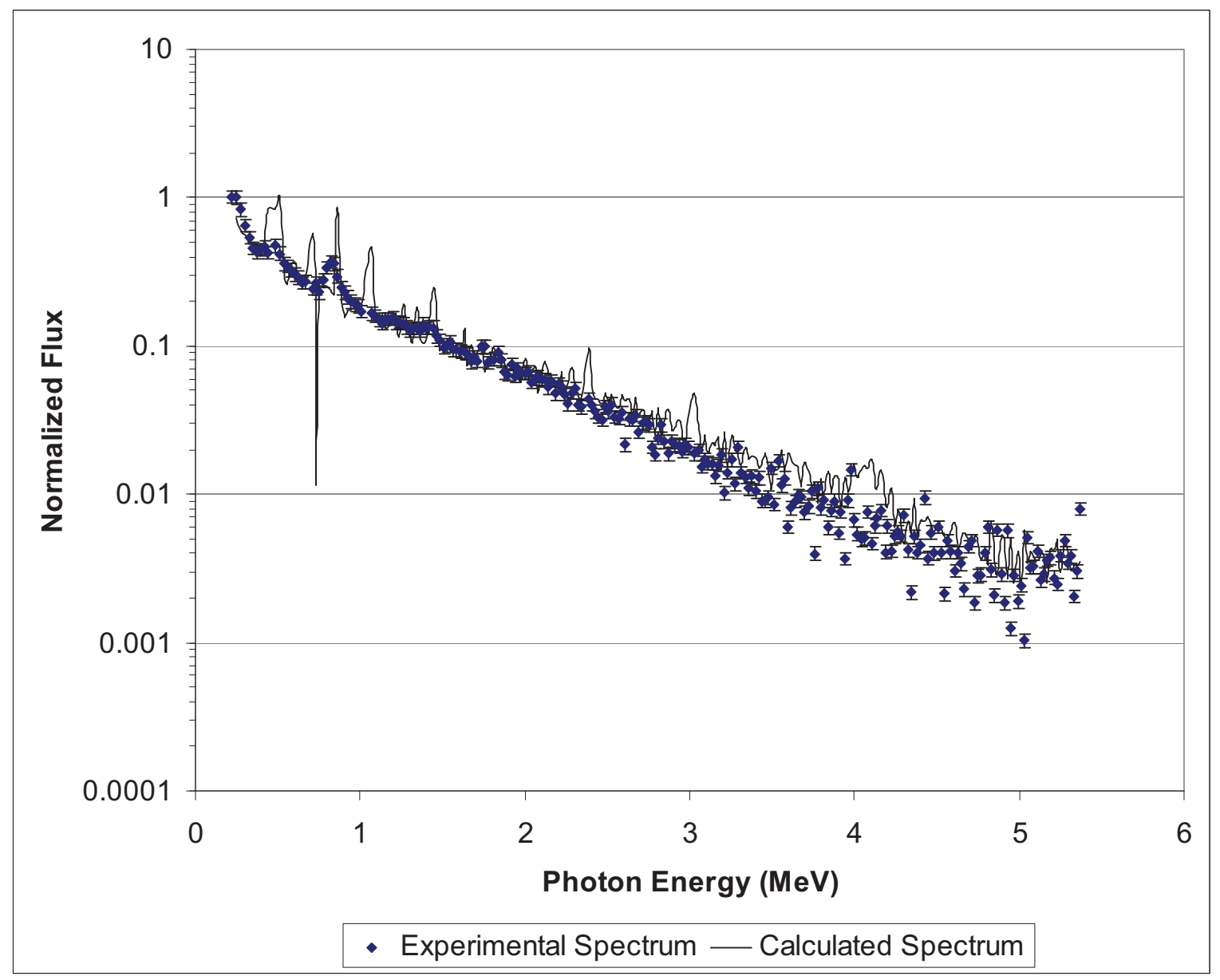

Figure 4.10.1. Low-Energy Gamma-Ray Spectrum in FFTF Core Center. 
NEA/NSC/DOC(2006)1

Liquid Metal Fast Reactor - LMFR

FFTF-LMFR-RESR-001

CRIT-SPEC-REAC-COEF-MISC

\subsection{REFERENCES}

1. R. A. Bennett, J. W. Daughtry, R. A. Harris, D. H. Jones, T. L. King, J. C. Midgett, J. L. Rathbun, R. B. Rothrock, and B. D. Zimmerman, "Initial Physics Measurements on FFTF," HEDL-SA-2163 (CONF-801107-56), Proc. ANS Annual Meeting, Las Vegas, NV, June 8-13 (1980).

2. R. A. Bennett, "Core Engineering Technical Progress Report: January, February, March 1980," HEDL-TME 80-37, (August 1980).

3. R. B. Rothrock, "FFTF Isothermal Physics Tests," TC-2044, (September 1981).

4. P. T. Choong, A. K. Hartman, and J. E. Wieging, "FFTF Cold Physics Analysis," GEFR-00706, (February 1984).

5. C. P. Cabell, "A Summary Description of the Fast Flux Test Facility," HEDL-400, (December 1980).

6. E. F. Bennett and T. J. Yule, "Summary of Neutron Spectrum Measurement Results at the Fast Flux Test Reactor," ZPR-TM-392, (March 1981).

7. R. A. Bennett, D. H. Jones, J. C. Midgett, O. A. Farabee, and T. L. King, "Initial Fuel Load and Criticality, Zero Power and Characterization Testing," HEDL-SA-2033 (CONF-800607-89), Proc. ANS Annual Meeting, Las Vegas, NV, June 8-13 (January 1980).

8. P. A. Ombrellaro, R. A. Harris, J. W. Daughtry, and R. A. Bennett, "Evaluation of MSM for Assessing Subcritical Reactivities in FFTF," HEDL-SA-2178-FP (CONF-801107-56), Proc. ANS Winter Meeting, Washington, D.C., November 17-21 (January 1980).

9. R. B. Rothrock, “Analysis of Initial FTR Zero-Power Physics Tests," HEDL-SA-2608-FP (CONF820609-61), Proc. ANS Annual Meeting, Los Angeles, CA, June 6-11 (May 1982).

10. J. L. Rathbun, R. A. Bennett, J. W. Daughtry, J. C. Midgett, and B. D. Zimmerman, "Initial Fuel Loading and First Criticality of the FFTF," HEDL-TME-82-31, (November 1982). 
NEA/NSC/DOC(2006)1

Liquid Metal Fast Reactor - LMFR

FFTF-LMFR-RESR-001

CRIT-SPEC-REAC-COEF-MISC

\section{APPENDIX A: COMPUTER CODES, CROSS SECTIONS, AND TYPICAL INPUT LISTINGS}

\section{A.1 Critical/Subcritical Configurations}

\section{A.1.1 Name(s) of code system(s) used.}

1. Monte Carlo n-Particle, version 5.1.40 (MCNP5).

2. NJOY-99.296.

\section{A.1.2 Bibliographic references for the codes used.}

1. X-5 Monte Carlo Team, "MCNP - a General Monte Carlo n-Particle Transport Code, version 5," LA-UR-03-1987, Los Alamos National Laboratory (2003).

2. R. E. MacFarlane and D. W. Muir, "The NJOY Nuclear Data Processing System Version 91," LA-12740-M (October 1994).

\section{A.1.3 Origin of Cross Section data.}

The Evaluated Neutron Data File library, ENDF/B-VII.0 $0^{\text {a }}$ processed by NJOY to $480 \mathrm{~K}$.

\section{A.1.4 Spectral calculations and data reduction methods used.}

Not applicable

\section{A.1.5 Number of energy groups or if continuous-energy cross sections are used in the different phases of the calculation.}

Continuous-energy cross sections

\section{A.1.6 Component calculations.}

- Type of cell calculation - Reactor core, reflectors, and shielding

- Geometry - HEX-Z homogenous with heterogeneous fuel and absorber pins

- Theory used - Not applicable

- Method used - Monte Carlo

- Calculation characteristics - histories/cycles/cycles skipped $=100,000 / 1,050 / 50$ continuous-energy cross sections

\section{A.1.7 Other assumptions and characteristics.}

Not applicable

\footnotetext{
${ }^{a}$ M. B. Chadwick, et al., "ENDF/B-VII.0: Next Generation Evaluated Nuclear Data Library for Nuclear Science and Technology," Nucl. Data Sheets, 107: 2931-3060 (2006). 
NEA/NSC/DOC(2006)1

Liquid Metal Fast Reactor - LMFR

FFTF-LMFR-RESR-001

CRIT-SPEC-REAC-COEF-MISC

\section{A.1.8 Typical input listings for each code system type.}

\section{MCNP5 Input Deck for the fully-loaded core configuration:}

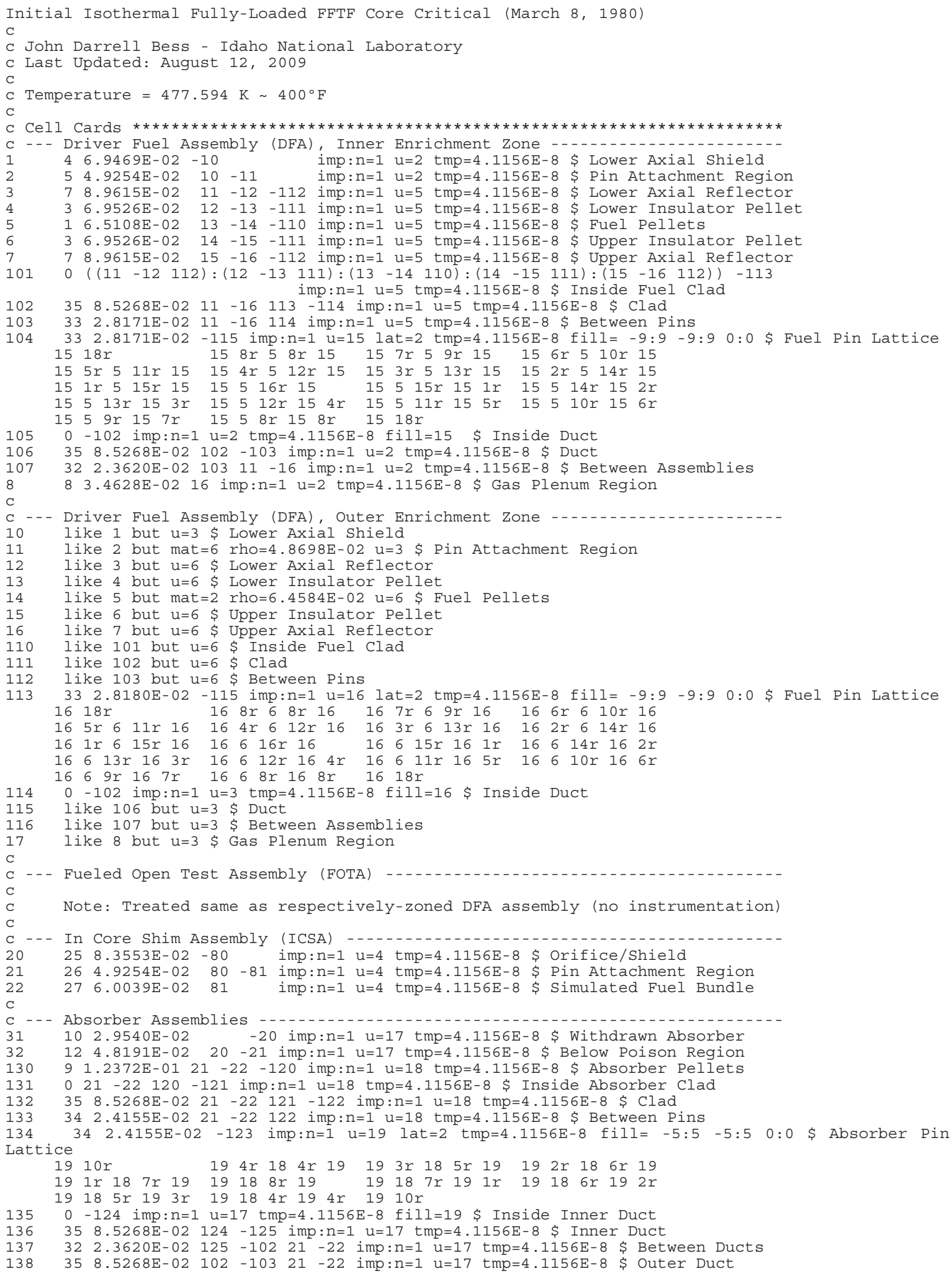

Revision: 0

Page 223 of 304

Date: March 31, 2010 
NEA/NSC/DOC(2006)1

\section{Liquid Metal Fast Reactor - LMFR \\ FFTF-LMFR-RESR-001 \\ CRIT-SPEC-REAC-COEF-MISC}

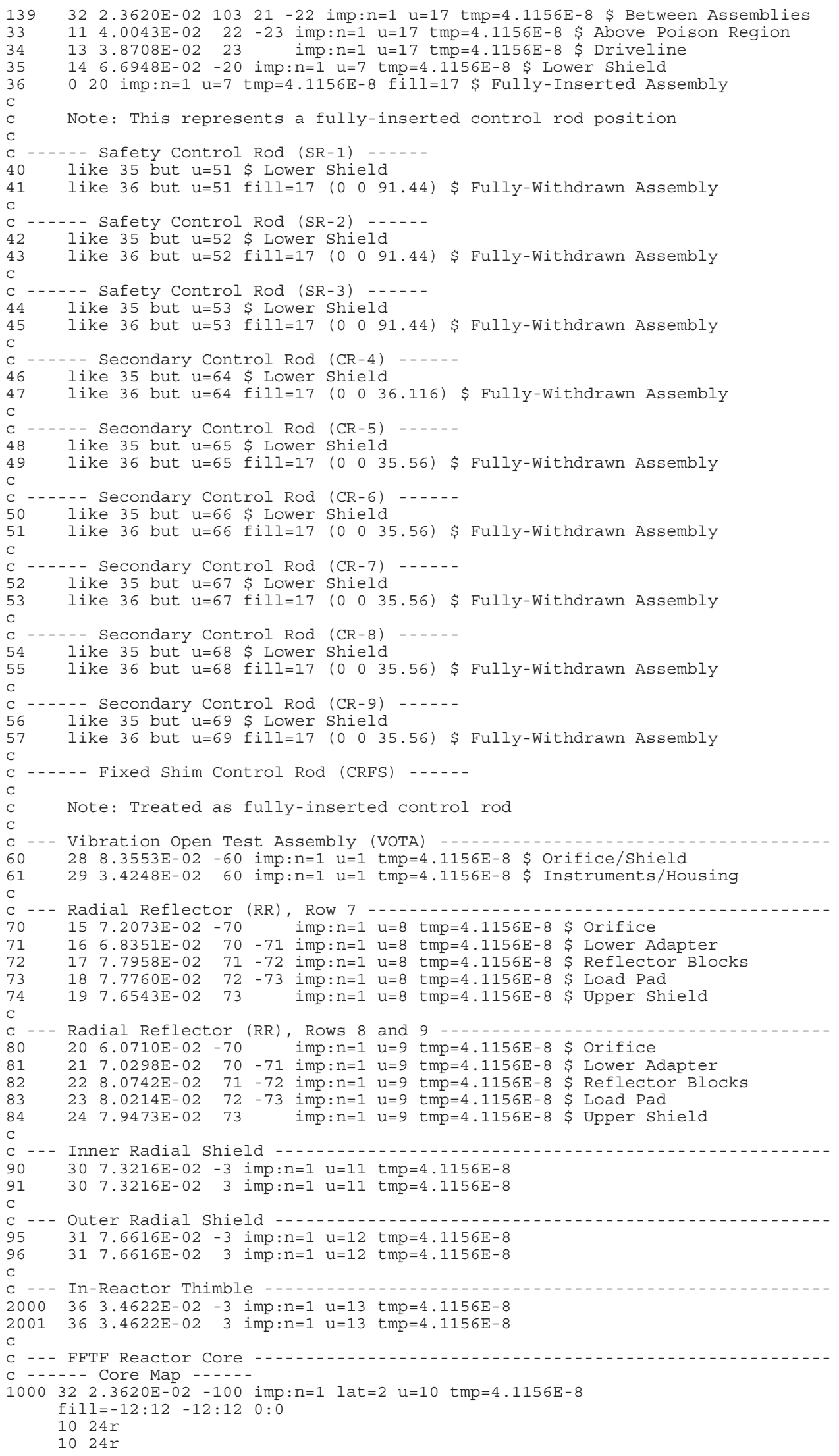

Revision: 0

Page 224 of 304

Date: March 31, 2010 
NEA/NSC/DOC(2006)1

\section{Liquid Metal Fast Reactor - LMFR}

FFTF-LMFR-RESR-001

CRIT-SPEC-REAC-COEF-MISC

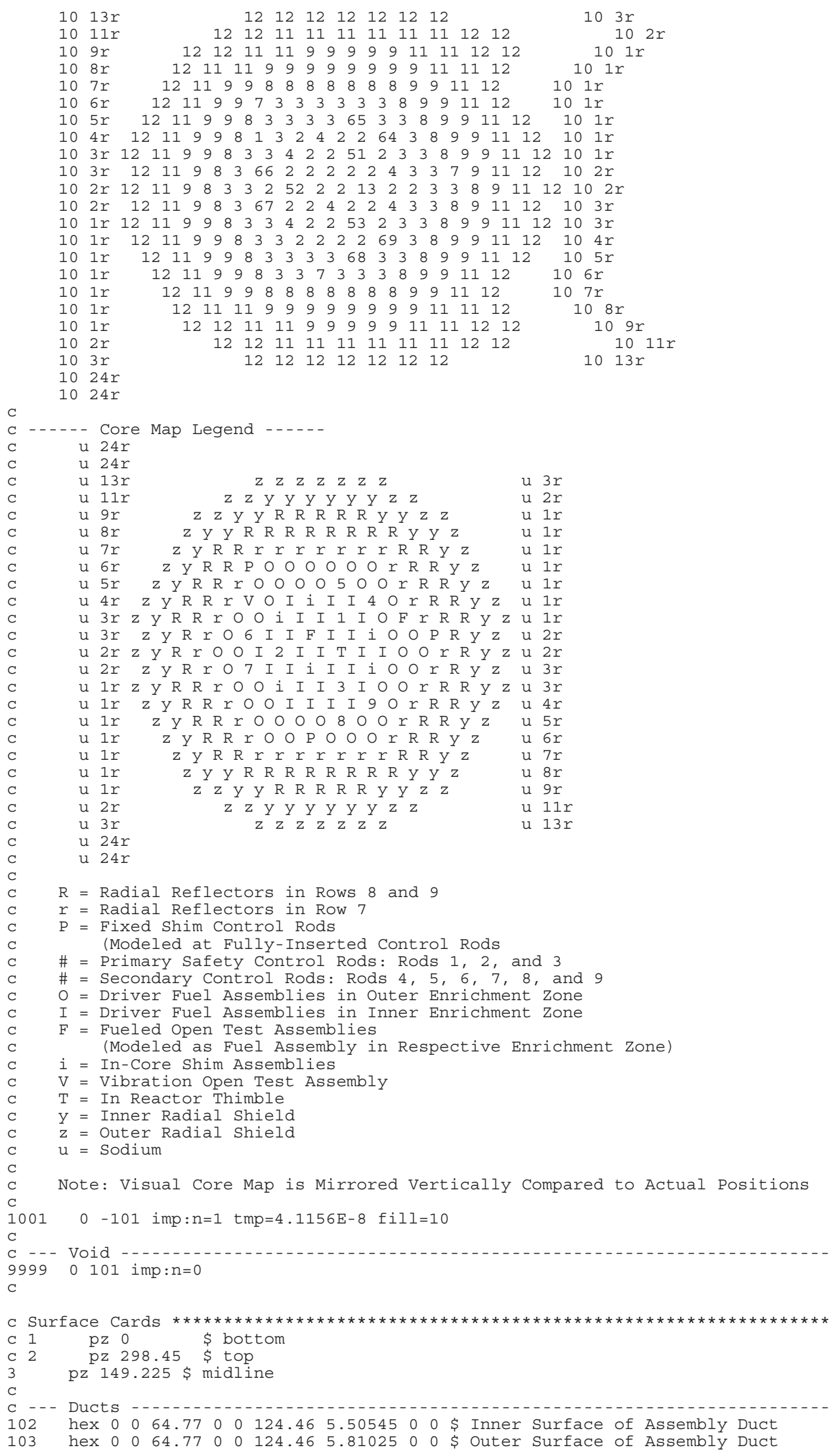

Revision: 0

Page 225 of 304

Date: March 31, 2010 
NEA/NSC/DOC(2006)1

\section{Liquid Metal Fast Reactor - LMFR}

FFTF-LMFR-RESR-001

CRIT-SPEC-REAC-COEF-MISC

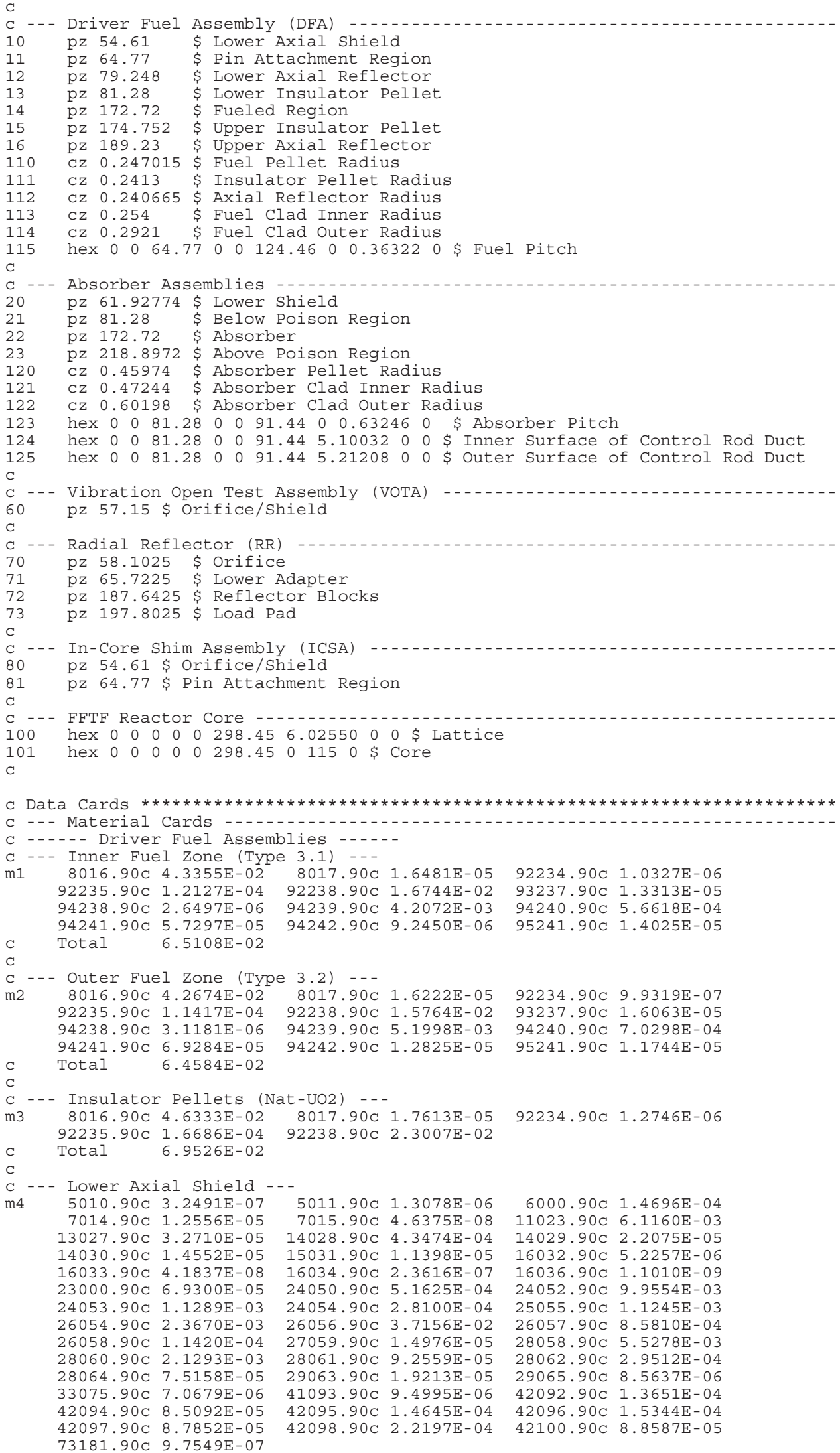

Revision: 0

Page 226 of 304

Date: March 31, 2010 
NEA/NSC/DOC(2006)1

\section{Liquid Metal Fast Reactor - LMFR}

FFTF-LMFR-RESR-001 CRIT-SPEC-REAC-COEF-MISC

\begin{tabular}{|c|c|c|c|c|c|c|}
\hline C & Total & $6.9469 E-02$ & & & & \\
\hline & & & & & & \\
\hline & Pin Atta & chment Regio & (Inner F & uel Zone) -. & & \\
\hline m5 & $5010.90 \mathrm{C}$ & $1.8159 E-07$ & $5011.90 \mathrm{C}$ & $7.3091 \mathrm{E}-07$ & $6000.90 \mathrm{C}$ & $8.2135 E-05$ \\
\hline & $7014.90 \mathrm{C}$ & $7.0171 E-06$ & $7015.90 \mathrm{C}$ & $2.5918 E-08$ & $11023.90 \mathrm{C}$ & $1.3847 E-02$ \\
\hline & $13027.90 \mathrm{C}$ & $1.8281 E-05$ & $14028.90 \mathrm{C}$ & $2.4297 E-04$ & $14029.90 \mathrm{C}$ & $1.2337 \mathrm{E}-05$ \\
\hline & $14030.90 \mathrm{C}$ & $8.1328 E-06$ & $15031.90 \mathrm{C}$ & $6.3699 \mathrm{E}-06$ & $16032.90 \mathrm{C}$ & $2.9206 \mathrm{E}-06$ \\
\hline & $16033.90 \mathrm{C}$ & $2.3382 E-08$ & $16034.90 \mathrm{C}$ & $1.3198 \mathrm{E}-07$ & $16036.90 \mathrm{C}$ & $6.1531 \mathrm{E}-10$ \\
\hline & $23000.90 \mathrm{C}$ & $3.8731 \mathrm{E}-05$ & $24050.90 \mathrm{C}$ & $2.8853 \mathrm{E}-04$ & $24052.90 \mathrm{C}$ & $5.5639 \mathrm{E}-03$ \\
\hline & $24053.90 \mathrm{C}$ & $6.3091 E-04$ & $24054.90 \mathrm{C}$ & $1.5705 \mathrm{E}-04$ & $25055.90 \mathrm{C}$ & $6.2848 E-04$ \\
\hline & $26054.90 \mathrm{C}$ & 1. $3229 \mathrm{E}-03$ & $26056.90 \mathrm{C}$ & $2.0766 \mathrm{E}-02$ & $26057.90 \mathrm{C}$ & $4.7958 E-04$ \\
\hline & $26058.90 \mathrm{C}$ & $6.3823 E-05$ & $27059.90 \mathrm{C}$ & $8.3697 \mathrm{E}-06$ & $28058.90 \mathrm{C}$ & $3.0894 E-03$ \\
\hline & $28060.90 \mathrm{C}$ & $1.1900 \mathrm{E}-03$ & $28061.90 \mathrm{C}$ & $5.1730 \mathrm{E}-05$ & $28062.90 \mathrm{C}$ & $1.6494 \mathrm{E}-04$ \\
\hline & $28064.90 \mathrm{C}$ & $4.2004 \mathrm{E}-05$ & $29063.90 \mathrm{C}$ & $1.0738 \mathrm{E}-05$ & $29065.90 \mathrm{C}$ & $4.7861 \mathrm{E}-06$ \\
\hline & $33075.90 \mathrm{C}$ & $3.9501 E-06$ & $41093.90 \mathrm{C}$ & $5.3091 E-06$ & $42092.90 \mathrm{C}$ & $7.6296 \mathrm{E}-05$ \\
\hline & $42094.90 \mathrm{C}$ & $4.7556 E-05$ & $42095.90 \mathrm{C}$ & $8.1849 E-05$ & $42096.90 \mathrm{C}$ & $8.5756 \mathrm{E}-05$ \\
\hline & $42097.90 \mathrm{C}$ & $4.9099 \mathrm{E}-05$ & $42098.90 \mathrm{C}$ & $1.2406 \mathrm{E}-04$ & $42100.90 \mathrm{C}$ & $4.9510 \mathrm{E}-05$ \\
\hline & $73181.90 \mathrm{C}$ & $5.4519 \mathrm{E}-07$ & & & & \\
\hline c & Total & $4.9254 \mathrm{E}-02$ & & & & \\
\hline c & & & & & & \\
\hline & Pin Atta & chment Regio & (Outer Fr & uel Zone) & & \\
\hline m6 & $5010.90 \mathrm{C}$ & $1.7764 E-07$ & $5011.90 \mathrm{C}$ & $7.1504 \mathrm{E}-07$ & $6000.90 \mathrm{c}$ & $8.0351 E-05$ \\
\hline & $7014.90 \mathrm{C}$ & $6.8647 E-06$ & $7015.90 \mathrm{C}$ & $2.5356 \mathrm{E}-08$ & $11023.90 \mathrm{C}$ & $1.4060 \mathrm{E}-02$ \\
\hline & $13027.90 \mathrm{C}$ & $1.7884 E-05$ & $14028.90 \mathrm{C}$ & $2.3769 E-04$ & $14029.90 \mathrm{C}$ & $1.2069 E-05$ \\
\hline & $14030.90 \mathrm{C}$ & $7.9562 E-06$ & $15031.90 \mathrm{C}$ & $6.2316 \mathrm{E}-06$ & $16032.90 \mathrm{C}$ & $2.8572 \mathrm{E}-06$ \\
\hline & $16033.90 \mathrm{C}$ & $2.2874 E-08$ & $16034.90 \mathrm{C}$ & $1.2912 \mathrm{E}-07$ & $16036.90 \mathrm{C}$ & $6.0195 \mathrm{E}-10$ \\
\hline & $23000.90 \mathrm{C}$ & $3.7890 \mathrm{E}-05$ & $24050.90 \mathrm{C}$ & $2.8226 \mathrm{E}-04$ & $24052.90 \mathrm{C}$ & $5.4431 \mathrm{E}-03$ \\
\hline & $24053.90 \mathrm{C}$ & $6.1720 E-04$ & $24054.90 \mathrm{C}$ & $1.5364 \mathrm{E}-04$ & $25055.90 \mathrm{C}$ & $6.1483 E-04$ \\
\hline & $26054.90 \mathrm{C}$ & 1.2941E-03 & $26056.90 \mathrm{C}$ & $2.0315 E-02$ & $26057.90 \mathrm{C}$ & $4.6916 \mathrm{E}-04$ \\
\hline & $26058.90 \mathrm{C}$ & $6.2437 E-05$ & $27059.90 \mathrm{C}$ & $8.1879 E-06$ & $28058.90 \mathrm{C}$ & $3.0223 E-03$ \\
\hline & $28060.90 \mathrm{C}$ & $1.1642 E-03$ & $28061.90 \mathrm{C}$ & $5.0606 \mathrm{E}-05$ & $28062.90 \mathrm{C}$ & $1.6135 \mathrm{E}-04$ \\
\hline & $28064.90 \mathrm{C}$ & $4.1092 E-05$ & $29063.90 \mathrm{C}$ & $1.0505 \mathrm{E}-05$ & $29065.90 \mathrm{C}$ & $4.6822 E-06$ \\
\hline & $33075.90 \mathrm{C}$ & $3.8643 E-06$ & $41093.90 \mathrm{C}$ & $5.1938 E-06$ & $42092.90 \mathrm{C}$ & $7.4639 \mathrm{E}-05$ \\
\hline & $42094.90 \mathrm{C}$ & $4.6524 E-05$ & $42095.90 \mathrm{C}$ & $8.0071 E-05$ & $42096.90 \mathrm{C}$ & $8.3893 E-05$ \\
\hline & $42097.90 \mathrm{C}$ & $4.8032 E-05$ & $42098.90 \mathrm{C}$ & $1.2136 \mathrm{E}-04$ & $42100.90 \mathrm{C}$ & $4.8435 E-05$ \\
\hline & $73181.90 \mathrm{C}$ & $5.3334 E-07$ & & & & \\
\hline C & Total & $4.8698 E-02$ & & & & \\
\hline $\mathrm{C}$ & & & & & & \\
\hline & Axial R & flectors (In & conel 600) & --- & & \\
\hline $\mathrm{m} 7$ & $6000.90 \mathrm{C}$ & $2.0908 E-03$ & $14028.90 \mathrm{C}$ & $4.1233 E-04$ & $14029.90 \mathrm{C}$ & $2.0937 E-05$ \\
\hline & $14030.90 \mathrm{C}$ & $1.3802 \mathrm{E}-05$ & $16032.90 \mathrm{C}$ & $1.1152 \mathrm{E}-05$ & $16033.90 \mathrm{C}$ & $8.9281 E-08$ \\
\hline & $16034.90 \mathrm{C}$ & $5.0397 E-07$ & $16036.90 \mathrm{C}$ & $2.3495 E-09$ & $24050.90 \mathrm{C}$ & $6.5053 E-04$ \\
\hline & $24052.90 \mathrm{C}$ & 1.2545E-02 & $24053.90 \mathrm{C}$ & $1.4225 E-03$ & $24054.90 \mathrm{C}$ & $3.5408 E-04$ \\
\hline & $25055.90 \mathrm{C}$ & $1.5998 \mathrm{E}-03$ & $26054.90 \mathrm{C}$ & $4.2054 \mathrm{E}-04$ & $26056.90 \mathrm{C}$ & $6.6015 E-03$ \\
\hline & $26057.90 \mathrm{C}$ & $1.5246 \mathrm{E}-04$ & $26058.90 \mathrm{C}$ & $2.0289 E-05$ & $27059.90 \mathrm{C}$ & $8.5222 \mathrm{E}-05$ \\
\hline & $28058.90 \mathrm{C}$ & $4.2900 \mathrm{E}-02$ & $28060.90 \mathrm{C}$ & $1.6525 \mathrm{E}-02$ & $28061.90 \mathrm{C}$ & $7.1832 E-04$ \\
\hline & $28062.90 \mathrm{C}$ & $2.2903 E-03$ & $28064.90 \mathrm{C}$ & $5.8328 \mathrm{E}-04$ & $29063.90 \mathrm{C}$ & $1.3667 E-04$ \\
\hline & $29065.90 \mathrm{C}$ & $6.0917 E-05$ & & & & \\
\hline C & Total & $8.9615 E-02$ & & & & \\
\hline $\mathrm{C}$ & & & & & & \\
\hline & Gas Plen & um --- & & & & \\
\hline m8 & $5010.90 \mathrm{C}$ & 1.2816E-07 & $5011.90 \mathrm{C}$ & $5.1585 \mathrm{E}-07$ & $6000.90 \mathrm{c}$ & $5.7968 \mathrm{E}-05$ \\
\hline & $7014.90 \mathrm{C}$ & $4.9525 E-06$ & $7015.90 \mathrm{C}$ & $1.8292 \mathrm{E}-08$ & $3.90 \mathrm{C}$ & $E-03$ \\
\hline & $13027.90 \mathrm{C}$ & $1.2902 \mathrm{E}-05$ & $14028.90 \mathrm{C}$ & $1.7148 \mathrm{E}-04$ & $14029.90 \mathrm{C}$ & $72 E-06$ \\
\hline & $14030.90 \mathrm{C}$ & $5.7399 \mathrm{E}-06$ & $15031.90 \mathrm{C}$ & $4.4957 \mathrm{E}-06$ & $16032.90 \mathrm{C}$ & $2.0612 E-06$ \\
\hline & $16033.90 \mathrm{C}$ & $1.6502 E-08$ & $16034.90 \mathrm{C}$ & $9.3150 \mathrm{E}-08$ & $16036.90 \mathrm{C}$ & $4.3427 \mathrm{E}-10$ \\
\hline & $23000.90 \mathrm{C}$ & $2.7335 E-05$ & $24050.90 \mathrm{C}$ & $2.0363 \mathrm{E}-04$ & $24052.90 \mathrm{C}$ & $3.9268 \mathrm{E}-03$ \\
\hline & $24053.90 \mathrm{C}$ & $4.4527 E-04$ & $24054.90 \mathrm{C}$ & $1.1084 \mathrm{E}-04$ & $25055.90 \mathrm{C}$ & $4.4356 \mathrm{E}-04$ \\
\hline & $26054.90 \mathrm{C}$ & $9.3362 E-04$ & $26056.90 \mathrm{C}$ & $1.4656 \mathrm{E}-02$ & $26057.90 \mathrm{C}$ & $3.3847 \mathrm{E}-04$ \\
\hline & $26058.90 \mathrm{C}$ & $4.5044 E-05$ & $27059.90 \mathrm{C}$ & $5.9070 E-06$ & $28058.90 \mathrm{C}$ & $2.1804 \mathrm{E}-03$ \\
\hline & $28060.90 \mathrm{C}$ & $8.3988 E-04$ & $28061.90 \mathrm{C}$ & $3.6509 E-05$ & $28062.90 \mathrm{C}$ & $1.1641 \mathrm{E}-04$ \\
\hline & $28064.90 \mathrm{C}$ & $2.9645 E-05$ & $29063.90 \mathrm{C}$ & $7.5786 \mathrm{E}-06$ & $29065.90 \mathrm{C}$ & $3.3779 E-06$ \\
\hline & $33075.90 \mathrm{C}$ & $2.7879 E-06$ & $41093.90 \mathrm{C}$ & $3.7470 E-06$ & $42092.90 \mathrm{C}$ & $7 E-05$ \\
\hline & $42094.90 \mathrm{C}$ & $3.3564 E-05$ & $42095.90 \mathrm{C}$ & $5.7766 \mathrm{E}-05$ & $42096.90 \mathrm{C}$ & $3 E-05$ \\
\hline & $42097.90 \mathrm{C}$ & $3.4652 \mathrm{E}-05$ & $42098.90 \mathrm{C}$ & $8.7556 \mathrm{E}-05$ & $42100.90 \mathrm{C}$ & $3.4942 \mathrm{E}-05$ \\
\hline & $73181.90 \mathrm{C}$ & $3.8477 E-07$ & & & & \\
\hline C & Total & $3.4628 \mathrm{E}-02$ & & & & \\
\hline C & & & & & & \\
\hline & - - Absol & ber Assembli & ----- & & & \\
\hline & Boron $\mathrm{Ca}$ & rbide (B4C) & --- & & & \\
\hline m9 & $5010.90 \mathrm{C}$ & 1.9979E-02 & $5011.90 \mathrm{C}$ & $8.0418 \mathrm{E}-02$ & $6000.90 \mathrm{C}$ & $2.3327 \mathrm{E}-02$ \\
\hline C & Total & $1.2372 \mathrm{E}-01$ & & & & \\
\hline C & & & & & & \\
\hline & Tith & n Absorber & & & & \\
\hline $\mathrm{m} 10$ & $5010.90 \mathrm{C}$ & $4.1900 \mathrm{E}-08$ & $5011.90 \mathrm{C}$ & $1.6865 \mathrm{E}-07$ & 6000 & $1.8952 \mathrm{E}-05$ \\
\hline & $7014.90 \mathrm{C}$ & $1.6192 \mathrm{E}-06$ & $7015.90 \mathrm{C}$ & $5.9806 E-09$ & 11023 & $2.1370 \mathrm{E}-02$ \\
\hline & $13027.90 \mathrm{C}$ & $4.2183 E-06$ & $14028.90 \mathrm{C}$ & $5.6064 \mathrm{E}-05$ & $14029.90 \mathrm{C}$ & $2.8468 E-06$ \\
\hline & $14030.90 \mathrm{C}$ & $1.8766 \mathrm{E}-06$ & $15031.90 \mathrm{C}$ & $1.4698 \mathrm{E}-06$ & $16032.90 \mathrm{C}$ & $6.7391 \mathrm{E}-07$ \\
\hline & $16033.90 \mathrm{C}$ & $5.3953 E-09$ & $16034.90 \mathrm{C}$ & $3.0455 E-08$ & $16036.90 \mathrm{C}$ & $1.4198 \mathrm{E}-10$ \\
\hline & $23000.90 \mathrm{C}$ & $8.9369 E-06$ & $24050.90 \mathrm{C}$ & $6.6576 \mathrm{E}-05$ & $24052.90 \mathrm{C}$ & $1.2839 \mathrm{E}-03$ \\
\hline & $24053.90 \mathrm{C}$ & $1.4558 \mathrm{E}-04$ & $24054.90 \mathrm{C}$ & $3.6238 E-05$ & $25055.90 \mathrm{C}$ & 1. $4502 \mathrm{E}-04$ \\
\hline & $26054.90 \mathrm{C}$ & $3.0524 E-04$ & $26056.90 \mathrm{C}$ & $4.7916 \mathrm{E}-03$ & $26057.90 \mathrm{C}$ & $1.1066 \mathrm{E}-04$ \\
\hline & $26058.90 \mathrm{C}$ & $1.4727 E-05$ & $27059.90 \mathrm{C}$ & $1.9313 \mathrm{E}-06$ & $28058.90 \mathrm{C}$ & $7.1286 \mathrm{E}-04$ \\
\hline & & & 280 & 05 & 28062 & 3. \\
\hline
\end{tabular}

Revision: 0

Page 227 of 304

Date: March 31, 2010 
NEA/NSC/DOC(2006)1

Liquid Metal Fast Reactor - LMFR

FFTF-LMFR-RESR-001 CRIT-SPEC-REAC-COEF-MISC

\begin{tabular}{|c|c|c|c|c|c|c|}
\hline & $28064.90 \mathrm{C}$ & $9.6923 \mathrm{E}-06$ & $29063.90 \mathrm{C}$ & $2.4778 \mathrm{E}-06$ & $29065.90 \mathrm{C}$ & $1.1044 E-06$ \\
\hline & $33075.90 \mathrm{C}$ & $9.1148 \mathrm{E}-07$ & $41093.90 \mathrm{C}$ & $1.2251 \mathrm{E}-06$ & $42092.90 \mathrm{C}$ & $1.7605 \mathrm{E}-05$ \\
\hline & $42094.90 \mathrm{C}$ & $1.0973 \mathrm{E}-05$ & $42095.90 \mathrm{C}$ & $1.8886 \mathrm{E}-05$ & $42096.90 \mathrm{C}$ & $1.9788 \mathrm{E}-05$ \\
\hline & $42097.90 \mathrm{C}$ & $1.1329 \mathrm{E}-05$ & $42098.90 \mathrm{C}$ & $2.8626 \mathrm{E}-05$ & $42100.90 \mathrm{C}$ & $1.1424 \mathrm{E}-05$ \\
\hline & $73181.90 \mathrm{C}$ & 1. $2580 \mathrm{E}-07$ & & & & \\
\hline C & Total & $2.9540 \mathrm{E}-02$ & & & & \\
\hline & & & & & & \\
\hline & Above-P & son Region & -- & & & \\
\hline $\mathrm{m} 11$ & $5010.90 \mathrm{C}$ & $1.6616 \mathrm{E}-07$ & $5011.90 \mathrm{C}$ & $6.6882 \mathrm{E}-07$ & $6000.90 \mathrm{C}$ & $7.5157 \mathrm{E}-05$ \\
\hline & $7014.90 \mathrm{C}$ & $6.4210 \mathrm{E}-06$ & $7015.90 \mathrm{C}$ & $2.3717 \mathrm{E}-08$ & $11023.90 \mathrm{C}$ & $7.6440 E-03$ \\
\hline & $13027.90 \mathrm{C}$ & $1.6728 \mathrm{E}-05$ & $14028.90 \mathrm{C}$ & $2.2233 E-04$ & $14029.90 \mathrm{C}$ & $1.1289 \mathrm{E}-05$ \\
\hline & $14030.90 \mathrm{C}$ & $7.4419 \mathrm{E}-06$ & $15031.90 \mathrm{C}$ & $5.8288 \mathrm{E}-06$ & $16032.90 \mathrm{C}$ & $2.6725 E-06$ \\
\hline & $16033.90 \mathrm{C}$ & $2.1396 \mathrm{E}-08$ & $16034.90 \mathrm{C}$ & $1.2077 \mathrm{E}-07$ & $16036.90 \mathrm{C}$ & $5.6304 \mathrm{E}-10$ \\
\hline & $23000.90 \mathrm{C}$ & $3.5440 \mathrm{E}-05$ & $24050.90 \mathrm{C}$ & $2.6401 \mathrm{E}-04$ & $24052.90 \mathrm{C}$ & $5.0913 E-03$ \\
\hline & $24053.90 \mathrm{C}$ & $5.7731 \mathrm{E}-04$ & $24054.90 \mathrm{C}$ & $1.4370 \mathrm{E}-04$ & $25055.90 \mathrm{C}$ & $5.7509 E-04$ \\
\hline & $26054.90 \mathrm{C}$ & $1.2105 \mathrm{E}-03$ & $26056.90 \mathrm{C}$ & $1.9002 \mathrm{E}-02$ & $26057.90 \mathrm{C}$ & $4.3883 E-04$ \\
\hline & $26058.90 \mathrm{C}$ & $5.8401 \mathrm{E}-05$ & $27059.90 \mathrm{C}$ & $7.6586 \mathrm{E}-06$ & $28058.90 \mathrm{C}$ & $2.8269 E-03$ \\
\hline & $28060.90 \mathrm{C}$ & $1.0889 \mathrm{E}-03$ & $28061.90 \mathrm{C}$ & $4.7335 \mathrm{E}-05$ & $28062.90 \mathrm{C}$ & $1.5092 \mathrm{E}-04$ \\
\hline & $28064.90 \mathrm{C}$ & $3.8436 \mathrm{E}-05$ & $29063.90 \mathrm{C}$ & $9.8258 \mathrm{E}-06$ & $29065.90 \mathrm{C}$ & $4.3795 E-06$ \\
\hline & $33075.90 \mathrm{C}$ & $3.6146 \mathrm{E}-06$ & $41093.90 \mathrm{C}$ & $4.8581 E-06$ & $42092.90 \mathrm{C}$ & $6.9814 E-05$ \\
\hline & $42094.90 \mathrm{C}$ & $4.3516 \mathrm{E}-05$ & $42095.90 \mathrm{C}$ & $7.4895 \mathrm{E}-05$ & $42096.90 \mathrm{C}$ & $7.8471 E-05$ \\
\hline & $42097.90 \mathrm{C}$ & $4.4928 \mathrm{E}-05$ & $42098.90 \mathrm{C}$ & $1.1352 \mathrm{E}-04$ & $42100.90 \mathrm{C}$ & $4.5304 E-05$ \\
\hline & $73181.90 \mathrm{C}$ & $4.9887 \mathrm{E}-07$ & & & & \\
\hline C & Total & $4.0043 E-02$ & & & & \\
\hline C & & & & & & \\
\hline & Below-Po & ison Region & --- & & & \\
\hline $\mathrm{m} 12$ & $5010.90 \mathrm{C}$ & $1.7405 \mathrm{E}-07$ & $5011.90 \mathrm{C}$ & $7.0057 \mathrm{E}-07$ & $6000.90 \mathrm{C}$ & $7.8725 E-05$ \\
\hline & $7014.90 \mathrm{C}$ & $6.7258 \mathrm{E}-06$ & $7015.90 \mathrm{C}$ & $2.4842 \mathrm{E}-08$ & $11023.90 \mathrm{C}$ & $1.4254 \mathrm{E}-02$ \\
\hline & $13027.90 \mathrm{C}$ & 1. $7522 \mathrm{E}-05$ & $14028.90 \mathrm{C}$ & $2.3288 E-04$ & $14029.90 \mathrm{C}$ & $1.1825 \mathrm{E}-05$ \\
\hline & $14030.90 \mathrm{C}$ & $7.7952 \mathrm{E}-06$ & $15031.90 \mathrm{C}$ & $6.1055 \mathrm{E}-06$ & $16032.90 \mathrm{C}$ & $2.7993 E-06$ \\
\hline & $16033.90 \mathrm{C}$ & $2.2411 \mathrm{E}-08$ & $16034.90 \mathrm{C}$ & 1.2651E-07 & $16036.90 \mathrm{C}$ & $5.8977 \mathrm{E}-10$ \\
\hline & $23000.90 \mathrm{C}$ & $3.7123 \mathrm{E}-05$ & $24050.90 \mathrm{C}$ & $2.7655 \mathrm{E}-04$ & $24052.90 \mathrm{C}$ & $5.3329 E-03$ \\
\hline & $24053.90 \mathrm{C}$ & $6.0471 \mathrm{E}-04$ & $24054.90 \mathrm{C}$ & $1.5053 \mathrm{E}-04$ & $25055.90 \mathrm{C}$ & $6.0239 E-04$ \\
\hline & $26054.90 \mathrm{C}$ & $1.2679 \mathrm{E}-03$ & $26056.90 \mathrm{C}$ & $1.9904 \mathrm{E}-02$ & $26057.90 \mathrm{C}$ & $4.5967 E-04$ \\
\hline & $26058.90 \mathrm{C}$ & $6.1173 E-05$ & $27059.90 \mathrm{C}$ & $8.0222 \mathrm{E}-06$ & $28058.90 \mathrm{C}$ & $2.9611 \mathrm{E}-03$ \\
\hline & $28060.90 \mathrm{C}$ & $1.1406 \mathrm{E}-03$ & $28061.90 \mathrm{C}$ & $4.9582 \mathrm{E}-05$ & $28062.90 \mathrm{C}$ & 1. $5809 \mathrm{E}-04$ \\
\hline & $28064.90 \mathrm{C}$ & $4.0261 E-05$ & $29063.90 \mathrm{C}$ & $1.0292 \mathrm{E}-05$ & $29065.90 \mathrm{C}$ & $4.5874 \mathrm{E}-06$ \\
\hline & $33075.90 \mathrm{C}$ & $3.7861 \mathrm{E}-06$ & $41093.90 \mathrm{C}$ & $5.0887 \mathrm{E}-06$ & $42092.90 \mathrm{C}$ & $7.3128 E-05$ \\
\hline & $42094.90 \mathrm{C}$ & $4.5582 \mathrm{E}-05$ & $42095.90 \mathrm{C}$ & $7.8450 \mathrm{E}-05$ & $42096.90 \mathrm{C}$ & $8.2196 \mathrm{E}-05$ \\
\hline & $42097.90 \mathrm{C}$ & $4.7060 \mathrm{E}-05$ & $42098.90 \mathrm{C}$ & $1.1891 \mathrm{E}-04$ & $42100.90 \mathrm{C}$ & $4.7455 E-05$ \\
\hline & $73181.90 \mathrm{C}$ & $5.2255 \mathrm{E}-07$ & & & & \\
\hline C & Total & $4.8191 \mathrm{E}-02$ & & & & \\
\hline C & & & & & & \\
\hline & Dri & --- & & & & \\
\hline $\mathrm{m} 13$ & $5010.90 \mathrm{C}$ & $1.0681 \mathrm{E}-07$ & $5011.90 \mathrm{C}$ & $4.2993 E-07$ & $6000.90 \mathrm{C}$ & $4.8313 E-05$ \\
\hline & $7014.90 \mathrm{C}$ & $4.1276 \mathrm{E}-06$ & $7015.90 \mathrm{C}$ & $1.5246 \mathrm{E}-08$ & $11023.90 \mathrm{C}$ & $1.7881 \mathrm{E}-02$ \\
\hline & $13027.90 \mathrm{C}$ & $1.0753 E-05$ & $14028.90 \mathrm{C}$ & 1. $4292 \mathrm{E}-04$ & $14029.90 \mathrm{C}$ & $7.2570 \mathrm{E}-06$ \\
\hline & $14030.90 \mathrm{C}$ & $4.7839 \mathrm{E}-06$ & $15031.90 \mathrm{C}$ & $3.7469 \mathrm{E}-06$ & $16032.90 \mathrm{C}$ & 1.7179E-06 \\
\hline & $16033.90 \mathrm{C}$ & $1.3754 \mathrm{E}-08$ & $16034.90 \mathrm{C}$ & $7.7636 \mathrm{E}-08$ & $16036.90 \mathrm{C}$ & $3.6194 E-10$ \\
\hline & $23000.90 \mathrm{C}$ & $2.2782 \mathrm{E}-05$ & $24050.90 \mathrm{C}$ & $1.6972 \mathrm{E}-04$ & $24052.90 \mathrm{C}$ & $3.2728 E-03$ \\
\hline & $24053.90 \mathrm{C}$ & 3.7111E-04 & $24054.90 \mathrm{C}$ & $9.2377 \mathrm{E}-05$ & $25055.90 \mathrm{C}$ & $3.6968 E-04$ \\
\hline & $26054.90 \mathrm{C}$ & 7. $7812 \mathrm{E}-04$ & $26056.90 \mathrm{C}$ & 1.2215E-02 & $26057.90 \mathrm{C}$ & $2.8210 E-04$ \\
\hline & $26058.90 \mathrm{C}$ & $3.7542 \mathrm{E}-05$ & $27059.90 \mathrm{C}$ & $4.9232 \mathrm{E}-06$ & $28058.90 \mathrm{C}$ & $1.8172 \mathrm{E}-03$ \\
\hline & $28060.90 \mathrm{C}$ & $6.9999 \mathrm{E}-04$ & $28061.90 \mathrm{C}$ & $3.0428 \mathrm{E}-05$ & $28062.90 \mathrm{C}$ & $9.7018 \mathrm{E}-05$ \\
\hline & $28064.90 \mathrm{C}$ & $2.4708 \mathrm{E}-05$ & $29063.90 \mathrm{C}$ & $6.3163 \mathrm{E}-06$ & $29065.90 \mathrm{C}$ & $2.8153 E-06$ \\
\hline & $33075.90 \mathrm{C}$ & $2.3235 E-06$ & $41093.90 \mathrm{C}$ & $3.1229 \mathrm{E}-06$ & $42092.90 \mathrm{C}$ & $4.4879 E-05$ \\
\hline & $42094.90 \mathrm{C}$ & $2.7974 \mathrm{E}-05$ & $42095.90 \mathrm{C}$ & $4.8145 \mathrm{E}-05$ & $42096.90 \mathrm{C}$ & $5.0443 E-05$ \\
\hline & $42097.90 \mathrm{C}$ & $2.8881 \mathrm{E}-05$ & $42098.90 \mathrm{C}$ & $7.2973 E-05$ & $42100.90 \mathrm{C}$ & $2.9123 E-05$ \\
\hline & $73181.90 \mathrm{C}$ & $3.2069 \mathrm{E}-07$ & & & & \\
\hline C & Total & $3.8708 \mathrm{E}-02$ & & & & \\
\hline C & & & & & & \\
\hline & Lower Sh & Leld --- & & & & \\
\hline m14 & $5010.90 \mathrm{C}$ & $3.0703 \mathrm{E}-07$ & $5011.90 \mathrm{C}$ & $1.2358 \mathrm{E}-06$ & $6000.90 \mathrm{c}$ & 1. $3888 E-04$ \\
\hline & 14 & $1.1865 \mathrm{E}$ & 7015 & $4.3824 \mathrm{E}$ & $11023.90 \mathrm{C}$ & $7.0810 \mathrm{E}-03$ \\
\hline & $13027.90 \mathrm{C}$ & $3.0910 \mathrm{E}-05$ & $14028.90 \mathrm{C}$ & $4.1081 \mathrm{E}-04$ & $14029.90 \mathrm{C}$ & $2.0860 \mathrm{E}-05$ \\
\hline & $14030.90 \mathrm{C}$ & $1.3751 \mathrm{E}-05$ & $15031.90 \mathrm{C}$ & $1.0770 \mathrm{E}-05$ & $16032.90 \mathrm{C}$ & $4.9382 E-06$ \\
\hline & $16033.90 \mathrm{C}$ & $3.9535 \mathrm{E}-08$ & $16034.90 \mathrm{C}$ & $2.2316 \mathrm{E}-07$ & $16036.90 \mathrm{C}$ & 1.0404E-09 \\
\hline & $23000.90 \mathrm{C}$ & $6.5487 \mathrm{E}-05$ & $24050.90 \mathrm{C}$ & $4.8785 \mathrm{E}-04$ & $24052.90 \mathrm{C}$ & $9.4076 \mathrm{E}-03$ \\
\hline & $24053.90 \mathrm{C}$ & $1.0668 \mathrm{E}-03$ & $24054.90 \mathrm{C}$ & $2.6554 \mathrm{E}-04$ & $25055.90 \mathrm{C}$ & $1.0627 \mathrm{E}-03$ \\
\hline & $26054.90 \mathrm{C}$ & $2.2367 \mathrm{E}-03$ & $26056.90 \mathrm{C}$ & 3. $5112 \mathrm{E}-02$ & $26057.90 \mathrm{C}$ & $8.1088 E-04$ \\
\hline & $26058.90 \mathrm{C}$ & 1.0791E-04 & $27059.90 \mathrm{C}$ & $1.4152 \mathrm{E}-05$ & $28058.90 \mathrm{C}$ & $5.2236 E-03$ \\
\hline & 28060 & $2.0121 \mathrm{E}-03$ & $28061.90 \mathrm{C}$ & $8.7466 \mathrm{E}-05$ & $28062.90 \mathrm{C}$ & $2.7888 E-04$ \\
\hline & 28064 . & $7.1022 \mathrm{E}-05$ & $29063.90 \mathrm{C}$ & $1.8156 \mathrm{E}-05$ & $29065.90 \mathrm{C}$ & $8.0925 E-06$ \\
\hline & $33075.90 \mathrm{C}$ & $6.6790 \mathrm{E}-06$ & $41093.90 \mathrm{C}$ & $8.9768 \mathrm{E}-06$ & $42092.90 \mathrm{C}$ & 1.2900E-04 \\
\hline & $42094.90 \mathrm{C}$ & $8.0410 E-05$ & $42095.90 \mathrm{C}$ & $1.3839 \mathrm{E}-04$ & $42096.90 \mathrm{C}$ & 1. $4500 \mathrm{E}-04$ \\
\hline & $42097.90 \mathrm{C}$ & $8.3018 E-05$ & $42098.90 \mathrm{C}$ & $2.0976 \mathrm{E}-04$ & $42100.90 \mathrm{C}$ & $8.3713 E-05$ \\
\hline & 73181. & $9.2181 \mathrm{E}-07$ & & & & \\
\hline C & Total & $6.6948 \mathrm{E}-02$ & & & & \\
\hline C & & & & & & \\
\hline & dic & Reflect & Row 7 & & & \\
\hline & & Region --- & & & & \\
\hline m15 & $5010.90 \mathrm{C}$ & $3.4337 \mathrm{E}-07$ & $5011.90 \mathrm{C}$ & $1.3821 \mathrm{E}-06$ & $6000.90 \mathrm{c}$ & 1.5531E-04 \\
\hline & $7014.90 \mathrm{C}$ & $1.3269 \mathrm{E}-05$ & $7015.90 \mathrm{C}$ & $4.9010 \mathrm{E}-08$ & $11023.90 \mathrm{C}$ & $5.1210 \mathrm{E}-03$ \\
\hline & $13027.90 \mathrm{C}$ & $3.4568 \mathrm{E}-05$ & $14028.90 \mathrm{C}$ & $4.5943 E-04$ & $14029.90 \mathrm{C}$ & $2.3329 E-05$ \\
\hline
\end{tabular}

Revision: 0

Page 228 of 304

Date: March 31, 2010 
NEA/NSC/DOC(2006)1

\section{Liquid Metal Fast Reactor - LMFR}

FFTF-LMFR-RESR-001 CRIT-SPEC-REAC-COEF-MISC

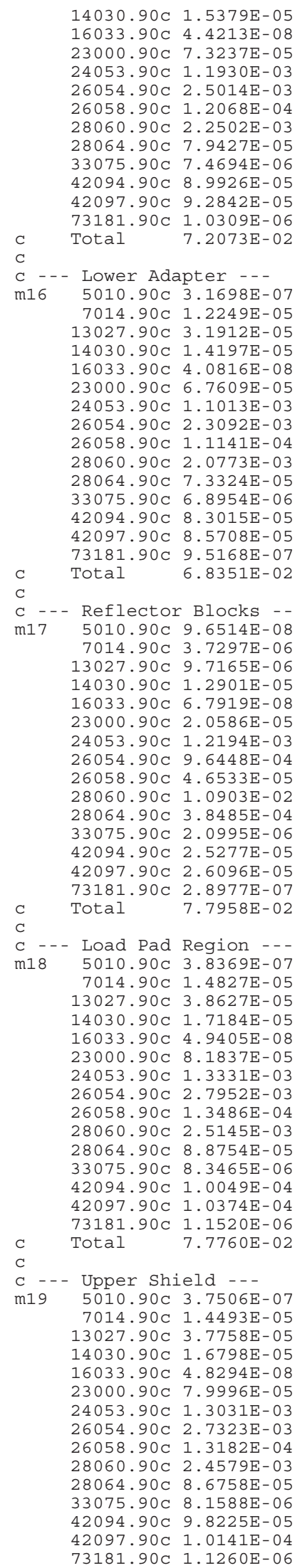

$14030.90 \mathrm{C} \quad 1.5379 \mathrm{E}-05$ $24053.90 \mathrm{C} 1.1930 \mathrm{E}-03$ $26054.90 \mathrm{C} 2.5014 \mathrm{E}-03$ 26058.90C $1.2068 \mathrm{E}-04$ $28064.90 \mathrm{C} 7.9427 \mathrm{E}-05$ $33075.90 \mathrm{C} 7.4694 \mathrm{E}-06$ $9.2842 \mathrm{E}-05$ . $3027.90 \mathrm{C} 3.1912 \mathrm{E}-05$ $14030.90 \mathrm{C} 1.4197 \mathrm{E}-05$ $23000.90 \mathrm{C} 6.7609 \mathrm{E}-05$ $4053.90 \mathrm{C} 1.1013 \mathrm{E}-03$ . $28060.90 \mathrm{C} 2.0773 \mathrm{E}-03$ $7.3324 \mathrm{E}-05$ $15 \mathrm{E}-05$ $9.5168 \mathrm{E}-07$

Reflector Blocks - 5010.90C 9.6514E-08 $3014.90 \mathrm{C} 3.7297 \mathrm{E}-06$ $14030.90 \mathrm{C} 1.2901 \mathrm{E}-05$ $033.90 \mathrm{C} 6.7919 \mathrm{E}-08$ $4053.90 \mathrm{C} 1.2194 \mathrm{E}-03$ $6054.90 \mathrm{C} 9.6448 \mathrm{E}-04$ $26058.90 \mathrm{C} 4.6533 \mathrm{E}-05$ $28064.90 \mathrm{C} 3.8485 \mathrm{E}-04$ $33075.90 \mathrm{C} 2.0995 \mathrm{E}-06$ $94.90 \mathrm{C} 2.5277 \mathrm{E}-05$ $97.90 \mathrm{C} 2.6096 \mathrm{E}-05$ $3181.90 \mathrm{C} 2.8977 \mathrm{E}-07$ Load Pad Region -$5010.90 \mathrm{C} 3.8369 \mathrm{E}-07$ $7014.90 \mathrm{C} \quad 1.4827 \mathrm{E}-05$ $4030.90 \mathrm{C} 1.7184 \mathrm{E}-05$ . $4033.90 \mathrm{C} 4.9405 \mathrm{E}-08$ $3000.90 \mathrm{C} 8.1837 \mathrm{E}-05$ $4053.90 \mathrm{C} 1.3331 \mathrm{E}-03$ $6058.90 \mathrm{C} 2.7952 \mathrm{E}-03$ $28060.90 \mathrm{C} 2.5145 \mathrm{E}-03$ $28064.90 \mathrm{C} 8.8754 \mathrm{E}-05$ $3075.90 \mathrm{C} 8.3465 \mathrm{E}-06$ $2097.90 \mathrm{C} 1.0374 \mathrm{E}-04$ $73181.90 \mathrm{C} 1.1520 \mathrm{E}-06$ Upper Shield -.$7010.90 \mathrm{C} 3.7506 \mathrm{E}-07$ $4.90 \mathrm{C} 1.4493 \mathrm{E}-05$ $14030.90 \mathrm{C} 1.6798 \mathrm{E}-05$ $16033.90 \mathrm{C} 4.8294 \mathrm{E}-08$ $0.90 \mathrm{C} 7.9996 \mathrm{E}-05$ $0 \mathrm{C} 1.3031 \mathrm{E}-03$ $54.90 \mathrm{C} 2.7323 \mathrm{E}-03$ $28060.90 \mathrm{C} 2.4579 \mathrm{E}-03$ 8064.90 $8.6758 \mathrm{E}-05$ $42094.90 \mathrm{C} 9.8225 \mathrm{E}-05$ $73181.90 \mathrm{C} 1.1260 \mathrm{E}-06$

$5011.90 \mathrm{C} \quad 3.8848 \mathrm{E}-07$ $7015.90 \mathrm{C} \quad 1.3776 \mathrm{E}-08$ $14028.90 \mathrm{C} \quad 3.8541 \mathrm{E}-04$ $15031.90 \mathrm{C} 3.3856 \mathrm{E}-06$ $16034.90 \mathrm{C} 3.8338 \mathrm{E}-07$ $24050.90 \mathrm{C} 5.5768 \mathrm{E}-04$ $24054.90 \mathrm{C} 3.0355 \mathrm{E}-04$ $26056.90 \mathrm{C}$ 1.5140E-02 $27059.90 \mathrm{C} 5.7417 \mathrm{E}-05$ $28061.90 \mathrm{C} 4.7396 \mathrm{E}-04$ $29063.90 \mathrm{C} 9.0654 \mathrm{E}-05$ $41093.90 \mathrm{C} 2.8218 \mathrm{E}-06$ $42095.90 \mathrm{C} 4.3503 \mathrm{E}-05$ $42098.90 \mathrm{C} 6.5938 \mathrm{E}-05$

$5011.90 \mathrm{C} \quad 1.5444 \mathrm{E}-06$ $7015.90 \mathrm{C} 5.4765 \mathrm{E}-08$ $14028.90 \mathrm{C} \quad 5.1338 \mathrm{E}-04$ $15031.90 \mathrm{C} 1.3459 \mathrm{E}-05$ $16034.90 \mathrm{C} 2.7888 \mathrm{E}-07$ $24050.90 \mathrm{C} \quad 6.0965 \mathrm{E}-04$ $24054.90 \mathrm{C} \quad 3.3183 \mathrm{E}-04$ $26056.90 \mathrm{C} 4.3878 \mathrm{E}-02$ $27059.90 \mathrm{C} 1.7685 \mathrm{E}-05$ $28061.90 \mathrm{C} 1.0930 \mathrm{E}-04$ $29063.90 \mathrm{C} 2.2689 \mathrm{E}-05$ $41093.90 \mathrm{C} 1.1218 \mathrm{E}-05$ $42095.90 \mathrm{C} 1.7294 \mathrm{E}-04$ $42098.90 \mathrm{C} 2.6213 \mathrm{E}-04$

$5011.90 \mathrm{C} 1.5097 \mathrm{E}-06$ $7015.90 \mathrm{C} 5.3533 \mathrm{E}-08$ $14028.90 \mathrm{C} 5.0183 \mathrm{E}-04$ $15031.90 \mathrm{C} 1.3157 \mathrm{E}-05$ $16034.90 \mathrm{C} 2.7261 \mathrm{E}-07$ $24050.90 \mathrm{C} 5.9593 \mathrm{E}-04$ $24054.90 \mathrm{C} \quad 3.2437 \mathrm{E}-04$ $26056.90 \mathrm{C} 4.2891 \mathrm{E}-02$ $27059.90 \mathrm{C} 1.7287 \mathrm{E}-05$ $28061.90 \mathrm{C} 1.0684 \mathrm{E}-04$ $29063.90 \mathrm{C} 2.2179 \mathrm{E}-05$ $41093.90 \mathrm{C} 1.0966 \mathrm{E}-05$ $42095.90 \mathrm{C} 1.6905 \mathrm{E}-04$ $42098.90 \mathrm{C} \quad 2.5623 \mathrm{E}-04$
$16032.90 \mathrm{C} \quad 5.5226 \mathrm{E}-06$ $16036.90 \mathrm{C} 1.1635 \mathrm{E}-09$ $24052.90 \mathrm{C} 1.0521 \mathrm{E}-02$ $25055.90 \mathrm{C} 1.1884 \mathrm{E}-03$ $26057.90 \mathrm{C} 9.0684 \mathrm{E}-04$ $28058.90 \mathrm{C} 5.8418 \mathrm{E}-03$ $28062.90 \mathrm{C} 3.1188 \mathrm{E}-04$ $29065.90 \mathrm{C} 9.0502 \mathrm{E}-06$ $42092.90 \mathrm{C} \quad 1.4427 \mathrm{E}-04$ $42096.90 \mathrm{C} 1.6216 \mathrm{E}-04$ $42100.90 \mathrm{C} 9.3620 \mathrm{E}-05$

$6000.90 \mathrm{C} 1.4338 \mathrm{E}-04$ $11023.90 \mathrm{C} \quad 6.5440 \mathrm{E}-03$ $14029.90 \mathrm{C} 2.1536 \mathrm{E}-05$ $16032.90 \mathrm{C} 5.0982 \mathrm{E}-06$ $16036.90 \mathrm{C} 1.0741 \mathrm{E}-09$ $24052.90 \mathrm{C} 9.7125 \mathrm{E}-03$ $25055.90 \mathrm{C} 1.0971 \mathrm{E}-03$ $26057.90 \mathrm{C} 8.3716 \mathrm{E}-04$ $28058.90 \mathrm{C} 5.3929 \mathrm{E}-03$ $28062.90 \mathrm{C} 2.8792 \mathrm{E}-04$ $29065.90 \mathrm{C} 8.3547 \mathrm{E}-06$ $42092.90 \mathrm{C} 1.3318 \mathrm{E}-04$ $42096.90 \mathrm{C} 1.4970 \mathrm{E}-04$ $42100.90 \mathrm{C} 8.6426 \mathrm{E}-05$

$6000.90 \mathrm{C} \quad 1.3432 \mathrm{E}-03$ $11023.90 \mathrm{C} 3.4400 \mathrm{E}-03$ $14029.90 \mathrm{C} 1.9570 \mathrm{E}-05$ $16032.90 \mathrm{C} 8.4836 \mathrm{E}-06$ $16036.90 \mathrm{C} 1.7873 \mathrm{E}-09$ $24052.90 \mathrm{C} 1.0754 \mathrm{E}-02$ $25055.90 \mathrm{C} 1.3284 \mathrm{E}-03$ $26057.90 \mathrm{C} 3.4966 \mathrm{E}-04$ $28058.90 \mathrm{C} 2.8306 \mathrm{E}-02$ $28062.90 \mathrm{C} 1.5112 \mathrm{E}-03$ $29065.90 \mathrm{C} 4.0406 \mathrm{E}-05$ $42092.90 \mathrm{C} 4.0552 \mathrm{E}-05$ $42096.90 \mathrm{C} 4.5580 \mathrm{E}-05$ $42100.90 \mathrm{C} 2.6315 \mathrm{E}-05$

$6000.90 \mathrm{C} \quad 1.7355 \mathrm{E}-04$ $11023.90 \mathrm{C} 2.9460 \mathrm{E}-03$ $14029.90 \mathrm{C} 2.6068 \mathrm{E}-05$ $16032.90 \mathrm{C} 6.1711 \mathrm{E}-06$ $16036.90 \mathrm{C} 1.3001 \mathrm{E}-09$ $24052.90 \mathrm{C} 1.1756 \mathrm{E}-02$ $25055.90 \mathrm{C} 1.3280 \mathrm{E}-03$ $26057.90 \mathrm{C} 1.0133 \mathrm{E}-03$ $28058.90 \mathrm{C} 6.5278 \mathrm{E}-03$ $28062.90 \mathrm{C} 3.4851 \mathrm{E}-04$ $29065.90 \mathrm{C} 1.0113 \mathrm{E}-05$ $42092.90 \mathrm{C} 1.6121 \mathrm{E}-04$ $42096.90 \mathrm{C} 1.8120 \mathrm{E}-04$ $42100.90 \mathrm{C} 1.0461 \mathrm{E}-04$

$6000.90 \mathrm{C} 1.6965 \mathrm{E}-04$ $11023.90 \mathrm{C} 3.4120 \mathrm{E}-03$ $14029.90 \mathrm{C} 2.5482 \mathrm{E}-05$ $16032.90 \mathrm{C} 6.0323 \mathrm{E}-06$ $16036.90 \mathrm{C} 1.2709 \mathrm{E}-09$ $24052.90 \mathrm{C} 1.1492 \mathrm{E}-02$ $25055.90 \mathrm{C} 1.2981 \mathrm{E}-03$ $26057.90 \mathrm{C} 9.9054 \mathrm{E}-04$ $28058.90 \mathrm{C} 6.3809 \mathrm{E}-03$ $28062.90 \mathrm{C} 3.4067 \mathrm{E}-04$ $29065.90 \mathrm{C} 9.8854 \mathrm{E}-06$ $42092.90 \mathrm{C} \quad 1.5758 \mathrm{E}-04$ $42096.90 \mathrm{C} 1.7712 \mathrm{E}-04$ $42100.90 \mathrm{C} 1.0226 \mathrm{E}-04$

Revision: 0

Page 229 of 304

Date: March 31, 2010 
NEA/NSC/DOC(2006)1

\section{Liquid Metal Fast Reactor - LMFR}

FFTF-LMFR-RESR-001 CRIT-SPEC-REAC-COEF-MISC

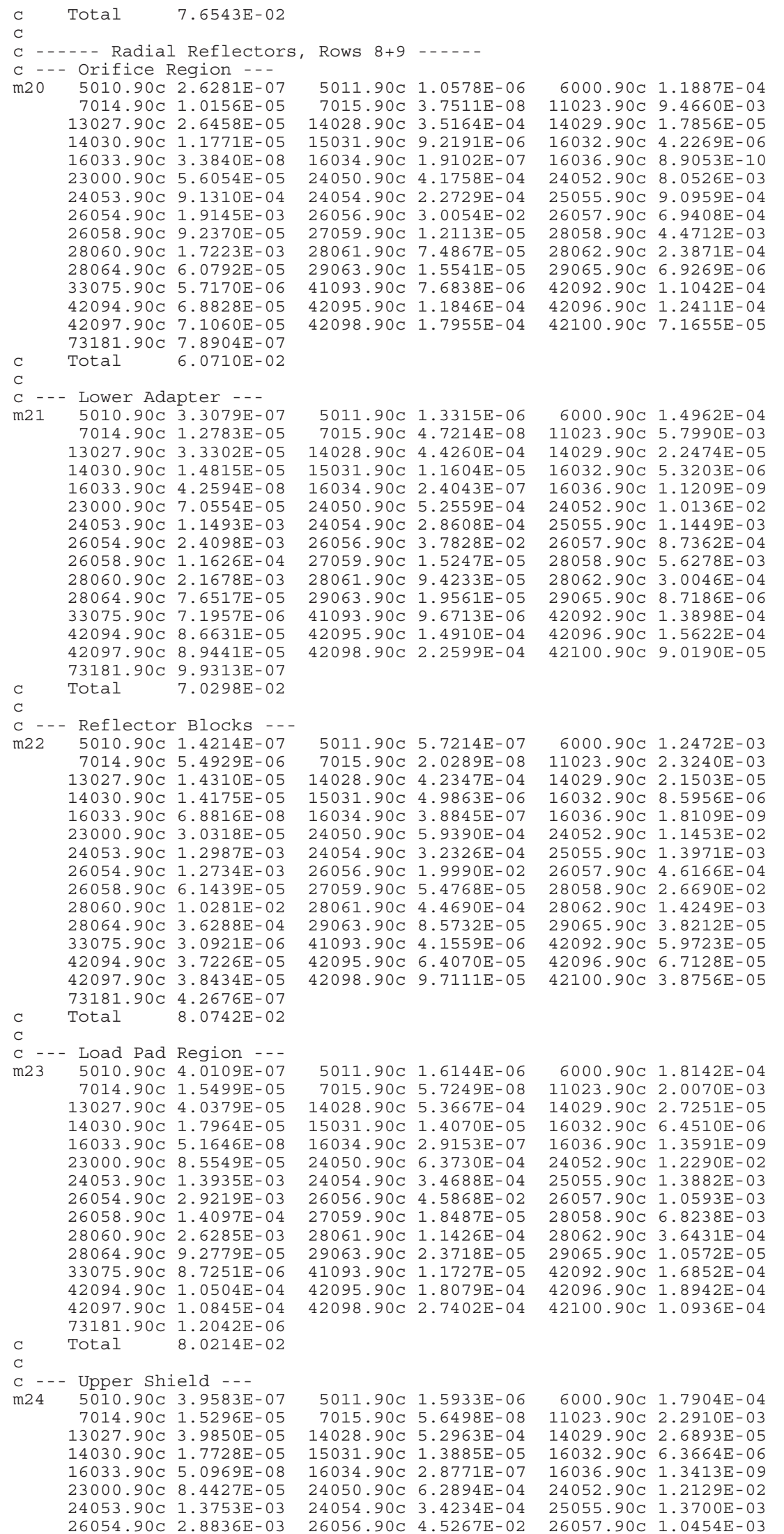

Revision: 0

Page 230 of 304

Date: March 31, 2010 
NEA/NSC/DOC(2006)1

\section{Liquid Metal Fast Reactor - LMFR}

FFTF-LMFR-RESR-001 CRIT-SPEC-REAC-COEF-MISC

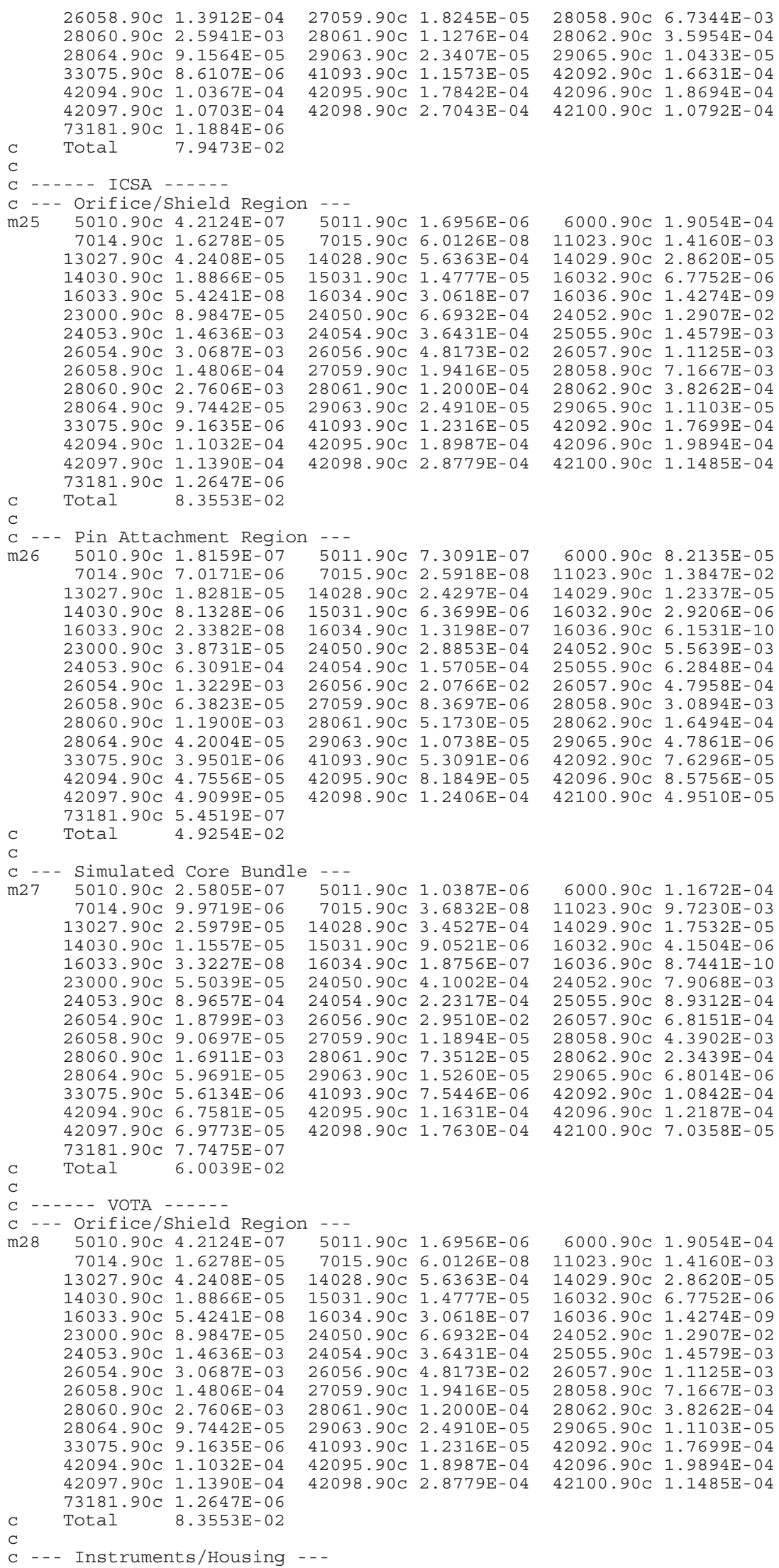

Revision: 0

Page 231 of 304

Date: March 31, 2010 
NEA/NSC/DOC(2006)1

\section{Liquid Metal Fast Reactor - LMFR}

FFTF-LMFR-RESR-001 CRIT-SPEC-REAC-COEF-MISC

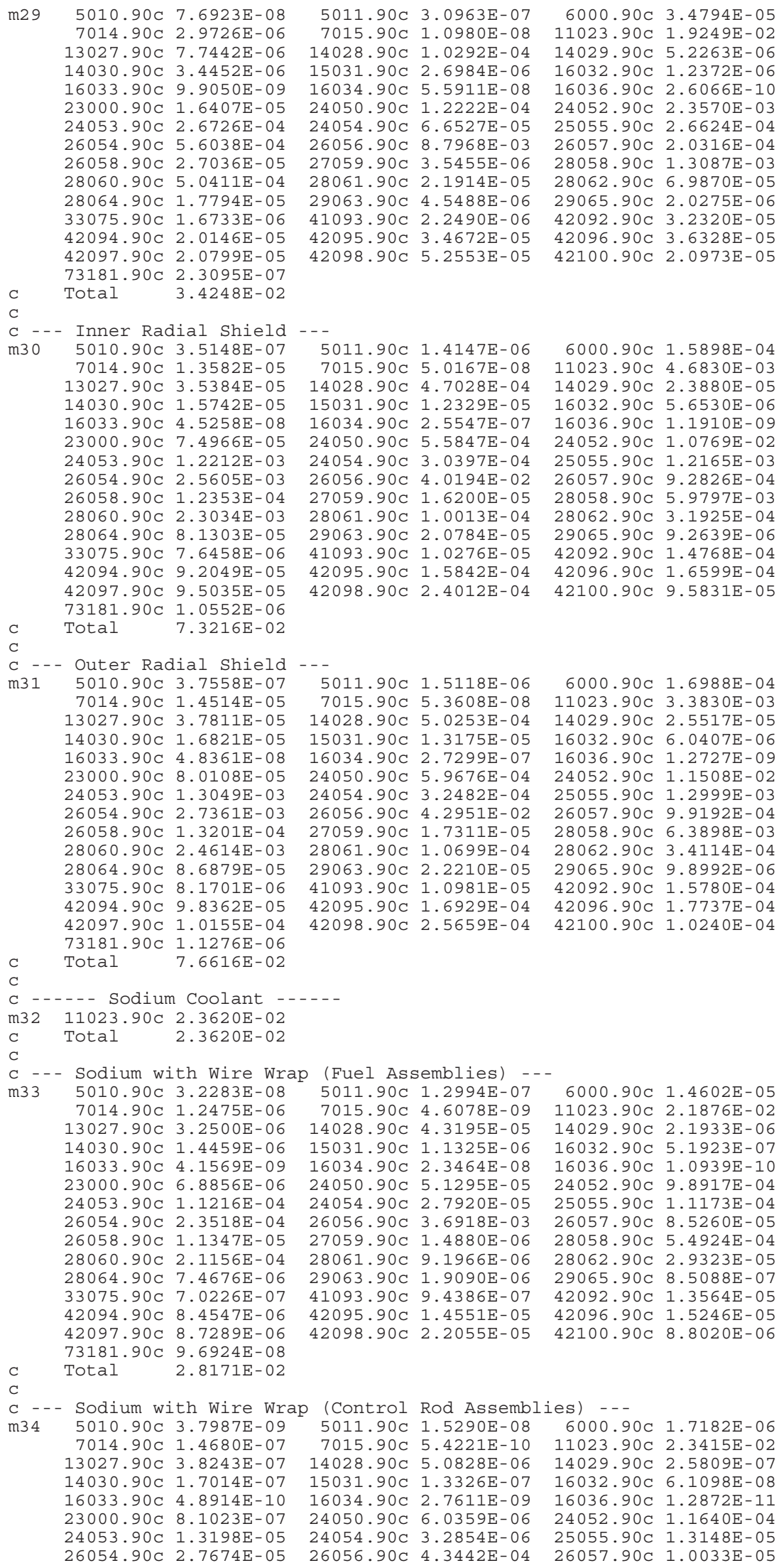

$7015.90 \mathrm{C} 1.0980 \mathrm{E}-08$ $14028.90 \mathrm{C}$ 1.0292E-04 $16034.90 \mathrm{C} 5.5911 \mathrm{E}-08$ $24054.90 \mathrm{C} \quad 6.6527 \mathrm{E}-05$ $27059.90 \mathrm{C} 3.5455 \mathrm{E}-06$ $29063.90 \mathrm{C} 4.5488 \mathrm{E}-06$ $41093.90 \mathrm{C} 2.2490 \mathrm{E}-06$ $42098.90 \mathrm{C} 5.2553 \mathrm{E}-05$

$5011.90 \mathrm{C} 1.4147 \mathrm{E}-06$

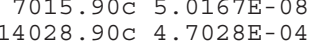

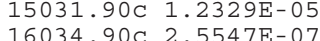
$\begin{array}{ll}24050.90 \mathrm{C} & 5.5847 \mathrm{E}-04 \\ 24054.90 \mathrm{C} & 3.0397 \mathrm{E}-04\end{array}$

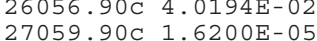

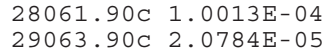
$41093.90 \mathrm{C} 1.0276 \mathrm{E}-05$ $42098.90 \mathrm{C} 2.4012 \mathrm{E}-04$

$5011.90 \mathrm{C} 1.5118 \mathrm{E}-06 \quad 6000.90 \mathrm{C} 1.6988 \mathrm{E}-04$ $7015.90 \mathrm{C} 5.3608 \mathrm{E}-08 \quad 11023.90 \mathrm{C} 3.3830 \mathrm{E}-03$ $14028.90 \mathrm{C}$ 5.0253E-04 14029.90C 2.5517E-05 $15031.90 \mathrm{C} \quad 1.3175 \mathrm{E}-05 \quad 16032.90 \mathrm{C} \quad 6.0407 \mathrm{E}-06$ $27059.90 \mathrm{C} 1.7311 \mathrm{E}-05 \quad 28058.90 \mathrm{C} 6.3898 \mathrm{E}-03$ $42092.90 \mathrm{C} 1.5780 \mathrm{E}-04$

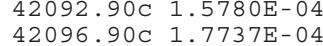
$42100.90 \mathrm{C} 1.0240 \mathrm{E}-04$ $6000.90 \mathrm{C} \quad 1.4602 \mathrm{E}-05$ $11023.90 \mathrm{C} 2.1876 \mathrm{E}-02$ $14029.90 \mathrm{C} 2.1933 \mathrm{E}-06$ $16032.90 \mathrm{C} 5.1923 \mathrm{E}-07$ $16036.90 \mathrm{C} 1.0939 \mathrm{E}-10$ $24052.90 \mathrm{C} 9.8917 \mathrm{E}-04$ $25055.90 \mathrm{C} 1.1173 \mathrm{E}-04$ $26057.90 \mathrm{C} 8.5260 \mathrm{E}-05$ $28058.90 \mathrm{C} \quad 5.4924 \mathrm{E}-04$ $28062.90 \mathrm{C} 2.9323 \mathrm{E}-05$ $29065.90 \mathrm{C} 8.5088 \mathrm{E}-07$ $42092.90 \mathrm{C} 1.3564 \mathrm{E}-05$ $42096.90 \mathrm{C} 1.5246 \mathrm{E}-05$ $42100.90 \mathrm{C} 8.8020 \mathrm{E}-06$

(Control Rod Assemblies) - -

$5011.90 \mathrm{C} 1.5290 \mathrm{E}-08 \quad 6000.90 \mathrm{C} \quad 1.7182 \mathrm{E}-06$ $7015.90 \mathrm{C} 5.4221 \mathrm{E}-10 \quad 11023.90 \mathrm{C} 2.3415 \mathrm{E}-02$ $14028.90 \mathrm{C} 5.0828 \mathrm{E}-06 \quad 14029.90 \mathrm{C} 2.5809 \mathrm{E}-07$ $15031.90 \mathrm{C} 1.3326 \mathrm{E}-07 \quad 16032.90 \mathrm{C} \quad 6.1098 \mathrm{E}-08$ $16034.90 \mathrm{C} 2.7611 \mathrm{E}-09 \quad 16036.90 \mathrm{C} \quad 1.2872 \mathrm{E}-11$ $24050.90 \mathrm{C} 6.0359 \mathrm{E}-06 \quad 24052.90 \mathrm{C}$ 1.1640E-04 $24054.90 \mathrm{C} 3.2854 \mathrm{E}-06 \quad 25055.90 \mathrm{C} 1.3148 \mathrm{E}-05$ $26056.90 \mathrm{C} \quad 4.3442 \mathrm{E}-04 \quad 26057.90 \mathrm{C} \quad 1.0033 \mathrm{E}-05$ 
NEA/NSC/DOC(2006)1

Liquid Metal Fast Reactor - LMFR

FFTF-LMFR-RESR-001 CRIT-SPEC-REAC-COEF-MISC

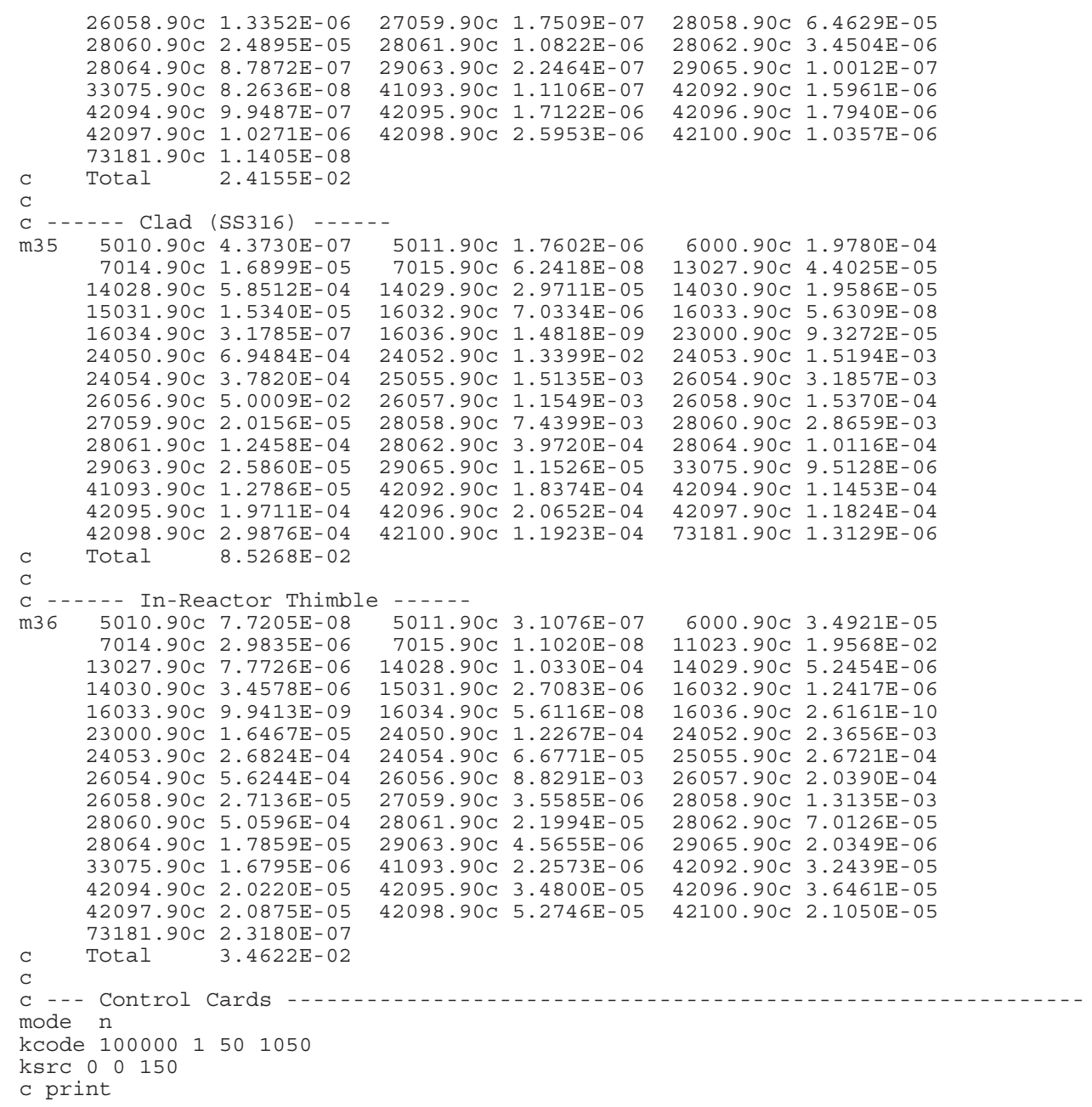

Revision: 0

Page 233 of 304

Date: March 31, 2010 
Liquid Metal Fast Reactor - LMFR

FFTF-LMFR-RESR-001

CRIT-SPEC-REAC-COEF-MISC

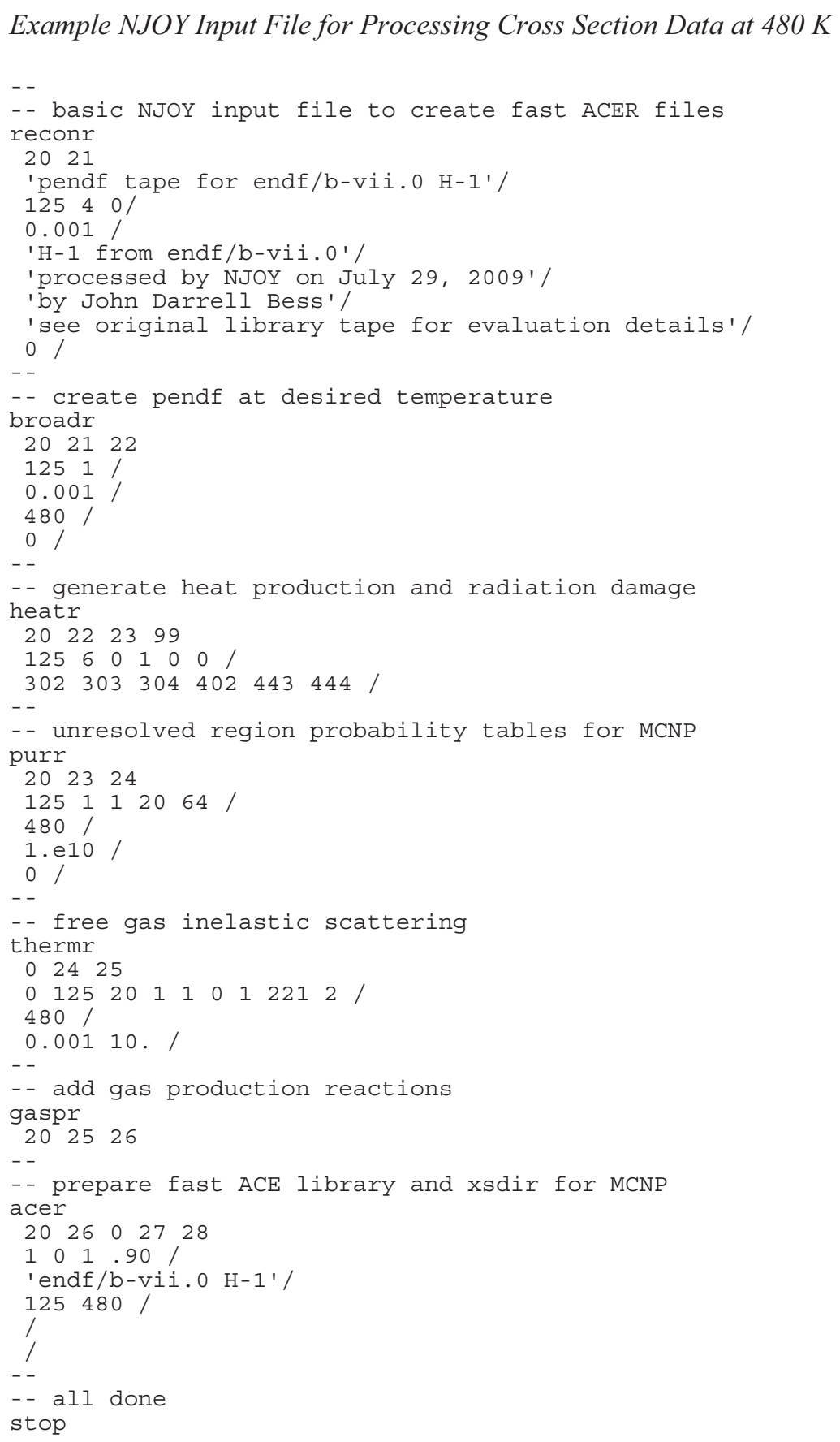


NEA/NSC/DOC(2006)1

Liquid Metal Fast Reactor - LMFR

FFTF-LMFR-RESR-001

CRIT-SPEC-REAC-COEF-MISC

\section{A.2 Buckling and Extrapolation Length Configurations}

Buckling and extrapolation length measurements were not made.

\section{A.3 Spectral-Characteristics Configurations}

\section{MCNP5 Input Deck for Evaluating Neutron Spectra Measurements:}

The input deck for analysis of the neutron spectra measurements is that of the fully-loaded critical configuration (Appendix A.1) with modifications to the IRT for a detector tally and tally-card specifications appended to the end of the input deck. The entire modified input deck is included here:

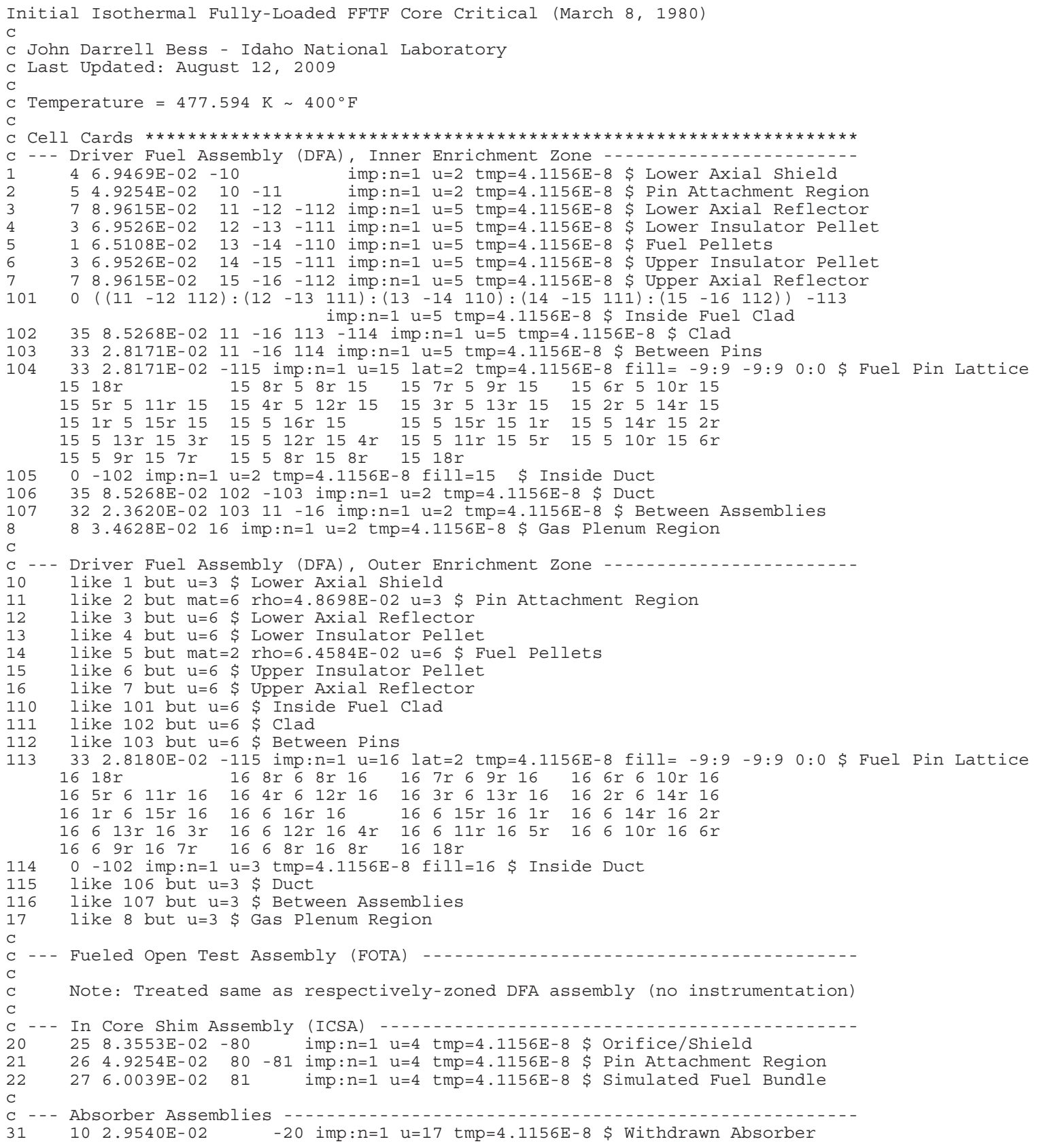

Revision: 0

Page 235 of 304

Date: March 31, 2010 
NEA/NSC/DOC(2006)1

\section{Liquid Metal Fast Reactor - LMFR}

FFTF-LMFR-RESR-001

CRIT-SPEC-REAC-COEF-MISC

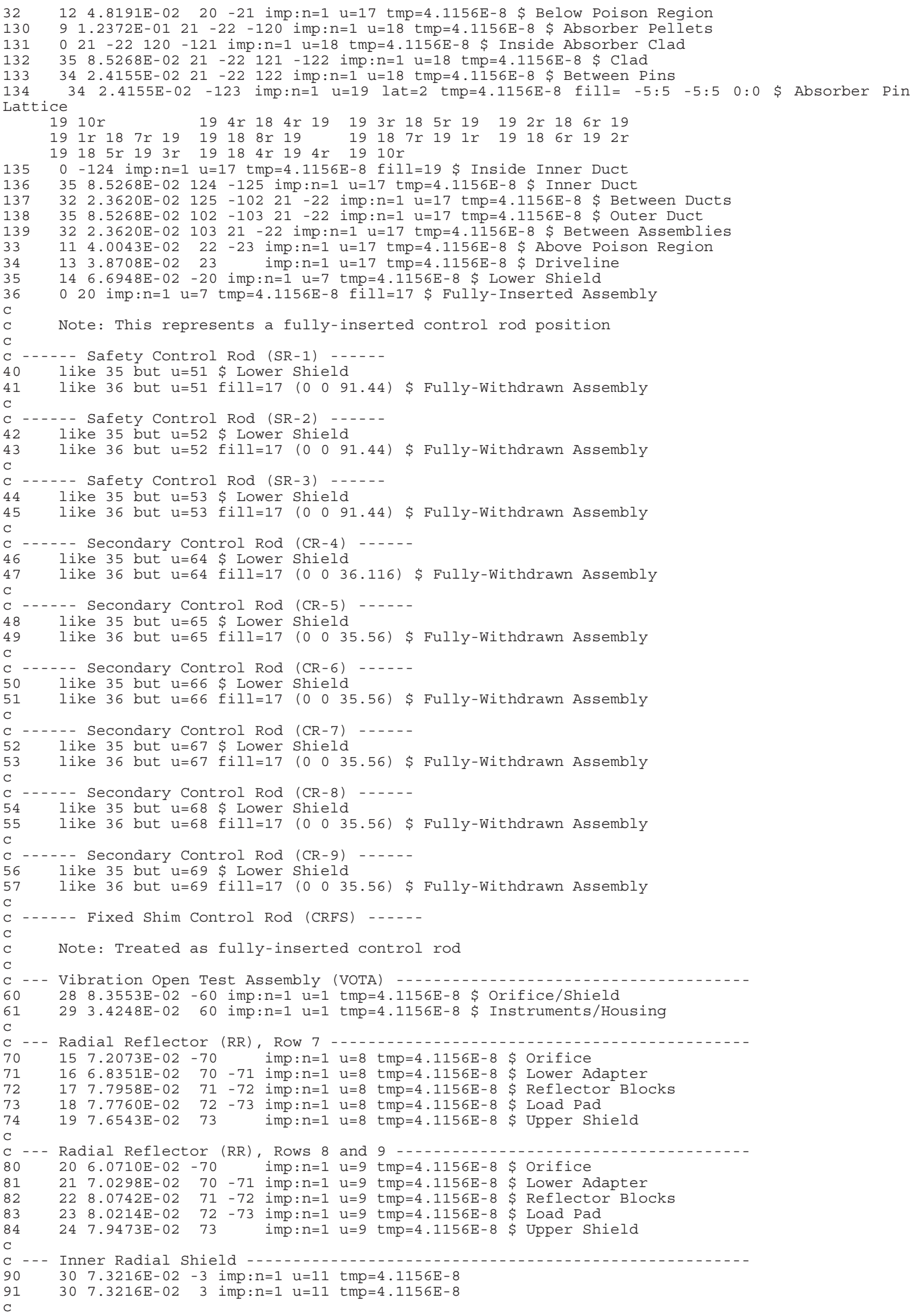

Revision: 0

Page 236 of 304

Date: March 31, 2010 
NEA/NSC/DOC(2006)1

Liquid Metal Fast Reactor - LMFR

FFTF-LMFR-RESR-001

CRIT-SPEC-REAC-COEF-MISC

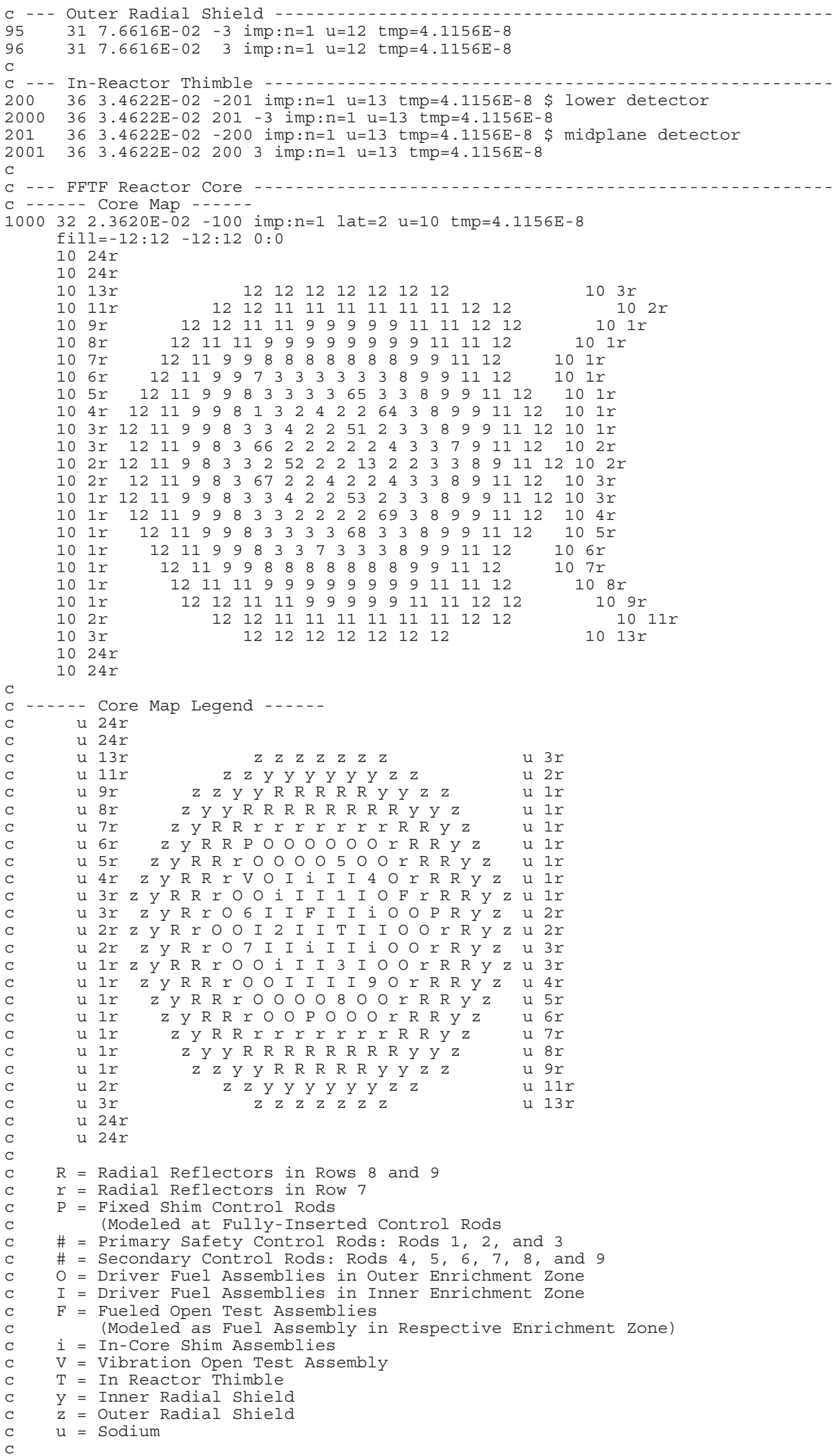

Revision: 0

Page 237 of 304

Date: March 31, 2010 
NEA/NSC/DOC(2006)1

\section{Liquid Metal Fast Reactor - LMFR}

FFTF-LMFR-RESR-001

CRIT-SPEC-REAC-COEF-MISC

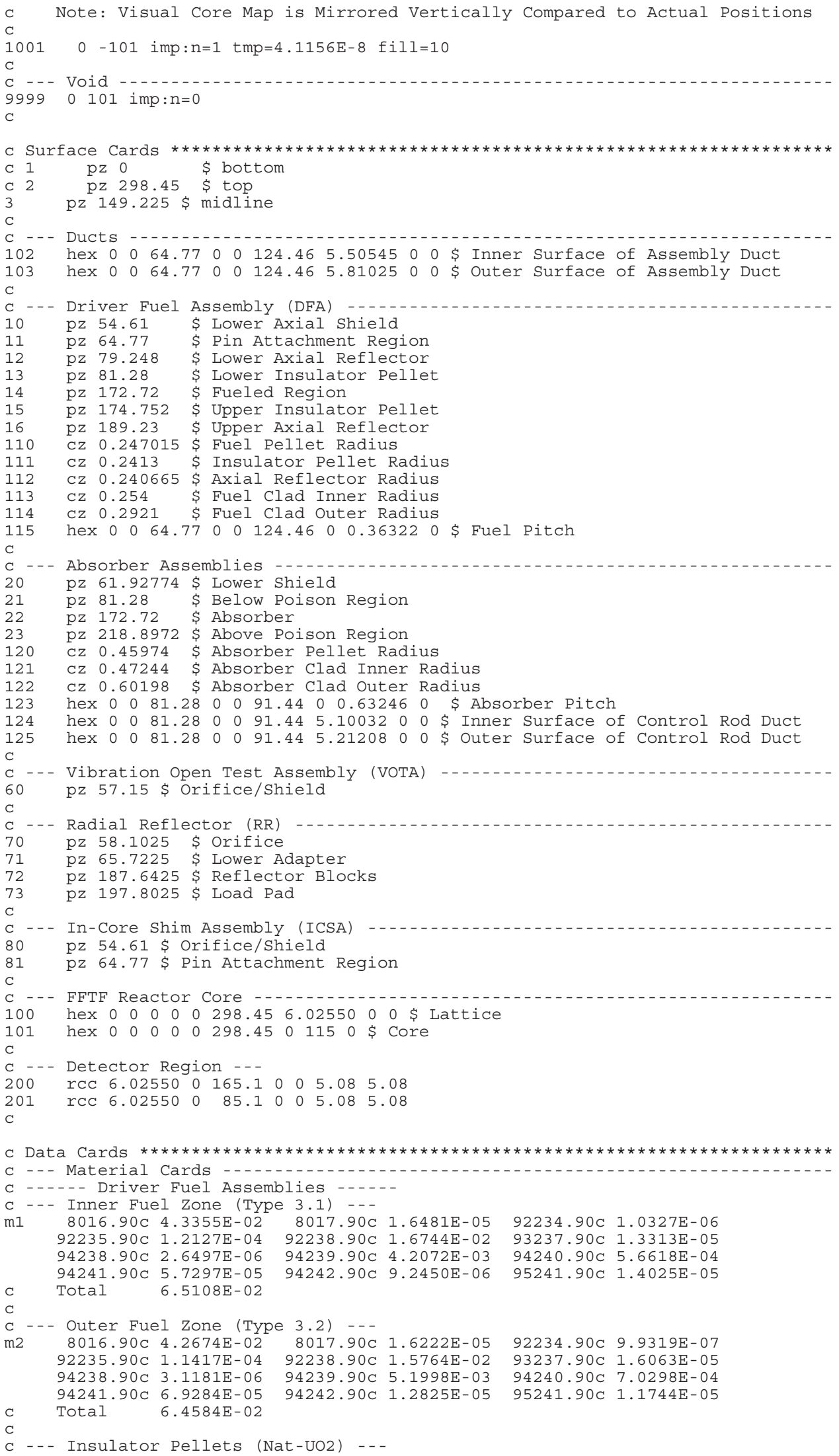

Revision: 0

Page 238 of 304

Date: March 31, 2010 
NEA/NSC/DOC(2006)1

\section{Liquid Metal Fast Reactor - LMFR}

FFTF-LMFR-RESR-001 CRIT-SPEC-REAC-COEF-MISC

\begin{tabular}{|c|c|c|c|c|c|c|}
\hline m3 & $\begin{array}{r}8016.90 \mathrm{C} \\
92235.90 \mathrm{C}\end{array}$ & $\begin{array}{l}4.6333 E-02 \\
1.6686 E-04\end{array}$ & $\begin{array}{r}8017.90 \mathrm{C} \\
92238.90 \mathrm{C}\end{array}$ & $\begin{array}{l}1.7613 \mathrm{E}-05 \\
2.3007 \mathrm{E}-02\end{array}$ & $92234.90 \mathrm{C}$ & 1. $2746 \mathrm{E}-06$ \\
\hline C & Total & $6.9526 \mathrm{E}-02$ & & & & \\
\hline & & & & & & \\
\hline & Lower Ax & ial Shield & & & & \\
\hline m4 & $5010.90 \mathrm{C}$ & $3.2491 E-07$ & $5011.90 \mathrm{C}$ & $1.3078 \mathrm{E}-06$ & $6000.90 \mathrm{C}$ & $1.4696 \mathrm{E}-04$ \\
\hline & $7014.90 \mathrm{C}$ & $1.2556 \mathrm{E}-05$ & $7015.90 \mathrm{C}$ & $4.6375 \mathrm{E}-08$ & $11023.90 \mathrm{C}$ & $6.1160 \mathrm{E}-03$ \\
\hline & $13027.90 \mathrm{C}$ & $3.2710 E-05$ & $14028.90 \mathrm{C}$ & $4.3474 E-04$ & $14029.90 \mathrm{C}$ & $2.2075 E-05$ \\
\hline & $14030.90 \mathrm{C}$ & $1.4552 \mathrm{E}-05$ & $15031.90 \mathrm{C}$ & $1.1398 \mathrm{E}-05$ & $16032.90 \mathrm{C}$ & $5.2257 \mathrm{E}-06$ \\
\hline & $16033.90 \mathrm{C}$ & $4.1837 E-08$ & $16034.90 \mathrm{C}$ & $2.3616 \mathrm{E}-07$ & $16036.90 \mathrm{C}$ & $1.1010 \mathrm{E}-09$ \\
\hline & $23000.90 \mathrm{C}$ & $6.9300 E-05$ & $24050.90 \mathrm{C}$ & $5.1625 \mathrm{E}-04$ & $24052.90 \mathrm{C}$ & $9.9554 \mathrm{E}-03$ \\
\hline & $24053.90 \mathrm{C}$ & $1.1289 \mathrm{E}-03$ & $24054.90 \mathrm{C}$ & $2.8100 \mathrm{E}-04$ & $25055.90 \mathrm{C}$ & $1.1245 \mathrm{E}-03$ \\
\hline & $26054.90 \mathrm{C}$ & $2.3670 E-03$ & $26056.90 \mathrm{C}$ & $3.7156 \mathrm{E}-02$ & $26057.90 \mathrm{C}$ & $8.5810 \mathrm{E}-04$ \\
\hline & $26058.90 \mathrm{C}$ & $1.1420 \mathrm{E}-04$ & $27059.90 \mathrm{C}$ & $1.4976 \mathrm{E}-05$ & $28058.90 \mathrm{C}$ & $5.5278 E-03$ \\
\hline & $28060.90 \mathrm{C}$ & $2.1293 E-03$ & $28061.90 \mathrm{C}$ & $9.2559 \mathrm{E}-05$ & $28062.90 \mathrm{C}$ & $2.9512 \mathrm{E}-04$ \\
\hline & $28064.90 \mathrm{C}$ & $7.5158 \mathrm{E}-05$ & $29063.90 \mathrm{C}$ & $1.9213 \mathrm{E}-05$ & $29065.90 \mathrm{C}$ & $8.5637 \mathrm{E}-06$ \\
\hline & $33075.90 \mathrm{C}$ & $7.0679 \mathrm{E}-06$ & $41093.90 \mathrm{C}$ & $9.4995 \mathrm{E}-06$ & $42092.90 \mathrm{C}$ & $1.3651 \mathrm{E}-04$ \\
\hline & $42094.90 \mathrm{C}$ & $8.5092 E-05$ & $42095.90 \mathrm{C}$ & $1.4645 \mathrm{E}-04$ & $42096.90 \mathrm{C}$ & $1.5344 \mathrm{E}-04$ \\
\hline & $42097.90 \mathrm{C}$ & $8.7852 \mathrm{E}-05$ & $42098.90 \mathrm{C}$ & $2.2197 \mathrm{E}-04$ & $42100.90 \mathrm{C}$ & $8.8587 E-05$ \\
\hline & $73181.90 \mathrm{C}$ & $9.7549 \mathrm{E}-07$ & & & & \\
\hline C & Total & $6.9469 \mathrm{E}-02$ & & & & \\
\hline C & & & & & & \\
\hline & Pin Att & iment & (Inner & el Zone) & & \\
\hline m5 & $5010.90 \mathrm{C}$ & $1.8159 \mathrm{E}-07$ & $5011.90 \mathrm{C}$ & $7.3091 \mathrm{E}-07$ & $6000.90 \mathrm{C}$ & $8.2135 E-05$ \\
\hline & $7014.90 \mathrm{C}$ & $7.0171 \mathrm{E}-06$ & $7015.90 \mathrm{C}$ & $2.5918 \mathrm{E}-08$ & $11023.90 \mathrm{C}$ & $1.3847 \mathrm{E}-02$ \\
\hline & $13027.90 \mathrm{C}$ & $1.8281 \mathrm{E}-05$ & $14028.90 \mathrm{C}$ & $2.4297 \mathrm{E}-04$ & $14029.90 \mathrm{C}$ & $1.2337 \mathrm{E}-05$ \\
\hline & $14030.90 \mathrm{C}$ & $8.1328 \mathrm{E}-06$ & $15031.90 \mathrm{C}$ & $6.3699 \mathrm{E}-06$ & $16032.90 \mathrm{C}$ & $2.9206 \mathrm{E}-06$ \\
\hline & $16033.90 \mathrm{C}$ & $2.3382 \mathrm{E}-08$ & $16034.90 \mathrm{C}$ & $1.3198 \mathrm{E}-07$ & $16036.90 \mathrm{C}$ & $6.1531 \mathrm{E}-10$ \\
\hline & $23000.90 \mathrm{C}$ & $3.8731 E-05$ & $24050.90 \mathrm{C}$ & $2.8853 E-04$ & $24052.90 \mathrm{C}$ & $5.5639 \mathrm{E}-03$ \\
\hline & $24053.90 \mathrm{C}$ & $6.3091 \mathrm{E}-04$ & $24054.90 \mathrm{C}$ & $1.5705 \mathrm{E}-04$ & $25055.90 \mathrm{C}$ & $6.2848 E-04$ \\
\hline & $26054.90 \mathrm{C}$ & $1.3229 \mathrm{E}-03$ & $26056.90 \mathrm{C}$ & $2.0766 \mathrm{E}-02$ & $26057.90 \mathrm{C}$ & $4.7958 E-04$ \\
\hline & $26058.90 \mathrm{C}$ & $6.3823 \mathrm{E}-05$ & $27059.90 \mathrm{C}$ & $8.3697 \mathrm{E}-06$ & $28058.90 \mathrm{C}$ & $3.0894 \mathrm{E}-03$ \\
\hline & $28060.90 \mathrm{C}$ & $1.1900 \mathrm{E}-03$ & $28061.90 \mathrm{C}$ & $5.1730 \mathrm{E}-05$ & $28062.90 \mathrm{C}$ & $1.6494 \mathrm{E}-04$ \\
\hline & $28064.90 \mathrm{C}$ & $4.2004 \mathrm{E}-05$ & $29063.90 \mathrm{C}$ & $1.0738 \mathrm{E}-05$ & $29065.90 \mathrm{C}$ & $4.7861 E-06$ \\
\hline & $33075.90 \mathrm{C}$ & $3.9501 \mathrm{E}-06$ & $41093.90 \mathrm{C}$ & $5.3091 \mathrm{E}-06$ & $42092.90 \mathrm{C}$ & $7.6296 \mathrm{E}-05$ \\
\hline & $42094.90 \mathrm{C}$ & $4.7556 \mathrm{E}-05$ & $42095.90 \mathrm{C}$ & $8.1849 \mathrm{E}-05$ & $42096.90 \mathrm{C}$ & $8.5756 E-05$ \\
\hline & $42097.90 \mathrm{C}$ & $4.9099 E-05$ & $42098.90 \mathrm{C}$ & $1.2406 \mathrm{E}-04$ & $42100.90 \mathrm{C}$ & $4.9510 \mathrm{E}-05$ \\
\hline & $73181.90 \mathrm{C}$ & $5.4519 \mathrm{E}-07$ & & & & \\
\hline C & Total & $4.9254 \mathrm{E}-02$ & & & & \\
\hline C & & & & & & \\
\hline & $\mathrm{P}$ & $\mathrm{ame}$ & (Outer & del Zone) & & \\
\hline m6 & $5010.90 \mathrm{C}$ & $1.7764 \mathrm{E}-07$ & $5011.90 \mathrm{C}$ & $7.1504 \mathrm{E}-07$ & $6000.90 \mathrm{C}$ & $8.0351 E-05$ \\
\hline & $7014.90 \mathrm{C}$ & $6.8647 \mathrm{E}-06$ & $7015.90 \mathrm{C}$ & $2.5356 \mathrm{E}-08$ & $11023.90 \mathrm{C}$ & $1.4060 \mathrm{E}-02$ \\
\hline & $13027.90 \mathrm{C}$ & $1.7884 \mathrm{E}-05$ & $14028.90 \mathrm{C}$ & $2.3769 \mathrm{E}-04$ & $14029.90 \mathrm{C}$ & $1.2069 \mathrm{E}-05$ \\
\hline & $14030.90 \mathrm{C}$ & $7.9562 \mathrm{E}-06$ & $15031.90 \mathrm{C}$ & $6.2316 \mathrm{E}-06$ & $16032.90 \mathrm{C}$ & $2.8572 \mathrm{E}-06$ \\
\hline & $16033.90 \mathrm{C}$ & $2.2874 \mathrm{E}-08$ & $16034.90 \mathrm{C}$ & $1.2912 \mathrm{E}-07$ & $16036.90 \mathrm{C}$ & $6.0195 \mathrm{E}-10$ \\
\hline & $23000.90 \mathrm{C}$ & $3.7890 \mathrm{E}-05$ & $24050.90 \mathrm{C}$ & $2.8226 \mathrm{E}-04$ & $24052.90 \mathrm{C}$ & $5.4431 E-03$ \\
\hline & $24053.90 \mathrm{C}$ & $6.1720 \mathrm{E}-04$ & $24054.90 \mathrm{C}$ & $1.5364 \mathrm{E}-04$ & $25055.90 \mathrm{C}$ & $6.1483 E-04$ \\
\hline & $26054.90 \mathrm{C}$ & $1.2941 \mathrm{E}-03$ & $26056.90 \mathrm{C}$ & $2.0315 \mathrm{E}-02$ & $26057.90 \mathrm{C}$ & $4.6916 \mathrm{E}-04$ \\
\hline & $26058.90 \mathrm{C}$ & $6.2437 \mathrm{E}-05$ & $27059.90 \mathrm{C}$ & $8.1879 \mathrm{E}-06$ & $28058.90 \mathrm{C}$ & $3.0223 E-03$ \\
\hline & $28060.90 \mathrm{C}$ & $1.1642 \mathrm{E}-03$ & $28061.90 \mathrm{C}$ & $5.0606 \mathrm{E}-05$ & $28062.90 \mathrm{C}$ & $1.6135 E-04$ \\
\hline & $28064.90 \mathrm{C}$ & $4.1092 \mathrm{E}-05$ & $29063.90 \mathrm{C}$ & $1.0505 \mathrm{E}-05$ & $29065.90 \mathrm{C}$ & $4.6822 E-06$ \\
\hline & $33075.90 \mathrm{C}$ & $3.8643 E-06$ & $41093.90 \mathrm{C}$ & $5.1938 E-06$ & $42092.90 \mathrm{C}$ & $7.4639 \mathrm{E}-05$ \\
\hline & $42094.90 \mathrm{C}$ & $4.6524 \mathrm{E}-05$ & $42095.90 \mathrm{C}$ & $8.0071 \mathrm{E}-05$ & $42096.90 \mathrm{C}$ & $8.3893 E-05$ \\
\hline & $42097.90 \mathrm{C}$ & $4.8032 E-05$ & $42098.90 \mathrm{C}$ & $1.2136 \mathrm{E}-04$ & $42100.90 \mathrm{C}$ & $4.8435 E-05$ \\
\hline & $73181.90 \mathrm{C}$ & $5.3334 \mathrm{E}-07$ & & & & \\
\hline C & Total & $4.8698 \mathrm{E}-02$ & & & & \\
\hline C & & & & & & \\
\hline & Axial $R$ & flectors (Ir & conel 600) & -- & & \\
\hline m7 & $6000.90 \mathrm{C}$ & $2.0908 E-03$ & $14028.90 \mathrm{C}$ & $4.1233 E-04$ & $14029.90 \mathrm{C}$ & $2.0937 E-05$ \\
\hline & $14030.90 \mathrm{C}$ & $1.3802 \mathrm{E}-05$ & $16032.90 \mathrm{C}$ & $1.1152 \mathrm{E}-05$ & 16033 & $8.9281 E-08$ \\
\hline & $16034.90 \mathrm{C}$ & $5.0397 E-07$ & $16036.90 \mathrm{C}$ & $2.3495 \mathrm{E}-09$ & $24050.90 \mathrm{C}$ & $6.5053 E-04$ \\
\hline & $24052.90 \mathrm{C}$ & $1.2545 \mathrm{E}-02$ & $24053.90 \mathrm{C}$ & $1.4225 \mathrm{E}-03$ & $24054.90 \mathrm{C}$ & $3.5408 E-04$ \\
\hline & 25055 & $1.5998 \mathrm{E}-03$ & $26054.90 \mathrm{C}$ & $4.2054 \mathrm{E}-04$ & $26056.90 \mathrm{C}$ & $6.6015 E-03$ \\
\hline & $26057.90 \mathrm{C}$ & $1.5246 \mathrm{E}-04$ & $26058.90 \mathrm{C}$ & $2.0289 \mathrm{E}-05$ & $27059.90 \mathrm{C}$ & $8.5222 \mathrm{E}-05$ \\
\hline & $28058.90 \mathrm{C}$ & $4.2900 \mathrm{E}-02$ & $28060.90 \mathrm{C}$ & $1.6525 \mathrm{E}-02$ & $28061.90 \mathrm{C}$ & $7.1832 \mathrm{E}-04$ \\
\hline & $28062.90 \mathrm{C}$ & $2.2903 E-03$ & $28064.90 \mathrm{C}$ & $5.8328 \mathrm{E}-04$ & $29063.90 \mathrm{C}$ & $1.3667 \mathrm{E}-04$ \\
\hline & $29065.90 \mathrm{C}$ & $6.0917 E-05$ & & & & \\
\hline C & Total & $8.9615 E-02$ & & & & \\
\hline c & & & & & & \\
\hline & Gas Ple & um --- & & & & \\
\hline m8 & $5010.90 \mathrm{C}$ & $1.2816 \mathrm{E}-07$ & $5011.90 \mathrm{C}$ & $5.1585 \mathrm{E}-07$ & $6000.90 \mathrm{C}$ & $5.7968 \mathrm{E}-05$ \\
\hline & 7014 & $4.9525 \mathrm{E}$ & $7015.90 \mathrm{C}$ & $1.8292 \mathrm{E}-08$ & 11023 & 9.63 \\
\hline & $13027.90 \mathrm{C}$ & $1.2902 \mathrm{E}-05$ & $14028.90 \mathrm{C}$ & $1.7148 \mathrm{E}-04$ & $14029.90 \mathrm{C}$ & $8.7072 \mathrm{E}-06$ \\
\hline & $14030.90 \mathrm{C}$ & $5.7399 \mathrm{E}-06$ & $15031.90 \mathrm{C}$ & $4.4957 \mathrm{E}-06$ & $16032.90 \mathrm{C}$ & $2.0612 E-06$ \\
\hline & $16033.90 \mathrm{C}$ & $1.6502 \mathrm{E}-08$ & $16034.90 \mathrm{C}$ & $9.3150 \mathrm{E}-08$ & $16036.90 \mathrm{C}$ & $4.3427 \mathrm{E}-10$ \\
\hline & $23000.90 \mathrm{C}$ & $2.7335 E-05$ & $24050.90 \mathrm{C}$ & $2.0363 E-04$ & $24052.90 \mathrm{C}$ & $3.9268 E-03$ \\
\hline & $24053.90 \mathrm{C}$ & $4.4527 E-04$ & $24054.90 \mathrm{C}$ & $1.1084 \mathrm{E}-04$ & $25055.90 \mathrm{C}$ & $4.4356 \mathrm{E}-04$ \\
\hline & $26054.90 \mathrm{C}$ & $9.3362 \mathrm{E}-04$ & $26056.90 \mathrm{C}$ & $1.4656 \mathrm{E}-02$ & $26057.90 \mathrm{C}$ & $3.3847 E-04$ \\
\hline & $26058.90 \mathrm{C}$ & $4.5044 E-05$ & $27059.90 \mathrm{C}$ & $5.9070 \mathrm{E}-06$ & $28058.90 \mathrm{C}$ & $2.1804 \mathrm{E}-03$ \\
\hline & 28060 & $8.3988 E-04$ & $28061.90 \mathrm{C}$ & $3.6509 \mathrm{E}-05$ & $28062.90 \mathrm{C}$ & $1.1641 \mathrm{E}-04$ \\
\hline & $28064.90 \mathrm{C}$ & $2.9645 E-05$ & $29063.90 \mathrm{C}$ & $7.5786 \mathrm{E}-06$ & $29065.90 \mathrm{C}$ & $3.3779 E-06$ \\
\hline & $33075.90 \mathrm{C}$ & $2.7879 E-06$ & $41093.90 \mathrm{C}$ & $3.7470 \mathrm{E}-06$ & $42092.90 \mathrm{C}$ & $5.3847 E-05$ \\
\hline & $42094.90 \mathrm{C}$ & $3.3564 \mathrm{E}-05$ & $42095.90 \mathrm{C}$ & $5.7766 \mathrm{E}-05$ & $42096.90 \mathrm{C}$ & $6.0523 E-05$ \\
\hline
\end{tabular}

Revision: 0

Page 239 of 304

Date: March 31, 2010 
NEA/NSC/DOC(2006)1

\section{Liquid Metal Fast Reactor - LMFR}

FFTF-LMFR-RESR-001 CRIT-SPEC-REAC-COEF-MISC

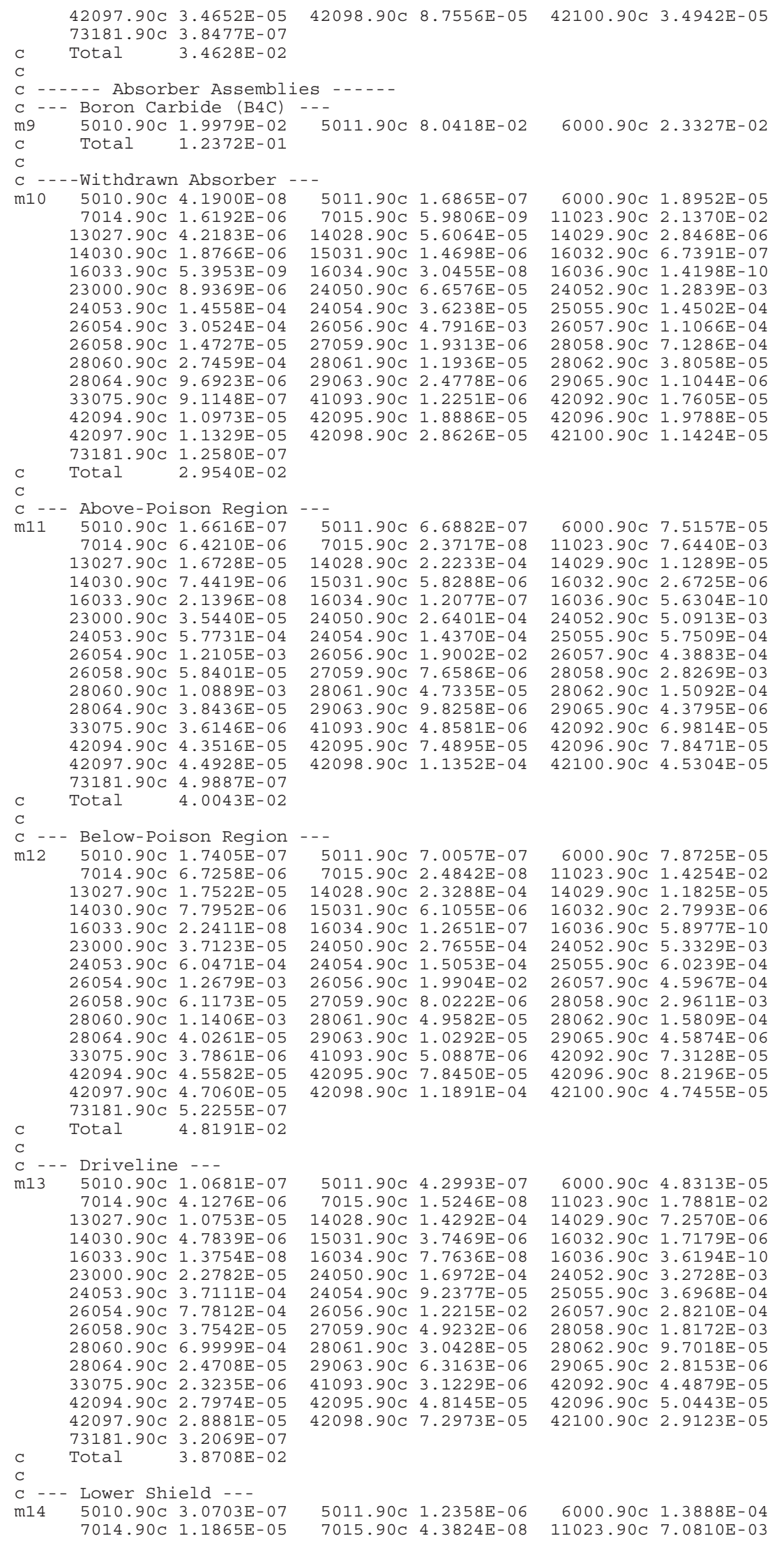

Revision: 0

Page 240 of 304

Date: March 31, 2010 
NEA/NSC/DOC(2006)1

\section{Liquid Metal Fast Reactor - LMFR}

FFTF-LMFR-RESR-001 CRIT-SPEC-REAC-COEF-MISC

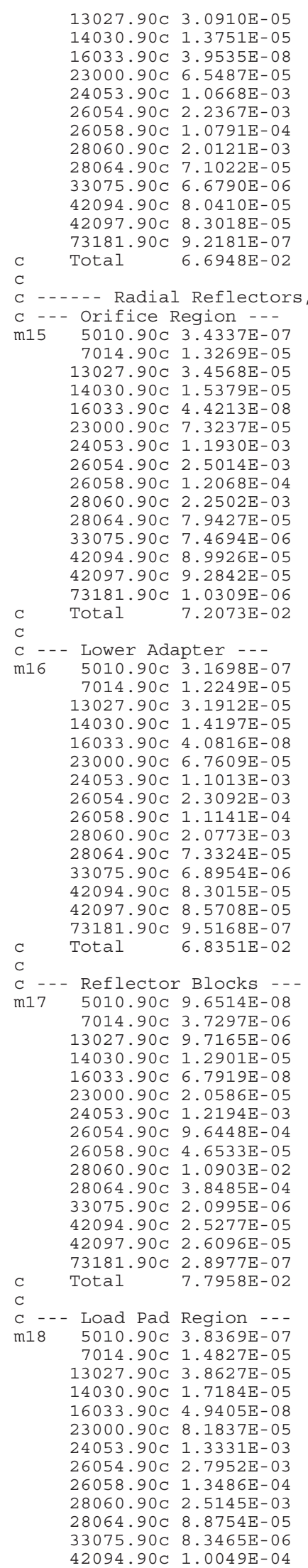

$14027.90 \mathrm{C} 3.0910 \mathrm{E}-05$

$16033.90 \mathrm{C} 3.9535 \mathrm{E}-08$

$3000.90 \mathrm{C} 6.5487 \mathrm{E}-05$

$791 \mathrm{E}-04$

$28060.90 \mathrm{C} 2.0121 \mathrm{E}-03$

$28064.90 \mathrm{C} 7.1022 \mathrm{E}-05$

$42094.90 \mathrm{C} 8.0410 \mathrm{E}-05$

$097.90 \mathrm{C} 8.3018 \mathrm{E}-05$

$73181.90 \mathrm{C} 9.2181 \mathrm{E}-07$

..... Radial Reflectors, Row 7 .....

C - - Orifice Region -..

$5010.90 \mathrm{C} 3.4337 \mathrm{E}-07$

$7014.90 \mathrm{C} \quad 1.3269 \mathrm{E}-05$

$14030.90 \mathrm{C} 1.5379 \mathrm{E}-05$

$16033.90 \mathrm{C} 4.4213 \mathrm{E}-08$

$3000.90 \mathrm{C} 7.3237 \mathrm{E}-05$

$26054.90 \mathrm{C} 2.5014 \mathrm{E}-03$

(2058.90C $1.2068 \mathrm{E}-04$

$33075.90 \mathrm{C} 7.4694 \mathrm{E}-06$

$2094.90 \mathrm{C} 8.9926 \mathrm{E}-05$

(9.2842E-05

$73181.90 \mathrm{C} 1.0309 \mathrm{E}-06$

--- Lower Adapter ---

$5010.90 \mathrm{C} 3.1698 \mathrm{E}-07$

$3027.90 \mathrm{C} 3.1912 \mathrm{E}-05$

$14030.90 \mathrm{C} 1.4197 \mathrm{E}-05$

.

24053.90C $1.1013 \mathrm{E}-03$

$6054.90 \mathrm{C} 2.3092 \mathrm{E}-03$

$8060.90 \mathrm{C} 2.0773 \mathrm{E}-03$

$064.90 \mathrm{C} 7.3324 \mathrm{E}-05$

$33075.90 \mathrm{C} 6.8954 \mathrm{E}-06$

$2094.90 \mathrm{C} 8.3015 \mathrm{E}-05$

$3181.90 \mathrm{C} 9.5168 \mathrm{E}-07$

$5011.90 \mathrm{C} \quad 1.3821 \mathrm{E}-06$

$7015.90 \mathrm{C} 4.9010 \mathrm{E}-08$

$14028.90 \mathrm{C} 4.5943 \mathrm{E}-04$

$15031.90 \mathrm{C} 1.2045 \mathrm{E}-05$

$16034.90 \mathrm{C} 2.4957 \mathrm{E}-07$

$24050.90 \mathrm{C} 5.4558 \mathrm{E}-04$

$24054.90 \mathrm{C} 2.9696 \mathrm{E}-04$

$26056.90 \mathrm{C} 3.9267 \mathrm{E}-02$

$27059.90 \mathrm{C} 1.5826 \mathrm{E}-05$

$28061.90 \mathrm{C} 9.7817 \mathrm{E}-05$

$29063.90 \mathrm{C} 2.0305 \mathrm{E}-05$

$41093.90 \mathrm{C} 1.0039 \mathrm{E}-05$

$42095.90 \mathrm{C} 1.5477 \mathrm{E}-04$

$42098.90 \mathrm{C} 2.3458 \mathrm{E}-04$

$5011.90 \mathrm{C} 1.2759 \mathrm{E}-06$ $7015.90 \mathrm{C} \quad 4.5244 \mathrm{E}-08$ $14028.90 \mathrm{C} 4.2413 \mathrm{E}-04$ $15031.90 \mathrm{C} 1.1119 \mathrm{E}-05$ $16034.90 \mathrm{C} 2.3039 \mathrm{E}-07$ $24050.90 \mathrm{C} 5.0366 \mathrm{E}-04$ $24054.90 \mathrm{C} \quad 2.7414 \mathrm{E}-04$ $26056.90 \mathrm{C} \quad 3.6249 \mathrm{E}-02$ $27059.90 \mathrm{C} 1.4610 \mathrm{E}-05$ $28061.90 \mathrm{C} 9.0300 \mathrm{E}-05$ $29063.90 \mathrm{C} 1.8745 \mathrm{E}-05$ $41093.90 \mathrm{C} 9.2677 \mathrm{E}-06$ $42095.90 \mathrm{C}$ 1.4288E-04 $42098.90 \mathrm{C} 2.1656 \mathrm{E}-04$

$5011.90 \mathrm{C} \quad 3.8848 \mathrm{E}-07$ $7015.90 \mathrm{C} 1.3776 \mathrm{E}-08$ $14028.90 \mathrm{C} \quad 3.8541 \mathrm{E}-04$ $15031.90 \mathrm{C} 3.3856 \mathrm{E}-06$ $16034.90 \mathrm{C} 3.8338 \mathrm{E}-07$ $24050.90 \mathrm{C} 5.5768 \mathrm{E}-04$ $24054.90 \mathrm{C} \quad 3.0355 \mathrm{E}-04$ $26056.90 \mathrm{C}$ 1.5140E-02 $27059.90 \mathrm{C} 5.7417 \mathrm{E}-05$ $28061.90 \mathrm{C} 4.7396 \mathrm{E}-04$ $29063.90 \mathrm{C} 9.0654 \mathrm{E}-05$ $41093.90 \mathrm{C} 2.8218 \mathrm{E}-06$ $42095.90 \mathrm{C} 4.3503 \mathrm{E}-05$ $42098.90 \mathrm{C} \quad 6.5938 \mathrm{E}-05$

$5011.90 \mathrm{C} \quad 1.5444 \mathrm{E}-06$ $7015.90 \mathrm{C} 5.4765 \mathrm{E}-08$ $14028.90 \mathrm{C} 5.1338 \mathrm{E}-04$ $15031.90 \mathrm{C} 1.3459 \mathrm{E}-05$ $16034.90 \mathrm{C} 2.7888 \mathrm{E}-07$ $24050.90 \mathrm{C} 6.0965 \mathrm{E}-04$ $24054.90 \mathrm{C} \quad 3.3183 \mathrm{E}-04$ $26056.90 \mathrm{C} 4.3878 \mathrm{E}-02$ $27059.90 \mathrm{C} 1.7685 \mathrm{E}-05$ $28061.90 \mathrm{C} 1.0930 \mathrm{E}-04$ $29063.90 \mathrm{C} 2.2689 \mathrm{E}-05$ $41093.90 \mathrm{C} 1.1218 \mathrm{E}-05$ 42095.90C 1.7294E-04
$14029.90 \mathrm{C} \quad 2.0860 \mathrm{E}-05$ $16032.90 \mathrm{C} 4.9382 \mathrm{E}-06$ $16036.90 \mathrm{C} 1.0404 \mathrm{E}-09$ $24052.90 \mathrm{C} 9.4076 \mathrm{E}-03$ $25055.90 \mathrm{C} 1.0627 \mathrm{E}-03$ $26057.90 \mathrm{C} 8.1088 \mathrm{E}-04$ $28058.90 \mathrm{C} 5.2236 \mathrm{E}-03$ $28062.90 \mathrm{C} 2.7888 \mathrm{E}-04$ $29065.90 \mathrm{C} 8.0925 \mathrm{E}-06$ $42092.90 \mathrm{C} 1.2900 \mathrm{E}-04$ $42096.90 \mathrm{C}$ 1.4500E-04 $42100.90 \mathrm{C} 8.3713 \mathrm{E}-05$

$6000.90 \mathrm{C} 1.5531 \mathrm{E}-04$ $11023.90 \mathrm{C} 5.1210 \mathrm{E}-03$ $14029.90 \mathrm{C} 2.3329 \mathrm{E}-05$ $16032.90 \mathrm{C} 5.5226 \mathrm{E}-06$ $16036.90 \mathrm{C} 1.1635 \mathrm{E}-09$ $24052.90 \mathrm{C} 1.0521 \mathrm{E}-02$ $25055.90 \mathrm{C} 1.1884 \mathrm{E}-03$ $26057.90 \mathrm{C} 9.0684 \mathrm{E}-04$ $28058.90 \mathrm{C} 5.8418 \mathrm{E}-03$ $28062.90 \mathrm{C} 3.1188 \mathrm{E}-04$ $29065.90 \mathrm{C} 9.0502 \mathrm{E}-06$ $42092.90 \mathrm{C}$ 1.4427E-04 $42096.90 \mathrm{C} 1.6216 \mathrm{E}-04$ $42100.90 \mathrm{C} 9.3620 \mathrm{E}-05$

$6000.90 \mathrm{C} \quad 1.4338 \mathrm{E}-04$ $11023.90 \mathrm{C} 6.5440 \mathrm{E}-03$ $14029.90 \mathrm{C} 2.1536 \mathrm{E}-05$ $16032.90 \mathrm{C} 5.0982 \mathrm{E}-06$ $16036.90 \mathrm{C} 1.0741 \mathrm{E}-09$ $24052.90 \mathrm{C} 9.7125 \mathrm{E}-03$ $25055.90 \mathrm{C} 1.0971 \mathrm{E}-03$ $26057.90 \mathrm{C} 8.3716 \mathrm{E}-04$ $28058.90 \mathrm{C} 5.3929 \mathrm{E}-03$ $28062.90 \mathrm{C} 2.8792 \mathrm{E}-04$ $29065.90 \mathrm{C} 8.3547 \mathrm{E}-06$ $42092.90 \mathrm{C} \quad 1.3318 \mathrm{E}-04$ 42096.90C 1.4970E-04 $42100.90 \mathrm{C} 8.6426 \mathrm{E}-05$

$6000.90 \mathrm{C} \quad 1.3432 \mathrm{E}-03$ $11023.90 \mathrm{C} 3.4400 \mathrm{E}-03$ $14029.90 \mathrm{C} 1.9570 \mathrm{E}-05$ $16032.90 \mathrm{C} 8.4836 \mathrm{E}-06$ $16036.90 \mathrm{C} 1.7873 \mathrm{E}-09$ $24052.90 \mathrm{C} 1.0754 \mathrm{E}-02$ $25055.90 \mathrm{C} 1.3284 \mathrm{E}-03$ $26057.90 \mathrm{C} \quad 3.4966 \mathrm{E}-04$ $28058.90 \mathrm{C} 2.8306 \mathrm{E}-02$ $28062.90 \mathrm{C} 1.5112 \mathrm{E}-03$ $29065.90 \mathrm{C} 4.0406 \mathrm{E}-05$ $42092.90 \mathrm{C} 4.0552 \mathrm{E}-05$ $42096.90 \mathrm{C} 4.5580 \mathrm{E}-05$ $42100.90 \mathrm{C} 2.6315 \mathrm{E}-05$

$6000.90 \mathrm{C} \quad 1.7355 \mathrm{E}-04$ $11023.90 \mathrm{C} 2.9460 \mathrm{E}-03$ $14029.90 \mathrm{C} 2.6068 \mathrm{E}-05$ $16032.90 \mathrm{C} 6.1711 \mathrm{E}-06$ $16036.90 \mathrm{C} 1.3001 \mathrm{E}-09$ $24052.90 \mathrm{C} 1.1756 \mathrm{E}-02$ $25055.90 \mathrm{C} 1.3280 \mathrm{E}-03$ 26057.90C 1.0133E-03 $28058.90 \mathrm{C} 6.5278 \mathrm{E}-03$ $28062.90 \mathrm{C} \quad 3.4851 \mathrm{E}-04$ $29065.90 \mathrm{C} 1.0113 \mathrm{E}-05$ $42092.90 \mathrm{C} 1.6121 \mathrm{E}-04$ $42096.90 \mathrm{C} 1.8120 \mathrm{E}-04$

Revision: 0

Page 241 of 304

Date: March 31, 2010 
NEA/NSC/DOC(2006)1

\section{Liquid Metal Fast Reactor - LMFR}

FFTF-LMFR-RESR-001 CRIT-SPEC-REAC-COEF-MISC

\begin{tabular}{|c|c|c|c|c|c|c|}
\hline$C$ & $\begin{array}{l}42097.90 \mathrm{C} \\
73181.90 \mathrm{C}\end{array}$ & $\begin{array}{l}1.0374 \mathrm{E}-04 \\
1.1520 \mathrm{E}-06\end{array}$ & $42098.90 \mathrm{C}$ & $2.6213 E-04$ & $42100.90 \mathrm{C}$ & $1.0461 \mathrm{E}-04$ \\
\hline C & & & & & & \\
\hline C & Upper Shj & eld -.- & & & & \\
\hline m19 & $5010.90 \mathrm{C}$ & $3.7506 \mathrm{E}-07$ & $5011.90 \mathrm{C}$ & $1.5097 \mathrm{E}-06$ & $6000.90 \mathrm{C}$ & $1.6965 E-04$ \\
\hline & $7014.90 \mathrm{C}$ & $1.4493 \mathrm{E}-05$ & $7015.90 \mathrm{C}$ & $5.3533 \mathrm{E}-08$ & $11023.90 \mathrm{C}$ & $3.4120 E-03$ \\
\hline & $13027.90 \mathrm{C}$ & $3.7758 \mathrm{E}-05$ & $14028.90 \mathrm{C}$ & $5.0183 \mathrm{E}-04$ & $14029.90 \mathrm{C}$ & $2.5482 \mathrm{E}-05$ \\
\hline & $14030.90 \mathrm{C}$ & $1.6798 \mathrm{E}-05$ & $15031.90 \mathrm{C}$ & $1.3157 \mathrm{E}-05$ & $16032.90 \mathrm{C}$ & $6.0323 E-06$ \\
\hline & $16033.90 \mathrm{C}$ & $4.8294 \mathrm{E}-08$ & $16034.90 \mathrm{C}$ & $2.7261 \mathrm{E}-07$ & $16036.90 \mathrm{C}$ & 1.2709E-09 \\
\hline & $23000.90 \mathrm{C}$ & $7.9996 \mathrm{E}-05$ & $24050.90 \mathrm{C}$ & $5.9593 E-04$ & $24052.90 \mathrm{C}$ & 1.1492E-02 \\
\hline & $24053.90 \mathrm{C}$ & $1.3031 \mathrm{E}-03$ & $24054.90 \mathrm{C}$ & $3.2437 \mathrm{E}-04$ & $25055.90 \mathrm{C}$ & 1.2981E-03 \\
\hline & $26054.90 \mathrm{C}$ & $2.7323 E-03$ & $26056.90 \mathrm{C}$ & $4.2891 \mathrm{E}-02$ & $26057.90 \mathrm{C}$ & $9.9054 \mathrm{E}-04$ \\
\hline & $26058.90 \mathrm{C}$ & 1. $3182 \mathrm{E}-04$ & $27059.90 \mathrm{C}$ & $1.7287 \mathrm{E}-05$ & $28058.90 \mathrm{C}$ & $6.3809 E-03$ \\
\hline & $28060.90 \mathrm{C}$ & $2.4579 E-03$ & $28061.90 \mathrm{C}$ & $1.0684 \mathrm{E}-04$ & $28062.90 \mathrm{C}$ & $3.4067 E-04$ \\
\hline & $28064.90 \mathrm{C}$ & $8.6758 \mathrm{E}-05$ & $29063.90 \mathrm{C}$ & $2.2179 \mathrm{E}-05$ & $29065.90 \mathrm{C}$ & $9.8854 \mathrm{E}-06$ \\
\hline & $33075.90 \mathrm{C}$ & $8.1588 \mathrm{E}-06$ & $41093.90 \mathrm{C}$ & $1.0966 \mathrm{E}-05$ & $42092.90 \mathrm{C}$ & $1.5758 \mathrm{E}-04$ \\
\hline & $42094.90 \mathrm{C}$ & $9.8225 \mathrm{E}-05$ & $42095.90 \mathrm{C}$ & $1.6905 \mathrm{E}-04$ & $42096.90 \mathrm{C}$ & $1.7712 \mathrm{E}-04$ \\
\hline & $42097.90 \mathrm{C}$ & 1.0141E-04 & $42098.90 \mathrm{C}$ & $2.5623 \mathrm{E}-04$ & $42100.90 \mathrm{C}$ & $1.0226 \mathrm{E}-04$ \\
\hline & $73181.90 \mathrm{C}$ & $1.1260 \mathrm{E}-06$ & & & & \\
\hline C & Total & $7.6543 \mathrm{E}-02$ & & & & \\
\hline C & & & & & & \\
\hline & -.- Radia & Reflector & Rows $8+9$ & $-\cdots--$ & & \\
\hline & Orifice & Region -.. & & & & \\
\hline $\mathrm{m} 20$ & $5010.90 \mathrm{C}$ & $2.6281 \mathrm{E}-07$ & $5011.90 \mathrm{C}$ & $1.0578 \mathrm{E}-06$ & $6000.90 \mathrm{C}$ & $1.1887 E-04$ \\
\hline & $7014.90 \mathrm{C}$ & $1.0156 \mathrm{E}-05$ & $7015.90 \mathrm{C}$ & $3.7511 \mathrm{E}-08$ & $11023.90 \mathrm{C}$ & $0 E-03$ \\
\hline & $13027.90 \mathrm{C}$ & $2.6458 \mathrm{E}-05$ & $14028.90 \mathrm{C}$ & $3.5164 \mathrm{E}-04$ & $14029.90 \mathrm{C}$ & $1.7856 \mathrm{E}-05$ \\
\hline & $14030.90 \mathrm{C}$ & $1.1771 \mathrm{E}-05$ & $15031.90 \mathrm{C}$ & $9.2191 \mathrm{E}-06$ & $16032.90 \mathrm{C}$ & $9 E-06$ \\
\hline & $16033.90 \mathrm{C}$ & $3.3840 \mathrm{E}-08$ & $16034.90 \mathrm{C}$ & $1.9102 \mathrm{E}-07$ & $16036.90 \mathrm{C}$ & $8.9053 E-10$ \\
\hline & $23000.90 \mathrm{C}$ & $5.6054 \mathrm{E}-05$ & $24050.90 \mathrm{C}$ & $4.1758 \mathrm{E}-04$ & $24052.90 \mathrm{C}$ & $8.0526 E-03$ \\
\hline & $24053.90 \mathrm{C}$ & $9.1310 \mathrm{E}-04$ & $24054.90 \mathrm{C}$ & $2.2729 \mathrm{E}-04$ & $25055.90 \mathrm{C}$ & $9.0959 E-04$ \\
\hline & $26054.90 \mathrm{C}$ & 1. $9145 \mathrm{E}-03$ & $26056.90 \mathrm{C}$ & $3.0054 \mathrm{E}-02$ & $26057.90 \mathrm{C}$ & $6.9408 E-04$ \\
\hline & $26058.90 \mathrm{C}$ & $9.2370 \mathrm{E}-05$ & $27059.90 \mathrm{C}$ & $1.2113 \mathrm{E}-05$ & $28058.90 \mathrm{C}$ & $4.4712 \mathrm{E}-03$ \\
\hline & $28060.90 \mathrm{C}$ & $1.7223 E-03$ & $28061.90 \mathrm{C}$ & $7.4867 \mathrm{E}-05$ & $28062.90 \mathrm{C}$ & $2.3871 E-04$ \\
\hline & $28064.90 \mathrm{C}$ & $6.0792 \mathrm{E}-05$ & $29063.90 \mathrm{C}$ & $1.5541 \mathrm{E}-05$ & $29065.90 \mathrm{C}$ & $6.9269 E-06$ \\
\hline & $33075.90 \mathrm{C}$ & $5.7170 \mathrm{E}-06$ & $41093.90 \mathrm{C}$ & $7.6838 \mathrm{E}-06$ & $42092.90 \mathrm{C}$ & $2 E-04$ \\
\hline & $42094.90 \mathrm{C}$ & $6.8828 \mathrm{E}-05$ & $42095.90 \mathrm{C}$ & $1.1846 \mathrm{E}-04$ & $42096.90 \mathrm{C}$ & 1.2411E-04 \\
\hline & $42097.90 \mathrm{C}$ & $7.1060 \mathrm{E}-05$ & $42098.90 \mathrm{C}$ & $1.7955 \mathrm{E}-04$ & $42100.90 \mathrm{C}$ & $7.1655 E-05$ \\
\hline & $73181.90 \mathrm{C}$ & $7.8904 \mathrm{E}-07$ & & & & \\
\hline C & Total & $6.0710 \mathrm{E}-02$ & & & & \\
\hline C & & & & & & \\
\hline & Lower $A$ & pter --- & & & & \\
\hline m21 & $5010.90 \mathrm{C}$ & $3.3079 \mathrm{E}-07$ & $5011.90 \mathrm{C}$ & $1.3315 \mathrm{E}-06$ & $6000.90 \mathrm{C}$ & $1.4962 \mathrm{E}-04$ \\
\hline & $7014.90 \mathrm{C}$ & $1.2783 \mathrm{E}-05$ & 7015 & $4.7214 \mathrm{E}-08$ & $11023.90 \mathrm{C}$ & $5.7990 E-03$ \\
\hline & $13027.90 \mathrm{C}$ & $3.3302 \mathrm{E}-05$ & $14028.90 \mathrm{C}$ & $4.4260 \mathrm{E}-04$ & $14029.90 \mathrm{C}$ & $2.2474 E-05$ \\
\hline & $14030.90 \mathrm{C}$ & $1.4815 \mathrm{E}-05$ & $15031.90 \mathrm{C}$ & $1.1604 \mathrm{E}-05$ & $16032.90 \mathrm{C}$ & $5.3203 E-06$ \\
\hline & $16033.90 \mathrm{C}$ & $4.2594 \mathrm{E}-08$ & $16034.90 \mathrm{C}$ & $2.4043 E-07$ & $16036.90 \mathrm{C}$ & $1.1209 \mathrm{E}-09$ \\
\hline & $23000.90 \mathrm{C}$ & $7.0554 \mathrm{E}-05$ & $24050.90 \mathrm{C}$ & $5.2559 \mathrm{E}-04$ & $24052.90 \mathrm{C}$ & $1.0136 \mathrm{E}-02$ \\
\hline & $24053.90 \mathrm{C}$ & $1.1493 \mathrm{E}-03$ & $24054.90 \mathrm{C}$ & $2.8608 \mathrm{E}-04$ & $25055.90 \mathrm{C}$ & $1.1449 \mathrm{E}-03$ \\
\hline & $26054.90 \mathrm{C}$ & $2.4098 E-03$ & $26056.90 \mathrm{C}$ & $3.7828 \mathrm{E}-02$ & $26057.90 \mathrm{C}$ & $8.7362 E-04$ \\
\hline & $26058.90 \mathrm{C}$ & $1.1626 \mathrm{E}-04$ & $27059.90 \mathrm{C}$ & $1.5247 \mathrm{E}-05$ & $28058.90 \mathrm{C}$ & $5.6278 E-03$ \\
\hline & $28060.90 \mathrm{C}$ & $2.1678 \mathrm{E}-03$ & $28061.90 \mathrm{C}$ & $9.4233 \mathrm{E}-05$ & $28062.90 \mathrm{C}$ & $3.0046 E-04$ \\
\hline & $28064.90 \mathrm{C}$ & $7.6517 \mathrm{E}-05$ & $29063.90 \mathrm{C}$ & $1.9561 \mathrm{E}-05$ & $29065.90 \mathrm{C}$ & $8.7186 E-06$ \\
\hline & $33075.90 \mathrm{C}$ & $7.1957 \mathrm{E}-06$ & $41093.90 \mathrm{C}$ & $9.6713 \mathrm{E}-06$ & $42092.90 \mathrm{C}$ & 1. $3898 E-04$ \\
\hline & $42094.90 \mathrm{C}$ & $8.6631 \mathrm{E}-05$ & $42095.90 \mathrm{C}$ & $0 E-04$ & $42096.90 \mathrm{C}$ & 1. $5622 \mathrm{E}-04$ \\
\hline & $42097.90 \mathrm{C}$ & $8.9441 \mathrm{E}-05$ & $42098.90 \mathrm{C}$ & $2.2599 \mathrm{E}-04$ & $42100.90 \mathrm{C}$ & $9.0190 \mathrm{E}-05$ \\
\hline & $73181.90 \mathrm{C}$ & $9.9313 \mathrm{E}-07$ & & & & \\
\hline C & Total & $7.0298 \mathrm{E}-02$ & & & & \\
\hline C & & & & & & \\
\hline & Reflector & r Blocks -- & & & & \\
\hline $\mathrm{m} 22$ & $5010.90 \mathrm{C}$ & $1.4214 \mathrm{E}-07$ & $5011.90 \mathrm{C}$ & $5.7214 \mathrm{E}-07$ & $6000.90 \mathrm{C}$ & 1. $2472 \mathrm{E}-03$ \\
\hline & $7014.90 \mathrm{C}$ & $5.4929 \mathrm{E}-06$ & 7015 & $2.0289 \mathrm{E}-08$ & $11023.90 \mathrm{C}$ & $2.3240 E-03$ \\
\hline & $13027.90 \mathrm{C}$ & $1.4310 \mathrm{E}-05$ & $14028.90 \mathrm{C}$ & $4.2347 \mathrm{E}-04$ & $14029.90 \mathrm{C}$ & $2.1503 E-05$ \\
\hline & 14030 & $1.4175 \mathrm{E}-05$ & 15031. & $4.9863 \mathrm{E}-06$ & $16032.90 \mathrm{C}$ & $8.5956 E-06$ \\
\hline & $16033.90 \mathrm{C}$ & $6.8816 \mathrm{E}-08$ & $16034.90 \mathrm{C}$ & $3.8845 \mathrm{E}-07$ & $16036.90 \mathrm{C}$ & $1.8109 E-09$ \\
\hline & $23000.90 \mathrm{C}$ & $3.0318 \mathrm{E}-05$ & $24050.90 \mathrm{C}$ & $5.9390 \mathrm{E}-04$ & $24052.90 \mathrm{C}$ & 1.1453E-02 \\
\hline & $24053.90 \mathrm{C}$ & $1.2987 \mathrm{E}-03$ & $24054.90 \mathrm{C}$ & $3.2326 \mathrm{E}-04$ & $25055.90 \mathrm{C}$ & $1.3971 E-03$ \\
\hline & $26054.90 \mathrm{C}$ & $1.2734 \mathrm{E}-03$ & $26056.90 \mathrm{C}$ & $1.9990 \mathrm{E}-02$ & $26057.90 \mathrm{C}$ & $4.6166 \mathrm{E}-04$ \\
\hline & $26058.90 \mathrm{C}$ & $6.1439 \mathrm{E}-05$ & $27059.90 \mathrm{C}$ & $5.4768 \mathrm{E}-05$ & $28058.90 \mathrm{C}$ & $2.6690 \mathrm{E}-02$ \\
\hline & $28060.90 \mathrm{C}$ & $1.0281 \mathrm{E}-02$ & $28061.90 \mathrm{C}$ & $4.4690 \mathrm{E}-04$ & $28062.90 \mathrm{C}$ & $1.4249 \mathrm{E}-03$ \\
\hline & $28064.90 \mathrm{C}$ & $3.6288 E-04$ & $29063.90 \mathrm{C}$ & $8.5732 \mathrm{E}-05$ & $29065.90 \mathrm{C}$ & $3.8212 E-05$ \\
\hline & $33075.90 \mathrm{C}$ & $3.0921 \mathrm{E}-06$ & $41093.90 \mathrm{C}$ & $4.1559 \mathrm{E}-06$ & $42092.90 \mathrm{C}$ & $5.9723 E-05$ \\
\hline & $42094.90 \mathrm{C}$ & $3.7226 \mathrm{E}-05$ & $42095.90 \mathrm{C}$ & $6.4070 \mathrm{E}-05$ & $42096.90 \mathrm{C}$ & $6.7128 E-05$ \\
\hline & $42097.90 \mathrm{C}$ & $3.8434 \mathrm{E}-05$ & $42098.90 \mathrm{C}$ & $9.7111 \mathrm{E}-05$ & $42100.90 \mathrm{C}$ & $3.8756 \mathrm{E}-05$ \\
\hline & $73181.90 \mathrm{C}$ & $4.2676 \mathrm{E}-07$ & & & & \\
\hline C & Total & $8.0742 \mathrm{E}-02$ & & & & \\
\hline C & & & & & & \\
\hline & Load Pad & Region --- & & & & \\
\hline m23 & $5010.90 \mathrm{C}$ & 4.0109E-07 & $5011.90 \mathrm{C}$ & $1.6144 \mathrm{E}-06$ & $6000.90 \mathrm{C}$ & $1.8142 \mathrm{E}-04$ \\
\hline & 7014 & $1.5499 \mathrm{E}-05$ & 7015 & $5.7249 \mathrm{E}-08$ & $11023.90 \mathrm{C}$ & $2.0070 \mathrm{E}$ \\
\hline & $13027.90 \mathrm{C}$ & $4.0379 E-05$ & 14028 & $5.3667 \mathrm{E}-04$ & $14029.90 \mathrm{C}$ & $2.7251 E-05$ \\
\hline & $14030.90 \mathrm{C}$ & $1.7964 \mathrm{E}-05$ & $15031.90 \mathrm{C}$ & $1.4070 \mathrm{E}-05$ & $16032.90 \mathrm{C}$ & $6.4510 \mathrm{E}-06$ \\
\hline & $16033.90 \mathrm{C}$ & $5.1646 \mathrm{E}-08$ & $16034.90 \mathrm{C}$ & $2.9153 \mathrm{E}-07$ & $16036.90 \mathrm{C}$ & $1.3591 \mathrm{E}-09$ \\
\hline & $23000.90 \mathrm{C}$ & $8.5549 \mathrm{E}-05$ & $24050.90 \mathrm{C}$ & $6.3730 \mathrm{E}-04$ & $24052.90 \mathrm{C}$ & $1.2290 \mathrm{E}-02$ \\
\hline
\end{tabular}

Revision: 0

Page 242 of 304

Date: March 31, 2010 
NEA/NSC/DOC(2006)1

\section{Liquid Metal Fast Reactor - LMFR}

FFTF-LMFR-RESR-001 CRIT-SPEC-REAC-COEF-MISC

\footnotetext{
$24053.90 \mathrm{C} 1.3935 \mathrm{E}-03$ $26054.90 \mathrm{C} 2.9219 \mathrm{E}-03$ $26058.90 \mathrm{C} 1.4097 \mathrm{E}-04$ $28060.90 \mathrm{C} 2.6285 \mathrm{E}-03$ $28064.90 \mathrm{C} 9.2779 \mathrm{E}-05$ $33075.90 \mathrm{C} 8.7251 \mathrm{E}-06$ $42094.90 \mathrm{C} 1.0504 \mathrm{E}-04$ $42097.90 \mathrm{C} 1.0845 \mathrm{E}-04$ $73181.90 \mathrm{C} 1.2042 \mathrm{E}-06$$$
\text { C }
$$

$5011.90 \mathrm{C} \quad 1.0387 \mathrm{E}-06$ $7015.90 \mathrm{C} 3.6832 \mathrm{E}-08$ $14028.90 \mathrm{C} \quad 3.4527 \mathrm{E}-04$ $15031.90 \mathrm{C} 9.0521 \mathrm{E}-06$ $16034.90 \mathrm{C} 1.8756 \mathrm{E}-07$ $24050.90 \mathrm{C} 4.1002 \mathrm{E}-04$ $24054.90 \mathrm{C} 2.2317 \mathrm{E}-04$ $26056.90 \mathrm{C} 2.9510 \mathrm{E}-02$ $27059.90 \mathrm{C} 1.1894 \mathrm{E}-05$ $28061.90 \mathrm{C} 7.3512 \mathrm{E}-05$ $29063.90 \mathrm{C} 1.5260 \mathrm{E}-05$ $41093.90 \mathrm{C} 7.5446 \mathrm{E}-06$ $42095.90 \mathrm{C}$ 1.1631E-04 $42098.90 \mathrm{C} 1.7630 \mathrm{E}-04$
}

$25055.90 \mathrm{C} \quad 1.3882 \mathrm{E}-03$ $26057.90 \mathrm{C} 1.0593 \mathrm{E}-03$ $28058.90 \mathrm{C} 6.8238 \mathrm{E}-03$ $28062.90 \mathrm{C} 3.6431 \mathrm{E}-04$ $29065.90 \mathrm{C} 1.0572 \mathrm{E}-05$ $42092.90 \mathrm{C} 1.6852 \mathrm{E}-04$ $42096.90 \mathrm{C} 1.8942 \mathrm{E}-04$ $42100.90 \mathrm{C} 1.0936 \mathrm{E}-04$

$6000.90 \mathrm{C} \quad 1.7904 \mathrm{E}-04$ $11023.90 \mathrm{C} 2.2910 \mathrm{E}-03$ $14029.90 \mathrm{C} 2.6893 \mathrm{E}-05$ $16032.90 \mathrm{C} 6.3664 \mathrm{E}-06$ $16036.90 \mathrm{C} 1.3413 \mathrm{E}-09$ $24052.90 \mathrm{C} 1.2129 \mathrm{E}-02$ $25055.90 \mathrm{C} 1.3700 \mathrm{E}-03$ $26057.90 \mathrm{C} 1.0454 \mathrm{E}-03$ $28058.90 \mathrm{C} \quad 6.7344 \mathrm{E}-03$ $28062.90 \mathrm{C} \quad 3.5954 \mathrm{E}-04$ $29065.90 \mathrm{C} 1.0433 \mathrm{E}-05$ $42092.90 \mathrm{C} 1.6631 \mathrm{E}-04$ $42096.90 \mathrm{C} 1.8694 \mathrm{E}-04$ $42100.90 \mathrm{C} 1.0792 \mathrm{E}-04$

$6000.90 \mathrm{C} 1.9054 \mathrm{E}-04$ $11023.90 \mathrm{C} 1.4160 \mathrm{E}-03$ $14029.90 \mathrm{C} 2.8620 \mathrm{E}-05$ $16032.90 \mathrm{C} 6.7752 \mathrm{E}-06$ $16036.90 \mathrm{C} 1.4274 \mathrm{E}-09$ $24052.90 \mathrm{C} 1.2907 \mathrm{E}-02$ $25055.90 \mathrm{C} 1.4579 \mathrm{E}-03$ $26057.90 \mathrm{C} 1.1125 \mathrm{E}-03$ $28058.90 \mathrm{C} \quad 7.1667 \mathrm{E}-03$ $28062.90 \mathrm{C} 3.8262 \mathrm{E}-04$ $29065.90 \mathrm{C} 1.1103 \mathrm{E}-05$ $42092.90 \mathrm{C} 1.7699 \mathrm{E}-04$ $42096.90 \mathrm{C} 1.9894 \mathrm{E}-04$ $42100.90 \mathrm{C} 1.1485 \mathrm{E}-04$

$6000.90 \mathrm{C} 8.2135 \mathrm{E}-05$ $11023.90 \mathrm{C} 1.3847 \mathrm{E}-02$ $14029.90 \mathrm{C} 1.2337 \mathrm{E}-05$ $16032.90 \mathrm{C} 2.9206 \mathrm{E}-06$ $16036.90 \mathrm{C} 6.1531 \mathrm{E}-10$ $24052.90 \mathrm{C} 5.5639 \mathrm{E}-03$ $25055.90 \mathrm{C} 6.2848 \mathrm{E}-04$ $26057.90 \mathrm{C} 4.7958 \mathrm{E}-04$ $28058.90 \mathrm{C} 3.0894 \mathrm{E}-03$ $28062.90 \mathrm{C} 1.6494 \mathrm{E}-04$ $29065.90 \mathrm{C} 4.7861 \mathrm{E}-06$ $42092.90 \mathrm{C} 7.6296 \mathrm{E}-05$ 42096 $42100.90 \mathrm{C} 4.9510 \mathrm{E}-05$

$6000.90 \mathrm{C} \quad 1.1672 \mathrm{E}-04$ $11023.90 \mathrm{C} 9.7230 \mathrm{E}-03$ $14029.90 \mathrm{C} 1.7532 \mathrm{E}-05$ $16032.90 \mathrm{C} 4.1504 \mathrm{E}-06$ $16036.90 \mathrm{C} 8.7441 \mathrm{E}-10$ $24052.90 \mathrm{C} 7.9068 \mathrm{E}-03$ $25055.90 \mathrm{C} 8.9312 \mathrm{E}-04$ $26057.90 \mathrm{C} 6.8151 \mathrm{E}-04$ $28058.90 \mathrm{C} 4.3902 \mathrm{E}-03$ $28062.90 \mathrm{C} 2.3439 \mathrm{E}-04$ $29065.90 \mathrm{C} 6.8014 \mathrm{E}-06$ 42092.90C 1.0842E-04 $42096.90 \mathrm{C} 1.2187 \mathrm{E}-04$ $42100.90 \mathrm{C} 7.0358 \mathrm{E}-05$ 
NEA/NSC/DOC(2006)1

\section{Liquid Metal Fast Reactor - LMFR}

FFTF-LMFR-RESR-001 CRIT-SPEC-REAC-COEF-MISC

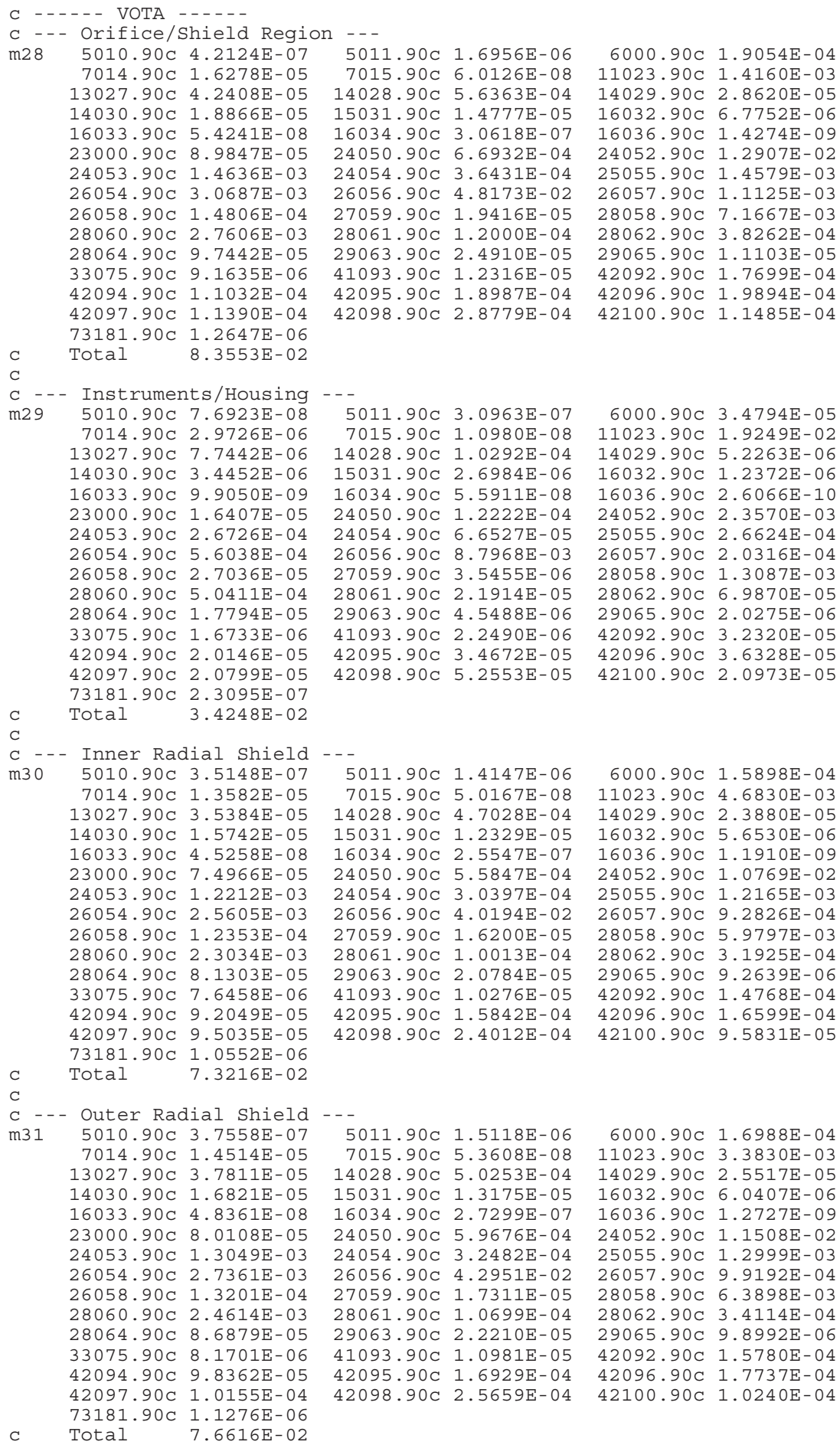
$42100.90 \mathrm{C} 9.5831 \mathrm{E}-05$

$6000.90 \mathrm{C} \quad 1.9054 \mathrm{E}-04$ $11023.90 \mathrm{C} 1.4160 \mathrm{E}-03$ $14029.90 \mathrm{C} 2.8620 \mathrm{E}-05$ $16032.90 \mathrm{C} 6.7752 \mathrm{E}-06$ $16036.90 \mathrm{C} 1.4274 \mathrm{E}-09$ $24052.90 \mathrm{C} 1.2907 \mathrm{E}-02$ 25055.90C 1.4579E-03 $26057.90 \mathrm{C} 1.1125 \mathrm{E}-03$ $28058.90 \mathrm{C} 7.1667 \mathrm{E}-03$ $28062.90 \mathrm{C} 3.8262 \mathrm{E}-04$ $29065.90 \mathrm{C} 1.1103 \mathrm{E}-05$ $42092.90 \mathrm{C} 1.7699 \mathrm{E}-04$ $42096.90 \mathrm{C} 1.9894 \mathrm{E}-04$ $42100.90 \mathrm{C} 1.1485 \mathrm{E}-04$

$6000.90 \mathrm{C} 3.4794 \mathrm{E}-05$ $11023.90 \mathrm{C} 1.9249 \mathrm{E}-02$ $14029.90 \mathrm{C} 5.2263 \mathrm{E}-06$ $16032.90 \mathrm{C} 1.2372 \mathrm{E}-06$ $16036.90 \mathrm{C} 2.6066 \mathrm{E}-10$ $24052.90 \mathrm{C} 2.3570 \mathrm{E}-03$ $25055.90 \mathrm{C} 2.6624 \mathrm{E}-04$ $26057.90 \mathrm{C} 2.0316 \mathrm{E}-04$ $28058.90 \mathrm{C} 1.3087 \mathrm{E}-03$ $28062.90 \mathrm{C} 6.9870 \mathrm{E}-05$ $29065.90 \mathrm{C} 2.0275 \mathrm{E}-06$ $42092.90 \mathrm{C} 3.2320 \mathrm{E}-05$ $42096.90 \mathrm{C} 3.6328 \mathrm{E}-05$ $42100.90 \mathrm{C} 2.0973 \mathrm{E}-05$

$6000.90 \mathrm{C} \quad 1.5898 \mathrm{E}-04$ $11023.90 \mathrm{C} 4.6830 \mathrm{E}-03$ $14029.90 \mathrm{C} 2.3880 \mathrm{E}-05$ $16032.90 \mathrm{C} 5.6530 \mathrm{E}-06$ $16036.90 \mathrm{C} 1.1910 \mathrm{E}-09$ $24052.90 \mathrm{C} 1.0769 \mathrm{E}-02$ $25055.90 \mathrm{C} 1.2165 \mathrm{E}-03$ $26057.90 \mathrm{C} 9.2826 \mathrm{E}-04$ $28058.90 \mathrm{C} 5.9797 \mathrm{E}-03$ $28062.90 \mathrm{C} 3.1925 \mathrm{E}-04$ $29065.90 \mathrm{C} 9.2639 \mathrm{E}-06$ $42092.90 \mathrm{C}$ 1.4768E-04

$6000.90 \mathrm{C} \quad 1.6988 \mathrm{E}-04$ $11023.90 \mathrm{C} 3.3830 \mathrm{E}-03$ $14029.90 \mathrm{C} 2.5517 \mathrm{E}-05$ $16032.90 \mathrm{C} 6.0407 \mathrm{E}-06$ $16036.90 \mathrm{C} 1.2727 \mathrm{E}-09$ $24052.90 \mathrm{C} 1.1508 \mathrm{E}-02$ $25055.90 \mathrm{C} 1.2999 \mathrm{E}-03$ 26057.90C 9.9192E-04 $28058.90 \mathrm{C} \quad 6.3898 \mathrm{E}-03$ $28062.90 \mathrm{C} 3.4114 \mathrm{E}-04$ $29065.90 \mathrm{C} 9.8992 \mathrm{E}-06$ $42092.90 \mathrm{C} 1.5780 \mathrm{E}-04$ $42096.90 \mathrm{C} 1.7737 \mathrm{E}-04$ $42100.90 \mathrm{C} 1.0240 \mathrm{E}-04$ 
NEA/NSC/DOC(2006)1

\title{
Liquid Metal Fast Reactor - LMFR
}

FFTF-LMFR-RESR-001 CRIT-SPEC-REAC-COEF-MISC

\author{
$24053.90 \mathrm{C} 1.1216 \mathrm{E}-04$ \\ $26054.90 \mathrm{C} 2.3518 \mathrm{E}-04$ \\ $26058.90 \mathrm{C} 1.1347 \mathrm{E}-05$ \\ $28060.90 \mathrm{C} 2.1156 \mathrm{E}-04$ \\ $28064.90 \mathrm{C} 7.4676 \mathrm{E}-06$ \\ $33075.90 \mathrm{C} 7.0226 \mathrm{E}-07$ \\ $42094.90 \mathrm{C} 8.4547 \mathrm{E}-06$ \\ $42097.90 \mathrm{C} 8.7289 \mathrm{E}-06$ \\ $73181.90 \mathrm{C} 9.6924 \mathrm{E}-08$ \\ C Total $2.8171 \mathrm{E}-02$

Revision: 0

Page 245 of 304

Date: March 31, 2010 
NEA/NSC/DOC(2006)1

Liquid Metal Fast Reactor - LMFR

FFTF-LMFR-RESR-001

CRIT-SPEC-REAC-COEF-MISC

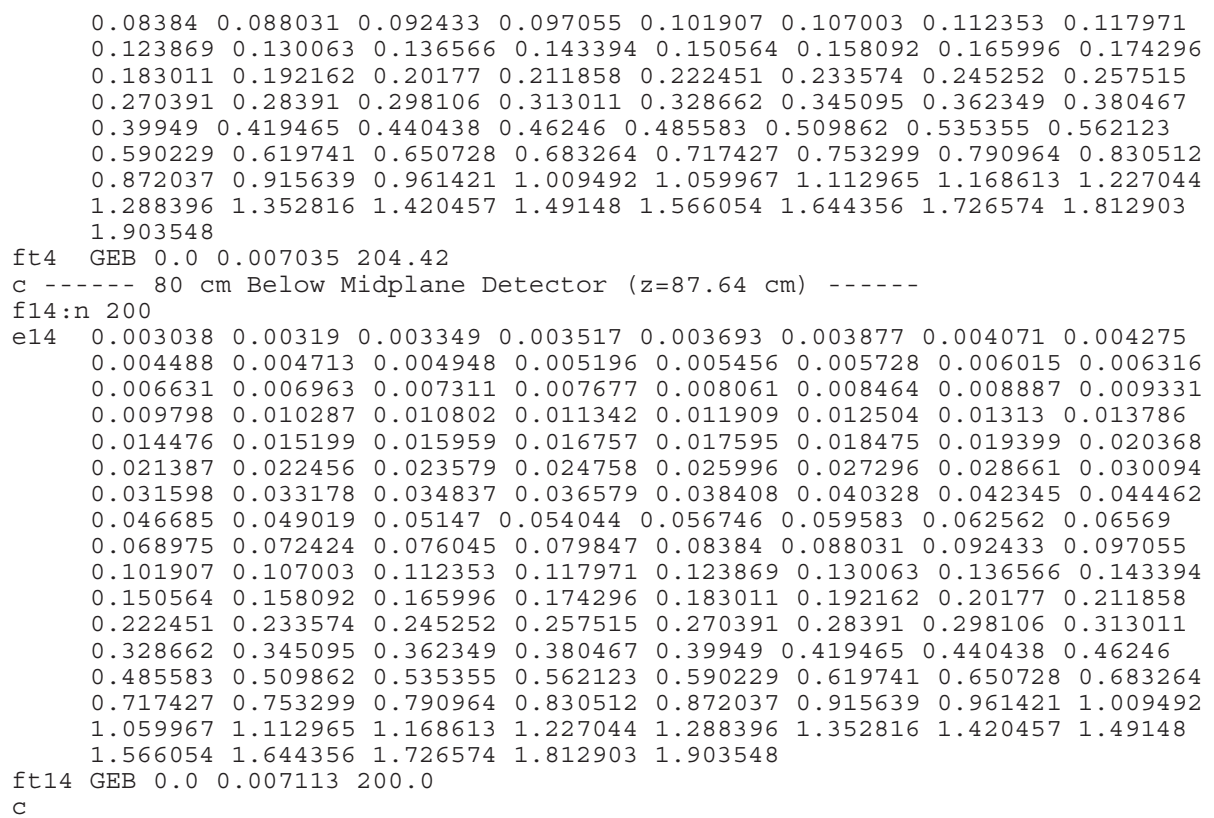

A.4 Reactivity-Effects Configurations

MCNP5 Input for Evaluating Control Rod Worths:

The input decks for analysis of the control rod worths are that of the fully-loaded critical configuration with control rod adjustments discussed in Section 3.4.2.1.

MCNP5 Input for Evaluating Control Rod Bank Worths:

The input decks for analysis of the control rod bank worths are that of the fully-loaded critical configuration with control rod adjustments discussed in Section 3.4.2.2.

\section{MCNP5 Input for Evaluating Differential Control Rod Worths:}

The input decks for analysis of the differential control rod worths are that of the fully-loaded critical configuration with control rod adjustments discussed in Section 3.4.2.3.

MCNP5 Input for Evaluating Shutdown Margin:

The input decks for analysis of the shutdown margin are that of the fully-loaded critical configuration with control rod adjustments discussed in Section 3.4.2.4.

MCNP5 Input for Evaluating Excess Reactivity:

The input decks for analysis of excess reactivity are that of the fully-loaded critical configuration with control rod adjustments discussed in Section 3.4.2.5.

\section{A.5 Reactivity Coefficient Configurations}

MCNP5 Input for Evaluating the Isothermal Temperature Coefficient:

Revision: 0

Page 246 of 304

Date: March 31, 2010 
NEA/NSC/DOC(2006)1

\author{
Liquid Metal Fast Reactor - LMFR \\ FFTF-LMFR-RESR-001 \\ CRIT-SPEC-REAC-COEF-MISC
}

The input decks for analysis of the isothermal temperature coefficient are that of the fully-loaded critical configuration discussed in Section 3.5.4 with temperature adjustments.

Example NJOY Input File for Processing Cross Section Data at 455 and $505 \mathrm{~K}$

The input files for processing of neutron cross section libraries at 455 and $505 \mathrm{~K}$ are identical to that used for processing the data at $480 \mathrm{~K}$ but at the desired temperature.

\title{
A.6 Kinetics Parameter Configurations
}

Kinetics measurements were not made.

\section{A.7 Reaction-Rate Configurations}

Reaction-rate distribution measurements were not evaluated.

\section{A.8 Power Distribution Configuration}

Power distribution measurements were not made.

\section{A.9 Isotopic Configurations}

Isotopic measurements were not made.

\section{A.10 Configurations of Other Miscellaneous Types of Measurements}

\section{MCNP5 Input Deck for Evaluating the Low-Energy Electron and Gamma-Ray Spectra:}

The input deck for analysis of the low-energy electron and gamma-ray spectra is that of the fully-loaded critical configuration (Appendix A.1) with the following mode change and tally-card specifications appended to the end of the input deck:

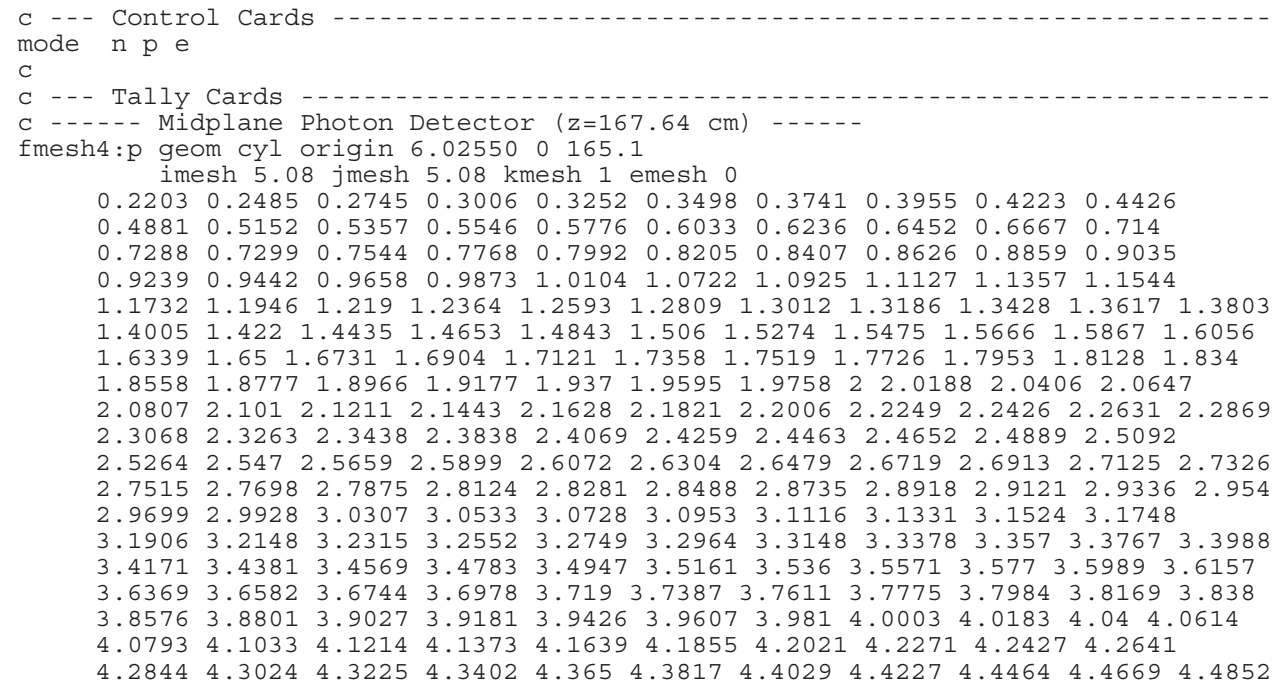

Revision: 0

Page 247 of 304

Date: March 31, 2010 
NEA/NSC/DOC(2006)1

Liquid Metal Fast Reactor - LMFR

FFTF-LMFR-RESR-001

CRIT-SPEC-REAC-COEF-MISC

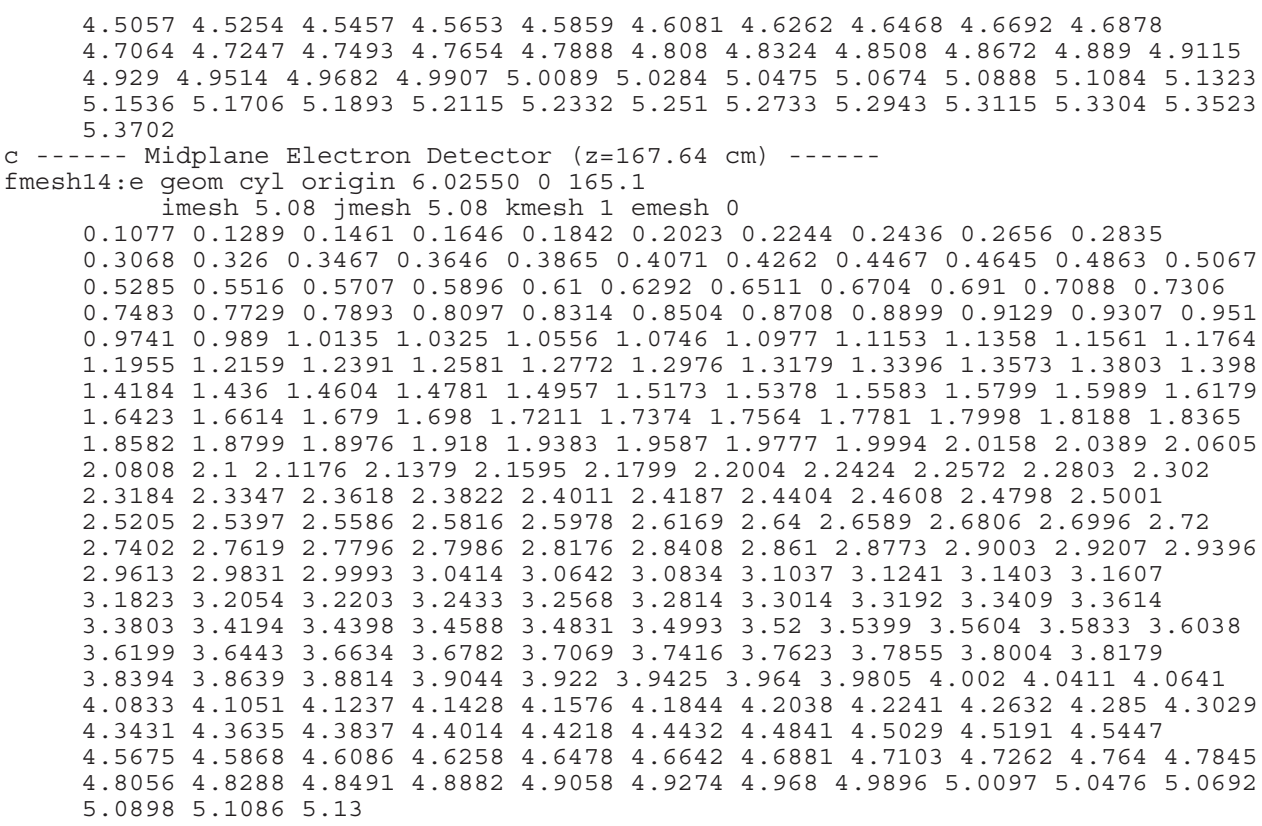

Revision: 0 
NEA/NSC/DOC(2006)1

Liquid Metal Fast Reactor - LMFR

FFTF-LMFR-RESR-001

CRIT-SPEC-REAC-COEF-MISC

\section{APPENDIX B: ADDITIONAL ILLUSTRATIONS OF INTEREST}

\section{B.1 Illustration Archive}

During the course of identifying experimental data for the benchmark, some additional figures or photographs were located and included in this appendix.

Figure B.1.1 is a map of the Hanford site and shows the location of the FFTF near the lower right-hand corner. The Hanford site ( 586 sq. miles) is located in southeastern Washington, United States, near the Columbia River. ${ }^{\mathrm{a}}$

Figure B.1.2 is a scanned copy of a foldout from a 1985 edition of Nuclear Engineering International. ${ }^{\mathrm{b}}$

Figure B.1.3 is a scanned copy of a compilation of photographs of the FFTF.

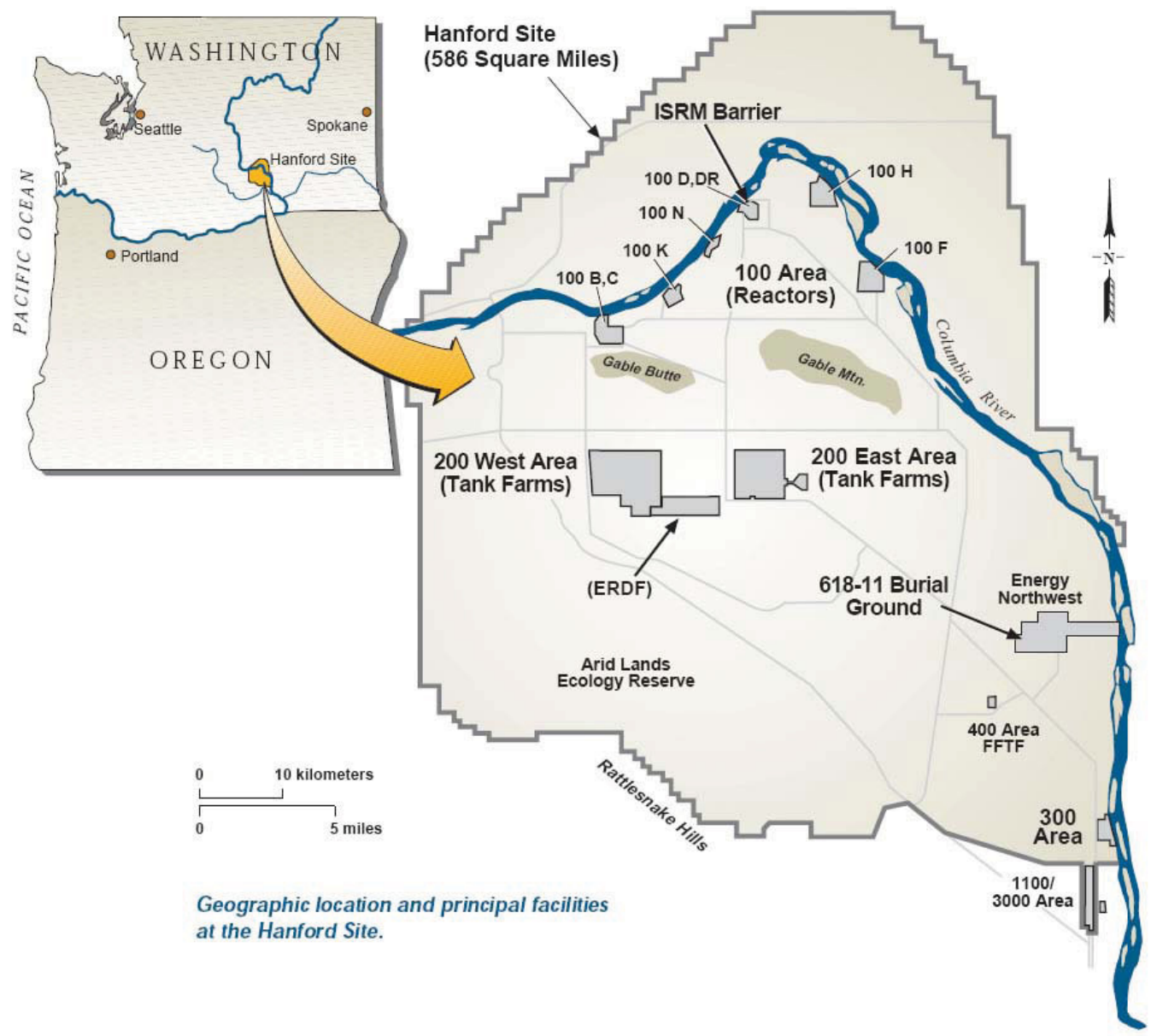

Figure B.1.1. Geographic Location and Principal Facilities at the Hanford Site. ${ }^{a}$

\footnotetext{
${ }^{\text {a }}$ State of Oregon, Nuclear Safety, http://www.oregon.gov/ENERGY/NUCSAF/index.shtml, Accessed 07/16/2009.
}

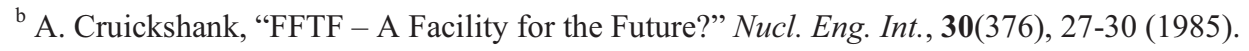




\section{FFTF (FAST FLUX TEST FACILITY)}

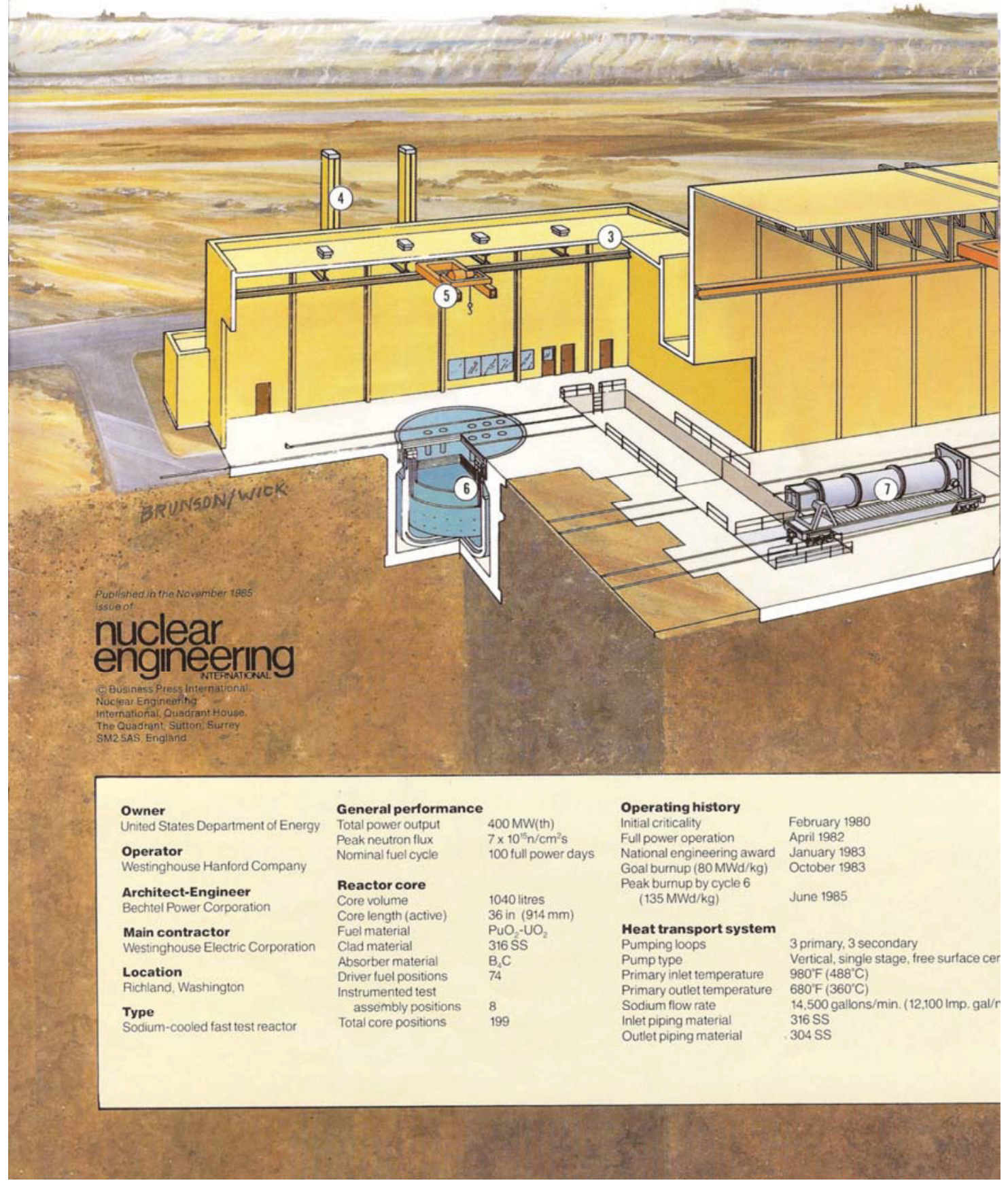

Figure B.1.2. Foldout of the FFTF. ${ }^{\mathrm{a}}$

a A. Cruickshank, "FFTF - A Facility for the Future?" Nucl. Eng. Int., 30(376), 27-30 (1985).

Revision: 0 
FFTF-LMFR-RESR-001

\section{CRIT-SPEC-REAC-COEF-MISC}

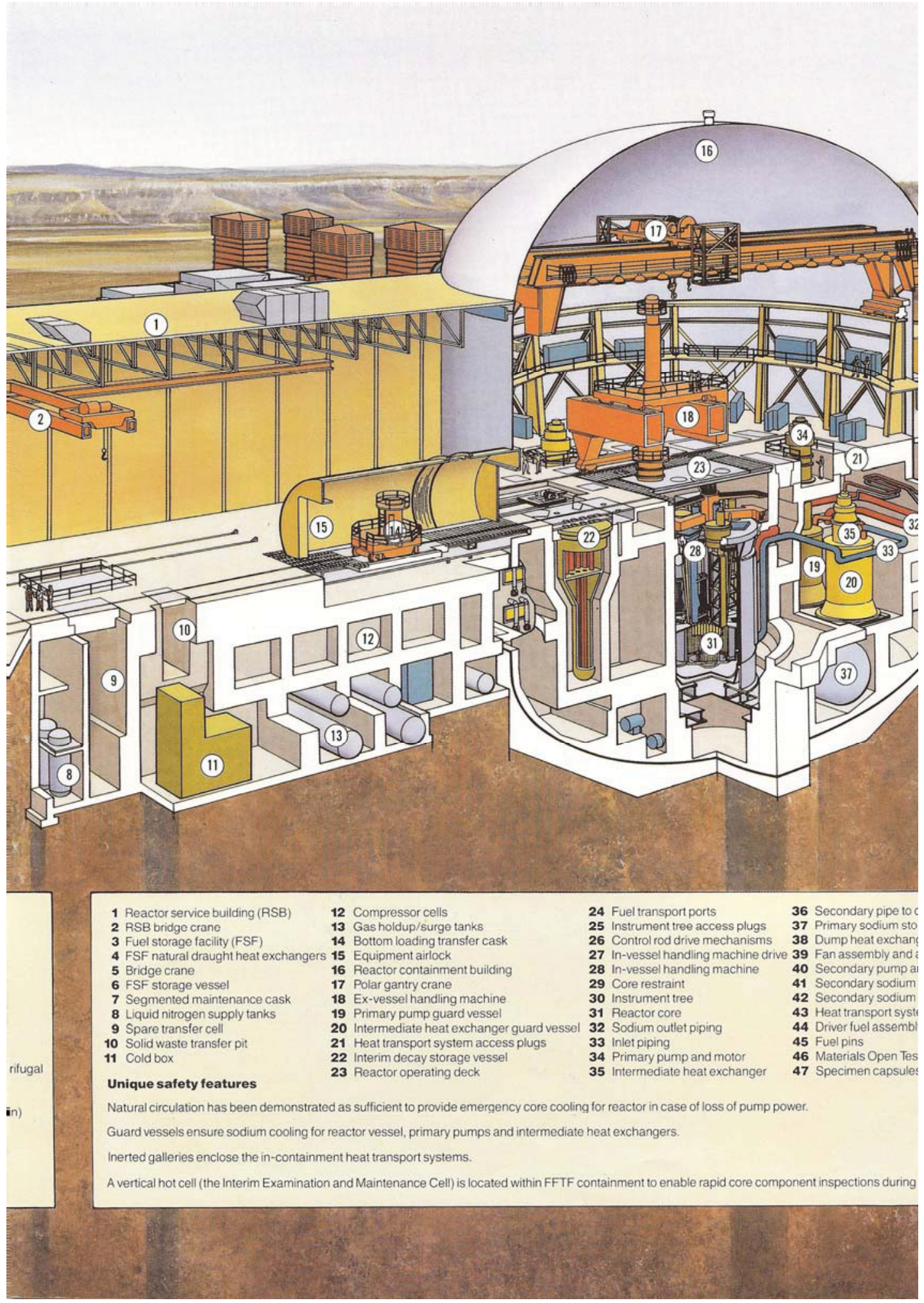

Figure B.1.2 (cont'd.). Foldout of the FFTF. ${ }^{\mathrm{a}}$

a A. Cruickshank, "FFTF - A Facility for the Future?" Nucl. Eng. Int., 30(376), 27-30 (1985).

Revision: 0 
NEA/NSC/DOC(2006)1

Liquid Metal Fast Reactor - LMFR

FFTF-LMFR-RESR-001

CRIT-SPEC-REAC-COEF-MISC

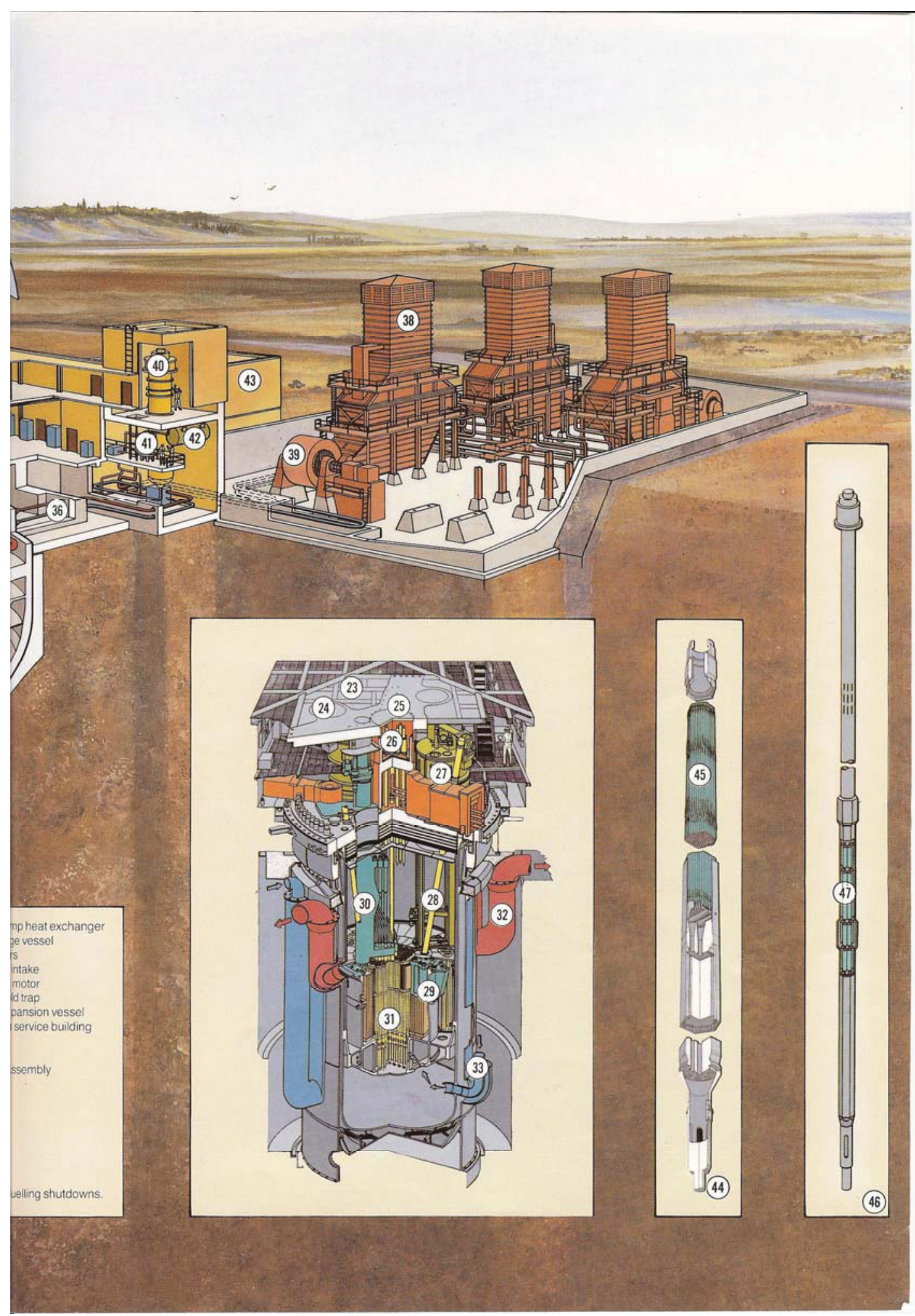

Figure B.1.2 (cont'd.). Foldout of the FFTF. ${ }^{\mathrm{a}}$

${ }^{a}$ A. Cruickshank, "FFTF - A Facility for the Future?" Nucl. Eng. Int., 30(376), 27-30 (1985).

Revision: 0

Page 252 of 304

Date: March 31, 2010 
NEA/NSC/DOC(2006)1

Liquid Metal Fast Reactor - LMFR

FFTF-LMFR-RESR-001

CRIT-SPEC-REAC-COEF-MISC
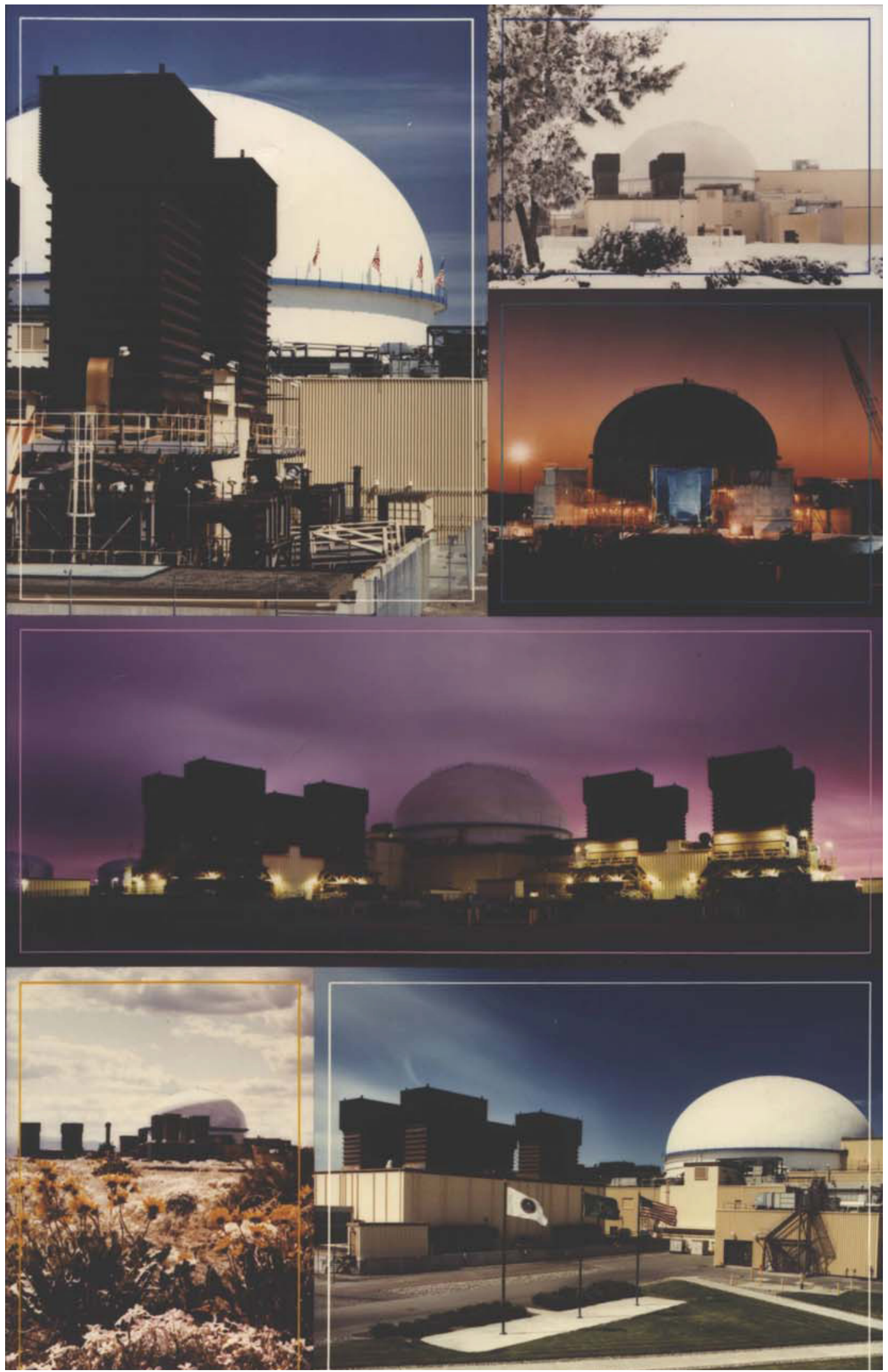

Figure B.1.3. Photographs of the FFTF. 
FFTF-LMFR-RESR-001

CRIT-SPEC-REAC-COEF-MISC

\section{APPENDIX C: CALCULATED SPECTRAL DATA}

\section{C.1 Spectral Data for the Critical and Subcritical Configurations}

Data generated in the MCNP5 output files include information regarding the energy of the average lethargy causing fission (EALF) and the percentages of fission caused by thermal, intermediate, and fast energy neutrons for each case shown in Table 4.1.1; results are shown in Table C.1.1 for the fully-loaded critical configuration.

Table C.1.1. Spectral Data for the FFTF Fully-Loaded Core Critical.

\begin{tabular}{||c|c|c|c|c||}
\hline \hline $\begin{array}{c}\text { Neutron } \\
\text { Cross section } \\
\text { Library }\end{array}$ & \multirow{2}{*}{$\begin{array}{c}\text { EALF } \\
(\mathrm{MeV})\end{array}$} & \multicolumn{3}{|c|}{ Percentage of Neutrons Causing Fission } \\
$<0.625 \mathrm{eV}$ & $\begin{array}{c}0.625 \mathrm{eV}- \\
100 \mathrm{keV}\end{array}$ & $>100 \mathrm{keV}$ \\
\hline \hline END/B-VII.0 & 0.100 & 0.01 & 40.97 & 59.02 \\
\hline
\end{tabular}


NEA/NSC/DOC(2006)1

Liquid Metal Fast Reactor - LMFR

FFTF-LMFR-RESR-001

CRIT-SPEC-REAC-COEF-MISC

\section{APPENDIX D: DATA FROM THE $16^{\mathrm{TH}}$ EDITION CHART OF THE NUCLIDES ${ }^{\mathrm{a}}$}

\section{D.1 Isotopic Abundances and Atomic Weights}

This evaluation incorporated atomic weights and isotopic abundances found in the $16^{\text {th }}$ edition of the Chart of the Nuclides. A list of the values used in the benchmark model or in the generation of the MCNP input deck is compiled in Table D.1.

Table D.1. Summary of Data Employed from the $16^{\text {th }}$ Ed. of the Chart of the Nuclides.

\begin{tabular}{|c|c|c|c|c|c|}
\hline $\begin{array}{c}\text { Isotope or } \\
\text { Element }\end{array}$ & $\begin{array}{l}\text { Atomic } \\
\text { Weight } \\
\end{array}$ & $\begin{array}{c}\text { Isotopic } \\
\text { Abundance } \\
\end{array}$ & $\begin{array}{c}\text { Isotope or } \\
\text { Element }\end{array}$ & $\begin{array}{l}\text { Atomic } \\
\text { Weight } \\
\end{array}$ & $\begin{array}{c}\text { Isotopic } \\
\text { Abundance } \\
\end{array}$ \\
\hline $\mathrm{H}$ & 1.00794 & -- & $\mathrm{Mg}$ & 24.305 & -- \\
\hline${ }^{1} \mathrm{H}$ & -- & 99.9885 & ${ }^{24} \mathrm{Mg}$ & -- & 78.99 \\
\hline${ }^{2} \mathrm{H}$ & -- & 0.0115 & ${ }^{25} \mathrm{Mg}$ & -- & 10 \\
\hline $\mathrm{He}$ & 4.002602 & -- & ${ }^{26} \mathrm{Mg}$ & -- & 11.01 \\
\hline${ }^{3} \mathrm{He}$ & -- & 0.000137 & $\mathrm{Al}$ & 26.981538 & -- \\
\hline${ }^{4} \mathrm{He}$ & -- & 99.999863 & $\mathrm{Si}$ & 28.0855 & -- \\
\hline $\mathrm{Li}$ & 6.941 & -- & ${ }^{28} \mathrm{Si}$ & -- & 92.2297 \\
\hline${ }^{6} \mathrm{Li}$ & -- & 7.59 & ${ }^{29} \mathrm{Si}$ & -- & 4.6832 \\
\hline${ }^{7} \mathrm{Li}$ & -- & 92.41 & ${ }^{30} \mathrm{Si}$ & -- & 3.0872 \\
\hline $\mathrm{Be}$ & 9.012182 & -- & $\mathrm{P}$ & 30.973761 & -- \\
\hline B & 10.811 & -- & S & 32.065 & -- \\
\hline${ }^{10} \mathrm{~B}$ & 10.0129370 & 19.9 & ${ }^{32} \mathrm{~S}$ & -- & 94.93 \\
\hline${ }^{11} \mathrm{~B}$ & 11.0093055 & 80.1 & ${ }^{33} \mathrm{~S}$ & -- & 0.76 \\
\hline $\mathrm{C}$ & 12.0107 & -- & ${ }^{34} \mathrm{~S}$ & -- & 4.29 \\
\hline $\mathrm{N}$ & 14.0067 & -- & ${ }^{36} \mathrm{~S}$ & -- & 0.02 \\
\hline${ }^{14} \mathrm{~N}$ & -- & 99.632 & $\mathrm{Cl}$ & 35.453 & -- \\
\hline${ }^{15} \mathrm{~N}$ & -- & 0.368 & ${ }^{35} \mathrm{Cl}$ & -- & 75.78 \\
\hline $\mathrm{O}$ & 15.9994 & -- & ${ }^{37} \mathrm{Cl}$ & -- & 24.22 \\
\hline${ }^{16} \mathrm{O}$ & -- & 99.757 & K & 39.0983 & -- \\
\hline${ }^{17} \mathrm{O}$ & -- & 0.038 & ${ }^{39} \mathrm{~K}$ & -- & 93.2581 \\
\hline${ }^{18} \mathrm{O}^{\mathrm{a}}$ & -- & 0.205 & ${ }^{40} \mathrm{~K}$ & -- & 0.0117 \\
\hline $\mathrm{F}$ & 18.9984032 & -- & ${ }^{41} \mathrm{~K}$ & -- & 6.7302 \\
\hline $\mathrm{Na}$ & 22.989770 & -- & & & \\
\hline
\end{tabular}

(a) Neutronically, ${ }^{18} \mathrm{O}$ is treated as ${ }^{16} \mathrm{O}$.

${ }^{\mathrm{a}}$ Nuclides and Isotopes: Chart of the Nuclides, $16^{\text {th }}$ edition, (2002). 
NEA/NSC/DOC(2006)1

Liquid Metal Fast Reactor - LMFR

FFTF-LMFR-RESR-001

CRIT-SPEC-REAC-COEF-MISC

Table D.1 (cont'd). Summary of Data Employed from the $16^{\text {th }}$ Ed. of the Chart of the Nuclides.

\begin{tabular}{|c|c|c|c|c|c|}
\hline $\begin{array}{c}\text { Isotope or } \\
\text { Element }\end{array}$ & $\begin{array}{l}\text { Atomic } \\
\text { Weight }\end{array}$ & $\begin{array}{c}\text { Isotopic } \\
\text { Abundance }\end{array}$ & $\begin{array}{c}\text { Isotope or } \\
\text { Element }\end{array}$ & $\begin{array}{l}\text { Atomic } \\
\text { Weight }\end{array}$ & $\begin{array}{c}\text { Isotopic } \\
\text { Abundance }\end{array}$ \\
\hline $\mathrm{Ca}$ & 40.078 & -- & ${ }^{65} \mathrm{Cu}$ & -- & 30.83 \\
\hline${ }^{40} \mathrm{Ca}$ & -- & 96.941 & $\mathrm{Zn}$ & 65.409 & -- \\
\hline${ }^{42} \mathrm{Ca}$ & -- & 0.647 & As & 74.9216 & -- \\
\hline${ }^{43} \mathrm{Ca}$ & -- & 0.135 & $\mathrm{Rb}$ & 85.4678 & -- \\
\hline${ }^{44} \mathrm{Ca}$ & -- & 2.086 & ${ }^{85} \mathrm{Rb}$ & -- & 72.17 \\
\hline${ }^{46} \mathrm{Ca}$ & -- & 0.004 & ${ }^{87} \mathrm{Rb}$ & -- & 27.83 \\
\hline${ }^{48} \mathrm{Ca}$ & -- & 0.187 & $\mathrm{Zr}$ & 91.224 & -- \\
\hline $\mathrm{Ti}$ & 47.867 & -- & ${ }^{90} \mathrm{Zr}$ & -- & 51.45 \\
\hline${ }^{46} \mathrm{Ti}$ & -- & 8.25 & ${ }^{91} \mathrm{Zr}$ & -- & 11.22 \\
\hline${ }^{47} \mathrm{Ti}$ & -- & 7.44 & ${ }^{92} \mathrm{Zr}$ & -- & 17.15 \\
\hline${ }^{48} \mathrm{Ti}$ & -- & 73.72 & ${ }^{94} \mathrm{Zr}$ & -- & 17.38 \\
\hline${ }^{49} \mathrm{Ti}$ & -- & 5.41 & ${ }^{96} \mathrm{Zr}$ & -- & 2.8 \\
\hline${ }^{50} \mathrm{Ti}$ & -- & 5.18 & $\mathrm{Nb}$ & 92.90638 & -- \\
\hline $\mathrm{V}$ & 50.9415 & -- & Mo & 95.94 & -- \\
\hline $\mathrm{Cr}$ & 51.9961 & -- & ${ }^{92} \mathrm{Mo}$ & -- & 14.84 \\
\hline${ }^{50} \mathrm{Cr}$ & -- & 4.345 & ${ }^{94} \mathrm{Mo}$ & -- & 9.25 \\
\hline${ }^{52} \mathrm{Cr}$ & -- & 83.789 & ${ }^{95} \mathrm{Mo}$ & -- & 15.92 \\
\hline${ }^{53} \mathrm{Cr}$ & -- & 9.501 & ${ }^{96} \mathrm{Mo}$ & -- & 16.68 \\
\hline${ }^{54} \mathrm{Cr}$ & -- & 2.365 & ${ }^{97} \mathrm{Mo}$ & -- & 9.55 \\
\hline $\mathrm{Mn}$ & 54.938049 & -- & ${ }^{98} \mathrm{Mo}$ & -- & 24.13 \\
\hline $\mathrm{Fe}$ & 55.845 & -- & ${ }^{100} \mathrm{Mo}$ & -- & 9.63 \\
\hline${ }^{54} \mathrm{Fe}$ & - & 5.845 & $\mathrm{Ag}$ & 107.8682 & -- \\
\hline${ }^{56} \mathrm{Fe}$ & -- & 91.754 & ${ }^{107} \mathrm{Ag}$ & -- & 51.839 \\
\hline${ }^{57} \mathrm{Fe}$ & -- & 2.119 & ${ }^{109} \mathrm{Ag}$ & -- & 48.161 \\
\hline${ }^{58} \mathrm{Fe}$ & -- & 0.282 & $\mathrm{Cd}$ & 112.411 & -- \\
\hline Co & 58.9332 & -- & ${ }^{106} \mathrm{Cd}$ & -- & 1.25 \\
\hline $\mathrm{Ni}$ & 58.6934 & -- & ${ }^{108} \mathrm{Cd}$ & -- & 0.89 \\
\hline${ }^{58} \mathrm{Ni}$ & -- & 68.0769 & ${ }^{110} \mathrm{Cd}$ & -- & 12.49 \\
\hline${ }^{60} \mathrm{Ni}$ & -- & 26.2231 & ${ }^{111} \mathrm{Cd}$ & -- & 12.8 \\
\hline${ }^{61} \mathrm{Ni}$ & -- & 1.1399 & ${ }^{112} \mathrm{Cd}$ & -- & 24.13 \\
\hline${ }^{62} \mathrm{Ni}$ & -- & 3.6345 & ${ }^{113} \mathrm{Cd}$ & -- & 12.22 \\
\hline${ }^{64} \mathrm{Ni}$ & -- & 0.9256 & ${ }^{114} \mathrm{Cd}$ & -- & 28.73 \\
\hline \multirow{2}{*}{$\begin{array}{c}\mathrm{Cu} \\
{ }^{63} \mathrm{Cu}\end{array}$} & \multirow{2}{*}{$\begin{array}{c}63.546 \\
-- \\
\end{array}$} & \multirow{2}{*}{$\begin{array}{c}-- \\
69.17\end{array}$} & ${ }^{116} \mathrm{Cd}$ & -- & 7.49 \\
\hline & & & & & \\
\hline
\end{tabular}


NEA/NSC/DOC(2006)1

Liquid Metal Fast Reactor - LMFR

FFTF-LMFR-RESR-001

CRIT-SPEC-REAC-COEF-MISC

Table D.1 (cont'd). Summary of Data Employed from the $16^{\text {th }}$ Ed. of the Chart of the Nuclides.

\begin{tabular}{|c|c|c|c|c|c|}
\hline $\begin{array}{c}\text { Isotope or } \\
\text { Element }\end{array}$ & $\begin{array}{l}\text { Atomic } \\
\text { Weight }\end{array}$ & $\begin{array}{c}\text { Isotopic } \\
\text { Abundance }\end{array}$ & $\begin{array}{c}\text { Isotope or } \\
\text { Element }\end{array}$ & $\begin{array}{l}\text { Atomic } \\
\text { Weight }\end{array}$ & $\begin{array}{c}\text { Isotopic } \\
\text { Abundance }\end{array}$ \\
\hline Sn & 118.71 & -- & ${ }^{154} \mathrm{Gd}$ & -- & 2.18 \\
\hline${ }^{112} \mathrm{Sn}$ & -- & 0.97 & ${ }^{155} \mathrm{Gd}$ & -- & 14.8 \\
\hline${ }^{114} \mathrm{Sn}$ & -- & 0.66 & ${ }^{156} \mathrm{Gd}$ & -- & 20.47 \\
\hline${ }^{115} \mathrm{Sn}$ & -- & 0.34 & ${ }^{157} \mathrm{Gd}$ & -- & 15.65 \\
\hline${ }^{116} \mathrm{Sn}$ & -- & 14.54 & ${ }^{158} \mathrm{Gd}$ & -- & 24.84 \\
\hline${ }^{117} \mathrm{Sn}$ & -- & 7.68 & ${ }^{160} \mathrm{Gd}$ & -- & 21.86 \\
\hline${ }^{118} \mathrm{Sn}$ & -- & 24.22 & Dy & 162.5 & -- \\
\hline${ }^{119} \mathrm{Sn}$ & -- & 8.59 & ${ }^{156} \mathrm{Dy}$ & -- & 0.06 \\
\hline${ }^{120} \mathrm{Sn}$ & -- & 32.58 & ${ }^{158} \mathrm{Dy}$ & -- & 0.1 \\
\hline${ }^{122} \mathrm{Sn}$ & -- & 4.63 & ${ }^{160} \mathrm{Dy}$ & -- & 2.34 \\
\hline${ }^{124} \mathrm{Sn}$ & -- & 5.79 & ${ }^{161}$ Dy & -- & 18.91 \\
\hline $\mathrm{Cs}$ & 132.90545 & -- & ${ }^{162} \mathrm{Dy}$ & -- & 25.51 \\
\hline $\mathrm{Ba}$ & 137.327 & -- & ${ }^{163} \mathrm{Dy}$ & -- & 24.9 \\
\hline${ }^{130} \mathrm{Ba}$ & -- & 0.106 & ${ }^{164} \mathrm{Dy}$ & -- & 28.18 \\
\hline${ }^{132} \mathrm{Ba}$ & -- & 0.101 & $\mathrm{Ta}$ & 180.9479 & -- \\
\hline${ }^{134} \mathrm{Ba}$ & -- & 2.417 & W & 183.84 & -- \\
\hline${ }^{135} \mathrm{Ba}$ & -- & 6.592 & ${ }^{180} \mathrm{~W}^{\mathrm{b}}$ & -- & 0.12 \\
\hline${ }^{136} \mathrm{Ba}$ & -- & 7.854 & ${ }^{182} \mathrm{~W}$ & -- & 26.5 \\
\hline${ }^{137} \mathrm{Ba}$ & -- & 11.232 & ${ }^{183} \mathrm{~W}$ & -- & 14.31 \\
\hline${ }^{138} \mathrm{Ba}$ & -- & 71.698 & ${ }^{184} \mathrm{~W}$ & -- & 30.64 \\
\hline $\mathrm{Sm}$ & 150.36 & -- & ${ }^{186} \mathrm{~W}$ & -- & 28.43 \\
\hline${ }^{144} \mathrm{Sm}$ & -- & 3.07 & $\mathrm{Au}$ & 196.96655 & -- \\
\hline${ }^{147} \mathrm{Sm}$ & -- & 14.99 & $\mathrm{~Pb}$ & 207.2 & -- \\
\hline${ }^{148} \mathrm{Sm}$ & -- & 11.24 & ${ }^{204} \mathrm{~Pb}$ & -- & 1.4 \\
\hline${ }^{149} \mathrm{Sm}$ & -- & 13.82 & ${ }^{206} \mathrm{~Pb}$ & -- & 24.1 \\
\hline${ }^{150} \mathrm{Sm}$ & -- & 7.38 & ${ }^{207} \mathrm{~Pb}$ & -- & 22.1 \\
\hline${ }^{152} \mathrm{Sm}$ & -- & 26.75 & ${ }^{208} \mathrm{~Pb}$ & -- & 52.4 \\
\hline${ }^{154} \mathrm{Sm}$ & -- & 22.75 & $\mathrm{Bi}$ & 208.98038 & -- \\
\hline $\mathrm{Eu}$ & 151.964 & -- & ${ }^{234} \mathrm{U}$ & 234.040946 & $0.0055^{\mathrm{c}}$ \\
\hline${ }^{151} \mathrm{Eu}$ & -- & 47.81 & ${ }^{235} \mathrm{U}$ & 235.043923 & $0.7200^{\mathrm{c}}$ \\
\hline${ }^{153} \mathrm{Eu}$ & -- & 52.19 & ${ }^{238} \mathrm{U}$ & 238.050783 & $99.2745^{\mathrm{c}}$ \\
\hline $\mathrm{Gd}$ & 157.25 & -- & ${ }^{237} \mathrm{~Np}$ & 237.048167 & -- \\
\hline${ }^{152} \mathrm{Gd}$ & -- & 0.2 & & & \\
\hline
\end{tabular}




\section{Liquid Metal Fast Reactor - LMFR \\ FFTF-LMFR-RESR-001 \\ CRIT-SPEC-REAC-COEF-MISC}

Table D.1 (cont'd). Summary of Data Employed from the $16^{\text {th }}$ Ed. of the Chart of the Nuclides.

\begin{tabular}{|c|c|c|c|c|c|}
\hline $\begin{array}{c}\text { Isotope or } \\
\text { Element }\end{array}$ & $\begin{array}{l}\text { Atomic } \\
\text { Weight } \\
\end{array}$ & $\begin{array}{c}\text { Isotopic } \\
\text { Abundance } \\
\end{array}$ & $\begin{array}{c}\text { Isotope or } \\
\text { Element }\end{array}$ & $\begin{array}{l}\text { Atomic } \\
\text { Weight } \\
\end{array}$ & $\begin{array}{c}\text { Isotopic } \\
\text { Abundance } \\
\end{array}$ \\
\hline${ }^{238} \mathrm{Pu}$ & 238.049553 & -- & ${ }^{241} \mathrm{Pu}$ & 241.016118 & -- \\
\hline${ }^{239} \mathrm{Pu}$ & 239.052157 & -- & ${ }^{242} \mathrm{Pu}$ & 242.058737 & -- \\
\hline${ }^{240} \mathrm{Pu}$ & 240.053807 & -- & ${ }^{241} \mathrm{Am}$ & 241.056823 & -- \\
\hline
\end{tabular}

(a) Neutronically, ${ }^{180} \mathrm{~W}$ is treated as ${ }^{186} \mathrm{~W}$.

(b) Natural isotopic abundance of $U$. 
NEA/NSC/DOC(2006)1

Liquid Metal Fast Reactor - LMFR

FFTF-LMFR-RESR-001

CRIT-SPEC-REAC-COEF-MISC

\section{APPENDIX E: PRESERVATION OF ADDITIONAL FFTF DATA}

\section{E.1 General Facility Description and Additional Experimental Data}

\section{E.1.1 Geometry of the Experiment Configuration and Measurement Procedure}

Information is preserved in this appendix regarding the general FFTF facility and experimental measurements not assessed in this benchmark evaluation such as initial fuel loading, partially-loaded core critical, and subcritical configurations.

\section{E.1.1.1 General Facility Description}

The FFTF included the reactor, equipment, and structures necessary for heat removal, containment, core component handling and examination, instrumentation and control, and for supplying utilities and other essential services. It was a complex array of buildings and equipment arranged around a central reactor containment building (Figure E.1.1). The surrounding buildings were constructed from steel and concrete and were structurally independent of the reactor containment building. The reactor itself was contained within a shielded cavity within a $186 \mathrm{ft} 8 \mathrm{in} .(56.896 \mathrm{~m})$ high, $135 \mathrm{ft}(41.148 \mathrm{~m})$ diameter containment vessel made of carbon steel (Figures E.1.2 and E.1.3) and designed for an internal pressure of $10 \mathrm{psig}(68.9476 \mathrm{kPa})$. The operations floor was at grade level with $77 \mathrm{ft} 10.5 \mathrm{in}$. $(23.7363 \mathrm{~m})$ of the containment below grade level (Ref. 5, pp. 2-2 through 2-5, 2-12, and 11-4). 
NEA/NSC/DOC(2006)1

Liquid Metal Fast Reactor - LMFR

FFTF-LMFR-RESR-001

CRIT-SPEC-REAC-COEF-MISC

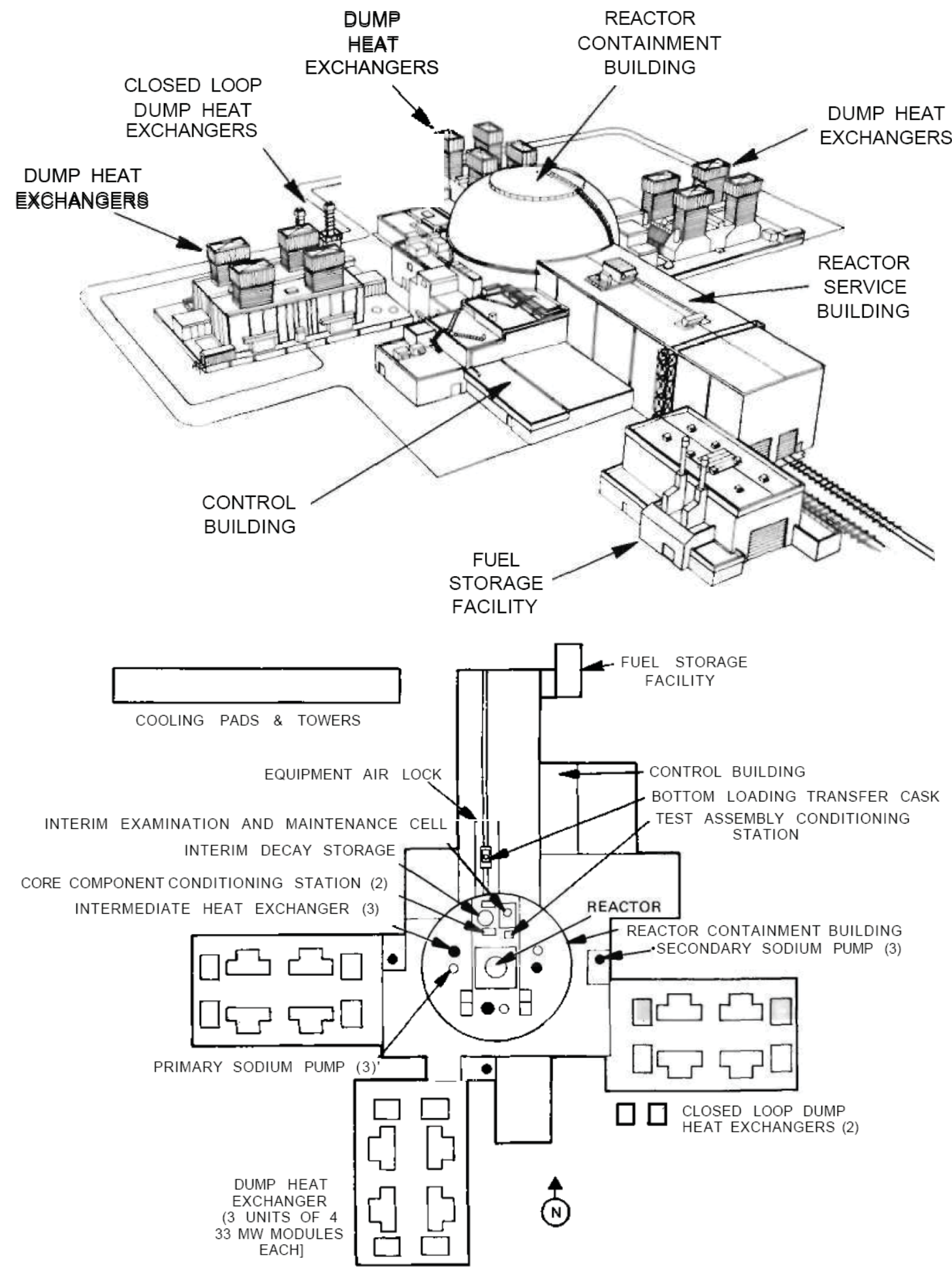

Figure E.1.1. Fast Flux Test Facility Plant Arrangement (Ref. 5, Fig. 2-1 and 2-2). 
NEA/NSC/DOC(2006)1

Liquid Metal Fast Reactor - LMFR

FFTF-LMFR-RESR-001

CRIT-SPEC-REAC-COEF-MISC
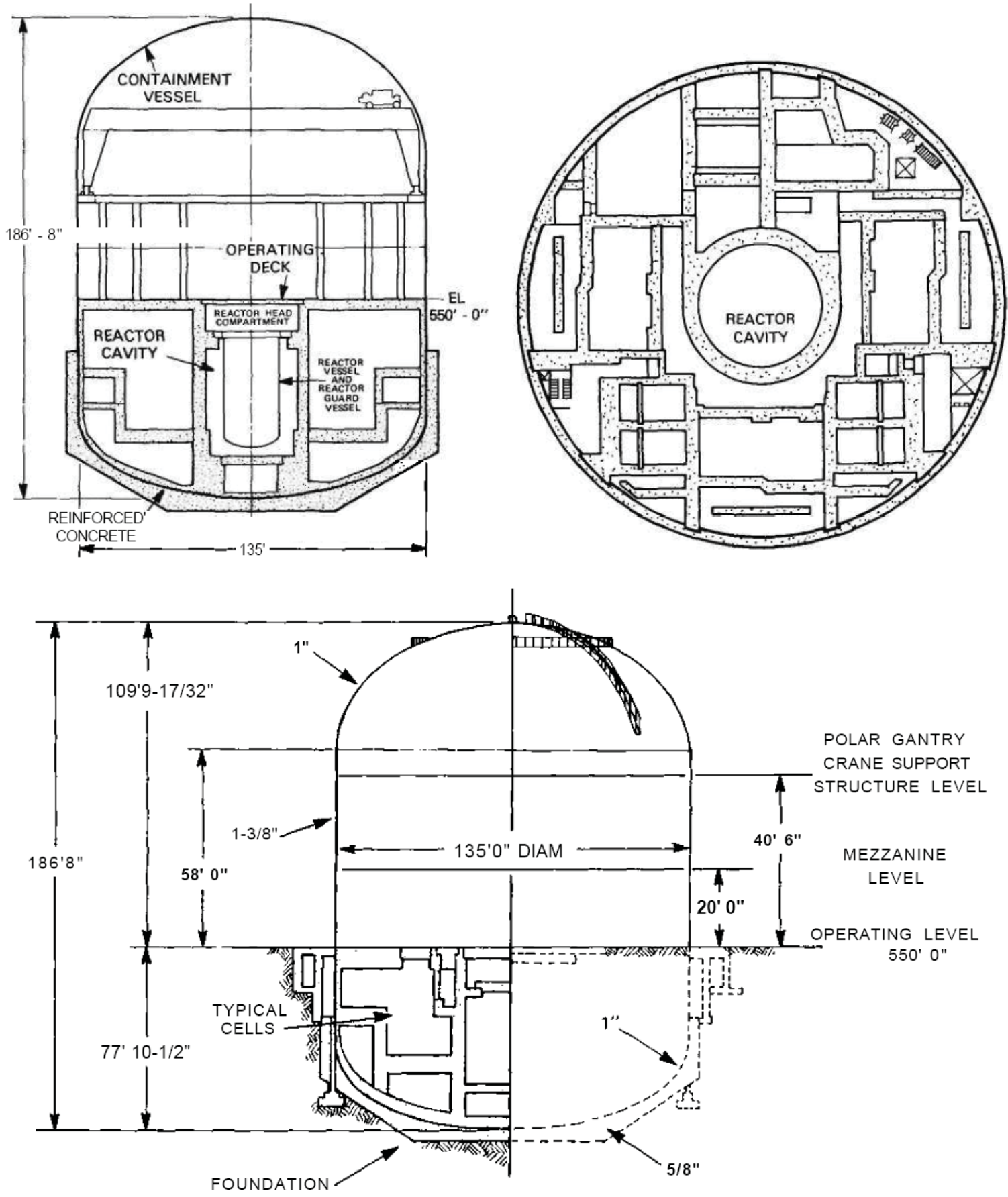

Figure E.1.2. FFTF Containment Vessel (Ref. 5, Fig. 2-3 and 11-2). 
NEA/NSC/DOC(2006)1

Liquid Metal Fast Reactor - LMFR

FFTF-LMFR-RESR-001

CRIT-SPEC-REAC-COEF-MISC

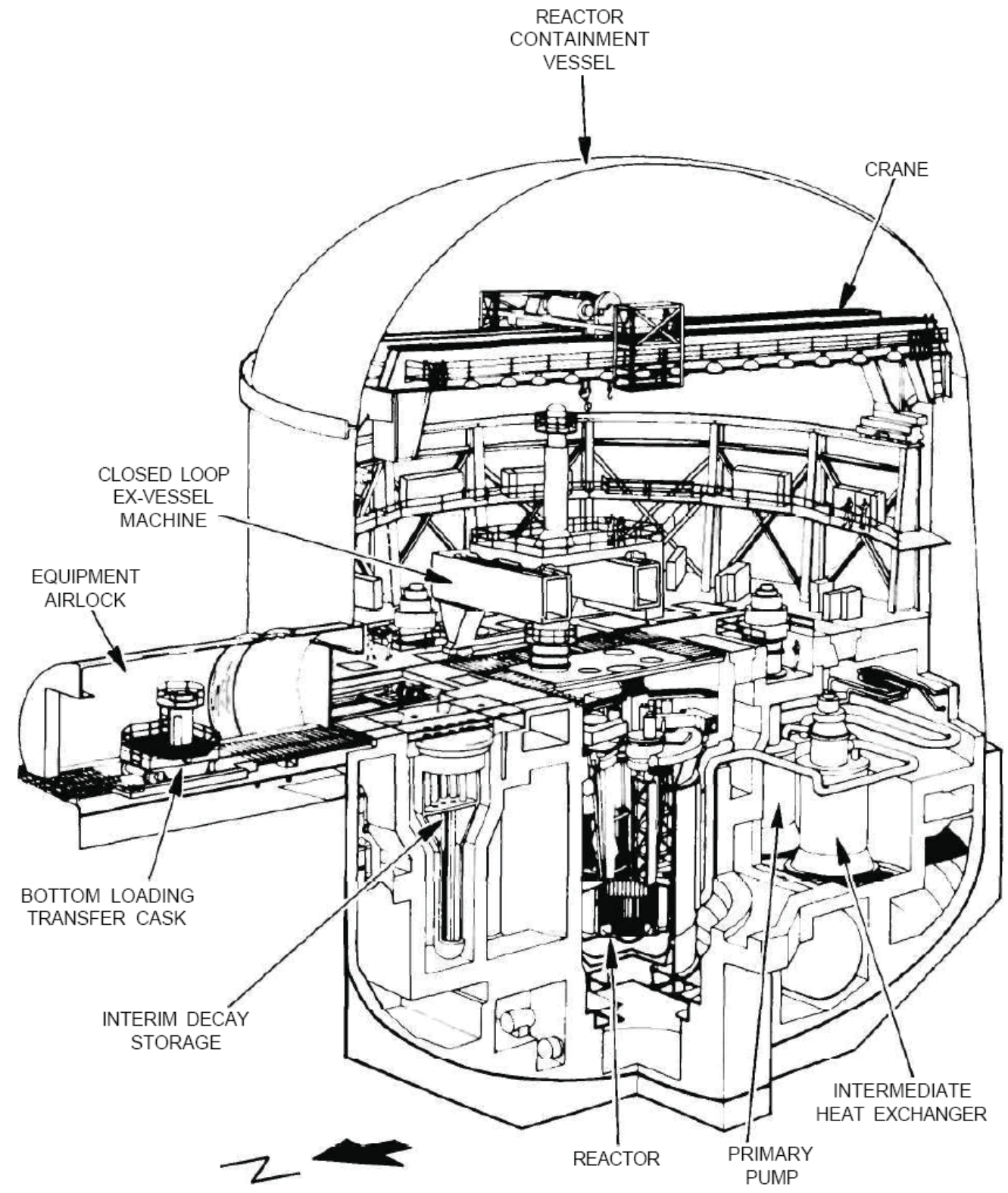

Figure E.1.3. Reactor Containment Building Cutaway (Ref. 5, Fig. 2-4).

The reactor vessel was constructed from stainless steel 304 and is approximately $43 \mathrm{ft}(13.1064 \mathrm{~m})$ high with an inside diameter of $20 \mathrm{ft}(6.096 \mathrm{~m})$. The reactor vessel wall thickness varied between 2-3/8 inches to $2-3 / 4$ inches $(6.0325 \mathrm{~cm}$ to $6.985 \mathrm{~cm})$. A vessel liner with an inner diameter of $19 \mathrm{ft}-10.5$ in $(6.0579$ $\mathrm{m})$ and an annulus between the vessel and liner of approximately $1-1 / 4$ inches $(3.175 \mathrm{~cm})$ protected the vessel wall from severe temperature transients. A pool of liquid sodium filled the reactor vessel from the bottom with a zone of argon cover gas near the top. Three 16-inch $(40.64-\mathrm{cm})$ nozzles in the lower part of the vessel provided inlets for the sodium coolant. The coolant flowed through the core and other reactor components and then out three 28 -inch $(71.12-\mathrm{cm})$ outlet nozzles located approximately at the mid-height level of the vessel. All nozzles were located $120^{\circ}$ apart, around the reactor vessel. A schematic of the reactor vessel is shown in Figure E.1.4 (Ref. 5, pp. 2-6 and 4-3). 
NEA/NSC/DOC(2006)1

Liquid Metal Fast Reactor - LMFR

FFTF-LMFR-RESR-001

CRIT-SPEC-REAC-COEF-MISC

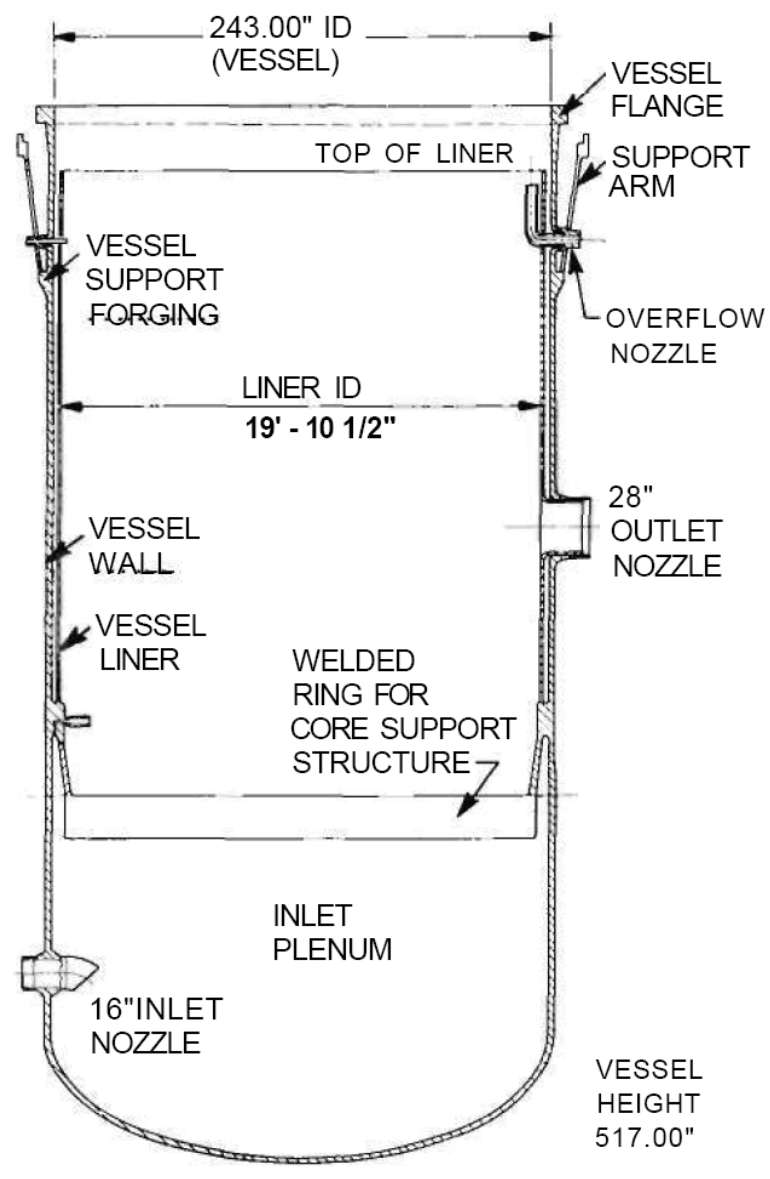

Figure E.1.4. Reactor Vessel (Ref. 5, Fig. 4-3).

The reactor vessel was suspended from its upper section by 30 support arms welded to the vessel and bolted to the main support structure, and nestled in a bottom-mounted, stainless steel 304, reactor guard vessel. The guard vessel provided an 8 -inch $(20.32-\mathrm{cm})$ annulus with the purpose of keeping the vessel nozzles submerged in sodium in the event of a sodium leak. The thickness varied between 1 and 2.5 inches $(2.54$ and $6.35 \mathrm{~cm})$ to provide structural integrity at its design temperature of $1050{ }^{\circ} \mathrm{F}\left(\sim 566^{\circ} \mathrm{C}\right)$ with a full head of sodium coolant. The vessel was insulated on the outer surface to protect reactor cavity walls and to reduce heat loads to the heating and ventilation systems (Ref. 5, pp. 2-6 through 2-7 and 4-4 through 4-5).

The reactor head closed the vessel and has a diameter of $25 \mathrm{ft}(6.7056 \mathrm{~m})$, measures about 22 inches $(55.88 \mathrm{~cm})$ thick, and weighs 214 tons. It was fabricated from two SA-508, Class 2 forgings (low-alloy carbon steel) that were butt-welded together as a Class A vessel. The shielding beneath the head was comprised of four thick metallic plates: two 4-inch $(10.16-\mathrm{cm})$ carbon steel plates, one 4-inch $(10.16-$ $\mathrm{cm})$ stainless steel 410 plate, and one 4-inch $(10.16-\mathrm{cm})$ Inconel 600 plate. Below the shield plates were eight stainless steel 304 thermal reflectors and a segmented gas entrainment suppressor plate of stainless steel 316. The head assembly was supported by the main vessel support structure and secured by 82 peripheral hold-down stud bolts on a 291.5-inch (7.4041-m) diameter bolt circle. The head was sealed by an SB-168 Inconel omega seal weld and two concentric metallic O-rings (copper-plated Inconel 600). The reactor head contained 50 major penetrations (Figure E.1.5). Equipment was mounted on the head, including drive mechanisms, seals, and shielding for openings that provided in-vessel access, and coolant piping for independently cooled test loops. The head compartment area for the equipment was $36 \mathrm{ft}$ by 
NEA/NSC/DOC(2006)1

Liquid Metal Fast Reactor - LMFR

FFTF-LMFR-RESR-001

CRIT-SPEC-REAC-COEF-MISC

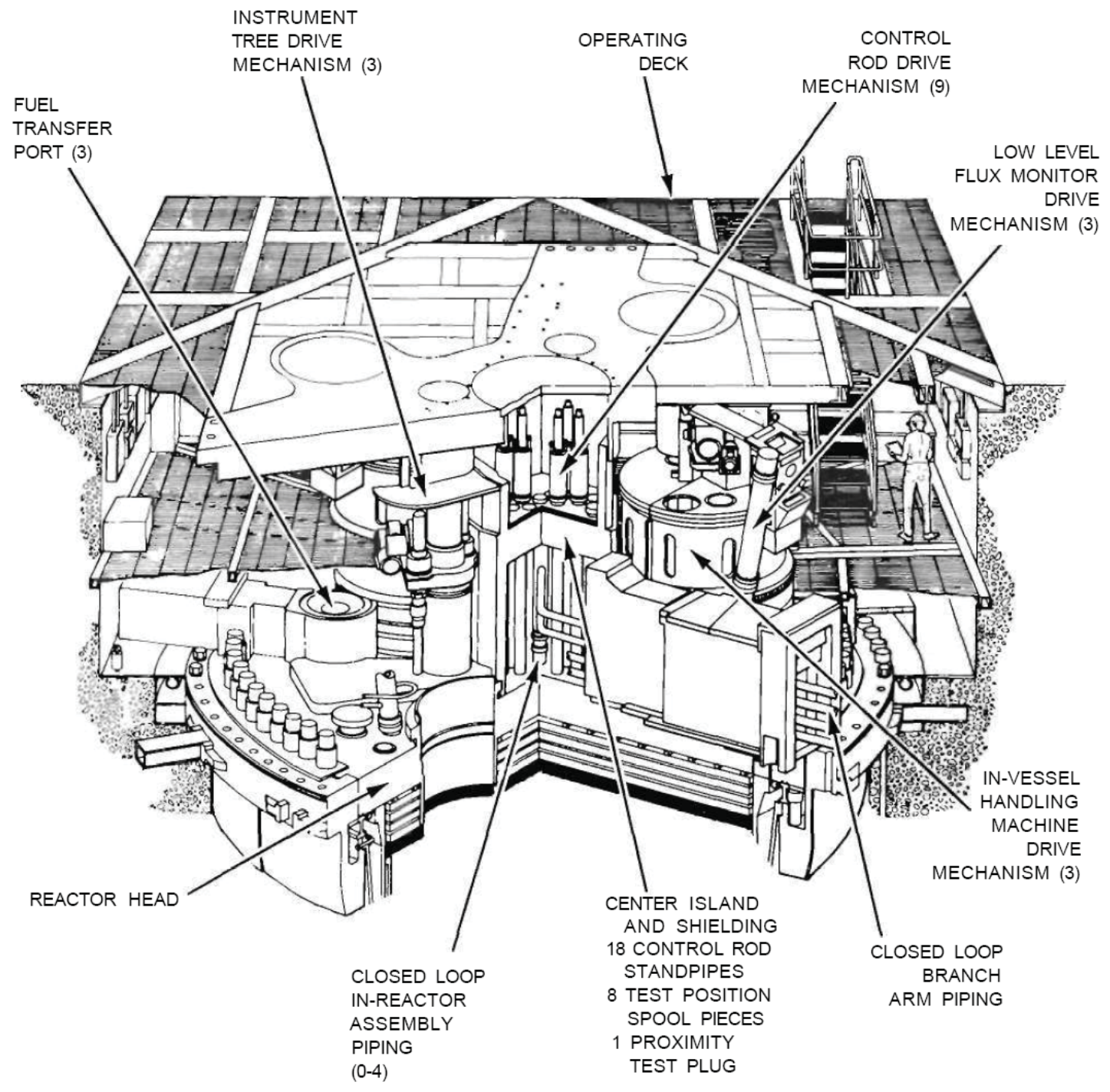

Figure E.1.6. Head Compartment Arrangement (Ref. 5, Fig. 4-9). 
NEA/NSC/DOC(2006)1

Liquid Metal Fast Reactor - LMFR

FFTF-LMFR-RESR-001

CRIT-SPEC-REAC-COEF-MISC

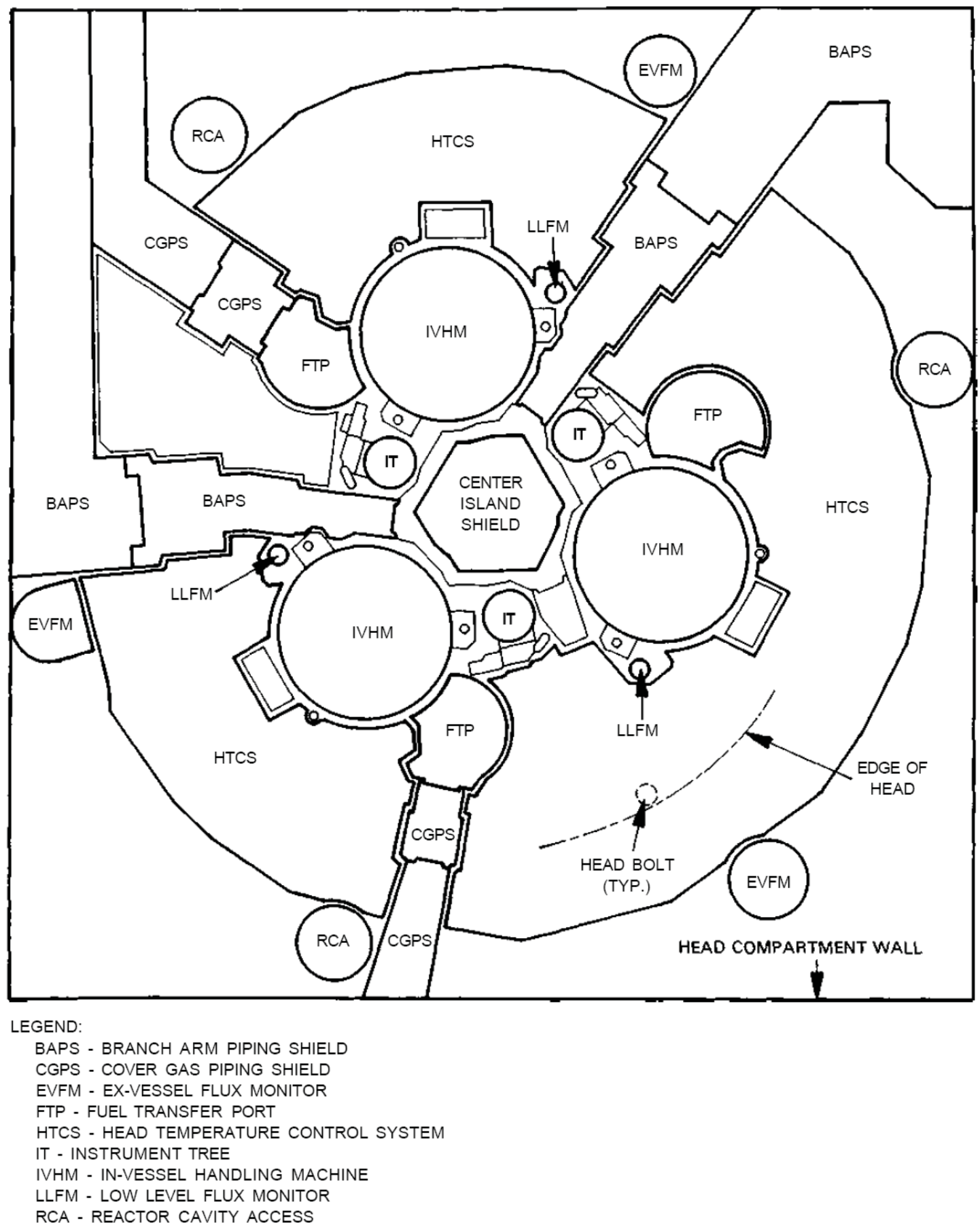

Figure E.1.7. Head Compartment Plan View (Ref. 5, Fig. 4-10). 
NEA/NSC/DOC(2006)1

Liquid Metal Fast Reactor - LMFR

FFTF-LMFR-RESR-001

CRIT-SPEC-REAC-COEF-MISC

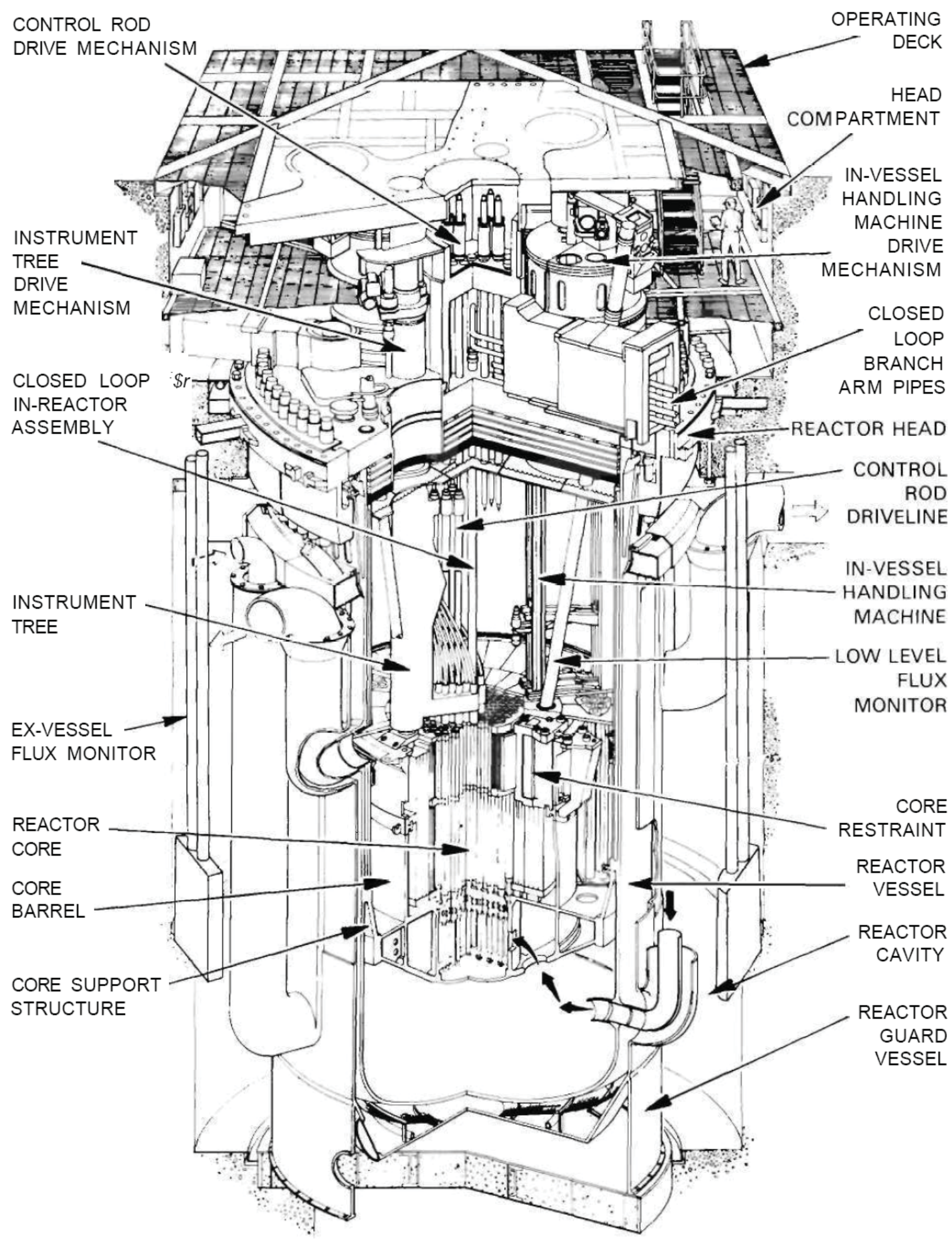

Figure E.1.8. Reactor Vessel Cutaway (Ref. 5, Fig. 2-5). 
NEA/NSC/DOC(2006)1

Liquid Metal Fast Reactor - LMFR

FFTF-LMFR-RESR-001

CRIT-SPEC-REAC-COEF-MISC

Various above-core, in-vessel components in the FFTF, including nine control rod drivelines, three instrument trees that supported flow and temperature monitoring sensors, three temperature and liquid level monitors, one outlet temperature monitor plug, three low-level neutron flux monitors, and three invessel handling machines. Additional equipment in the FFTF included ex-vessel flux monitors and surveillance cameras. Neutron flux monitors used for the automatic shutdown system were suspended between the reactor guard vessel and the reactor cavity wall. Each of these three vessels consisted of two thimbles installed in graphite thermal blocks and covered the reactor power range from approximately 4 $\mathrm{W}$ up to $500 \mathrm{MW}$. Close-circuit television surveillance equipment was placed between the reactor vessel and guard vessel to provide remote visual examination of the welds on the outer surface of the reactor vessel and nozzles, as well as the inner surface of the welds on the reactor guard vessel (Ref. 5, p. 2-8).

Heat was removed from the reactor by liquid sodium circulated through three primary loops within the containment vessel. Secondary sodium loops then transported the reactor heat from the intermediate heat exchangers to the air-cooled tubes of the dump heat exchangers. Nominal conditions for the primary system were $133 \mathrm{psig}(0.917 \mathrm{MPa})$ reactor inlet pressure, $680^{\circ} \mathrm{F}\left(\sim 360^{\circ} \mathrm{C}\right)$ reactor inlet temperature, and $980^{\circ} \mathrm{F}\left(\sim 527^{\circ} \mathrm{C}\right)$ reactor outlet temperature. The sodium was monitored continuously for purity and contamination, and purified using on-line, NaK-cooled, cold traps. Argon as used as a cover gas for the heat removal loops and as the atmosphere for cells where reactor components were handled. Nitrogen was used as the atmosphere for cells and pipeways which housed piping and/or equipment containing primary system sodium. A schematic of the heat removal facilities and overall plan view of the heat transport system are shown in Figures E.1.9 and E.1.10, respectively (Ref. 5, pp. 2-4 and 2-9 through 211).

A summary of the major FFTF design parameters is shown in Table E.1.1.

\section{FFTF REACTOR HEAT TRANSPORT SYSTEM ONE OF THREE PARALLEL COOLANT CIRCUITS}

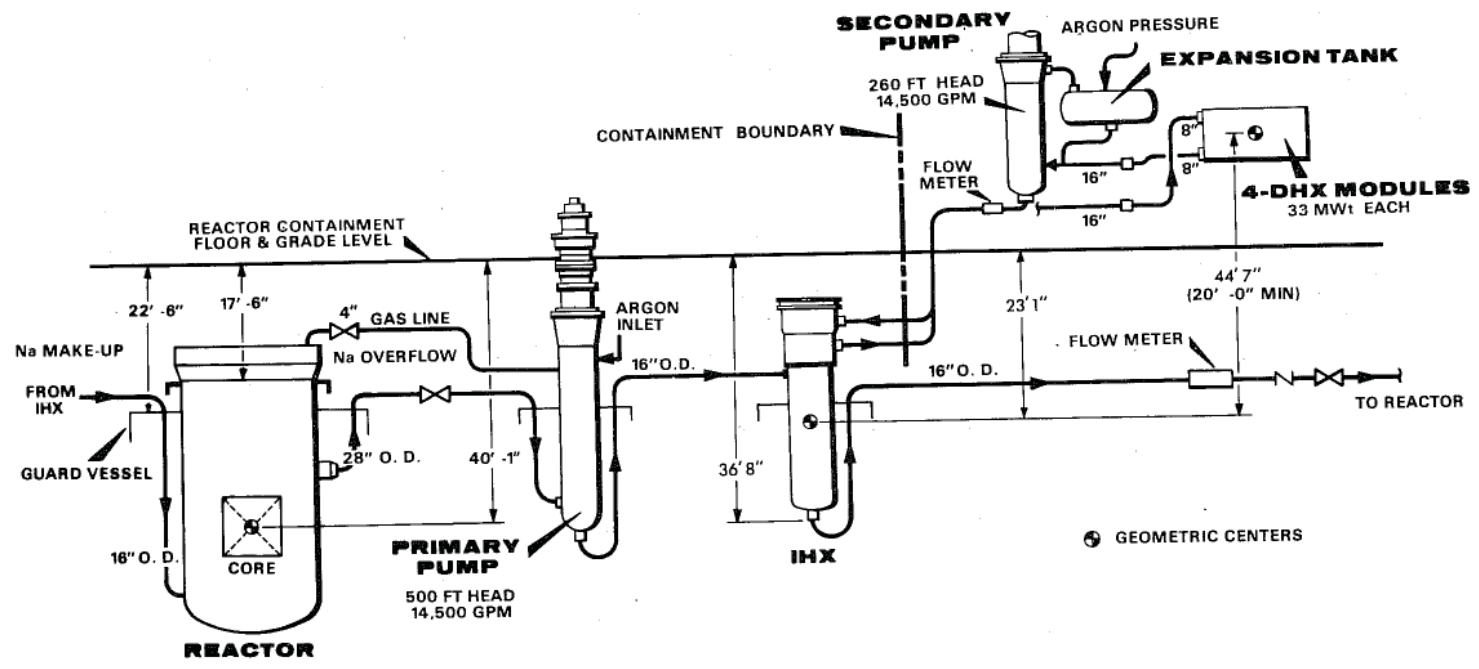

Figure E.1.9. Schematic of Heat Removal Facilities. ${ }^{a}$

\footnotetext{
${ }^{\text {a }}$ Figure II.B.2.10 (p. II-44) from United States Atomic Energy Commission, "Environmental Statement: Fast Flux Test Facility, Richland, Washington,” WASH-1510, (May 1972).
} 
FFTF-LMFR-RESR-001

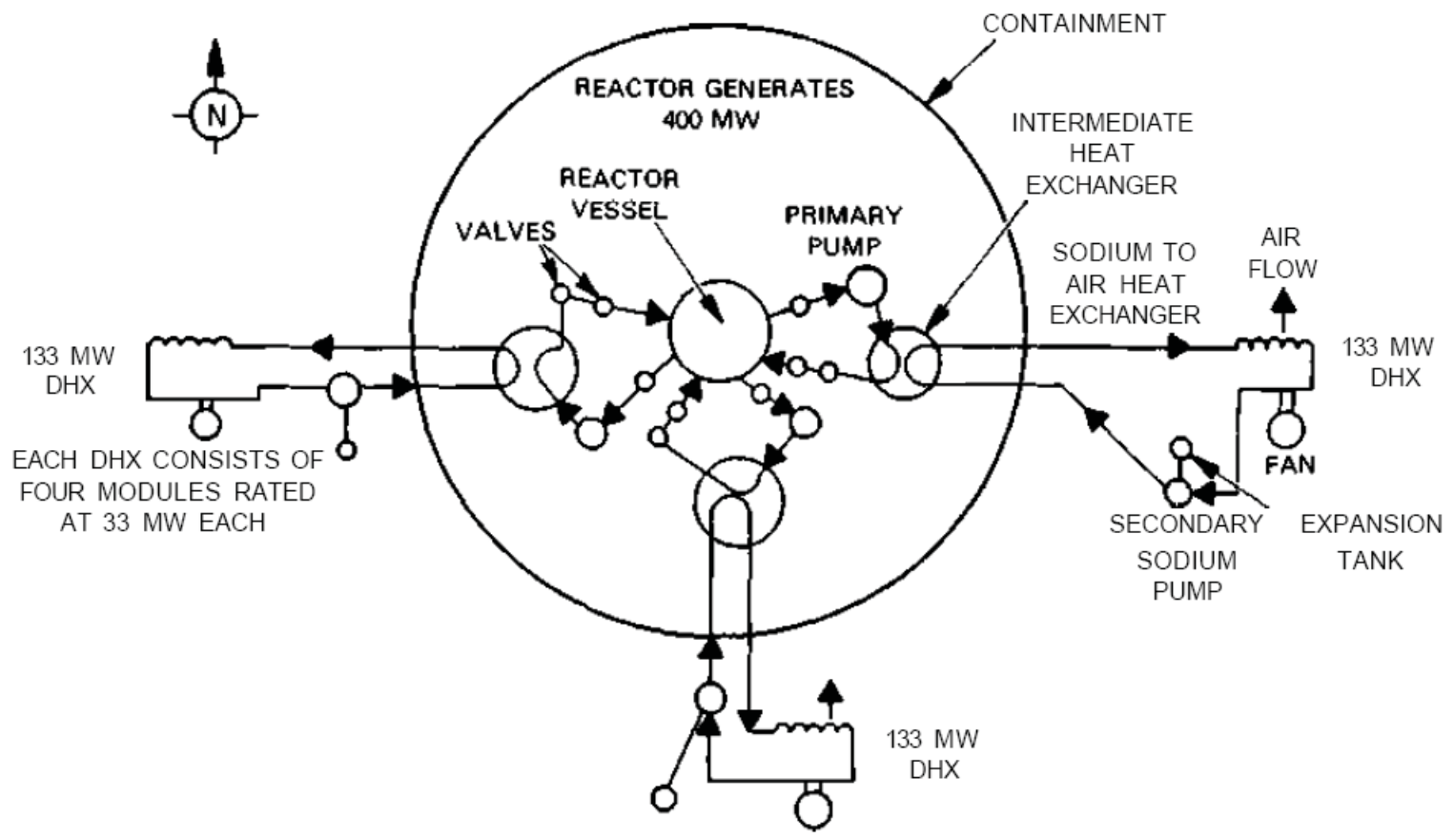

Figure E.1.10. Plan View of Heat Transport System (Ref. 5, Fig. 2-7). 
FFTF-LMFR-RESR-001

CRIT-SPEC-REAC-COEF-MISC

Table E.1.1. FFTF Design Parameters (Ref. 5, p. 2-14).

REACTOR CONTAINMENT VESSEL

Material

Diameter

Overall Height

Depth Below Operating Floor

Wall Thickness Above Grade

Internal Design Pressure

\section{REACTOR VESSEL}

Material

Internal Diameter

Height

Wall Thickness

Vessel Liner Internal Diameter

REACTOR GUARD VESSEL

Material

Wall Thickness

Annulus Between Reactor Vessel

and Reactor Guard Vessel

REACTOR VESSEL HEAD

Material

Diameter

Thickness

Weight

GENERAL

Power, Excluding Closed Loops

Closed Loops Capability

\section{NUCLEAR CONTROLS}

Type of Absorber

Boron-10 per Assembly

Pins per Assembly

Pin Cladding - OD

$$
\text { - Thickness }
$$

Pellet Diameter

Duct - Wall Thickness

- Length

- Material

Cladding Material

Wire Wrap Material

HEAT REMOVAL - MAIN HEAT TRANSPORT SYSTEM

Circuits

Rating per Circuit

$\mathrm{DHX}$ Modules

Rating per DHX Module

Sodium Flow Rate

Reactor Vessel

- Inlet Temperature

- Outlet Temperature

- Inlet Pressure
Carbon steel

$135 \mathrm{ft} 0$ in.

$186 \mathrm{ft} 8 \mathrm{in}$

$77 \mathrm{ft} 10-1 / 2 \mathrm{in}$.

1 to $1-3 / 8$ in.

10 psig

304 stainless steel

$243 \mathrm{in}$. (about $20 \mathrm{ft}$ )

517 in. (about $43 \mathrm{ft}$ )

$2-3 / 8$ to $2-3 / 4$ in.

$19 \mathrm{ft} 10-1 / 2 \mathrm{in}$.

304 stainless steel

1 to $2-1 / 2$ in.

8-in.

Low carbon alloy steel

$25 \mathrm{ft}$

about 22 in. (plus

shielding plates)

214 tons

$400 \mathrm{MW}$

4 at $2.3 \mathrm{MW}$ each

$\mathrm{B}_{4} \mathrm{C}$ Pellets

1.16 to $1.28 \mathrm{~kg}$

61

$0.474 \mathrm{in}$

0.051 in.

0.362 in.

0.120 in.

$12 \mathrm{ft}$

316 SS $20 \%$ CW

316 SS $20 \%$ CW

316 SS $17 \%$ CW

3

$133 \mathrm{MW}$

12

$33 \mathrm{MW}$

$43,500 \mathrm{gal} / \mathrm{min}$

$680^{\circ} \mathrm{F}$ nominal

$980^{\circ} \mathrm{F}$ nominal

133 psig nominal 
FFTF-LMFR-RESR-001

CRIT-SPEC-REAC-COEF-MISC

Table E.1.1 (cont'd.). FFTF Design Parameters (Ref. 5, p. 2-14).

CORE CONFIGURATION

$\begin{array}{lll}\text { Core Positions } & \text { - Rows 1-6 (Fueled Zone) } & 91 \\ & - \text { Rows 7-9 (Reflector Zone) } & 108 \\ \text { Core Assembly } & - \text { Total Positions } & 199 \\ & - \text { Shape } & \text { Hexagonal } \\ & - \text { Dimension Across Load Pads } & 4.715 \mathrm{in} . \\ \text { Active Core } & - \text { Length (for Most) } & 12 \mathrm{ft} \\ & - \text { Fueled Height } & 36 \mathrm{in} . \\ \text { Positions for Driver Fuel } & 47.2 \mathrm{in} . \\ \text { Primary Control Rods (Boron Carbide) } & 1034 \text { liters } \\ \text { Secondary Control Rods (Boron Carbide) } & 74 \\ \text { Fixed Peripheral Absorber Assemblies } & 3 \\ \text { Positions } & 6\end{array}$

Positions for 40-ft Independently Instrumented Test Assemblies

\section{CORE PHYSICS}

Total Fissile Mass

Neutron Flux (Peak)

$$
0.1 \mathrm{MeV}
$$

Conversion Ratio

Fuel Cycle, Nominal

Average Discharge Burnup

Limiting Peak Burnup

Power Density

Doppler Coefficient

Delayed Neutron Fraction

\section{DRIVER FUEL}

Fuel Assembly Power

Assembly Width at Load Pads

Assembly Length

Fuel Pin Linear Average Power

Driver Fuel Assembly Average Coolant Flow

Initial Core Average Coolant Velocity

Fuel Type

$\mathrm{Pu} \%$ (of $\mathrm{Pu}$ and $\mathrm{U}$ ) - Rows $1-4$

$\mathrm{Pu} \%$ (of Pu and U) - Rows 5-6

Pu Fissile Content (239Pu + 241Pu)

Uranium Type

Duct Wall Thickness

Duct Material

Fuel Pins per Fuel Assembly

Fuel Pin Cladding -OD

- Thickness

- Material

Fuel Pin Wire Wrap - Diameter

- Material

Fuel Pellets per Pin

Fuel Pin

- Length

- Fueled Length
3 to $7 \mathrm{MN}$

4.715 in.

$12 \mathrm{ft}$

$7.3 \mathrm{~kW} / \mathrm{ft}$

$18.7 \times 10^{4} \mathrm{lb} / \mathrm{hr}$

$21 \mathrm{ft} / \mathrm{s}$

PUO2-UO2

about $22.4 \%$

about $27.4 \%$

88 wt \%

Natural

0.120 in.

316 SS 20\% CW

217

0.23 in.

0.015 in.

316 SS $20 \% \mathrm{CW}$

0.056 in.

316 SS 17\% CW

about 150

about 94 in.

36 in. 
NEA/NSC/DOC(2006)1

Liquid Metal Fast Reactor - LMFR

FFTF-LMFR-RESR-001

CRIT-SPEC-REAC-COEF-MISC

\section{E.1.1.2 Detailed Reactor Description}

\section{Reactor Core External Components}

A core support structure was welded to the reactor vessel and used to maintain the position of the reactor core within the vessel (Figure E.1.11). A core basket was located in the center of the support structure so as to provide vertical support for the assemblies in the reactor core. The basket was a closed cylinder with 151 tubular receptacles connecting the upper and lower tube sheets. The cylindrical section of the basket had twelve rectangular flow holes that were fitted with strainers and served as the inlet for the sodium flow. The core barrel was a cylinder approximately $10 \mathrm{ft}(3.048 \mathrm{~m})$ high and 11-1/2 ft (3.5052 $\mathrm{m})$ in diameter. The barrel surrounded the radial shield of the core and was supported by, and welded to, the core support structure (Ref. 5, p. 3-6).

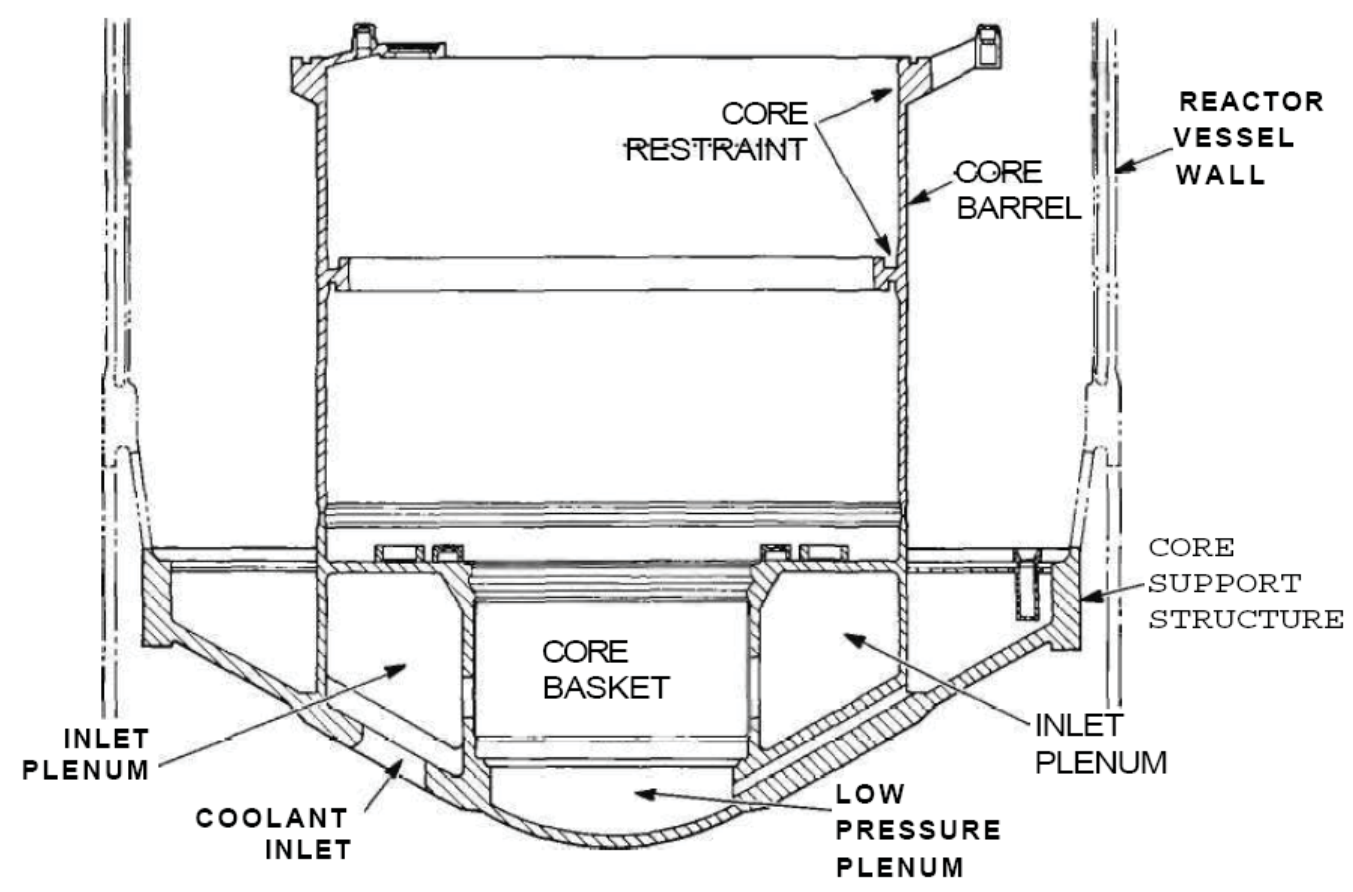

Figure E.1.11. Core Support Structure and Core Barrel (Ref. 5, Fig. 3-4).

The radial shield consisted of six inner blocks and six outer blocks arranged around the periphery of the radial reflector region of the core (Ref. 5, p. 3-6). The modular radial shielding was constructed of parallel stainless steel plates separated by coolant passages, with a structure volume fraction of $92 \%$ for the inner shield modules. The average thickness of the shielding was 23.6 inches $(59.944 \mathrm{~cm})$, and it extended 44 inches $(1.1176 \mathrm{~m})$ below and 70 inches $(1.778 \mathrm{~m})$ above the core midplane (Ref. 3, p. 17).

Core restraint mechanisms were used to apply radial forces to the load pads of the assemblies within the core so as to hold them into position for operation. Six restraint mechanisms were mounted on the core barrel, equally spaced around the periphery of the core. They were actuated by shafts extending through the reactor head. The core restraint mechanisms are shown in Figure E.1.12. A horizontal baffle was mounted on the upper end of the core barrel to act as a thermal insulator and flow barrier between the hot outlet plenum of the reactor vessel and the cooler inlet plenum. The baffle was sealed to the reactor vessel liner (Ref. 5, p. 3-7). 
NEA/NSC/DOC(2006)1

Liquid Metal Fast Reactor - LMFR

FFTF-LMFR-RESR-001

CRIT-SPEC-REAC-COEF-MISC

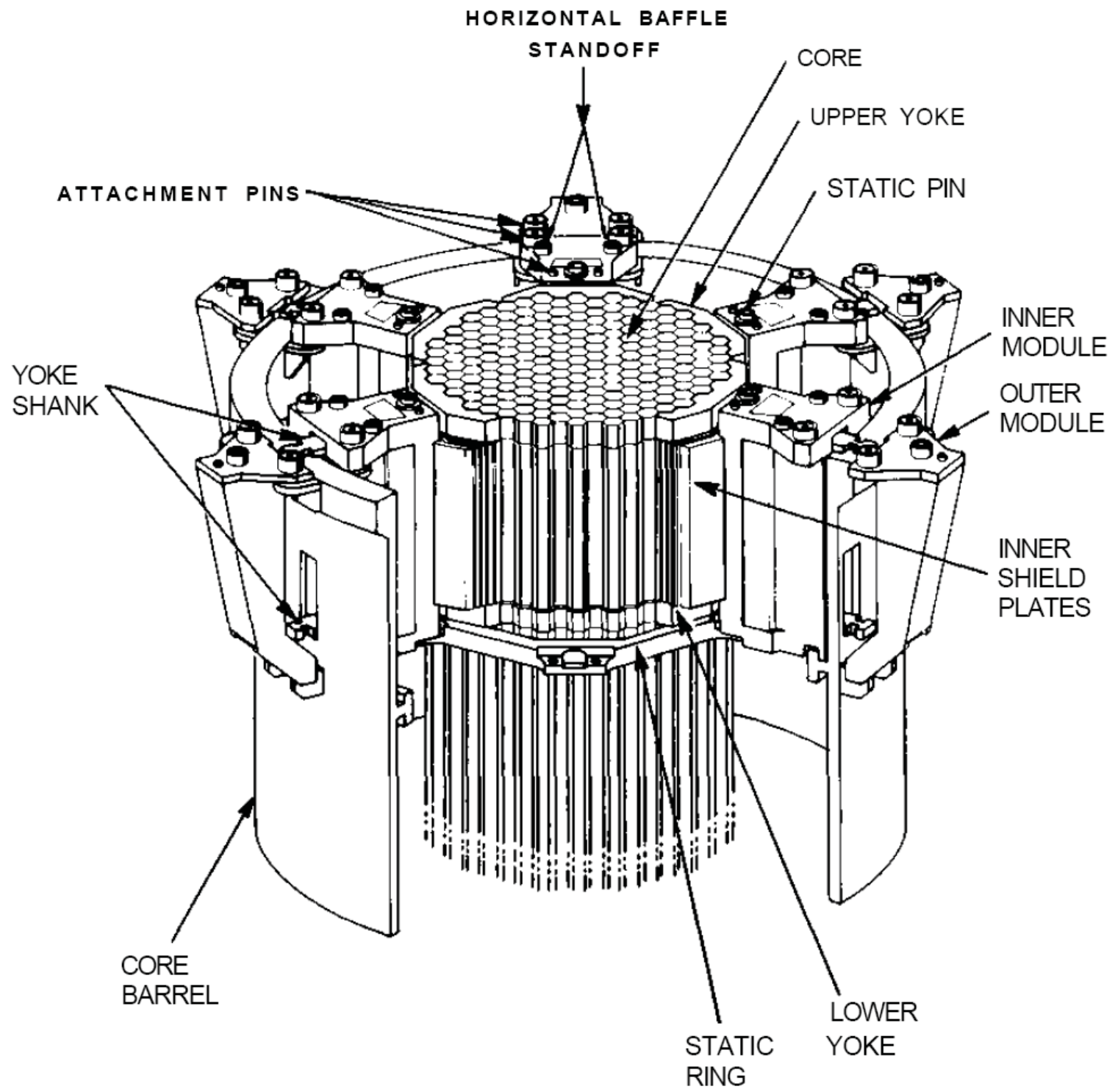

Figure E.1.12. Core Restraint Mechanisms around Core (Ref. 5, Fig. 3-5).

In-vessel storage modules (Figure E.1.13) were available in three sections of the annular region between the core barrel and the reactor vessel thermal liner (Figure E.1.14). Each module provided 19 naturalconvection-cooled, stainless steel 304 receptacles for core components and one transfer port position for the core component pot. Assemblies used for material surveillance samples were also installed in the invessel storage modules, exposing them to the sodium, thermal, and radiation environment during reactor operations (Ref. 5, p. 3-8). 
NEA/NSC/DOC(2006)1

Liquid Metal Fast Reactor - LMFR

FFTF-LMFR-RESR-001

CRIT-SPEC-REAC-COEF-MISC

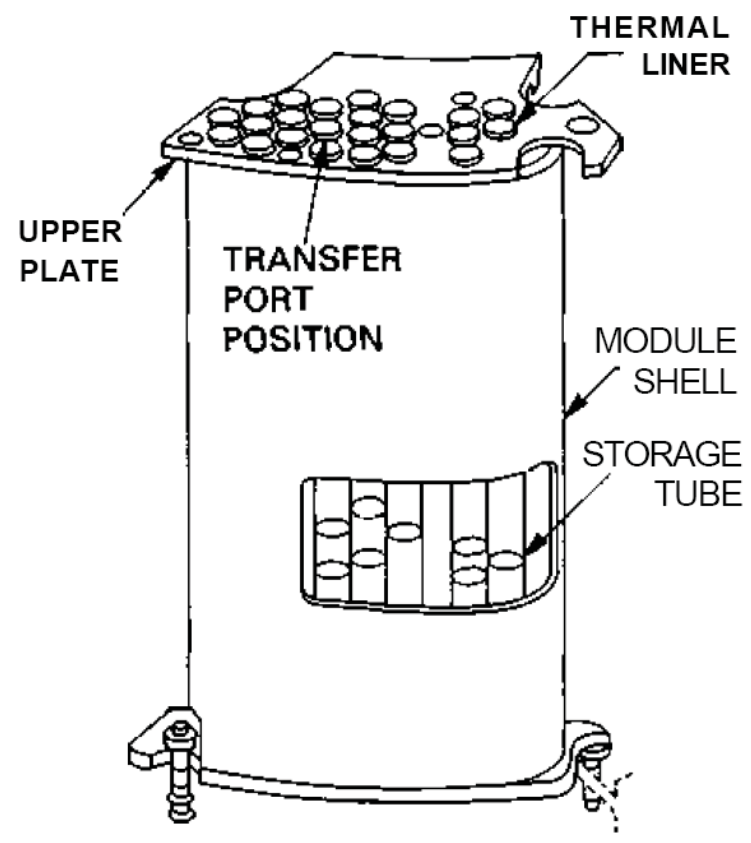

Figure E.1.13. In-Vessel Storage Module (Ref. 5, Fig. 3-6).

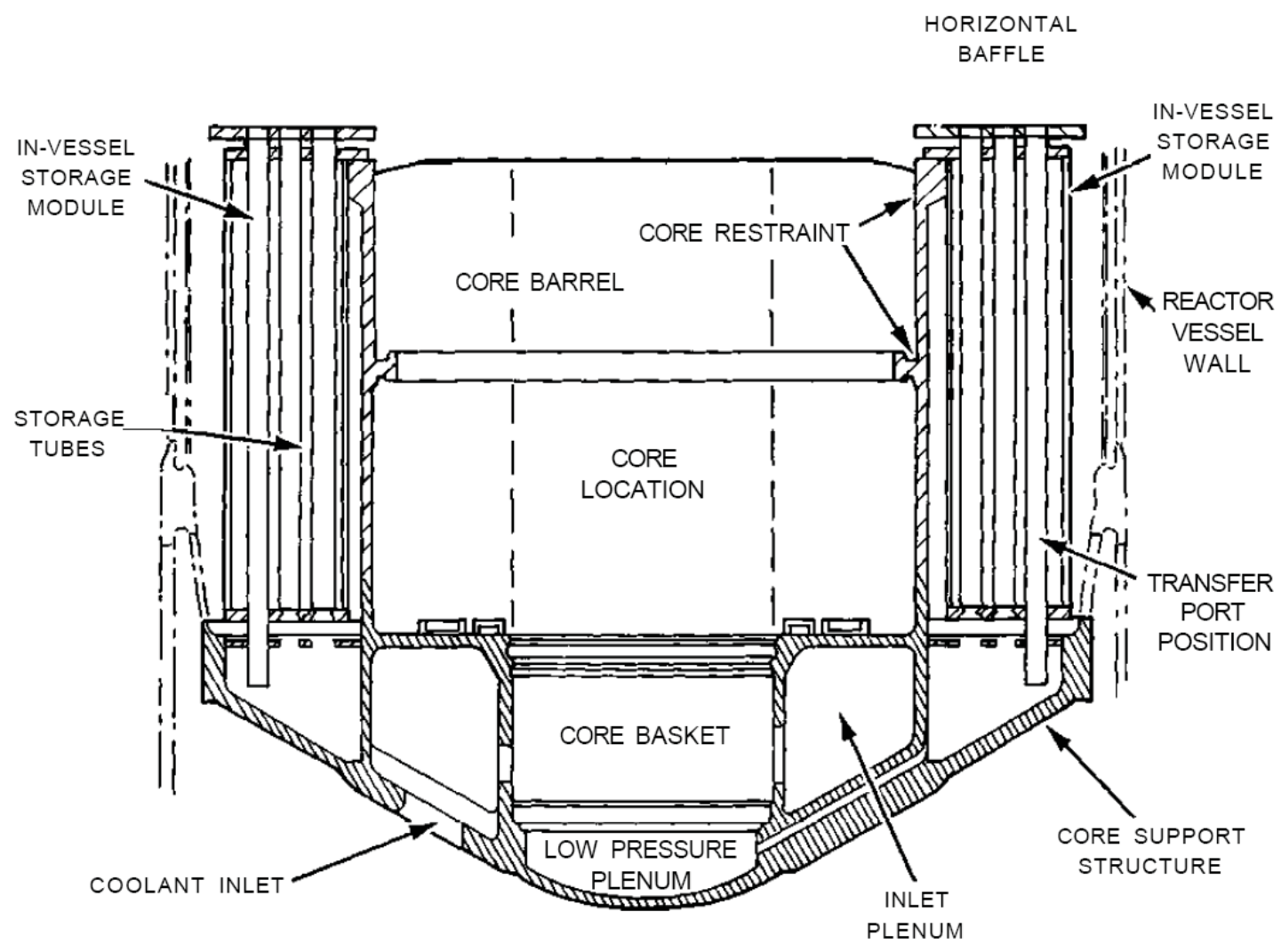

Figure E.1.14. Locations of In-Vessel Storage Modules (Ref. 5, Fig. 3-8). 
NEA/NSC/DOC(2006)1

Liquid Metal Fast Reactor - LMFR

FFTF-LMFR-RESR-001

CRIT-SPEC-REAC-COEF-MISC

Three low level flux monitors (LLFMs) were located $120^{\circ}$ apart within the reactor vessel. The instrumentation was used to determine the reactivity status during subcritical and low-power conditions in the core. Each assembly (Figure E.1.15) was made up of a composite thimble, a sensor drive unit, a sensor with cable, and a nitrogen gas cooling system that maintains the neutron sensor and its cabling below $300^{\circ} \mathrm{F}\left(\sim 149^{\circ} \mathrm{C}\right)$, (Ref. 5, p. 3-9). The LLFM thimbles extended down from the reactor head into the radial shielding surrounding the core. The thimbles were angled, with a radius from the vessel centerline of about 94 inches $(2.3876 \mathrm{~m})$ at the head, reducing to 43 inches $(1.0922 \mathrm{~m})$ at the elevation of the core midplane. At the core midplane, the three thimbles were located at azimuthal angles of $77.2^{\circ}$, $197.2^{\circ}$, and $317.2^{\circ}$ clockwise from north as shown later in Figure E.1.20. Each thimble housed a ${ }^{235} \mathrm{U}$ fission chamber that was normally positioned at the core midplane during subcritical operations and retracted upward prior to power ascent. The isothermal physics test results were obtained with the detectors located at approximately the core midplane elevation (Ref. 3, p. 17).

The LLFMs were symmetrically located at the core midplane at three positions in the surrounding radial shielding $113 \mathrm{~cm}^{\text {a }}$ from the core centerline. The LLFMs were capable of being retracted to a position approximately five feet above the core midplane, thereby extending the power level monitoring range capability of the LLFMs. Three neutron monitors, temporary startup chambers, were used for initial fuel loading. One of the chambers was located at the core midplane and the other two were located vertically above and below it, near the top and bottom of the active fuel region in the In-Reactor Thimble (Ref. 1, p. $3)$.

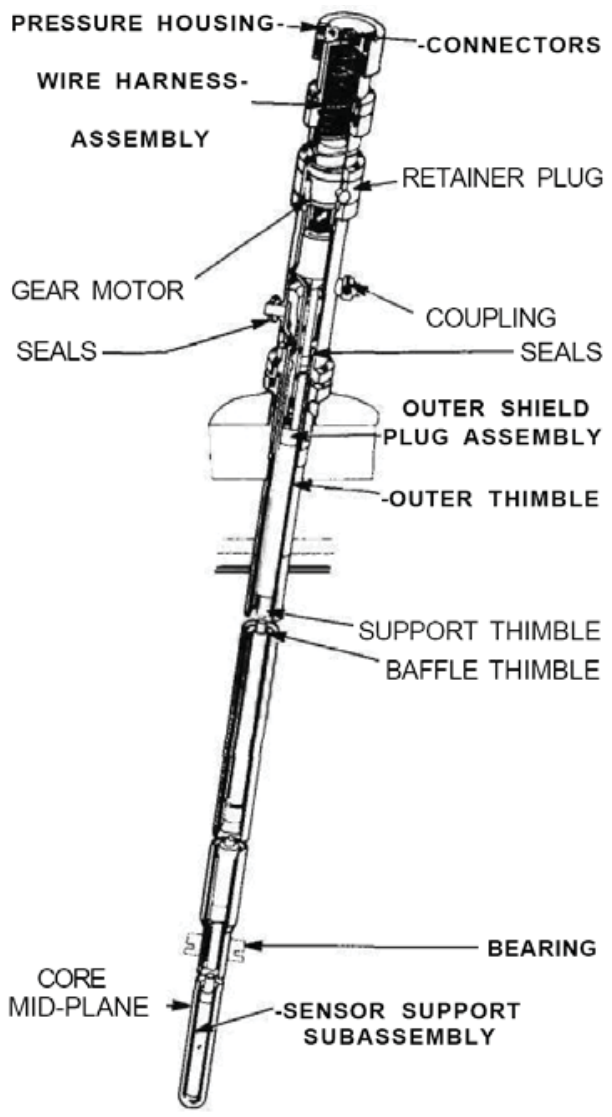

Figure E.1.15. Low Level Flux Monitor (Ref. 5, Fig. 3-7).

\footnotetext{
${ }^{a}$ Reported as approximately 43 in $(109.22 \mathrm{~cm})$ in HEDL-SA-2608.
} 
NEA/NSC/DOC(2006)1

\section{Liquid Metal Fast Reactor - LMFR}

FFTF-LMFR-RESR-001

CRIT-SPEC-REAC-COEF-MISC

Three instrument trees (Figure E.1.16) were implemented in the FFTF. Each tree covered a $120^{\circ}$ section of the core, was approximately 33 feet $(10.0584 \mathrm{~m})$ high, and weighed about 30 tons. An instrument tree had a variety of functions: provided space and support for reactor core and reflector instrumentation assemblies that measure flow and temperature; directed the coolant flow from the in-core assemblies to the instrumentation; provided secondary hold-down for the fuel, reflector, and control rod absorber duct assemblies, limiting the axial movement of in-core assemblies to two inches $(5.08 \mathrm{~cm})$, and provided support, location, and translation to the control rod driveline disconnect assemblies. Each instrument tree contained guide tubes for up to six control rod drivelines and included its own mechanism for raising and lowering the tree with respect to the reactor core. The lift and rotational drives were designed to lift the tree 15 inches $(38.1 \mathrm{~cm})$ vertically at a nominal rate of 1.8 inches $/ \min (0.0762 \mathrm{~cm} / \mathrm{s})$, and rotated the tree $184^{\circ}$ at a nominal rate of 11.4 degrees per minute. The tree could be lifted vertically 16.75 inches $(42.545 \mathrm{~cm})$ for removal from the core. The radius of rotation was 65.142 in $(1.6546068 \mathrm{~m})$ (Ref. 5, pp. 3-4, 3-5, and 3-21 through 3-23).

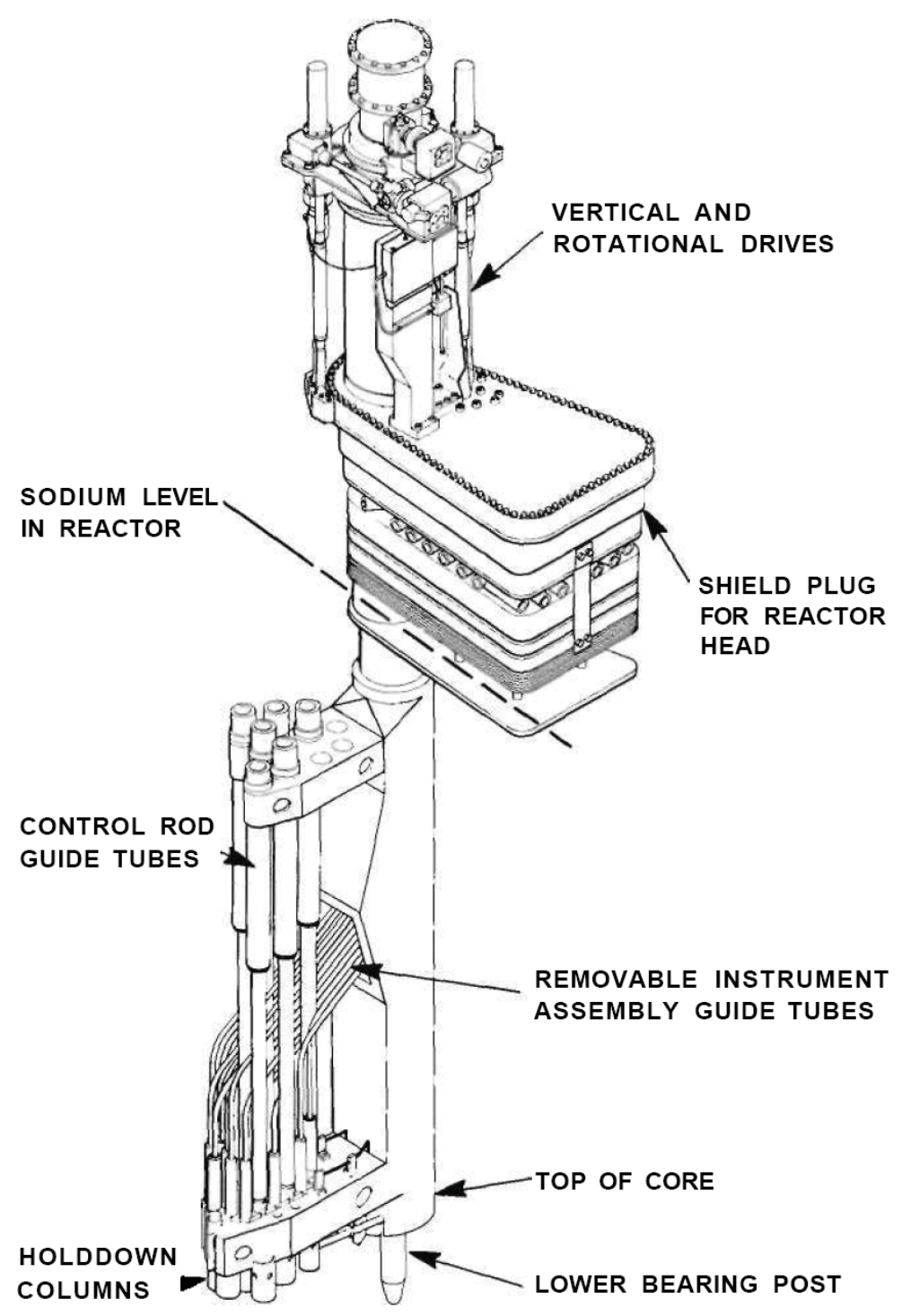

Figure E.1.16. Instrument Tree Schematic (Ref. 5, Fig. 3-2). 
NEA/NSC/DOC(2006)1

\section{Liquid Metal Fast Reactor - LMFR \\ FFTF-LMFR-RESR-001 \\ CRIT-SPEC-REAC-COEF-MISC}

The control rod drive mechanism (CRDM), shown in Figure E.1.17, was an electro-mechanical roller-nut actuating device that moved and positioned the control rod absorber pin bundle within the active core to control the reactor and also dropped the control rod into the core when reactor shutdown was needed. The CRDM was nitrogen cooled and consisted of three subassemblies separately installed into the reactor vessel head nozzle and connected to the control rod driveline assembly. These subassemblies were the lower CRDM assembly, the rotor assembly, and the stator assembly. The CRDM was operated by signals from the reactor control room, which caused the roller nuts on the rotor to engage the threaded portion of the leadscrew on the lower CRDM assembly. Rotation of the electrical field of the stator caused rotation of the roller nuts with respect to the leadscrew and raised or lowered the leadscrew to any desired position. De-energizing the stator caused the roller nuts to disengage and allowed the leadscrew to fall. This action, under spring-assisted gravity forces, dropped the control rod into the core and shut down the nuclear fission reaction. The shafts of this system were hollow, so as to allow for insertion of tools needed for connecting and disconnecting the control rod absorber pin bundle assemblies with control rod drivelines. Eighteen penetrations were provided in the head for control rod assemblies (Ref. 5, pp. 3-4, 3-5, 3-22, and 3-23).

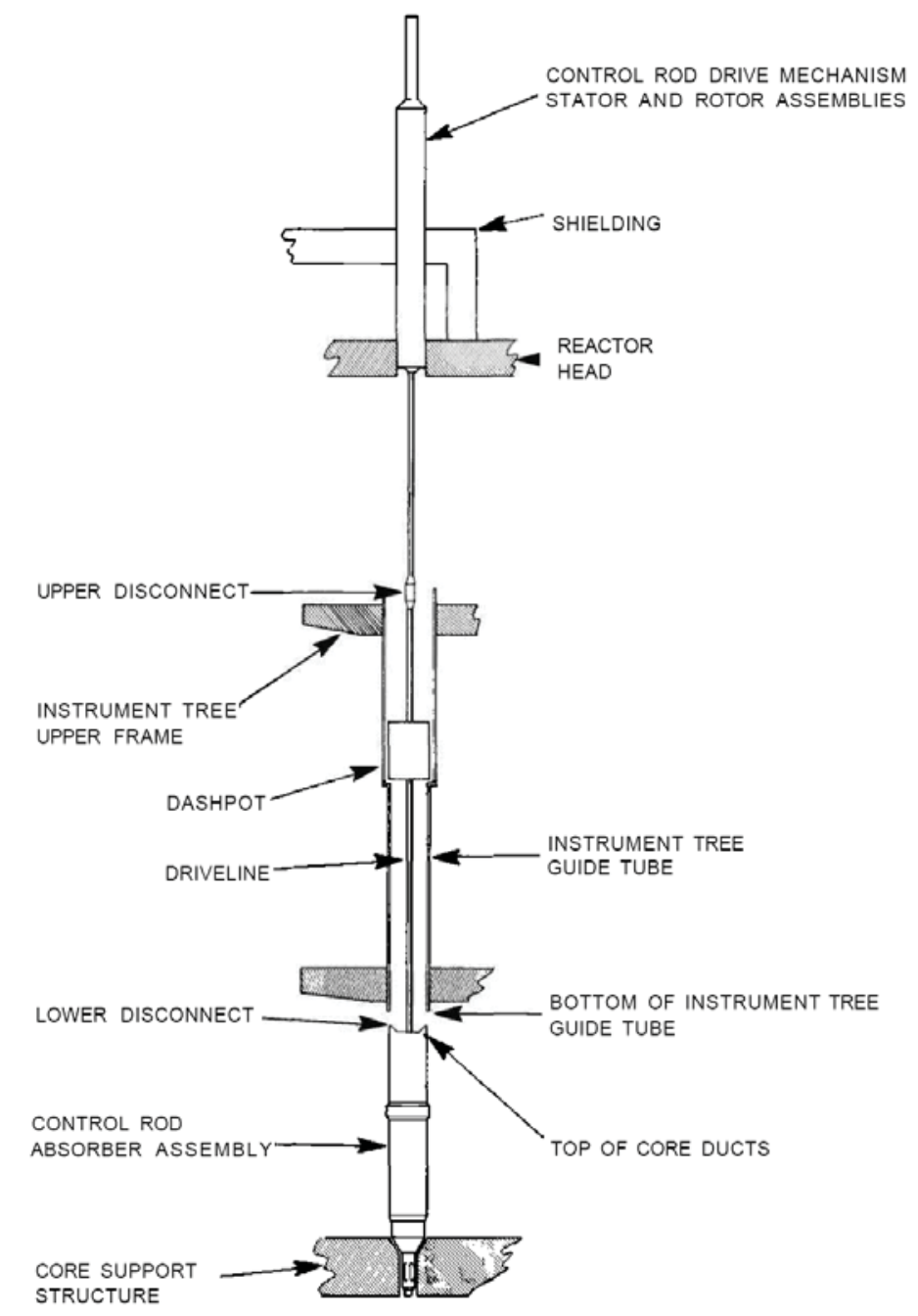

Figure E.1.17. Nuclear Control System (Ref. 5, Fig. 3-3). 
NEA/NSC/DOC(2006)1

Liquid Metal Fast Reactor - LMFR

FFTF-LMFR-RESR-001

CRIT-SPEC-REAC-COEF-MISC

Three in-vessel handling machines (Figure E.1.18) were used to move 12-ft (3.6576 m) core assemblies back and forth between the reactor core, the in-vessel storage modules, and the core component pots beneath the fuel transfer ports. All movements were under sodium. Each of these units serviced its own $120^{\circ}$ sector of the reactor core (Figure E.1.19) and was mounted to a large plug installed in the reactor head. An arm structure with a grappling device was used to grasp core components. Both the plug and arm structure could be rotated by independent ex-vessel drive systems and the arm structure could be raised or lowered. A computer-programmed control console was used to coordinate movements and allow access to any location within the core or storage area. During reactor operation, the IVHM arm was stored radially adjacent to the core, near the vessel wall (Ref. 5, pp. 12-14 through 12-17).

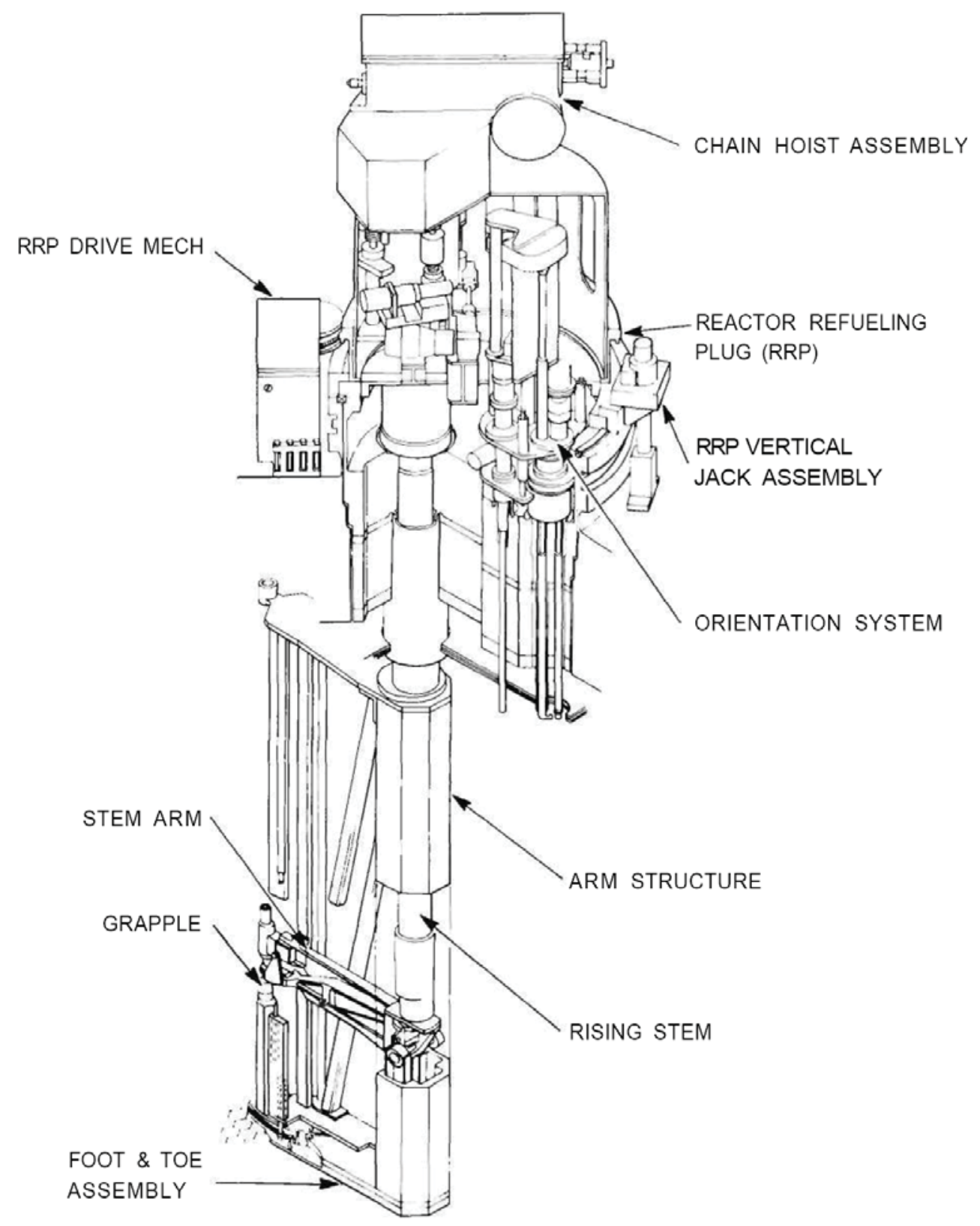

Figure E.1.18. In-Vessel Handling Machine (Ref. 5, Fig. 12-12). 
FFTF-LMFR-RESR-001

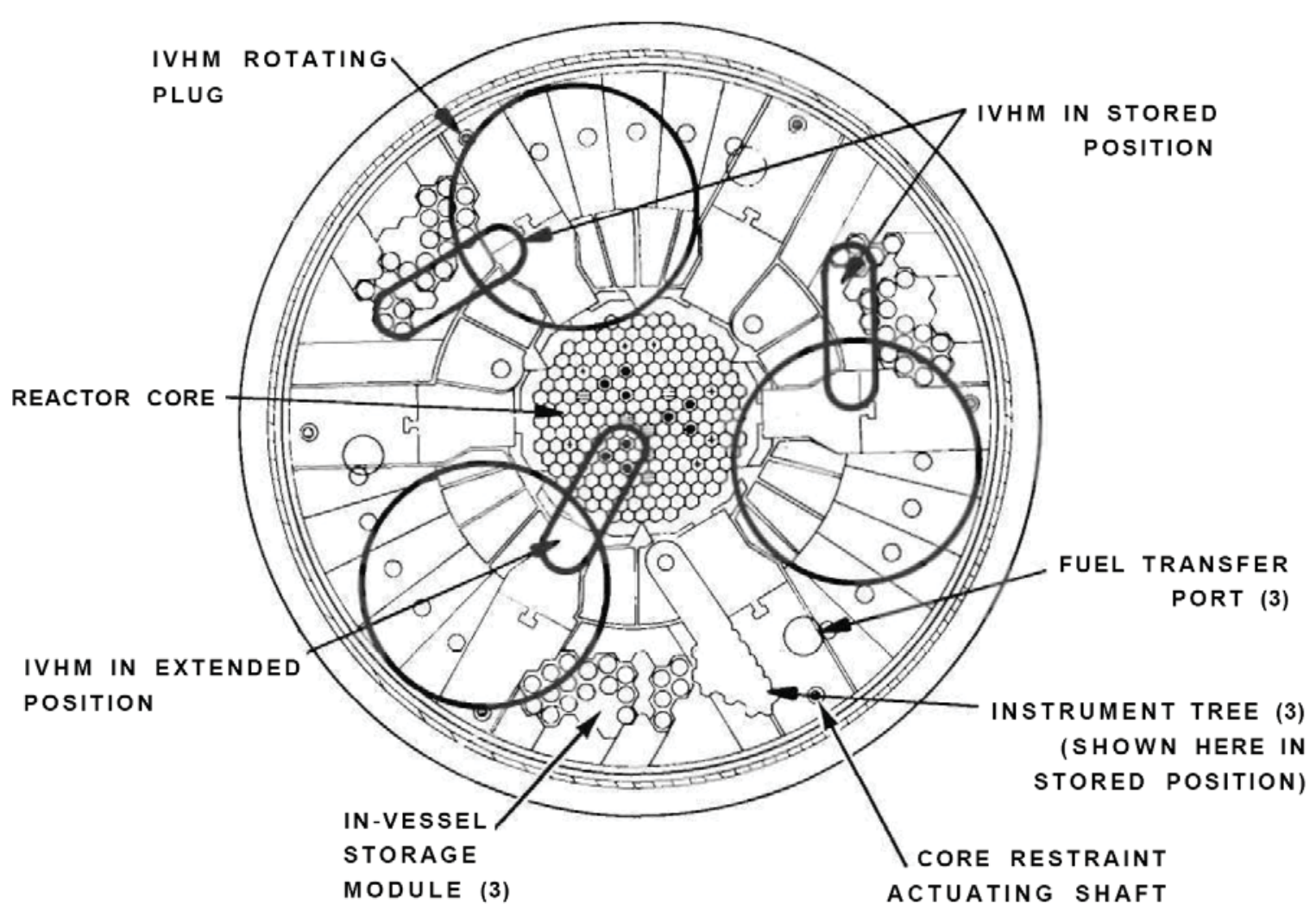

Figure E.1.19. Locations of In-Vessel Handling Machines (Ref. 5, Fig. 12-13).

\section{Simulated Core Assembly (SCA)}

The initial, nonfueled, core array installed in the reactor prior to fuel loading was comprised of SCAs. They were replaced, one at a time, by DFAs. A number of SCAs were present in the core at the time of the initial physics measurements on the partially-loaded core, but they were all removed in order to complete the fully-loaded core design. The main portion of the SCA was a 4-inch $(10.16-\mathrm{cm})$ diameter pipe with a nozzle and orifice assembly attached to the bottom and a handling socket with hexagonal spacer pad assembly at the top. Near the top of the assembly (above the normal fueled region) was a filter and particle trap assembly for coolant system cleanup prior to fuel loading. The overall length of the assembly was the same as a standard core component (Ref. 3, p. 17).

\section{E.1.1.3 Description of Additional Measurements}

The initial approach to criticality commenced on November 27, 1979, when the first fuel assembly was inserted into a core position. All nonfuel assemblies, including reflector assemblies, control rods, safety rods, shim rods, and specially instrumented assemblies, were installed into the hexagonal array core by November 13, 1979. All core positions planned for fuel assemblies had been loaded with Simulated Core Assemblies, which were replaced with fuel assemblies, one at a time, in a preplanned manner. Final check-out of the instrumentation that would monitor the nuclear status of the reactor throughout the fuel loading process had been completed (Ref. 1, pp. 2-3).

Counting data from the LLFMs and IRTs were collected, analyzed, and recorded by a small computer and printer system. The input to the computer consisted of real time, integrated counts from six 
NEA/NSC/DOC(2006)1

Liquid Metal Fast Reactor - LMFR

FFTF-LMFR-RESR-001

CRIT-SPEC-REAC-COEF-MISC

individual scalers, and manual entries of selected plant parameters. Each scaler received ${ }^{235} \mathrm{U}$ fission chamber pulse signals from one of the six monitors. The scalers were gated simultaneously by a single timer (Ref. 1, p. 3).

The reactor was loaded such that after the first four test assemblies and nonfuel assemblies were installed into the core the active core could be loaded on a trisector by trisector basis. During the loading, the neutron multiplication in the core was monitored with the three fission chambers placed in the IRT. Three LLFM fission chambers placed in-vessel but external to the reflector also monitored the neutron multiplication during core loading (Ref. 7, p. 2).

The first fuel assembly loaded was inserted into the central position of the hexagonal array core. Just prior to insertion of the fuel assembly, a background assessment was performed. During and after the insertion of the first fuel assembly, counts from each of the six monitors were collected, analyzed, and recorded, as was the case with all subsequent fuel assembly insertions (Ref. 1, p. 5).

The second fuel assembly was inserted into the core on November 29, 1979, into a test position over which an instrumented stalk was then installed. The next two fuel assemblies installed were FOTA instrumented to provide later confirmation of natural circulation heat removal capability. All remaining fuel to be loaded could then be identified as belonging to one of three trisectors. After the fourth fuel assembly was installed, count rates were obtained that served as the normalization point for beginning the plotting of inverse count rates and predictions of the minimum critical loading of fuel (Ref. 1, p. 5).

Prior to any core component changeout in a given trisector, it was necessary to disengage the instrument tree part of the control rod driveline from the drive motor above and absorber assembly below. This freed the instrument tree (for that trisector) to be swung out, allowing the IVHM access to that part of the core. Because of this arrangement, it was most efficient to load fuel into one trisector at a time. As each trisector loading was completed, the instrument tree for that trisector was rotated and lowered over the core, the control rod drivelines were connected, and the control rods in that trisector could then be manipulated through electrical mechanisms located above the reactor vessel closure head (Ref. 1, p. 5).

The first trisector loaded with fuel was Trisector \#3, adjacent to LLFM B (see Figure E.1.20). Loading commenced on December 11, 1979, with the loading of the fifth fuel assembly into the core, and the trisector was completed on December 23, 1979, with the loading of the twenty-seventh fuel assembly into the core (Ref .1, p. 9). Between December 24, 1979 and January 10, 1980, final preparations were completed that enabled the FFTF to operate as a nuclear reactor facility, including containment isolation, plant protection system actuation, and insertion and withdrawal capability of the three absorber rods of Trisector \#3. Checks on the worths of the three absorber rods in Trisector \#3 (rods 3, 8, and 9) were made, compared to predictions, and accepted according to specified criteria (Ref. 1, p. 11).

The second trisector loaded with fuel was Trisector \#1, adjacent to LLFM C (see Figure E.1.20). Loading commenced on January 10, 1980, with the loading of the twenty-eighth fuel assembly into the core, and the trisector was completed on January 26, 1980, when the fiftieth fuel assembly was loaded into the core (Ref. 1, p. 11). Following the loading of the second trisector, checks on the worths of the three absorber rods in Trisector \#1 (rods 1, 4, and 5) were made by collecting and analyzing count data with each rod fully withdrawn and comparing with prediction. Acceptance criteria were satisfied (Ref. 1, p. 26).

The third and final trisector loaded with fuel was Trisector \#2, adjacent to LLFM A (see Figure E.1.20). Loading of the final trisector commenced on January 30, 1980, with the loading of the fifty-first fuel assembly into the core. Fuel loading was halted temporarily on February 3, 1980, after the fifty-ninth fuel assembly was loaded into the core, to prepare for the initial criticality attempt. Figure E.1.20 shows the core loading pattern for the FFTF (Ref. 1, p. 26). 
FFTF-LMFR-RESR-001

CRIT-SPEC-REAC-COEF-MISC
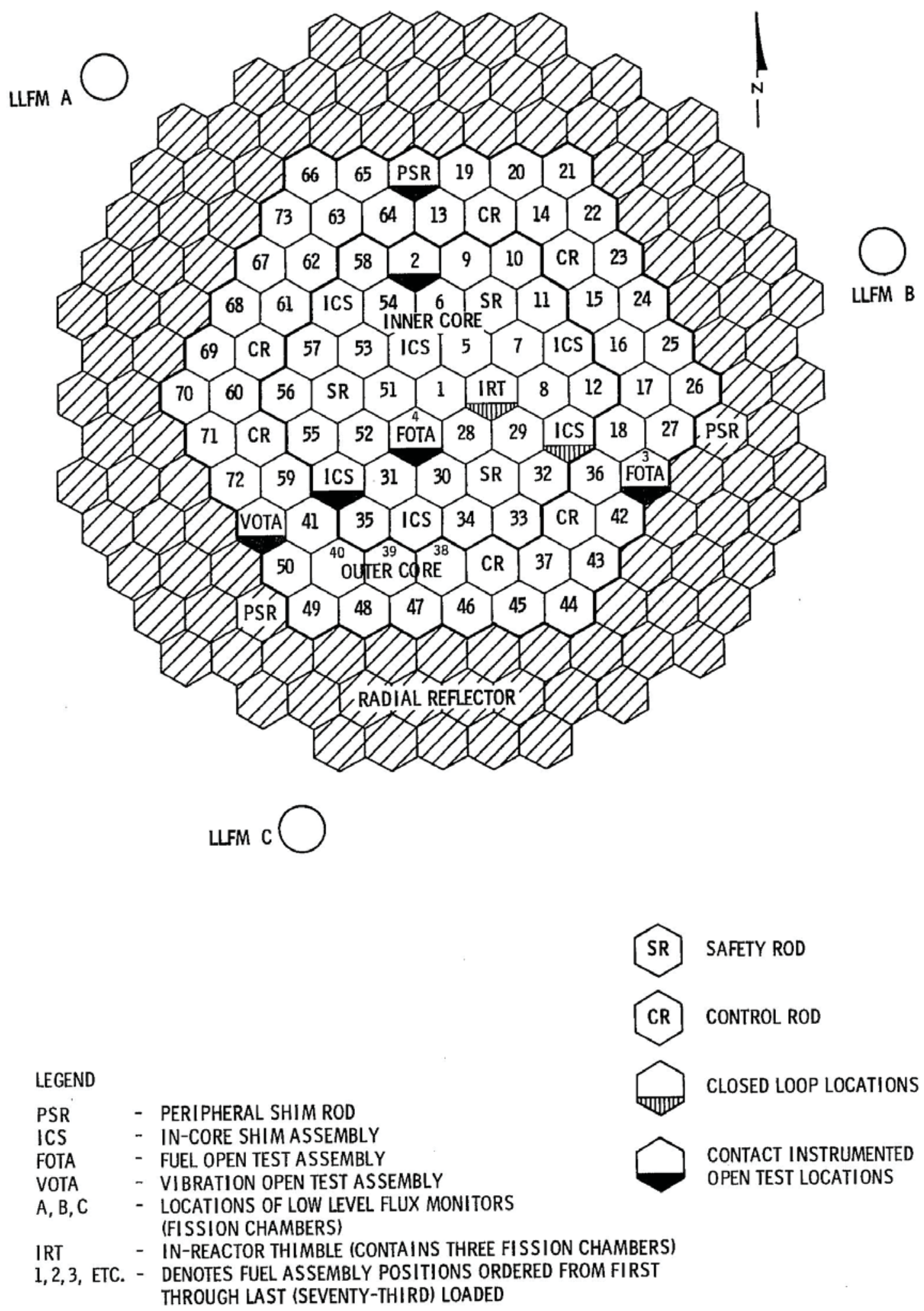

Figure E.1.20. Fuel Loading Pattern for the FFTF (Ref. 2, Fig. 4). 


\section{Liquid Metal Fast Reactor - LMFR \\ FFTF-LMFR-RESR-001 \\ CRIT-SPEC-REAC-COEF-MISC}

The inverse count rates for the first 32 fuel assemblies, normalized to the count rate after loading the fourth fuel assembly, when all control rods are fully inserted is shown in Figure E.1.21. Figure E.1.22 presents the inverse count rate renormalized to the $29^{\text {th }}$ fuel assembly with the three control rods in Trisector 3 fully withdrawn and Figure E.1.23 presents inverse count rate data renormalized to the $50^{\text {th }}$ fuel assembly with six control rods in Trisectors 1 and 3 fully withdrawn (Ref. 2, pp. 5-11). Specific data with uncertainties for the count rates can be found elsewhere (Ref. 10).

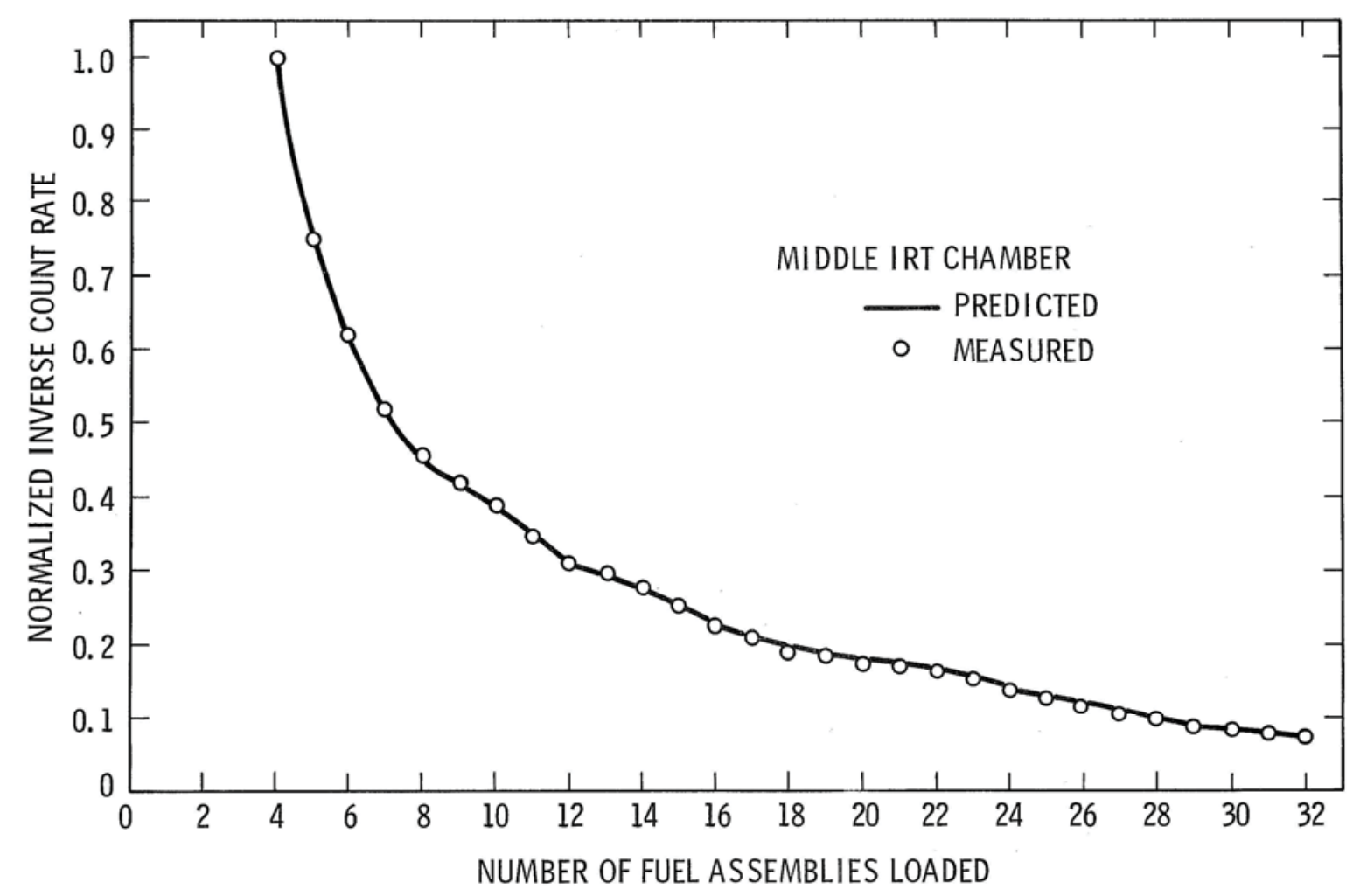

Figure E.1.21. Inverse Count Rate vs. Fuel Assemblies Loaded; First Trisector Loaded; All Control Rods Inserted (Ref. 2, Fig. 1). 
NEA/NSC/DOC(2006)1

Liquid Metal Fast Reactor - LMFR

FFTF-LMFR-RESR-001

CRIT-SPEC-REAC-COEF-MISC

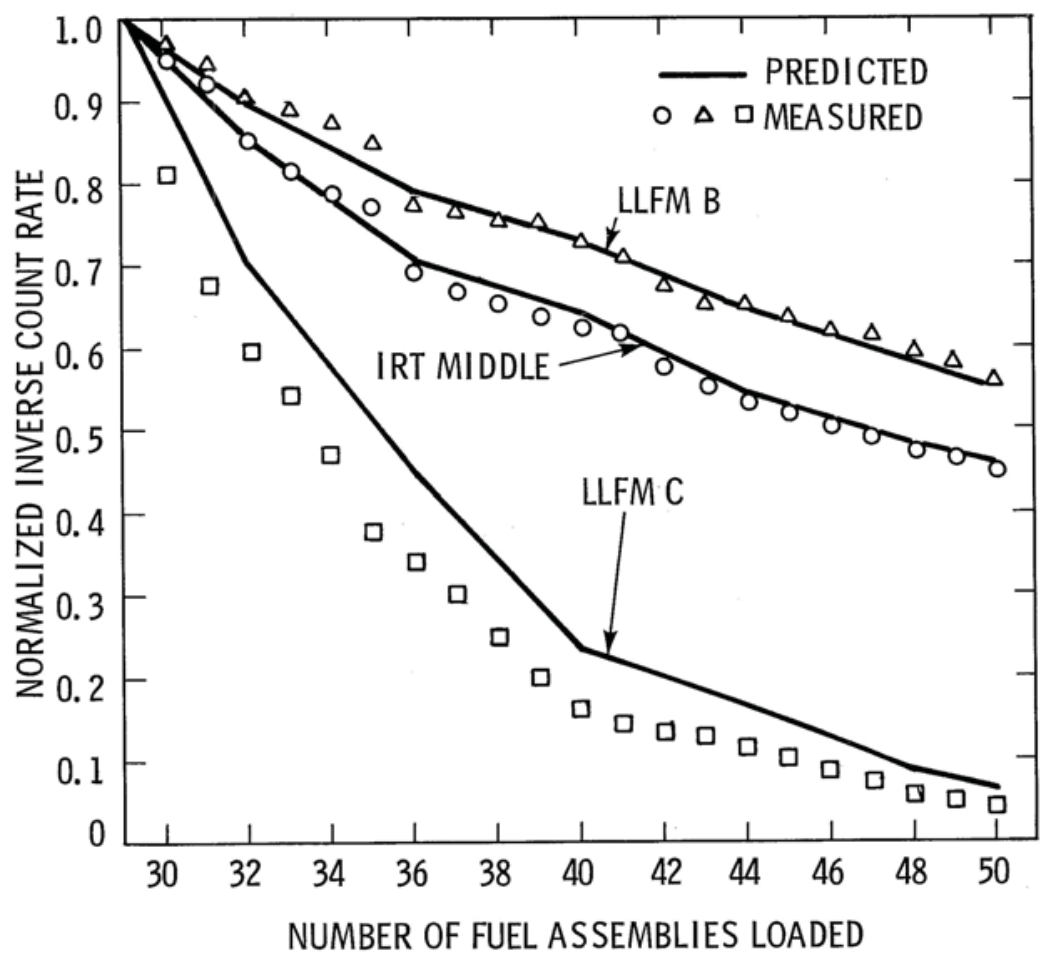

Figure E.1.22. Inverse Count Rate vs. Fuel Assemblies Loaded; Second Trisector Loaded;

Three Control Rods Withdrawn (Ref. 2, Fig. 2).

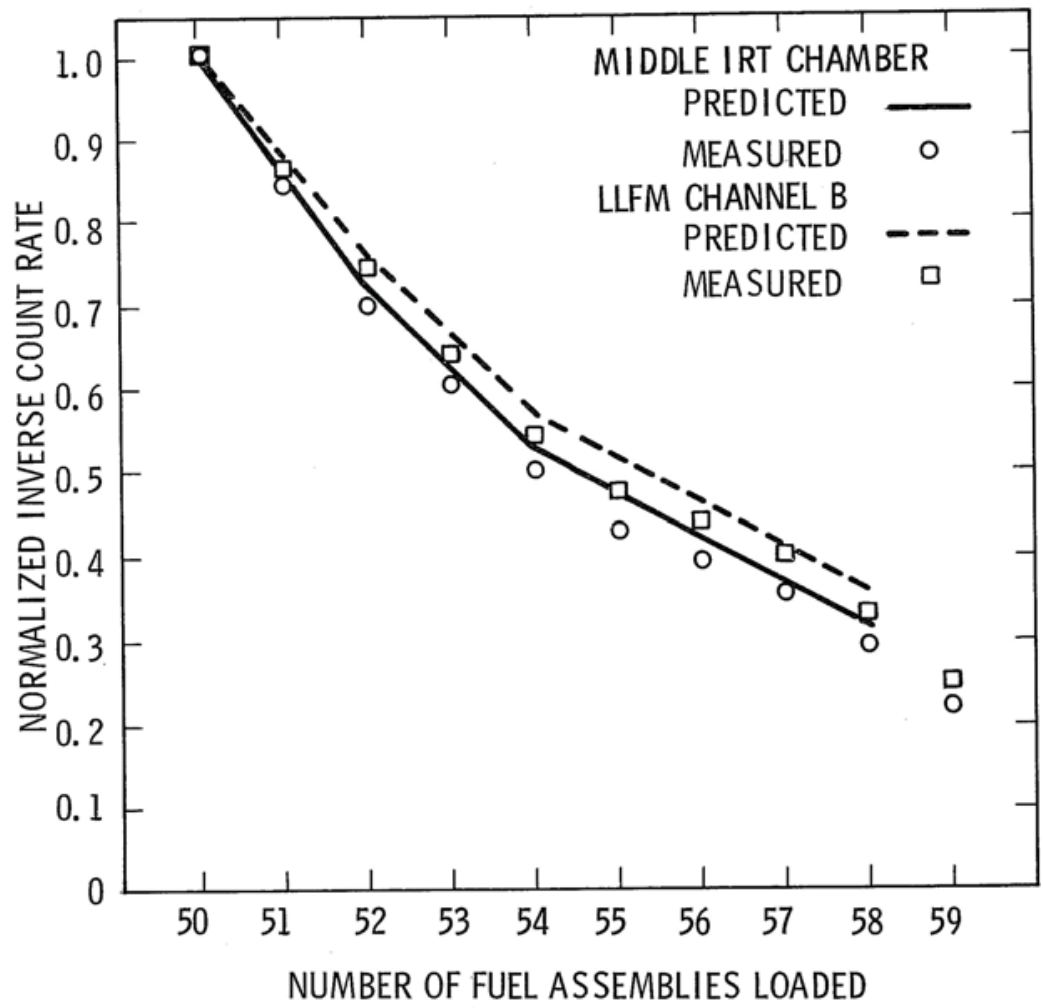

Figure E.1.23. Inverse Count Rate vs. Fuel Assemblies Loaded; Third Trisector Loaded; Six Control Rods Withdrawn (Ref. 2, Fig. 3). 
NEA/NSC/DOC(2006)1

Liquid Metal Fast Reactor - LMFR

FFTF-LMFR-RESR-001

CRIT-SPEC-REAC-COEF-MISC

On February 3, 1980, after fifty-nine fuel assemblies had been installed in the core, further fuel loading was suspended and the third instrument tree, for Trisector \#2, was rotated and lowered over the core. The drivelines for the last three absorber assemblies (rods 2,6, and 7) were connected and the fuel transfer ports through the reactor vessel head were closed and sealed. All mechanical actions needed for the initial approach to criticality were completed by February 6, 1980 (Ref. 1, p. 36).

On February 9, 1980, the rod withdrawals to achieve criticality began. The primary safety rods (rods 1, 2 , and 3) were fully withdrawn and count data were recorded that provided the normalization point for the inverse count rate as a function of secondary rod bank height. At 9:30 am the plot of inverse count rate as a function of secondary rod bank height had begun. The secondary rods (rods 4, 5, 6, 7, 8, and 9) were then withdrawn, one at a time, a preplanned distance or until one or more of the observed count rates doubled. Rod pulls were made in three-inch $(7.62-\mathrm{cm})$ increments until a bank height of twentyseven inches $(68.58 \mathrm{~cm})$ from full insertion was achieved. At each three-inch $(7.62-\mathrm{cm})$ increment in secondary rod bank height, count data were recorded and plotted. The secondary rods were then pulled to bank heights of $29,30,30.6$, and 31 inches $(73.66,76.2,77.724$, and $78.74 \mathrm{~cm})$, with count data taken at each bank height. By 2:30 pm, control rod bank withdrawal had proceeded to the height of thirty-one inches $(78.74 \mathrm{~cm})$, the height from which the next rod bank movement was expected to achieve criticality. Figure E.1.24 shows the inverse count rate as a function of secondary rod bank height. The extrapolated critical rod bank height, shown in Figure E.1.24 and Table E.1.2, was 31.3 inches (79.502 cm) (Ref. 1, pp. 36-37). The core configuration of the partially-loaded core is shown in Figure E.1.25.

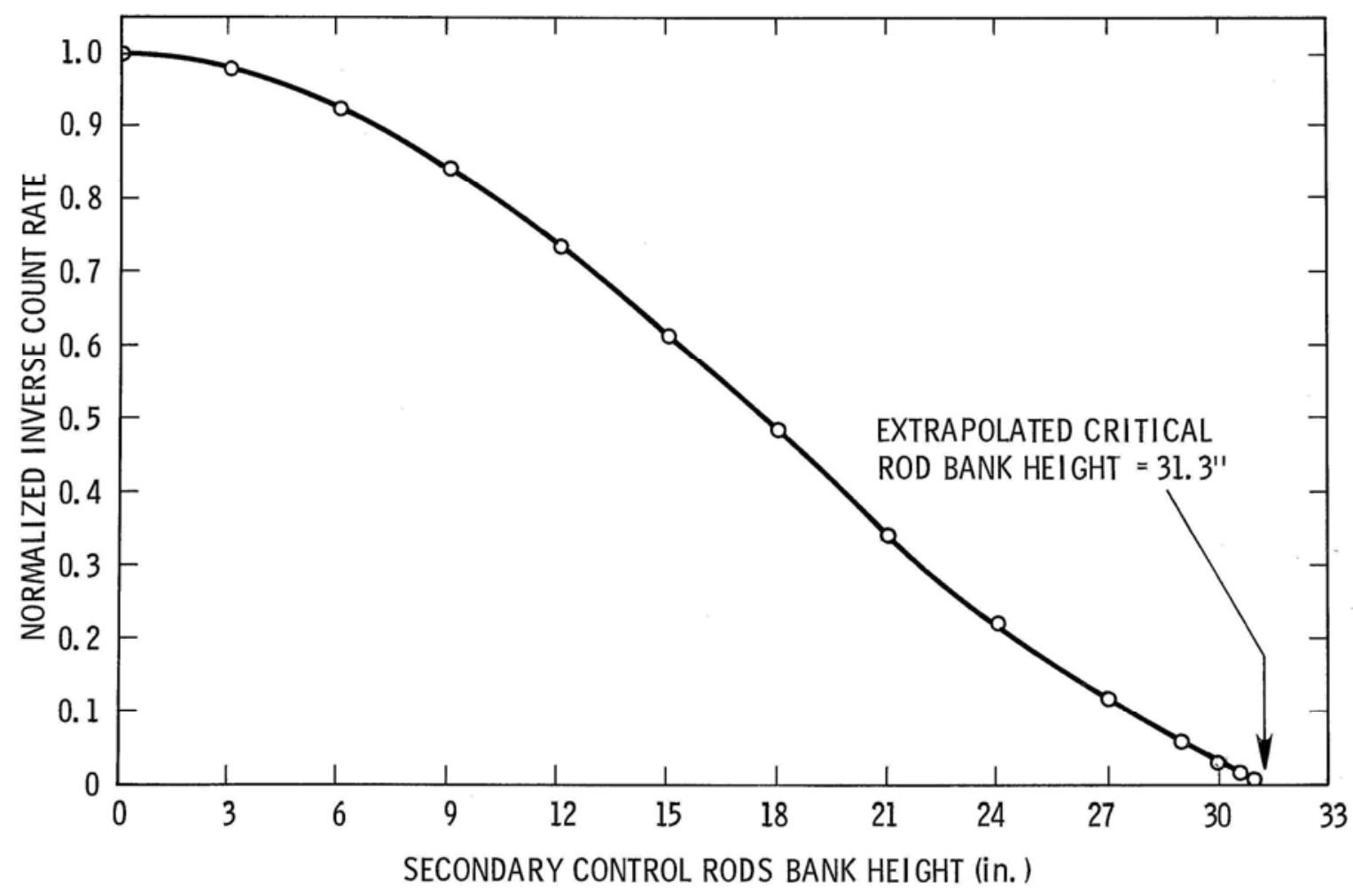

Figure E.1.24. FFTF Initial Approach to Critical (Ref. 1, p. 38, Fig. 26, Ref. 2, Fig. 5, and Ref. 7). 


\section{NEA/NSC/DOC(2006)1}

Liquid Metal Fast Reactor - LMFR

FFTF-LMFR-RESR-001

CRIT-SPEC-REAC-COEF-MISC

Table E.1.2. Extrapolated Critical Rod Bank Positions (Ref. 3, p. 34).

\begin{tabular}{||c|c|c||}
\hline Number of Fuels Loaded & Temperature & $\begin{array}{c}\text { Critical Rod Position } \\
\text { (distance withdrawn) }\end{array}$ \\
\hline \hline 59 (Partial Core) & $406^{\circ} \mathrm{F}\left(\sim 208^{\circ} \mathrm{C}\right)$ & 31.3 in. $(79.502 \mathrm{~cm})$ \\
73 (Fully-Loaded Core) & $404^{\circ} \mathrm{F}\left(\sim 207^{\circ} \mathrm{C}\right)$ & 14.1 in. $(35.814 \mathrm{~cm})$ \\
\hline
\end{tabular}

\section{FFTF CORE MAP}

\section{INITIAL CFITICAL CONFIGURATION}

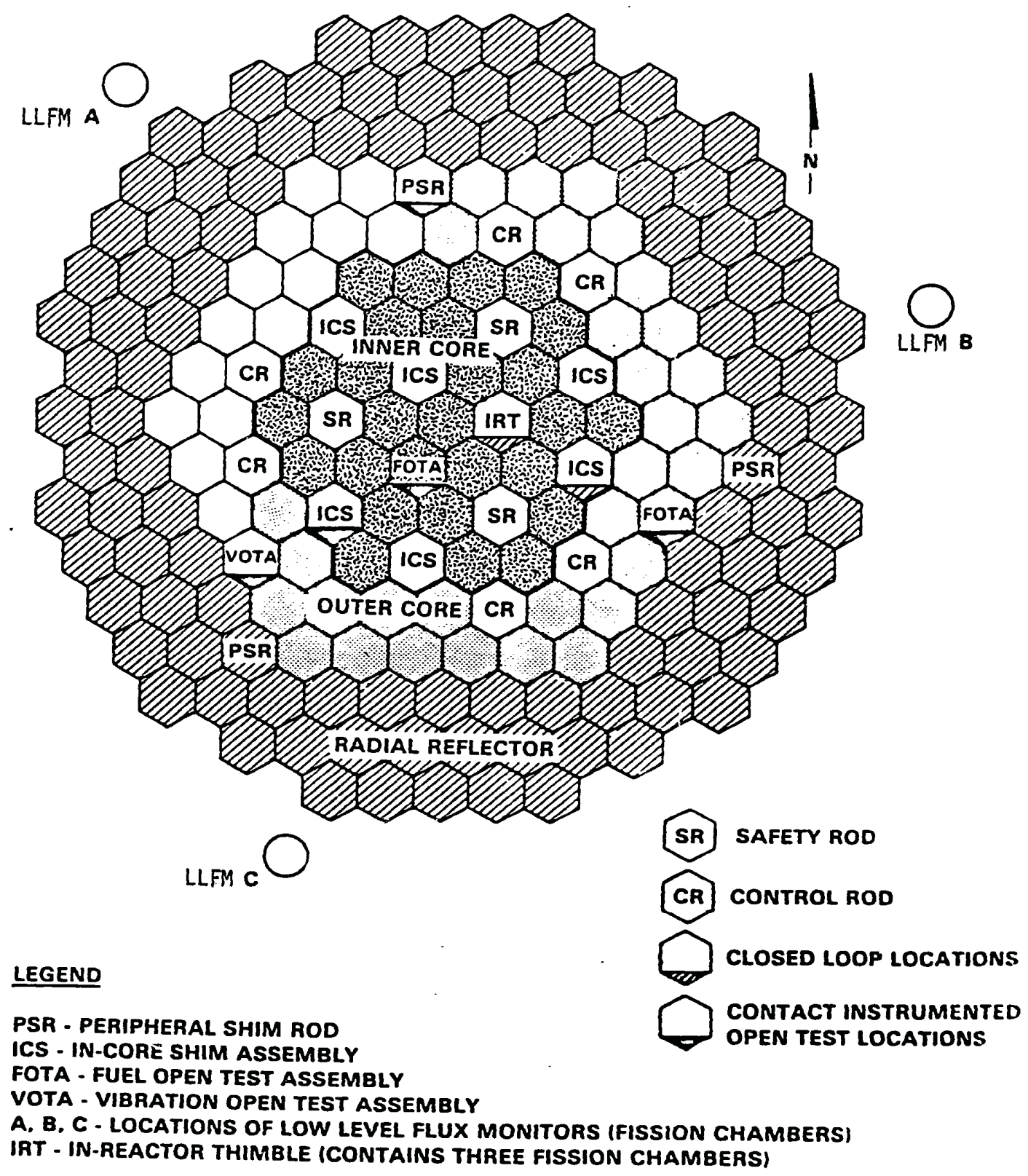

Figure E.1.25. Partially-Loaded Core Layout (Ref. 1, Fig. 18 and Ref. 3, Fig. 7). 
NEA/NSC/DOC(2006)1

Liquid Metal Fast Reactor - LMFR

FFTF-LMFR-RESR-001

CRIT-SPEC-REAC-COEF-MISC

For efficiency purposes it was decided not to pull the secondary rods further as a bank to achieve criticality, but to pull one rod from the bank. Rod 4 was selected and at 3:45 pm was pulled from the bank sufficient to achieve initial criticality at a startup rate of approximately 0.9 decade per minute. Startup was terminated by reinsertion of rod 4 when approximately $1 \mathrm{~kW}$ of fission power was reached (Ref. 1, p. 37).

With the three primary rods fully withdrawn to 36.5 inches $(92.71 \mathrm{~cm})$ and the six secondary control rods banked at 31 inches $(78.74 \mathrm{~cm})$, secondary rod 4 was withdrawn to a height of approximately 34.3 inches $(87.122 \mathrm{~cm})$ to achieve criticality with a 29 -second period (Ref. 2, p. 12).

Relatively few measurements were performed on the initial critical loading of the FFTF because they were less important than those for the fully-loaded core. However, the results from the initial criticality demonstrated good agreement with calculations based on analytical techniques for fully-loaded cores. ${ }^{\text {a }}$

Subsequent to initial criticality, Trisector \#2 was reopened and fuel loading continued to complete the loadout of the core. The sixtieth fuel assembly was inserted into the core on February 12, 1980, and core loading was completed by inserting the seventy-third fuel assembly into the core on February 19, 1980. Figure E.1.26 shows the final, fully loaded core arrangement. During the loading of the last fourteen fuel assemblies, count rate data were recorded for two rod configurations; all-rod-in and rod 3 out. During final loadout of the core, the secondary system shutdown margin was also estimated, and a criterion for continuation of fuel loading based on margin assessment was invoked. The margin value was to remain negative at all times. The criterion was not violated (Ref. 1, p. 37).

On February 22, 1980, the reactor was again taken to a near-critical state; the first time since the completion of fuel loading. By this time the special startup chambers in the IRT had served their purpose and had been removed from the reactor. Consequently, count rate data were recorded for the LLFMs only. At 12:30 pm on February 22 the three primary rods had been withdrawn and the plot of inverse count rate as a function of secondary rod bank height had begun. The secondary rods were withdrawn, one at a time, a preplanned distance or until the count rate doubled. Rod pulls were made in two-inch $(5.08-\mathrm{cm})$ increments until a bank height of twelve inches $(30.48 \mathrm{~cm})$ from full insertion was achieved. At each two-inch $(5.08-\mathrm{cm})$ increment in secondary rod bank height, count data were recorded and plotted. The secondary rods were then pulled to bank heights of 13,13.5, 13.8, and 13.9 inches (33.02, $34.29,35.052$, and $35.306 \mathrm{~cm}$ ), with count data taken at each bank height. By 5:40 pm the rod pulls had proceeded to the secondary rod bank height of 13.9 inches $(35.306 \mathrm{~cm})$, from which it was expected that one more rod pull would achieve criticality. Figure E.1.27 shows the inverse count rate as a function of secondary rod bank height. The extrapolated critical rod bank height, shown in Figure E.1.27 and Table E.1.2, was 14.1 inches $(35.814 \mathrm{~cm})$, (Ref. 1, p. 44).

The objective of the February 22 approach to criticality was not to achieve criticality but to assess the subcritical reactivity state by the inverse kinetics method analysis of a rod drop experiment. This analysis was performed by the IKRD computer program. The reactivity state, as determined by dropping rod 3 and analyzing the results, was $-16 \phi$. This experiment set the stage for performing subsequent subcritical reactivity measurements, rod worth measurements, and shutdown system margin measurements (Ref. 1, p. 46 and Ref. 2, p. 12).

\footnotetext{
${ }^{a}$ R. B. Rothrock, J. W. Daughtry, B. D. Zimmerman, N. E. Petrowicz, R. A. Bennett, and P. A. Ombrellaro, "FFTF Initial Fuel Loading, Preanalysis, and Comparison with Preliminary Results," HEDL-SA-2104 (CONF-800942-18), Proc. Advances in Reactor Physics and Shielding, Sun Valley, ID, September 14 (February 1980).
}

Revision: 0

Page 286 of 304

Date: March 31, 2010 
FFTF-LMFR-RESR-001

CRIT-SPEC-REAC-COEF-MISC

Figure 28. FULLY LOADED FFTF CORE

FEBRUARY 19, 1980
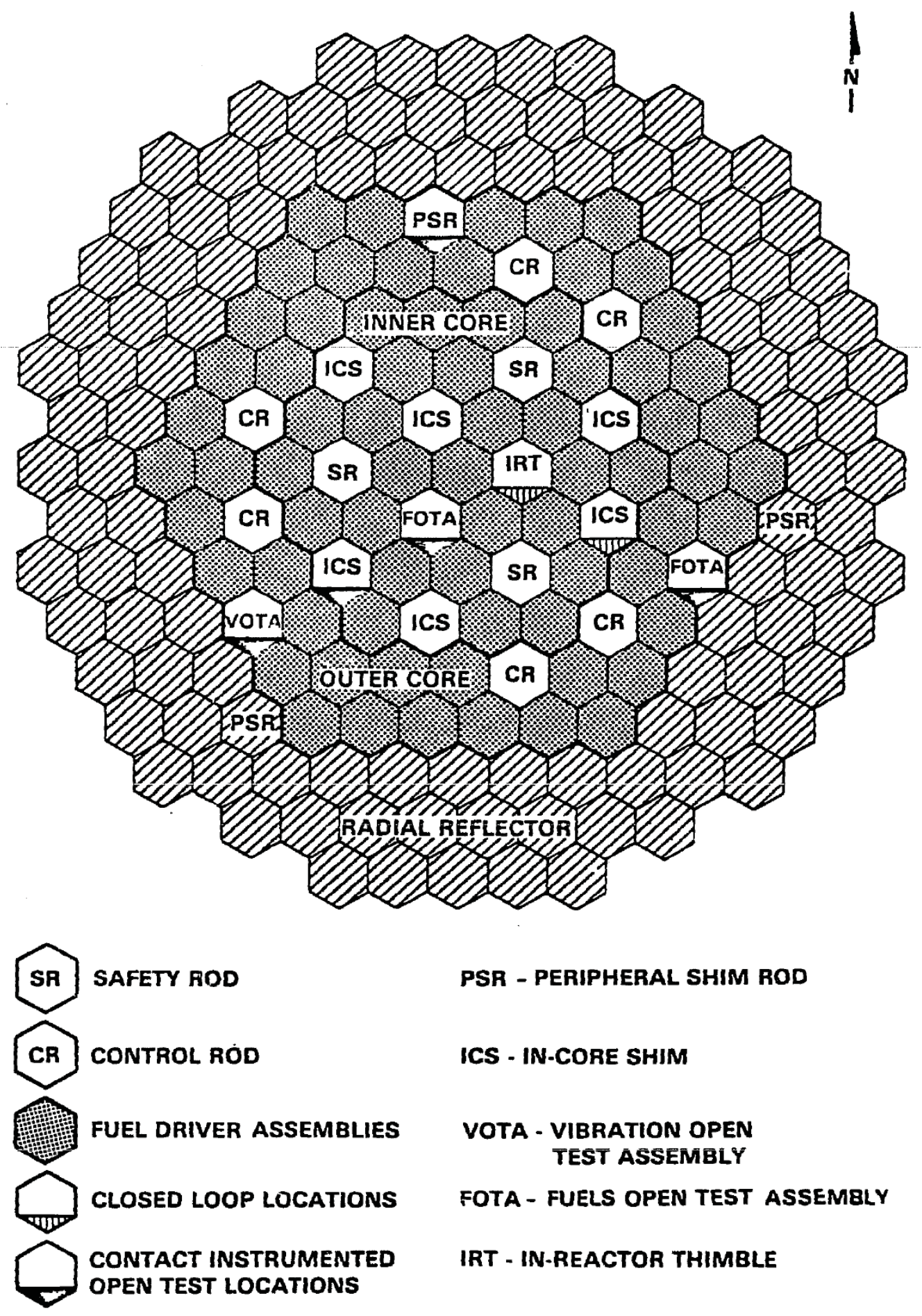

Figure E.1.26. Fully-Loaded Core Layout (Ref. 1, Fig. 28). 
NEA/NSC/DOC(2006)1

Liquid Metal Fast Reactor - LMFR

FFTF-LMFR-RESR-001

CRIT-SPEC-REAC-COEF-MISC

FTR CORE MAP

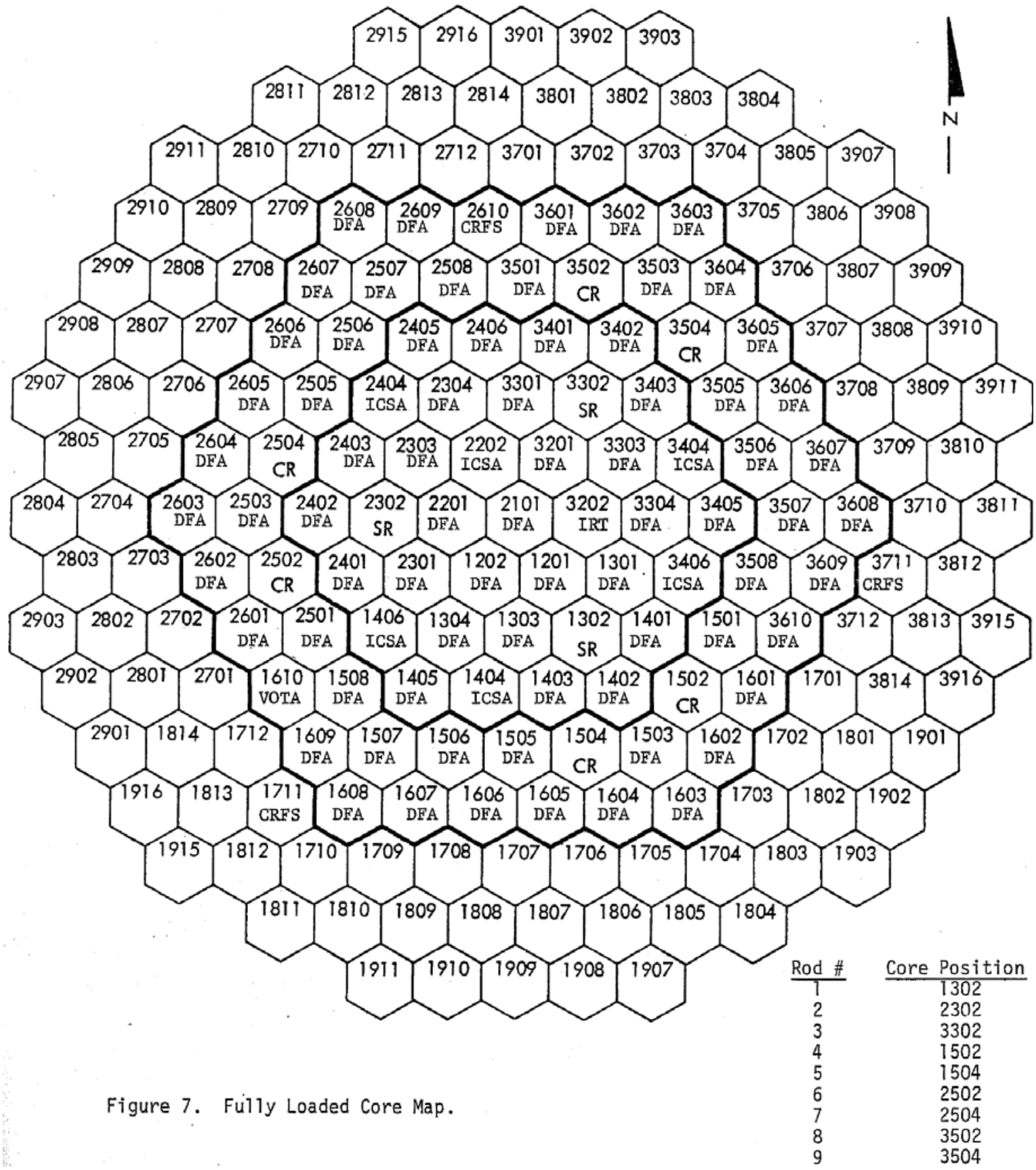

Figure E.1.26 (cont'd.). Fully-Loaded Core Layout (Ref. 1, Fig. 2 and Ref. 3, Fig. 7). 
NEA/NSC/DOC(2006)1

Liquid Metal Fast Reactor - LMFR

FFTF-LMFR-RESR-001

CRIT-SPEC-REAC-COEF-MISC

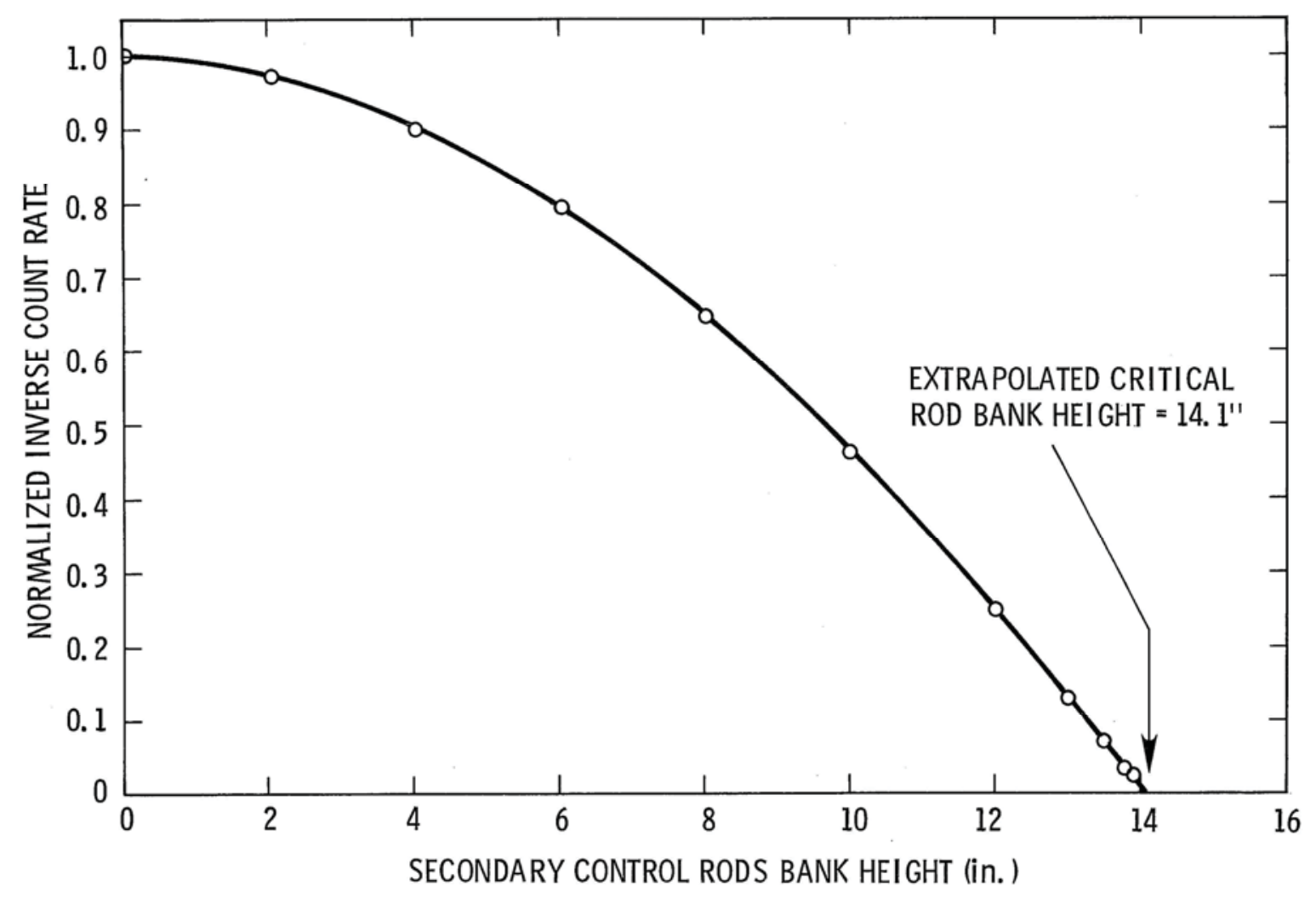

Figure E.1.27. FFTF Approach to Critical After Core Fully Loaded

(Ref. 1, Fig. 32, Ref. 2, p.6, and Ref. 7).

\section{E.1.1.4 Description of Subcriticality Measurements}

Modified source multiplication (MSM) was the method that was used to monitor the reactivity of the FFTF during all subcritical operations such as startup, refueling, and reactor component changeout. The method was based on measured neutron count rates and core configuration factors calculated for the neutron detectors. These factors account for neutron detection efficiency changes resulting from core configuration changes, such as rod movements. Before MSM was fully implemented, its accuracy was determined from reactivity comparisons with inverse kinetics rod drops (IKRD). This latter method is a technique of measuring control rod worths by rod-drop experiments performed with the reactor near critical (Ref. 1, pp. 46-47).

A systematic difference of $2.67 \%$ was found between count rates measured with the on-line computer in the dedicated IKRD mode, and those measured in a multitask mode, as in the case for MSM measurements. This is attributed to the slight difference in the timing of counting intervals due to the overhead operations required for the multitask mode, and not performed in the dedicated mode. Since the average detector count rates reported for MSM measurements were collected in the multitask mode, the appropriate LLFM calibration constants for use with these data are $2.67 \%$ larger than the values obtained by the IKRD processing (Ref. 3, p. 34). 
NEA/NSC/DOC(2006)1

Liquid Metal Fast Reactor - LMFR

FFTF-LMFR-RESR-001

CRIT-SPEC-REAC-COEF-MISC

\section{Inverse Kinetics Rod Drop (IKRD)}

The IKRD method was used at the FFTF for precise measurements of subcriticality, for worth measurements of control rods, and to establish a calibration point for shutdown reactivity measurements using the modified source multiplication (MSM) technique (Ref. 3, p. 26).

Further description of the IKRD method used in the FFTF can be found in Section 1.4.2.

\section{Modified Source Multiplication (MSM)}

The majority of operational subcritical reactivity measurements at the FFTF were obtained by the MSM technique, once a calibration of the effective neutron source strength for each detector had been made. Details of the HEDL calculational methods for MSM applications are provided elsewhere (Ref. 3, p. 28 and Ref. 8 pp. 1-4). ${ }^{\mathrm{a}}$

Subcriticality measurements by MSM were implemented at the FFTF with the on-line computer, which was used to collect count rate data from the three LLFMs for a preset time interval, performed statistical checks on the data, and computed the subcriticality using appropriate configuration factors keyed in by the operator. Separate results were provided for each detector, along with an estimated uncertainty (Ref. 3 , p. 29)

\section{Experimental Results}

Results of the MSM subcriticality measurements are given in Table E.1.3 for core configurations with all nine rods in, three rods out, and six rods out.

\footnotetext{
a P. A. Ombrellaro and R. A. Harris, "Method of Monitoring Subcritical Reactivity During Core Refueling," HEDLSA-2612-FP, Proc. Advances in Reactor Physics and Core Thermal Hydraulics, Kiamesha Lake, NY, September 22-24 (May 1982).
} 
NEA/NSC/DOC(2006)1

Liquid Metal Fast Reactor - LMFR

FFTF-LMFR-RESR-001

CRIT-SPEC-REAC-COEF-MISC

Table E.1.3. MSM Subcriticality Measurements (Ref. 3, p. 36 and Ref. 9, p. 8).

\begin{tabular}{|c|c|c|c|c|c|c|}
\hline \multicolumn{2}{|c|}{ Rod Configuration } & \multirow{2}{*}{ Temperature $^{\mathrm{a}}$} & & \multicolumn{3}{|c|}{ LLFMs } \\
\hline Row 3 Rods & Row 5 Rods & & & A & $\mathrm{B}$ & $\mathrm{C}$ \\
\hline \multirow[t]{4}{*}{ Out } & \multirow[t]{4}{*}{ In } & \multirow{4}{*}{$\begin{array}{c}400^{\circ} \mathrm{F} \\
\left(\sim 204^{\circ} \mathrm{C}\right)\end{array}$} & F-factor & 1.000 & 1.000 & 1.000 \\
\hline & & & Avg. Count Rate & 649 & 433 & 623 \\
\hline & & & Calibration Constant & 4731.9 & 2316.3 & 4472.4 \\
\hline & & & Subcriticality & $\$ 7.29$ & $\$ 7.43$ & $\$ 7.18$ \\
\hline \multirow[t]{4}{*}{ In } & \multirow[t]{4}{*}{ In } & \multirow{4}{*}{$\begin{array}{c}404^{\circ} \mathrm{F} \\
\left(\sim 207^{\circ} \mathrm{C}\right)\end{array}$} & F-factor & 1.1036 & 1.1053 & 1.0929 \\
\hline & & & Avg. Count Rate & 222 & 147 & 210 \\
\hline & & & Calibration Constant & 4731.9 & 3216.3 & 4472.4 \\
\hline & & & Subcriticality & $\$ 23.5$ & $\$ 24.2$ & $\$ 23.3$ \\
\hline \multirow[t]{4}{*}{ In } & \multirow[t]{4}{*}{ Out } & \multirow{4}{*}{$\begin{array}{c}404^{\circ} \mathrm{F} \\
\left(\sim 207^{\circ} \mathrm{C}\right)\end{array}$} & F-factor & 1.022 & 1.036 & 1.058 \\
\hline & & & Avg. Count Rate & $\mathrm{b}$ & b & b \\
\hline & & & Calibration Constant & 4731.9 & 3216.3 & 4472.4 \\
\hline & & & Subcriticality & \multicolumn{3}{|c|}{$\$ 3.68$ average } \\
\hline
\end{tabular}

(a) Results can be adjusted using the measured isothermal temperature coefficient of $-0.6 \phi /{ }^{\circ} \mathrm{F}$.

(b) Individual counter readings not available.

Values for $\mathrm{k}_{\mathrm{eff}}$ were reported for four cases: two MSM cases from Table E.1.3 and two IKRD measurements. The cases and their configurations are reported in Table E.1.4. These cases had been selected for evaluation of the criticality bias, and covered a subcriticality range from near-critical to $\$ 7.3$ shutdown (Ref. 9, p. 11).

Table E.1.4. HEDL Criticality Results

(Ref. 3, p. 46, Ref. 4, pp. 31-36, and Ref. 9, p. 12).

\begin{tabular}{|c|c|c||}
\hline Case \# & Configuration & Measured $\mathrm{k}_{\text {eff }}{ }^{\mathrm{a}}$ \\
\hline \hline 1 & $1,2,3$ out & 0.9773 \\
& 4 thru 9 in & \\
2 & $1,2,3$ in & 0.9884 \\
& 4 thru 9 out & 0.9991 \\
$3^{\mathrm{b}}$ & $4,6,8,9$ in & 0.9900 \\
& $1,2,3,5,7$ out \\
4 & $4,6,7,8,9$ in \\
\end{tabular}

(a) Measured subcritical reactivities were converted using a value of $\beta=0.00318$. Cases 1 and 2 are based on MSM measurements, and Cases 3 and 4 are from IKRD results.

(b) Rod \#7 withdrawn to 28 inches $(71.12 \mathrm{~cm})$; a bias of $\$ .47$ $(0.0015 \Delta \mathrm{k})$ accounts for the difference between the partially inserted and fully withdrawn position of this rod. 
NEA/NSC/DOC(2006)1

Liquid Metal Fast Reactor - LMFR

FFTF-LMFR-RESR-001

CRIT-SPEC-REAC-COEF-MISC

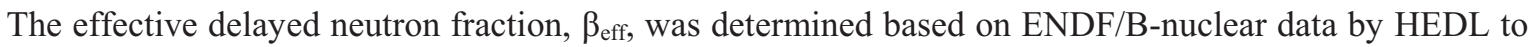
be 0.00318 and was used to convert measured reactivities into $\Delta \mathrm{k}$ (Ref. 4, p. 15). General Electric utilized a $\beta_{\text {eff }}$ value of 0.003107 or 0.003097 in its calculations when calculating rod worths (Ref. 4, pp. 15 and 39-43).

The first subcritical configuration is described by the first entry in Table E.1.3 and first entry in Table E.1.4. The core description was that of a fully loaded core (Figure E.1.26) with the three SRs in Row 3 completely out of the core and the CRs in Row 5 completely inserted into the core.

The second subcritical configuration is described by the second entry in Table E.1.3. The core description was that of a fully loaded core (Figure E.1.26) with the three SRs in Row 3 completely inserted into the core and the CRs in Row 5 completely inserted into the core.

The third subcritical configuration is described by the third entry in Table E.1.3 and the second entry in Table E.1.4. The core description was that of a fully loaded core (Figure 1.1.26) with the three SRs in Row 3 completely inserted into the core and the CRs in Row 5 completely out of the core.

The fourth subcritical configuration is described by the third entry in Table E.1.4. The core description was that of a fully loaded core (Figure E.1.26) with rods 4, 6, 8, and 9 completely inserted into the core; rods $1,2,3$, and 5 completely out of the core; and rod 7 withdrawn to 28 inches $(71.12 \mathrm{~cm})$.

The fifth subcritical configuration is described by the fourth entry in Table E.1.4. The core description was that of a fully loaded core (Figure E.1.26) with rods 4, 6, 7, 8, and 9 completely inserted into the core and rods $1,2,3$, and 5 completely out of the core.

Two additional near-critical configurations were specified when the rod worth experiments were performed. A $\$ 0.15$ subcritical state was achieved with the three primary rods fully withdrawn and the six secondary rods in a bank withdrawn to a height of 13.9 inches $(35.306 \mathrm{~cm})$. A $\$ 0.28$ subcritical state was achieved with the three primary control rods and control rod 5 fully withdrawn and control rod 7 withdrawn to a height of 28 inches $(71.12 \mathrm{~cm})$ above the base of the fuel (Ref. 2, pp. 24-25). This latter configuration had a recorded temperature of $404^{\circ} \mathrm{F}\left(\sim 207^{\circ} \mathrm{C}\right)$ (Ref. 3, p. 29). Additional subcritical states can be obtained from the data provided for rod worth measurements in Section 1.4.2.

\section{E.1.2 Material Data}

\section{E.1.2.1 General Facility Compositions}

The FFTF building and surrounding structures were constructed from steel and concrete. The shielded cavity with the reactor complex holds a carbon-steel containment vessel (Ref. 5, pp. 2-2 through 2-5, 212, and 11-4). The shielded cell containing the reactor was filled with nitrogen gas and the reactor vessel was constructed from type 304 stainless steel. The reactor vessel was filled with liquid sodium at the bottom with argon cover gas on top (Ref. 5, pp. 2-6 and 4-3). The reactor guard vessel made of type 304 stainless steel supported the reactor vessel (Ref. 5, pp. 2-6, 2-7, and 4-4 through 4-5). The reactor head formed from low-alloy carbon steel caps the reactor vessel with shielding. The shielding beneath the head was comprised of four thick metallic plates: two 4-inch $(10.16-\mathrm{cm})$ carbon steel plates, one 4-inch $(10.16-\mathrm{cm})$ stainless steel 410 plate, and one 4-inch $(10.16-\mathrm{cm})$ Inconel 600 plate. Below the shield plates were eight stainless steel 304 thermal reflectors and a segmented gas entrainment suppressor plate of stainless steel 316 (Ref. 5, pp. 2-6, 2-7, and 4-5 through 4-10). 
FFTF-LMFR-RESR-001

CRIT-SPEC-REAC-COEF-MISC

\section{E.1.2.2 Detailed Reactor Compositions}

\section{Reactor Core External Components}

The core support structure and core basket were composed of stainless steel 304. The core barrel was comprised of stainless steel 304. The inner and outer shield blocks were composed of vertical standing stainless steel 304 plates, and were supported by the core support structure (Ref. 5, p. 3-6). The in-vessel storage modules contained stainless steel 304 receptacles (Ref. 5, p. 3-8).

The LLFMs were manufactured by Westinghouse Electric Corporation, Model WL-23831, each containing 2.3 grams of Uranium-235 (Ref. 1, p. 3). The neutron sensitivity was estimated as 1.3 cps/equivalent neutron flux at a $1 \mathrm{cps}$ alpha-plus-noise cutoff discriminator setting. The operating range capability of the LLFM was $\sim 1$ to $10^{6} \mathrm{cps}$. Above $10^{5} \mathrm{cps}$, dead-time corrections to the counting rates were required (Ref. 10, p. 19).

\section{Simulated Core Assembly (SCA)}

The main portion of the SCA pipe was schedule 40, stainless steel 304 (Ref. 3, p. 17). 
NEA/NSC/DOC(2006)1

Liquid Metal Fast Reactor - LMFR

FFTF-LMFR-RESR-001

CRIT-SPEC-REAC-COEF-MISC

\section{APPENDIX F: HEX-Z HOMOGENIZED REFERENCE MODEL}

\section{F.1 General HEX-Z Homogenized Model Provided for Isothermal Physics Calculations}

\section{F.1.1 Homogenized Model Dimensions for Nuclear Analysis Studies}

Various simplified models of the FFTF were developed to allow for computational modeling of the reactor experiments and further analyses of the results for development of the computer codes and database libraries. The choice of codes was dependent upon convenience and costs of performing the computations for a particular analysis. The calculated eigenvalue was found to be very sensitive to the modeling approach. Models using an R-Z representation or a 3-D model demonstrated the most accurate correlation between calculated and experimental data. Furthermore, the FFTF core was found to be very similar to a heterogeneous core, and special heterogeneity features should be properly accounted for in order to accurately predict core performance parameters (Ref. 4, pp. 8,21, and 22).

A heterogeneous configuration of the FFTF would involve modeling of all core components as accurately as possible with exact dimensions and compositions. Many of the drawings for components in the FFTF are not publicly available and a heterogeneous description of the core is not feasible.

Simplified core models had been developed for FFTF end-users. One dimensional axial and radial reactor models, an R,Z zone map, and a 3-D Hex model had been used in computational analyses of the initial isothermal physics experiments. The axial regions employed for nuclear analysis in the 3-D model are shown in Figure F.1.1. All components contained additional hardware above and below the active core region, often with structural detail. These regions had been coalesced into a minimum number needed for 3-D and R-Z nuclear analyses (Ref. 3, pp. 11, 15-16, and 18). 
NEA/NSC/DOC(2006)1

Liquid Metal Fast Reactor - LMFR

FFTF-LMFR-RESR-001

CRIT-SPEC-REAC-COEF-MISC
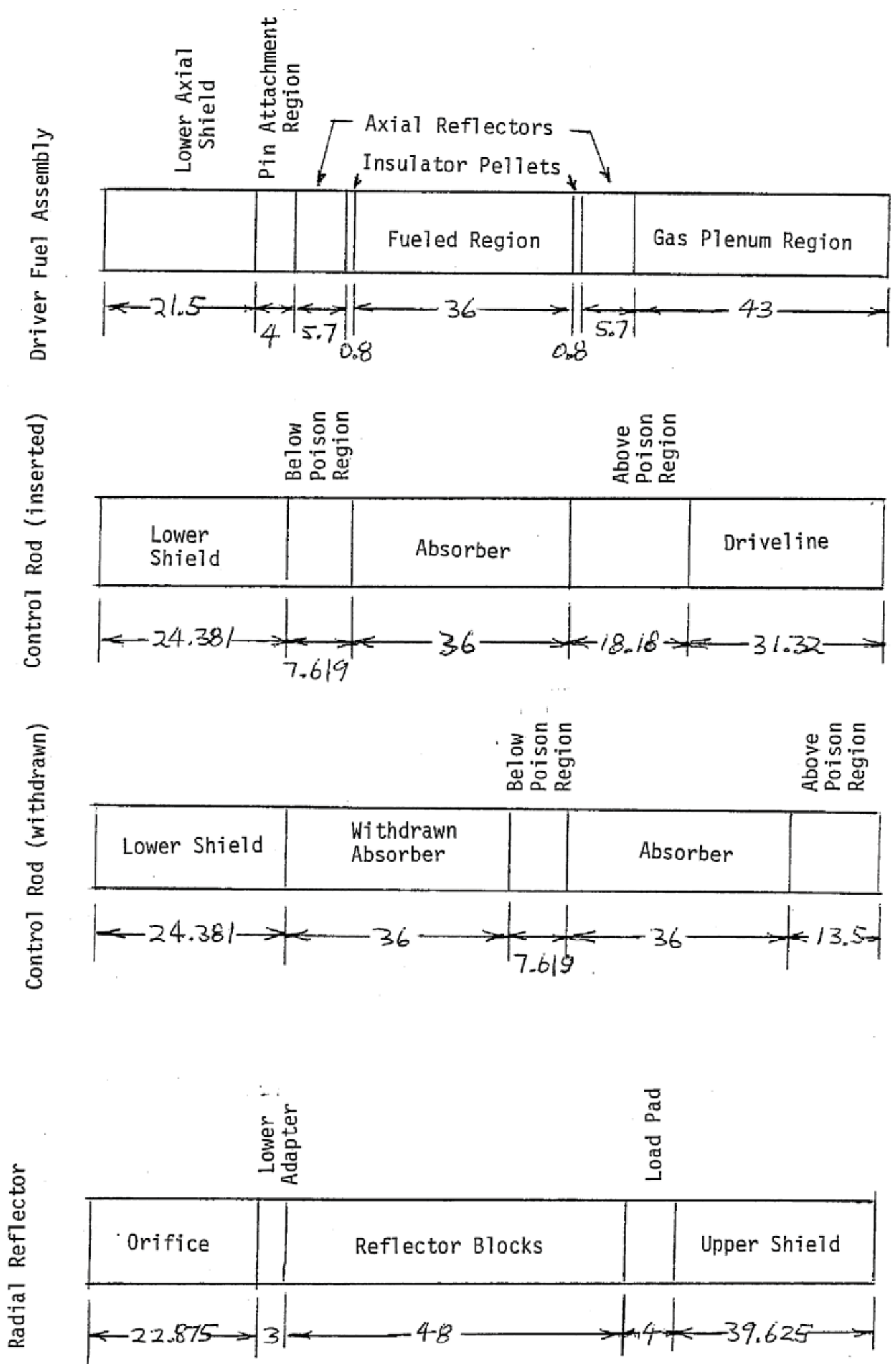

Figure F.1.1. Axial Zones of Core Components, (dimensions are in inches)

(Ref. 3, Figs. 6A and B). 
FFTF-LMFR-RESR-001

CRIT-SPEC-REAC-COEF-MISC
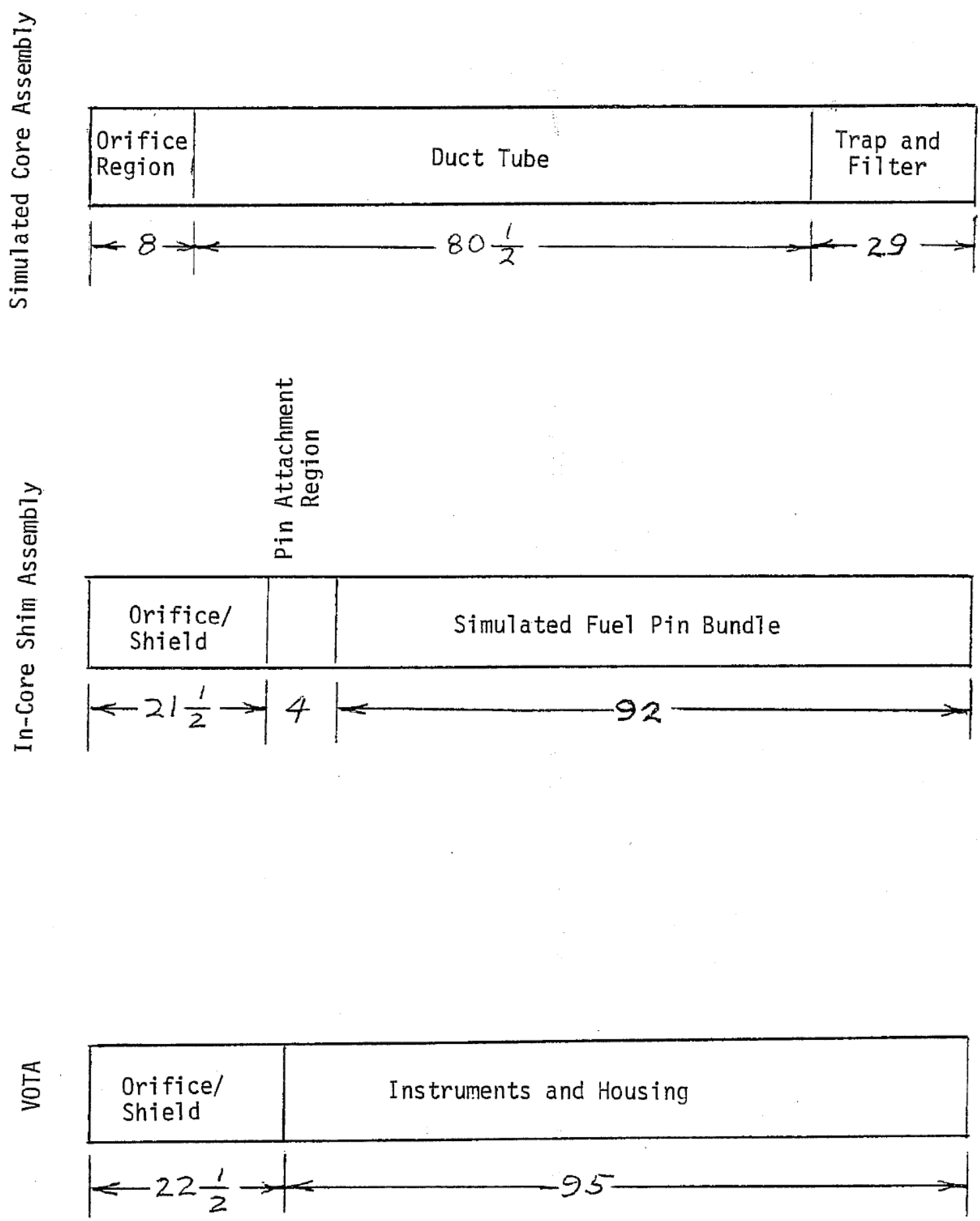

Figure F.1.1 (cont'd.). Axial Zones of Core Components, (dimensions are in inches) (Ref. 3, Figs. 6A and B). 
NEA/NSC/DOC(2006)1

Liquid Metal Fast Reactor - LMFR

FFTF-LMFR-RESR-001

CRIT-SPEC-REAC-COEF-MISC

\section{F.1.2 Homogenized Model Materials for Nuclear Analysis Studies}

The homogenized atom densities are given in Table F.1.1. The coolant and structure volume fractions for core components were obtained from FFTF design documentation, relying largely on the nuclear analyses models used in the Core 1 fuel enrichment calculations. These volume fractions were based on a room temperature cell dimension of 4.715 inches $(11.9761 \mathrm{~cm})$, flat-to-flat (the load pad dimension). However, for isothermal conditions, the core component spacing was assumed to be controlled by the core support plate hole pitch of 4.730 inches $(12.0142 \mathrm{~cm})$ at room temperature. Consequently, the original volume fractions were modified to conform to a room temperature cell dimension of 4.73 inches $(12.0142 \mathrm{~cm})$ across flats. Atom densities for structure and coolant constituents were then calculated using $400^{\circ} \mathrm{F}\left(\sim 204^{\circ} \mathrm{C}\right)$ density values from the Nuclear Systems Materials Handbook. Pseudo-isotopic cross sections are used at the HEDL to represent common alloys, and the atom densities shown in Table 2.1.2 for types 304 and 316 stainless steel and for Inconel 600 represent such pseudo-isotopes. The atom fractions of each of the major constituents of these alloys are given in Table F.1.2, along with its equivalent atomic weight and density. These alloy compositions were obtained from material certification and chemical analysis data for FFTF 'first core steel' (type 316); the In-Reactor Thimble (type 304); and the radial reflectors (Inconel 600), (Ref. 3, pp. 18-23).

In the case of fuel and absorber assemblies, the coolant and structural atom densities were obtained as described above, based on nominal design dimensions; and the manufacturing records for individual assemblies were consulted to determine the as-built inventories of uranium and plutonium isotopes, ${ }^{10} \mathrm{~B}$, and $\mathrm{B}_{4} \mathrm{C}$. Because the assembly-to-assembly variation in most isotopes was small, averaged isotopic compositions were used in most HEDL analyses to represent all unirradiated fuel or control assemblies of a specific type, so as to reduce the effort required in setting up and checking computer input data. The homogenized atom densities given in Table F.1.1 for fuel and absorber materials are based on these averaged assembly inventories, with a total cell volume determined by the $12.051 \mathrm{~cm}$ hexagonal cell dimension at $400^{\circ} \mathrm{F}\left(\sim 204^{\circ} \mathrm{C}\right)$ and a fuel column active length of $91.56 \mathrm{~cm}$.

Table F.1.1. Homogenized Atom Densities (a/b-cm) of FFTF Core Components (Ref. 3, p. 19 and Ref. 4, p. 25).

\begin{tabular}{||cccc||}
\hline \hline Fuel Assemblies Material & Insulator Pellets & Inner Fuel Zone & Outer Fuel Zone \\
\hline \hline Pu-239 & -- & 0.001402 & 0.001714 \\
$\mathrm{Pu}-240$ & -- & 0.000190 & 0.000231 \\
$\mathrm{Pu}-241$ & -- & 0.000020 & 0.000025 \\
$\mathrm{Pu}-242$ & -- & 0.000003 & 0.000004 \\
$\mathrm{Am}-241$ & -- & 0.000006 & 0.000007 \\
$\mathrm{U}-235$ & 0.000051 & 0.000040 & 0.000038 \\
$\mathrm{U}-238$ & 0.007285 & 0.005674 & 0.005341 \\
Oxygen & 0.014672 & 0.014377 & 0.014426 \\
SS 316 & 0.020221 & 0.020221 & 0.020221 \\
Sodium & 0.009723 & 0.009723 & 0.009723 \\
\hline
\end{tabular}


NEA/NSC/DOC(2006)1

Liquid Metal Fast Reactor - LMFR

FFTF-LMFR-RESR-001

CRIT-SPEC-REAC-COEF-MISC

Table F.1.1 (cont'd.). Homogenized Atom Densities (a/b-cm) of FFTF Core Components (Ref. 3, p. 19 and Ref. 4, p. 25).

\begin{tabular}{||ccc||}
\hline Absorber Assemblies Material & Inserted Absorber & Withdrawn Absorber \\
\hline \hline B-10 & 0.006473 & -- \\
B-11 & 0.026219 & -- \\
Carbon & 0.007393 & -- \\
SS 316 & 0.028596 & 0.008170 \\
Sodium & 0.007637 & 0.021370 \\
\hline
\end{tabular}

Table F.1.1 (cont'd.). Homogenized Atom Densities (a/b-cm) of FFTF Core Components (Ref. 3, p. 19 and Ref. 4, p. 25).

\begin{tabular}{|c|c|c|c|}
\hline Structural, Shielding, and Special Components & Stainless Steel & Inconel & Sodium \\
\hline \multicolumn{4}{|l|}{ Driver Fuel Assembly - Nonfueled Regions } \\
\hline Lower Axial Shield & 0.063353 & & 0.006116 \\
\hline Pin Attachment Region (Inner Fuel Zone) & 0.035407 & & 0.013847 \\
\hline Pin Attachment Region (Outer Fuel Zone) & 0.034638 & & 0.014060 \\
\hline Axial Reflectors & 0.020221 & 0.027739 & 0.009723 \\
\hline Gas Plenum & 0.024989 & & 0.009639 \\
\hline \multicolumn{4}{|l|}{$\underline{\text { Control Rod Assembly }}$} \\
\hline Above-Poison Region & 0.032399 & & 0.007644 \\
\hline Below-Poison Region & 0.033937 & & 0.014254 \\
\hline Driveline & 0.020827 & & 0.017881 \\
\hline Lower Shield & 0.059867 & & 0.007081 \\
\hline \multicolumn{4}{|l|}{ Radial Reflectors, Row 7} \\
\hline Orifice Region & 0.066952 & & 0.005121 \\
\hline Lower Adapter & 0.061807 & & 0.006544 \\
\hline Reflector Blocks & 0.018819 & 0.055699 & 0.003440 \\
\hline Load Pad Region & 0.074814 & & 0.002946 \\
\hline Upper Shield & 0.073131 & & 0.003412 \\
\hline \multicolumn{4}{|l|}{$\underline{\text { Radial Reflectors, Rows } 8 \text { and } 9}$} \\
\hline Orifice Region & 0.051244 & & 0.009466 \\
\hline Lower Adapter & 0.064499 & & 0.005799 \\
\hline Reflector Blocks & 0.027716 & 0.050702 & 0.002324 \\
\hline Load Pad Region & 0.078207 & & 0.002007 \\
\hline Upper Shield & 0.077182 & & 0.002291 \\
\hline Inner Radial Shield & 0.068533 & & 0.004683 \\
\hline Outer Radial Shield & 0.073233 & & 0.003383 \\
\hline
\end{tabular}

Revision: 0

Page 298 of 304

Date: March 31, 2010 
NEA/NSC/DOC(2006)1

Liquid Metal Fast Reactor - LMFR

FFTF-LMFR-RESR-001

CRIT-SPEC-REAC-COEF-MISC

Table F.1.1 (cont'd.). Homogenized Atom Densities (a/b-cm) of FFTF Core Components (Ref. 3, p. 19, and Ref. 4, p. 25).

\begin{tabular}{||ccc||}
\hline Structural, Shielding, and Special Components & Stainless Steel $^{(\mathrm{a})}$ & Sodium \\
\hline \hline$\underline{\text { In-Core Shim Assembly (ICSA) }}$ & & \\
Orifice/Shield Region & 0.082137 & 0.001416 \\
Pin Attachment Region & 0.035407 & 0.013847 \\
Simulated Fuel Pin Bundle & 0.050316 & 0.009723 \\
\hline \hline$\underline{\text { In-Reactor Thimble (IRT) }}$ & 0.015054 & 0.019568 \\
\hline \hline Simulated Core Assemblies (SCA) & & \\
Orifice Region & 0.043292 & 0.011927 \\
Duct Tube & 0.014311 & 0.019769 \\
Particle Trap and Filter Region & 0.022716 & 0.017495 \\
\hline \hline Vibration Open Test Assembly (VOTA) & & \\
Orifice/Shield Region & 0.082137 & 0.001416 \\
Instruments and Housing & 0.014999 & 0.019249 \\
\hline
\end{tabular}

(a) Type 304 for SCA and IRT: Type 316 for all other components.

Table F.1.2. Alloy Composition Data (Ref. 3, p. 23).

\begin{tabular}{|cccc|}
\hline $\begin{array}{c}\text { Element } \\
\text { (Atom Fractions) }\end{array}$ & $\begin{array}{c}\text { SS-316 } \\
\text { (clad \& ducts) }\end{array}$ & $\begin{array}{c}\text { SS-304 } \\
\text { (IRT) }\end{array}$ & $\begin{array}{c}\text { Inconel 600 } \\
\text { (Reflectors) }\end{array}$ \\
\hline $\mathrm{Fe}$ & Balance & Balance & 0.087 \\
$\mathrm{Cr}$ & 0.188 & 0.193 & 0.162 \\
$\mathrm{Ni}$ & 0.130 & 0.080 & Balance \\
$\mathrm{Mo}$ & 0.013 & -- & -- \\
$\mathrm{Mn}$ & 0.016 & 0.017 & 0.004 \\
$\mathrm{Si}$ & 0.011 & 0.014 & 0.002 \\
$\mathrm{C}$ & 0.002 & 0.003 & 0.002 \\
\hline \hline Equivalent Atomic Weight, amu & 55.6 & 54.8 & 57.2 \\
Density @ $400^{\circ} \mathrm{F}, \mathrm{g} / \mathrm{cm}^{3}$ & 7.89 & 7.95 & 8.34 \\
Mean thermal expansion coefficient, & $9.2 \times 10^{-6}$ & & \\
RT to 400 ${ }^{\circ} \mathrm{F},\left({ }^{\circ} \mathrm{F}\right)^{-1}$ & & & \\
\hline \hline
\end{tabular}


NEA/NSC/DOC(2006)1

Liquid Metal Fast Reactor - LMFR

FFTF-LMFR-RESR-001

CRIT-SPEC-REAC-COEF-MISC

\section{APPENDIX G: DISCUSSION OF MODEL HOMOGENIZATION}

\section{G.1 HOMOGENIZATION EFFECTS}

\section{G.1.1 General Homogenization Bias and Uncertainty}

The dominant constituents of the physical configuration of the reactor core are the fuel and absorber pin assemblies. These assemblies have been modeled as heterogeneous components in the benchmark model to reduce bias and uncertainty margins related to the effects of homogenizing these components. An assessment of the magnitude of the bias for homogenizing these core components is discussed in Section 3.1.1. Many of the non-fuel and non-absorber components, for which inadequate detail was provided in the available documentation, were represented as homogenous materials in the benchmark model. These components were judged to be of insignificant contribution to the total uncertainty of the benchmark model, as the uncertainties in other geometrical properties (Section 2.1.2) and density of the primary structural components (SS316, Inconel 600, and sodium in Sections 2.1.3.1 through 2.1.3.3) are relatively small.

\section{G.1.2 Homogenization of Fuel and Absorber Pins}

The inner and outer fuel pins were homogenized, as shown in Figure G.1.1, with the composition shown in Table G.1.1 (compare with Figures 3.1.1 through 3.1.3 and Tables 3.1.7, 3.1.8, and 3.1.19). The absorber pins were homogenized, as shown in Figure G.1.2, with the composition shown in Table G.1.2 (compare with Figures 3.1.4 through 3.1.6 and Tables 3.1.8, 3.1.9, and 3.1.19). The effective bias for homogenizing the fuel and absorber pin lattice structures was determined to be $-0.01096 \pm 0.00008$, or about a $-1.1 \%$ bias.

The homogenization of the fuel and absorber pellet lattices and their bias are not included in the benchmark model. They were provided here for evaluation demonstration only. 
NEA/NSC/DOC(2006)1

Liquid Metal Fast Reactor - LMFR

FFTF-LMFR-RESR-001

CRIT-SPEC-REAC-COEF-MISC

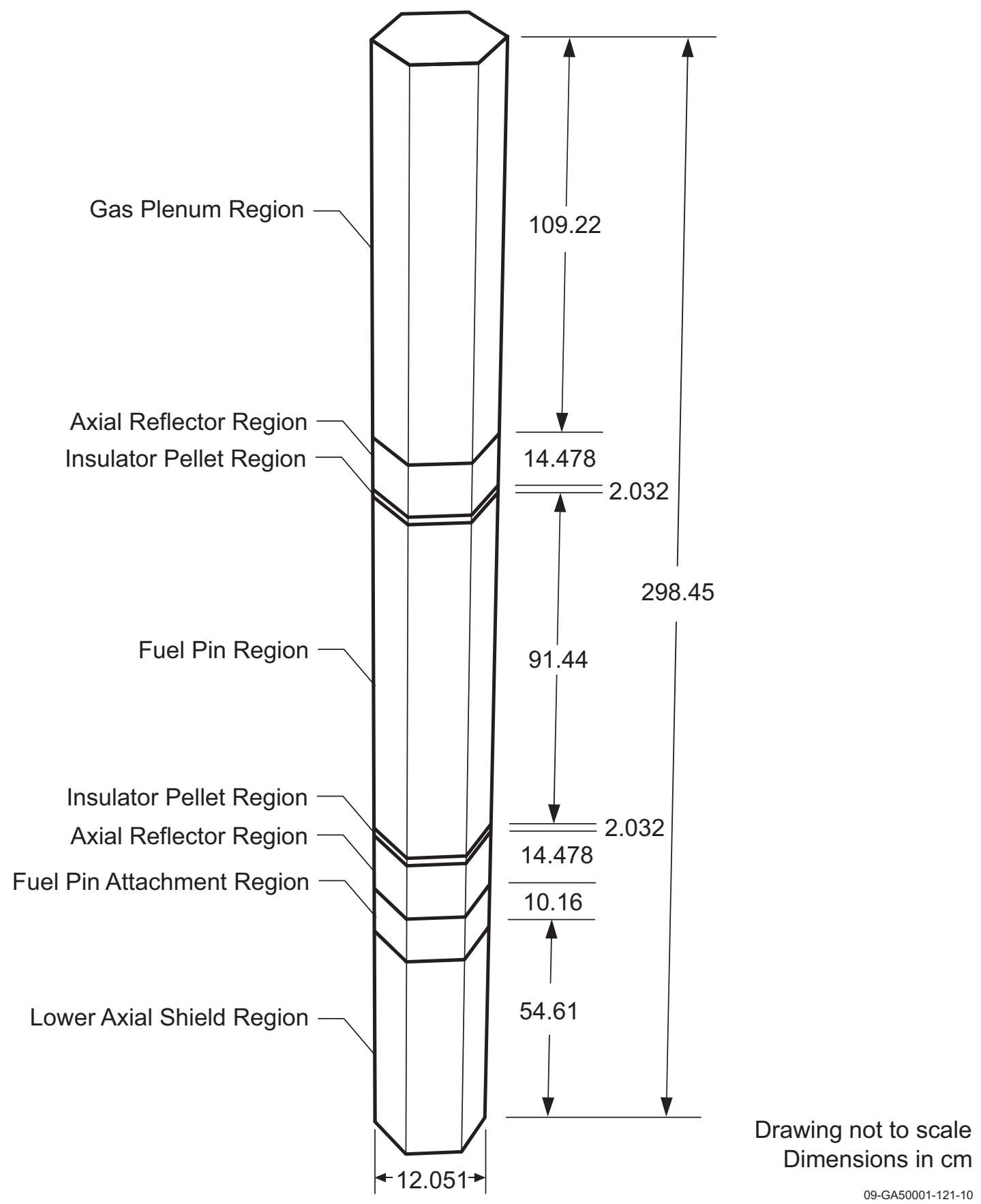

Figure G.1.1. Diagram of Homogenized Driver Fuel Assembly. 
NEA/NSC/DOC(2006)1

Liquid Metal Fast Reactor - LMFR

FFTF-LMFR-RESR-001

CRIT-SPEC-REAC-COEF-MISC

Table G.1.1. Homogenized Composition of the Fuel Pins.

\begin{tabular}{|c|c|c|c|c|}
\hline \multirow[b]{2}{*}{$\begin{array}{l}\text { Element } \\
\text { or Isotope }\end{array}$} & \multicolumn{4}{|c|}{ Atom Density (a/b-cm) } \\
\hline & $\begin{array}{l}\text { Inner Fuel } \\
\text { (Type 3.1) }\end{array}$ & $\begin{array}{l}\text { Outer Fuel } \\
\text { (Type 3.2) }\end{array}$ & $\begin{array}{c}\mathrm{UO}_{2} \\
\text { Insulator } \\
\text { Pellets } \\
\end{array}$ & $\begin{array}{c}\text { Axial } \\
\text { Reflectors }\end{array}$ \\
\hline $\mathrm{O}$ & $1.4345 \mathrm{E}-02$ & $1.4119 \mathrm{E}-02$ & $1.4629 \mathrm{E}-02$ & -- \\
\hline${ }^{234} U$ & 3.4154E-07 & $3.2848 \mathrm{E}-07$ & $4.0229 \mathrm{E}-07$ & -- \\
\hline${ }^{235} \mathrm{U}$ & 4.0109E-05 & 3.7761E-05 & $5.2663 \mathrm{E}-05$ & -- \\
\hline${ }^{238} \mathrm{U}$ & $5.5377 \mathrm{E}-03$ & $5.2136 \mathrm{E}-03$ & 7.2613E-03 & -- \\
\hline${ }^{237} \mathrm{~Np}$ & 4.4030E-06 & $5.3125 \mathrm{E}-06$ & -- & -- \\
\hline${ }^{238} \mathrm{Pu}$ & $8.7633 \mathrm{E}-07$ & $1.0313 \mathrm{E}-06$ & -- & -- \\
\hline${ }^{239} \mathrm{Pu}$ & $1.3915 \mathrm{E}-03$ & $1.7198 \mathrm{E}-03$ & -- & -- \\
\hline${ }^{240} \mathrm{Pu}$ & $1.8726 \mathrm{E}-04$ & $2.3250 \mathrm{E}-04$ & -- & -- \\
\hline${ }^{241} \mathrm{Pu}$ & $1.8950 \mathrm{E}-05$ & $2.2915 \mathrm{E}-05$ & -- & -- \\
\hline${ }^{242} \mathrm{Pu}$ & $3.0576 \mathrm{E}-06$ & 4.2416E-06 & -- & -- \\
\hline${ }^{241} \mathrm{Am}$ & 4.6386E-06 & $3.8842 \mathrm{E}-06$ & -- & -- \\
\hline${ }^{10} \mathrm{~B}$ & $1.0288 \mathrm{E}-07$ & $1.0288 \mathrm{E}-07$ & $1.0288 \mathrm{E}-07$ & $1.0288 \mathrm{E}-07$ \\
\hline${ }^{11} \mathrm{~B}$ & $4.1412 \mathrm{E}-07$ & 4.1412E-07 & 4.1412E-07 & $4.1412 \mathrm{E}-07$ \\
\hline $\mathrm{C}$ & 4.6536E-05 & 4.6536E-05 & 4.6536E-05 & $7.0295 \mathrm{E}-04$ \\
\hline $\mathrm{N}$ & 3.9905E-06 & 3.9905E-06 & $3.9905 \mathrm{E}-06$ & $3.9905 \mathrm{E}-06$ \\
\hline $\mathrm{Na}$ & $9.8029 \mathrm{E}-03$ & $9.8029 \mathrm{E}-03$ & $9.8029 \mathrm{E}-03$ & $9.8029 \mathrm{E}-03$ \\
\hline $\mathrm{Al}$ & $1.0358 \mathrm{E}-05$ & $1.0358 \mathrm{E}-05$ & $1.0358 \mathrm{E}-05$ & $1.0358 \mathrm{E}-05$ \\
\hline $\mathrm{Si}$ & $1.4926 \mathrm{E}-04$ & $1.4926 \mathrm{E}-04$ & $1.4926 \mathrm{E}-04$ & $2.8962 \mathrm{E}-04$ \\
\hline $\mathrm{P}$ & 3.6091E-06 & 3.6091E-06 & $3.6091 \mathrm{E}-06$ & $3.6091 \mathrm{E}-06$ \\
\hline S & $1.7431 \mathrm{E}-06$ & $1.7431 \mathrm{E}-06$ & $1.7431 \mathrm{E}-06$ & $5.4312 \mathrm{E}-06$ \\
\hline $\mathrm{V}$ & $2.1944 \mathrm{E}-05$ & $2.1944 \mathrm{E}-05$ & $2.1944 \mathrm{E}-05$ & $2.1944 \mathrm{E}-05$ \\
\hline $\mathrm{Cr}$ & $3.7624 \mathrm{E}-03$ & $3.7624 \mathrm{E}-03$ & $3.7624 \mathrm{E}-03$ & $8.4628 \mathrm{E}-03$ \\
\hline $\mathrm{Mn}$ & $3.5609 \mathrm{E}-04$ & $3.5609 \mathrm{E}-04$ & $3.5609 \mathrm{E}-04$ & $8.5836 \mathrm{E}-04$ \\
\hline $\mathrm{Fe}$ & $1.2823 \mathrm{E}-02$ & $1.2823 \mathrm{E}-02$ & $1.2823 \mathrm{E}-02$ & $1.5082 \mathrm{E}-02$ \\
\hline Co & 4.7421E-06 & 4.7421E-06 & $4.7421 \mathrm{E}-06$ & $3.1498 \mathrm{E}-05$ \\
\hline $\mathrm{Ni}$ & $2.5712 \mathrm{E}-03$ & $2.5712 \mathrm{E}-03$ & $2.5712 \mathrm{E}-03$ & $2.2355 \mathrm{E}-02$ \\
\hline $\mathrm{Cu}$ & $8.7958 \mathrm{E}-06$ & $8.7958 \mathrm{E}-06$ & $8.7958 \mathrm{E}-06$ & $7.0829 \mathrm{E}-05$ \\
\hline As & $2.2381 \mathrm{E}-06$ & $2.2381 \mathrm{E}-06$ & $2.2381 \mathrm{E}-06$ & $2.2381 \mathrm{E}-06$ \\
\hline $\mathrm{Nb}$ & $3.0081 \mathrm{E}-06$ & $3.0081 \mathrm{E}-06$ & $3.0081 \mathrm{E}-06$ & $3.0081 \mathrm{E}-06$ \\
\hline Mo & $2.9129 \mathrm{E}-04$ & 2.9129E-04 & $2.9129 \mathrm{E}-04$ & $2.9129 \mathrm{E}-04$ \\
\hline $\mathrm{Ta}$ & 3.0889E-07 & $3.0889 \mathrm{E}-07$ & $3.0889 \mathrm{E}-07$ & $3.0889 \mathrm{E}-07$ \\
\hline Total & $5.1397 \mathrm{E}-02$ & $5.1224 \mathrm{E}-02$ & $5.1807 \mathrm{E}-02$ & $5.7999 \mathrm{E}-02$ \\
\hline
\end{tabular}

Revision: 0 
NEA/NSC/DOC(2006)1

Liquid Metal Fast Reactor - LMFR

FFTF-LMFR-RESR-001

CRIT-SPEC-REAC-COEF-MISC

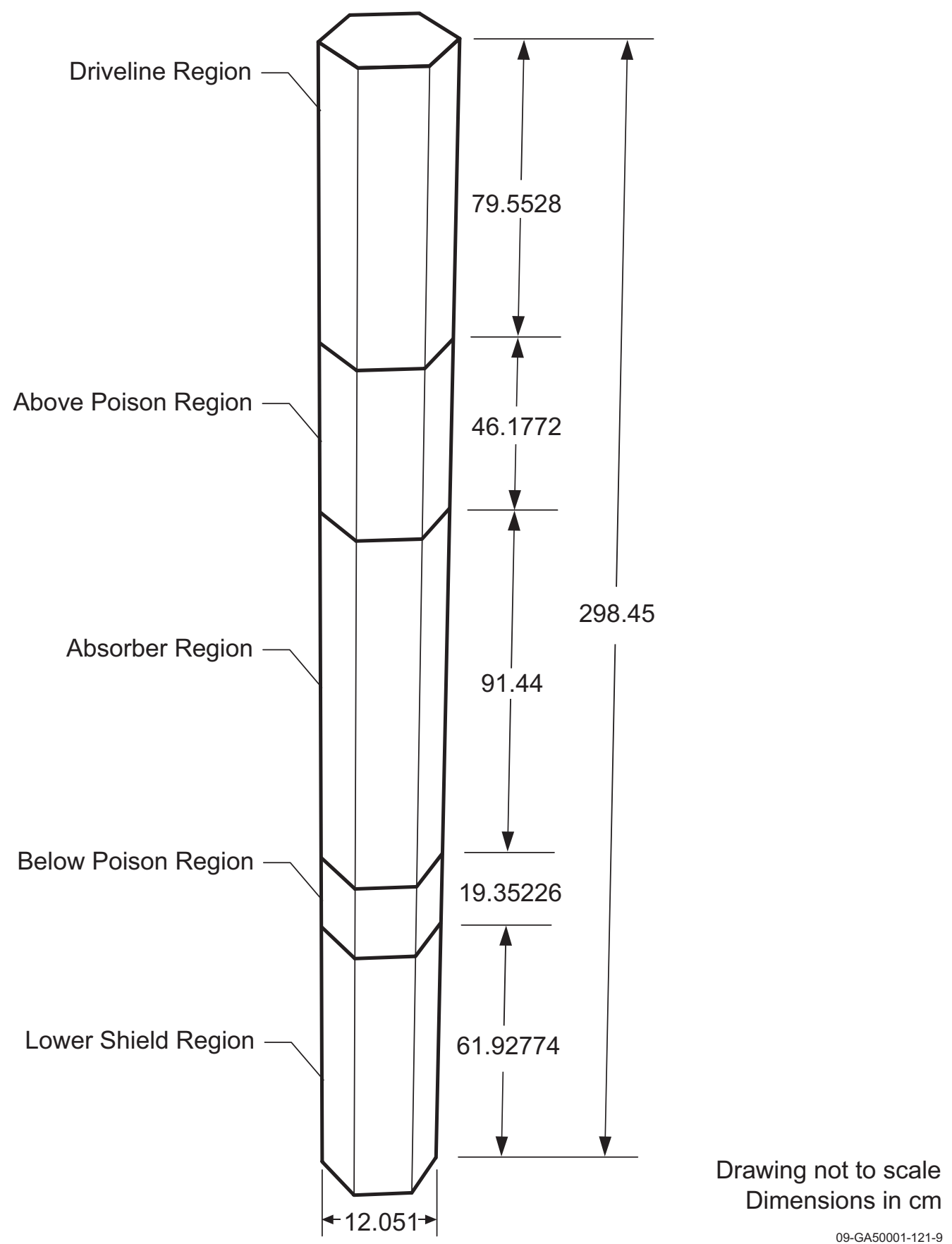

Figure G.1.2. Diagram of Homogenized, Fully-Inserted Control Rod. 


\section{NEA/NSC/DOC(2006)1}

Liquid Metal Fast Reactor - LMFR

FFTF-LMFR-RESR-001

CRIT-SPEC-REAC-COEF-MISC

Table G.1.2. Homogenized Composition of the Absorber Pin Region.

\begin{tabular}{|c|c|}
\hline $\begin{array}{c}\text { Element } \\
\text { or Isotope }\end{array}$ & $\begin{array}{c}\text { Atom Density } \\
(\mathrm{a} / \mathrm{b}-\mathrm{cm})\end{array}$ \\
\hline \hline${ }^{10} \mathrm{~B}$ & $6.5370 \mathrm{E}-03$ \\
${ }^{11} \mathrm{~B}$ & $2.6312 \mathrm{E}-02$ \\
$\mathrm{C}$ & $7.6994 \mathrm{E}-03$ \\
$\mathrm{~N}$ & $5.7471 \mathrm{E}-06$ \\
$\mathrm{Na}$ & $7.5837 \mathrm{E}-03$ \\
$\mathrm{Al}$ & $1.4917 \mathrm{E}-05$ \\
$\mathrm{Si}$ & $2.1496 \mathrm{E}-04$ \\
$\mathrm{P}$ & $5.1978 \mathrm{E}-06$ \\
$\mathrm{~S}$ & $2.5105 \mathrm{E}-06$ \\
$\mathrm{~V}$ & $3.1604 \mathrm{E}-05$ \\
$\mathrm{Cr}$ & $5.4185 \mathrm{E}-03$ \\
$\mathrm{Mn}$ & $5.1284 \mathrm{E}-04$ \\
$\mathrm{Fe}$ & $1.8468 \mathrm{E}-02$ \\
$\mathrm{Co}$ & $6.8296 \mathrm{E}-06$ \\
$\mathrm{Ni}$ & $3.7030 \mathrm{E}-03$ \\
$\mathrm{Cu}$ & $1.2668 \mathrm{E}-05$ \\
$\mathrm{As}$ & $3.2233 \mathrm{E}-06$ \\
$\mathrm{Nb}$ & $4.3322 \mathrm{E}-06$ \\
$\mathrm{Mo}$ & $4.1952 \mathrm{E}-04$ \\
$\mathrm{Ta}$ & $4.4487 \mathrm{E}-07$ \\
\hline $\mathrm{Total}$ & $7.6956 \mathrm{E}-02$ \\
\hline
\end{tabular}

